\title{
Use of wavelet-packet transforms to develop an engineering model for multifractal characterization of mutation dynamics in pathological and nonpathological gene sequences
}

\author{
David Lee Walker \\ West Virginia University
}

Follow this and additional works at: https://researchrepository.wvu.edu/etd

\author{
Recommended Citation \\ Walker, David Lee, "Use of wavelet-packet transforms to develop an engineering model for multifractal \\ characterization of mutation dynamics in pathological and nonpathological gene sequences" (1999). \\ Graduate Theses, Dissertations, and Problem Reports. 3139. \\ https://researchrepository.wvu.edu/etd/3139
}

This Dissertation is protected by copyright and/or related rights. It has been brought to you by the The Research Repository @ WVU with permission from the rights-holder(s). You are free to use this Dissertation in any way that is permitted by the copyright and related rights legislation that applies to your use. For other uses you must obtain permission from the rights-holder(s) directly, unless additional rights are indicated by a Creative Commons license in the record and/ or on the work itself. This Dissertation has been accepted for inclusion in WVU Graduate Theses, Dissertations, and Problem Reports collection by an authorized administrator of The Research Repository @ WVU. For more information, please contact researchrepository@mail.wvu.edu. 
Use Of Wavelet Packet Transforms to Develop an Engineering Model for Multi-Fractal Characterization of Mutation Dynamics in Pathological and Non-pathological Gene Sequences

\author{
David L. Walker
}

Dissertation Submitted to the College of Engineering and Mineral Resources at West Virginia University in partial fulfillment of the requirements for the degree of

Doctor of Philosophy in Engineering Science

\author{
Alfred H. Stiller, Ph.D., Chair \\ Charles M. Jaffe, Ph.D. \\ Charter Stinespring, Ph.D. \\ Caulton L. Irwin, Ph.D. \\ Thomas R. Long, Ph.D. \\ Michael R. Miller, Ph.D.
}

Morgantown, West Virginia 1999

Keywords: Chaos, DNA, wavelets, fractals, Hurst Copyright 1999 David L.Walker 


\title{
ABSTRACT
}

\author{
Use Of Wavelet Packet Transforms to Develop an \\ Engineering Model for Multi-Fractal Characterization of \\ Mutation Dynamics in Pathological and \\ Non-pathological Gene Sequences
}

\section{David L. Walker}

This study uses dynamical analysis to examine in a quantitative fashion the information coding mechanism in DNA sequences. This exceeds the simple dichotomy of either modeling the mechanism by comparing DNA sequence walks as Fractal Brownian Motion (fbm) processes. The 2-D mappings of the DNA sequences for this research are from Iterated Function System (IFS) (Also known as the "Chaos Game Representation" (CGR)) mappings of the DNA sequences. This technique converts a 1-D sequence into a 2-D representation that preserves subsequence structure and provides a visual representation. The second step of this analysis involves the application of Wavelet Packet Transforms, a recently developed technique from the field of signal processing. A multi-fractal model is built by using wavelet transforms to estimate the Hurst exponent, $\mathrm{H}$. The Hurst exponent is a non-parametric measurement of the dynamism of a system. This procedure is used to evaluate gene-coding events in the DNA sequence of cystic fibrosis mutations. The $\mathrm{H}$ exponent is calculated for various mutation sites in this gene. The results of this study indicate the presence of anti-persistent, random walks and persistent "sub-periods" in the sequence. This indicates the hypothesis of a multi-fractal model of DNA information encoding warrants further consideration.

This work examines the model's behavior in both pathological (mutations) and non-pathological (healthy) base pair sequences of the cystic fibrosis gene. These mutations both natural and synthetic were introduced by computer manipulation of the original base pair text files. The results show that disease severity and system "information dynamics" correlate. These results have implications for genetic engineering as well as in mathematical biology. They suggest that there is scope for more multi-fractal models to be developed. 


\section{Curriculum Vitae}

DAVID L. WALKER

\section{EMPLOYMENT}

\begin{tabular}{|c|c|c|}
\hline ANSER & Senior Research Scientist & 1998-present \\
\hline GTE & $\begin{array}{l}\text { Senior Member Technical } \\
\text { Staff III }\end{array}$ & 1996-1998 \\
\hline Geo-Com, Inc. & $\begin{array}{l}\text { Chief Engineer for } \\
\text { Signal Processing }\end{array}$ & 1995-1996 \\
\hline CIA/OTC/SDG & $\begin{array}{l}\text { Senior Software System } \\
\text { Project Manager }\end{array}$ & 1995 \\
\hline CIA/OTC/COSC & $\begin{array}{l}\text { Software Engineer and } \\
\text { Intelligence Analyst }\end{array}$ & 1993-1995 \\
\hline $\begin{array}{l}\text { CIA/ORD/Counter-Intelligence } \\
\text { \& Security }\end{array}$ & R/D Project Manager & 1992-1993 \\
\hline CIA/ORD/SIGINT (COMINT) & R/D Project Manager & 1983-1992 \\
\hline National Security Agency & Electrical Engineer & $1979-1983$ \\
\hline
\end{tabular}

\section{EDUCATION}

M.S., Applied Physics, George Mason University, 1995

M.S., Computer Science, Johns Hopkins University, 1982

M.S., Electrical Engineering, West Virginia University, 1979

B.S., Geophysics, Virginia Polytechnic Institute, 1977

\section{PROFESSIONAL AFFILIATIONS}

Society of Industrial and Applied Mathematicians, Society of Exploration Geophysicists, Institute of Electronic and Electrical Engineers. 


\section{Dedication}

There are many that made this work possible. This feeble attempt at a dedication in no ways pays my debt to them. My first dedication is to my daughter, Rachel Lee Tani Walker. Who every day is the twinkle in my eye, the reason to smile and who shows me all things are possible. My second dedication is to my wife who I love and who has supported me in this effort even though this work and I have tried her patience. My third dedication is to my four "horsemen" of the academy. The first and leader of the quartet is Dr. Alfred H. Stiller. Dr. Stiller is a brilliant researcher, instructor and the consummate academician. He is also a man of vision. The second of the four is Dr. Thomas R. Long whom faith in me and whose vision kept this project alive. The third is Dr. Charter D. Stinespring whose vision and courage all college professors should emulate. The fourth member of the advisory team is Dr. Mike R. Miller another man of vision and also one who was patient with my initial ignorance of biology. The fifth dedication is one that is especially meaningful. Being number five in order in no way diminishes their importance in my life and how it is lived. I dedicate this work to my parents who gave me every tool that I needed to see the job through. These are not material tools but the strength of character and dogged determination to finish no matter what the odds. The sixth dedication is to Dr. H. Price Kagey whose faith in me and my talents always kept my spirits up when they might flag. The final dedication is to my uncle, Judge Dennis R. Knapp who believed in me and this effort who is unfortunately no longer here to see the end result. 
The Author Wishes To Acknowledge and Extend Hearfelt Thanks To Dr. Mike R. Miller West Virginia University Department of Biochemistry for the Cystic Fibrosis Data. Also the Author Wishes To Thank ANSER, Inc. And Particularly Dr. Helena S. Wisniewski for the Interest and Support Shown By Her and ANSER in This Endeavor. 


\section{Table of Contents}

Page Number

I Introduction 1

(A) Scope of the Problem 2

(B) Thesis Statement 4

II. Literature Review

$\begin{array}{lr}\text { (A) Introduction } & 6\end{array}$

$\begin{array}{lr}\text { (B) Non-linear or Chaotic Dynamics } & 10\end{array}$

(C) Appropriate Literature Review on Transform Based Pattern Analysis 54

(D) Analysis Based on Non-Linear Dynamics 60

(E) Chaotic Modeling of Data Sequences 61

(F) DNA Data Sequence Analysis Based on Visualization 74

$\begin{array}{ll}\text { III. } & \text { Background }\end{array}$

$\begin{array}{ll}\text { (A) DNA Review } & 90\end{array}$

(B) L-Systems (IFS mapping is an example of an L-System) 93

$\begin{array}{ll}\text { (C) Elements of Fractals } & 102\end{array}$

(D) Fractal Geometry 103

$\begin{array}{lr}\text { (E) Fractal Sequences } & 106\end{array}$

(F) Recent Fractal Analysis Applied to DNA 111

(G) Elements of DNA Sequences and the Concept of Dynamic Disease 114

(H) A New Non-parametric Statistic: The Hurst Exponent 119 
$\begin{array}{ll}\text { (I) Multi-Fractal Approach to DNA } & 127\end{array}$

$\begin{array}{ll}\text { (J) Wavelet Based Analysis } & 131\end{array}$

IV Methodology
(A) Introduction

(B) Theoretical Background: Methodology for Fractal-based Analysis

of DNA Sequences

(C) Theoretical Background: Wavelet Transforms 142

(D) Wavelet Packet Transforms $\quad 154$

(E) Minimum Entropy Method of Estimating the Global and Local 156

Hurst Exponent Using Best Basis Wavelet packet Transforms

(F) Biological Data Set: Cystic Fibrosis DNA Sequence 164

$\begin{array}{lll}\text { V. Discussion and Results } & 175\end{array}$

$\begin{array}{ll}\text { VI. Conclusions } & 250\end{array}$

VII Recommendations

$\begin{array}{ll}\text { (A) Introduction } & 259\end{array}$

(B) A Hypothetical Genetic Engineering Example of How This 260

Research Might be Applied

(C) Further Non-linear Time-frequency Analysis 
(D) Sequence Complexity 273

(E) Sequence Kolmogorov Entropy 275

(F) L-Systems and DNA Grammars 276

(G) The Dynamical System Basis of Information Transference in the 277

Action of Antibiotics and Anti-cancer drugs

$\begin{array}{ll}\text { (H) DNA Computing } & 279\end{array}$

(I) Protein Engineering 282

(J) Forensic DNA 284

(K) Evolution Studies 285

$\begin{array}{ll}\text { VIII. Bibliography } & 287\end{array}$

IX. Appendix
A. Gene Data Set
296
B. Mutation Data Set 300
$\begin{array}{ll}\text { C. Matlab Script Files } & 304\end{array}$ 


\section{List of Tables}

Page

Number

I Introduction

II Literature Review
(A) Table II-1
Mapping Information for the Sierpinski
83

Triangle

III Background
(A) Table III-1
Example Production Rule Application
95
(B) Table III-2
Model of a Biological Formal
101
(L-System) System ${ }^{49}$

IV Methodology
(A) Table IV-1
Mapping Information for the Sierpinski
139
Carpet
(B) Table IV.2
DNA Sequence Mutations In The CFTR
168
Gene Coding Region

V. Discussion and Results

VI. Conclusions
(A) Table VI-1 Legend for Figure VI-1
258
(B) Table VI-2 Legend for Figure VI-2
259
(C) Table VI-3 Legend for Figure VI-3 


\section{Page Number}

VII Recommendations

(A) Table VII.1 Genetic Code as a Tabular 269

Algorithm to Specify Amino Acid Residues as

Triplet Codons

(B) Table VII.2 64 "Trial" Amino Acids 271

(C) Table VII-3 Plot Legend For Figure VII-1 And 276

All Subsequent Figures 


\section{List Of Figures}

Page Number

I Introduction

III Literature Review

III Background

(1) Figure III-1 Classical Spiral Helix Of The DNA Sequence 92

(2) Figure III-2 A Pictorial Representation Of The Production 100 Rules For AN RNA Sequence.

(3) Figure III-3 Depicts A Fractal Representation Of A 104 Mountain Range Generated By A Computer Algorithm.

(4) Figure III-4 The Classic Fractal Fern Showing Self-Similarity 105

(5) Figure III-5a Daily GTE Stock Prices For One Month 107

(6) Figure III-5b Weekly GTE Stock Prices For One Year 108

(7) Figure III-5c Monthly GTE Stock Prices For Three Years 108

(8) Figure III-6 Example Of A Stable (Gaussian) Distribution 110

(9) Figure III-7 A General Brownian Sequence. 113

$\begin{array}{lll}\text { (10) Figure III-8 DNA Spiral Sequence } & 115\end{array}$

IV Methodology

(1) Figure IV-1 IFS Representation Of A Sickle Cell Anemia Gene 142

(2) Figure IV-2a Basic Wavelet Function 147 
(3) Figure IV-2b Basic Wavelet Function W(X,1) 147

(4) Figure IV-2c Basic Wavelet Function W(X,-1) 147

(5) Figure IV-2d ${ }^{69}-152$

(6) Figure IV.3a Tree Representation Of A Wavelet Transform ${ }^{69} 157$

(7) Figure IV.3a Tree Representation Of A Wavelet Packet 157 Transform $^{69}$

(8) Figure IV.4 Wavelet Packet Transform To Level $10 \quad 163$

(9) Figure IV.5a Wavelet Tree Representation Of The Original 165

(10) Cystic Fibrosis Sequence. This Figure Is The Full Wavelet Decomposition

(11) Figure IV.5b This Figure Is The "Best-Basis" Representation 166 Of The Original Cystic Fibrosis Sequence.

V. Discussion And Results

(1) Figure V-1a IFS Mapping Of The Original Sequence 178

(2) Figure V-1b Histogram Of The Of Base Pairs 179

(3) Figure V-2a Wavelet Packet Decomposition 180

(4) Figure V-2b Best Basis Representation 180

(5) Figure V-3 Plot Coefficients Of The Continuous Wavelet Transform 181

(6) Figure V-4 Log-Log Plot Of The Best Basis Wavelet Packet Energy Vs. 182 The Coefficients Index A Least Square Approximation Of The Slope Is 
Calculated. $|\delta+1|$ Is The Global Hurst Exponent

(7) Figure V-5 Log-Log Plot Of The Local Slopes Best Basis Wavelet 183 Packet Energy Vs. The Coefficients

(8) Figure V-6a IFS Mapping Of The Cftr1.Txt Sequence 184

(9) Figure V-6b Histogram Of The Base Pairs 184

(10) Figure V-7a Difference In The Two IFS Mappings "Original" - "Mutation" 185

(11) Figure V-7b Difference In The Wavelet Coefficients 185

(12) Figure V-8 Best Basis Representation 186

(13) Figure V-9 Loglog Plot For Global Hurst Exponent Cftr1 187

(14) Figure V-10 Loglog Plot For Local Hurst Exponent 187

(15) Figure V-11a IFS Mapping Of The Cftr2.Txt Sequence 188

(16) Figure V-11b Histogram Of The Base 188

(17) Figure V-12a Difference In The Two IFS Mappings "Original" - "Mutation" 189

(18) Figure V-12b Difference In The Wavelet Coefficients 189

(19) Figure V-13 Best Basis Representation 190

(20) Figure V-14 Log Log Plot For The Global Hurst Exponent 191

(21) Figure V-15 Log Log Plot For The Local Hurst Exponent 191

(22) Figure V-16a IFS Mapping Of The Cftr3.Txt Sequence 193

(23) Figure V-16b Histogram Of The Base-Pairs 193

(24) Figure V-17a Difference In The Two IFS Mappings "Original" - "Mutation" 194

(25) Figure V-17b Difference In The Wavelet Coefficients 194 
(26) Figure V-18 Best Basis Representation 195

(27) Figure V-19 Loglog Plot For The Global Hurst Exponent 195

(28) Figure V-20 Loglog Plot For The Local Hurst Exponent 196

(29) Figure V-21 IFS Mapping Of The Cftr4.Txt Sequence 197

(30) Figure V-22a Histogram Of The Base-Pairs 197

(31) Figure V-22b Difference In The Two IFS Mappings "Original" - "Mutation" 198

(32) Figure V-23a Difference In The Wavelet Coefficients 198

(33) Figure V-23b Best Basis Representation 199

(34) Figure V-24 Loglog Plot For The Global Hurst Exponent 199

(35) Figure V-25 Loglog Plot For The Local Hurst Exponent 200

(36) Figure V-26a IFS Mapping Of The Cftr5.Txt Sequence 201

(37) Figure V-26b Histogram Of The Base Pairs 201

(38) Figure V-27a Difference In The Two IFS Mappings "Original" - Mutation" 202

(39) Figure V-27b Difference In The Wavelet Coefficients 202

(40) Figure V-28 Best Basis Representation 203

(41) Figure V-29 Loglog Plot For The Global Hurst Exponent 203

(42) Figure 5-30 Loglog Plot For The Local Hurst Exponent 204

(43) Figure V-31 IFS Mapping Of The Cftr5.Txt Sequence 205

(44) Figure V-32 Difference In IFS Mappings "Original" - "Mutation" 206

(45) Figure V-33 Difference In The Wavelet Coefficients 206 
(46) Figure V-34 Best Basis Representation

(47) Figure V-35 Loglog Plot For The Global Hurst Exponent 207

(48) Figure V-36 Loglog Plot For The Local Hurst Exponent 208

(49) Figure V-37 IFS Mapping Of The Cftr7.Txt Sequence 209

(50) Figure V-38 Difference In IFS Mappings "Original" - "Mutation" 209

(51) Figure V-39 Difference In The Wavelet Coefficients 209

(52) Figure V-40 Best Basis Representation 210

(53) Figure V-41 Loglog Plot For The Global Hurst Exponent 210

(54) Figure V-42 Loglog Plot For The Local Hurst Exponent 211

(55) Figure V-43 IFS Mapping Of The Cftr8.Txt Sequence 211

(56) Figure V-44 Difference In IFS Mappings "Original” - "Mutation" 212

(57) Figure V-45 Difference In The Wavelet Coefficients 212

(58) Figure V-46 Best Basis Representation 213

(59) Figure V-47 Loglog Plot For The Global Hurst Exponent 213

(60) Figure V-48 Loglog Plot For The Local Hurst Exponent 214

(61) Figure V-49 IFS Mapping Of The Cftr9.Txt Sequence 214

(62) Figure V-50 Difference In IFS Mapings "Original" - "Mutation" 215

(63) Figure V-51 Difference In The Wavelet Coefficients 215

(64) Figure V-52 Best Basis Representation 216

(65) Figure V-53 Loglog Plot For The Global Hurst Exponent 216

(66) Figure V-54 Loglog Plot For The Local Hurst Exponent 217 
Page Number

(67) Figure V-55 IFS Mapping Of The Cftr10.Txt Sequence 218

(68) Figure V-56 Difference In IFS Mappings "Original" - "Mutation" 219

(69) Figure V-57 Difference In The Wavelet Coefficients 219

(70) Figure V-58 Best Basis Representation 220

(71) Figure V-59 Loglog Plot For The Global Hurst Exponent 220

(72) Figure V-60 Loglog Plot For The Local Hurst Exponent 221

(73) Figure V-61 IFS Maping Of The The Cftr10.Txt Sequence 222

(74) Figure V-62 Difference In IFS Mapings “Original” - "Mutation' 222

(75) Figure V-64 Difference In The Wavelet Coefficients 223

(76) Figure V-65 Best Basis Representation 223

(77) Figure V-66 Loglog Plot For The Global Hurst Exponent 223

(78) Figure V-67 Loglog Plot For The Local Hurst Exponent 224

(79) Figure V-68 IFS Maping Of The The Cftr12.Txt Sequence 224

(80) Figure V-69 Difference In IFS Mapings "Original" - "Mutation" 225

(81) Figure V-70 Difference In The Wavelet Coefficients 225

(82) Figure V-71 Best Basis Representation 225

(83) Figure V-72 Loglog Plot For The Global Hurst Exponent 226

(84) Figure V-73 Loglog Plot For The Local Hurst Exponent 226

(85) Figure V-74 IFS Maping Of The The Cftr13.Txt Sequence 227

(86) Figure V-75 Difference In IFS Mapings "Original” - "Mutation" 227

(87) Figure V-76 Difference In The Wavelet Coefficients 228 
(88) Figure V-77 Best Basis Representation 228

(89) Figure V-78 Loglog Plot For The Global Hurst Exponent 229

(90) Figure V-79 Loglog Plot For The Local Hurst Exponent 229

(91) Figure V-80 Original Sequence 230

(92) Figure V-81 Base Pair Distribution 230

(93) Figure V-82 Artificially Mutated Sequence 1000 -> 1009 Order Reversed 231

(94) Figure V-83 Base Pair Distribution 231

(95) Figure V-84 Difference In IFS Representations I.E. "Cftr0 - Cftr09" 232

(96) Figure V-85 Continuous Wavelet Transform Of Original Sequence 232

(97) Figure V-86 Continuous Wavelet Transform Of The Mutated Sequence 233

(98) Figure V-87 Difference In The Wavelet Transforms 233

(99) Figure V-88 Best Basis Representation Of The Mutated Sequence 234

(100) Figure V-89 Global Hurst Exponent Of The Mutated Sequence 234

(101) Figure V-90 Local Hurst Exponent Of The Mutated Sequence 235

(102) Figure V-91 Original Sequence 235

(103) Figure V-92 Base Pair Distribution 236

(104) Figure V-93 Artificially Mutated Sequence 1000 -> 1030 Order Reversed 236

(105) Figure V-94 Difference In IFS Representation 237

(106) Figure V-95 Continuous Wavelet Transform Of Original Sequence 237

(107) Figure V-96 Continuous Wavelet Transform Of Mutated Sequence 238 1000 -> 1030 Order Reversed 
(109) Figure V-97 Difference In The Continuous Wavelet Transforms

Of The Two Sequences

(110) Figure V-98 Best Basis Representation Of The Mutated Sequence

(111) Figure V-99 Global Hurst Exponent Of The Mutated Sequence

(112) Figure V-100 Local Hurst Exponent Of The Mutated

Sequence 1000 -> 1030

(113) Figure V-101 Original Sequence

(114) Figure V-102 Base Pair Distribution

(115) Figure V-103 Mutated Sequence 1000 ->1300

(116) Figure V-104 Base Pair Distribution 1000 ->1300

(117) Figure V-105 Difference In The IFS Representations between

Original And Mutated Sequence 1000 -> 1300 Order Reversed

(118) Figure V-106 Continuous Wavelet Transform Of The Original Sequence

(119) Figure V-107 Continuous Wavelet Transform Of The Mutated Sequence Hfh 1000 -> 1300 Order Reversed

(120) Figure V-108 Difference In The Continuous Wavelet Transform Of The Mutated Sequence And The Original Sequence

(121) Figure V-109 Best Basis Representation 
(123) Figure V-111 Local Hurst Exponent Of The Mutated Sequence

(124) Figure V-112 Original Sequence

(125) Figure V-113 Base Pair Distribution

(126) Figure V-114 Mutated Sequence 1000 ->4000

(127) Figure V-115 Base Pair Distribution 1000 ->4000

(128) Figure V-116 Difference In The IFS Representations Between Original And Mutated Sequence 1000 -> 4000 Order Reversed

(129) Figure V-117 Continuous Wavelet Transform Of The Original Sequence

(130) Figure V-118 Continuous Wavelet Transform Of The Mutated Sequence 1000 -> 4000 Order Reversed

(131) Figure V-119 Difference In The Continuous Wavelet Transform Of The Mutated Sequence And The Original Sequence

(132) Figure V-120 Best Basis Representation Of The Mutated Sequence 250 1000 -> 4000 Order Reversed

(133) Figure V-121 Global Hurst Exponent Of The Mutated Sequence

(134) Figure V-122 Local Hurst Exponent Of The Mutated Sequence 1000 -> 4000 Order Reversed

VI. Conclusions

(1) Figure VI-1 
(2) Figure $\mathrm{Vl}-2 \quad 254$

(3) Figure $\mathrm{Vl}-3 \quad 256$

VII Recommendations

$\begin{array}{ll}\text { (A) Figure VII-1 } & 275\end{array}$

$\begin{array}{ll}\text { (B) Figure VII-2 } & 277\end{array}$

$\begin{array}{ll}\text { (C) Figure VII-3 } & 278\end{array}$ 


\section{List of Symbols, Abbreviations, and/or Nomenclature}

\begin{tabular}{|c|c|}
\hline DNA Deoxoribnucleic Acid & C Cytosine, one of the DNA base-pairs \\
\hline $\mathrm{T} \sim$ Thymine, one of the DNA base-pairs & G Guanine, one of the DNA base-pairs \\
\hline A Adenine, one of the DNA base-pairs & $\begin{array}{l}\text { Chaos A deterministic non-linear dynamical system that can } \\
\text { produce random looking results. It must have a fractal dimension } \\
\text { and show sensitivity to independence. }\end{array}$ \\
\hline $\begin{array}{l}\text { Fractional Brownian Motion Processes A biased random walk; } \\
\text { comparable to shooting craps with loaded dice. Unlike standard } \\
\text { brownian motion, the odds are biased in one direction or the } \\
\text { other. }\end{array}$ & $\begin{array}{l}\text { Classical Euclidean Geometry The traditional geometry derived } \\
\text { from Greek thought gave us the point, the one-dimensional } \\
\text { straight line, the two-dimensional plane, and the three- } \\
\text { dimensional solid }{ }^{38} \text {, i.e the "geometry of man". }\end{array}$ \\
\hline $\begin{array}{l}\text { Fractal It comes from the Latin "fractus" for fragmented or } \\
\text { irregular. An object is fractal if it displays the property of "self- } \\
\text { similarity and non-integer dimensionality }\end{array}$ & $\begin{array}{l}\text { Fractal Geometry A geometry concerned with fractal shapes and } \\
\text { is often described as the geometry of nature }\end{array}$ \\
\hline $\begin{array}{l}\text { Genome The complete set of instructions for making an } \\
\text { organism is called its genome. It contains the master blueprint for } \\
\text { all cellular structures and activities for the lifetime of the cell or } \\
\text { organism. Found in every nucleus of a persons many trillions of } \\
\text { cells, the human genome consists of tightly coiled threads of } \\
\text { deoxyribonucleic acid (DNA) and associated protein molecules, } \\
\text { organized into structures called chromosomes }\end{array}$ & $\begin{array}{l}\text { Fractal Dimension A number that describes how an object fills a } \\
\text { space. In Euclidean geometry, objects are solid and continous, } \\
\text { they have no holes or gaps }\end{array}$ \\
\hline $\begin{array}{l}\text { Spectrogram An object of class Spectrogram represents an } \\
\text { acoustic time-frequency representation of a sound: the power } \\
\text { spectral density } P(f, t) \text {, expressed in } \mathrm{Pa}^{2} / \mathrm{Hz} \text {. It is sampled into a } \\
\text { number of points centred around equally spaced times } t_{i} \text { and } \\
\text { frequencies } f_{j} \text {. }\end{array}$ & $\begin{array}{l}\text { Self-Similarity A descriptive of parts of an object that are } \\
\text { quantitatively the same as or simialr to the whole object at smaller } \\
\text { scales. In determinsitic fractals the small pieces look the same as } \\
\text { the larger objects. In statistical fractals the small details are } \\
\text { statistically similar. }\end{array}$ \\
\hline $\begin{array}{l}\text { Fourier Transform The Fourier Transform is a generalization of } \\
\text { the Fourier Series. Strictly speaking it only applies to continous } \\
\text { and aperiodic functions. (See Fourier Series) }\end{array}$ & $\begin{array}{l}\text { Stable Pareto-Lévy Distributions A characteristic function of the } \\
\text { fractal family of distributiuons, the characteristic component alpha } \\
\text { can range from between } 1 \text { and } 2 \text {. (See Fractal Disreibutions) }\end{array}$ \\
\hline $\begin{array}{l}\text { Wavelet Transforms The fundamental idea behind wavelets is to } \\
\text { analyze according to scale. Wavelets are functions that satisfy } \\
\text { certain mathematical requirements and are used in representing } \\
\text { data or other functions. }\end{array}$ & $\begin{array}{l}\text { Gaussian } \sim \text { A system whose properties are well described by the } \\
\text { normal distributions, or bell-shped curve. }\end{array}$ \\
\hline $\begin{array}{l}\text { Fourier Series Theory developed by Joseph Fourier for function } \\
\text { representation by the use of sums of scaled trigometric series } \\
\text { (sines \& cosines). For many functions } f \text {, can reconstruct from its } \\
\text { Fourier coefficients, by using the Fourier series. In the case of a } \\
\text { string fixed between two points, and initially held in a shape which } \\
\text { is the graph of } f \text {, the Fourier (sine or cosine) coefficients have a } \\
\text { physical interpretation; they represent the amount of the } \\
\text { corresponding harmonic in the resulting vibration. }\end{array}$ & $\begin{array}{l}\text { Kurtosis (or kurt) } \sim \text { A non-dimensional quantity. It measures the } \\
\text { relative peakedness or flatness of a distribution, relative to a } \\
\text { normal distribution. }\end{array}$ \\
\hline $\begin{array}{l}\text { Wavelet Packet Transforms The objective of wavelet packet } \\
\text { transform analysis is to find the "best" representation of a digital } \\
\text { signal through its adequate display with a relatively small number } \\
\text { of coefficients. }\end{array}$ & $\begin{array}{l}\text { Fractal distributions A probalbility distribution that is statistically } \\
\text { self-similar. That is, in different increments of time, the statistical } \\
\text { characteristics remain the same. }\end{array}$ \\
\hline \multicolumn{2}{|c|}{$\begin{array}{l}\text { L-Systems A symbolic dynamical systems called Lindenmayer systetrotokturtic Distribution with positive kurtosis. (See Kurtosis). An } \\
\text { systems, after Aristid Lindenmayer who first used them to model bioloigidtween distribution is termed mesokurtic.) } \\
\text { phenomena. Froma Formal Language Theory viewpoint } \\
\text { L-Systems have production rules or grammars. }\end{array}$} \\
\hline $\begin{array}{l}\text { IFS Algorithm Visualization technique that is a type of L-System } \\
\text { that is a 2-Dimensional mapping of linear data. }\end{array}$ & Platykurtic $\sim$ Distribution with negative kurtosis. (See Kurtosis) \\
\hline $\begin{array}{l}\text { Context-Free Process A type of L-system that has production } \\
\text { rules that have no memory. It takes account only of an individual } \\
\text { symbol, and not of what their neighbors are. Example -Fibonacci } \\
\text { system. }\end{array}$ & Mesokurtic In-between Leptokurtic and Platykurtic \\
\hline
\end{tabular}




\section{List of Symbols, Abbreviations, and/or Nomenclature continued}

\begin{tabular}{|l|l|}
\hline $\begin{array}{l}\text { Hurst Exponent } ~ A \text { mesure of the bias in fractional brownian } \\
\text { motion. } \mathrm{H}=0.5 \text { for brownian motion; } 0.50<\mathrm{H}<=1.00 \text { for a persistent } \\
\text { series; } 0<=\mathrm{H}<0.50 \text { for am anti-persistent series. The inverse of } \\
\text { the Hurst exponent is alpha, the characteristic exponent for a } \\
\text { fractal or a Pareto distribution }\end{array}$ & $\begin{array}{l}\text { Re-Scaled Range Analysis ( R/S Analysis) the method } \\
\text { developed by Hurst to determine long-term memory effects and } \\
\text { fractional brownian motion. A measurement of how the distance } \\
\text { covered by a particle increases over longer and longer times } \\
\text { scales. }\end{array}$ \\
\hline Introns Information bearing portion of the genome & $\begin{array}{l}\text { Exons "Non-information" portion of the geome. Thoug is thought } \\
\text { to be important from a L-System grammar viewpoint }\end{array}$ \\
\hline $\begin{array}{l}\text { Production Rules The "grammar rules" of a L-System. (See L- } \\
\text { Systems }\end{array}$ & RNA Ribonuclic acid \\
\hline $\begin{array}{l}\text { Context-Sensitive Process A type of L-system where the } \\
\text { production rules in the system have memory. The production rules } \\
\text { apply to a particular symbol and only if the symbol has certain } \\
\text { neighbors. Example-DNA }\end{array}$ & Codons Triplets of base-pairs that code for amono acids. \\
\hline
\end{tabular}


"I intend to fight it out on this line even if it takes all

\author{
summer!" \\ Ulysses S. Grant \\ Battle of the Wilderness
}

May 1864 


\section{Introduction}

The modern mathematical ideas concerning non-linear systems are applied to a wide range of scientific problems in biology ${ }^{96}$, chemistry ${ }^{27}$, physics ${ }^{23}$, and engineering. Modern science tells us that if one can describe a physical system with mathematics accurately, one can understand the systems properties and make predictions about the system's future state. ${ }^{6,15,22,37}$ Examples of this can be shown for a wide variety of phenomena such as planetary motion or particle physics. ${ }^{47}$ The phenomena in biological systems are peculiar examples of non-linear systems. ${ }^{48,122}$

Biological patterns are a cornerstone of living systems. Some patterns continue throughout life and any break in these patterns could be lethal. ${ }^{52}$ Other patterns some of these are controlled by the organism, and other patterns that are not controlled appear and disappear throughout the organism's existence. ${ }^{46}$ These patterns interact with one another and with the external environment. ${ }^{47}$ Variations of the patterns, outside normal boundary conditions, or the introduction of new patterns, could represent the presence of disease. ${ }^{122}$

Understanding the mechanisms of biological patterns requires an approach that integrates the engineering of non-linear systems theory and the various sub-disciplines of biology ${ }^{64}$. Although Poincare established the foundations of non-linear dynamics in the last century they could not be applied to these highly non-linear biological systems until the advancement of digital computers. There has been a remarkable resurgence of non- 
linear dynamic analysis in the last 20 years. Unfortunately the engineering of non-linear dynamics is outside the training paradigm for most biological scientists as is the understanding of biological processes for engineers. The premise of this research is that ideas from the field of non-linear dynamics could lead to deeper understanding for biological scientists. Such advances will not follow without an inter-disciplinary approach. ${ }^{69,70,71}$

\section{A) Scope of the Problem}

The search for patterns in the sequence of DNA bases is an active topic in molecular engineering. Periodic and non-periodic patterns affect physical, chemical and biological properties of the DNA but the role of many of these patterns are unknown. In order to gain an understanding of these patterns, one must integrate the engineering modeling of non-linear systems theory with biology ${ }^{47}$. Insight into the meaning of these patterns could follow by treating their parent DNA sequences as a "time-series" and then

applying the methods of non-linear engineering analysis methodologies to the series. ${ }^{45,90}$ An example, a human bladder cancer gene could be treated as if it were a speech waveform, which is an acoustic energy time-series. ${ }^{90}$

DNA's double helix structure contains the basic genetic information for biological systems. ${ }^{59,60}$ DNA contains all the basic genetic information of all living cells. It is in a fashion an organism's 'computer program'. The sequences of bases of DNA probably hold information for protein synthesis as well as information for signals to regulate the inner 
workings of the organism. The sequence of bases are often described as a four letter code for its bases: A, C, G and T (adenine, cytosine, guanine and thymine A, C, G, and T respectively). These letters can be mapped to a simple numerical representation to create a data sequence. For example an AT rich region is thought to be involved in the programming for transcription. It is also known that certain specific viral sequences cause carcinogenic changes in living cells and hence cancerous tumors in living creatures. DNA sequences and composition also control the physical properties of the DNA e.g. DNA melting temperature ${ }^{90}$ and different conformations.

The present work examines pathological and non-pathological gene sequences for information using the strength of linear and non-linear analytical and visualization methods. Analytical techniques will be applied as if the DNA sequence was a time series of the acoustic speech signal. ${ }^{90}$ The linear techniques used will involve modern operator methods such as Wavelet Packet Transforms. ${ }^{24}$, The non-linear techniques will involve using the IFS algorithm (IFS mapping).$^{50,84,90}$ IFS mappings are a two dimension mapping that converts data which is basically a single dimension set of inputs into a graphical form that presents the data in a visual fashion. This allows the embedded patterns in the data to be recognized. This technique captures the dynamics of how individual base's position relates to the position of every other base in the sequence. The DNA considered in the present study is not a time-series but a position series therefore dynamics must be redefined to mean the way that the bases $T, A, G$, and $C$ change due to mutation with respect to each other and to their position in the sequence. These changes can occur both in a linear and in a non-linear fashion. Furthermore the non-linear condition is either 
"random" or "chaotic". Each of these sub-conditions could have a specific biological interpretation. The visualization methods will be important to consider, since by their very nature they concentrate on preserving structure of the sequence information. The primary research goal is to develop analytical tools to examine the information encoding ${ }^{49,119}$ of a DNA sequence which could be useful in understanding the biological consequences of the system pattern.

B) Thesis Statement

The hypothesis for this work is that a multi-fractal model for DNA information encoding can be built and analyzed using non-traditional and traditional Information Engineering Theory ${ }^{117}$ techniques. The title of this study is "Use of Wavelet Packet Transforms to Develop an Engineering Model for Multi-Fractal Characterization of Mutation Dynamics in Pathological and Non-Pathological Gene Sequences". This study investigates a unified framework of the information encoding mechanism. The multifractal approach attempts to build a model by comparing these sequences as fractional Brownian motion processes. Using this approach, cystic fibrosis sequences both healthy and pathological were modeled as a two dimensional approximation of a formal grammar called an IFS (Iterated Function System). Through this approach a number of observed changes in DNA sequences by mutations will characterized. The second step of this information analysis procedure involves the application of Wavelet Packet Transforms. This method is used to calculate scaling relationships in the data. These relationships 
are then used to calculate the Hurst exponent that gives a quantitative measure of the information complexity stored in the gene sequence. In this dissertation the Hurst exponent will detect perturbation to the system as a result of mutations in the gene.

The research goals are:

1. Can non-linear analysis detect changes of information complexity in DNA sequences. Specifically can different states of "randomness" or "chaos" be categorized?

2. Can information structures be identified, quantified and the change in these structures be identified and quantified?

3. Can one distinguish pathogenic from non-pathogenic?

4. Can one distinguish degrees of pathogenicity? Can one distinguish minor illness from catastrophic illness?

5. Can one alter the gene pattern using genetic engineering methods and measure the changes in information content?

6. Can one use the above techniques on diseased sequences and alter the catastrophic nature of the serious defect? 


\section{Literature Review}

A. Introduction

The physical world was once symbolized by stable periodic motion e.g. the dynamics of planetary motion. This is the classic modeling paradigm of classical mechanics. This notion of linear systems governed by differential equations where given a set of equations and the proper boundary conditions and system behavior was known for all time. It was acknowledged that this was a model and hence only approximated the system's behavior but the differences in reality and the model were viewed as insignificant. Science and engineering applied this model to the physical world with obviously astounding success. This modeling methodology for good and ill also permeated the social sciences. However when one really examines the physical world things are not as simple as the earlier models would have us to believe. The physical world is a world of instabilities and the difference between the linear models and the 'real' world as not as insignificant as once believed. Reality is fundamentally non-linear. The instabilities and fluctuations the one observes in the physical world yield the rich variety to the forms and structures that make up this 'real' world. New concepts and new tools are necessary to explore this 'real' world. It must be acknowledged that the old linear methods are inadequate. Non-linear dynamical theory developed by Poincare and others must now be considered. 
The modern mathematics of non-linear systems can be applied to a wide range of scientific problems in biology, chemistry, physics ${ }^{17}$, and engineering. Modern engineering lets one describe a physical system with mathematics. One can then understand the system's characteristics and predictions its time evolution. The systems characteristics or structure might be the rate that certain measurable values in the system appear and disappear. The rate that these values change 'state' that is turn into other values or how each value relates to past values or future values. In short patterns in the data that indicate a underlying structure. This approach can be applied to a wide range of phenomena such as planetary motion, particle physics or biological systems.

Biological patterns are the foundation of living systems. Some patterns indicate continued life for a organism and any break in these patterns could mean death ${ }^{2,3}$. Other patterns occur, some of these the organism can control and other patterns the organism can not control. They come and go throughout the organism's existence. These patterns interact with one another maybe even form new patterns. They also can interact with the external environment. Changes in these patterns outside normal boundary conditions, or the introduction of new patterns, could represent the presence of disease. ${ }^{19,50,58}$

Modeling biological patterns requires an approach that combines the engineering of non-linear systems theory and the various sub-disciplines of biology. This builds foundations of non-linear dynamics developed by Poincare in the last century. Unfortunately the engineering of non-linear dynamics is outside the normal biological scientist's domain as is the understanding of biological processes for engineers. Non- 
linear dynamics could lead to better methods for biological scientists to understand their field, however advances will not follow without an inter-disciplinary approach. ${ }^{16,51}$

Pattern analysis of the DNA base pair sequences is an important topic in molecular biology. Patterns that show periodicity and non-periodicity and how they relate to the physical, chemical and biological properties of DNA are important. To understand these patterns, one must integrate engineering modeling of non-linear systems theory and biology ${ }^{25}$. Patterns could be analyzed by treating their parent DNA sequences as a timeseries $^{30}$ and then applying non-linear engineering analysis to the series. Again consider the example of the human bladder cancer gene. Pickover shows how this gene could be treated as if it was a speech waveform. ${ }^{62}$ Again consider Figure II-1 which shows DNA's double helix. This figure shows structure that contains the basic genetic information for biological systems. It is a four letter code: A, C, G and T (adenine, cytosine, guanine and thymine A, C, G, and T respectively) These letters are mapped to a simple numerical representation. DNA has all the basic genetic information of all living cells. It is an organism's computer program. The sequences of bases of DNA hold information for protein synthesis as well as information for signals to regulate the inner workings of the organism. The AT rich region is thought to be involved in the programming for transcription. It is also know in that certain specific viral sequences are carcinogenic They cause cancerous tumors in living creatures. DNA sequences and composition also control the physical properties of the molecule. Detailed comparisons are useful and can be achieved by several traditional statistical approaches. However these statistical techniques 
usually leave one with no feel for the structure of the sequence. Furthermore differences between sequences may overwhelm their similarities. Also testing for the "randomness" of a DNA sequences is difficult to do..$^{8,11}$

The goal of this research is look for patterns in pathologic and nonpathologic DNA sequences with linear/non-linear/chaotic mathematical analysis. The DNA sequence will be treated analytically like a time series e.g. $x(t)$ but instead of time being the abscissa its unit distance along the sequence. The ordinate is the base pair, represented as one of four numbers. DNA or deoxyribonucleic acid consists of four nucleotide or bases: thymine $(\mathrm{T})$, adenine $(A)$, guanine $(G)$, cytosine $(C)$. These base pair representations are mapped non-linearly by an algorithm into a two dimensional plane thus revealing any underlying patterns. Periodicity and non-periodicity patterns in the DNA affect physical, chemical and biological properties of the DNA. An operator transform method that is sensitive to non-linear patterns used to analyze the data, e.g. Wavelet Transform Theory. Earlier work by Pickover, etc. using the operator method called Fourier Transforms demonstrated linear features such as periodicity in bladder cancer data. Sudden changes of patterns in the data indicate changes in the arrangement of the base pairs and their relationship to each other. Patterns indicate structure and structure is related to genetic information. Wavelets have a relationship to the Hurst complexity statistic, this statistic can be used to show changes 'information' in the DNA data due to deviations, i.e. mutations from the normal sequence. 
An engineering modeling approach using non-linear systems theory, methods for multivariate data visualization integrated with biology will be used to relate biological meaning to these patterns. The product of this research has potential to shed light on information content of the base patterns which is important to understanding coding and non-coding regions in the sequence. This knowledge could lead to better therapies for a host of diseases both genetic and non-genetic based. It also could lead to a deeper understanding of evolutionary processes.

B. Non-linear or Chaotic Dynamics

Dynamics is the study of motion. By "motion" it mean fluctuations in a variables value $^{65}$ These fluctuations can be time-varying or position(spatial) varying fluctuations in whatever phenomenon that one has data. For example the time varying quantity might be the price of an economic indicator, concentration of a chemical reactant, or celestial motion or the incidence of flu infections in a human population. It does not matter what the conditions are, there is a common language with which fluctuations can be analyzed. .,43 $^{9}$

The methodology and language of this field of study are encapsulated in the science of non-linear dynamics. This branch of science has undergone extraordinary growth over the past fifteen years. It has moved beyond the province of a curiosity of 
interest to only mathematicians to one in which applied scientists and engineers are deeply involved. It clearly has deep roots in traditional mathematics and physics but the subject has moved well beyond the limits of classical inquiry. It is different from traditional dynamics in the following manner.

\section{1) Complex Dynamics}

Traditional dynamically systems theory focused on regular behavior. e.g. stable points and periodic orbits. Modern dynamics is more often concerned with turbulence ${ }^{49}$ and chaos. This is because the research emphasis has shifted to examining non-linear systems. The dynamics of linear systems represent very few "real" world cases. Their behavior is predictable and generally benign. Non-linear systems however introduce a bewildering diversity of behaviors including various forms of "chaos". ${ }^{13,14}$

2) Quantitative Behavior and Universality

Traditional books on differential equations emphasize that the classical techniques and methodologies for writing down "closed solutions" for specific equations. A newer approach emphasizes qualitative behavior. Sometimes very general results hold for entire classes of equations. Such properties are said to be universal, and it was this discovery of a "universality" in non-linear equations that led to the idea that there is indeed "order in chaos"27 $^{27}$ 
3) Importance of the Computational Methods

The vast majority of non-linear systems can not be "solved" in the traditional classical sense. This is where one writes down the general solution formula and tests it against boundary conditions. It is used to describe the systems evolution. These systems must be studied numerically. This fact introduces an experimental aspect to studying modern system dynamics in which the computer becomes a virtual laboratory..$^{29,47,48}$

\section{4) Application to Experimental Data}

Nonlinear equations are being used to account for erratic behavior of experimental data sets. These data sets are sets that are "odd" meaning that they do no seem to fit into the "norms" of conventional descriptions. These descriptions emphasize constant behaviors and strict periodicity, consider the following examples: ${ }^{31,32,33}$
a. Belousov-Zhabotinskii Reactions ${ }^{1}$
b. turbulent fluids ${ }^{6}$
c. celestial mechanics $^{5}$
d. mechanical engineering ${ }^{55}$
e. optics Gibbs $(1981)^{55}$

The above were examples of data generated from chemical, mechanical, i.e. nonliving systems. However there is considerable reason to believe that non-linear processes 
also account for behavior in living systems. In human physiology such as cardiology and neurology, it is theorized that normal cardiac and neurological functionality involves complicated non-linear behavior whereas pathological or diseased states are characterized by near periodic behavior. For Example cardiac dysfunction and epileptic seizures are characterized by nearly periodic states $\mathrm{s}^{50,51}$

\section{5) Dynamical Systems}

A dynamic system is in its most simple form a collection of behaviors that specify the evolution of one or more variables. This evolution could occur over space or time or both. These variables are referred to as "state variables" and their behaviors can be transformed into mathematical rules that are expressed as equations. For example the equations (1) and (2) below are examples of continuous and discrete dynamically systems. ${ }^{23,24}$

$$
\begin{aligned}
& \frac{d x(t)}{d t}=f[x(t)] \\
& x[i+1]=F[X(i)]
\end{aligned}
$$

One must remember that the right hand side of the above equations gives a complete description of the change of the state variables with respect to the independent variable which could be time or position. Therefore knowing the value of the state of $x(t)$ or 
$X[i]$ at specific t's or i's together with the functional form of the equation allows one to forecast future behavior of the system. Note that $x(t)$ and $X[i]$ may be vectors and that $x(t)$ is a continuous function while X[i] represents a discrete function.

6) Continuous Dynamical Systems ${ }^{67,68,69}$

Considering the continuous case first for the sake of simplicity. Therefore the above equations can be recast in vector form as:

$$
\begin{aligned}
& \frac{\mathrm{dx}_{1}(\mathrm{t})}{\mathrm{d}}=\mathrm{f}_{1}\left[\mathrm{x}_{1}(\mathrm{t}), \mathrm{x}_{2}(\mathrm{t}), \ldots \ldots . .\right] \\
& \frac{\mathrm{dx}_{2}(\mathrm{t})}{\mathrm{d}}=\mathrm{f}_{2}\left[\mathrm{x}_{1}(\mathrm{t}), \mathrm{x}_{2}(\mathrm{t}), \ldots \ldots . .\right]
\end{aligned}
$$

$"$

,

etc. 
Given that above vector-matrix equation representations one can now speak of this system evolution in terms of a phase space or in two-dimensional cases, the phase plane. The phase space is a Euclidean space whose axes are $x 1, x 2$, etc. Therefore in two dimensions one might write continuous first-order differential equations in the following manner:

$$
\text { (III.4) } \begin{aligned}
\frac{d x(t)}{d t} & =f(x, y) \\
\frac{d x(t)}{d t} & =g(x, y)
\end{aligned}
$$

In two dimensions ${ }^{44}$ the dynamics are represented by plotting coordinate pairs [x(t), $y(t)]$, in the $X-Y$ plane. If we connect the points in a temporal or a position sequence, we obtain a trajectory or a solution curve. The construction of this curve depends on the initial conditions

$$
\left[\mathrm{x}\left(\mathrm{t}_{0}\right), \mathrm{y}\left(\mathrm{t}_{\mathrm{t}}\right)\right]=\left[\mathrm{x}_{0}, \mathrm{y}_{0}\right]
$$


This means that different initial conditions produce different trajectories. ${ }^{40}$ Therefore one can say that the solution curve or trajectory is based at a particular initial condition. If one superimposes solution curves at numerous points one constructs a phase portrait, which represents the motion over the entire space

\section{7) Discrete Dynamical Systems}

In discrete dynamical systems, one goes forward with the analysis in much the same fashion. There is a important exception, it no longer makes sense to connect the points. In strict mathematical terms this is because in discrete systems state variables do not vary continuously and are only defined over a finitely countable number of points. Instead of trajectories one has point sequences sometimes called itineraries

$$
\sum_{\mathrm{i}} \mathrm{X}_{\mathrm{i}} \mathrm{i}=1,2,3, \ldots \ldots
$$

Granted this seems like a insignificant difference, however it has extraordinary consequences. In continuous systems, the basic existence and uniqueness theorems for differential equations preclude intersecting solutions.. This is because if solution curves cross, the same initial conditions would lead to different outcomes. This would violate determinism. This restriction places limitations on the degree of dynamical complexity ${ }^{36,45}$ that one can observe in low dimensional systems. Consider the following two 
dimensional case. It is easy to convince oneself that most complex system dynamics that are allowed correspond to closed curves. That is while two dimensional continuous systems can exhibit periodic behavior, a minimum of three dimensions is required for chaotic solution curves to appear which wind around in a complex fashion but do not repeat. However such restrictions do not apply to discrete systems because the solution curves as defined in the continuous case simply do not exist. Successive iterations of a discrete mapping can therefore be interwoven in a very complicated fashion The is because these points have zero volume and will not overlap. This is why even one dimension discrete systems such as the logistic equation can exhibit chaotic behavior.

8) Comparing Conservative Dynamical Systems with Dissipative Dynamical Systems

One can view dynamical systems by their affect that they have on volumes in the phase space. This can be examined by doing the following: Let a small region of the phase space (a volume in 3-D phase space and a plane in 2-D phase space) This region is populated with initial condition points. At each point a dynamic equations are solved for a specific interval (e.g. for a time-series its a time interval). The new volume is calculated and also a volume rate of change. This is called the Lie derivative or the divergence

$$
\frac{1}{V} \cdot \frac{d V}{d t}=\frac{\partial(d x / d t)}{\partial x}+\frac{\partial(d y / d t)}{\partial y}+\ldots . .
$$


The quantity can remain constant for all values of state variables. Usually the rate of contraction or expansion will vary. In these cases one must repeat the experiment everywhere in that state space and compute an average rate of change.

There are two conditions that arise from this:

1) There are systems that preserve volumes.

$$
\left\langle\frac{1}{V} \cdot \frac{d V}{d t}\right\rangle=0
$$

These systems are said to be conservative.

2) Systems where the volume is diminished:

$$
\text { (III.9) } \quad\left\langle\frac{1}{\mathrm{~V}} \cdot \frac{\mathrm{dV}}{\mathrm{dt}}\right\rangle<0
$$

These systems are known as Dissipative. \{ Note the $<>$ means a average is taken.\}

Conservative system examples are physical systems that conservative energy e.g. frictionless pendulum and planetary motion. If friction is added to the pendulum then conservative systems become dissipative systems. The most important difference 
between these two types of dynamical systems involves the long term behavior of solution curves based on different initial conditions. In the dissipative case, one can have attractors, i.e. sets of points (one or more) to which solution curves based on initial conditions tend to limit to as time increases. Conservative systems do not possess attractors. Therefore solution curves based at different points do not exhibit after their transient behavior the same long-term behavior of the dissipative system. In the conservative case each initial condition induces its own dynamics which depending on the system may differ from other either very little or a great deal.

Attractors are a compact set; they are bound and closed. They have the property that solution curves based within some neighborhood of the attractor will as $t \rightarrow \infty$ approach the attractor to an arbitrary degree of closeness. This neighborhood is called a basin of attraction. Importantly these solution curves based at points on the attractor will remain there forever and finally every attractor contains an orbit which comes arbitrarily close to every point on the attractor. This is saying that an attractor can not be decomposed into smaller sets.

9) Normal System Behavior Compared to Chaotic System Behavior

It's often very difficult to put a precise definition on chaos at the working level. Usually people look at a systems behavior and say if it is not periodic or quasi-periodic it must be "chaos". The behavior of the system in question is usually represented in the phase space, which if the dimension is greater then 3 may be extremely difficult to 
visualize. To get a proper system description that is a more quantitative then qualitative definition of chaos, it better to define the system in terms of the degree to which nearby solution curves separate or come together. If on the average the neighboring orbits diverge exponentially, it can be said that the system exhibits sensitivity to initial conditions. This means that small perturbations can have far-reaching consequences. This is the operative definition of chaos. Mathematicians as is their want require more precision in terminology and they test for the presence of invariant sets called horseshoes. The existence of these invariant sets guarantee solution curves that exhibit sensitive dependence. It is important to state that horseshoes are not attractors. Points near the horseshoe will always diverge from them. Horseshoes are building blocks of chaos and whenever chaotic dynamics are seen one can expect to see a horseshoe in the system dynamics.

It is fundamental to remember that systems that exhibit sensitivity to initial conditions are unpredictable over long time periods. Since one can not specify a systems location in phase space with infinite precision, therefore small errors in one's defining the system's initial state are amplified to a point that its is impossible to say where the system will be at a given time.

The term chaos in its most commonly used term is applied to asymptotic motions on attracting sets in dissiapative systems. This condition produces the term chaotic attractor. It must be remembered that chaos can be seen in a conservative system like planetary motion involving more then two bodies. ${ }^{38,44}$ However this is not the attracting flavor of 
"chaos", that is no attractors are present. This is the operative distinction that separates chaotic systems from stochastic systems. Poincare demonstrated this brand of chaos in 1899. There are more complete examinations of this in Henon's work ${ }^{69}$.

10) Ways to Characterize Chaotic Dynamical Systems

A method of characterizing a strange attractor which is independent of the coordinate system used to represent it is to calculate the following dynamical quantities ${ }^{41,44}$

a) Invariant Measure

This is a probability distribution specifying the frequency with which different regions of the attractor are visited. One can think of this as covering that atttractor with little boxes i.e. cubes (or hypercubes if a higher dimensional requires it) and then counting the number of points in each box. An interesting property of this situation, is if one decreases the size of the boxes a increasing number of points are contained in a ever smaller fraction of the boxes. One then can define a "attractor's core" which contains most of the dynamics of the system.

b) Dimension

The dimension of an attractor quantifies the number of independent quantities (i.e. degree's of freedom) required to specify motion. An example of this, is the dimension of a 
stable equilibrium (a point) is zero; the dimension of a limit cycle (closed curve) is one; the dimension of a attracting torus is two. It is important to recognize that an attractor's dimension may be lower then the dimension of the phase space. In the dynamics represented by partial differential equations, the dimension of the phase space is infinite. But one can have finite dimensional attractors such as fixed points and cycles. In chaotic systems it is interesting to note that the dimension of the attractor is non-integer. This means the attractors are fractals. ${ }^{26}$ This comes from the fact that diverging solution curves are re-united by folding or layering in the phase space. This comes from the fact that attractors are bounded. If solution curves diverge locally then there must be some global mechanism to keep them from moving to infinity. Complex systems exhibit similarity and can be scaled and their dimensions must be established and understood. The types of dimensions that are of most importance to complexity studies are:
a) Euclidean dimension, $D_{E}$
b) Topological dimensions, $D_{T}$
c) Fractal dimension, $D_{F}$

$D_{E}$ is familiar to all because it is the dimension concept that was taught in high school geometry. Topological dimensions $D_{T}$ are derived by dividing one geometric set into another. There are obvious examples of this, volumes subsume areas, areas 
subsume lines and lines subsume points. Now $D_{F}$, fractal dimensions such as the Hausdorff-Besocovitch $D_{H-B}$ or the capacity dimension, $D_{C}$

Given the above paragraph the relationships between the dimensions are $D_{E}>D_{F}>D_{T}$ and is not surprising in their paper on strange attractors point out that there are three basic types of fractal dimensions. ${ }^{67,68,69,81}$ The "metric" dimensions are defined to be the Hausdorff-Besocovitch $D_{H-B}$ or the capacity dimension, $D_{C}$. The metric dimensions are independent of the frequency with which the attractor is visited. The other class of fractal dimensions is said to be "probabilistic" because it does depend on the frequency in which an attractor site is visited. There is another type of dimension that is related to Shannon's Entropy Theorem called the information dimension, $D_{I}$ is a "probabilistic" fractal dimension

(1) Hausdorff-Besocovitch $D_{H-B}$ Dimension $67,68,69$

This is a generalized characteristic dimension also known as the capacity dimension. The size or effective dimension, $\mathrm{V}$, of a region increases with the linear dimension, L. This can be written in the following manner:

$$
\mathrm{V}(\mathrm{L})=\mathrm{L}^{\mathrm{D}}
$$


$D$ is the effective dimension that may be an integer or a non-integer number. If it is noninteger then it is a fractal dimension. This scaling is done on the line, the area and the volume by a magnification factor or scale ratio. The scaling can be done with any number integer or non-integer. It is analogous to zooming in or zooming out with a variable focal length lens. This is called the generalized scale ratio, $r$ let $\mathrm{N}$ be the number of parts of the data set. Therefore using the equation and Euclidean space for clarity:

(III.11) $\quad \mathrm{Nr}^{\mathrm{x}}=1$

Where "d" is defined as the dimension. So the above equation describes the following geometric objects:
a) $\quad \mathrm{Nr}^{1}=1 \quad$ This is a line.
b) $\quad \mathrm{Nr}^{2}=1 \quad$ This is a square.
c) $\quad \mathrm{Nr}^{3}=1 \quad$ This is a cube. 
In general $\mathrm{Nr}^{\mathrm{D}}=1$ and a capital $\mathrm{D}$ are substituted for $\mathrm{x}$ to maintain consistence with convention. Using the above line of reasoning we can re-write this for other geometries and we get:

$$
D=\frac{\log (N)}{\log (1 / r)}
$$

Since no restrictions were specified for the above derivation. D is size dimension of the object under scrutiny.

This generalized dimension concept goes back to the German mathematician Felix Hausdorff. The total size of a object is $L$ and it covers all the little hyper-cubes or hyperspheres $N$ in number and having size $r$. Let this be demoted as $N(r)$. Based on the argument presented in the previous paragraph, the Hausdorff dimension is descriptive of any type of line or curve having zero length, i.e. point sets. The extreme condition $L=1$ and letting $r \rightarrow 0$ then:

$$
D_{H-B}=\lim _{r \rightarrow 0} \frac{\log (N(r))}{\log (1 / r)}
$$


The $D_{H-B}=0$ for a point as in Euclidean space.

(2) Point-wise Dimension

If the data sequence is sampled so that a large number of points per orbit are generated. A sphere or cube of radius or length $r$ is placed at some point in the orbit. The number points that are contained in this geometric volume is expressed by $N(r)$. Let the probability of finding a particular point in this geometric volume be $\mathrm{P}(\mathrm{r})$. It is found by dividing the total number of points in the orbit, $\mathrm{N}_{0}$ into $\mathrm{N}(\mathrm{r})$ that is:

$$
P(r)=\frac{N(r)}{N_{0}}
$$

If the orbit is a one dimensional orbit, then the orbit is a closed periodic orbit and $\mathrm{P}(\mathrm{r})$ will be linear in $\mathrm{r}$ as $\mathrm{r} \rightarrow 0, N_{0} \rightarrow \infty$ and $P(r) \approx b r$. If the orbit was quasi-periodic then a toroidal surface is generated in a three-dimensional phase space. The probability of finding a point on the orbit in a small spatial probability cube or a spatial probability sphere of radius $\mathrm{r}$ is $\mathrm{P}(\mathrm{r}) \approx \mathrm{b} \mathrm{r}^{2}$. Then one defines a dimension of an orbit at a point $\overline{x_{\mathrm{i}}}$ where $\overline{x_{\mathrm{i}}}$ is 
a vector in phase space as the relative percentage of time that the orbit spends in that small probability sphere or cube. This is expressed as:

$$
d_{p}=\lim _{r \rightarrow 0} \frac{\log \left(P\left(r ; x_{i}\right)\right)}{\log (r)}
$$

This can then be recast in a form that is easier to calculate. It is based on choosing a arbritary subset of $M$ points distributed around a attractor, where $M<<N_{0}$. So this can be expressed as:

$$
\lim _{r \rightarrow 0} \frac{1}{M} \sum_{i=1}^{M} P\left(r ; x_{i}\right)=a r^{d_{p}}
$$

Now re-writing this in a more understandable form: 
(III.17) $d_{p}=\lim _{r \rightarrow 0} \frac{\log \left(\frac{1}{M}\right) \sum P(r)}{\log (r)}$

(3) Correlation Dimension ${ }^{28,67,68,69,81}$

Another type of fractal dimension is the correlation dimension It was developed and used by Schaffer (1989) and Moon(1984) and is related to the point-wise of dimension. Grassberger and Proccacia (1983) also studied the properties of this dimension extensively.

In the same manner as the point-wise dimension one discretizes the orbit into a set of $\mathrm{N}$ points $\overline{\mathrm{x}}_{\mathrm{i}}$ in the phase space. The distances between points in calculated between pairs of points that is:

$$
\mathrm{S}_{\mathrm{i}, \mathrm{j}}=\left|\overline{\mathrm{x}_{\mathrm{i}}}-\overline{\mathrm{x}_{\mathrm{j}}}\right|
$$

This is a conventional Euclidean distance measure or some equivalent measure. Defining a correlation function as: 
(III.19)

$$
C(r)=\lim _{N \rightarrow \infty} \frac{1}{N^{2}}\left(\begin{array}{l}
\# \text { of_pairs }(i, j) \_w i t h \\
\text { dis } \tan c e_{-} s_{i, j}<r
\end{array}\right)
$$

For many attractors this has been found to show a power law dependence on $r$ as $r \rightarrow 0$ which is

$$
\text { (III.20) } \quad \lim _{r \rightarrow 0} C(r)=a r^{d}
$$

Now redefining the above as a fractal or correlation dimension using of the $\ln (C(r))$ vs. $\ln (r)$ :

$$
\text { (III.21) } \quad d_{G}=\lim _{r \rightarrow 0} \frac{\log (C(r))}{\log (r)}
$$

$C(r)$ can be found more efficiently by constructing a sphere or a cube at each $\bar{x}_{i}$ in phase space and counting the number of points in each sphere that is: 


$$
C(r)=\lim _{r \rightarrow 0} \frac{1}{N^{2}} \sum_{i}^{N} \sum_{j}^{N} H\left(r-\left|\overline{x_{i}}-\overline{x_{j}}\right|\right)
$$

Where $\mathrm{H}(\mathrm{s})=1$ if $\mathrm{S}>0$ and $\mathrm{H}(\mathrm{s})=0$ if $\mathrm{s}<0$. This type of fractal dimension is different from point-wise dimension because the sum is performed about every point.

(4) Information Dimension

Another type of fractal dimension is the information dimension. It is similar in concept to the capacity or Hausdorff-Besocovitch $D_{H-B}$ dimension. However it tries to accommodate the frequency with which a trajectory visits each covering cube (or sphere). The information dimension is defined in a similar fashion as the capacity dimension. Its is a set of points each point covered one by a sphere or a cube This set of $\mathrm{N}$ cubes or spheres has a size $\varepsilon$. A uniform discretization is done of the continuous trajectory or path. A long enough trajectory must be used to cover the attractor whose dimension is to be measured. In the quasi-periodic case the trajectory has to go long enough to get to all the regions on the two-dimensional toroidal surface of the attractor. 
The information dimension is calculated in the following manner. The number of points $N_{i}$ in each of the $\mathrm{N}$ cells and the probability of a point in that cell called $P_{i}$ Expressing all this in mathematical form :

$$
\text { (III.23) } \quad P_{i}=\frac{N_{i}}{N_{0}} \quad \text { where } \sum_{i}^{N} P_{i}=1 N_{0} \neq N
$$

$\mathrm{N}_{0}$ is the total number of points in the set. The information entropy is thus defined to be:

$$
\text { (III.24) } \quad I(\varepsilon)=-\sum_{i}^{N} P_{i} \log _{2}\left(P_{i}\right)
$$

$I(\varepsilon)$ has units of bits and for $\varepsilon$ equation (23) becomes

$$
\text { (III.25) } \quad I \approx d_{I} \log \left(\frac{1}{\varepsilon}\right)
$$


where $d_{I}$ the information dimension is defined to be:

(III.26a) $\quad d_{I}=\lim _{\varepsilon \rightarrow 0} \frac{I(\varepsilon)}{\log _{2}\left(\frac{1}{\varepsilon}\right)}=\lim _{\varepsilon \rightarrow 0} \frac{-\sum_{i}^{N} P_{i} \log _{2}\left(P_{i}\right)}{\log _{2}\left(\frac{1}{\varepsilon}\right)}$

This reduces to:

(III.25b) $\quad d_{I}=\lim _{\varepsilon \rightarrow 0} \frac{\sum_{i}^{N} P_{i} \log _{2}\left(P_{i}\right)}{\log _{2}(\varepsilon)}$

The information dimension is related to the capacity or Hausdorff-Besocovitch $D_{H-B}$ dimension. The relationship is derived in the following manner.

The probabilities $\mathrm{P}_{\mathrm{i}}$ are equiprobable for all cells, that is: 
(III.26) $\quad \mathrm{P}_{\mathrm{i}}=\frac{\mathrm{N}_{\mathrm{i}}}{\mathrm{N}_{0}}=\frac{1}{\mathrm{~N}}$

The one can say:

(III.27) $\quad I=P_{i} \log \left(P_{i}\right)=-N \frac{1}{N} \log \left(\frac{1}{N}\right)=\log (N)$

From (27) it is clear that $d_{I}=d_{c}$ is true and is a upper bound. Farmer (1983) generalized this to say:

(III.28) $\quad d_{I} \leq d_{C}$

More detail in the information dimension concept can be found in Farmer. (1983), Grassberger and Proccacia (1983) and Shaw (1984) 
Information probability measures the unpredictability in a system. The assumption of uniform probability for each cell, .i.e. $P_{i}=\frac{N_{i}}{N_{0}}=\frac{1}{N}$, sets the upper bound for I. For maximum predictability all the points are located in one cell then $I=0$. This is demonstrated below:

If (III.29) $\mathrm{P}_{\mathrm{i}}=\frac{\mathrm{N}_{\mathrm{i}}}{\mathrm{N}_{0}}=\frac{1}{\mathrm{~N}}$ then (III.30) $\quad I=\log (N)$

but if (III.31) $P_{1}=1$ and (III.32) $P_{i}=1$ for all $i \neq 1$

then (III.33) $I=1 \cdot \log (1)=0$

The literature tends to be confusing with its symbols regarding the information dimension. Shaw (1981) uses the symbol $\mathrm{H}$ for entropy and I for negative entropy $(-\mathrm{H})$ or information. Using Shaw's notation the more predictable a system, i.e. the more distinct the $\mathrm{P}_{\mathrm{i}}$ distribution the higher the information content.

5. Relationship Between Fractal Dimensions and Lyapunov Exponents ${ }^{31,32,33}$

The following fractal dimensions have been defined in the preceding paragraphs: 

a) Capacity Dimension, $d_{c}$ (or Hausdorff-Besocovitch $D_{H-B}$ dimension)
b) Point-wise Dimension, $d_{p}$
c) Correlation Dimension, $d_{G}$
d) Information Dimension, $d_{I}$

Grassberger and Proccacia (1983) demonstrated that the information dimension, $d_{I}$ and correlation dimension, $d_{G}$ are the lower bounds to the capacity dimension, $d_{c}$. That is:

$$
\mathrm{d}_{\mathrm{G}} \leq \mathrm{d}_{\mathrm{I}} \leq \mathrm{d}_{\mathrm{c}}
$$

In summarizing the above relationships, it is clear that the capacity dimension does not consider the distribution of points between covering cells. However the information dimension measures the probability of finding a point in a cell. The correlation dimension measures the probability of finding two points in the same cell.

c) Lyapunov Exponents ${ }^{83}$ 
Lyapunov exponents quantify the rate at which nearby trajectories diverge or are contracted together. In a n-dimensional system there are $n$ exponents If these are considered as a whole then they are called the systems Lyapunov's spectrum. In a three dimensional system with a globally attracting fixed point, there will be three negative exponents indicating convergence in three directions. The Lyapunov spectrum can then be represented as (-, -, -) In another three-dimensional system with a globally attracting cycle this system will have a Lyapunov's spectrum of $(-,-, 0)$ while a attracting torus will have a spectrum of $(-, 0,0)$. The zero exponents correspond to motions on the attractors. However for chaotic attractors in three dimensions, the sign spectrum is $(-, 0,+)$. Here the positive exponent indicates that there is a direction where the trajectories diverge.

Its is important to understand that Lyapunov exponents are average quantities. These can actually be viewed as generalized eigenvalues evaluated over the entire attractor. It therefore is possible for local rates of expansion and attraction to vary dramatically. This inherent non-uniformity is why calculations of Lyapunov's exponents are slow to converge. This is why it is impossible to predict the future behavior of points on the attractor.

Lyapunov exponents can be used as a tool for diagnosing whether or not a system is chaotic. As was said in an earlier paragraph chaos in deterministic systems implies a sensitivity to initial conditions. For ease of discussion time and position in the data sequence will be synonymous. What this means if two trajectories start close together in phase space, on the average they will move exponentially apart from one another for small 
time values. Let $d_{0}$ be a measure of the initial distance between the two starting points at another point in time this small distance can be expressed as:

$$
d(t)=d_{0} 2^{\lambda t}
$$

Wolf, etc. (1986) wrote an excellent review on the use of Lyapunov exponents to diagnose chaotic motion.

The divergence of chaotic orbits is only locally exponential because the system is bounded, as are most physical experiments. In order to define a measure of this divergence of orbits an average of the exponential growth at many points along the trajectory must be done. To do this one begins with a reference trajectory called a fiduciary by Wolf etc. (1985) and a point on a nearby trajectory and then measure $\frac{d(t)}{d_{0}}$. When the condition occurs that $\mathrm{d}(\mathrm{t})$ grows too large, another nearby trajectory is then picked thus defining a new $d_{0}(t)$. Therefore the Lyanpunov exponent can be defined by the following expression: 
(III.36)

$$
\lambda=\frac{1}{t_{N}-t_{0}} \sum_{k}^{N} \log _{2} \frac{d\left(t_{k}\right)}{d_{0}\left(t_{k-1}\right)}
$$

where $\frac{1}{t_{N}-t_{0}}$ is sequence length.

Using this the criteria for chaos is:
a) $\lambda>0$ chaotic motion
b) $\lambda \leq 0$ regular motion

These numbers can only be calculated from data collected from physical experiments or simulations.

d) Lyapunov Dimension

Yorke (1978) demonstrated a relationship between the fractal dimension, information entropy and the Lyapunov exponents. The Lyapunov exponents are a 
measure of the rate at which trajectories that are off the attractor converge toward the attractor. There is a small spherical volume defined by the initial conditions centered at some point on the attractor in phase space is imagined to deform under a dynamic process into an ellipse.

Yorke (1978) have shown that one can calculate a dimension for a fractal attractor based on the Lyapunov exponents. In terms of two-dimensional maps this dimension is defined to be:

$$
d_{L}=1+\frac{\log \left(L_{1}\right)}{\log \left(\frac{1}{L_{2}}\right)}=1-\frac{\lambda_{1}}{\lambda_{2}}
$$

$L_{1}$ and $L_{2}$ are average over the whole attractor and are called Lyapunov numbers where $\lambda_{i}=\log \left(L_{i}\right)$ is done to get the Lyapunov exponent. Generalizing to higher dimensional maps in a $\mathrm{N}$-dimensional phase space a more complex procedure is required. The Lyapunov exponents must be ordered in the following manner:

$$
\mathrm{L}_{1}>\mathrm{L}_{2}>\ldots \ldots>\mathrm{L}_{\mathrm{k}}>\ldots>\mathrm{L}_{\mathrm{N}}
$$


Find $\mathrm{L}_{\mathrm{k}}$ such that the product is:

(III.40) $\quad \mathrm{L}_{1} \mathrm{~L}_{2} \cdots \mathrm{L}_{\mathrm{k}} \geq 1$

The Lyapunov dimension is thus defined to be:

(III.41) $\quad d_{L}=k+\frac{\log \left(L_{1} L_{2} \cdots L_{k}\right)}{\log \left(\frac{1}{L_{k+1}}\right)}$

Yorke (1978) have shown that this is a lower bound on the capacity dimension.

(III.42) $\quad d_{L} \leq d_{c}$ 
e) Hurst Exponents ${ }^{12,16,60,61,66}$

Standard statistical analysis makes the assumption that the system under study is primarily random. These means that the system under study is causal and has many complex interacting parts. Due to that complexity, the interaction of these parts can not be explained deterministically. Only the use of probability can aid in understanding this process and thus allow one to take advantage of the systems actions. The reasoning behind this approach is that randomness and determinsim can not simultaneously coexist. However we know of situations where the system is a mixture of non-linear stochastic and deterministic processes, e.g. a IFS based system. These systems are not describable by Gaussian statistics.

Standard Gaussian statistical analysis is effective only under a very restrictive set of assumptions. The Central Limit Theorem says as $n \rightarrow \infty$ where $n$ is the number of trials the limiting probability distribution of a random system is the normal distribution or the famous bell-shaped curve. Events measured must be "independent and identically distributed" (IID). The trials or events must not influence one another, and they must all be likely to occur. We people statistically model a large complex system an assumption of normality or near-normality is made. What if this assumption is not valid? For example what if nearby values influence the value of each individual data points, such as a DNA sequence. A system like this is not IID. 
If the system under study is not IID or even close, then a non-parametric method must be used. There is a very robust technique, which was developed by H.E. Hurst a famous British hyrdologist. He published this method in a 1951 paper, "The Long-Term Storage Capacity of Reservoirs". On the surface this paper only dealt with reservoir design, however Hurst extended his analysis to include many natural systems. His effort produced a new statistical methodology for distinguishing random and non-random systems, the presistence of trends and the duration of cycles. This method called R/S analysis (rescaled range analysis) can distinguish random series from a fractal series.

This test statistic was developed while Hurst worked on a Nile River Dam project around 1907 and remained to study the river for the next forty years. This dedicated effort propmted some Egyptians to nickname him, "The Father of the Nile". The reason for this study was reservoir control. Ideally a reservoir should never overflow because a control mechanism is in place that would always discharge the correct amount of water for that time of year. If the influx of water was too low then the reservoir would become too low thus endangering crops. Hurst modeled rainfall as a random walk since this was in his mind the one thing that he had no control over. Random walk models are a common way to depict a system that has many degrees of freedom. Hurst measured reservoir deviation from its average over time. The range of these deviations changes depending on the length of time used for measurement. If a random series the range, $r$ would increase with the square root of the time. This is cased the $\mathrm{T}^{\frac{1}{2}}$ rule. Hurst standardized this measurement over time by creating a dimensionless ratio by dividing the range by the standard deviations of the observations. This analysis is the definaition of re-scaled range 
analysis i.e. R/S analysis. Hurst showed that most nature phenomena, including river discharges, temperature, rainfall, and sunspots followed a "biased" random walk. These series were trends with noise. The strength of the trend and the noise level could be measured by how the re-scaled range scales with time. In other words how much greater then 0.5 .

This new statistic that Hurst developed is very robust. It makes no assumptions about the "state" of the data being studied and it can classify time series. It can tell a random series from a non-random series, even if the random series is non-Gaussian. Hurst found as was stated above that natural systems are not random walks, Gaussian or not.

This research intends uses Hurst's methodology to study DNA sequences after they have been mapped by a IFS algorithm. This was done to visually show any underlying structure in the data. As has been stated previously this data is not a times series but position series. The Hurst technique is used to determine whether they are biased random walks or not. It is shown that there are sections of the DNA sequence that are closer to the random walk value and sections that have a clear bias. The operating assumption is the more the bias the more important biological effect of the section of the sequence

Hurst's methods for a general data seriesa re shown by the formulas below: Consider a data series $t$ with $u$ observations 
(III.43) $\quad X_{t, N}=\sum_{u=1}^{t}\left(e_{u}-M_{N}\right)$

where $X_{t, N}=$ cumulative deviation over $\mathrm{N}$ periods

$e_{u} \quad=$ a measurement value at a particular observation $u$

$M_{N}=$ average $e_{u}$ over $\mathrm{N}$ periods

The range is then difference between the maximum and minimum values of $X_{t, N}$

$$
R=\operatorname{Max}\left(X_{t, N}\right)-\operatorname{Min}\left(X_{t, N}\right)
$$

where $\mathrm{R}=$ range of $X_{t, N}$

$$
\begin{aligned}
& \operatorname{Max}\left(X_{t, N}\right)=\text { maximum value of } X_{t, N} \\
& \operatorname{Min}\left(X_{t, N}\right)=\text { minimum value of } X_{t, N}
\end{aligned}
$$

To compare different data series the range is divided by the standard deviations of the original observations. This is mathematical definition of "re-scaled range" and this should increase with time. Hurst then developed the following relationship:

$$
R / S=(a \cdot N)^{H}
$$




$$
\text { where } \quad \begin{aligned}
& \mathrm{R} / \mathrm{S}=\text { re-scaled range } \\
& \mathrm{N} \quad=\text { number of observations or periods } \\
& \mathrm{a} \quad=\text { a constant } \\
& \mathrm{H} \quad=\text { Hurst exponent }
\end{aligned}
$$

From a statistical mechanical viewpoint if $\mathrm{H}=0.5$ then the series is a random walk. That is the range should increase with the square root of time, N. Hurst applied "creation" to the Nile River water discharge and found much to his surprise, $\mathrm{H}=.90$. He then applied his statistic on other rivers and consistently found that $H>0.5$. He then examined different physical phenomena and in all cases $H>0.5$. When $\mathrm{H}$ differs from 0.5 the observations are not independent, each observation contained a "memory" of all the events that preceded it. This is not short-term system memory usually referred to as "Markovian" memory. This memory is long-term, in theory it lasts the entire length of the data sequence. Recent events have greater impact then more then more distant events. A system that shows Hurst statistics is a system that is a result of long stream of interconnected events. The impact of the present on the future can be expressed as a correlation:

$$
\mathrm{C}=2^{(2 \mathrm{H}-1)}-1
$$




$$
\begin{aligned}
& \text { where } \mathrm{C}=\text { correlation } \\
& \mathrm{H}=\text { Hurst exponent }
\end{aligned}
$$

There are three distinct classification of the Hurst exponent $\mathrm{H}$ :

$$
\begin{array}{ll}
\text { (III.47) } & \text { 1) } H=0.50 \\
\text { 2) } 0 \leq H<0.50 \\
\text { 3) } 0.50<H<1.00
\end{array}
$$

$\mathrm{H}=0.5$ means a truly random sequence. Events in this sequence are random and uncorrelated and III.46 will equal zero. In this sequence the present does not influence the future. Its probability density function can be Gaussian but it doesn't have to be.

Consider the second Hurst classification $0 \leq H<0.50$. This type of system is antipersistent, i.e. an ergodic series. Third type of series is sometimes referred to as "mean reverting". If a system has been up in the previous period it is more likely to be down in the next period. The strength of this anti-persistent behavior is a function on of how close $\mathrm{H}$ is to zero. The closer $\mathrm{H}$ moves to zero the closer $\mathrm{C}$ moves to -0.50 or negative correlation. This kind of a series would be more volatile then a random series and it would have frequent reversals. 
The third classification $0.50<H<1.00$ is a condition which means that the series is a persistent or trend-reinforcing series. When the series has been up (or down) in the last period, the chances are it will continue to be up (or down) in the next. Trends in the data should be readily apparent. The strength of this trend reinforcing condition is a function of how $\mathrm{H}$ approaches 1.0. If $\mathrm{H}=1$ the data is $100 \%$ correlated. The closer $\mathrm{H}$ approaches 0.5 , then noisier the data it will be, and the less well defined the trends are. Persistent series examples are fractional Brownian motion or biased random walks. The strength of the bias is dependent on how far $\mathrm{H}$ is above zero. Dynamically speaking there is correlation between events across the series time or position scales. This relationship means the probability of two events following one another is not .50 . The true probability theorist would very likely object to use of the word "probability" in describing these Hurst values. They are essentially "confidence" measures more so then true probabilities. The Hurst exponent, $\mathrm{H}$ describes the likelihood that two consecutive events are likely to occur. If $\mathrm{H}=0.60$ then in a sense a one could think of the value as $60 \%$ confidence that the trend will be repeated with the next value. That the if the last move was positive the next move will also be positive.

Since each point in the sequence is not equally likely like a random walk, the fractal dimension of a probability distribution is not 2 but a number between 1 and 2 . Mandelbrot (1972) demonstrated that the fractal dimension is the inverse of $\mathrm{H}$. Consider the random walk again where $\mathrm{H}=0.5$, it will have a fractal dimension of 2 the inverse of $\mathrm{H}=0.5$. A random walk that is truly two-dimensional would fill up a plane. If $\mathrm{H}=0.7$ the fractal dimension is the inverse of $\mathrm{H}$ that is $H^{-1}=0.7^{-1}=1.43$. If $\mathrm{H}$ moves closer to 1 the series 
grows less noisy and has more consecutive observations with the same signs. As $\mathrm{H}$ increases the cumulative line becomes smoother and less jagged. There is less noise in the system and the "trends" or deviations from the average, become more pronounced. The Hurst exponent, $\mathrm{H}$, shows how jagged the time series is. A perfectly deterministic system produces a smooth curve. A fractal time series divides a pure random series from a deterministic system perturbed by random events.

\section{f) Information and Lyapunov Exponents}

The characteristic feature of chaotic systems is sensitivity to initial conditions. This implies divergence of initially dynamic adjacent states. The initial state could be known to a high but not infinite degree of precision. The ability to predict future states later decreases because of the diverging trajectories. This means that information is lost. Looking at it conversely, more information is needed to specify the system with the original initial precision. Therefore entropy has increased. ${ }^{46,47}$

In many systems the information function has a simple linear dependence. This is expressed in the following equation: $:^{34,36}$

$$
I(t)=I_{0}+K t
$$


$I(t)$ depends on the probabilities, $p_{i}$ that change as the system evolves and the length, $\varepsilon$, of the elemental unit that is used to partition the space. The rate of information change is the Kolmogorov Entropy, K. It is an average rate taken over long time periods, although in a numerical calculation T would have to be less than the time taken to cover the attractor. Using the above stated conditions $\mathrm{K}$ can then be defined as:

$$
K=\lim _{\varepsilon \rightarrow 0}\left[\lim _{T \rightarrow \infty}\left[\frac{I(\varepsilon T)}{T}\right]\right]
$$

Consider an example of Kolmogorov Entropy imagine a dynamic system that is capable of displaying three types of motion:
a) deterministic, non-chaotic motion,
b) deterministic, chaotic motion
c) random motion.

K's value is dependent on the type of motion. Again as stated before, the phase space is divided into $\mathrm{N}$ small regions, each with $p_{i}$. Let the system in a known particular initial 
phase region exist and let the initial probabilities be zero except the corresponding probability for that phase region and let it equal one. Therefore all the initial information entropy is zero. The above three kinds of motion cause different types of evolution and therefore different changes in the information function.

During a non-chaotic time evolution the system's phase trajectories remain close together. As time $\mathrm{T}$ increases nearby phase points are closely grouped in a small region of phase space. and there is no change in information. The Kolmogorov entropy is zero. In a chaotic time evolution the system's phase trajectories diverge, and the number of phase regions after time $T$ is

(III.50) $\quad \mathrm{e}^{\lambda_{+} \mathrm{T}} \lambda_{+}$is a positive Lyapunov exponent.

If all regions are as before assumed to be equi-probable, the information function becomes:

(III.51a) $\quad I(T)=-\sum_{i=1}^{N} p_{i}(T) \log \left(p_{i}(T)\right)=\log \left(e^{\lambda_{+} T}\right)$ 
(III.51b) $\quad I(T)=\lambda_{+} T$

Therefore the Kolmogorov entropy in the chaotic case is $\lambda_{+}$. In a very short time the random evolution for all phase space regions becomes possible, and assuming equal probabilities the information entropy is:

(III.52) $\quad I=\log (N) \mathrm{N}$ is the number of phase cells.

$\mathrm{N}$ is finite and each cell has a finite probability of being instantly accessed. This implies an infinite rate of information expansion. Therefore in the case of random evolution the Kolmgorov entropy is infinite.

The previous paragraph makes it evident that the Kolmogorov entropy for chaotic systems depends on the positive Lyapunov exponents. In system with several positive Lyapunov exponents $\mathrm{K}$ is:

$$
K \leq \sum_{i=1}^{j} \lambda_{i} \mathrm{j} \text { is the index of the smallest positive exponent }
$$


Grassberger and Procaccia (1983) have shown that (III.53) is usually a equality. Therefore knowledge of the Lyapunov exponents provides a good estimate of the Kolmogorov entropy .The categorization is as follows:
a) $K=0$ the motion is regular i.e. periodic, quasi-periodic, or stationary
b) $K>0$ the motion is chaotic
c) $K=\infty$ then the motion is random.

An important feature of Kolmgorov entropy is that it may be used to estimate the time for which future predictions of the state of a chaotic system may be valid After this prediction time the systems uncertainty has diverged to the size of the space phase space. The system can only be describes by a probability density that depends on location in phase space. So for prediction purposes the system is probabilistic.

A system that is characterized by a positive Lyapunov exponent $\lambda_{+}$and its initial state defined within a size $\varepsilon$ At time $T$ the uncertainty in the coordinates have expanded to the size of the attractor: 


$$
\text { (III.54a) } L \approx \varepsilon \cdot e^{\lambda_{+} T}
$$

or

$$
\text { (III.54b) } L \approx \varepsilon \cdot e^{K T}
$$

Either one of these two relations can be solved for the prediction time:

$$
\text { (III.55a) } T \approx \frac{1}{\lambda_{+}} \log _{2}\left(\frac{L}{\varepsilon}\right)
$$

or

$$
\text { (III.55b) } T \approx \frac{1}{K} \log _{2}\left(\frac{L}{\varepsilon}\right)
$$

The prediction time increases only logarithmically with the initial measurement. This is way chaotic states only allow short time prediction. 


\section{C) Appropriate Literature Review on Transform Based Pattern Analysis}

The search for patterns in the sequence of bases in long DNA sequences is an active topic in molecular graphics. Periodicity and various patterns affect physical,

chemical and biological properties of the DNA. ${ }^{62,69}$ The usual methods of analyzing a time series has been to expand the series into a set of Fourier modes using operational mathematics. If the number of modes is small, then the dynamics of the system can be expressed in terms of the dynamics of these modes. Mathematically this means that the times series for a quantity $\mathrm{X}(\mathrm{t})$ maybe expressed in the form

$$
\text { (III.56) } X(t)=\sum_{m=1}^{M} A_{m} e^{i \omega_{m} t}
$$

with $\mathrm{M}$ being a small integer. The power spectrum

$$
\text { (III.57) } P(\omega)=\left|\int X(t) e^{i \omega t}\right|^{2}
$$

is just a series of delta functions at $\omega=\omega_{m}$ (where $\mathrm{m}$ ranges from 1 to $\mathrm{M}$ ) of strength $\left|A_{m}\right|^{2}$ This method is useful if the power spectrum consists of a finite number of distinct peaks. In practice, the presence of external noise broadens the delta functions, but if the noise is not too strong, the peaks in $P(\omega)$ can still be recognized and hence $A_{m}$ and $\omega_{m}$ can be 
calculated. The above expression for $\mathrm{X}(\mathrm{t})$ then gives a smooth or noise-free representation of the original data.

The goal is how best to represent these smoothed data. One way is to represent these smoothed data. One way is to use the phase plane where $X(t)$, for example, is plotted against $\frac{d X}{d t}$. The latter quantity is readily obtained from the above equation, but not from the original noisy data. For $M=1$ which means one mode, such a plot would show a simple closed loop, whereas for $\mathrm{M}=m_{\omega}$ then $\left(m_{0}+1\right)$-dimensional loop or torus. This depends on whether or not the frequencies are harmonically related. If one using this type of analysis shows how a single time-varying quantity $X(t)$ can give multidimensional information. In most experimental data the power spectrum $P(\omega)$ has little structure. If this is the case, use of the above equation is not really appropriate although a Fourier transform integral does convey some information.

It is important to remember that Fourier analysis is based on finding the appropriate frequency modes using a basis function such as $\mathrm{e}^{\mathrm{i} \omega t}$, the complex representation of a sinusoids i.e. $(\cos (\omega)+j \sin (\omega))^{4}$. Fourier analysis unfortunately misses transient behavior in the series being studied. If a time series has transients, i.e. short duration signals, which are mathematically, like impulses. Fourier analysis will average these short duration signals through the rest of the series. This essentially smooths the data and disperses the transient information. This is why that classical Fourier analysis is inappropriate for data that requires a time-frequency approach. This problem is handled by using a different set of basis functions. A good example of this is the relatively new 
technique time-frequency analysis called wavelet transforms. In wavelet transforms a narrow function (the basis function) of width $\pm \Delta$ t centered on time $t$ is moved through the time series and convoluted with the data. One can think of this process as examining the data with a microscope whose field of view $\Delta t$ and position $t$ takes on all possible values. If the structure is confined primarily to a particular range of $\Delta t$, it means most of the variation occurs on that time or position scale. Systematic variations with $t$ can indicate a lack of stationarity in the data. Another time-frequency technique which will be examined is Wigner-Ville analysis. ${ }^{35}$

1) Analysis of DNA Sequences Based on Fourier Transforms

A 3-D Fourier transformation was performed on a 4000 base-pair human bladder ocogene sequence ${ }^{62}$. Ocogenes have been detected in tumors representative of each of the major forms of human cancer and have been shown to be able to induce malignant transformations in certain cell lines. The bladder carcinoma ocogene is derived from a sequence of similar structure present in the normal human genome Several prominent features can be seen on this mapping, these features correspond to biologically important area of the DNA sequence. The largest peak occurring roughly between base 590 and 900 correspond with a sequence which when deleted drastically reduces the transforming activity of the ocogene. This indicates that this non-coding sequence plays a crucial role. It may be possible to discover some other interesting periodicites in the DNA sequence by calculating other 3-D Fourier mappings where the base-to-number assignments are 
weighted by relative molecular weight, electrostatic potential or some other physical parameters. ${ }^{62,69}$ This could be done in automated fashion so then the human analyst could examine important features and parameters that he/she might not normally see.

$$
\text { (III.57) } F(\bar{\omega})=\frac{1}{(2 \pi)^{3}} \iiint f(\bar{x}) e^{-j \bar{\omega} \bar{x}} d \bar{x}
$$

A spectrogram is generated much like a speech spectrogram is done in signal processing of speech, A plot of 3-dimensional plot amplitude vs. time vs. frequency is calculated but instead of time being the one of the axis its replaced by the DNA base's position in the sequence. The other axis is defined to be frequency. Amplitude is usually defined by false color, grey scaling or by a numerical amplitude. This axis is perpendicular to the plane defined by frequency \& position

Two examples of methods to calculate a standard 2-D Fourier power spectrum ${ }^{4}$ is shown below. Power is arbitrary and the maximum frequency is the Nyquist critical frequency. (The Nyquist critical frequency is the reciprocal twice the interval between the data points, i.e. $1 / 2 \Delta t$. (Note $\Delta \mathrm{t}$ is position difference not a time difference.) The data sequence is truncated at the largest integer power of two and calculated in nonoverlapping segments to reduce spectral leaking from nearby frequency intervals. ${ }^{63}$. 
2) Wavelet Transformation Analysis ${ }^{20,35}$

In the wavelet transform, a narrow function of width $\pm \Delta t$ centered on $t$ is moved through the data sequence and convolved with the data. The amplitude of the convolution is plotted versus $\Delta t$ and using "t" which is scaled in some fashion. Typical transform functions are the Daubachies family of functions. These functions range from the two coefficients Haar that emphasizes the ability to detect impulsive events up to the 12 coefficient Daubachies which has traded off accuracy in locating transients for better frequency resolution.

This basically is a process in which the data is passed through a series of bandpass filters in which higher frequency components are filtered out and displayed. If you look at the above plot as you go toward the top of the page high frequency (rapidly changing) information is removed by the band-pass filter nature of the wavelet transform. The field of view is $\Delta t$ and it position $t$ is allowed to range from $\pm \infty$ The structure is held to a particular range of $\Delta t$. This is where the variation occurs on that time scale. Systematic variations with $\mathrm{t}$ can show a distinct lack of stationarity in the data. It is also important to vary the wavelet type.

3) Wigner-Ville Analysis ${ }^{35}$

The Wigner-Ville method of analysis is a technique, which has similarities to both the Fourier analysis and wavelet methods. It is a projection of a data signal onto a family 
of sinusoids as in the case of the Fourier transform; however it also depends on time or position translations of the series. The Wigner-Ville method is particularly good at uncovering periodic patterns in the data sets where the series is not stationary (i.e. the data may have a strong trend). By using the Wigner-Ville analysis, the data series can be decomposed into its frequency components which vary in temporal or spatial duration.

$$
\text { (III.58) } \Phi_{x x}(\omega, t)=\frac{1}{2 \pi} \int_{-\infty}^{\infty} x\left(t-\frac{\tau}{2}\right) x\left(t+\frac{\tau}{2}\right) e^{-i \omega t} d \tau^{\prime}
$$

$\Phi_{x x}(\omega, t)$ is called the Wigner distribution and is function of $\omega$ and $t$ for the series in question $x(t)$. Wigner first used this distribution in quantum mechanics and Ville used it in harmonic analysis hence the name Wigner-Ville. However there are some difficulties with this method because of the infinite range of integration. This makes it impossible to have a truly local spectral density because of the continuos nature of harmonic waves. Since it retains much of the character of Fourier analysis there is the fundamental uncertainty that 
is characteristic of time-dependent spectra, so high resolution in time and frequency is all but unobtainable.

\section{Analysis Based on Non-Linear Dynamics}

Chaotic dynamics have been known to exist as something separate from "noise" for some time now. Mathematicians such as Poincare at the tun of the century, then early efforts by Birkhoff, Cartwright, Littlewood, Levinson, Smale and Kolmogorov increased knowledge of this field. It remained a obscure mathematical curiosity until the advent of digital computers. In the mid-1970's this changed dramatically. Lorentz's work which shows the limits of weather prediction to Glass's work on chaotic rhythms in cardiac activity chaos has proved relevant to a wide range of scientific and engineering topics ${ }^{47,48}$.

The field of chaotic dynamics has matured enough to a point where the original goals of demonstrating that chaos occurs in a wide range of physical phenomena and is not to be dismissed as a "curiosity" The goal is no to show that in many cases, chaotic dynamics are a fundamental property of that phenomenon. Research has moved on to where a chaotic time series can be examined and characterized. In most cases the underlying dynamics are not known or they maybe known in principle but are too complex to solve directly. In these cases of analyzing an experimental time series data from chaotic systems methods for calculating dimensions, Lyanpunov exponents, Hurst exponents and orbits have been developed to characterize the hidden dynamics. 


\section{E. Chaotic Modeling of Data Sequences}

The objective of any experimental data analysis is to find a pattern or structure that models the data. The structure in itself may not be simple or easily decipherable. However once this structure is found the data has a new simplicity. The data that produces a Lissajous figure can be expressed as two Fourier modes with constant amplitude and frequency. Most of the time a simple picture is an incomplete picture. Consider the example of the turbulent behavior of fluids, such as water. The appropriate data here requires a large number of Fourier modes that form a continuum.

The mathematical study of dynamical systems has shown that the solution of simple, non-linear equations can exhibit complicated temporal and spatial behavior. The equations and system of equations show a range of behavior from simple periodic to chaotic. The concept that underlies this is the strange attractor, which was discussed. Simple attractors such as a system in thermodynamic equilibrium (point attractor) or those which decay into periodic states (limit cycles) have been known for some time. A strange attractor has the property that the system under study decays to (is attracted to) a final state, but this state is not periodic and is extremely complex. It is chaotic or pseudorandom but arises as the solution to a set of deterministic equations and is highly sensitive to initial conditions.

In applying non-linear dynamical analysis to analyzing time series, $X(t)$ or in the case of this study a position series, $X(r)$ two assumptions are made. ${ }^{22,30,44}$ The first 
assumption is the presence of a strange attractor can lead to a complicated time series. The second assumption that such phenomena can be described by simple equations. These equations may be differential equations in which the quantity $X$ varies continuously in time, $\mathrm{t}$ (or position, $\mathrm{r}$ ) is sampled at different discrete time intervals, or they may be difference equations in which the quantity $\mathrm{X}$ changes abruptly from one step to the next. The presence of a strange attractor is best revealed from phase-space plots; if the data were given at sufficiently short time intervals and did not have a significant noise component, then the phase-space plot can be constructed with components $X(t)$ and $\frac{d X(t)}{d t}$ and higher derivatives, then the whole data record is represented as a trajectory in phase space. The procedure is to consider higher and higher dimensional phase spaces until an increase in the dimension did not change the topology of the underlying structure. A plot in a two dimensional phase space of the trajectory for a simple loop would still remain a simple loop if plotted in a higher dimensional space.

Once a strange attractor has been identified, its nature dimension and its nature can be quantified by calculating various measures of its dimension and its Lyapunov exponent. The dimension is a measure of the complexity of the phase-space trajectory whereas the Lyapunov exponent is a measure of sensitivity to initial conditions. In order to get an accurate measure of the attractor's dimension, it is theoretically necessary to embed it in a space of dimension at least $2 d+1$ where $d$ is the least integral dimension containing the attractor. 
There are various (in fact, an infinite number of) ways to define the dimension of an attractor. The simplest are the Hausdorff and capacity dimensions, which describe the geometric difference of the attractor without regard to how frequently the trajectory visits various locations on the attractor.. The correlation dimension is a lower bound on the capacity dimension. The correlation dimension is more accurate for small data sets because it weighs more heavily those regions of the embedding space that contains the data. It is very difficult to calculate a capacity dimension when the dimension is greater that about two. The number of data points required to get a reliable estimate of the dimension is order of $M^{d}$ where $\mathrm{M}$ has been variously estimated to be in the range of about 10 to 42 . More recent estimates give a minimum number of data points of approximately $10^{2+0.4 d}$. Similar considerations apply for the calculation of the Lyapunov, although it appears that a somewhat smaller embedding dimensions suffices in practice. The reliability of the results depends on the system and no rigorous methods for determining errors are known that work for all cases.

1) Tests for Chaotic Structure Based on Singular Value Decomposition ${ }^{75}$

Another method of analysis is called singular value decomposition also known as principal component analysis. These methods originated in statistics and were used to study linear problems. They have recently been applied to non-linear problems. The technique replaces expansion in Fourier modes by an expansion in terms of another 
complete set of functions. A set of functions is generated from the data unlike the Fourier method in which trigonometric basis is imposed on the data set. In other words

$$
\text { (III.59) } \quad \mathrm{x}(\mathrm{t})=\sum_{\mathrm{m}=1}^{\infty} \psi_{\mathrm{m}}(\mathrm{t})
$$

where the functions $\psi_{\mathrm{m}}$ form a complete orthogonal set. Analogous to $P(\omega)$ we can make the following definition

$$
P_{m}=\left[\int x(t) \psi_{m} d t\right]^{2}
$$

which is simply

$$
\text { (III.61) } \quad P_{m}=\left(\int \psi_{m} d t\right)^{2}
$$

This we interpret as the probability of the system being in state $\psi_{\mathrm{m}}$. Analogous to Fourier modes we can see that there are conditions where $\mathrm{x}(\mathrm{t})$ can be represented by a few $\psi_{\mathrm{m}}$. functions. This is just like the case where a few peaks in the power spectrum show that a Fourier expansion can be done with a few numbers of modes. The existence of a few 
dominant values of $\mathrm{P}_{\mathrm{m}}$ shows that here too a expansion can be done with a few $\psi_{\mathrm{m}}$. functions.

Singular value decomposition is a way to generate a orthogonal set of such functions. The technique is performed in the following manner. The data is finite in length, $\mathrm{N}$ and is equally spaced in time. Therefore one can write $x_{m}=x(t)_{t=n \Delta t}$ for $n=1,2,3$. From this finite data set a set of $\mathrm{M}$-dimensional vectors $\mathrm{V}_{\mathrm{e}}$ are then constructed. They are defined such that

$$
V_{e}=\left[x_{l}, x_{l+1}, x_{l+2}, \ldots . ., x_{M+l-1}\right]
$$

An auto-correlation function is then constructed and is defined to be

$$
C(n)=\sum_{l=1}^{m} x_{l} x_{l+m}
$$

These values are then used to build a symmetric $M \times M$ correlation matrix $\overline{\bar{M}}$ with elements 


$$
M_{l p}=C(|l-p|)
$$

This matrix has $M$ eigenvalues denoted by $\lambda_{m}$ and its eigenfunctions $\alpha_{m}$. Using all this we can define the following function set

$$
\psi_{m}(t)_{t=n \Delta t}=\alpha_{m} \bullet V_{n}
$$

These functions are orthogonal and normalized such that

$$
\frac{1}{N} \sum_{l=1}^{N} \psi_{m}{ }^{2}(l \Delta t)=\lambda_{m}
$$

which leads to $P_{m}=\lambda_{m}^{2}$. The eigenvalue $\lambda_{m}$ is a measure of the importance of the mode $\psi_{m}$ in the expansion of $x(t)$. Analogous to expanding in a finite number of Fourier modes, we expand in a finite number, $d$ of the values for $\psi$ and only use those values for $\psi$ that has the largest values of $\lambda_{\mathrm{m}}$. Similar to a Fourier series expansion is an approximation to 
the data in a finite sense a new approximation of the data is generated based on the original data $\mathrm{x}(\mathrm{t})$ This expression is

$$
x_{d}(t)=\sum_{m=1}^{d} \psi_{m}(t)
$$

This gives the best set of orthogonal modes as was discussed in the above paragraph. This method involves some smoothing of the original data and this is desirable since most experimental data has significant noise present. This noise is a non-deterministic part of the signal of interest. If the data series was truly random, a correlation matrix $\overline{\bar{M}}$ would be generated which is a diagonal matrix $\left(C(n)=C_{0} \delta_{n, 0}\right)$ and its eigenvalues are all equal to $C_{0}$. If we have any other type of data the existence of eigenvalues are $\left(\lambda_{\mathrm{m}}\right)$ greater than $C_{0}$ reveals the presence of structure in the data. The above summation equation only sums "d" values so a great deal of the noise is removed. This creates a smoothed version of $\mathrm{x}(\mathrm{t})$. This smoothed version of $\mathrm{x}(\mathrm{t})$ is called $x_{d}(t)$. This method is similar to the Karhussen-Love expansion and was used to study turbulence ${ }^{15}$

A powerful method used to examine the underlying structure of the d-dimensional phase space, which is created by the $\psi_{1}, \psi_{2}, \psi_{3}, \ldots \ldots \ldots ., \psi_{m}$ which are sometimes called the topological functions. Plots of these function can show the topological structure of the solution. This structure is the same as the one constructed by using a phase obtained from 
the vectors $\mathbf{V}$ that has been created from the original data. The presence of noise will mask the topological details of these plots. The desired topological structure is conserved from V-based phase space to a $\psi$-based phase space because the $\psi$ functions are created in such a way as to be linear combinations of the V's. Another important aspect of this technique is the fact that a two-dimensional plot of $\psi_{1}$ vs. $\psi_{2}$ is a rotation of the attractor in a manner that one views from its broadest side. This makes the attractor more readily apparent.

The $\psi$ functions assume a set of equations of motion that have the following form

$$
\overline{\psi_{m}}(t+\Delta t)=F_{m}\left[\overline{\psi_{n}}(t)\right]
$$

The bar over the $\psi^{\prime}$ s is used to denote a value satisfying these model equations and $\mathrm{F}_{\mathrm{m}}$ is a non-linear function of the $\psi_{\mathrm{n}}$ values from a $\mathrm{n}=1$ to $\mathrm{d}$. Since we have assumed that these non-linear function are sufficient to produce chaotic behavior. The above equation can be re-written as

$$
J_{m}=\frac{1}{N} \sum_{s=1}^{N}\left\{\left[\psi_{m}(s+1) \Delta t\right]-F_{m}\left[\psi_{m}(s \Delta t)\right]\right\}^{2}
$$


Where $\mathrm{t}=s \Delta t$ and $J_{m}$ is minimized with respect to the coefficients "a" and "b".

The original data $x(t)$ exists over a finite time interval, $N$. However if the equations that describe the dynamics of the system have been solved for the then $\psi$ functions then they are solved for all time then using equation

$$
x_{d}(t)=\sum_{m=1}^{d} \psi_{m}(t)
$$

as an estimate of $\mathrm{x}(\mathrm{t})$. If it exists, it is called $x_{d}(t)$. This can be viewed as extrapolation method. It is important to keep in mind that the underlying system that produced the equation is chaotic and is very sensitive to initial conditions. Therefore it is a good estimate to $x(t)$ for only a short time after $t=N \Delta t$. The $\psi^{\prime}$ 's do capture the topology of the of the original data, but solutions of the model equation:

$$
\overline{\psi_{m}}(t+\Delta t)=F_{m}\left[\overline{\psi_{n}}(t)\right]
$$


Do not always yield good topological information.

Topological information depends on sufficient data. A large problem in calculating fractal information is $\mathrm{N}$ is too small, that is insufficient data. When the amounts of data are small, the model equation can be used to produce as many data points as need to calculate the dimensionality of the atttractor as well as the Lyapunov exponents. Thus the amount of numerical work to do is greatly reduced if the equation does indeed approximate the topology of the data.

In singular value decomposition there are two important quantities $\mathrm{M}$, the order of the correlation matrix and $d$, the number of significant eigenfunctions kept. These parameters must be modified to obtain the best fit of the real system under investigation and the model solution

$$
\overline{\psi_{m}}(t+\Delta t)=F_{m}\left[\overline{\psi_{n}}(t)\right]
$$

It is very likely that in applying this method there will be situations where the autocorrelation matrix shows very little structure. The complicated signal variation must then be attributed to the presence of an attractor. The parameters $M$ and $d$ must then be modified to best reproduce the features of the attractor 
2) Procedure to Analyze for Chaotic Structure ${ }^{54,56,57}$

In analyzing real experimental data ${ }^{11}$ its always best to try the simple things first. One should look very carefully at ones data and make certain there are no gaps or other anomalies. Real experimental data often shows trends, this could be simply a unstable sensor or could indicate some underlying dynamics that are an integral part of the system dynamics. Examples are the upward (downward) trend in stock market data that shows a prolonged bull (bear) market, climatic data, which shows seasonal variation. These trends might not be relevant so in analyzing the data so the data must be "detrended". This is particularly necessary if there is not sufficient data present to resolve these slowly varying trends. The data can be "detrended" by doing the following: one could subtract from each data point the value obtained from a low-order polynomial fit or a Fourier fit to the data. Also one could use wavelet approach and remove the low frequency coefficients. One could also take successive higher-order differences but it should be remembered that differentiation accentuates the noise, therefore it should only be used in cases of accurately measured data. Differencing works best in cases where data is developed from numerical simulations. Correlation time should be reached in 1 to 10 steps

Data that is "non-stationary" is often difficult to deal with. A system that has a lot of high frequency structure might suddenly change to one with low frequency structure. Behavior such as this can occur in a dynamical system. If it happens infrequently then it is 
very possible that the data set is not long enough to capture all the system dynamics. A test for "stationarity" is to split the data set in half. and verify that system statistics agree for both halves and with the entire data record.

3) Fractal Characterization of DNA Sequences ${ }^{51,59}$

An alternate way of representing and categorizing DNA sequences is to use the concepts of fractal geometry. B. Mandelbrot (1983) developed this field in his book "The Fractal Geometry of Nature ". An important concept developed by Mandelbrot is "selfsimilarity". Self-similarity shows up in the natural world in many patterns and objects. A range of scales can be used to view an original pattern in mountains and coastlines as well as some purely mathematical objects. This "scale invariance" concept was studied extensively by B. Mandelbrot (1975), and he created the term "fractal" to describe these shapes.

Fractal objects have a shape that becomes more and more detailed in its features with increasing magnification like mountain and coastlines which is stated self-similarity. Note the edge of a circle is not a fractal and is featureless with greater magnification, it becomes a straight-line. A single number can characterize random looking shapes of nature. This number is called a "fractal dimension". The different types of fractal dimensions were defined and discussed mathematically in section 2.10. A goal of this research will be to find a suitable fractal dimension and its value for DNA sequences. The fractal dimension of a DNA sequence is actually a measure on how jagged the sequence 
is. A straight-line has a fractal dimension of 1 , the same as its Euclidean dimension. Remember a random walk has a 50-50 chance of going up or going down. If a fractal dimension is between 1 and 1.5 the sequence is more of a line and less then a random walk. It is smoother then a random walk but more jagged than a line.

$$
\text { (III.73) } \quad D=\frac{\log (N)}{\log (1 / r)}
$$

The fractal dimension(s) can be solved as the slope of a log/log plot. In a data sequence, let the radius increase as time or position in the sequence increases and then the number of circles needed to cover the entire sequence as a function of time or position in the sequence. It is clear that the fractal dimension of a data sequence is a function of scaling in time.

However one wishes to define fractal dimensions of a sequence is important because it recognizes that a process can be somewhere between a deterministic sequence (fractal dimension, $d=1$ ) and random (fractal dimension, $d=1.5$ ). The fractal dimension of a line ranges from 1 to 2 . The values, $1.5<d<2$ means a sequence is more jagged than a random series or has more reversals. The statistics of such as sequence is very different from Gaussian statistics and is not normal distributions. 


\section{G. DNA Data Sequence Analysis Based on Visualization}

One of the methods of dealing with this explosion of data is visualization. Human vision is mankind's most powerful analytical tool. It has the largest "bandwidth" of all our senses and is our primary tool for dealing with the physical world. When one looks at a "picture" one, "processes" information and draws correlations that are inherently multidimensional though one is clearly not aware of all this computational activity. Looking at data in a visual sense lets one gain insights to this data. Most analytical techniques are ultimately a form of visualization. An important psychological aspect to consider is that because our reliance on vision, humans are predisposed to "understand" visually presented data rather then data presented through another sense. A spectrogram of speech data is a "visualization" of a time-dependent acoustic energy phenomenon, but the application of the Fourier transform allows the data to be displayed in a manner that shows its frequency dependency as well as its time-dependency. ${ }^{21}$

Visualization methods give the analyst new ways to represent phenomena. The analyst can show multidimensional relationships in data or physical models. Visualization draws on the skills of many disciplines such as computer logic, graphic arts, human interface design, signal processing and electronic publishing. Visualization integrates thinking expands understanding and clarifies communication. The core of visualization is the representation of numeric data into pictorial form. Data may come from many sources or from simulations. What one gets in it most elemental form is pages and pages of 
numbers. Raw numbers left to themselves are meaningless to human interpretation. These numbers are transformed through the use of graphical and other processing techniques into pictures that represent the underlying phenomena being studied..$^{10,21}$

Everyone has done some form of scientific visualization creating a simple $X-Y$ graph is a "visualization" of data. Graphing data has become an art and all can think of many examples of presenting data that has multidimensional dependencies on a single plot. Computer enables the analyst to plot data without the previous level of effort. A computer enables one to analyze large complex data sets. In representing multivariate and field data early main frame computers had problems handling these visually in ways that were meaningful. For these problems the only approaches available were changing character coding ranges and overprinting. ${ }^{18}$

As the size of computers decreased and their inherent computational abilities increased, the computer moved from a large shared resource to a more personal one. The capabilities for direct visual display increased dramatically. This advance was driven more by the video and computer game industry then by anything else. Vector-oriented and pixeloriented display screens permitted allowed easier graphical data. Imaging methods improved and systems were developed to allow the visual representation of static as well as dynamic data. Color and gray-scale monochrome became very popular techniques to represent structure in 2-D data. Commercial systems that use these features are very common now and are used in many disciplines, especially computational fluid dynamics and mechanical design. Important visualization methods became common such as color- 
mapping, graphics and imaging libraries; and graphical user interfaces improved. All one has to do is look at what's available to the personal computer user in the way of graphics to see where the state of the art is today.

The usefulness of a particular visual display method can be a function of many things but it must embody certain desirable features such as descriptive capacity, potential for comparison, aid in focusing the analysts attention and versatility. It is particularly important if the visualization method can reduce the dimensionality of the data.

Pickover (1992) calls visual methods that reduce the dimensionality of multidimensional "iconic graphs". The "graphs" that we are most familiar with displays data in 2-D or 3-D. These "iconic graphs" take higher dimensional relationships in the data and map them into visually apparent features. These visual features then change as the higher dimensional relationships change in the data. These "iconic graphs" such as those proposed below rely on the feature-integration abilities of the human visual system and our psychological pre-disposition to want to relate visually to the outside world.

1) Symmetrized Dot Pattern of DNA Sequences ${ }^{62}$

Data must be displayed in a way that is meaningful to the analyst in order for him to be able to interpret the process that he is studying. For example there are many ways to display acoustical data/ One of the most interesting and colorful that has been used with success is to map the data onto a figure with six-fold symmetry. The technique is to covert 
the sound (or any data) into a collection of dots which are then reflected through mirror planes. The resulting representation is a called a symmetrized dot pattern (SDP), it provides a stimulus in which local visual correlation's are integrated to form a global precept. It can potentially be applied to the detection and characterization of significant features of any sampled data. The symmetry, color and redundancy of the dot-patterns can be used to enhance the ability of a human analyst (Pickover 1986). A SDP can be implemented in the following fashion starting with a data sequence. The data's "jagged" trace on a graph represents how the data varies over time or space. The data is mapped to a six-fold pattern by comparing the loudness of pairs of adjacent points and plotting the results on a polar coordinate graph. The points are then reflected through the axis of the graph. The correlations between adjacent pairs of points determine the structure of the SDP.

This technique has been applied in several biological settings, speech research, and speaker identification and animal vocalization identification. An interesting application that has potential direct human concerns is in phonocardigraphy or electrocardiogram (ECG). The symmetrized dot-pattern display has been used to represent normal and pathological conditions of the heart.

2) Vectorgrams of DNA Sequences ${ }^{62}$

As was stated earlier in this document, DNA contains the basic genetic information of all living cells. The sequences of DNA bases ( $A, C, G$ and $T)$ may hold all the 
information necessary for protein synthesis as well as a variety of regulatory signals. Consider the following example, specific AT-rich regions are thought to be codes for beginning transcription. Also certain specific viral sequences select cancerous changes in cells in animals and in vitro. In addition to containing such regulatory codes and tumor producing codes, DNA sequence and composition are often correlated with physical properties of the DNA such as melting temperature.

Detailed comparisons of DNA sequences are useful and are normally done but computationally intensive statistical calculations, these however leave out information on the structure. Differences between sequences may obscure similarities. Even determining whether a particular sequence is random is very difficult. DNA vectorgrams are a methodology to represent and compare random and DNA vector sequences in such a way as to be more discernible to the analyst.

Vectorgrams look like the famed "random walk" that is so often used to model statistical physical phenomena such as Brownian motion or the stock market. This technique can be used to search for patterns in the sequence of DNA bases. This method involves transforming the DNA sequence to binary data and the subsequent mapping of the data onto a two dimensional cellular lattice. Consider the following example, triply bonded bases (GC) are differentiated from doubly bonded bases (AT) by assigning nucleotide values as follows $G=1, C=1, A=0, T=0$. The sequence generated by these assignments will still produce a structure in which a human would have difficulty telling what is going on let alone differentiate between different sequences. 
This problem is solved by letting the digit strings map into characteristic two dimensional patterns which are drawn on a unit cellular lattice. D.H. Green using basic computer shift registers in 1968 first applied this technique. Using Green's simple conversion method, three digits at a time are examined and assigned a direction of movement over the cellular lattice, e.g. 010 is up and to the right, 000 is up and to the left while 001 is straight up. One can clearly see that for each of the three digit combinations causes a vector to be drawn from a point on the lattice to one of the eight points immediately adjacent according to the above coding system. This procedure is then repeated using serial overlapping windows. Using this approach sequences with a predominance of G's or C's will show a overall movement toward the right lower diagonal. Sequences with high G-C content will show a downward tendency. For each combination of the three bases found in the sequence, there exists at some region another combination, which is the logical inverse e.g., a $G$ and $A$ interchanged 010 and 101 respectively. A repeating sequence such as...GGGGAAAGAATACGAGGGGAA.. generates a trace that returns to its starting point. ${ }^{62,69}$

3) Chernoff Faces ${ }^{62}$

It is often very difficult to represent in a meaningful way let alone interpret multidimensional data which involve complex relationships. Pseudo-color, animation, three-dimensional figures ad a variety of shading schemes are among the techniques 
used to demonstrate relationships not readily apparent from simple correlations based on two-dimensional linear theories.

To show a correlation between two or three variables is simple. One generates a two-dimensional or a three dimensional graph. However if one is trying four, five or ten different variables at once it becomes very difficult to do. Chernoff, a Harvard statistician, developed the Chernoff face method in 1973. The technique uses gradations of various facial features in a cartoon-like face. Such facial feature changes might be changes in degree of eyebrow slant or pupil size, a single face can convey the value of a multiplicity of variables. These "faces" have been shown to be more reliable and more memorable to the analyst then other symbolic representations. These "faces" allow the human analyst to grasp many of the essential regularities and irregularities in the data.

In general $\mathrm{n}$ data parameters are mapped into a figure with $\mathrm{n}$ features each feature varying in size or shape according to the point's coordinate in that dimension. The data sample variables are mapped to facial characteristics; thus, each multivariate observation is visualized as a computer drawn face. This aspect of the graphical point displays capabilities capitalizes on the feature integration abilities of the human visual system and is particular useful for higher level of cognitive processing.

Pickover (1985) applied this technique to speech processing and developed a "autocorrelation-face" which involved 10 facial parameters and each facial characteristic had 10 settings providing for 10 billion possible different faces. The controlled features are: 
1. head eccentricity,

2. eye eccentricity,

3. pupil size.

4. eyebrow slant

5. nose size,

6. mouth shape,

7. eyespacing

8. mouth length,,

9. mouth shape,

10. mouth opening.

Pickover proposed that Chernoff faces can be used to detect irregularities in DNA sequences. The deviation of the form there expected random values causes deviations of the facial parameters from their middle positions. The number of possible DNA characteristics that can be visualized is large (See Pickover 1984, 1989)

4) Visualization of DNA Sequences by IFS (Iterated Function Systems) $)^{39}$

Its very difficult for humans to visualize patterns or properties of long sequences of numbers or characters in one dimensional series. However humans are particularly adept at seeing patterns or structures in two dimensions. Converting these one-dimensional sequences into a graphical form that is inherently a higher dimension then the data is the 
basis of all data analysis. Using the technique of IFS one-dimensional sequences can be mapped into two-dimensional form that preserves the underlying subsequence structure. The subsequence structure may be in the case of DNA of primary importance to the investigator.

The IFS mathematically is "The Chaos Game" which one often sees referenced in the popular literature on chaos and fractals. An IFS is a set of linear mappings with the following form:

$$
\begin{aligned}
& x=a x+b y+e \\
& y=c x+d y+l
\end{aligned}
$$

The new value of $x$ and $y$ is computed from the above mapping. Consider the following example, if the vertices are at $(0,0),(0,1)$ and $(1,0)$ and a random number generator e.g. dice, yields a 3 then vertex 2 is indicated and the new coordinates of the new point are : $x=0.5 \cdot(x+0)=0.5 x$ and $y=0.5 \cdot(y+1.0)=0.5 y+0.5$. These equations can be re-written as:

$$
w(x, y)=(a x+b y+e, c x+d y+l)
$$


The "mapping" can be expressed in tabular form below e.g. for the Sierpinski Triangle

$\begin{array}{lllllll}\text { w1 } & 0.5 & 0 & 0 & 0.5 & 0 & 0 \\ \text { w2 } & 0.5 & 0 & 0 & 0.5 & 0 & 0.5 \\ \text { w3 } & 0.5 & 0 & 0 & 0.5 & 0.5 & 0.5\end{array}$

\section{Table III.1 Mapping Information for the Sierpinski Triangle}

In the case of the "Chaos Game" the die roll or the random number generator governs the choice of the "mapping". Each of these "maps" has an associated probability. For dice and a "good" random number generator the probabilities are equiprobable. If the probabilities are not equal, the shape of the attractor will not change but its details will. In tabular form this is known as a "IFS Code". If the data is generated by something other then a random number generator the attractor will display characteristics that are inherent to that sequence. Jeffrey (1992) refers to the IFS code as a CGR (Chaos Game Realization).

Non-randomness in a sequence implies that the sequence has a "structure" that may or may not be apparent. In a mathematical sense structure implies the presence in the sequence of internal subsequences. If a sequence of numbers has a underlying structure or a non-uniformity based on embedded subsequences. An "IFS Code" can show 
this "visually" by doing this mathematical mapping. Jeffery (1992) demonstrated this could be done on DNA sequences. The bases "T,A,G, and C or U,G,A, and C" are used to generate the probabilities for the "IFS Code". Its much like rolling a 4 sided die.. The 4 bases are associated with the four corners of a square and the following algorithm is applied:

1. The first "G" is plotted halfway between the center of the square and the "G" corner.

2. The next base "A" is plotted halfway between the point just plotted and the " $A$ " corner.

3. The base "A" is plotted halfway between the previous point and the "A" corner.

4. Next "T" is plotted halfway between the previous point and the "T" corner.

This procedure is continued until the sequence is exhausted.

Jeffrey (1992) expressed the above mathematically in the following two theorems: Like DNA an alphabet of 4 character is assumed, "a", "b", "c", and "d". These are assigned to the corners (vertices) of a square from the lower left-hand corner around in a clock-wise fashion.

\section{Theorem 1:}

There is a one-to-one map between the sequence and the interior square, in which the k-th point plotted on the GCR (or IFS Code) of a sequence corresponds to the 
first k-long initial subsequence of the sequence and no other subsequence (limited by the screen resolution). Thus, there is a on-to-one correspondence between the subsequences (anchored at the start) of a sequence and the points of the GCR.

\section{Theorem 2:}

In a GCR whose side is of length 1, two sequences with suffix of length $k$ are contained within the square with side of length $2^{-k}$. Further, the center of the square is given by the following recursive definition:

1. The center of the suffix of 0 length is $(1 / 2 /, 1 / 2)$.

2. If the center of the square containing sequences with suffix $w$ is at $(x, y)$ then

(a) the center of the square containing sequences with suffix wa is $(x / 2, y / 2)$;

(b) the center of the square containing sequences with suffix wb is $(\mathrm{x} / 2,(\mathrm{y}+1) / 2)$

(c) the center of the square containing sequences with suffix wc is $((x+1) / 2,(y+1) / 2) ;$

(d) the center of the square containing sequences with suffix wd is $((x+1) / 2, y / 2)$ 
Features in the IFS display to think about:

1. Note that a base will always be plotted somewhere in the quadrant.

2. The IFS plot displays self-similarity that is the same patterns re-occur at finer detail.

3. The self-similarity and the division into quadrants and sub-quadrants etc., is a property of the non-uniform in the probabilities of the sub-sequences and not a property of RNA.

One of the goals of this research is to examine the usefulness of these techniques for investigating DNA sequence structure. 


\section{Background}

The mathematical sciences have served engineering science as its language by which its language by which the natural world is modeled models the natural world. The focus of mathematical modeling was on smoothly varying and easily calculable quantities and objects. This was due to the limited computational capabilities of the past ${ }^{68,72}$. The ability to compute the behavior of many more dynamic systems has shown that "smoothness" is a very rare phenomenon in nature. Poincare and Mandelbrot were the first mathematicians to understand this and approach modeling of nature from a different perspective $e^{70,80}$. They accepted the discrete non-continuous nature of the world and understood the true geometry of nature is very different indeed. Mandelbrot named this geometry "fractal". It comes from the Latin "fractus" for fragmented or irregular. An object is fractal if it displays the property of "self-similarity"32. This means the object under question has parts that are related to the whole. An example is the branching network of a tree. Each branch and successive smaller branching is different but all are qualitatively similar to the structure of the whole tree ${ }^{10}$. This "qualitative measure" is usually expressed by a scaling relationship. Nature generally exhibits patterns of extreme intricacy and detail which represent scaling relationships.

Although Poincare developed this technique at the turn of the century progress in this field became moribund ${ }^{112}$. Modern high-speed numerical analysis brought this field out of the scientific "attic". High speed computing enables these techniques to be applied to more systems. This is timely because in the second half of the century scientific activity 
has created veritable mountains of data ${ }^{26}$. Scientists and engineers are collecting and creating data faster than methods and tools can be developed to analyze them. Research databases such as the Human Genome Project and NASA's Global Climate Change are approaching terabytes in size and promise to ultimately to grow to be petabytes in size $e^{14,26}$.

One of the methods of dealing with this explosion of data is visualization. Human vision is mankind's most powerful analytical tool. It has the largest "bandwidth" of all our senses and is our primary tool for dealing with the physical world ${ }^{14}$. When one looks at a "picture" one, "processes" information and draws correlations that are inherently multidimensional though one is clearly not aware of all this computational activity ${ }^{85}$. Looking at data in a visual sense lets one gain insight to this data. Most analytical techniques are ultimately a form of visualization. An important psychological aspect to consider is our reliance on vision. Humans are predisposed to "understand" visually presented data rather then data presented through another sense ${ }^{86}$. A spectrogram of speech data is a "visualization" of a time-dependent acoustic energy phenomenon, but the application of the Fourier transform allows the data to be visually displayed in a manner that shows its frequency dependency and its time-dependency. ${ }^{87,90}$

Visualization methods give the analyst the most easily interpreted way to represent phenomena. Visualization techniques can show can show the analyst multidimensional relationships in data or physical models ${ }^{14,26}$. Visualization draws on the skills of many disciplines such as computer logic, graphic arts, human interface design, signal 
processing and electronic publishing. Visualization integrates thinking, expands understanding, and clarifies communication. The core of visualization is the representation of numeric data into pictorial form ${ }^{14,26}$. Data may come from many sources or from simulations. Data in it most elemental form is pages and pages of numbers. Raw numbers left alone are meaningless to human interpretation. That interpretation can be accomplished when these numbers are transformed through the use of graphical and other processing techniques into pictures that represent the underlying phenomena being studied. $^{14,26,21}$

Everyone has done some form of scientific visualization. The creation of a simple $\mathrm{X}-\mathrm{Y}$ graph is a "visualization" of data. Graphing data has become an area of expertise and one can think of many examples of presenting data that has multidimensional dependencies on a single plot. Modern computers enable the analyst to plot data with less effort and permits the analysis of large complex data sets. ${ }^{22,25,26}$

The usefulness of a particular visual display method can be a function of many things but it must embody certain desirable features such as descriptive capacity, potential for comparison, aiding in focusing the analyst's attention, and versatility. It is particularly important that the visualization method reduce the dimensionality of the data $^{85}$.

A major portion of this study investigates a new mathematical methodology to increase the understanding of the way biological "information" is encoded in a sequence. 
Insight will also be gained on how information might adjust to mutations which force it to depart from its original form. In recent years, research into the nature and information bearing efficiency of DNA sequences has been producing more questions than it has been answering, and the need for a new method of interpretation has become apparent.

\section{(A) DNA Review}

Before introducing the details of the methodology used in this study, it is be appropriate to begin with a very brief review of the developments in the area of DNA information representation. This provides the background for the present study.

A DNA sequence is a linear arrangement of base pairs which "fully reflect" available information necessary to code for a particular biological function or characteristic ${ }^{23,27}$. This is analogous to the way a computer program is a code, which contains information structured in a manner so that the program can perform a function or conduct a process. The DNA code is described as "redundant and optimal"119 for the 20 amino acids that are coded from the four base pairs. It is redundant because information-bearing patterns of base pairs are repeated linearly and in an iterative fashion. It is iterative because genes overlap, since more then one protein can be transcribed from the same section of $\mathrm{DNA}^{35}$. It is also optimal because it makes economic use of the nucleotides to do that coding by using a minimal average code length ${ }^{119}$. Arrival of new 
information (i.e. a mutation) causes imperfection. In most cases, this "imperfection" can be weathered by the organism and even corrected by the transcription process. In other cases the introduction of this "new information" is catastrophic the organism dies or at least is impaired. A three dimensional example of the structure of DNA is shown in the figure below ${ }^{23,27,73}$.

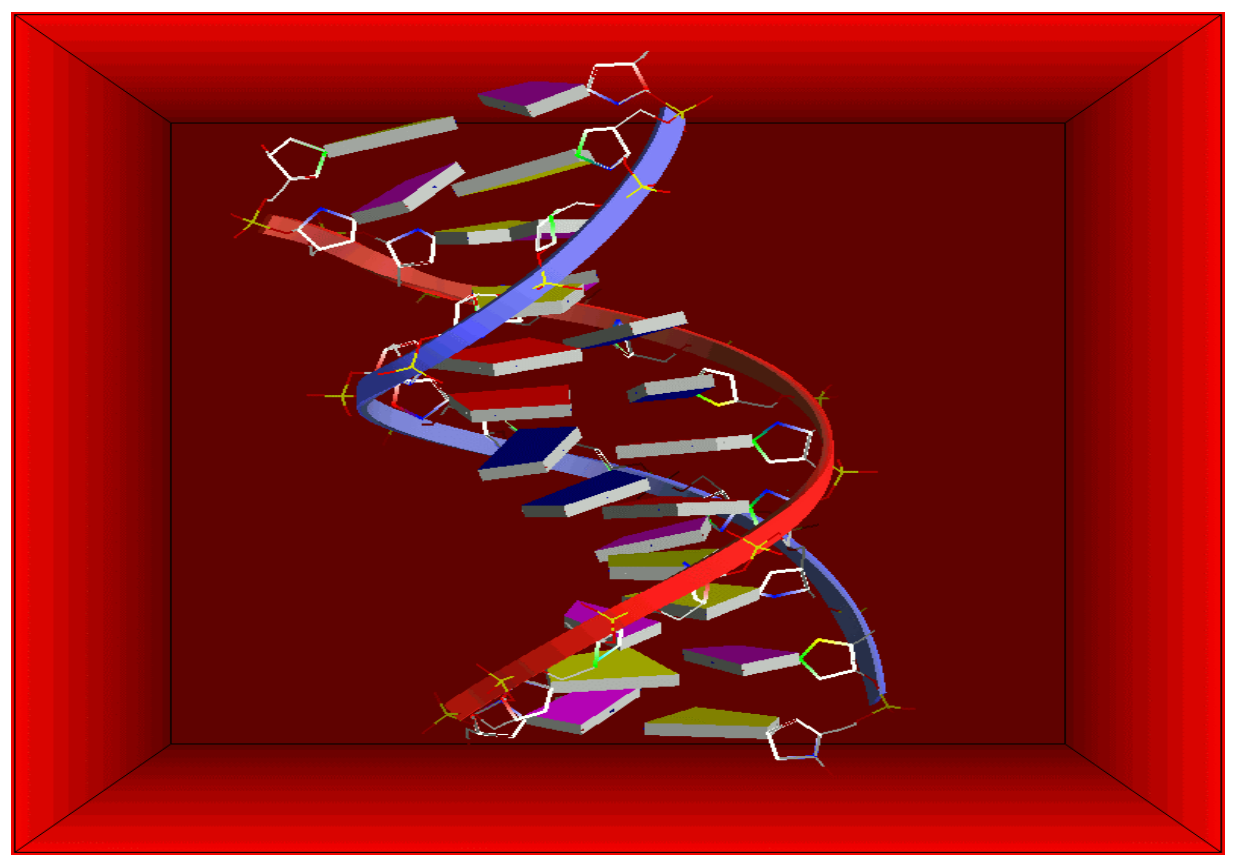

\section{Figure III-1 Classical Spiral Helix of the DNA sequence}

An alternate way of representing information in DNA sequences is to use the concepts of fractal geometry ${ }^{2,3,109}$. B. Mandelbrot (1983) developed this field in his book "The Fractal Geometry of Nature "65. As discussed earlier, an important concept developed by Mandelbrot is "self-similarity". It appears in the natural world in many 
patterns and objects. A range of scales can be used to view an original pattern in mountains and coastlines as well as some purely mathematical objects. This "scale invariance" concept was studied extensively by Mandelbrot $(1975)^{65}$; he created the term "fractal" to describe these shapes.

Since 1992 studies of the modeling of DNA sequences as fractal structures has grown exponentially ${ }^{2,3}$. This fractal representation and biologically interpretations of these representations is subject to debate. DNA walks (also known as vectorgrams ${ }^{45,86}$ ) and CGR (Chaos Game Representation) are the two major mathematical mapping techniques used to convert discrete base pair sequences into a mathematically analyzable form. CGR approaches have been used to examine sequence visualization and pattern recognition while $1-D$ and 2-D random walks have been used to explore long range internal correlations ${ }^{86.89}$.

The previously mentioned title of this dissertation is called "Use of Wavelet Packet Transforms to Develop an Engineering Model for Multi-Fractal Characterization of Mutation Dynamics in Pathological and Non-pathological Gene Sequences". This study uses non-linear dynamic methods to examine the DNA information encoding mechanism ${ }^{49}$. This multi-fractal approach models these sequences as a fractional Brownian motion processes, and will register the observed changes in DNA sequences caused by mutations. Cystic fibrosis DNA sequences were the focus used in this study. Cystic fibrosis sequences both healthy and pathological were modeled as a two dimensional approximation 
of an L-system called an IFS (Iterated Function System) ${ }^{50}$. Wavelet Packet Transforms ${ }^{24,51}$ are used to generate the quantitative measures of complexity of information from these two dimensional representations. IFS mappings are two dimension mappings where a single dimension data set is transformed into a two dimensional graphical representation that presents the data visually. Embedded patterns in the data are then displayed. Wavelet Transforms and Wavelet Packet Transforms historically have proven to be useful in the analysis of complicated signals and erratic time-series processes. In this research Wavelet Packet Transforms are then calculated and used to track changes in base pair pattern structure from the two dimensional mapping. The ability to quantify the information complexity embedded in these sequences will have implications in the future development of genetic engineering.

(B) L-Systems (IFS mapping is an example of an L-System)

In the first analytical part of this study the one-dimensional description of DNA will be transformed into a two dimensional representation using IFS mappings. DNA sequences are a coded information series with a set of combination or production rules, which govern how the sequence can be arranged one dimensionally. The DNA sequence is transformed by the IFS mapping into a two dimensional representation ${ }^{50}$. 
A dynamical system is a physical setting, governed by rules which control this procedures for changing from one "state" to another as time progresses. The goal of a mathematical theory of dynamical systems is to determine and/or characterize the longterm behavior of such systems. There are three types of dynamical systems. They are:

1. Symbolic dynamical systems ${ }^{34}$,

2. Discrete-time systems ${ }^{100}$,

3. Continuous-time systems ${ }^{100}$.

The analytic approach in this research is based on the first type of dynamic system.

In symbolic dynamical systems a physical setting is expressed as a string of formal symbols, which can commonly is taken to be letters a, b, c., d (or for DNA T, A, G, C). The rules for reproducing a string, take the form of rewriting sub-strings, or single letters by replacement strings. These are called rewriting rules. ${ }^{49}$

Consider the following "simple dynamical system" as an example, let there be exactly two symbols "a" and "b" and two rewriting rules, e.g.:

II. 1

$a \rightarrow a a$

and 

II.2
$b \rightarrow b a$

The process begins at the 0 th generation with the string $b$. Therefore by the second rule, Generation 1 is "ba", and after that Generation 2 is
II.3
baaa.

If one continues to use the production rules for a few symbol generations, the following is generated:

Generation String

$0 \quad b$

1 ba

2 baaa

3 baaaaa

4 baaaaaaaa

\section{Example Production Rule Application}

Table III.1 
Its clear after a few generations the long-term nature of the string is a single b followed by a lot of a's.

An aspect of the "fractal" model is that this intricacy is the result of sensitivity to initial conditions of simple dynamical systems over long periods of time. Simple dynamical systems can be expressed by using simple rules or simple grammars. It will be seen how fractal geometry arises from very simply-stated symbolic dynamical systems called Lindenmayer systems or L-systems, after Aristid Lindenmayer who first used them to model biological phenomena

\section{Basic definitions}

L-systems (Lindenmayer systems) are a particular type of symbolic dynamical system. They have the added feature of a geometrical interpretation of the evolution of the system. Aristid Lindenmayer invented this approach in 1968 to model biological growth. The limiting geometry of even very simple systems can be extraordinary fractals. The components of an L-system are as follows:

Alphabet:

The alphabet is a finite set $V$ of formal symbols, usually taken to be letters $a, b, c, d$ etc., or possibly some other characters. In this study the characters are T, A, G, C, i.e. the DNA bases! 
Axiom:

The axiom (also called the initiator) is a string of symbols from $\mathrm{V}$. The set of strings (also called words) from $\mathrm{V}$ is denoted. Given,

$$
\text { II.4 } V=\{a, b, c, d\}
$$

Some examples of "produced" words are aabca, caab, b, bbc, etc. The length $|w|$ of a word $w$ is the number of symbols in the word. In the case of DNA words would be codons and at a higher level codons would be amino acids. (Condons are subsets of DNA.)

\section{Productions:}

A production (or rewriting rule) is a mapping of a symbol $a \in V$ to a word $w \in V^{*}$. ( $V$ is a symbol space.) This will be labeled and written with notation: 


\section{II.5}

$$
p: a \rightarrow w
$$

Possible production mappings of "a" to the empty word, denoted $\varnothing$, or to "a" itself. If a symbol does not have an explicitly given production, we assume it is mapped to itself by default. In that case, "a" is a constant of the L-system.

Types of L-systems:

One type of L-system is a context-free process; an example of this is a Fibonacci system. This system uses production rules that have no memory. To take account only of an individual symbol, and not of what their neighbors are. Another type of L-system is a context-sensitive process. The production rules in this system have memory. The production rules apply to a particular symbol and only if the symbol has certain neighbors. The rules for grouping DNA in its codons are very similar to this. Consider the following example from 'Unraveling DNA' a book by noted DNA research scientist Maxim D. Frank-Kamenetskii ${ }^{35}$. Its 
graphical representation of the "rule set" for RNA, but the idea still holds for

DNA.

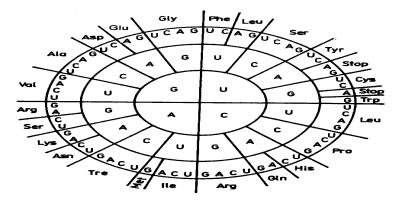

Figure III-2 A Pictorial Representation of the Production Rules for an RNA Sequence. (Page 24 Figure 7 Chapter 2 of 'Unraveling DNA' ${ }^{35}$ 


\begin{tabular}{|l|l|l|}
\hline $\begin{array}{l}\text { Coding } \\
\text { Level }\end{array}$ & Symbol Sets & $\begin{array}{l}\text { Function } \\
\text { (Production } \\
\text { Rules) }\end{array}$ \\
\hline 1 & $\begin{array}{l}\text { base-pairs (nuleotides; } \\
\text { T,A,G,and C) }\end{array}$ & transcription \\
\hline 2 & amino acids & translation \\
\hline 3 & proteins (primary structures) & gene expression \\
\hline 4 & proteins (tertiary structures) & gene expression \\
\hline 5 & protein clusters & $\begin{array}{l}\text { higher levels of } \\
\text { gene expression }\end{array}$ \\
\hline 6 & $? ? ?$ & $\begin{array}{l}\text { unknown levels of } \\
\text { gene expression }\end{array}$ \\
\hline 7 & "'" & $\begin{array}{l}\text { unknown levels of } \\
\text { gene expression }\end{array}$ \\
\hline$:$ & "'" "'" "'" \\
\hline$:$ & "'" & "'" \\
\hline$(\mathrm{N}-1)$ & & organism \\
\hline $\mathrm{N}$ & physical, mental, and \\
psychological traits & \\
\hline
\end{tabular}

III-2 Model of a Biological Formal (L-System) System ${ }^{49}$ 
In DNA at each coding level "production rules" or "grammars" are applied to manipulate the symbol sets to go to the next level. Then the process is repeated building a biological entity at each level. This continues until the organism is constructed.

(C) Elements of Fractals

The next part of this research the DNA will be considered for its fractal properties. The concept of fractals ${ }^{10}$ has caught the attention of researchers in many fields and there are a considerable number of publications on this topic.

In this section, the proposed piecewise fractional Brownian motion (fBm) model ${ }^{78}$ shows how DNA sequence patterns might adjust to new information e.g., mutations is discussed. Fractional Brownian motion, introduced by Mandelbrot and Van Ness (1968), has been applied in many diverse areas. Benoit Mandelbrot is, of course, most famous for the development of the field of fractal geometry or fractals (Mandelbrot $(1977,1982,1986))^{9,65}$. Essentially, there are two types of fractals: (i) fractal shapes, and (ii) fractal time-series ${ }^{44,80}$. This study will eventually attempt to compare DNA sequences to fractal series generated by fractional Brownian motion. This discussion touches upon fractal shapes since these help to readily describe the properties of fractals. 


\section{(D) Fractal Geometry}

Fractal geometry ${ }^{72,80}$ is concerned with fractal shapes and is often described as the geometry of nature. This definition of fractal geometry is in opposition to classical Euclidean geometry that is described as the "geometry of man". The traditional geometry derived from Greek thought gave us the point, the onedimensional straight line, the two-dimensional plane, and the three-dimensional solid $^{38}$. Symmetry and orderliness represented perfection to the ancient Greeks and they sought to explain structures in terms of perfect forms. This is the reason man-made objects are so symmetrical. Most of the shapes in nature are very complicated and not as well ordered as man-made creations. It seems then that there is a certain structure in the natural world that everyone is consciously aware of, but an understanding of these shapes has been denied to us because we only have had the opportunity to learn the language of classical Euclidean geometry. As it turns out, fractal geometry is the appropriate language to understand the complicated things we see in nature ${ }^{65,84,90}$.

There are two fundamental properties of fractals most easily appreciated geometrically. These properties are: (i) the fractal dimension and (ii) selfsimilarity. ${ }^{10,32,38}$ Fractal shapes best illustrate these two properties. If one thinks, for instance, of a mountain or a cloud, a first Euclidean approximation to the shape of a mountain that one imagines is a cone, and to a cloud is a sphere. However, there is more to the structure of mountains and clouds. Both have very 
undulating surfaces that jut out and fold in at many different places. Therefore, mountains and clouds are not really regular smooth three-dimensional cones and spheres. Their shapes fill up spaces that are somewhere between two and three dimensions. That is, their dimensions are fractional; they do not have integer dimensions ${ }^{20}$.

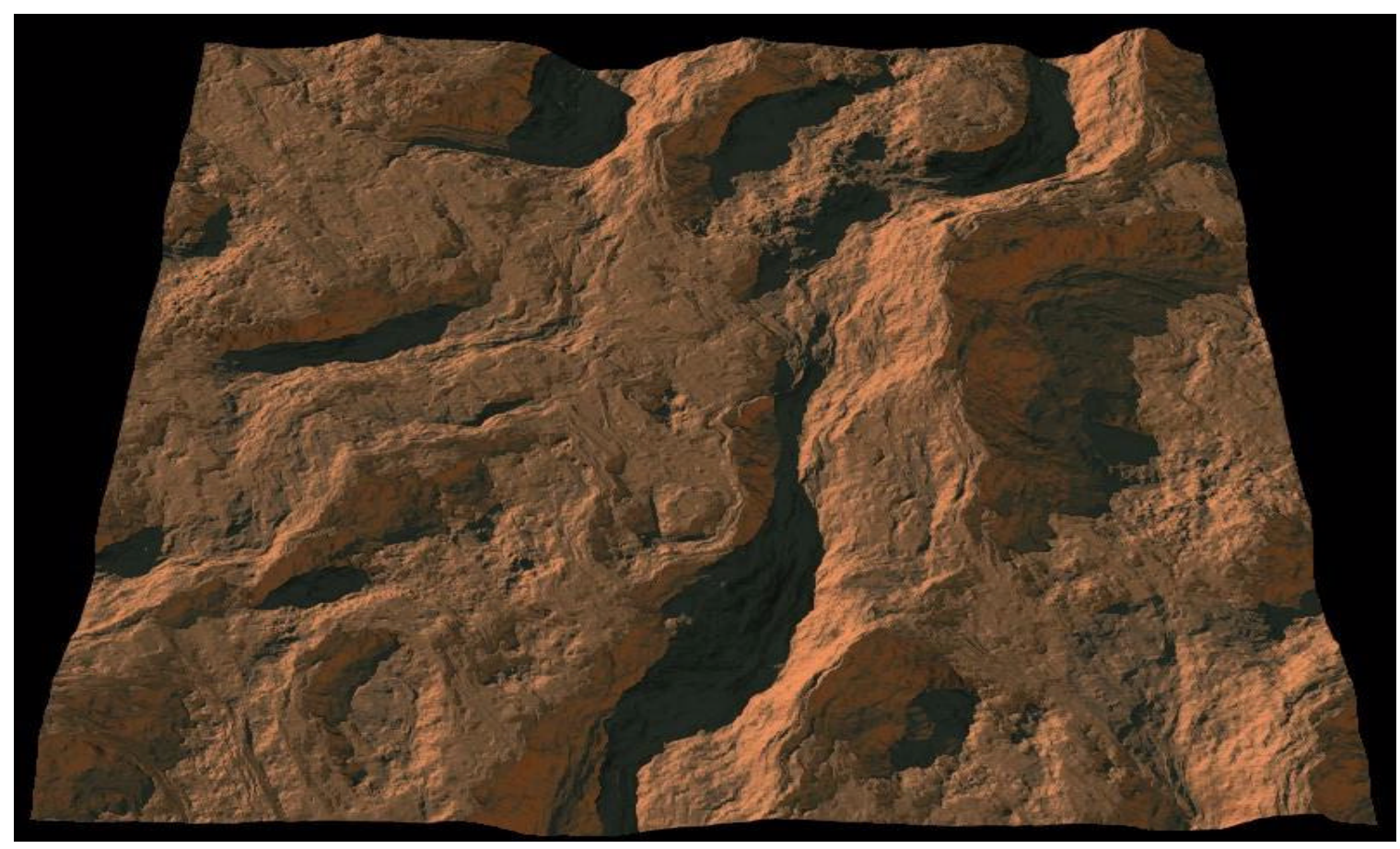

Figure III-3 Depicts A Fractal Representation Of A Mountain Range Generated By A Computer Algorithm.

A second mountain is rougher or more complex than the one above can be generated using the same computer program. The reason that this second range is different is because they have different fractal dimensions. The fractal dimension attempts to describe the roughness or jaggedness or complexity of a fractal shape. It tells us how well the fractal fills up the space in which it is 
placed. A fractal shape is one that occupies more than a certain number of dimensions but does not fill the next higher dimension; hence the fractal dimension has always a non-integer value $e^{20,80}$. Computer generated fractals are deterministic in the sense that the programmer has control over any aspect of the outcome, whereas real fractal shapes occurring in nature are random fractals. Lucas Films is the most famous commercial user of fractals to generate realistic looking artificial images

The second important property of fractals, self-similarity, refers to the idea that the parts of the fractal are similar to the whole.

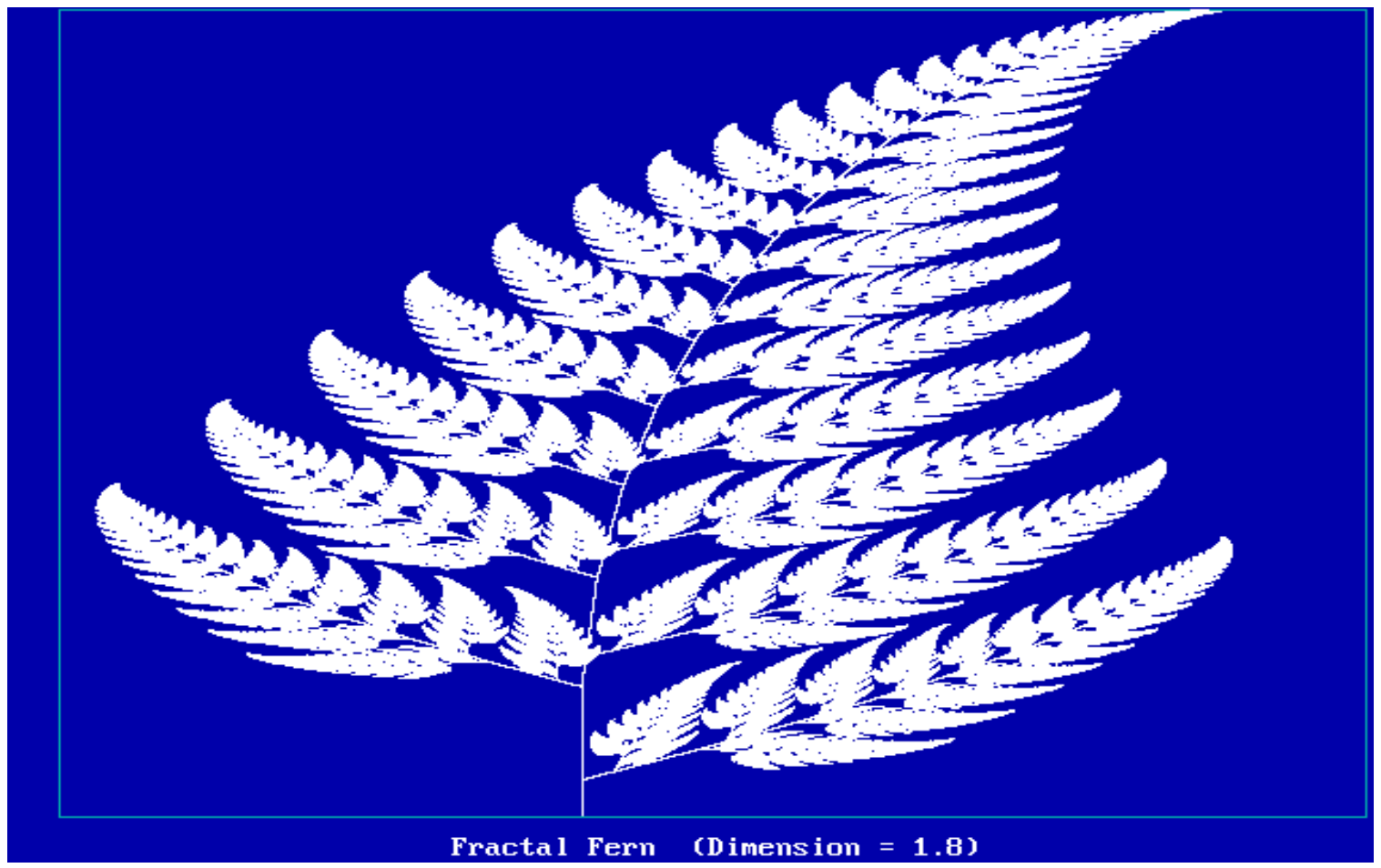

Figure III-4 The Classic Fractal Fern Showing Self-Similarity 
This is a computer version of a fern. In figure II-4, if one isolates one of the branches and looks at this branch at smaller and smaller scale. One sees each of the smaller branching pieces with a shape that is exactly similar to the bigger picture. If one would zoom in further on this piece, what one sees again is an exact replica of the original. If this were being viewed on a computer screen one could then go on further and further into the picture depending on the resolution power of the computer and the same intricate pattern would be noticed. This is the property of self-similarity. A deterministic fractal such as this fern would exhibit exact self-similarity, whereas a real fern that is a random fractal would show statistical self-similarity. An Euclidean shape does not possess the property of self-similarity. For instance, a segment of a circle, if magnified sufficiently, becomes a straight line ${ }^{44}$.

\section{(E) Fractal Sequences}

Self-similarity and non-integer fractal dimensions are properties also shared by fractal sequences. Consider as an example financial data, GTE stock prices over different time scales. The stock market is a fractal time-series it is a natural fractal because a computer does not generate $\mathrm{it}^{16}$. Statistical self-similarity in a random fractal time-series refers to the idea that the 'trail' of the time-series looks 'the same' when observed at different time intervals. In data such as the stock market meaningful time intervals are years, months, days, and occasionally hours. For other types of data these time intervals would be what 
ever has physical meaning necessary to describe the time dependency of the phenomenon being studied. It is important to remember that other types of fractal series are possible. Using time as the dependent variable is not sacred. This research will examine fractal series where data position is the dependent variable, e.g. DNA base-pair position. For purposes of clarity this discussion will start with fractal series where time is the dependent variable such as stock market data.

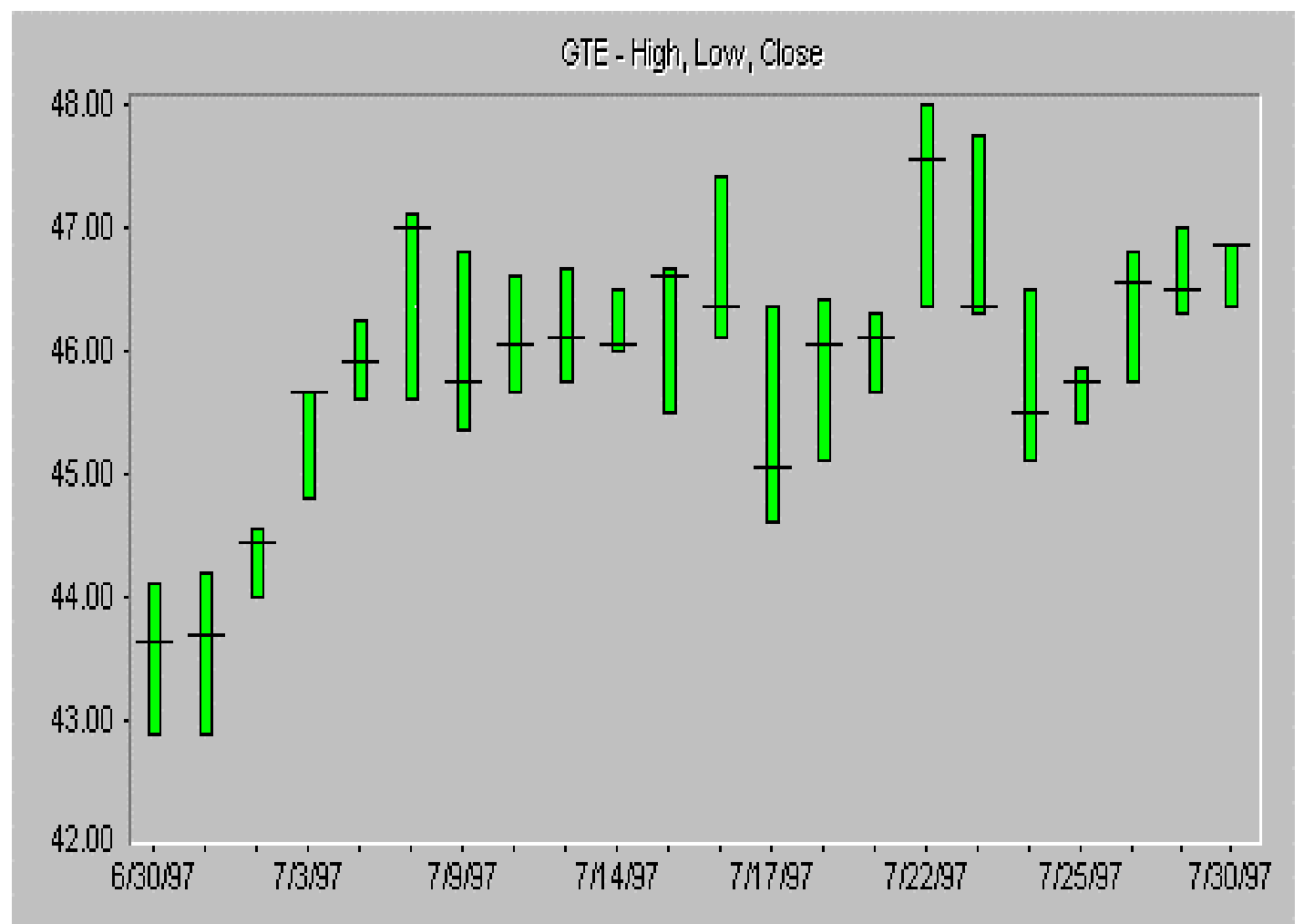

Figure III-5a Daily GTE Stock Prices For One Month 


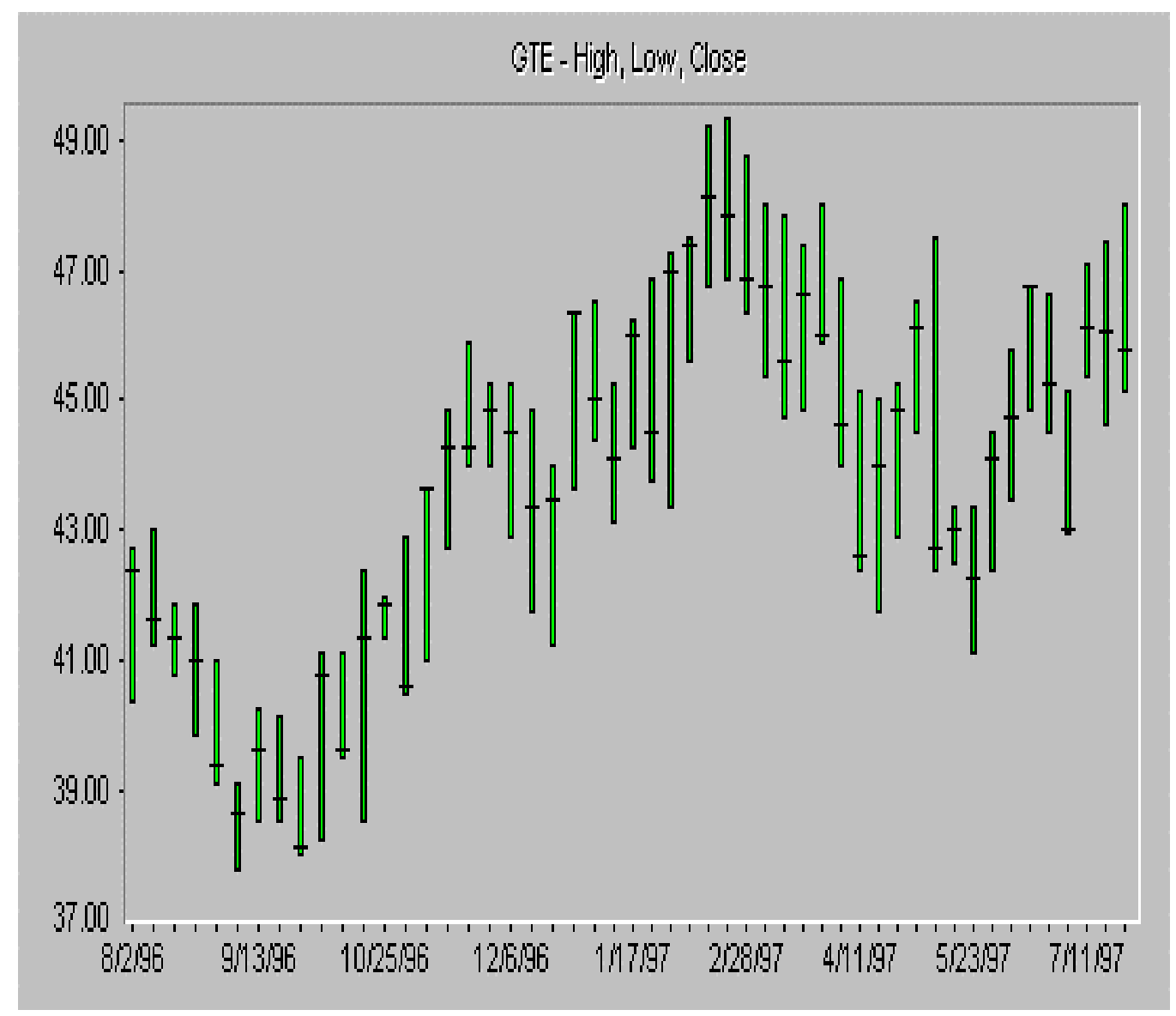

Figure III-5b Weekly GTE Stock Prices For One Year 


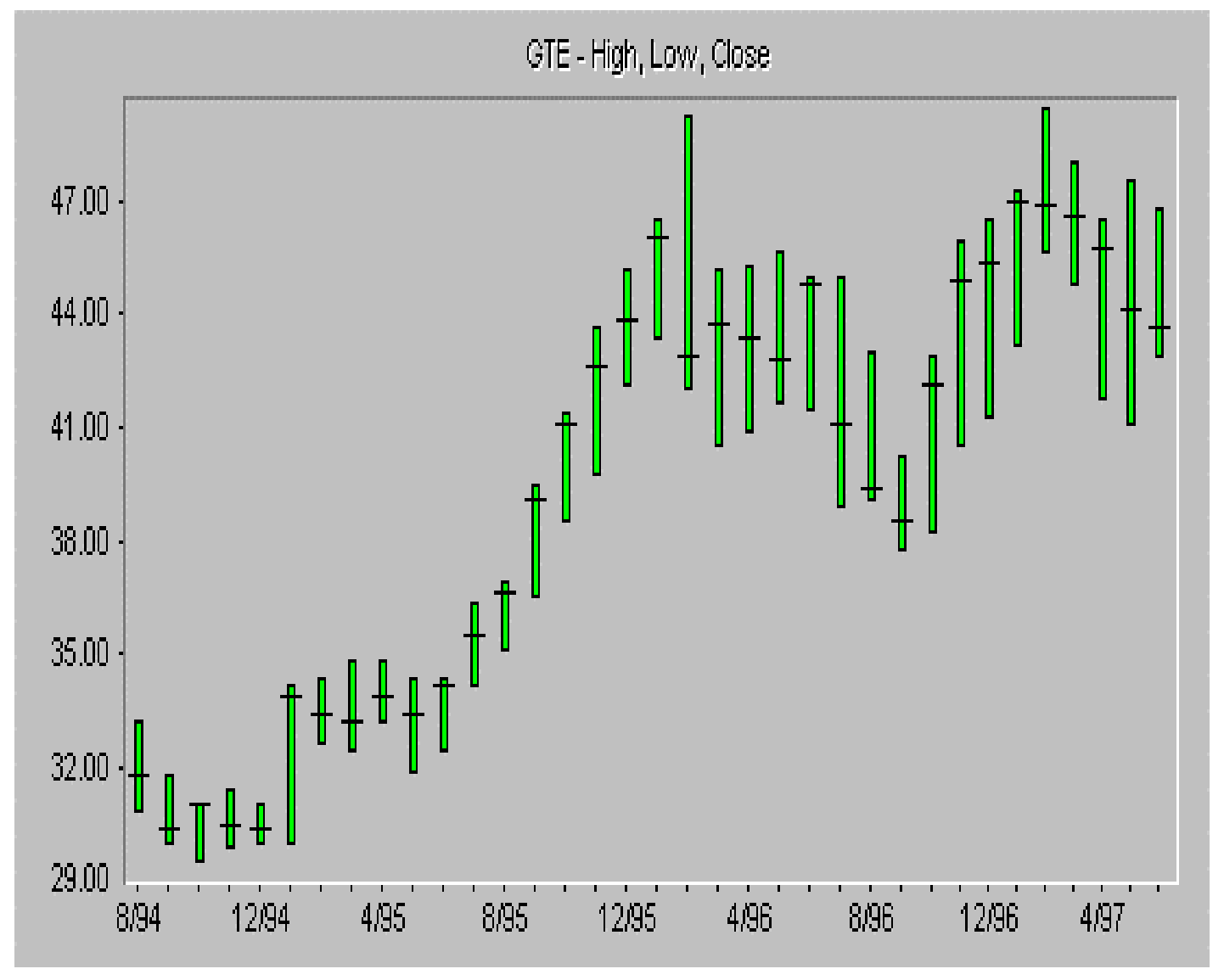

Figure III-5c Monthly GTE Stock Prices For Three Years 
For instance, if the time axes were removed from these graphs, one could not tell the difference among these various plots of the GTE stock prices. Statistical self-similarity in a fractal time-series or sequence is, therefore, a form of invariance with respect to changes in the time or position scale ${ }^{32}$. Also, from a geometric perspective, the 'trail' of the stock price series is not an ordinary onedimensional straight line, but nor is it quite a two-dimensional plane. In this sense, it can be said to have a fractional or fractal dimension between one and two ${ }^{80}$.

The notion of statistical self-similarity in "natural" time or position sequences is not new. As early as the 1960s, Mandelbrot $(1963,1965,1967)$ described a family of stable distributions called stable Paretian, or stable ParetoLévy distributions. Mandelbrot and Fama suggested that speculative markets might be described by Stable, or Levy, distributions ${ }^{65,78}$. This set of distributions includes the Gaussian, but also includes a variety of fractal distributions and all can be generated through Fractional Brownian Motion ${ }^{78,81,82}$. A fractal distribution is stable if two "rescaled" versions of the distribution are multiplied together they produce a distribution that is also a rescaled version of the original. This is similar to a grammar or production rule in an L-System. That is, $f$ is a stable distribution if for any A and B, there exists a $C$ such that 
II.5

$$
f(x / A)^{*} f(x / B)=f(x / C) .
$$

The name "stable" is used when multiple effects are applied to each distribution. The "net effect" will also generally be a stable distribution and be self-simliar. So, these distributions are the ones that can "survive" in systems that have numerous contributing factors. In L-Systems this becomes the basic grammar for the language. The Gaussian bell curve is one example of a stable distribution, unfortunately not the one believed to describe most markets.

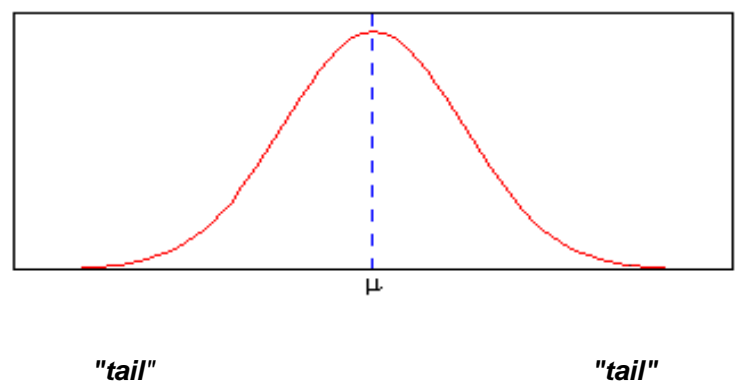

Figure III-6 Example Of A Stable (Gaussian) Distribution $\mu$ Is The Mean.

The 'Tails' Decrease Exponentially In Value.

Stable distributions can be parameterized with four parameters. That is, by continuously adjusting four parameters, all stable distributions can be achieved. Unfortunately, except for two or three special cases (such as the Gaussian), none of these distributions can be expressed in analytical form.

Fractal distributions have the property that when their corresponding timeseries is plotted, it will have a fractal dimension ${ }^{84}$. The time-series produced by a 
fractal distribution is (statistically) scale-independent. This property gives the system self-similarity. This is generally the case for stable distributions. (The Gaussian is an exception.) Fractional Brownian Motion occurs in time-series which obey a $" 1 / f^{-b}$ " rule. ${ }^{117,118}$ Their Fourier series decrease at a rate which is proportional to $1 / \mathrm{f}^{-\mathrm{b}}$, where $\mathrm{f}$ is the frequency of the time-series (the independent variable in Fourier-space), and b any constant. Interesting studies have shown that all stable distributions can be generated through Fractional Brownian Motion. Thus, all of these ideas correspond to the same kinds of time-series.

(F) Recent Fractal Analysis Applied to DNA

Allegrini, Barbi, Grigolini, West (1995) modeled DNA sequences as a diffusion process and described them as $\alpha$-stable Pareto-Levy distribution ${ }^{2}$. These stable distributions are a four-parameter family for which, aside from three special cases, there is no explicit expression for the densities of the distributions. Each distribution is usually expressed as the logarithm of its characteristic function. The characteristic exponent, $\alpha$, of this class of distributions determines the height of, or total probability contained in the extreme tails of the distributions. It can take any value in the interval $0<\alpha \leq 2$. When $\alpha=2$, the relevant class of distributions is the normal. When $\alpha$ is in the interval $0<\alpha<2$, the extreme tails of the distributions are higher than those of the normal distribution, with the total probability in the extreme tails larger the smaller the value of $\alpha$. The most important consequence of this is that the 
variance exists, or is finite, only in the limiting case, $\alpha=2$. The mean exists as long $\alpha>1$.

An everyday example of such a natural process again is financial data e.g., Standard \& Poor's 500 Index. If one plots the frequency distribution of 5day returns for the Standard \& Poor's 500 index one sees the following. The distribution has a high peak at the mean and additional observations in the tails are like the leptokurtic feature suggested by the class of stable distributions when $\alpha<2$. This observation was made by Mandalbrot and Fama in their 1965 study in which he investigated the distribution of stock market data ${ }^{65,78,81,82}$. Its important to remember that leptokurtic means a distribution with positive kurtosis. (The kurtosis or kurt, is also a non-dimensional quantity. It measures the relative peakedness or flatness of a distribution, relative to a normal distribution. A distribution with positive kurtosis is termed leptokurtic; a distribution with negative kurtosis is termed platykurtic. An in-between distribution is termed mesokurtic.) Fama found a significant $\alpha=1.93$, and most importantly, noticed also that this value remained unchanged for frequency plots of monthly, weekly, and daily time intervals. Fama's result can be regarded as the initial reference to statistical self-similarity in financial markets. Other researchers who have found leptokurtic distributions include Peters (1991) in equity markets ${ }^{81,82}$, Young and Graff (1993) in real estate markets, and Richardson's self-similar coast-lines (1960) in cartography and Clarke and Voss (1975) in voice \& speech processing ${ }^{90}$. 
Fat tails imply that information may be processed in infrequent clumps which means the data is not evenly distributed.

Instead of the normal procedure of processing the information in a smooth and continuous fashion. It seems then that fractional Brownian motion may be a worthwhile candidate to investigate as an appropriate model of the way mutations introduce new information into the DNA sequence. This hypothesis begins with the assertion that DNA sequences are piecewise fractional Brownian motion processes ${ }^{83,84,109}$. An example of Brownian motion is visually demonstrated by the Iterated Function System (IFS) mapping displayed in the next figure.

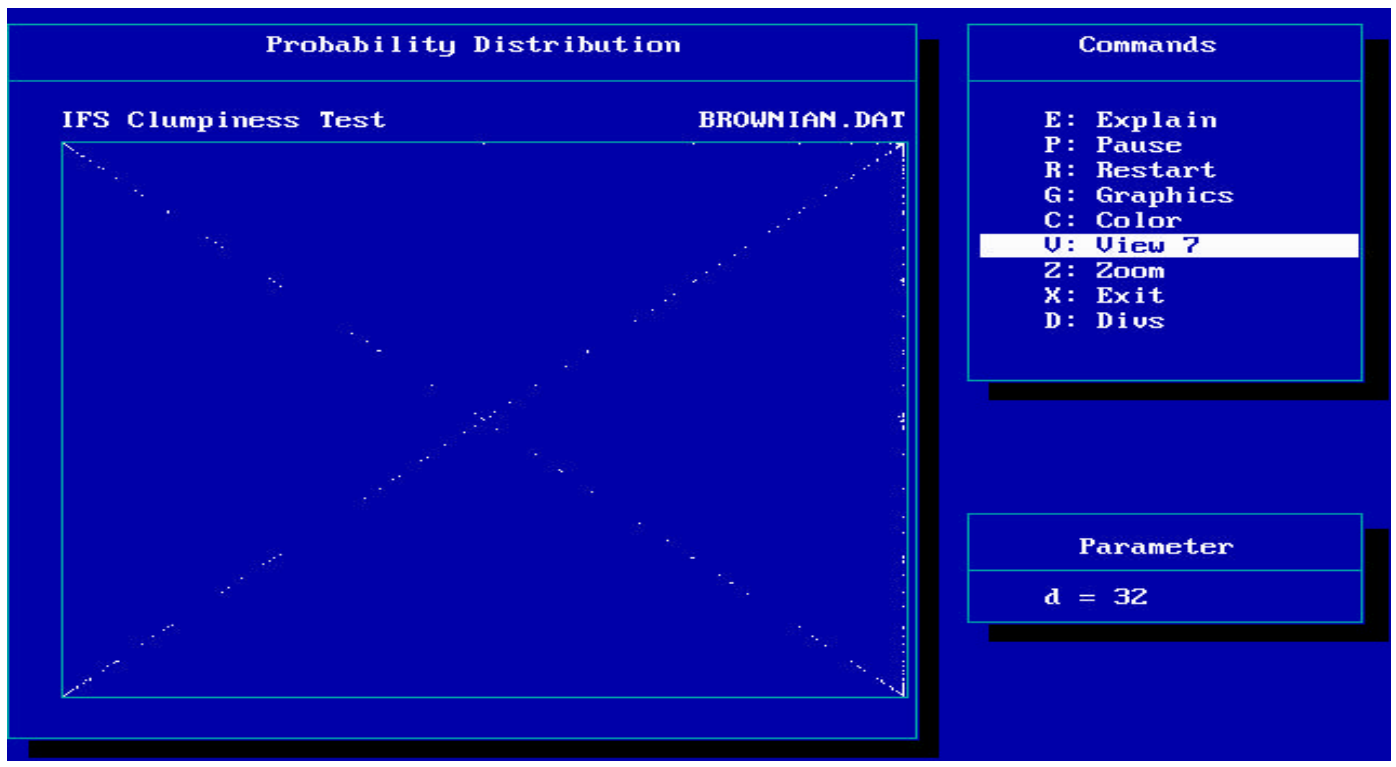

Figure III-7 A General Brownian Sequence. This Example Is From 'Chaos Data Analyzer 'By Dr. Julian C. Sprott ${ }^{108}$ 
(G)Elements of DNA Sequences and the Concept of Dynamic Disease ${ }^{50,53}$

DNA sequences in their most basic form are a type of code or "language" which consists of an alphabet of four symbols ${ }^{49}$. Using the language analogy these symbols are combined into groups of three called amino acids, these could be thought of as words. Amino acids are then combined to get proteins and proteins could be thought of as sentences. One could continue the analogy all the way up to the entire organism. The important point is that DNA sequences represent a structured hierarchy of information. These symbols can also be thought of in information theoretical terms, as a L-System grammar or in cellular automata terms. At the simplest level these symbols can be modeled as a one-dimensional cellular lattice four "states". A cellular site is defined to be a square that represents at the corners the four possible states. A "state" is defined in this case to be one of the DNA nucleotides. The view is of course hopelessly simplistic; the DNA molecule is much more then base pairs strung together. There are many other mechanisms present for information storage, e.g. spatial position in the double helix.

The research in this dissertation uses a first order approximation for the sake of simplicity. One can consider the DNA molecule like one dimensional cellular automata capable of self-reproduction having four states per cell, which represent the bases Adenine $(A)$, Cytosine $(C)$, Guanine $(G)$ and Thymine $(T)$. This study is going to concentrate on these "states" i.e. base pairs, and their linear arrangement represents information content in the sequence. This will be done computationally by calculating a quantitative measure of information content for normal sequences and then using the 
same procedure to calculate an information measurement on mutated versions of the same sequence.

Structurally the base pattern present on the DNA molecule is composed of a sequential arrangement of $A, C, G$ and $T$ along a strand that is twisted into an anti-parallel double-stranded helix. The alignment of these two strands is controlled by hydrogen bonding through the base pairs. This pairing always follows the following rules: A always pairs with $T$, and $G$ is always with $C$. Therefore, the base sequence of one strand completely determines the base sequence on the complimentary strand.

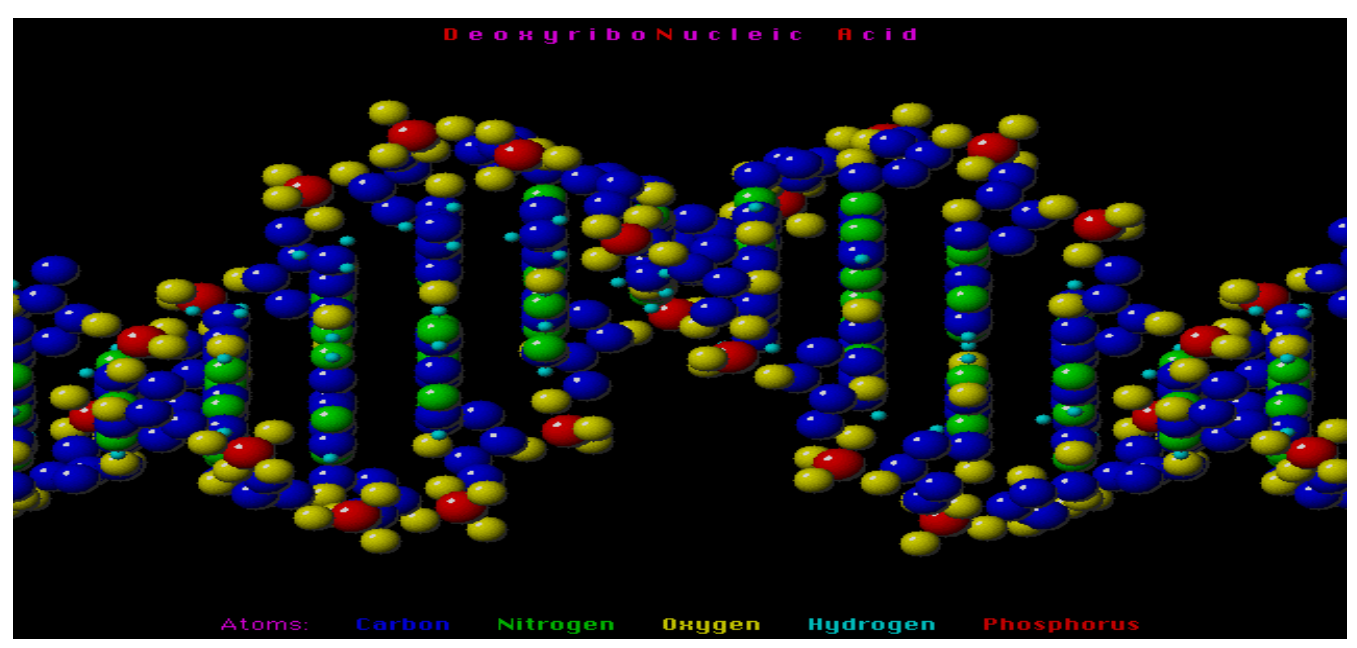

Figure III-8 DNA Spiral Sequence

Simple organisms have only a single DNA strand; higher organisms have separate bundles of DNA called chromosomes. The number of chromosomes varies according to species e.g., humans have 46 chromosomes and cows have 60 chromosomes. Each 
strand is further subdivided into sections called genes. A gene is by definition the amount of information needed to code for either one protein (a structure gene) or to induce or repress certain chemical operations of the cell (regulatory gene). Francis Crick has stated that the information in a cell flows from DNA to RNA to protein (phenotype). Information can not flow in the opposite direction from cellular nucleus (DNA storage center) as known as a genotype to the protein. This would represent a Lamarkian type of information flow. Crick's Rule or Law is a description of the way in which a DNA sequence specifies both its reproduction (replication) and the synthesis of proteins (transcription and translation $)^{27,35}$. The one exception to this law is for retroviruses where information flow is RNA to DNA to RNA to protein. ${ }^{58 .}$ Reproduction is done by the base-pairing of the complement of two DNA strands. The cellular DNA-synthesizing machinery reads each strand and then forms its complement strand. This protein synthesizing procedure goes in two steps ${ }^{76}$ :

1) In the transcription step, a messenger RNA (mRNA) strand is formed again as a complement of one of the DNA strands: but with a base element Uracil $(U)$ in place of Thymine (T). Messenger mRNA is a single stranded molecule.

2) The cellular protein-manufacturing machinery (the ribosomes) translates a section of the mRNA strand using the genetic code in order to form a protein strand. The RNA base sequence is read as a non-overlapping set of contiguous triplets called codons. Proteins are composed of amino acids of which there are some 20 different types. There is information redundancy built into the system 
since there are $=64$ possible codons. There is some redundancy in the translation of specific condons into amino acids

A normally healthy individual will display a complex interwoven pattern of rhythms in his body systems ${ }^{4}$. These rhythms are only very rarely periodic. In fact measurements of these rhythms such as heart rate and respiration rate show that variation does indeed occur $^{12}$. Mackey and Glass $(1977)^{64}$ have suggested that normal health is associated with chaotic dynamics and disease is associated with periodic behavior linear dynamics ${ }^{5}$. It is not always apparent in how to relate the physical conditions to the mathematics of the differing dynamical states ${ }^{23}$. In fact, " normal" system dynamics may be chaotic (or nonchaotic) and thus a change to some other type of dynamics may indicate a diseased state. It is clear that many pathologies can be identified from abnormal rythmicities ${ }^{98}$.

The main identifier of a dynamical disease is a marked change in the dynamics of some variable. Three types of possible qualitative changes can be observed ${ }^{122}$ :

1) Variables that are constant or undergoing a relative small-amplitude "random" change that then show a large amplitude oscillation but are more or less regular,

2) New periodicities that arise in already known periodic processes, and 
3) Rhythmic processes can disappear and be replaced by relatively constant dynamics or aperiodic dynamics

Unfortunately most work involving studies of periodic behaviors in biology involve time series data on disease $e^{98,122}$.

DNA sequences are the subject matter proposed in this study. The dynamics to be studied in this system are not time-dependent but dynamics based on changes in the base pairs of the DNA alphabet of T, A, G, and C as well as position in the sequence ${ }^{2}$. Attention is given to patterns of linear periodic behavior or non-linear aperiodic behavior i.e. "noise or chaos" in the DNA sequence $^{3,45}$. Both the linear sequence frequency and non-linear sequence have definite meanings. Linear periodic frequency occurrences of base pairs have a specific interpretation ${ }^{59,60}$. An example of this is the work on the bladder cancer oncogene done by Pickover ${ }^{90,101}$. While sections with "aperiodic" or chaotic occurrence hold important biological information ${ }^{47}$. These sections are known as the "coding regions" or exons. It is important to remember that the exon DNA segment carries the information. Non-linear frequency occurrences could mean sections with purely random sequences that carry little genetic information ${ }^{3}$. These sections commonly referred to as the "non-coding region" or introns. The intron is usually thought to be a connecting segment or a "place holder" that ties the exons together. However it is interesting to note that this 
non-coding region displays long range power law correlation and have some features common to a hierarchically structure languages ${ }^{109}$, i.e. an L-System.

Some researchers claim that linguistic analysis indicates that these noncoding regions are more similar to a language then the coding regions. That is to say that the coding region lacks the statistical properties of a structured language. This implies that most of the grammatical structure lies in the noncoding region. This is not to say that there is no grammatical structure in the coding regions but that it is more pronounced in the non-coding regions. The coding regions are the "words" and the non-coding regions are the punctuation. One could think of the coding region as a "pidgin" form of the overall language with the non-coding regions providing detailed rules to give the language a "richness" or accuracy to minimize misunderstanding ${ }^{49,109}$.

It is important to remember that time is a mathematical convenience in sequence analysis. In this particular analysis time is replaced by base pair position with no effect to the underlying mathematics.

(H) A New Non-parametric Statistic: The Hurst Exponent ${ }^{81,82,84,90}$

An important test statistic for measuring the complexity of a data sequence was developed by a hydrologist who worked on a Nile River Dam project around 1907 and remained to study the river for the next forty years ${ }^{81,82}$. His principle reason for this study 
was to devise some means of reservoir control. In the ideal case a reservoir will never overflow because a control mechanism would be in place that would always discharge the right amount of water for the particular time of year. If the influx of water were too low then the reservoir would get way too low thus endangering crops that the region depended on. He modeled rainfall as a random walk since rainfall was the single parameter that he had no control over. This is a common procedure in modeling a system that clearly has many degrees of freedom. Hurst measured how the reservoir fluctuated around its average over time. The range of this fluctuation would change depending on the length of time used for measurement. If the series were random, the range would increase with the square root of the time. This is cased the rule. Hurst standardized this measurement over time through the creation of a dimensionless ratio, by dividing the range by the standard deviations of the observations. This analysis is called re-scaled range analysis i.e. R/S analysis. Hurst found that most nature phenomena, including river discharges, temperature, rainfall, and sunspots followed a "biased" random walk. In short these series were trends with noise. The strength of the trend and the level of noise could be measured by how the re-scaled range scales with time. In other words how much greater then 0.5 .

This new statistic that Hurst developed is very robust. It contains almost no underlying assumption about the state being studied, and it can classify time series. It can differentiate a random series from a non-random series, even if the random series is nonGaussian. Hurst found, as was stated above, that natural systems are not random walks. It doesn't make any difference whether or not they are Gaussian ${ }^{82}$. 
This research used Hurst's methodology to study DNA sequences. As has been stated these sequences are not a time series but a position series. Still the Hurst technique can be used to determine whether or not they are biased random walks or not. It is expected that there will be sections of the DNA sequence that will be closer to the random walk value and sections will show clear bias. The operating assumption is the more the bias the more important biological effect of the section of the sequence because those sections are the maim bearers of the biological information.

Hurst's methods ${ }^{81,82}$ can be formulated for a general data series in the following fashion. Consider a data series $t$ with $u$ observations

$$
\text { II.6 } X_{t, N}=\sum_{u=1}^{t}\left(e_{u}-M_{N}\right)
$$

Where $X_{t, N}=$ cumulative deviation over N periods

$e_{u} \quad=$ A measurement value at a particular observation $\mathrm{u}$

$M_{N}=$ Average $e_{u}$ over $\mathrm{N}$ periods

The range is then difference between the maximum and minimum values of $X_{t, N}$

$$
\text { II.7 } R=\operatorname{Max}\left(X_{t, N}\right)-\operatorname{Min}\left(X_{t, N}\right)
$$


Where $\mathrm{R}=$ range of $X_{t, N}$

$\operatorname{Max}\left(X_{t, N}\right)=$ maximum value of $X_{t, N}$

$\operatorname{Min}\left(X_{t, N}\right)=$ minimum value of $X_{t, N}$

To compare different types of data series the range is divided by the standard deviations of the original observations. This is the "re-scaled range" and this should increase with time. Hurst then developed the following relationship ${ }^{81,82}$ :

$$
\begin{aligned}
& \text { II.8 } R / S=(a \cdot N)^{H} \\
& \text { Where } \mathrm{R} / \mathrm{S}=\text { re-scaled range } \\
& \mathrm{N} \quad=\text { number of observations or periods } \\
& \mathrm{a} \quad=\text { a constant } \\
& \mathrm{H} \quad=\text { Hurst exponent }
\end{aligned}
$$

Statistical mechanics states that $\mathrm{H}=0.5$ if the series is a random walk. In true random situations the range should increase with the square root of time, N. Hurst applied his statistic to the Nile River water discharge and found much to his surprise, $\mathrm{H}=.90$. He then tried this statistic on other rivers and consistently found that $H>0.5$. He then tried different physical phenomena and in all cases $H>0.5$. When $\mathrm{H}$ differs from 0.5 the observations are not independent; each observation contained a "memory" of all the events that preceded it. This is not short-term system memory usually referred to as 
"Markovian" memory. This memory is long-term, in theory it lasts forever. More recent events have greater impact than more distant events. A system that exhibits Hurst statistics is the result of long stream of interconnected events. The impact of the present on the future can be expressed as a correlation ${ }^{81,82}$ :

\section{II.8 $C=2^{\left(2 H^{-1}\right)}-1$}

$$
\begin{aligned}
& \text { Where } \mathrm{C}=\text { correlation } \\
& \mathrm{H}=\text { Hurst exponent }
\end{aligned}
$$

There are three distinct classifications of the Hurst exponent $\mathrm{H}$ :
1) $H=0.50$
2) $0 \leq H<0.50$
3) $0.50<H<1.00$

$\mathrm{H}=0.5$ means a truly random series. Here events are random and uncorrelated. The above equation will equal zero. The present does not influence the future. Its probability density function can be Gaussian but doesn't have to be. 
Consider the second Hurst classification $0 \leq H<0.50$. This type of system is antipersistent that is an ergodic series. It is sometimes referred to as "mean reverting". If a system has been up in the previous period it is more likely to be down in the next period. The strength of this anti-persistent behavior depends on how close $\mathrm{H}$ is to zero. The closer $\mathrm{H}$ will move to zero the closer $\mathrm{C}$ moves to -0.50 or negative correlation. This kind of a series would be more volatile than a random series and it would have frequent reversals.

The third classification $0.50<H<1.00$ is a condition indicating a persistent or trendreinforcing series. When the series has been up (or down) in the last period, the chances are it will continue to be up (or down) in the next. Trends in the data should be readily apparent. The strength of this trend reinforcing is as $\mathrm{H}$ approaches 1.0 in the above equation then the data approach $100 \%$ correlation. The closer $\mathrm{H}$ approaches 0.5 , the noisier the data it will be, and the less well defined the trends are. Persistent series is a fractional Brownian motion (or biased random walks) in which trends tend to continue. The strength of the bias depends on how far $\mathrm{H}$ is above zero. Dynamically speaking, there is correlation between events across the series time or position scales. This relationship means the probability of two events following one another is not .50 . The Hurst exponent, $\mathrm{H}$ describes the likelihood that two consecutive events are likely to occur. If $\mathrm{H}=0.60$ then in a sense a one could think of the value as approximately $60 \%$ confidence that the trend will be repeated with the next value. If the last move was positive the next move it will probably also be positive. 
Mandelbrot (1972) defined a "fractal dimension" ${ }^{44,90}$, Df is the inverse of H. (This research will use a definition of fractal dimension that relates fractal dimension to the underlying pattern topology called the "topological fractal dimension”,, $\mathrm{D}=2-\mathrm{H}$.) The Mandelbrot definition is still useful. The main point is that $\mathrm{H}$ is linearly related to the fractal dimension. Fractal dimensions like much in this field is an evolving concept. The various definitions of fractal dimension will be discussed later in this dissertation.) In systems that are equiprobable such as a random walk the fractal dimension, Df $=2$ since $\mathrm{Df}=\mathrm{H}^{-1}$ and $\mathrm{H}=1 / 2$. For natural systems $\mathrm{H}>1 / 2$, the fractal dimension of the probability distribution is not 2 but a number between 1 and 2. Consider the random walk again where $\mathrm{H}=0.5$, it would have a fractal dimension of 2 the inverse of $\mathrm{H}=0.5$. A random walk is truly two-dimensional and would fill up a plane. If $\mathrm{H}=0.7$ the fractal dimension is the inverse of $\mathrm{H}$ that is $H^{-1}=0.7^{-1}=1.43$. If $\mathrm{H}$ moves closer to 1 the series becomes less noisy and has more consecutive observations with the same signs. As $\mathrm{H}$ increases, the cumulative line becomes smoother and less jagged. There is less noise in the system and the "trends" or deviations from the average, become more pronounced. The Hurst exponent, $\mathrm{H}$, measures how jagged the time series is. A perfectly deterministic system would produce a smooth curve. A fractal time series separates a pure random series from a deterministic system perturbed by random events..$^{81,82,84,90}$

It is important to realize that the Hurst exponent can be used to characterize scaling relationships for sub-sequences as well as characterize a global scaling relationship for the whole sequence. For the rest of the discussion let $\mathrm{H}$ stand for the global Hurst exponent as well as the local Hurst exponent. It 
depends totally on the observing perspective. If one is concerned about the trending and power law scaling relationships between the sub-sequences then the local Hurst exponent is used. If one is considering the sequence as a whole then it's the global Hurst exponent. $H>1 / 2$, there is positive correlation $(C(t)>$ 0) and the multi-fractal system is in a persistent trend. In this case, if for some time in the past there had been an increase, and if $\mathrm{H}=0.7$ for instance; it means there is a $70 \%$ chance that there will be also an upward trend in the sequence in the future. In financial terms e.g. the stock market; this corresponds to the bullish type of market ${ }^{81,82}$. In DNA terms this means a rich region of the "higher numerical" valued base pair sequences. Consider the following example, $A$ and T are coded as 1 and 2 respectively, and $G$ and $C$ are coded as 3 and 4 respectively. A $\mathrm{H}=0.7$ implies a "bullish trend" toward base pairs of $\mathrm{G}$ and $\mathrm{C}$ to use stock market parlance. Conversely, a decreasing trend in the past implies on the average a continued downward trend in the future. This means movement toward a region of A's and G's. The Hurst exponent could thus be used to identify trends toward regions rich in either pyrimidines (i.e. T, C) or regions of purines (i.e. $A, G)^{90}$.

Anti-persistence in the multi-fractal series ${ }^{32}$ is the term for the case $\mathrm{H}$ $<1 / 2$. In this case, there is negative correlation $(C(s)<0)$. That is, if for instance $\mathrm{H}=0.3$, then there is a $70 \%$ chance that the trend will proceed in an opposite direction from what is currently observed. In other words using the language of the financial markets - " a bear market" ${ }^{\text {"1,82 }}$. 
(I) Multi-Fractal Approach to DNA

It may be possible to account for a number of observed DNA sequence "patterns" by modeling them as a multifractal or piecewise fractal Brownian motion process $^{2,38}$. Below are five arguments as to why these approaches seem reasonable.

(1) The multi-fractal approach allows these sequences to experience a range of modes - persistence, anti-persistence, and random walk - depending upon how the value of $\mathrm{H}$ for the local fbm within a particular subinterval affects the correlation function $\mathrm{C}(\mathrm{s})$. In this way, subintervals that contain 'memory effects' can be included, as well as random walk subintervals ${ }^{31}$. Such a model is consistent both with evidence from studies that have found long range correlation in DNA data as well as studies that have found correlation's in random walks. ${ }^{2,83,109}$

(2) DNA sequences can be shown to be statistically analogous to financial market data. ${ }^{2,78,97,109}$ Therefore mathematical arguments to financial data can be applied to DNA sequences. Fractional Brownian Motion may be a possible alternative explanation for the nonlinear dependency found in financial market data and DNA data as opposed to chaos models. Tests of nonlinear dependency, such as the BDS and Grassberger and Proccacia (1983) tests ${ }^{39,40}$, have no way of distinguishing between a time series generated by chaos 
dynamics or a time series generated by fractional Brownian motion. Thus, it is quite possible that the nonlinear dynamics that have been detected in DNA sequences may in fact be coming from fractional Brownian motion then from chaos dynamics ${ }^{41}$.

Furthermore, researchers in this field of mathematics suggest that chaos dynamics are driven by an underlying system of differential equations ${ }^{42}$. In other words, chaos models are deterministic models. On the other hand, fractional Brownian motion is a probabilistic model in the sense that the $\mathrm{H}$ exponent describes the likelihood of sequence trending. There may be an equation of motion driving the DNA dynamics, the element of chance drives the model, and this is important in building models of mutation dynamics ${ }^{9,77}$. In the financial world, efforts to build a model of price fluctuations may have an underlying differential equation driving the model but the element of chance may be of lessor importance ${ }^{16}$.

(3) Fractional Brownian motion processes generate the class of Stable Paretian distributions. When $\mathrm{H} \neq 1 / 2$, these distributions have a high peak at the mean and additional observations in the tails. Examples of these are DNA sequence data and financial data

(4) The span of interdependence between time intervals can be long-or short-range. By way of contrast, other functions have been used to model 
DNA sequences such as Markov processes ${ }^{59,60}$. They can capture only shortrange correlations, but other researchers have shown that DNA sequences display long-range correlations ${ }^{2,109}$.

(5) It may be possible to explain trends that arise from uneven assimilation of information introduced by mutations by either the feature of persistence or anti-persistence. Also, fbm's are self-similar processes, which refers to the idea that the trail of the fbm series looks similar in a statistical sense when observed at different sequence intervals. Thus, fbm may also be able to explain trends over smaller scales. In time series data these scales would be temporal trends that cannot be explained by other models.

The multi-fractal model will generate a multi-fractal series of base pair representations having the related properties of self-similarity and multiple noninteger fractal dimensions. The GTE stock market plots in an earlier section demonstrated this. Self-similarity refers to the idea that the 'trail' of the series looks similar in a statistical sense when observed at different time intervals. This scale invariance property of fractal time-series may be expressed in an explicit way by changing the time scale by any constant factor $\mathrm{C}$, and by changing the displacement scale or increment by a factor of $\mathrm{C} \mathrm{H}$. Self-similarity is what gives a fractal series its fractal dimension. The fractal dimension $D$ describes the geometric dimension of the 'trail' left by the fractal series 
The proposed hypothesis can, therefore, be tested by the estimation of a single variable - either the $\mathrm{H}$ exponent, or the fractal dimension $\mathrm{D}$. There are many ways to estimate $\mathrm{H}$ or $\mathrm{D}$ for fractional Brownian motion systems. In this study the methodology used is that of Wavelet Transforms and Wavelet Packet Transforms $\mathrm{s}^{24,51,108,118}$. The following chapter outlines this methodology and also the data sample that will be used in the three categories of the Hurst exponent $\mathrm{H}$ $<1 / 2, H>1 / 2$ and $H \neq 1 / 2$ these categories hold for the global Hurst exponent which

does give total sequence trending and scaling relationships. The local Hurst exponent gives local sub-sequence and scaling behavior.

It is a premise of this work that DNA is a fractal sequence and scale invariance is a property of fractal sequences. It is a logical next step to select a technique that is sensitive to scale analysis. The technique that immediately comes to mind again is wavelets which very early in its development was called "scale-space filtering". This technique grew out of time-frequency analysis ${ }^{25,92}$ research and in some quarters was called quadrature-mirror filtering ${ }^{18}$. It was first developed for geophysical processing and has found application in many other areas. 
(J) Wavelet Based Analysis ${ }^{25}$

The fundamental idea behind wavelets is to analyze according to scale. Indeed, some researchers in the wavelet field feel that, by using wavelets, one is adopting a whole new mindset or perspective in processing data.

Wavelets are functions that satisfy certain mathematical requirements and are used in representing data or other functions. This idea is not new. Approximation using superposition of functions has existed since the early 1800 's, when Joseph Fourier discovered that he could sum scaled sines and cosines to represent other functions ${ }^{6}$. It is important to remember the "window (scale)" in Fourier analysis is fixed. However in wavelet analysis the scale plays a special role. Wavelet algorithms process data at different scales or resolutions. If we look at a signal with a large "window (scale)," we would notice gross features (the forest). Similarly, if we look at a signal with a small "window (scale)," we would notice small features (the trees). The result in wavelet analysis ${ }^{25,69.108}$ is to see both the "forest and the trees", so to speak. This makes wavelets interesting and useful. For many decades, scientists have wanted more appropriate functions than the sines and cosines that comprise the bases of Fourier analysis and better "windowing (scaling) methods", to approximate choppy or transient signals ${ }^{116}$. Choppy or transient signals by definition are functions that are non-local (and stretch out to infinity). Fourier analysis does a 
very poor job in approximating sharp discontinuities such as noise spikes. Wavelets are well suited for approximating data with sharp discontinuities.

The wavelet analysis procedure is to adopt a wavelet prototype function, called an analyzing wavelet or mother wavelet. Temporal analysis is performed with a contracted, high frequency version of the prototype wavelet i.e. small scale narrow widow version, while frequency analysis is performed with a dilated, low-frequency version of the same wavelet i.e. a large scale wide window version. Because the original signal or function can be represented in terms of a wavelet expansion (using coefficients in a linear combination of the wavelet functions), data operations can be performed using just the corresponding wavelet coefficients. And if you further choose the best wavelets adapted to your data, or truncate the coefficients below a threshold, your data is sparsely represented. This sparse coding makes wavelets an excellent tool in the field of data compression. ${ }^{24,25}$

Other applied fields that are making use of wavelets include astronomy, quantum mechanics, acoustics, nuclear engineering, sub-band coding, signal and image processing, neurophysiology, music, magnetic resonance imaging, speech discrimination, optics, fractals, turbulence, earthquake-prediction, radar, human vision, and pure mathematics applications such as solving partial differential equations ${ }^{24,25}$. This research will use wavelets as applied to fractals 
to calculate dynamic properties from non-linear data sequences the final result will the Hurst exponent for trending information ${ }^{51}$. 


\section{Methodology}

A. Introduction

Thanks to modern biological research we realize that DNA contains an amazing amount of information: the equivalent of thirteen sets of the Encyclopedia Britannica ${ }^{35,90}$. This biological sequence commonly known as the genetic code can be considered similar to a programming language. A pseudoprogramming language with four symbols, A, C, G, and T: the chemical bases adenine, cytosine, guanine, and thymine. This language programs the birth, development, growth and death for all living things. Some sections in this sequence define the structure of proteins, others define commands for biological regulation, but the purpose of most of the DNA is unknown. Some authors have said that the protein-coding regions in DNA are analogous to sailing ships at sea on a world where sailing is understood but not the ocean ${ }^{35}$.

B. Theoretical Background: Fractal-based Analysis of DNA Sequences ${ }^{32,38}$

Cystic fibrosis sequences both healthy and pathological are modeled as a two dimensional approximation of an L-system called an IFS (Iterated Function System $)^{50}$. The theory behind this model was discussed previously in this document. It is presented again in its entirety for the sake of clarity to the reader. 
A sequence is feed through as set of production rules (the CGR) which then produces a visual $x y$-plane representation. These sets of $(x, y)$ points are in the terminology of L-system theory, two-dimensional words. The "information" this represents is then captured by an Euclidean distance measure; i.e. all "points" are then referenced distance-wise to a point of origin on the xy-plane. A multi-fractal is thus generated. A wavelet packet transform is calculated and a minimum entropy ${ }^{51}$ representation is also calculated. Global and local Hurst exponents are determined this gives information about local and global trending. This not only shows how information flows through the IFS production rule set but also how it affects the "unknown" rule set. These are the rules that say this is a healthy cystic fibrosis gene and this other one is pathological. The use of this approach will visually display any self-similarity that is imbedded in the sequence. Again self-similarity refers to the idea that the "trail" which is the "system dynamics" of the sequence has the same apperance in a statistical sense when viewed at difference scales. This demonstrates the scale invariance property of a fractal series ${ }^{3,9,20,32}$.

Humans are good at visualizing complex two-dimensional patterns They generally have problems detecting patterns in long sequences of numbers or characters that are arrayed in a one dimensional series. Converting these one-dimensional sequences into a graphical form that is inherently of a higher dimension then the data is the basis of all data analysis. Using the IFS algorithm one-dimensional sequences can be mapped into two- 
dimensional form that preserves the underlying subsequence structure. In the case of DNA the subsequence structure may be of primary importance to the investigator.

This material on the IFS algorithm is presented again in its entirety for the sake clarity to the reader. The IFS algorithm or "The Chaos Game" is the first step in transforming the data. It is the step that traps the relationship of every base pair in the sequence with every other base pair in the sequence. An IFS algorithm is a set of linear mappings of the following form:

$$
\text { (IV.1) } \begin{aligned}
& x=a x+b y+e \\
& y=c x+d y+l
\end{aligned}
$$

The new value of $x$ and $y$ is computed from relation (IV.1). For example if the reader examines the Sierpinski Triangle example that was cited previously in the literature review. The reader sees that the vertices are at $(0,0),(0,1)$ and $(1,0)$ and a random number generator is used such as a die. The following experiment can be performed. The die is thrown and a 3 is shown. This says that the vertex 2 is indicated and the new coordinates of the new point are: $x=0.5 \cdot(x+0)=0.5 x$ and $y=0.5 \cdot(y+1.0)=0.5 y+0.5$. These equations can be re-written as:

$(\mathrm{IV} .2) \quad w(x, y)=(a x+b y+e, c x+d y+l)$ 
The coefficients can be grouped in a table. The table, Table IV-1, represents the "mapping" and is shown below.

$\begin{array}{lllllll}\text { w1 } & 0.5 & 0 & 0 & 0.5 & 0 & 0 \\ \text { w2 } & 0.5 & 0 & 0 & 0.5 & 0 & 0.5 \\ \text { w3 } & 0.5 & 0 & 0 & 0.5 & 0.5 & 0.5\end{array}$

\section{Table IV-1 Mapping for a Sierpinski Carpet}

In the "Chaos Game" the choice of the above "maps" is governed by a probability. The probability measure could be found by any means of generating a random sequence of numbers. Each "mapping" has an associated probability. For "honest" dice and a "good" random number generator the probabilities are all the same. If the probabilities are not equal then there is a bias in the number distribution. The shape of the attractor will not change but its details will. Again this table is known as a "IFS Code". If the data is generated is not generated artificially by a die or a random number generator the attractor will display characteristics that are inherent to that sequence. Jeffrey (1992) calls the IFS code a CGR (Chaos Game Realization).

Bias in a data sequence implies that the data sequence has a "structure" that may be visible or may be hidden. Mathematically, structure implies the presence in the sequence of internal subsequences. An "IFS Code" is a visual representation of a mathematical mapping. Jeffery (1992) demonstrated this could be done on DNA 
sequences. The bases "T, A, G, and C or U, G, A, and C" are used to generate the statistics for the "IFS Code". It's the equivalent of rolling a 4-sided die. For this research the four bases of DNA are associated with the 4-corners of a square. The IFS algorithm as was introduced in the literature review section is applied. Again for the sake of clarity it is restated below:

IFS Algorithm

1. The first " $G$ " is plotted halfway between the center of the square and the "G" corner.

2. The next base "A" is plotted halfway between the point just plotted and the "A" corner.

3. The base " $A$ " is plotted halfway between the previous point and the " $A$ " corner.

4. Next "T" is plotted halfway between the previous point and the "T" corner.

This procedure is continued until the sequence is exhausted.

Jeffrey (1992) generalized this mapping mathematically in the two theorems. These two theorems are restated again in their entirety for the sake of continuity of discussion. He considered an alphabet of 4 characters. These characters are, "a", "b", "c", and "d". They are assigned to the corners (vertices) of a square from the lower left-hand corner around in a clock-wise fashion. He later applied this to chicken hemoglobin DNA by assigning the 4-corners to the DNA base-pairs, T,A,G,C. 


\section{Theorem $1^{50}$ :}

There is a one-to-one map between the sequence and the interior square, in which the k-th point plotted on the GCR (or IFS Code) of a sequence corresponds to the first k-long initial sub-sequence of the sequence and no other subsequence (limited by the screen resolution). Thus, there is an on-to-one correspondence between the subsequences (anchored at the start) of a sequence and the points of the GCR.

Theorem $2^{50}$ :

In a GCR whose side is of length 1 , two sequences with suffix of length $k$ are contained within the square with side of length $2^{-k}$. Furthermore, the center of the square is given by the following recursive definition:

1. The center of the suffix of 0 length is $(1 / 2 /, 1 / 2)$.

2. If the center of the square containing sequences with suffix $w$ is at $(x, y)$ then

(a) the center of the square containing sequences with suffix wa is $(x / 2, y / 2)$;

(b) the center of the square containing sequences with suffix wb is $(\mathrm{x} / 2,(\mathrm{y}+1) / 2)$ 
(c) the center of the square containing sequences with suffix wc is $((\mathrm{x}+1) / 2,(\mathrm{y}+1) / 2)$

(d) the center of the square containing sequences with suffix wd is $((x+1) / 2, y / 2)$

This algorithm has been applied to the gene responsible for sickle cell anemia

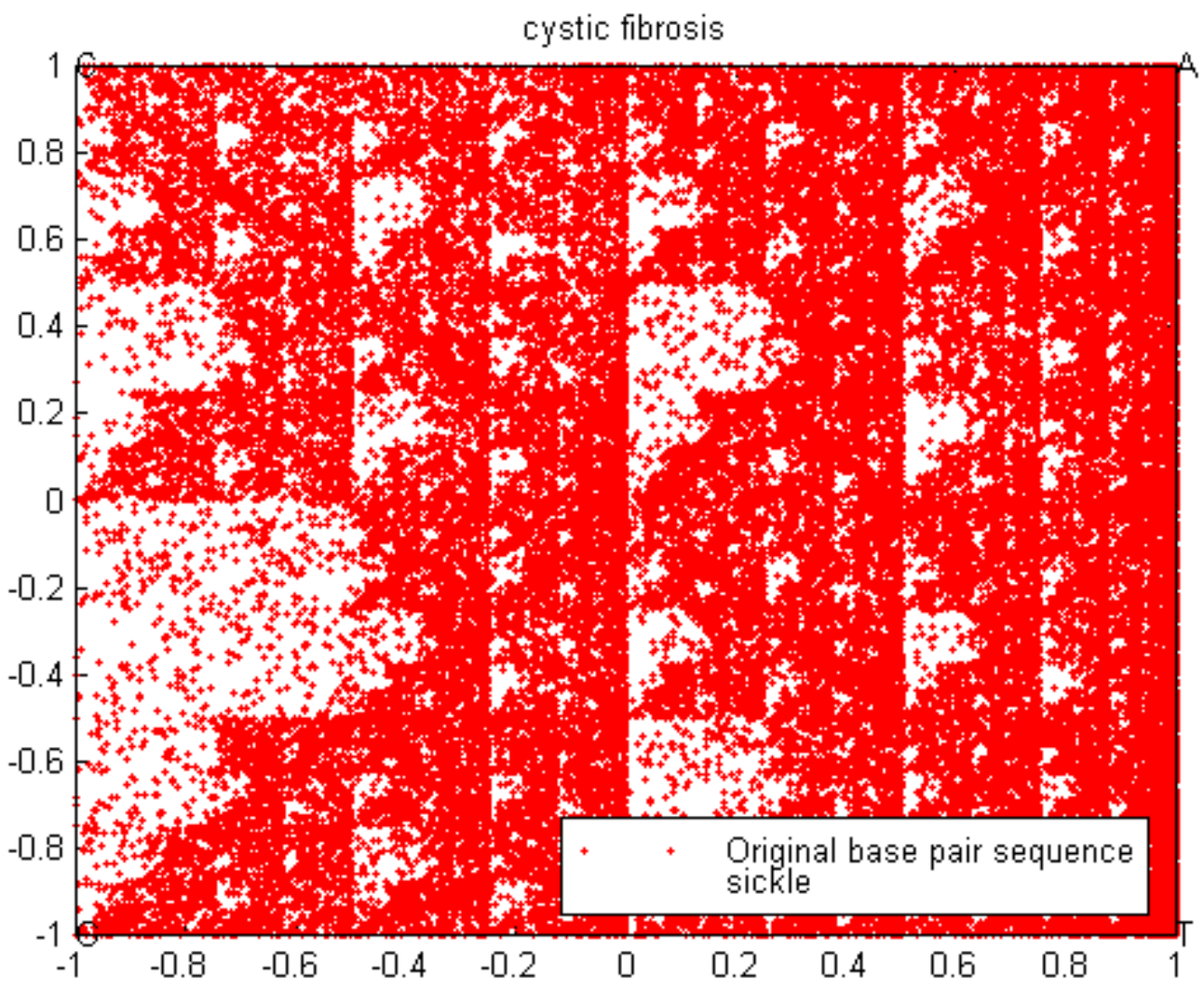

Figure IV-1

IFS Representation of a Sickle Cell Anemia Gene 
Features in the IFS display to consider:

1. The most obvious feature of the IFS plot is the sparseness in the lower left-hand corner. This is because the sequence is rich in " $\mathrm{A}$ " and "G" (particularly "A's") and their combinations and is low in "C's"

2. Note that a base will always be plotted somewhere in the quadrant.

3. The IFS plot displays self-similarity that is the same patterns re-occur at finer detail.

4. The self-similarity and the division into quadrants and sub-quadrants etc. are a property of the non-uniform in the probabilities of the subsequences and not a property of DNA.

One of the goals of this proposal is to examine the usefulness of these techniques for investigating DNA sequence structure.

In order to capture quantitatively the spatial extent of the IFS mappings, a concept first introduced by Pickover ${ }^{89,90}$ (1984) will be applied. This concept was used originally to capture the time-dependent low frequency mutations of the globular protein, "bovine pancreatic trypsin inhibitor". These proteins actually change their spatial extent over time. These fluctuations were quantified by calculating the "radius of gyration", Rg. 
(IV.3)

$$
\operatorname{Rg}[n]=\frac{\left(\sum_{i=1}^{n} r(i)-R(i)^{2}\right)}{n}
$$

Where $\quad r(i)=\left(x(i)^{2}+y(i)^{2}\right)^{.5}$

and $\mathrm{R}(\mathrm{i})$ is the "center of gravity" of the IFS mapping. The radius of gyration is a parameter useful for the comparing of the spatial extent of a collection of points (e.g. x, y points). Given this Rg's will be calculated for the original sequence and for all subsequent mutated sequences, then a comparison will be made.

(C) Theoretical Background: Wavelet Transforms $3,24,25,92,108$

The basic approach in testing the hypothesis is to estimate the $\mathrm{H}$ exponent for different DNA sequences the original non-mutated sequence and then various mutated sequences using the technique of Wavelet Transforms. The alternate hypothesis that is tested is that the sequence is multifractal, and this is tested against the null that the sequence follows random walk only, that is,

$H_{0}$ : The sequence is non-biased random walk, $(H=1 / 2)$.

$\mathrm{Hmf}$ : The sequence is multifractal $(0<\mathrm{H}<1)$ "mf multifractal" 
Wavelet transforms are a recently developed technique from the field of signal processing that has been designed to analyze complicated functions such as signals and erratic time-series processes (e.g., see Chui (1992), Cohen(1996), Jones (1996), Qian (1996)and Sprott( 1995)). Wavelet theory is very complicated and much of the wavelet literature requires that the reader understand the mathematics of functional analysis, group representation theory, Hilbert space theory, the Frobenious-Schur-Godement theorem, etc.. The coverage of wavelet transforms in this section, therefore, is in the form of an expository overview which is deemed necessary to introduce the topic and establish the groundwork for its application. The reader who is interested in a more rigorous treatment of wavelets is encouraged to refer to the sources cited. Wavelet transforms help to transform a time-series process into a more "wellbehaved" expression that yields certain useful properties. Wavelet transforms do this by breaking the time-series process down into many interrelated component pieces. Starting with a fundamental component or a "building block", called a basic wavelet, segments of the time-series process are replicated using variations of the basic wavelet obtained by shifting it left or right (translating) and dilating or compressing it (scaling). Transforming the timeseries process into its wavelet components is called wavelet decomposition. To get a graphical picture of how variations of a basic wavelet can be generated by translating and scaling it, consider the basic wavelet function $\psi(x)$ in Figure IV2a. Let $a$ and $b$ be scaling and translating factors, respectively. A set of wavelets, 
$\psi([\mathrm{x}-\mathrm{b}] / \mathrm{a})$ can then be generated by varying values for $\mathrm{a}$ and $\mathrm{b}$. Consider the following example, where $b$ the translation factor is set to 1 and the w scaling factor a is allowed to vary as $2^{j}$. The wavelet transform of a function of time provides information that is localized in time and at a particular scale. To see how this works, consider a family of simple quadratic wavelets. First we define a basic wavelet connecting four quadratic pieces ${ }^{91}$ :

$$
\begin{gathered}
\mathrm{f} 1(\mathrm{x}):=3 \cdot(\mathrm{x}+1)^{2} \\
\mathrm{f} 2(\mathrm{x}):=\frac{3}{4}+3 \cdot\left(\mathrm{x}+\frac{1}{2}\right)-9 \cdot\left(\mathrm{x}+\frac{1}{2}\right)^{2} \\
\mathrm{~F}(\mathrm{x}):=\mathrm{if}(\mathrm{x}<-.5, \mathrm{f} 1(\mathrm{x}), \mathrm{f} 2(\mathrm{x}))
\end{gathered}
$$

For the sake of this example let $\psi([x-b] / a)=G\left([x-1] / 2^{j}\right)$

$$
\mathrm{G}(\mathrm{x}):=\mathrm{if}(|\mathrm{x}|>1,0, \mathrm{if}(\mathrm{x}<0, \mathrm{~F}(\mathrm{x}),-\mathrm{F}(-\mathrm{x})))
$$

$\mathbf{G}$ has the appearance to a localized pulse that is zero outside the interval $[-1,1]$.

$$
\mathrm{x}:=-2,-1.99 . .2 .2
$$

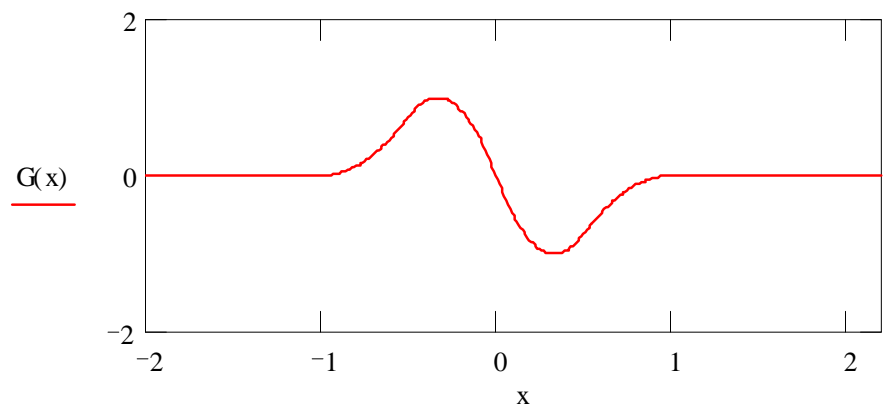

Figure IV-2a Basic Wavelet Function $\psi([\mathrm{X}-\mathrm{B}] / \mathrm{A})$.

Now we define a family of dyadic wavelets:

$$
W(x, j):=\frac{1}{\sqrt{2^{j}}} \cdot G\left(\frac{x-1}{2^{j}}\right)
$$


As $\mathbf{j}$ ranges over the integers, the wavelets $\mathbf{W}$ change in scale by a factor of 2 . For example, here are $\mathbf{W}(\mathbf{x}, \mathbf{1})$, which is twice as wide as the basic wavelet $\mathbf{G}$, and $\mathbf{W}(\mathbf{x},-\mathbf{1})$ which is half as wide. The scale factor in front maintains a constant integral for $\mathbf{W}^{2}$.

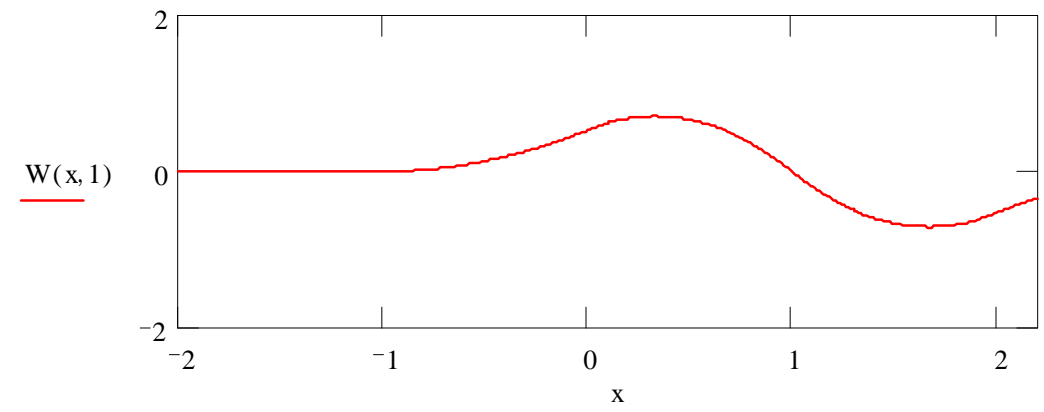

Figure IV-2b Basic Wavelet Function W(X,1) Where $B=1 \& A=1 / 2$

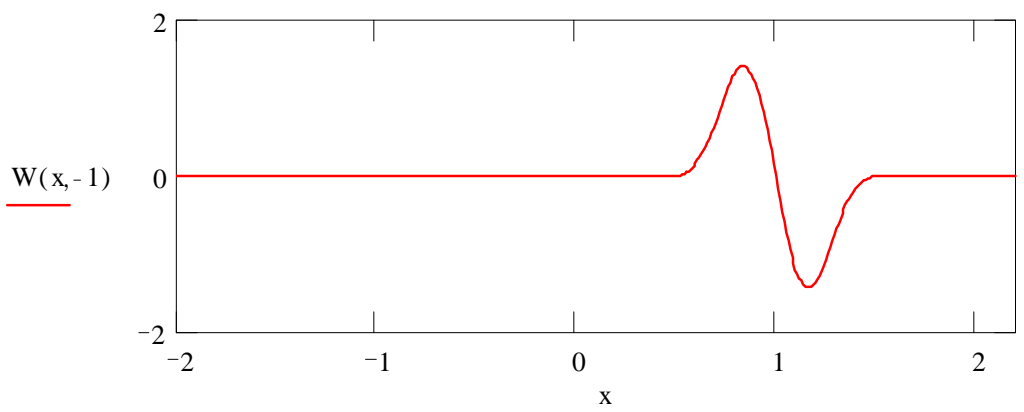

Figure IV-2c Basic Wavelet Function W(X,-1) Where $B=1 \& A=2$

The translation factor $b$ is currently set to 1 for ease of demonstration. In reality its value runs the entire length of the data set being analyzed. Each time $\mathrm{j}$ is incremented, the data is analyzed at the "coarse" setting (Figure IV-5a) where gross structure of the data are captured. Then process is repeated at the "fine" setting where the data's details are captured. 
The set of scaled and translated wavelets generated from a particular basic wavelet is then used to reconstruct an approximation of the time-series process. Essentially a time-series process is approximated by a weighted sum (or integral) over the wavelet set. To obtain the appropriate weight for each element of the wavelet set, the process being approximated is projected onto each element in the wavelet set. The result if each projection is a scalar number is called a wavelet coefficient that measures how well the original time-series process is approximated by the wavelet transforms. The building back process is called wavelet reconstruction. Why break a complicated process apart and then put it back together? This is because significant insights, gains and efficiencies can be obtained through the analysis process and operating on pieces rather than on the original time-series function.

Fractional brownian motion possesses the property of being statistically self-similar, which means that any portion of a given fbm can be viewed (from a statistical point of view) as a scaled version of a larger part of the same process. A fbm process, therefore, immediately lends itself to description in terms of wavelet transforms that are all deduced from one elementary wavelet by means of shifts and scalings. The application that will be used in this study to estimate the fractal dimension of a fbm via wavelet transforms follows the algorithm laid out by Wornell ${ }^{118}$ and Oppenheim (1992) and modified by Pancham $^{78}$ (1994) and later Jones ${ }^{51}$ (1996). 
The integral or continuous wavelet transform CWT $(a, \tau)$ of the fbm process

$f(x)$ relative to the basic wavelet $\psi$ at scale a and translation $\tau$ is defined by the following integral transform relationship,

$$
C W T(a, \tau)=|a|^{-1 / 2} \int_{-\infty}^{\infty} f(x) \cdot \psi\left(\frac{x-\tau}{a}\right) \cdot d x
$$

If $\psi(\mathrm{t})$ exist in such a way that this transform is invertible then

$$
s(t)=\frac{1}{c_{\psi}} \cdot \int_{-\infty \alpha>0}^{\infty} \int^{\infty} C W T(a, \tau) \cdot \frac{1}{\sqrt{a}} \cdot \psi\left(\frac{t-\tau}{a}\right) \cdot \frac{1}{a^{2}} d a \cdot d \tau
$$

where $\mathrm{C}_{\psi}$ is a constant that depends only on $\psi(\mathrm{t})$ and a is positive. This constant has a value expressed by the following:

(IV.6) $\quad c_{\psi}=\int_{0}^{\infty} \frac{\left|\psi(\omega)^{2}\right|}{\omega}<\infty$

This in turns imposes an admissibility condition on $\psi(\mathrm{t})$. For $\mathrm{c}_{\psi}<\infty$ $\psi(\mathrm{t})$ must be such that

(IV.7) $\quad|\psi(\omega)|<\infty$ 
for any $\omega$ and $\psi(0)=0$ implying that

(IV.8) $\int \psi(t) \cdot d t=0$

Discrete representations of the above relationships will be discussed later.

One can see from the above relationships that the wavelet transform of a data sequence or signal produces the following expression, CWT $(a, \tau)$. This expression is dependent on two variables "a" and " $\tau$ " which are actually indices. The characteristics of these indices will be discussed below. The are in general three types of data sequences that can be analyzed:

- continuous time/space data sequences,

- sampled time/space data sequences,

- discrete time/space signal recorded in discrete time.

And two types of transforms:

- continuous transform,

- discrete transform. 
From a laymen's viewpoint the wavelet decomposition consists of calculating a "resemblance index" between the data sequence and the wavelet. If the index is large the resemblance is strong. If the index is small the resemblance is slight. The indices of CWT $(a, \tau)$ are called coefficients.

The nature of the coefficients are defined by the nature of the analysis being done Since there are three types of analysis that can be done coefficients can come in three forms:

a) Continuous time "continuous analysis"

(IV.9)

$$
\begin{aligned}
& C(a, b)=\int_{R} s(t) \cdot \frac{1}{\sqrt{a}} \cdot \psi\left(\frac{x-b}{a}\right) \cdot d t \\
& a \in R^{+}-\{0\}, b \in R
\end{aligned}
$$

b) Continuous time "discrete analysis"

(IV.10)

$$
\begin{aligned}
& C(a, b)=\int_{R} s(t) \cdot \frac{1}{\sqrt{a}} \cdot \psi\left(\frac{x-b}{a}\right) \cdot d t \\
& a=\Delta 2^{j}, b=\Delta k 2^{j},(j, k)=Z
\end{aligned}
$$


c) Discrete time $(\Delta=1)$ \{e.g. DNA sequences\}

"discrete analysis"

(IV.11)

$$
\begin{aligned}
& C(a, b)=C(j, k)=\sum_{n \in Z} s(n) \cdot g_{j, k}(n) \\
& a=2^{j}, b=k 2^{j}, j \in N, k \in Z
\end{aligned}
$$

Earlier efforts used continuous analysis. The following examples will show how continuous analysis is the better choice even if the data being analyzed is clearly discrete.
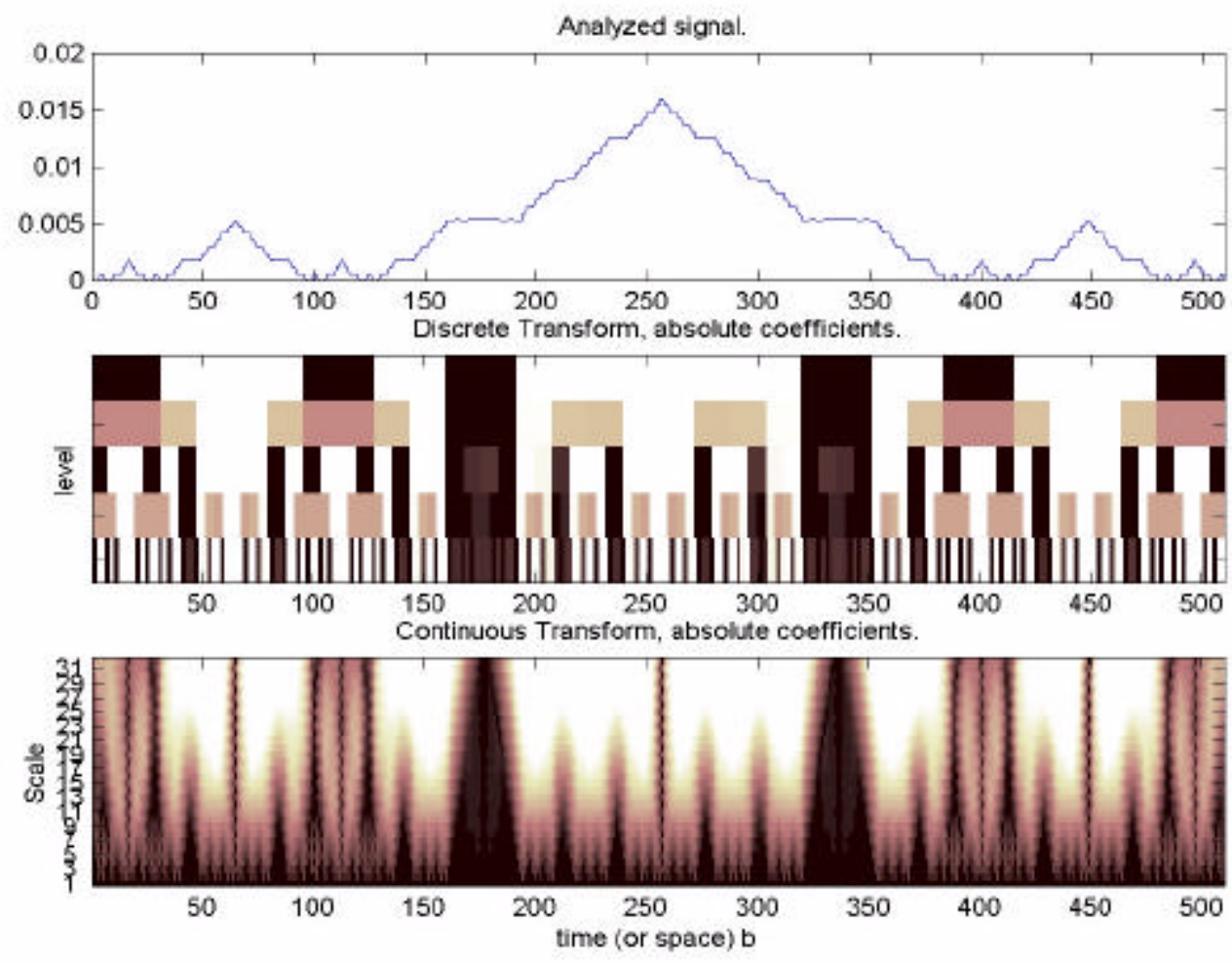

Figure IV-2 $d^{69}$ 
Which analysis is more appropriate continuous analysis or discrete analysis? Before this can be answered, ask the following questions; 'Are all the values of a continuous decomposition needed to reconstruct the data sequence exactly?' Can then a non-redundant analysis be done?

Consider the following, when the energy of the signal is finite, not all values of a decomposition are needed to exactly reconstruct the original data sequence, provided that are wavelet that satisfies some admissibility condition. Usual wavelets satisfy this condition. If this is the case then a continuous type of sequence can be entirely characterized by the knowledge of the discrete transform..$^{24,25,69,92}$

In $\mathrm{C}(\mathrm{j}, \mathrm{k}),(\mathrm{j}, \mathrm{k}) \in \mathrm{Z}^{2}$ cases i.e. discrete cases like DNA sequences, discrete analysis is sufficient and continuous analysis is redundant. However redundancy in visualizing data is often helpful So which should be used? The answer like most things in life is 'It depends!' Consider the following:

- Discrete analysis ensures space-saving coding and is sufficient for the synthesis.

- Continuous analysis as we can see in the above plot is visually more appealing and thus makes for easier interpretation. This is because of the aforementioned redundancy. This redundancy 
reinforces features in that data and makes them more visible This is very important when you are looking for very subtle information changes. What is lost is compactness in information encoding, which is not important for this type of analysis.

This clearly why Misti ${ }^{69}$ etc (1996) used continuous analysis in the 1996 Matlab Wavelet Toolbox Manual and will likewise be used unless otherwise stated in this project.

The global quantitative description of the irregularity (or 'jaggedness') observed in these profiles was solved by estimating the self-affine fractal dimension with the Hurst exponent and is similar to the numerical methods used to quantify 'DNA Walks' and heartbeat interval fluctuations ${ }^{3}$. This experimental approach to describe scale invariance in spatially extended systems has popularly been termed a 'statistical mechanical approach', in that micro-level scaling correlations are thought to impact on macro-level structural patterns. This viewpoint shares common features with adaptive walk models on fitness landscapes and the emergence of self-organized criticality to explain a possible mechanism underlying such patterns of fluctuation activity. A local Hurst exponent can be defined which shows local scale invariance. Changes in this exponent will reveal regions where the "self-similar state" changes. This local value reveals changes in a local fractional Brownian motion process. The local 
Hurst exponent can be estimated from the slope of the simple linear regression expression:

(IV.12)

$$
\log _{2}(\operatorname{Var}[W \Psi(i)])=\alpha+\beta i i=2,3, \ldots, \mathrm{N}
$$

where $\beta=2 H-1$. However, for data samples ${ }^{1}$ that are not large $(\mathrm{N}<30)$, the variance progression of the wavelet coefficients can be biased and this error may lead to an underestimation of the parameter $\mathrm{H}$.

For the algorithm by Wornell-Oppenheim (1992) that uses maximum likelihood estimation, the variance of the estimate for the $\mathrm{H}$ exponent is obtained from their information matrix and is given as,

$$
\operatorname{Var}(\widehat{H})=\frac{\sum_{i=1}^{N} 2^{N-i}}{2 \cdot(\ln 2)^{2}\left\{\sum_{i=1}^{N} 2^{N-i} \sum_{i=1}^{N} i^{2} \cdot 2^{N-i}-\left[\sum_{i=1}^{N} i \cdot 2^{N-i}\right]^{2}\right\}}
$$

Once $H^{\wedge}$ and $\operatorname{Var}\left(H^{\wedge}\right)$ are obtained, both can then be used in a conventional ttest to determine the statistical significance of the $\mathrm{H}$ estimate. The t-statistic to 
test whether $H^{\wedge}$ is significantly different from one-half is calculated $\mathrm{as}^{78}$,

$$
t-\text { statistic }=\frac{\widehat{H}-\frac{1}{2}}{\sqrt{\operatorname{Var}(\widehat{H})}}
$$

In this application, to estimate and test for the significance of the local $\mathrm{H}$ exponent of fbm using wavelet transforms of the Cystic Fibrosis sequences

After spending time describing what wavelet analysis is. The author thinks he is now justified in now generalizing wavelet decomposition, this is called wavelet packet decomposition .

\section{(D) Wavelet Packet Transforms}

In wavelet analysis, a data sequence is split into a approximation and a detail. The approximation is then split into a second-level approximation and detail this process can then be repeated if necessary to the limit of data granularity i.e. single data point. This would be where no more information about the data is obtainable. For an $n$-level decomposition there are $n+1$ possible ways to decompose (Note: Some authors use the term encode.) the data sequence. In wavelet packet analysis, 


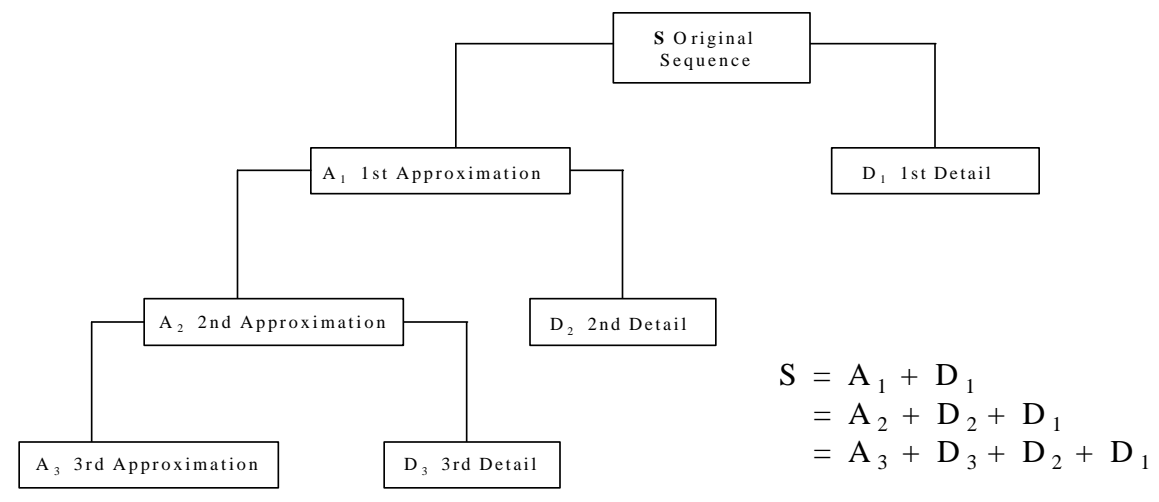

Figure IV.3a Tree Representation of a Wavelet Transform ${ }^{69}$

the details as well as the approximations can be decomposed.. This yields $2^{\mathrm{D}}$ different ways to decompose the sequence. This is wavelet packet decomposition tree.

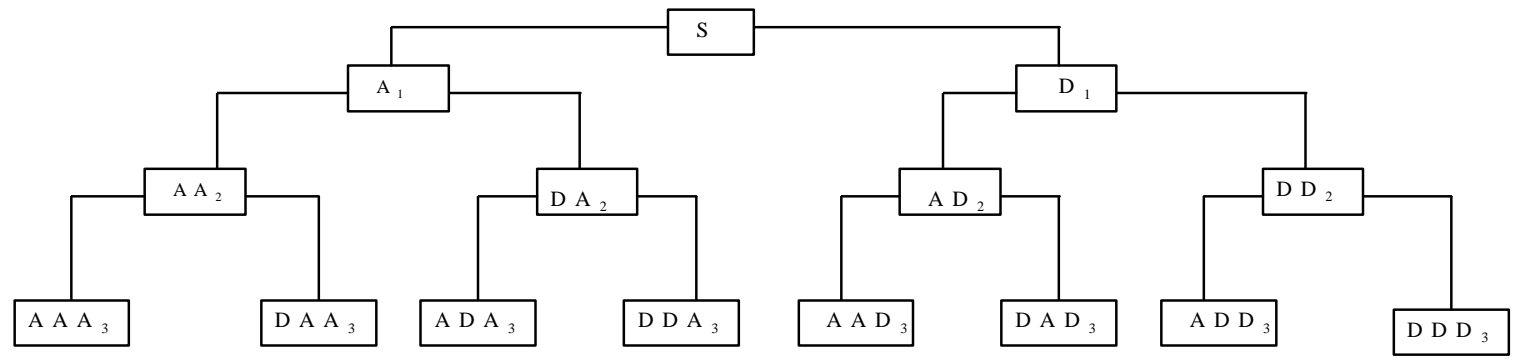

S - - O riginal Sequence

A - - approxim ation

D - - detail

Figure IV.3b Tree Representation of a Wavelet Packet Transform ${ }^{69}$ 
If one looks at Figure IV.2b wavelet packet analysis allows the data sequence to represented by lower branches of the tree structure as $S=A_{1}+A A D_{3}+D A D_{3}+$ $\mathrm{DD}_{2}$. It also clear that this is not the only possible representation. This type of representation is not possible with "common" wavelet analysis. Each transformation into the next level is an energy conservation process, and the total energy is considered to be the sum of all the individual energies mapping out the feature fluctuations.

(E) Minimum Entropy Method ${ }^{51}$ for Estimating the Global and Local $\mathrm{H}$ Exponent Using Best Basis Wavelet Packet Transforms

How does one choose and by what criteria does one choose a representation. A common criterion and one used in this study is an entropybased selection methodology to select a decomposition representation. An entropy-based approach looks at each node in the decomposition tree and quantifies the information to be gained by doing the split. An optimum decomposition can be selected using "best basis" of the wavelet packet transform. Jones in his 1996 paper used the Coifman and Wickerhauser ${ }^{51}$ algorithm to calculate the Hurst exponent via a wavelet packet decomposition.. They developed this adaptive filtering algorithm and used it mostly in optimal signal encoding and data compression. This approach is used to calculate the Hurst exponent in this study. To select an optimal transform of bases from the 
wavelet packet table, the best basis algorithm of Coifman and Wickerhauser is used. This algorithm is adaptive in that it minimizes a cost function by finding the minimum entropy for coefficients that belong to the best basis.

The goal of wavelet packet analysis is to create a binary tree decomposition of the signal into a set of energy levels, called "octave" windows where the frequency domain has been divided logarithmically. During the segmentation, each scale retains the dominant signal features while minimizing the wavelet coefficient amplitude of their representation. Because the wavelet packet coefficients contain information about the energy magnitude contribution for each discrete feature in terms of scale, frequency and position, this provides a robust method for feature and singularity detection. It is notable that determination of the singularity spectrum of fractal functions by wavelet analysis provides a microscopic statistical description of the scaling behavior in terms of thermodynamic energy functions.

The "best basis" method is more fundamental to wavelet packet analysis for signal approximation when compared with the more common discrete wavelet transform (DWT). In the wavelet packet transform procedure the input signal is decomposed with the $d 4$ wavelet (see Figure IV-3a \& Figure IV-3b). On the left, the DWT provides good decomposition of the coarse signal features but the WPT on the right results in superior decomposition and localization of fine, detail signal features. For the DWT, each detail resolution level is indexed by $D$ and the 
smooth signal by $\mathrm{S}$. The greater number of best basis function blocks for the best basis decomposition results in superior detection of local scaling trends. We then combine these coefficients into a single listing, and estimate the power law decay of sorted coefficient energies to determine the homogeneous Hurst exponent.

The computation methods for wavelet packets generation, is simple when using a orthogonal wavelet like the Haar wavelet. One starts with two filters of length $2 N$, called $h(n)$ and $g(n)$ corresponding to the wavelet These are the reversed versions of the low-pass decomposition filter and the high-pass decomposition filter divided by $\sqrt{ } 2$.

Using deduction the following sequence of functions can be defined: $\left(W_{n}(x), n=0,1,2,3,4 \ldots\right)$ by:

$$
W_{2 n}(x)=2 \cdot \sum_{k=0}^{2 N-1} h(k) \cdot W_{n}(2 x-k)
$$

(IV.15b)

$$
W_{2 n+1}(x)=2 \cdot \sum_{k=0}^{2 N-1} g(k) \cdot W_{n}(2 x-k)
$$


where $\mathrm{W}_{0}(\mathrm{x})=\phi(\mathrm{x})$ is the scaling function and $\mathrm{W}_{1}(\mathrm{x})=\psi(\mathrm{x})$ is the wavelet function. The mathematical representation of the Haar wavelet is

$N=1, h(0)=h(1)=1 / 2$ and $g(0)=-g(1)=1 / 2$

the equations then become:

(IV.16a) $\quad W_{2 n}(x)=W_{n}(2 x)+W_{n}(2 x-1)$

and

(IV.16b) $\quad W_{2 n+1}(x)=W_{n}(2 x)-W_{n}(2 x-1)$

$W_{0}(x)=\varphi(x)$ is the Haar scaling function and $W_{1}(x)=\psi(x)$ is the Haar wavelet it has compact support on the inteval $[0,1] . W_{2 n}$ by adding two $1 / 2$-scaled versions 
of $W_{n}$ which has compact support on the interval $[0,1 / 2]$ and $[1 / 2,1]$ and obtain $W_{2 n+1}$ subtracting the same versions of $W_{n}$.

By choosing a set of mother and father wavelet functions (a QMF) with known oscillation index, one can decompose an input signal to create a wavelet packet table. Figure IV. 4 shows the wavelet packet table created using the d4 wavelet function for an input fractional Brownian motion (fBm)-type sequence

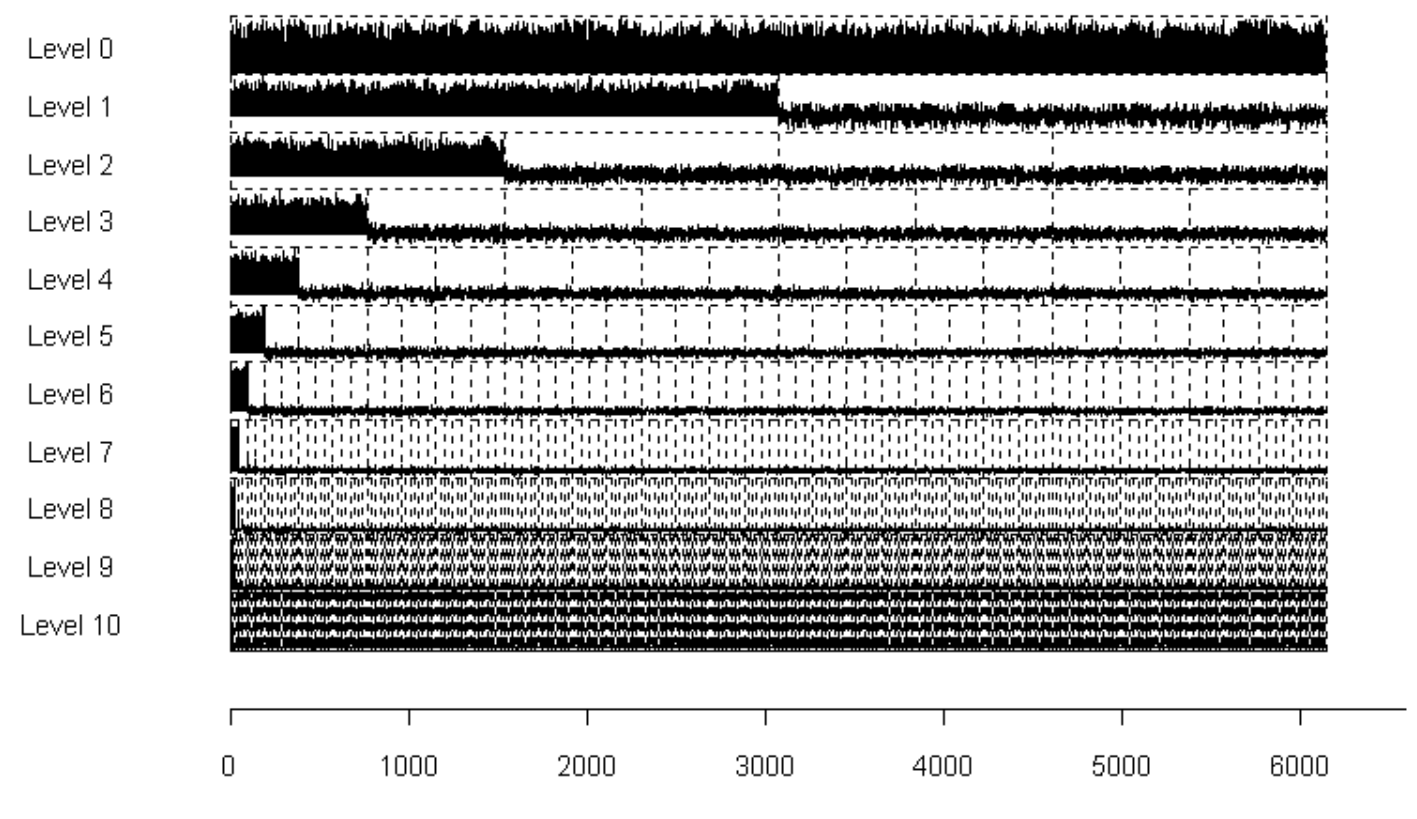

Figure IV.4 Wavelet Packet Transform to Level 10

For a signal in the case of the cystic fibrosis non-mutated sequence

$L=6129$ Each resolution level, $m$ has 6129 coefficients and is subdivided into 
blocks which contain the wavelet packet coefficients with an oscillation index $b=0,1, \ldots 2 \mathrm{~m}-1$. Level 0 reproduces the input, and resolution levels 1 to 10 are plotted in descending order. For each level, the left most block corresponds to an oscillation index $b=0$ and contains the low frequency, approximation coefficients. The block (or blocks) on the extreme right have an oscillation index $b=2^{m-1}$ and contain the high frequency, detail coefficients. Notably, the wavelet packet table is a redundant approximation of the sequence and for an input sequence of $L=6129$ the table contains $(M+1) \times 6129$ coefficients, $M$ is equivalent to the total number of resolution scales, $\mathrm{m}$ - which in this case is eight. We can now select a subset of coefficients from the wavelet packet table to create a unique, orthogonal wavelet packet transform.

The observed scaling between discrete coefficient energy magnitude and position is modeled in a sorted listing using the Korcak number-size frequency distribution power law. This shares a similar mathematical formalism to the Pareto distribution ${ }^{2}$ and the Mandelbrot-Zipf ${ }^{65}$ generalization, where one considers the number $\mathrm{N}$ of objects of size," A "greater than some minimum size ,a and relates this function to a frequency distribution. Rank and frequency can be introduced by making number analogous with probability where the overall power law can be expressed with ${ }^{52,51}$ :

$$
N(A>a) \propto k \cdot a^{-\delta}
$$


where $\mathrm{k}$ is a constant and $\delta$ is the slope exponent. Since we are considering a wavelet packet expansion which returns combinations of approximation and detail coefficients that minimize the entropy of their expansion (i.e. following best basis selection), this leads to a power-law relationship between number, $\mathrm{N} \mathrm{r}$ of coefficients $\mathrm{Cn}$ m having a particular energy size magnitude, and index position, $\mathrm{N}^{51}$ :

$$
N_{c}\left(C_{n}^{m}>N\right) \propto k \cdot N^{-\delta}=k \cdot N^{-(1+H)}
$$

The Hurst exponent parameter $\mathrm{H}$ is bounded by $0<\mathrm{H}<1$ for a graph of the function with a least squares linear regression fit through the data points. Since the slope exponent in (IV.17) and (IV.18) is equivalent to $-(1+\mathrm{H})$, the Hurst exponent may be determined from the rearrangement of terms ${ }^{51}$ :

$$
\delta+1=|H|
$$

or

$$
H=|\delta+1|
$$


By sorting the coefficients $C_{n}^{m}$ into decreasing order of discrete energy $\varepsilon$ following ${ }^{51}$ :

(IV.20)

$$
C_{1} \geq C_{2} \geq C_{3} \geq \ldots . .>C_{E}
$$

one can include best basis coefficients up to a particular energy value $\mathrm{E}$.

Therefore all included coefficients having discrete energies $\varepsilon$ will be $\geq \mathrm{E}$. It was found that for the $\mathrm{d} 2$ wavelet, the cutoff for smallest $E$ was to reject coefficients having discrete energies $\varepsilon \leq-0.25^{51}$.

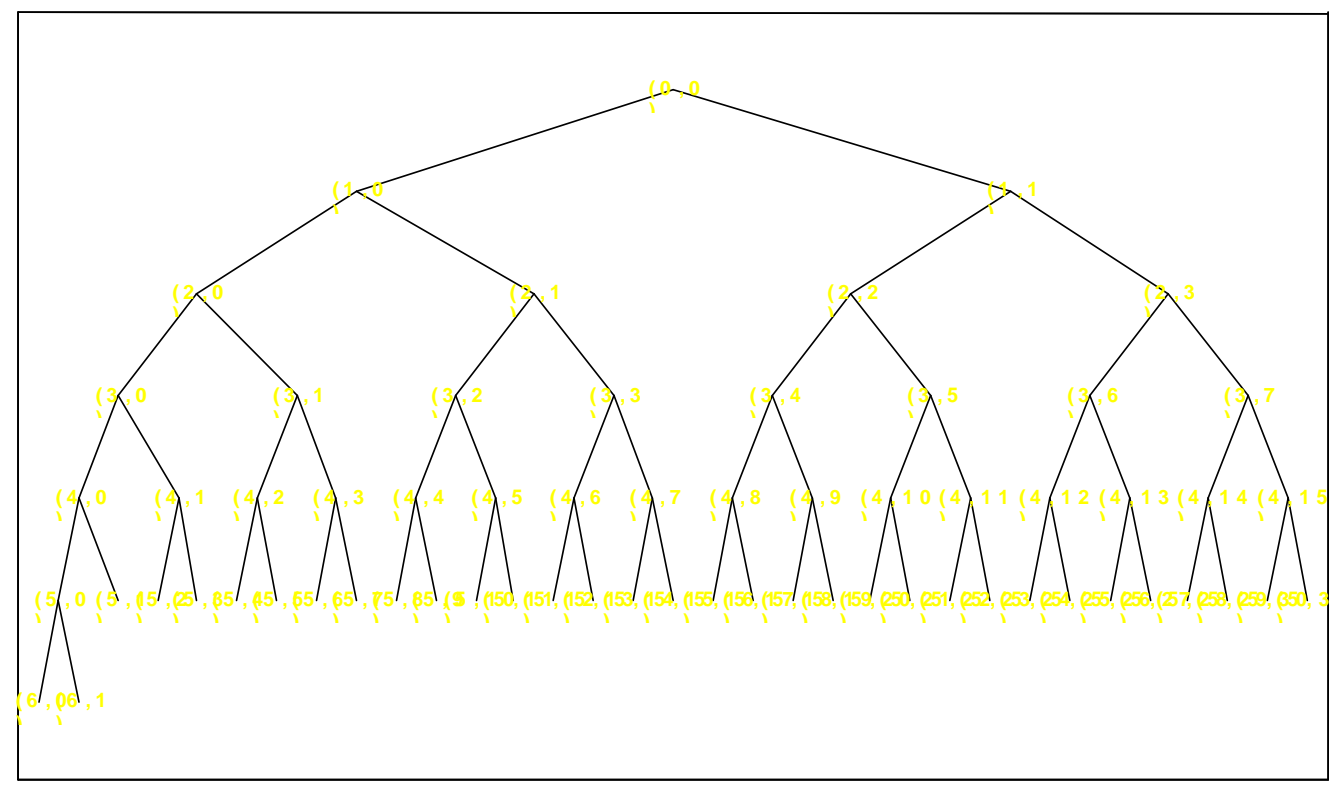

Figure IV.5a Wavelet Tree Representation of the Original Cystic Fibrosis Sequence. This Figure Is the Full Wavelet Decomposition. 


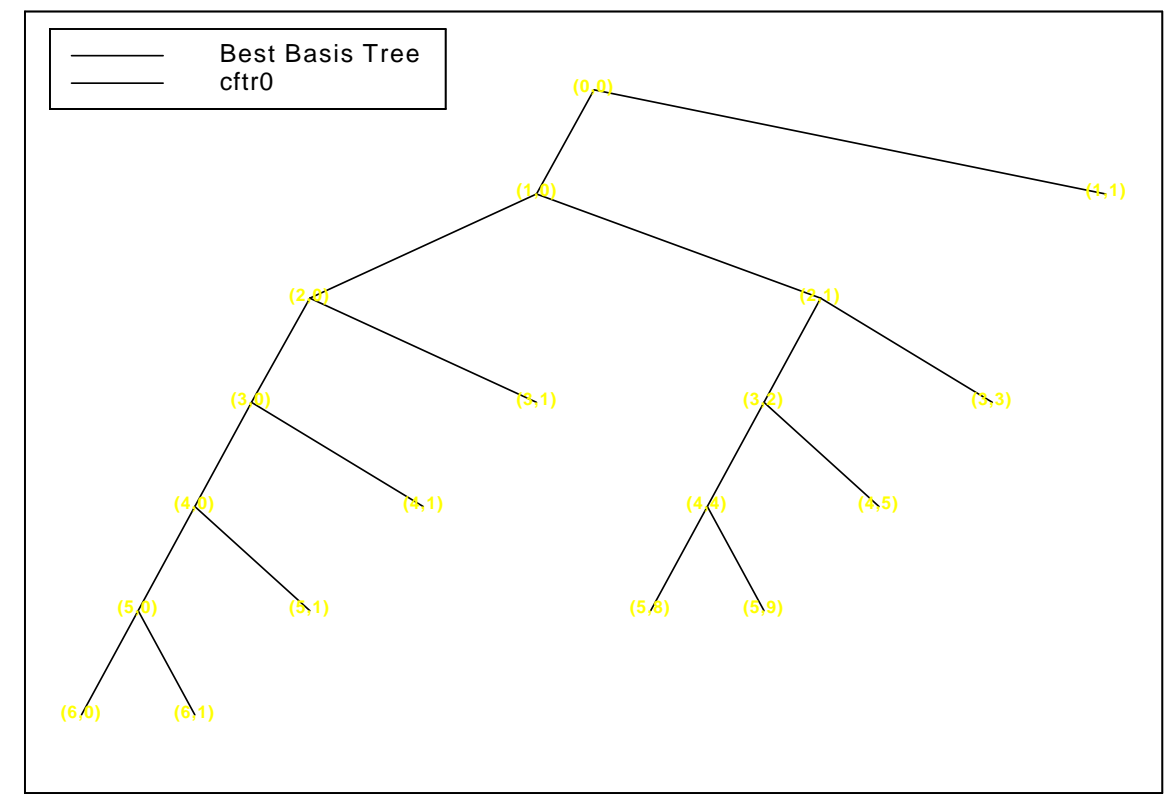

Figure IV.5b This Figure Is The "Best-Basis" Representation of the Original Cystic Fibrosis Sequence.

(F) Biological Data Set: Cystic Fibrosis DNA Sequence ${ }^{58,121}$

(1) Introduction

The DNA sequence chosen for this study was cystic fibrosis. It is a life threatening genetic disease of childhood. It is characterized by respiratory and digestive problems and is always fatal. The gene sequence is well known and the sequence location of the most damaging mutations are understood. The nongenomic sequence was analyzed. This was done to eliminate the possibility of confusion between the "information" content that distinguishes between coding regions (introns) from non-coding regions (extrons) and the information 
difference introduced by mutations. The next few paragraphs will discuss in general terms the state of current knowledge of the disease of cystic fibrosis as well as current forms of treatment. This is done to explain the biological origin of the sequence being studied its importance and the normal and pathologic cases.

(2) Biological Background

One of the earliest references to cystic fibrosis is an old saying, from northern European folklore,

"Woe to that child which when kissed on the forehead tastes salty. He is bewitched and soon must die. "121

Genetic diseases such as cystic fibrosis reflect gene mutations or abnormalities in chromosome structure or number and resulting in functional or anatomical changes. The faulty gene involved must be inherited from both parents. The disease is the most common inherited disease among Caucasians. Cystic fibrosis apparently is caused by the inability of chloride ions to cross the specialized epithelial cells of salivary, mucus, and sweat glands, and the pancreas. Effects include heavy production of thick mucus in respiratory tracts, which increases susceptibility to respiratory infections; 90 percent of all patients die of chronic lung disease. Secretions that block pancreatic ducts cause important digestive enzymes to fail to reach the small intestine. Treatment is directed toward relief of symptoms, and no cure is yet known. The discovery in

\footnotetext{
${ }^{1}$ 121. Welsh, M.J. and Smith, A. E. , 'Cystic Fibrosis', Scientific American, December 1995
} 
1989 of the gene that causes cystic fibrosis offers hope for improved treatments and genetic screening, but finding a cure is complicated by the increasing number of gene mutations found to be capable of causing the disease. The secretions become viscous, and an abnormally large amount of salt is present in the sweat; the latter characteristic is used as a diagnostic indicator. The disease affects the glands throughout the body, but pulmonary complications are very common.

As the medieval saying implies, the disorder once routinely killed children in infancy and is often identifiable by excessive salt in sweat. A salty brow is one of the more benign manifestations. The inherited genetic abnormality can also destroy the lungs and cause serious impairment of the pancreas, intestines and liver. Advances in therapy over the past few decades have brightened the outlook for afflicted children, enabling more than half of them to survive into their late twenties or beyond. However none of the approved treatments can yet correct the biochemical abnormality at the root of the condition, and none can remove the probability of an early death. The following table shows examples of 63 mutations

\section{Table IV.2 DNA Sequence Mutations In The CFTR Gene Coding Region}

Nucleotide position

$15 \mathrm{G} / \mathrm{A}$
Amino acid change

5' untranslated region
Exon

1
Reference

Claustres et al (NL\#67) 


\begin{tabular}{|c|c|c|c|}
\hline $156 \mathrm{G} / \mathrm{A}$ & $\begin{array}{l}\text { Lys at amino acid } \\
\text { position } 8 \text { (no change) }\end{array}$ & 1 & Claustres et al (NL\#67) \\
\hline $163 \mathrm{G} / \mathrm{A}$ & $\begin{array}{l}\text { Val or lle at amino acid } \\
\text { position } 11\end{array}$ & 1 & (NL\#50) Shackleton \& Harris \\
\hline $223 \mathrm{C} / \mathrm{T}$ & Arg or Cys at 31 & 2 & Ghanem et al. 1994 \\
\hline $263 \mathrm{~A} / \mathrm{T}$ & Asp or Val at 44 & 2 & Fanen et al. 1992 \\
\hline $345 \mathrm{~T} / \mathrm{C}$ & Asn at 71 (no change) & 3 & Claustres et al. (NL\#55) \\
\hline $356 \mathrm{G} / \mathrm{A}$ & Arg or Gln at 75 & 3 & Zielenski et al. 1991a \\
\hline $492 \mathrm{G} / \mathrm{A}$ & Ala at 120 (no change) & 4 & Dörk et al (NL\#67) \\
\hline $545 \mathrm{~T} / \mathrm{C}$ & Leu or Pro at 138 & 4 & Dörk et al. (NL\#50) \\
\hline $641 \mathrm{G} / \mathrm{A}$ & Arg or His at 170 & 5 & Devoto et al. (NL\#46) \\
\hline $676 \mathrm{~A} / \mathrm{G}$ & Ser or Gly at 182 & 5 & Claustres et al. (NL\#55) \\
\hline $741 \mathrm{C} / \mathrm{T}$ & no change (lle at 203) & $6 a$ & Casals et al. (NL\#65) \\
\hline $873 \mathrm{C} /$ & no change (Tyr at 247) & $6 a$ & TGasparini et al. 1991a \\
\hline $1047 \mathrm{C} / \mathrm{T}$ & no change (Phe at 305) & 7 & Edkins (NL\#54) \\
\hline $\begin{array}{l}\mid \mathrm{t} 11059 \mathrm{C} / \mathrm{G} \\
\text { 1096G/A }\end{array}$ & $\begin{array}{l}\text { no change (Ala at 309) } \\
\text { Val or Met at } 322\end{array}$ & $\begin{array}{l}7 \\
7\end{array}$ & $\begin{array}{l}\text { Férec et al. (NL\#45) } \\
\text { Férec et al. (NL\#51) }\end{array}$ \\
\hline $1104 \mathrm{C} / \mathrm{G}$ & No change (Pro at 324) & 7 & Petreska et al. (NL\#62) \\
\hline $1184 \mathrm{C} / \mathrm{G}$ & Thr or Ser at 351 & 7 & Mercier et al. 1993a \\
\hline $1191 \mathrm{~A} / \mathrm{C}$ & Gln or His at 353 & 7 & Férec et al. (NL\#65) \\
\hline $1531 \mathrm{C} / \mathrm{T}$ & Leu or Phe at 467 & 10 & Ghanem et al. (NL\#42) \\
\hline $1540 \mathrm{~A} / \mathrm{G}$ & Met or Val at 470 & 10 & Kerem et al. 1990 \\
\hline $1572 \mathrm{~T} / \mathrm{C}$ & no change (Gly at 480) & 10 & Ferec et al. (NL\#54) \\
\hline $1648 \mathrm{~A} / \mathrm{G}$ & Ile or Val at 506 & 10 & $\begin{array}{l}\text { Kobayashi et al. 1990; Vidaud } \\
\text { et al. per.comm }\end{array}$ \\
\hline $1650 \mathrm{C} / \mathrm{G}$ & Ile or Met at 506 & 10 & Chavelier \& Bozon (NL\#50) \\
\hline $1651 \mathrm{~A} / \mathrm{G}$ & Ile or Val at 507 & 10 & Will et al. 1992 \\
\hline $1655 \mathrm{~T} / \mathrm{G}$ & Phe or Cys at 508 & 10 & Kobayashi et al. 1990 \\
\hline
\end{tabular}




\begin{tabular}{|c|c|c|c|}
\hline $1713 \mathrm{~A} / \mathrm{G}$ & no change (Glu at 527) & 10 & Cutting et al. 1992 \\
\hline $1716 \mathrm{G} / \mathrm{A}$ & no change (Glu at 528) & 10 & Kerem et al. 1990 \\
\hline $1773 \mathrm{~A} / \mathrm{T}$ & no change (Thr at 547) & 11 & Gasparini et al. 1991 \\
\hline $1816 \mathrm{G} / \mathrm{A}$ & Val or lle at 562 & 12 & Fanen et al. 1992 \\
\hline $1859 G / C$ & Gly or Ala at 576 & 12 & Fanen et al. 1992 \\
\hline $2092 A / G$ & Ser or Gly at 654 & 13 & Claustres et al. (NL\#55) \\
\hline $2134 \mathrm{C} / \mathrm{T}$ & Arg or Cys at 668 & 13 & Fanen et al. 1992 \\
\hline 2209T/C & Phe or Leu at 693 & 13 & Ferec et al. (NL\#63) \\
\hline $2238 C / G$ & no change (Leu at 702) & 13 & Bienvenu et al. (NL\#61) \\
\hline $2553 A / G$ & Ile or Met at 807 & 13 & Ferec et al. (NL\#49) \\
\hline $2694 \mathrm{~T} / \mathrm{G}$ & no change (Thr at 854) & $14 a$ & Zielenski et al. 1991a \\
\hline $2736 \mathrm{G} / \mathrm{A}$ & no change (Val at 868) & $14 a$ & Quere et al. (NL\#40); \\
\hline $2839 \mathrm{~T} / \mathrm{C}$ & Tyr or His at 903 & 15 & $\begin{array}{l}\text { Balassopoulou \& } \\
\text { Hatzipanaiotou (NL\#66) }\end{array}$ \\
\hline $2858 \mathrm{G} / \mathrm{T}$ & Ser or lle at 909 & 15 & Schwartz et al. (NL\#59) \\
\hline $2901 \mathrm{C} / \mathrm{T}$ & Ala at 923 (no change) & 15 & Edkins \& Creegan (NL\#60) \\
\hline $3030 \mathrm{G} / \mathrm{A}$ & no change (Thr at 966) & 15 & Chillón et al. 1992b \\
\hline $3032 \mathrm{~T} / \mathrm{C}$ & Leu or Ser at 967 & 15 & Claustres et al. 1993 \\
\hline $3123 \mathrm{G} / \mathrm{C} \&$ & Leu or Phe at 997 & $17 a$ & Fanen et al. 1992 \\
\hline $3309 A / G$ & & & \\
\hline $3212 \mathrm{~T} / \mathrm{C}$ & $\begin{array}{l}\text { Ile or Thr at } 1027 \text { and no } \\
\text { change (Leu at 1059) }\end{array}$ & $17 b$ & Fanen et al. 1992 (NL\#50) \\
\hline $3332 \mathrm{C} / \mathrm{T}$ & Ala or Val at 1067 & $17 \mathrm{~b}$ & Costes et al., 1993 \\
\hline $3333 \mathrm{C} / \mathrm{T}$ & no change (Ala at 1067) & $17 b$ & Jezequel et al. (NL\#62) \\
\hline $3336 \mathrm{C} / \mathrm{T}$ & no change (Phe at 1068) & $17 \mathrm{~b}$ & Audrezet et al. (NL\#43) \\
\hline $3384 \mathrm{~A} / \mathrm{G}$ & no change (Leu at 1084) & $17 \mathrm{~b}$ & Bienvenu et al. (NL\#66) \\
\hline $3417 \mathrm{~A} / \mathrm{T}$ & no change (Thr at 1095) & $17 \mathrm{~b}$ & Dörk et al. 1994b \\
\hline $3471 \mathrm{~T} / \mathrm{C}$ & $\begin{array}{l}\text { No change (Ala at } 1113 \mathrm{~T}->\mathrm{C} \\
\text { change accompanying } \\
\text { Q1071P) }\end{array}$ & $17 \mathrm{~b}$ & Ghanem et al. 1994 \\
\hline $3617 \mathrm{G} / \mathrm{T}$ & Arg or Leu at 1162 & 19 & Fanen et al. 1992 \\
\hline
\end{tabular}




\begin{tabular}{|c|c|c|c|}
\hline $3690 A / G$ & no change (Gln at 1186 ) & 19 & Férec et al. (NL\#45) \\
\hline $3726 \mathrm{G} / \mathrm{T}$ & no change (Val at 1198) & 19 & Claustres et al. 1993 \\
\hline $3791 \mathrm{C} / \mathrm{T}$ & Thr or lle at 1220 & 19 & Ghanem et al. 1994 \\
\hline $3867 A / G$ & no change (Arg at 1245 ) & 20 & Pignatti et al. (NL\#15) \\
\hline $4002 A / G$ & no change (Pro at 1290 ) & 20 & $\begin{array}{l}\text { Férec et al. (NL\#34); } \\
\text { Ivaschenko et al. } 1993\end{array}$ \\
\hline 4029A/G & no change (Thr at 1299) & 21 & Fanen et al. 1992 \\
\hline $4050 \mathrm{C} / \mathrm{T}$ & no change (Pro at 1306) & 21 & Férec et al. (NL\#52) \\
\hline $4086 \mathrm{~T} / \mathrm{C}$ & no change (Val at 1318) & 21 & Claustres et al. (NL\#58) \\
\hline $4356 \mathrm{G} / \mathrm{A}$ & Leu at 1408 (no change) & 23 & 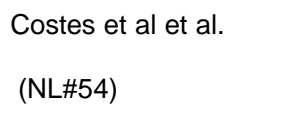 \\
\hline $4404 \mathrm{C} / \mathrm{T}$ & no change (Tyr at 1424$)$ & 24 & Shoshani et al. (NL\#37) \\
\hline $4521 \mathrm{G} / \mathrm{A}$ & no change (Gln at 1463 ) & 24 & Gasparini et al. 1991a \\
\hline
\end{tabular}

In the early 1980 s efforts were made to identify the specific genetic alteration that gives rise to cystic fibrosis. After about a decade of searching, the affected gene was isolated and then the mutation that most often leads to the disease was pinpointed. In those days genetic researchers could only guess at the gene's normal function that is, at the function performed by the protein produced from the healthy DNA. However a series of discoveries have been made where researchers have learned that the protein serves as a channel through which chloride, one component of salt, enters and leaves cells. These discoveries have explained how damage to the gene block chloride transport. These research efforts examined how the loss of chloride movement brings on 
the overt signs of cystic fibrosis. It is hoped that these findings will suggest new ideas for therapy. Some of the results this research may one day lead to a cure for the disorder.

One of the first major breakthroughs in cystic fibrosis research came in 1938 from Dorothy $\mathrm{H}$. Andersen of Columbia University. She had the sad but scientifically important task of performing autopsies on infants and children. Then she reviewed the youngsters' case histories. This provided the first comprehensive description of the symptoms of cystic fibrosis and of the changes the disease produced in organs. She discovered this almost always included destruction of the pancreas (even in infants) and infection and damage to the lung airways. Andersen coined the name for the disease. She called it "cystic fibrosis of the pancreas," on the basis of microscopic features she observed in pancreatic tissue.

After World War II physicians discovered that ductal systems and other passageways in the organs affected by cystic fibrosis generally become blocked with unusually thick secretions. In the pancreas, for instance, ducts that transport digestive enzymes to the intestines almost always become occluded. This action impairs the body's ability to break down food and extract nutrients from it.

The bronchial tubes in the lungs and the bronchioles become obstructed. In healthy patients these passages are usually bathed with a thin layer of mucus 
that traps inhaled particles and carries them to the throat for removal. However patients with cystic fibrosis, the mucus becomes very thick and resistant to removal. This change by itself narrows air passages and disrupts normal breathing. Also when bacteria remain in the air passages infections readily set in. These infections, which often recur, harm lung tissue by recruiting immune cells that secrete injurious chemicals and enzymes. As time marches on, chronic infection progressively destroys the bronchial passages and progressively plugs the airways; this of course ultimately leads to respiratory failure.

In 1946 studies of patients showed something about the genetics of cystic fibrosis. The pattern of disease inheritance is examined in families, researchers decided that cystic fibrosis is a recessive condition, caused by mutation of a single gene. If an infant inherited a damaged copy of the gene from both parents and this infant made no normal molecules of the protein specified by the gene, the child would then became ill; however, receipt of one good copy and one damaged copy did not produce disease.

Cystic fibrosis is now known to be among the most common genetic diseases and to strike mostly whites. About 5 percent of white Americans are asymptomatic carriers, harboring a single mutant version of the gene in their cells. One child in approximately 2,500 of European descent carries two defective copies and has the disease. In the U.S. such numbers translate into 
about 1,000 new cases a year and a total of some 30,000 people live with the disorder today.

Seven years after the inheritance pattern was identified, there was a heat wave in New York City. Hospitals saw a large number of children with cystic fibrosis, who apparently became dehydrated more readily than other youngsters. Paul di Sant' Agnese and his colleagues at Columbia University then found that boys and girls with cystic fibrosis lose an excessive amount of salt in sweat. This is what gave rise to the medieval saying. The reason for this increase in saltiness would not be discovered for many years, but the observation was of great clinical value. A test was developed that remains the basis of diagnosis: measurement of the chloride content in perspiration.

The earlier clinical work led to more accurate diagnosis and better treatments. Pancreatic failure is rarely life threatening today because patients replace their missing digestive enzymes with capsules taken when they eat. These digestive problems can generally be controlled. Lung impairment accounts for more than 90 percent of the disability and death in patients with cystic fibrosis. Treatment methods for the lung disease have greatly increased in recent years. Current therapy does include old techniques called postural drainage and chest percussion. Patients lie down with their head is tilted downward; then someone pounds gently and rapidly on their back or chest as if hitting the bottom of a catsup bottle. This is done to clear mucus from the airways. Patients can also benefit from a range of antibiotics that help to control the repeated infections, 
however it does not eliminate them. Recently a new treatment has become available. The patient inhales a drug called DNase. This compound breaks up mucus by digesting long, sticky strands of DNA released from dying cells.

Research into the biochemistry of cystic fibrosis yielded slower progress in information gained by clinical work. The pace picked up in the first half of the 1980s. Scientists realized that malfunction of epithelial tissue was at fault in every organ impaired by cystic fibrosis. (An epithelium is a sheet of cells that form a barrier between different compartments of the body; such sheets, which often secrete mucus, line the intestines and many ducts.) This revealed two important facts:

(1) epithelia of patients with cystic fibrosis were relatively impermeable to chloride.

(2) This discovery implied that some chloride-transporting channel in epithelial tissue was malfunctioning.

Paul M. Quinton of the University of California at Riverside found that the epithelia lining the ducts of sweat glands failed to take up chloride efficiently from cavities (lumen) of the glands. This explained why people with cystic fibrosis have unusually salty sweat. Sweat is normally produced at the base of sweat glands; it then flows to the skin surface through a narrow duct. Initially the sweat is a solution rich in sodium and chloride ions that are the constituents of salt. 
When fluid traverses the duct, the ions escape into the epithelium, leaving the water behind. Thus, the sweat that emerges to cool the skin surface is only slightly salty. In patients with cystic fibrosis, in contrast, the inability of epithelial tissue to absorb chloride and the consequent impairment of sodium absorption from the duct lumen cause sweat to retain excess sodium and chloride and to become abnormally salty. 


\section{Discussion and Results}

In a sections II, III and IV the Hurst exponent is described as a measure of the bias in fractional Brownian motion where $\mathrm{H}=0.5$ for pure Brownian motion; $0.50<\mathrm{H} \leq 1.00$ for a persistent or trend-reinforcing series; $0 \leq \mathrm{H}<0.50$ a antipersistent or trend reversing series. The inverse of the $\mathrm{H}$ exponent is equal to the alpha, $\alpha$ which is the characteristic exponent for fractal ${ }^{117}$ or Parteo distributions ${ }^{2,3}$. All fractals scale according to a power law. The underlying subsequence structure was shown using the IFS mapping procedure. Then this was captured from the two-dimensional IFS structure by a Euclidean distance measurement $r=\left(x^{2}+y^{2}\right)^{5}$, to the $x y$-origin, $(0,0)$. This $r$ results in a series of numbers that are subjected to wavelet packet decomposition. This decomposition is shown below as a binary tree representation. The "binary" breakdown of the sequence is into numbers (wavelet coefficients) that represent "gross approximations " and "details" of the sequence. This of course is done at ever increasing smaller power of 2 scales. The lowest level of the binary tree, the "leaves" represent all the information that exists about the signal at that level of detail or scale.

An optimal way of representing the "information" content in the binary tree is using an optimization technique developed by Wickenhauser and Coiffman ${ }^{51,118}$. They had a need for optimal representations of these decompositions in order to use wavelets for signal compression. A cost function is used to minimize "entropy". Changes in the "entropy" regime of the sequence 
drove the binary representation, this in general simplified the decomposition tree structure to a minimal entropy representation. This technique provides this research effort with a method of examining entropy i.e. "information" changes due to changes from the original DNA sequence. These are of course mutations. Such changes manifests themselves in visual changes like a different optimal tree structure called "best basis" but would also show up in different local scalings. A local Hurst exponent is calculated. Mutations are then examined using the aforementioned methodologies for their ability to perturb the "information" state of the original sequence.

Results:

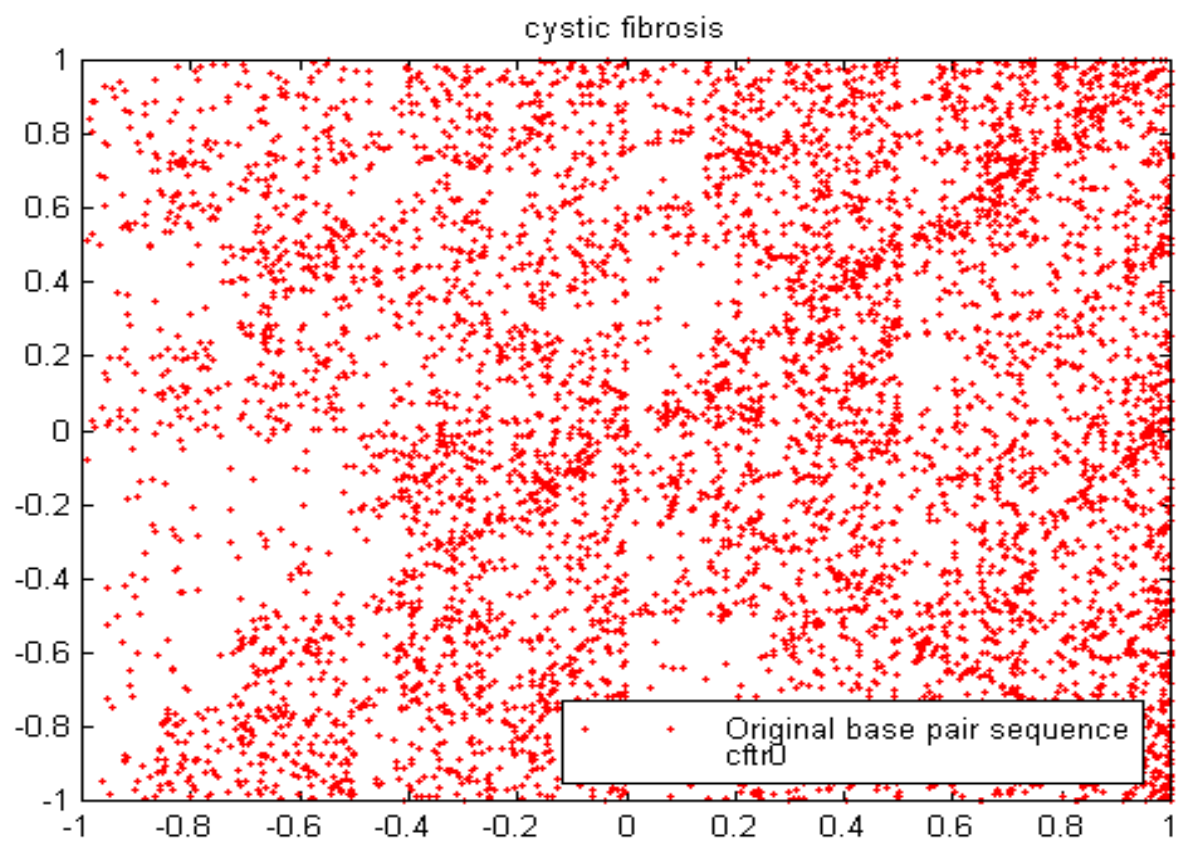

Figure V-1a IFS Mapping of the original sequence 


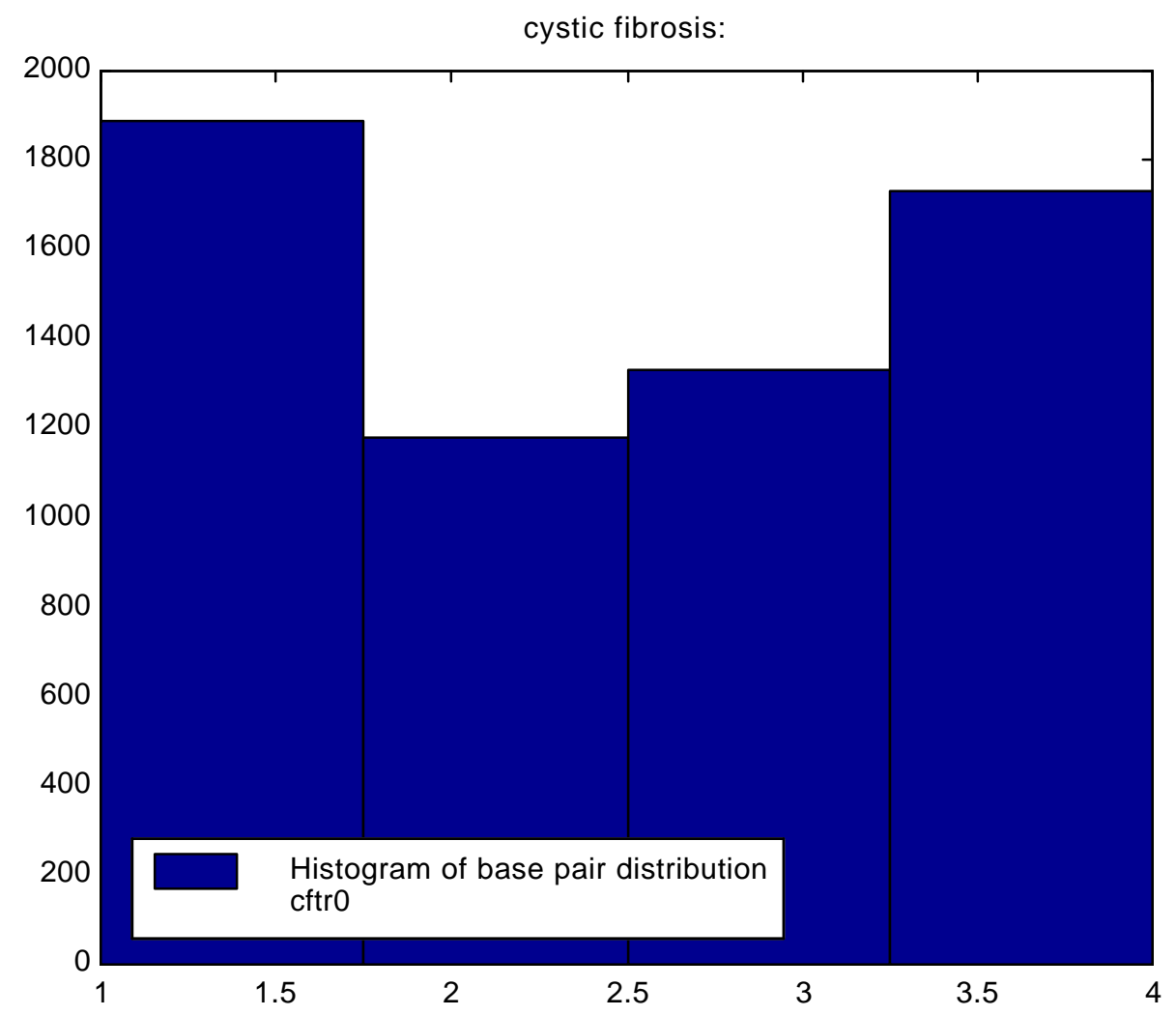

Figure V-1b Histogram of the of base pairs

In the IFS mapping of the original sequence one can clearly see that mutifractal nature of the DNA sequence. In Figure 5-1a there is a repeated two-dimensional pattern that re-occurs but at a larger scale going from right to left. Figure $5-1 \mathrm{~b}$ is included to simply show the original base pair distribution. This only changes with mutations that are deletions 


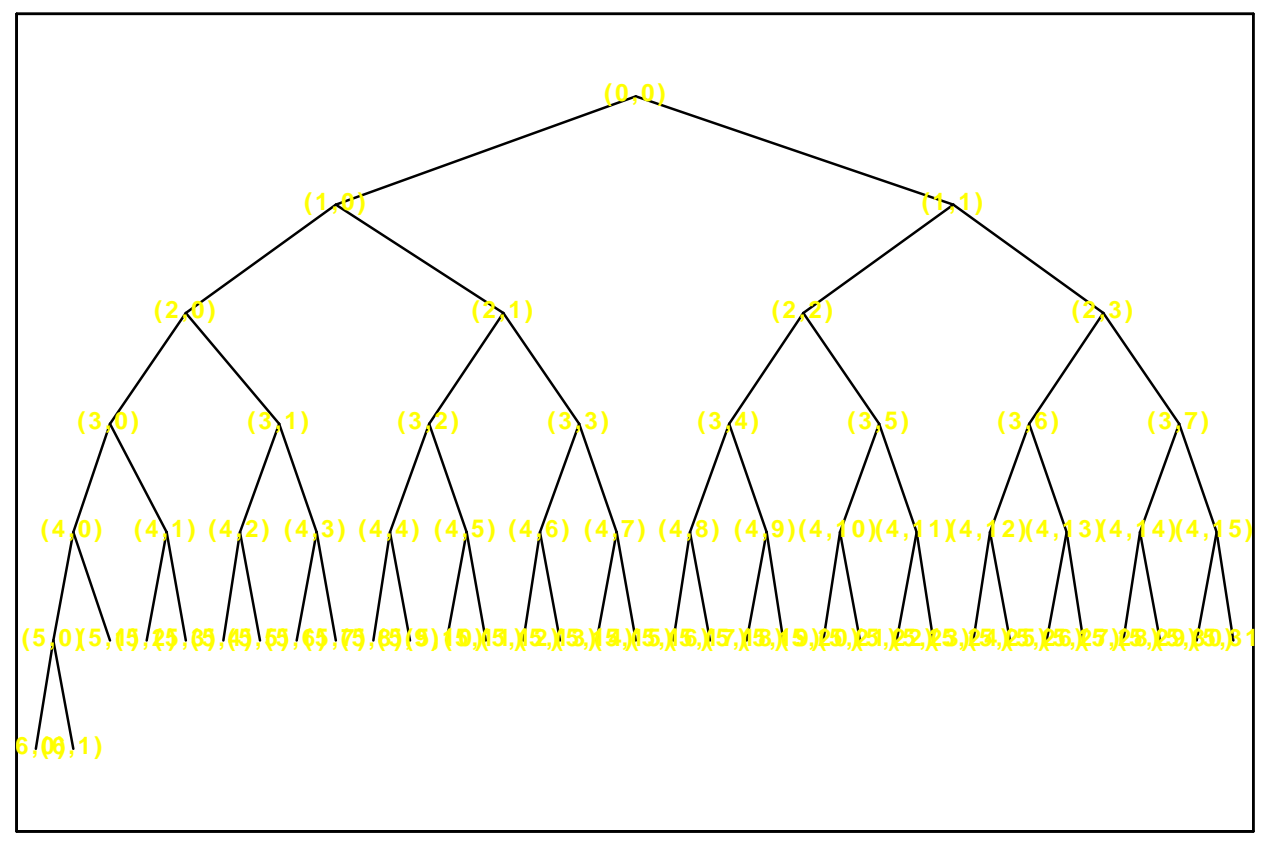

Figure V-2a Wavelet Packet Decomposition

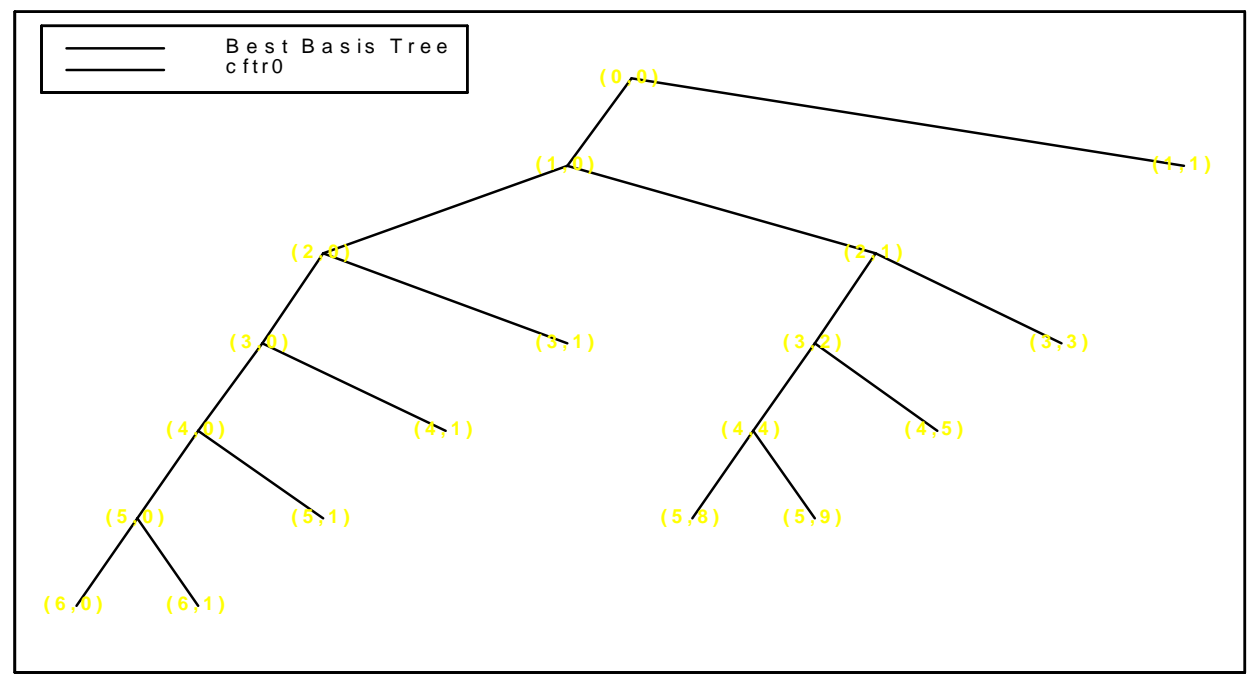

Figure V-2b Best Basis representation 
Again the "leaves" hold all the information that is necessary for a complete reconstruction of the original sequence.

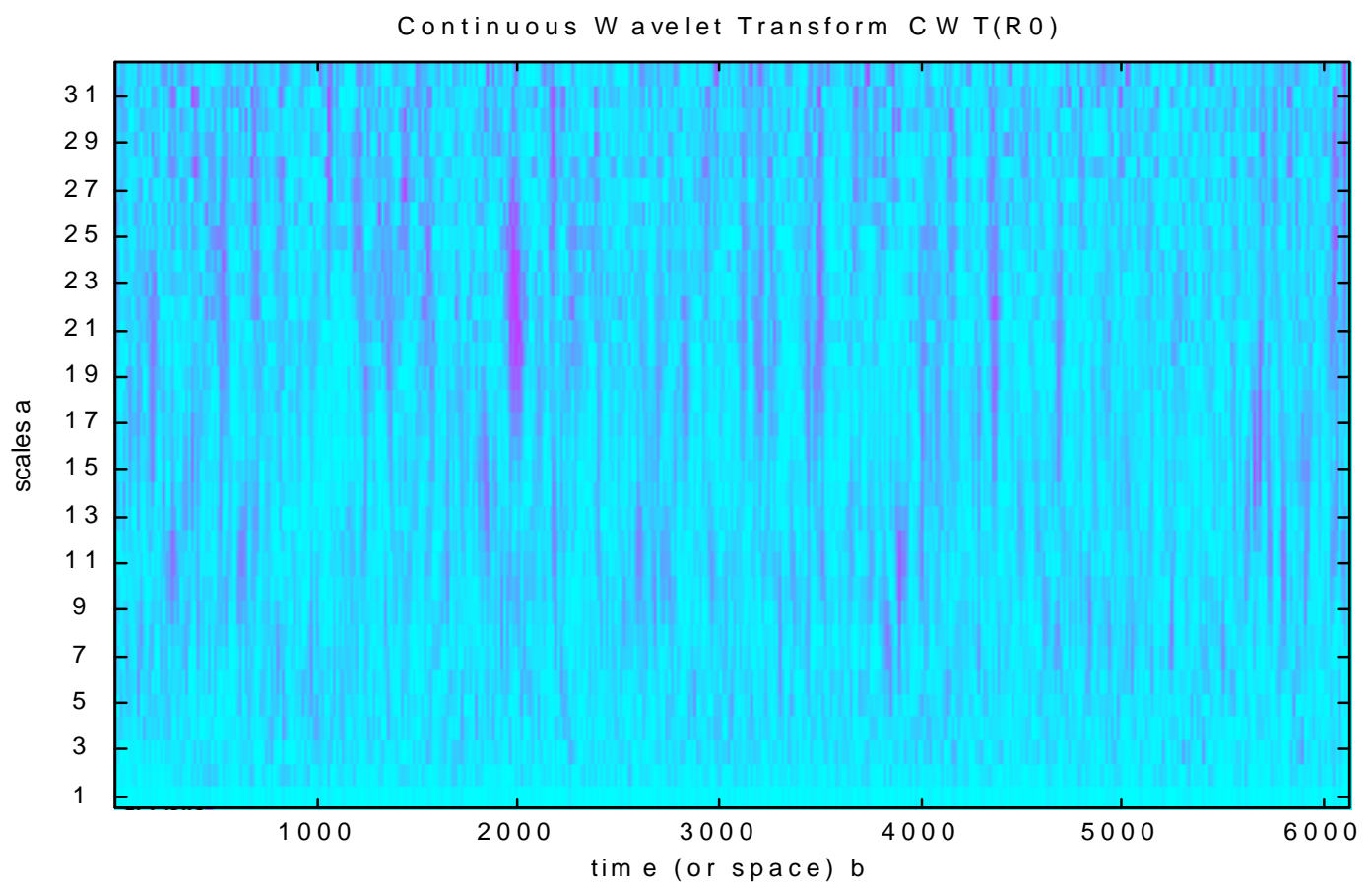

Figure V-3 Plot Coefficients of the Continuous Wavelet Transform

The uniformity along the scaling axis shows the essentially multifractal nature the sequence. The disturbances or "bright spots" show up in the above plots, e.g. at approximately 2000 on the pseudo-time (base pair position) axis. They range from 27 to 15 on the scale axis. The observation that they seem to repeat 5 times at about every 1500 sequences is from the production rules which reproduce the scaled pattern in the original sequence. 


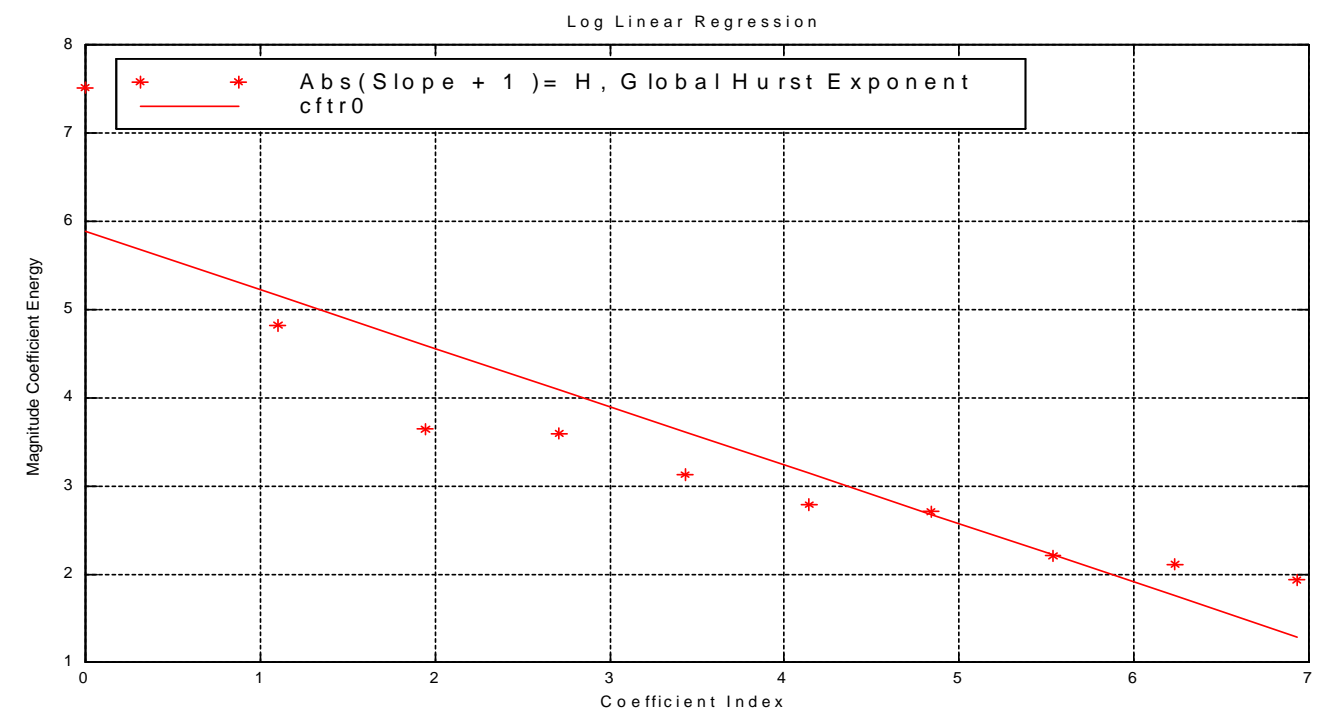

Figure V-4 Log-Log Plot Of The Best Basis Wavelet Packet Energy Vs. The Coefficients Index A Least Square Approximation Of The Slope Is Calculated. $|\delta+1|$ Is The Global Hurst Exponent

If you observe the sequence as a whole, it with the global Hurst exponent displays "anti-persistent behavior. If the system has been trending upward or downward, it will probably reverse. The number $\mathrm{H}_{\text {global }}$ is the "relative strength" of the sequence's tendency not to reverse. Note an anti-persistent sequence covers less distance then a random one. Remember a random sequence is a sequence where $H=1 / 2$. This sequence must reverse itself more frequently then a random process. Statistical theorists would call this a "mean-reverting process". However that notion assumes that the system under study has stable mean. No such assumption is made here. 


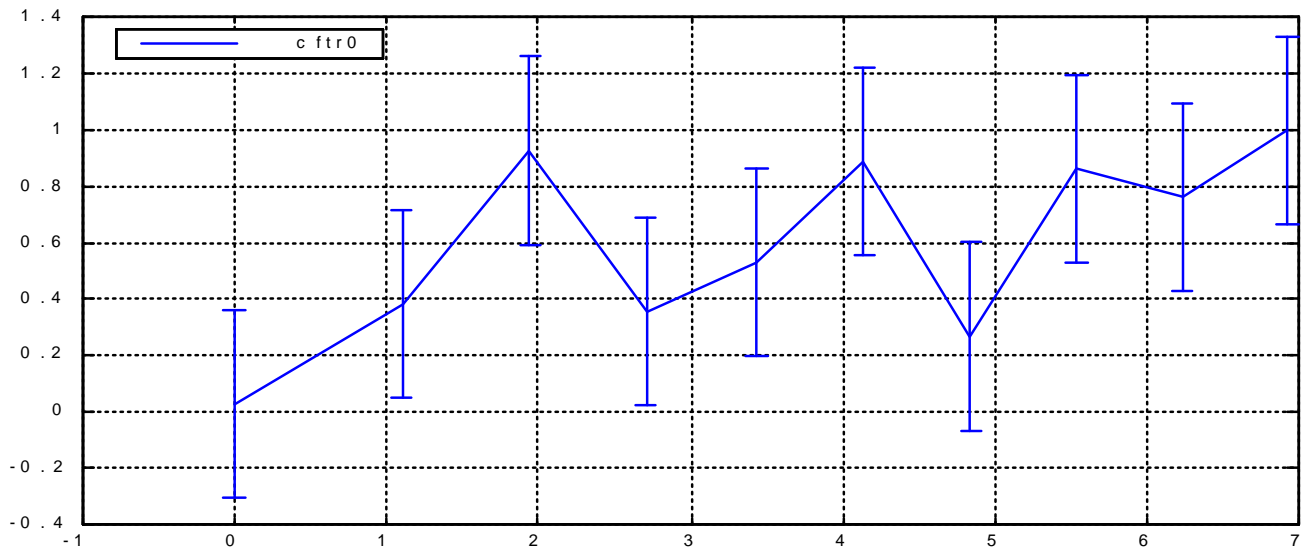

Figure V-5 Log-Log Plot Of The Local Slopes Best Basis Wavelet Packet Energy Vs. The Coefficients

A particular observation of this plot is that the Hurst exponent changes state from anti-persistent to persistent and back again several times. The persistent state represents the building of a "2-D pattern". The pattern is then lost as the sequence transitions to a new scaling state. This scaling state is gained and a re-scaled version of the pattern is thus repeated.

The following data sets will show how mutations effect plots by changing the "information" content of the sequence, which changes the entropy. This entropy change then drives the selection of the best basis representation. This is used to calculate the both global and local Hurst exponents. 


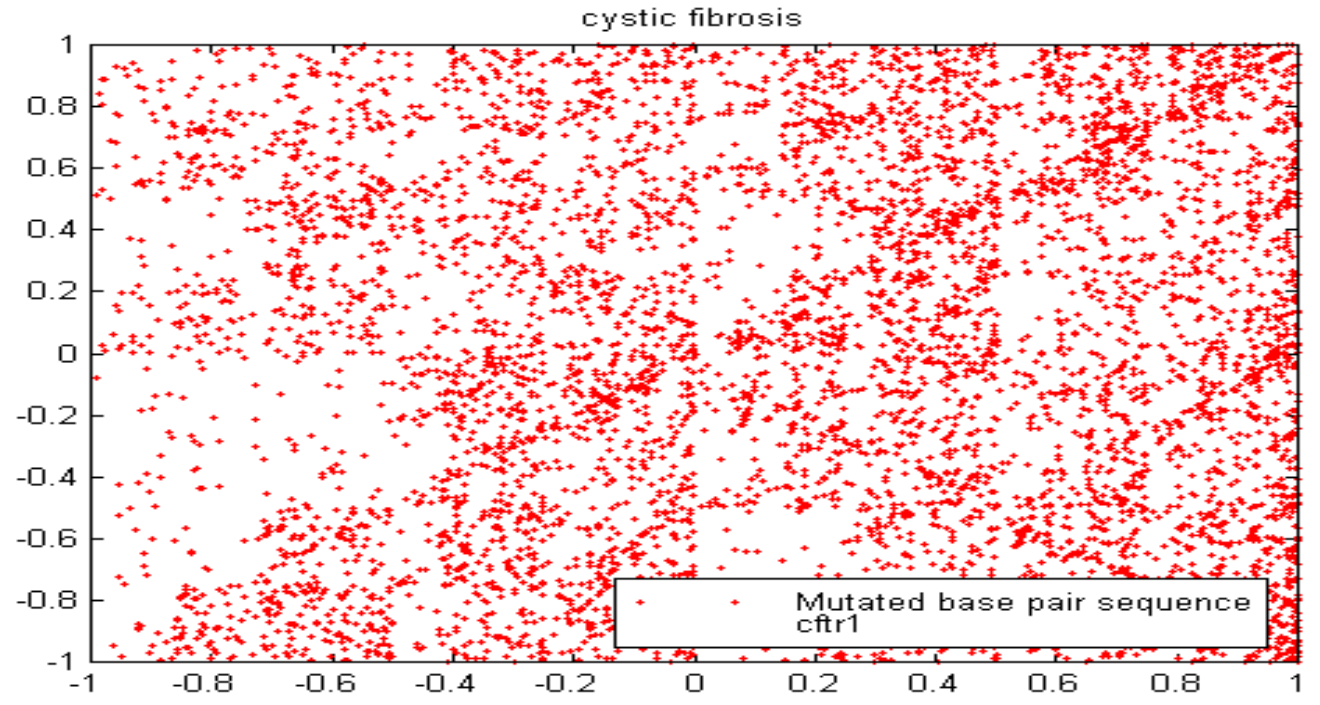

Figure V-6a IFS Mapping Of the Cftr1.Txt Sequence

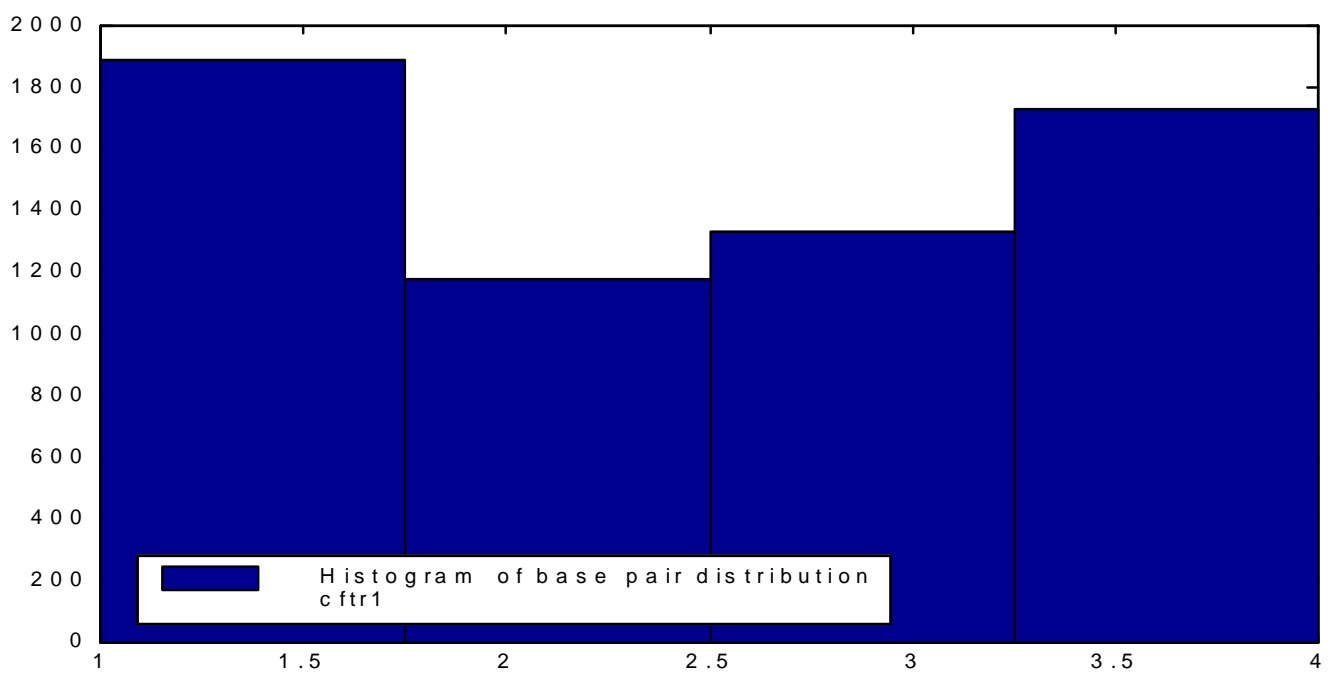

Figure V-6b Histogram Of The Base Pairs 
In line 1621 at base pair positions $1654-1657$ a amino acid i.e. 3 base pairs are deleted Specifically it is the following ' tc atCTTtggtg ttt' i.e. CTT is deleted

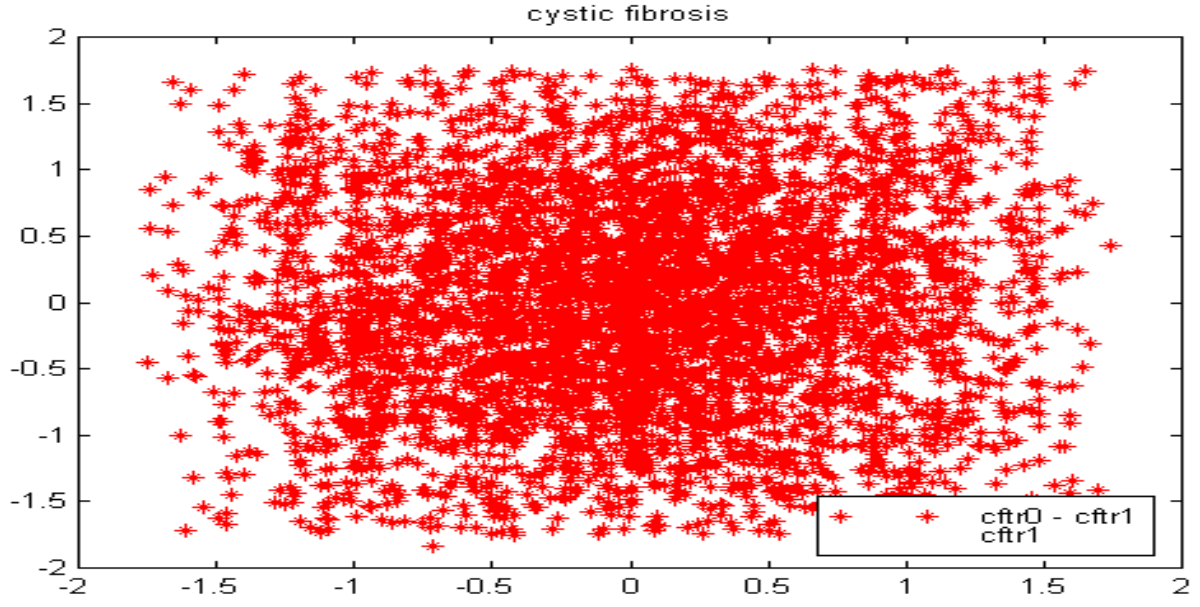

Figure V-7a Difference In The Two IFS Mappings "Original" - "Mutation"

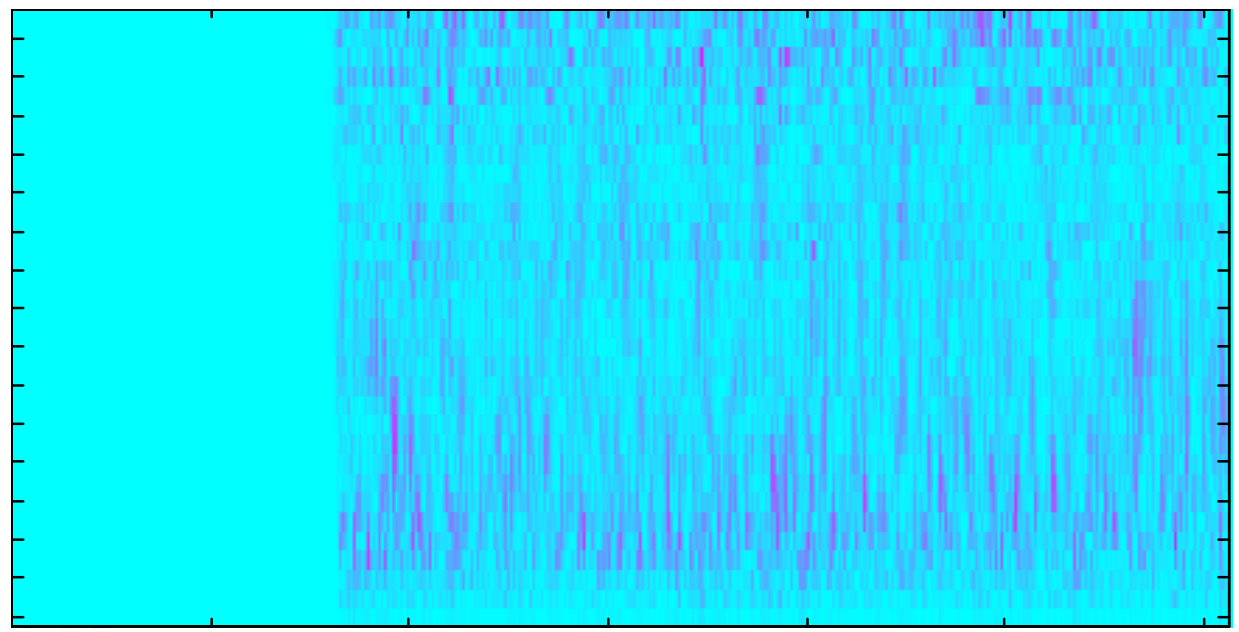

Figure V-7b Difference In The Wavelet Coefficients 
The radius of gyration of the mutated sequence is $\mathrm{Rg}=1.9920$. When a spatial difference is taken between the two sequences, it is clear that the loss of the 3 base pairs (one amino acid) has disturbed the spatial distribution of IFS mapping. This should not be surprising because biologically, the sequence "closes ranks" and all subsequent base pairs fill it for the missing three. This leaves the sequence "off by 3 " when its paired with its "brother" sequence to make the famous double helix. This "disturbance" is easily picked up by wavelet analysis and at the precise spot where the deletion occurred.

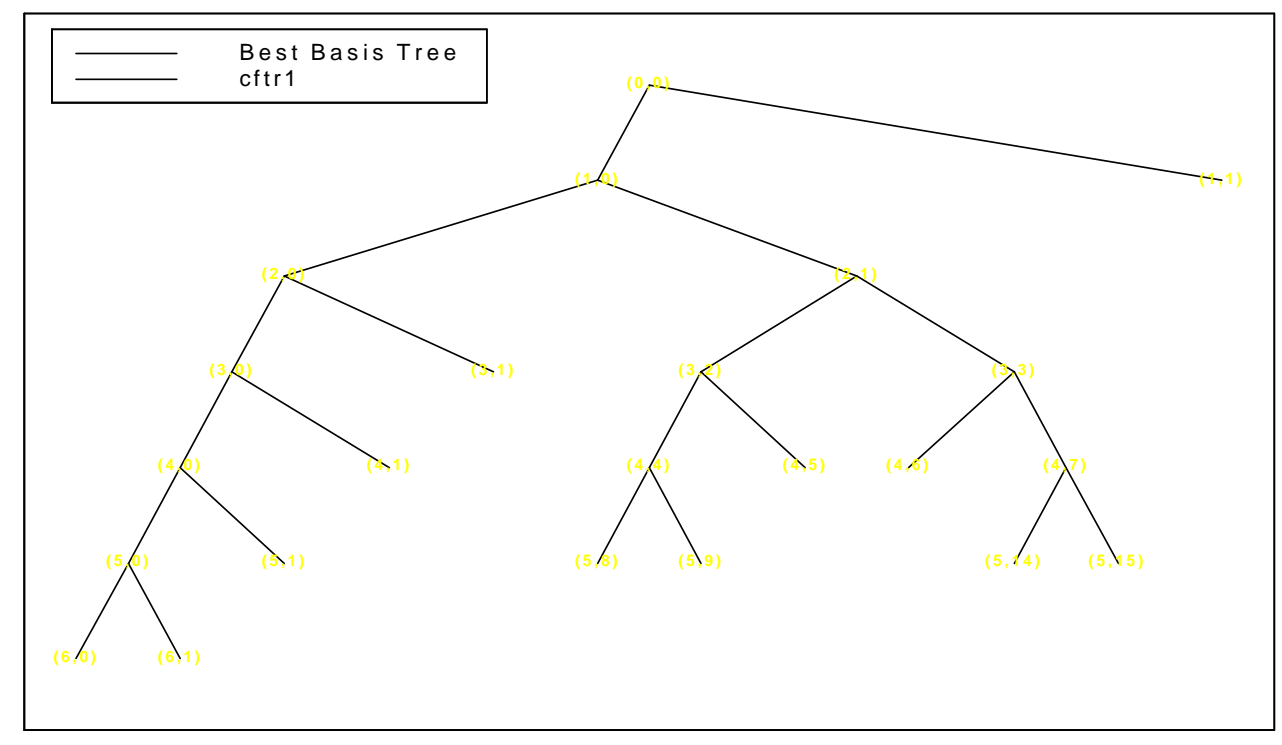

Figure V-8 Best Basis Representation

Since this deletion was so disturbing to the wavelet coefficients it again is not surprising that it changes the entropy and therefore produces a different best basis presentation. 


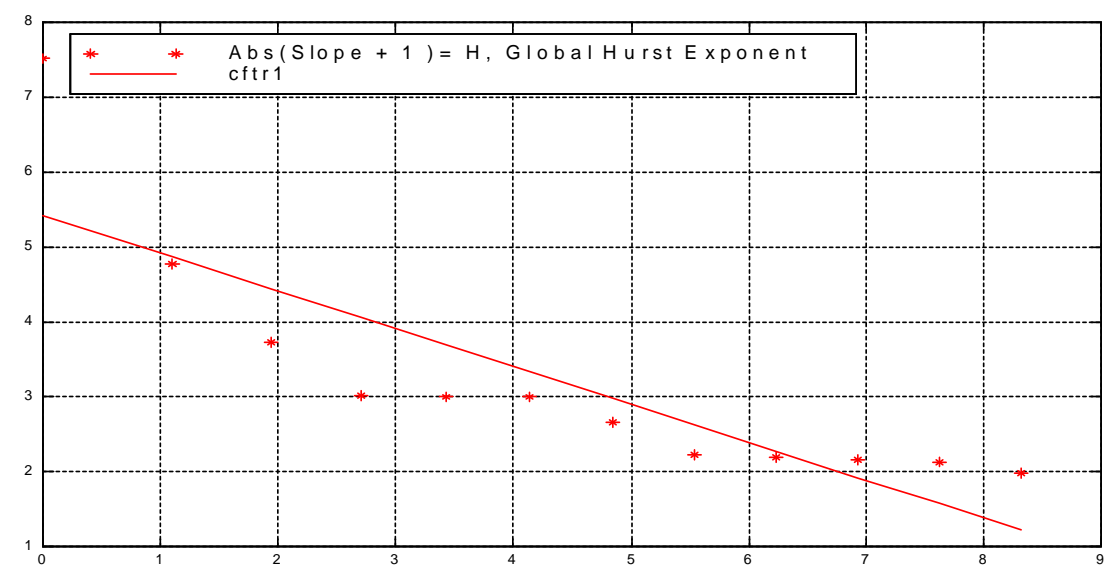

Figure V-9 loglog Plot for Global Hurst Exponent

cftr1

Notice how the deletion has driven the sequence's global Hurst exponent closer to random i.e. $\mathrm{H}=1 / 2$.

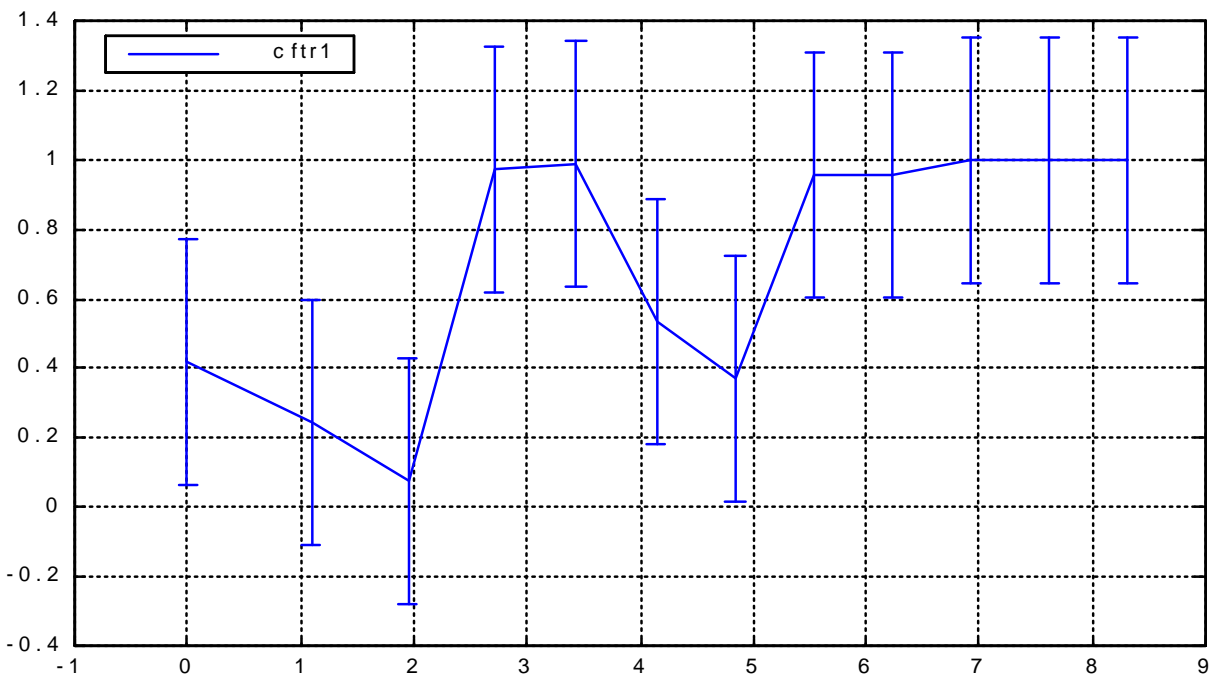

Figure V-10 loglog Plot for Local Hurst Exponent

The deletion has clearly affected the behavior of local trending. 
cystic fibrosis

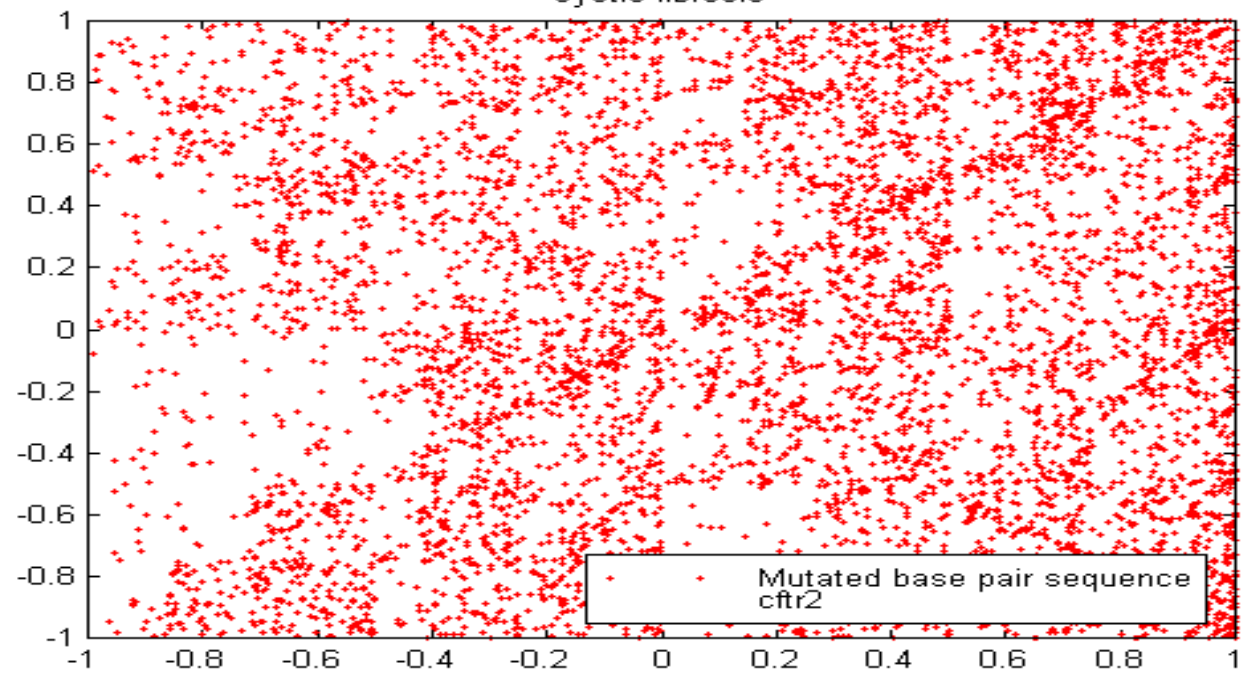

Figure V-11a IFS Mapping of the cftr2.txt sequence

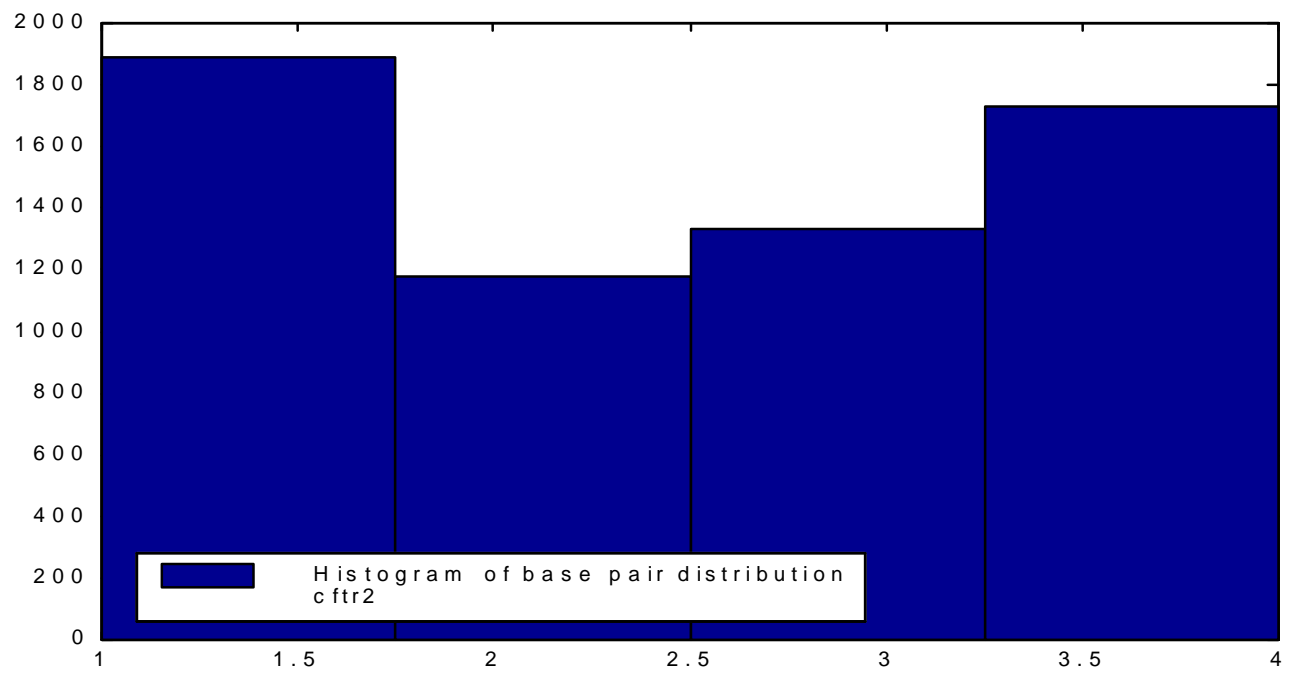

Figure V-11b Histogram Of The Base 
In line 1741 the following amino acid is mutated. A AGA mutation occurs a stop condon is created, i.e. ' $g$--> $a$ '

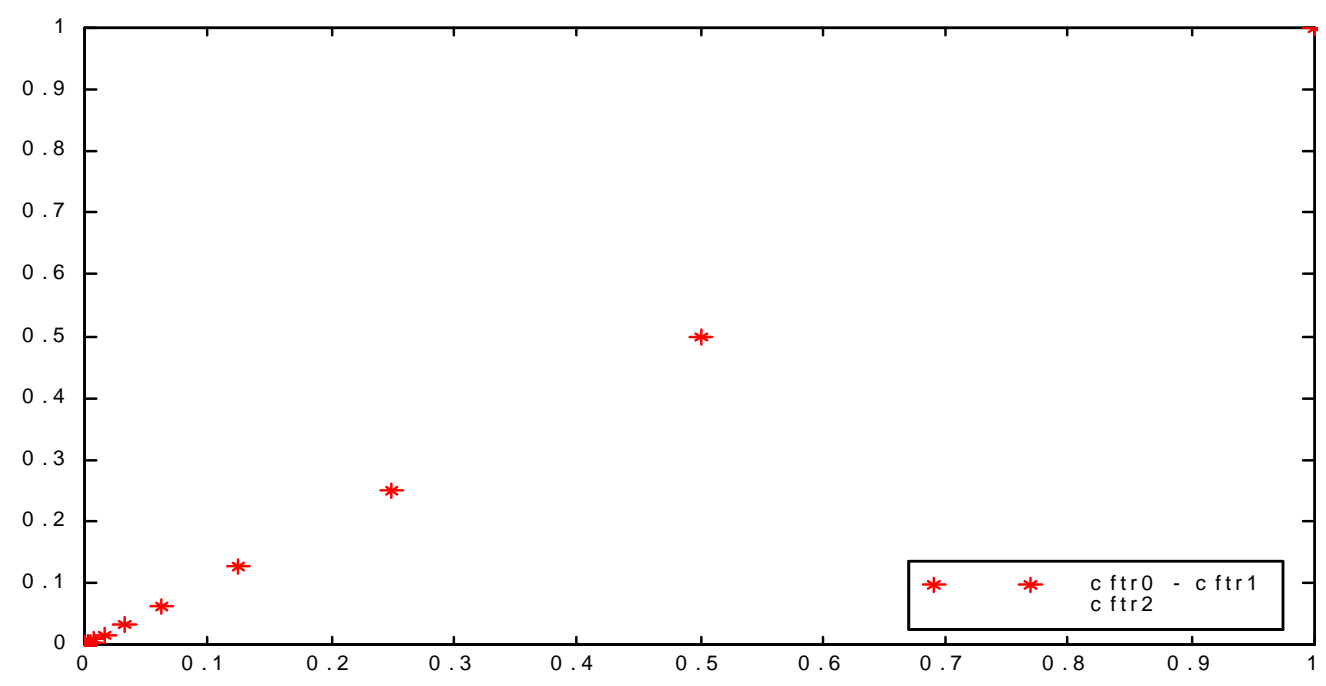

Figure V-12a Difference in the two IFS Mappings

"Original" - "mutation"

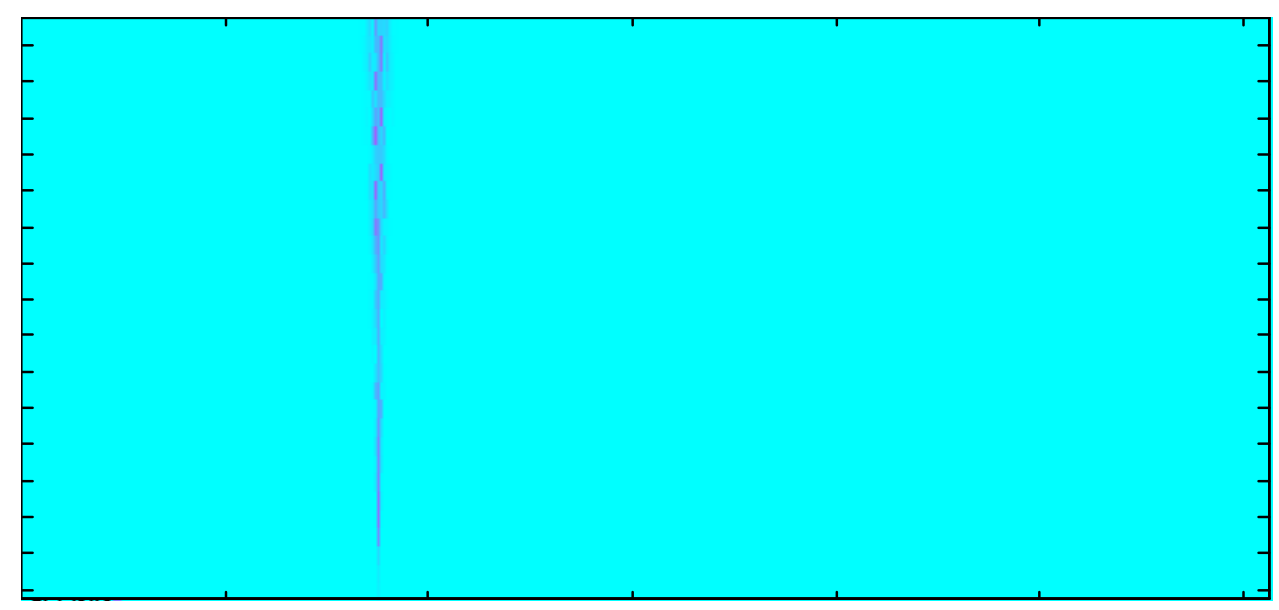

Figure V-12b Difference in the

Wavelet coefficients 
Change in one base pair which is a numerical change of 3 to 1 that is a difference of 2. This effects the IFS mapping but as Figure 5.14a shows the disturbance eventually damps out. It's important to note that this magnitude change can be picked up by wavelet analysis.

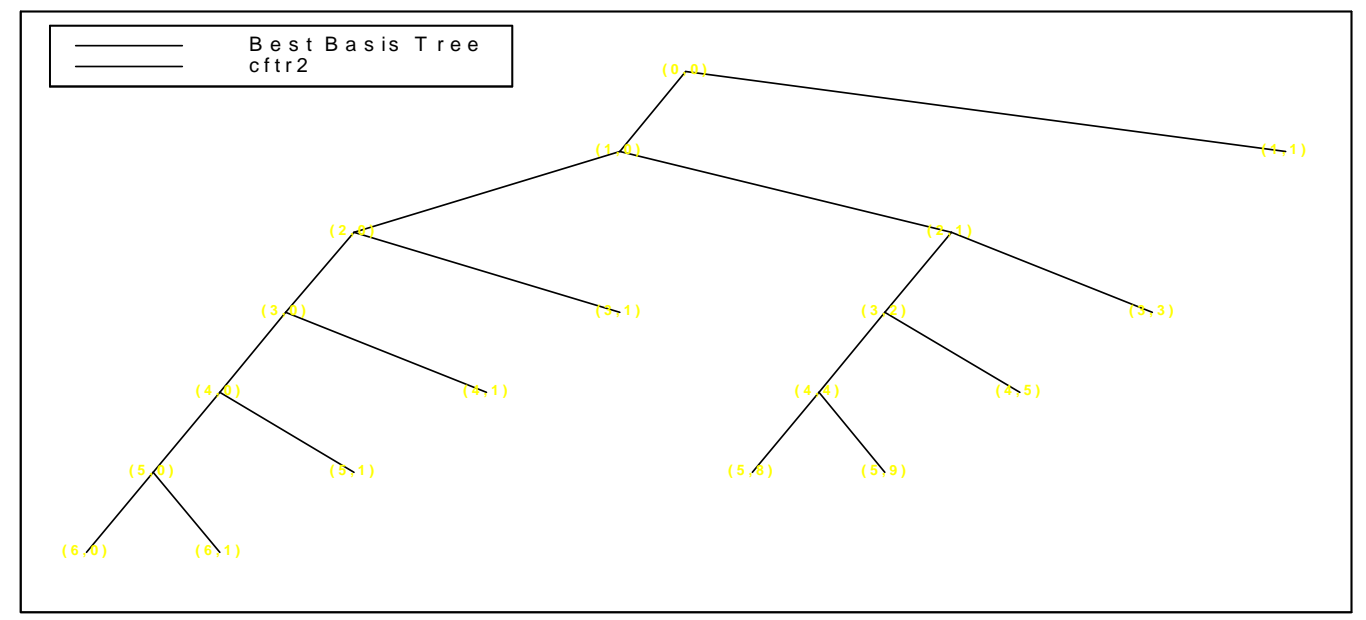

Figure V-13 Best Basis Representation

However this change was significant enough to change the best basis representation from the original best basis binary tree. This is because it did not produce enough of a change in entropy to trip the threshold, which governs the best basis optimization algorithm. 


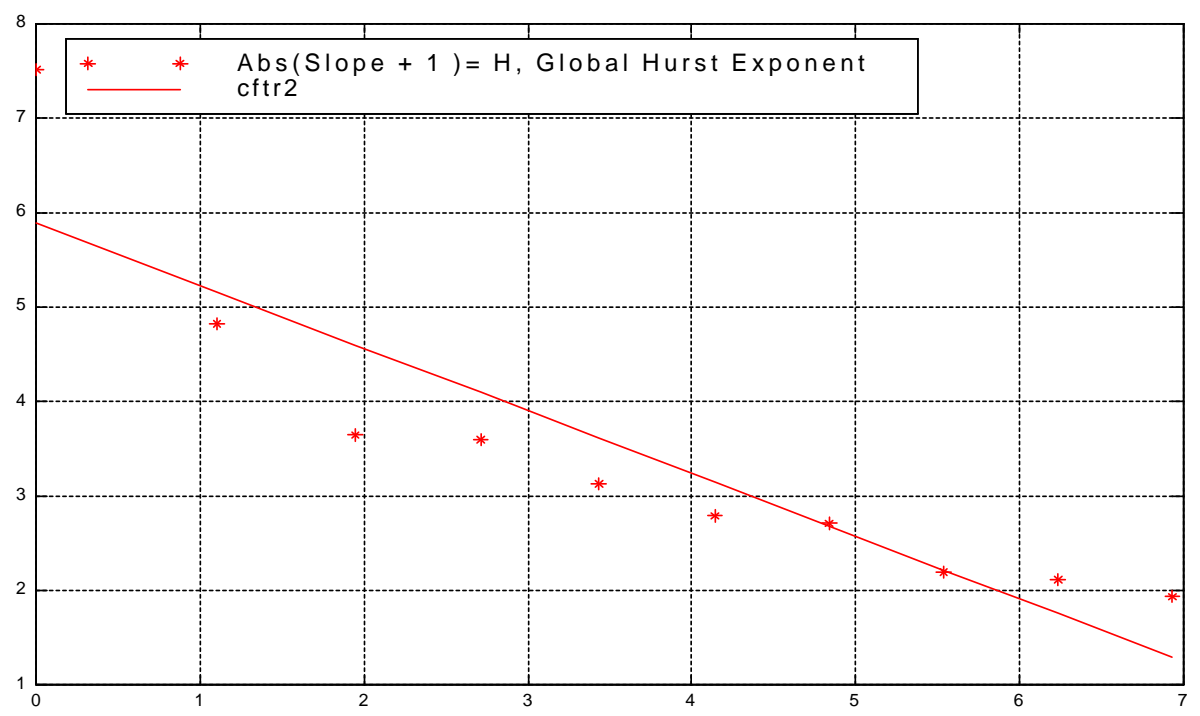

Figure V-14 Log Log Plot For The Global Hurst Exponent

Not much change in the global Hurst exponent from the original, again this because of the lack of significant change in the best basis.

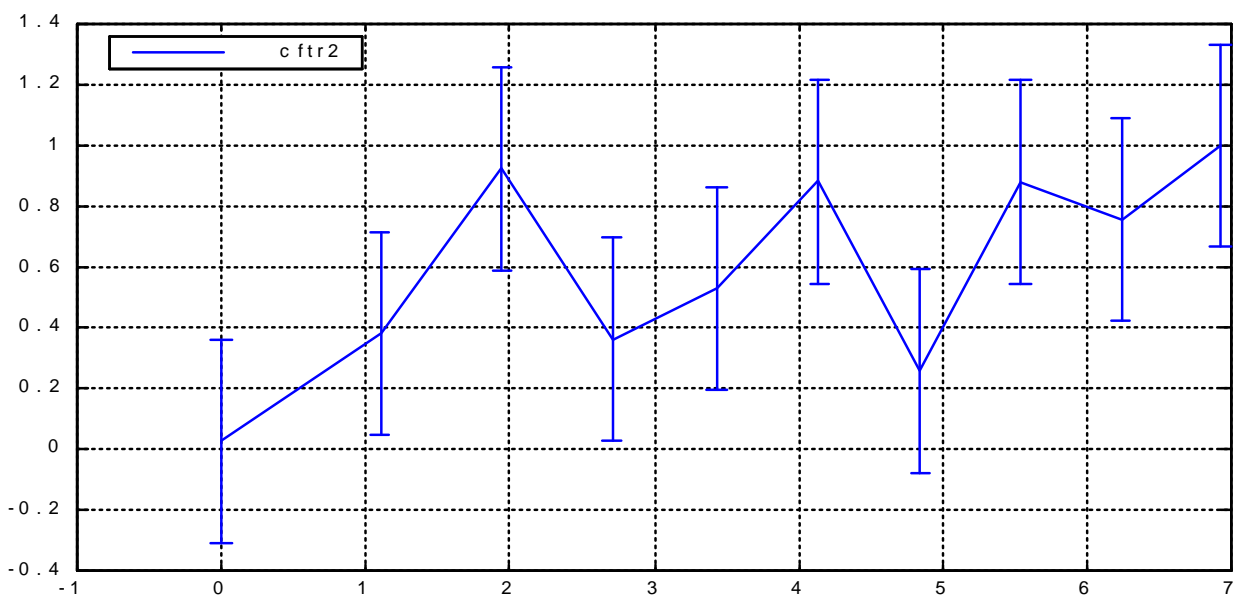

Figure V-15 Log Log Plot For The Local Hurst Exponent 
Since there is little change in the entropy of the original there will be little change in the behavior of this plot. 


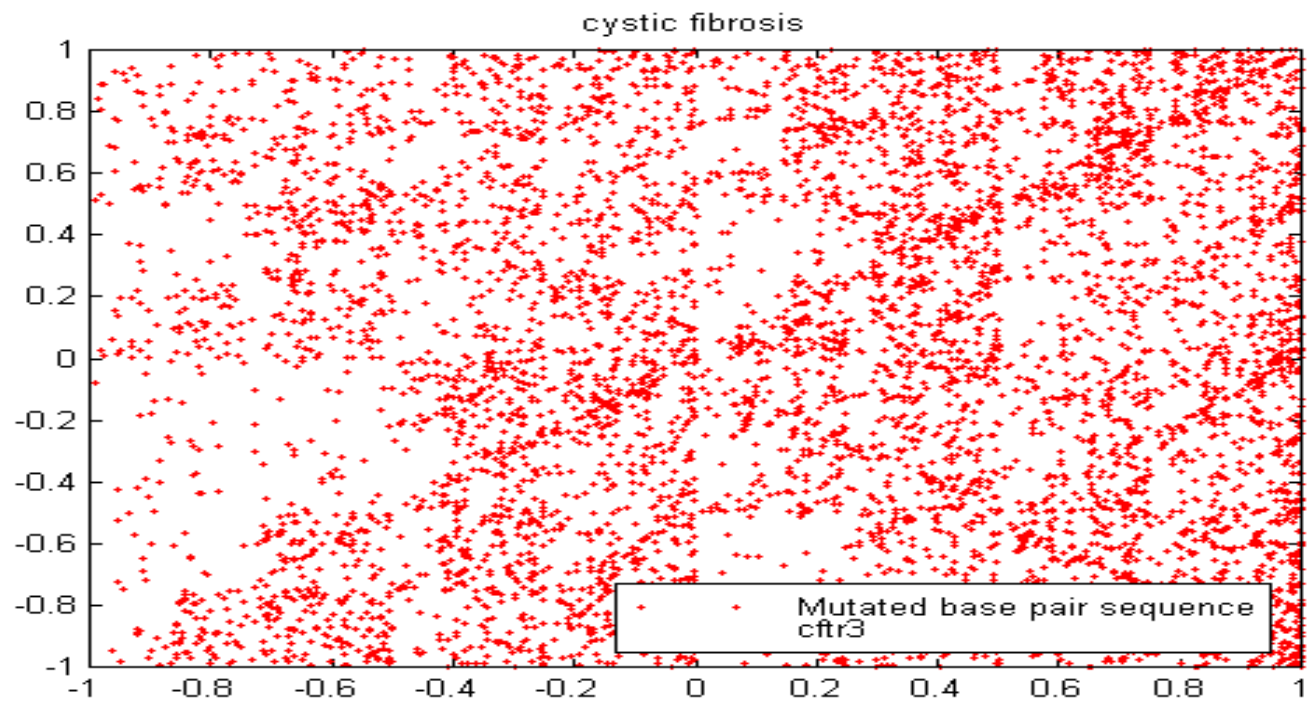

Figure V-16a IFS mapping of the cftr3.txt sequence

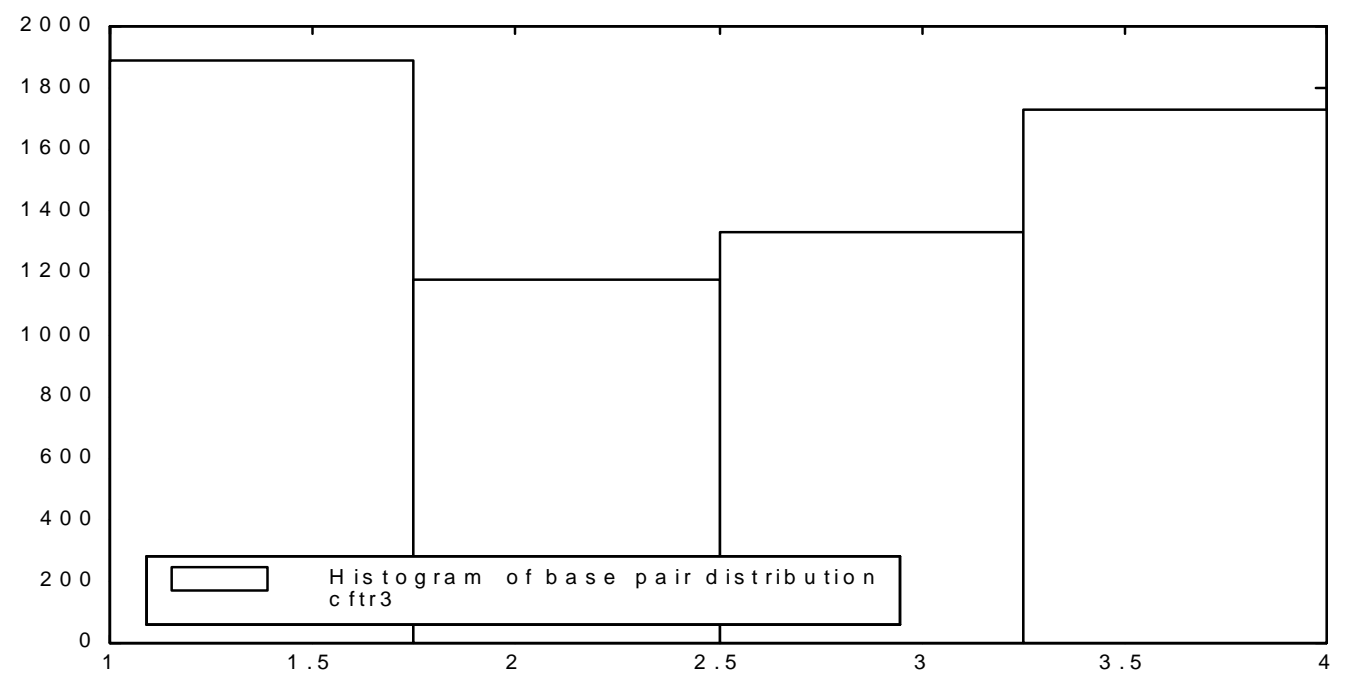

Figure V-16b Histogram of the base

In line 1741 the following amino acid is mutated. The GaA mutation occurs 
i.e. Asp replaced by Gly 'tg gagAtcaacg' goes to ' tg gagGtcaacg'

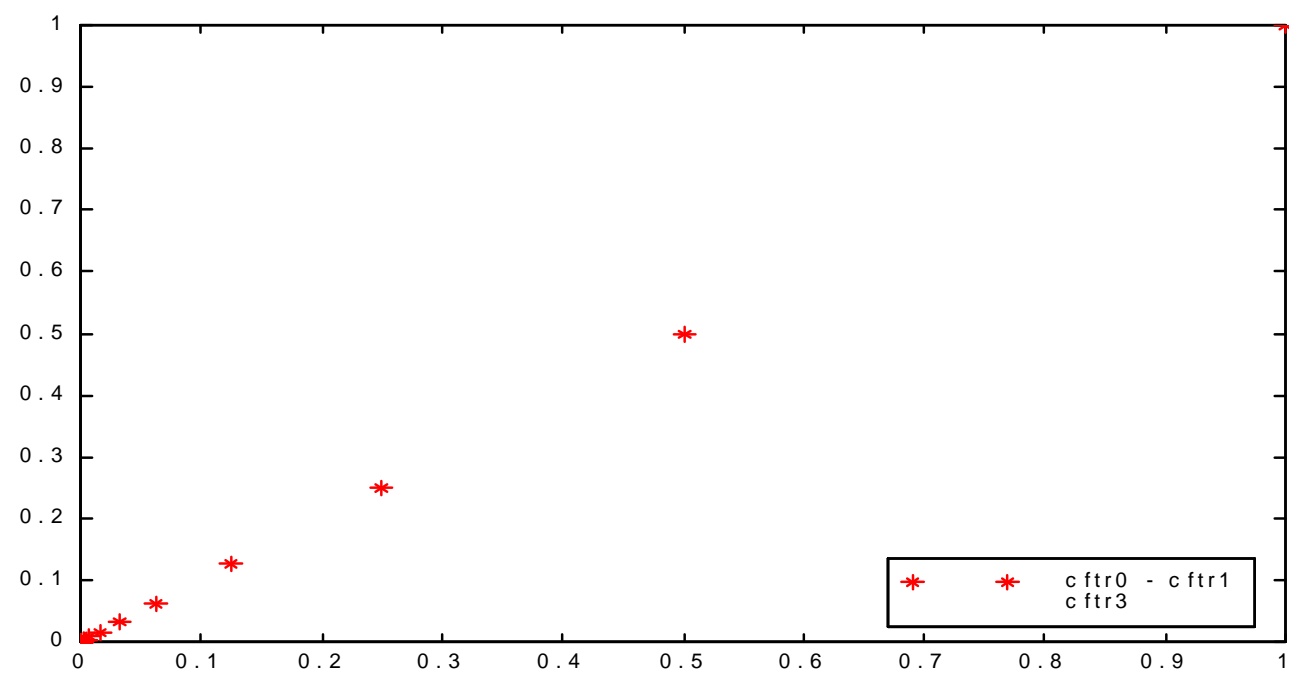

Figure V-17a Difference in the two IFS Mappings

"Original" - "mutation"

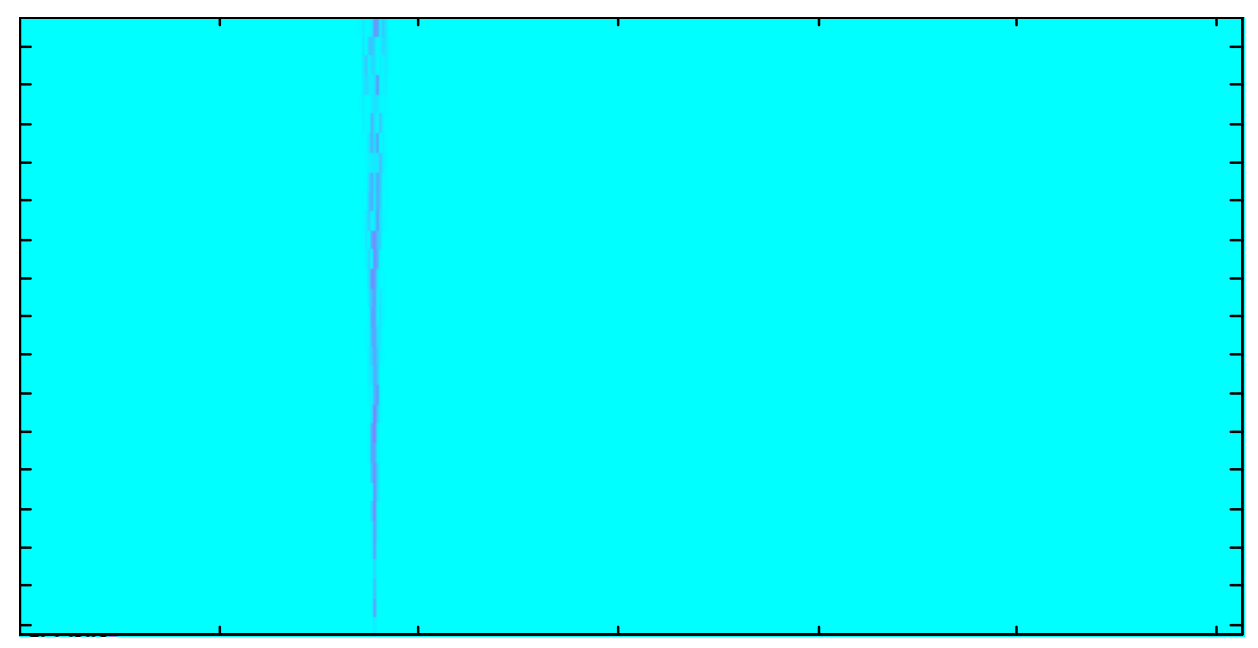

Figure V-17b Difference in the

Wavelet coefficients 
Change in one base pair that is a numerical change of 1 to 3 that is a difference of -2. Again this effects the IFS mapping but as Figure $5.14 \mathrm{a}$ and the disturbances eventually damped out. This magnitude change is picked up by wavelet analysis.

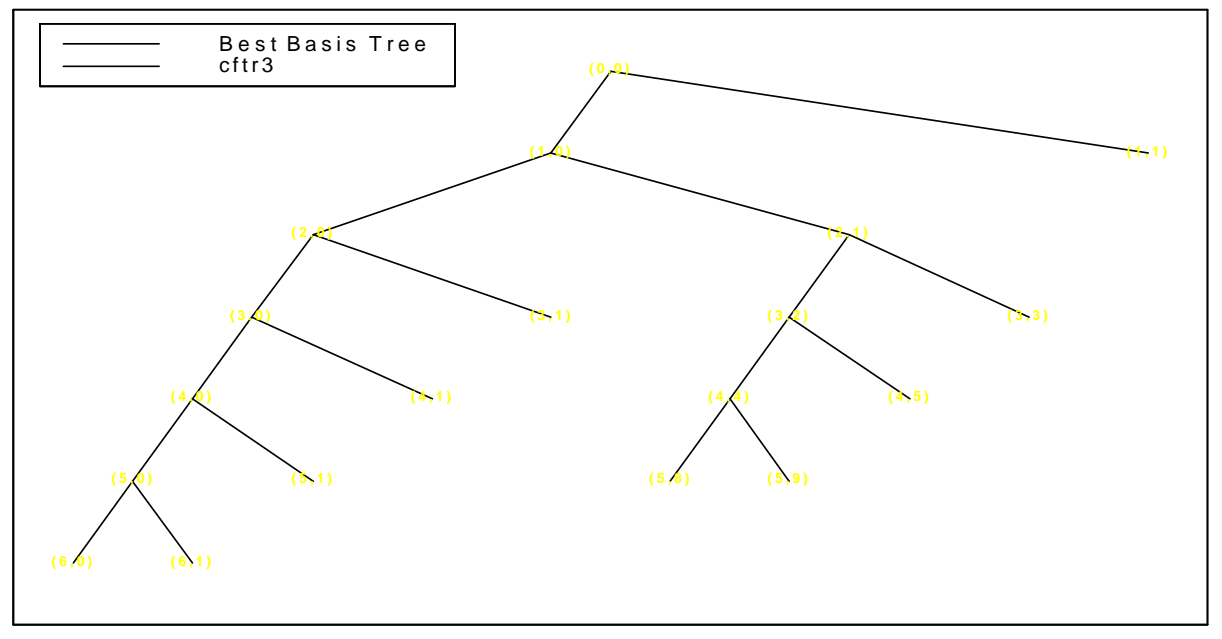

Figure V-18 Best Basis Representation

The "tree" is the same as in the normal sequence, for the same reasons as before.

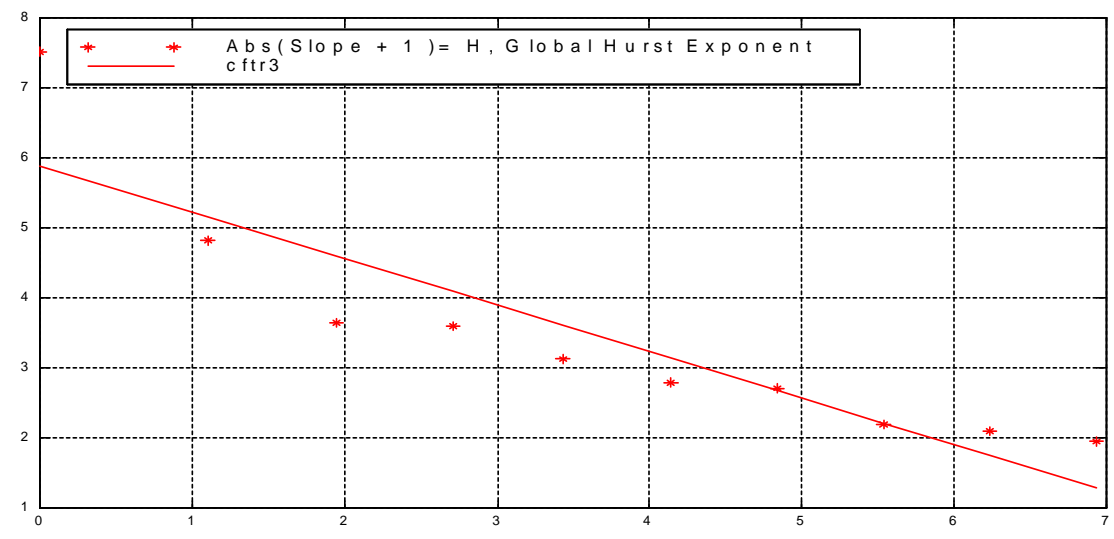

Figure V-19 loglog plot for the global Hurst exponent 
As one would expect not a significant change here

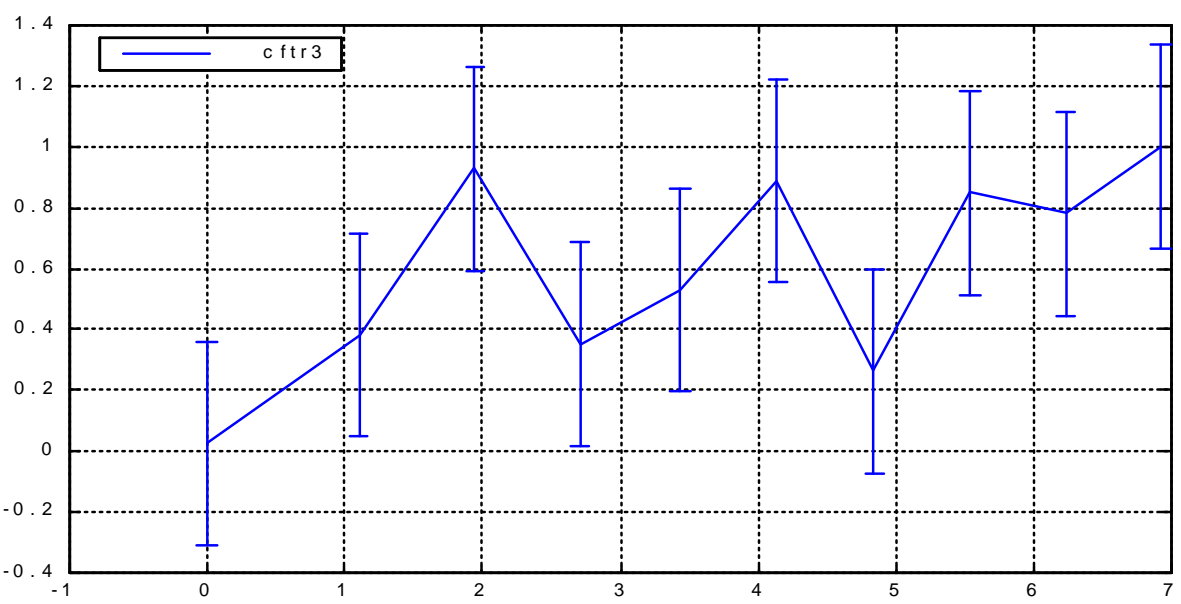

Figure V-20 loglog plot for the local Hurst exponent

The behavior of the above figure is the same as before 


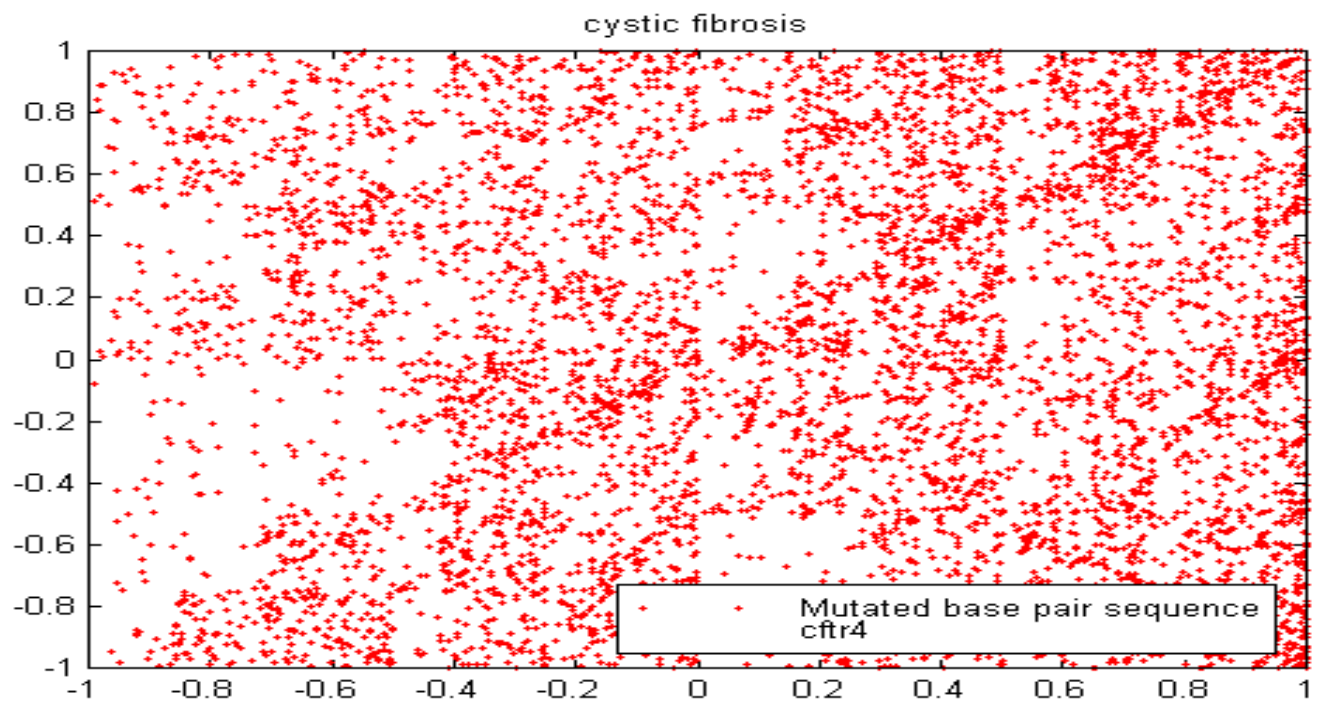

Figure V-21 IFS mapping of the cftr4.txt sequence

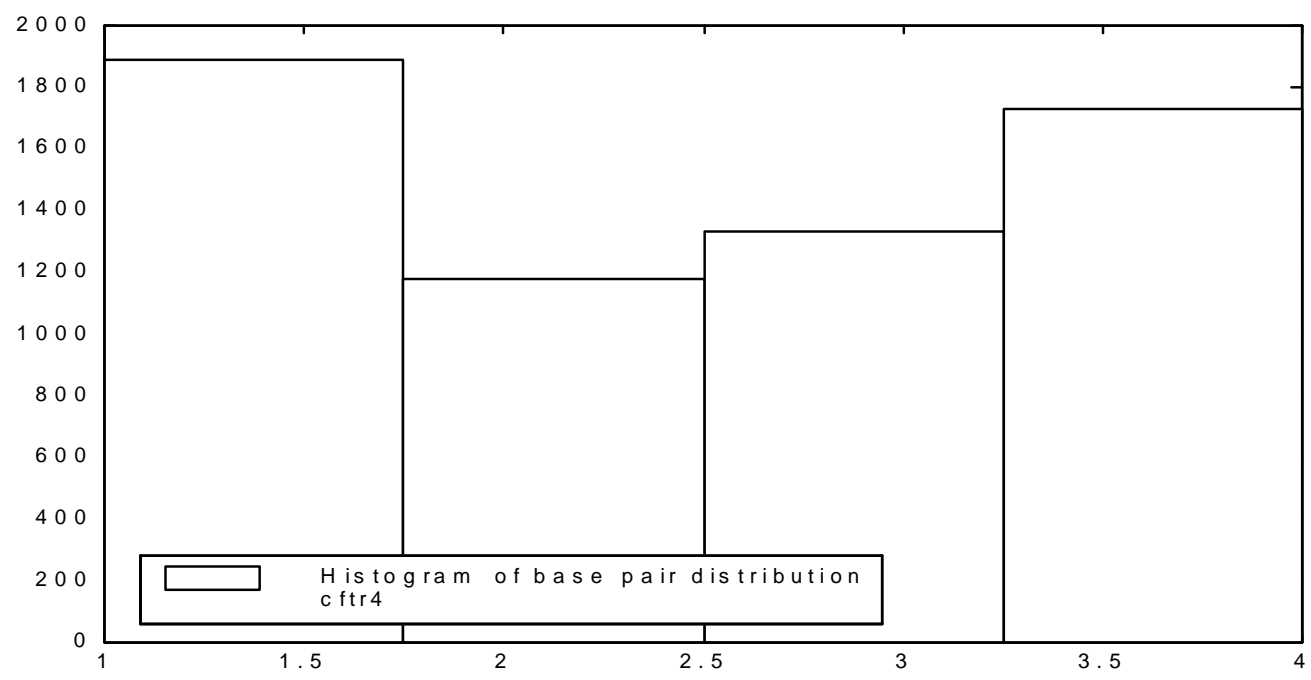

Figure V-21 Histogram of the Base-Pairs

In line 3601 the following amino acid is mutated. The CaT at codon 1162 this is a stop codon i.e. ' $\mathrm{c}-->$ t' that is 2 goes to a 4 . 


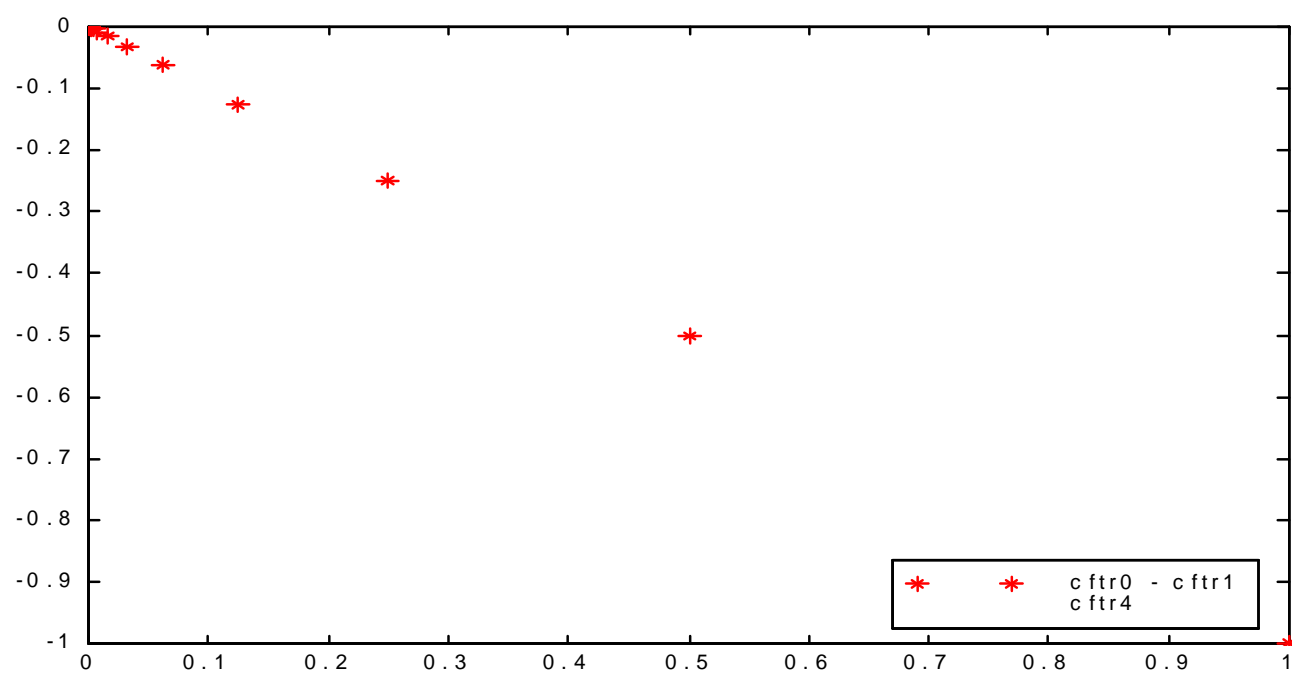

Figure V-22a Difference in the two IFS Mappings

"Original" - "mutation"

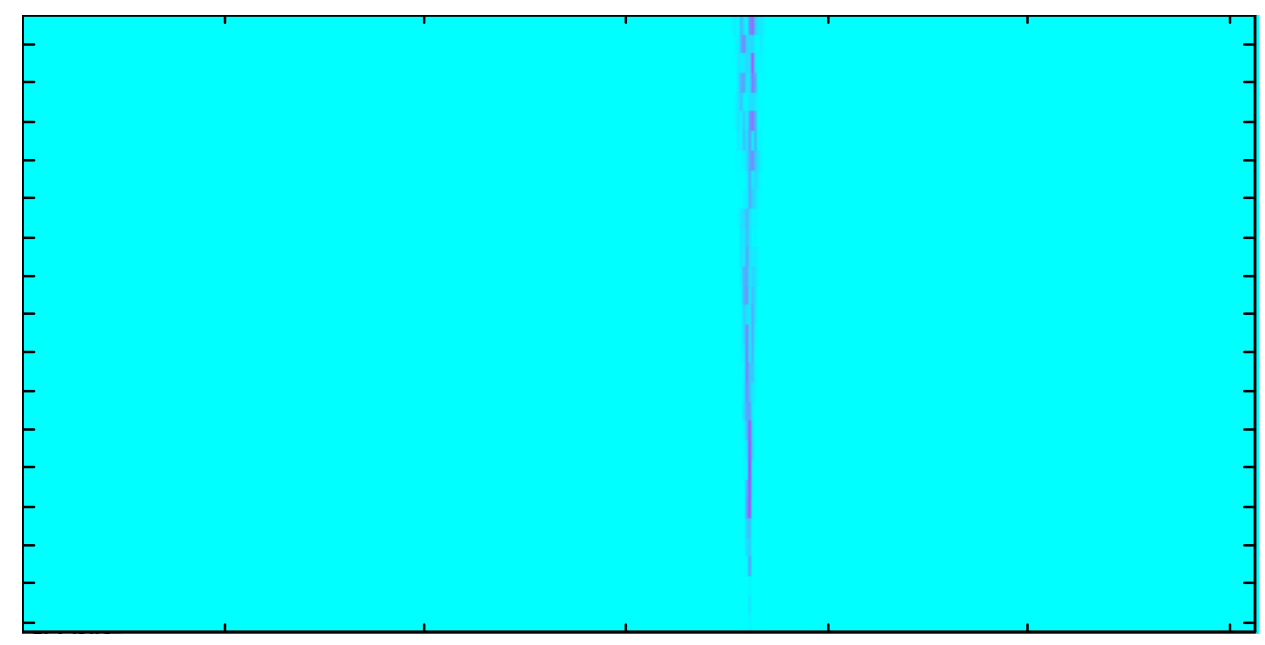

Figure V-22b Difference in the

Wavelet coefficients

Change in one base pair that is a numerical change of 2 to 4 that is a difference of 2. Again this effects the IFS mapping but as Figure 5.23a and the disturbance 
is eventually damped out. This magnitude change is picked up by wavelet analysis.

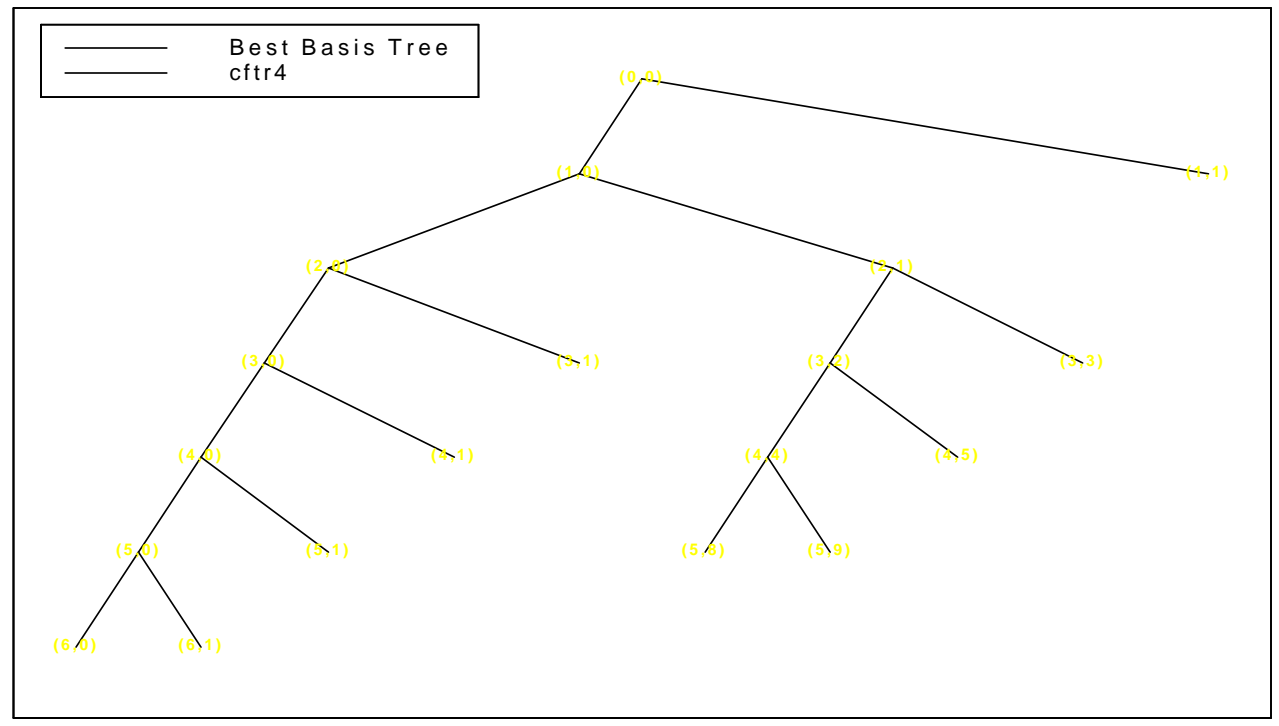

Figure V-23 Best Basis Representation

The "tree" same as in the non-mutated sequence, for the same reasons as before

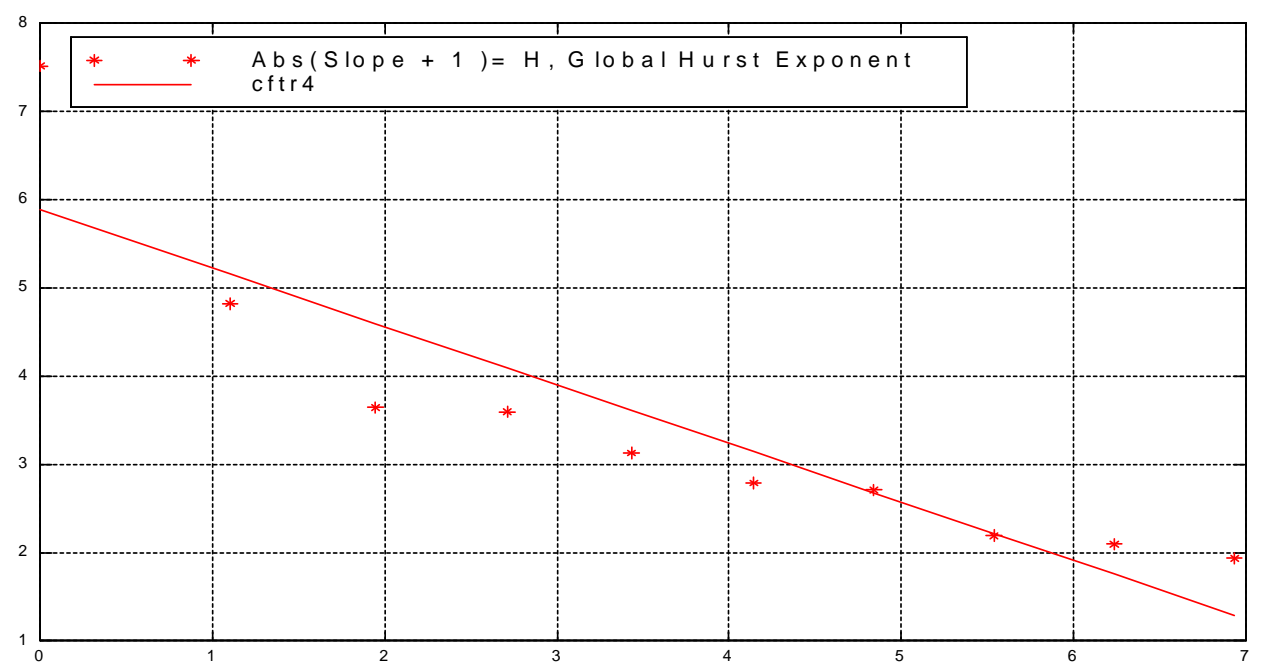

Figure V-24 loglog plot for the global Hurst exponent 
As one would expect not a significant change here

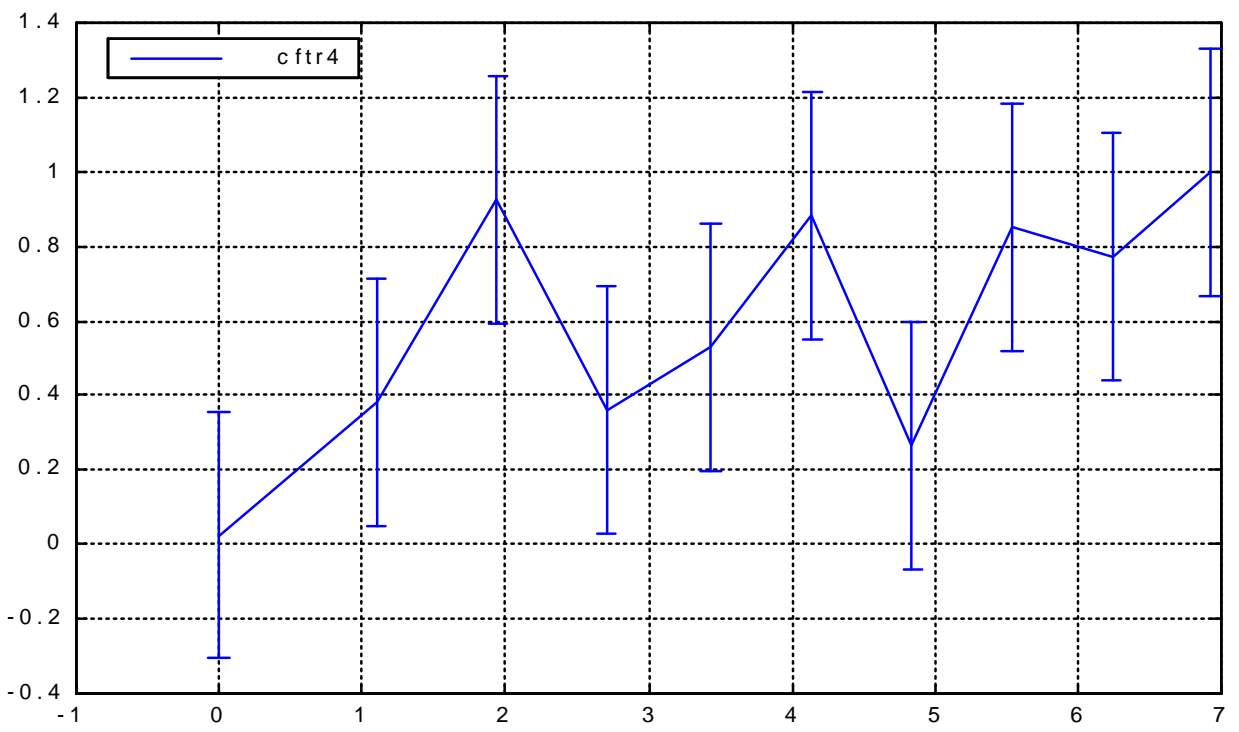

Figure V-25 loglog plot for the local Hurst exponent

The behavior of the above figure is the same as before 


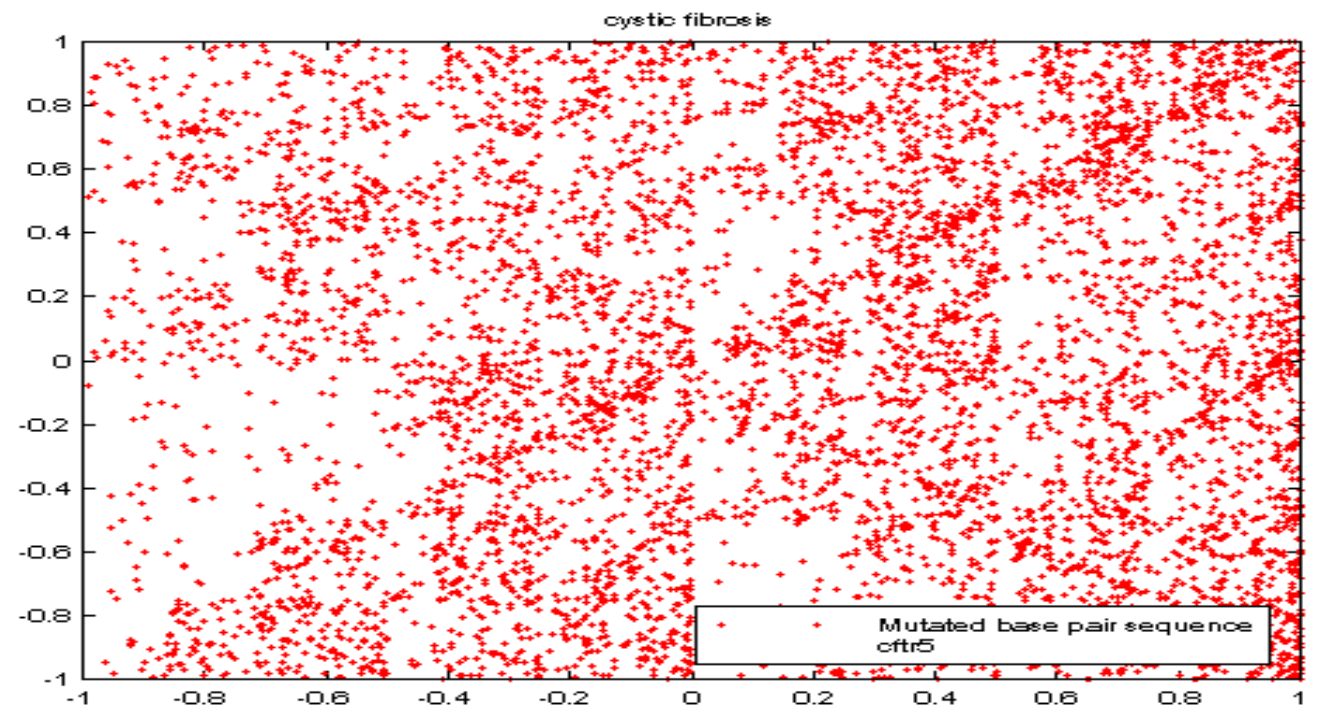

Figure V-26a IFS mapping of the cftr5.txt sequence

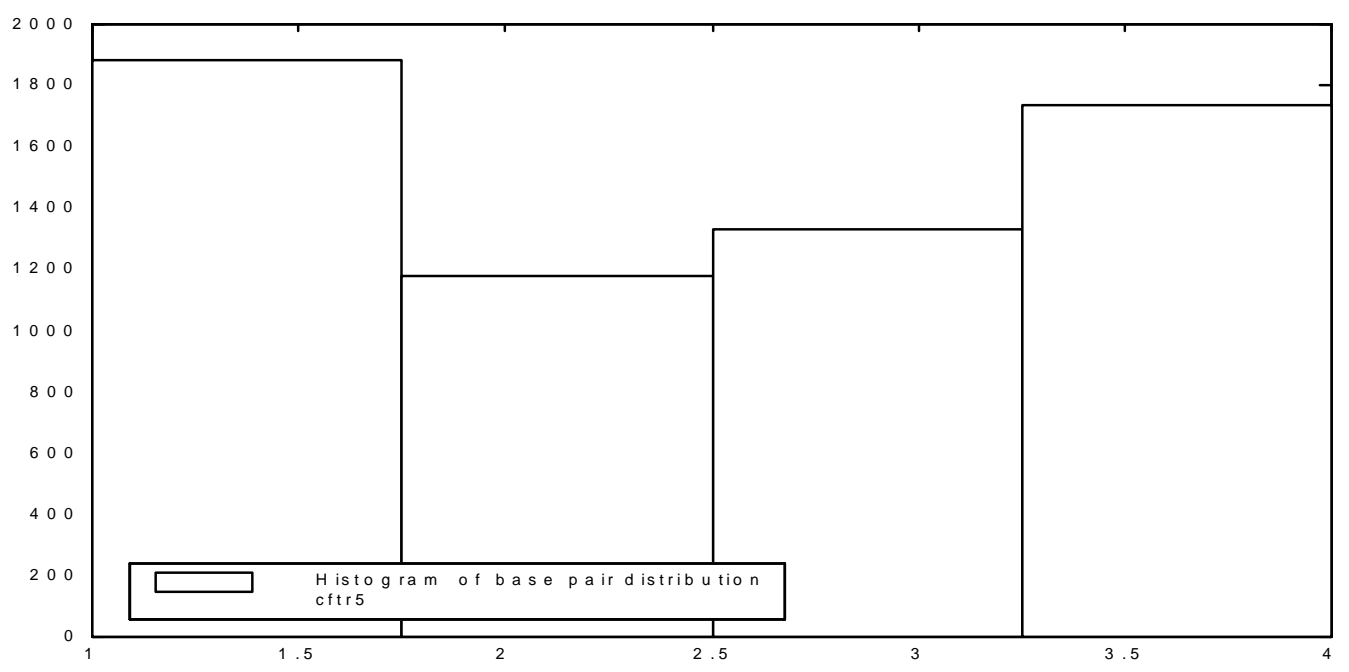

Figure V-26b Histogram of the base pairs

In line 1621 another deletion occurs at 1651 to 1654 that is 'attatgcctg gcaccattaa agaaaatATC atctttggtg tttcctatga 'tgaatataga' the ATC is deleted 


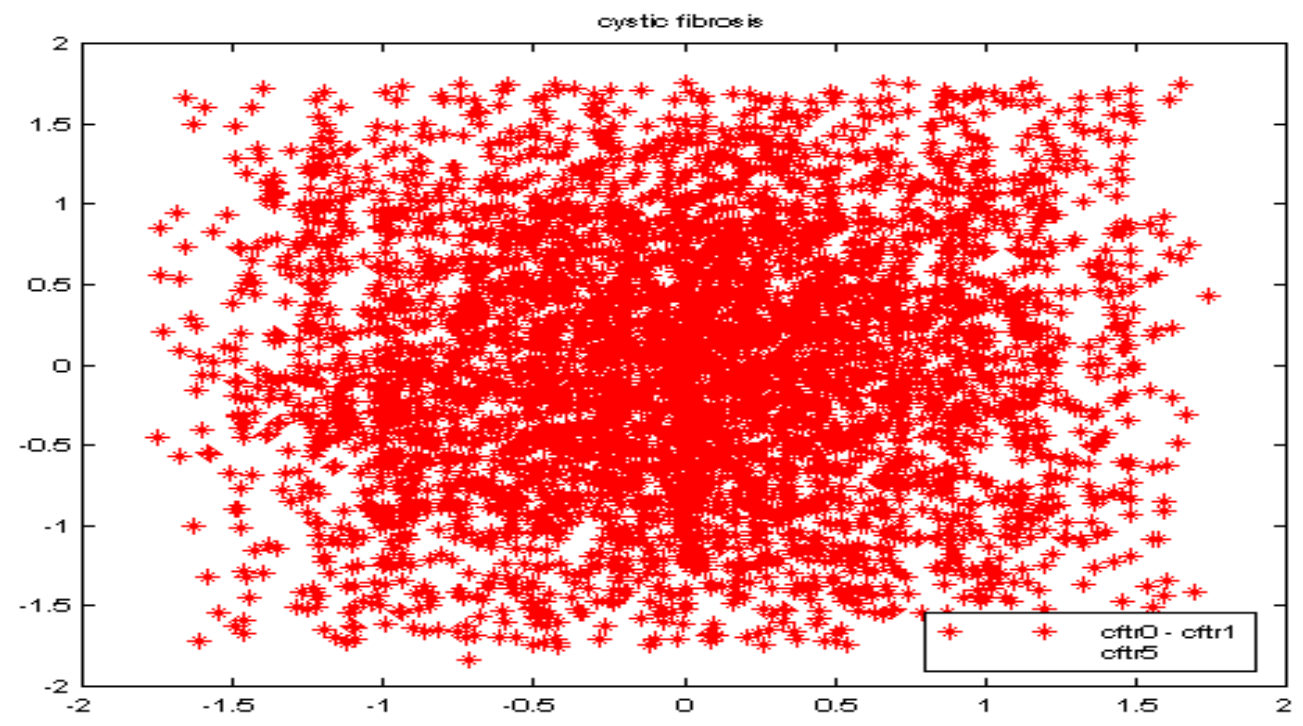

Figure V-27a Difference in the two IFS Mappings

"Original" - mutation"

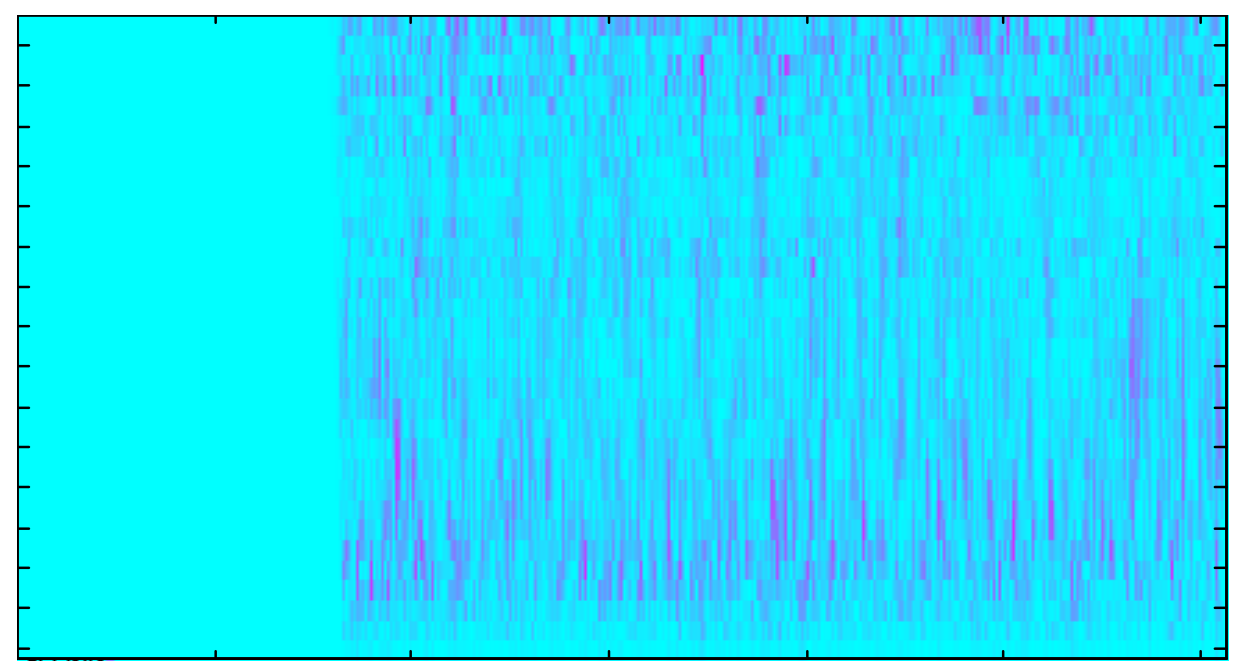

Figure V-27b Difference in the Wavelet coefficients

As in cftr1.txt a deletion even one base pair causes a considerable disturbance. 


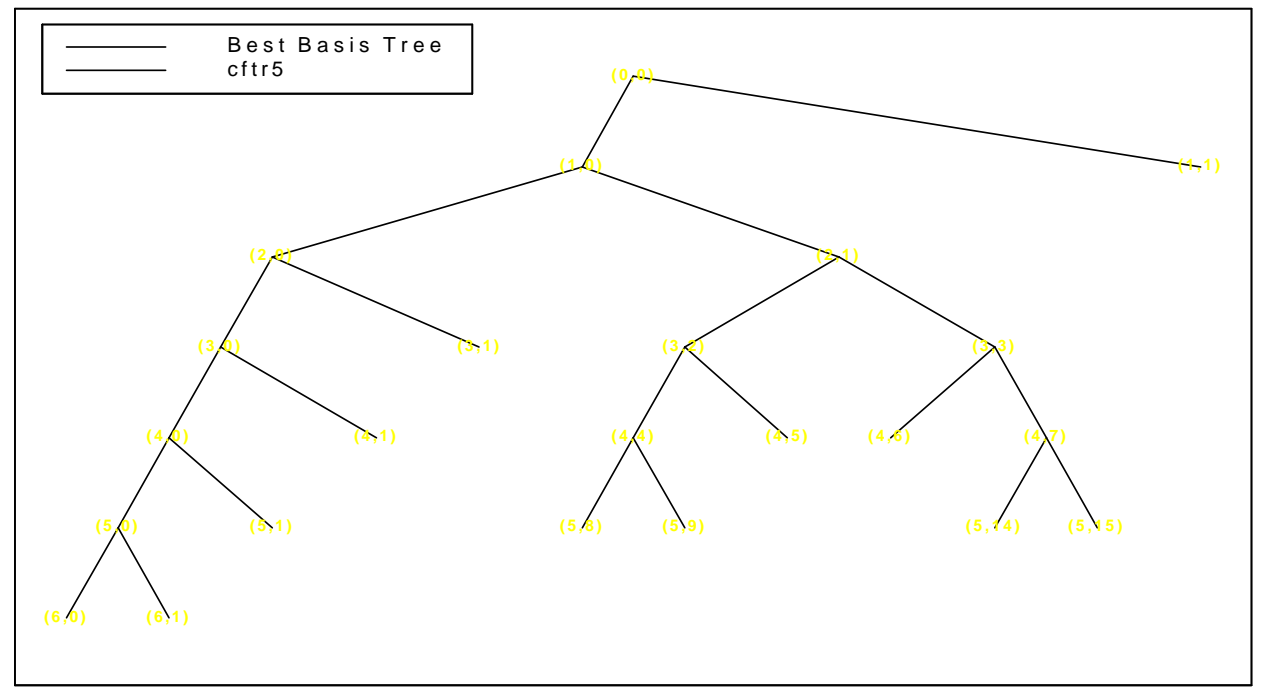

Figure V-28 Best Basis Representation

This of course produces a new best basis tree.

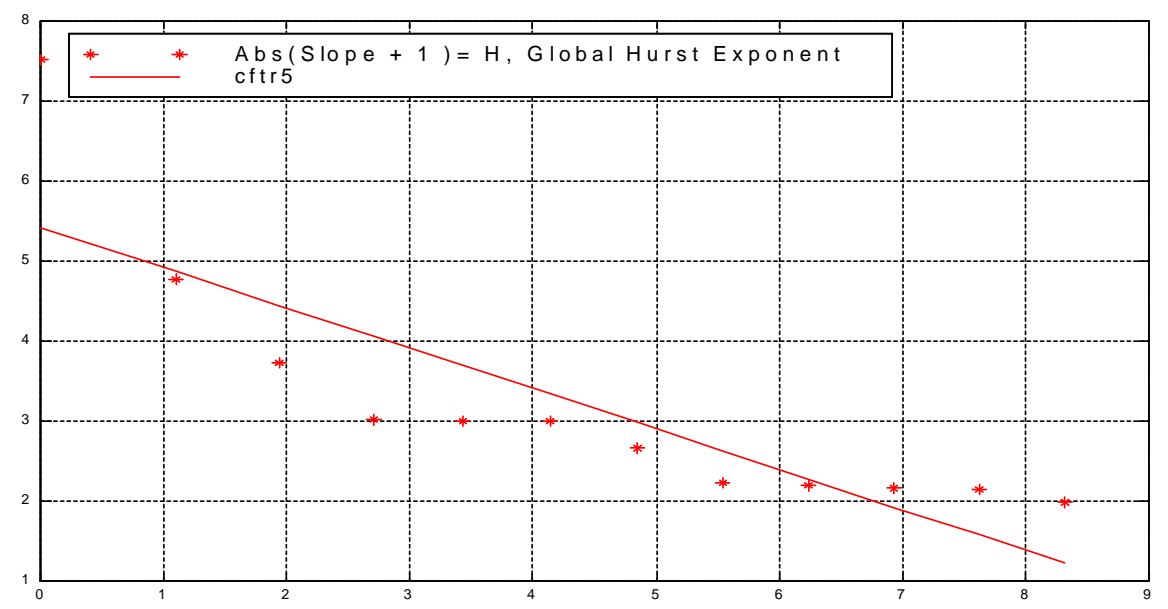

Figure V-29 loglog plot for the global Hurst exponent

It also drives the global Hurst exponent back toward randomness. 


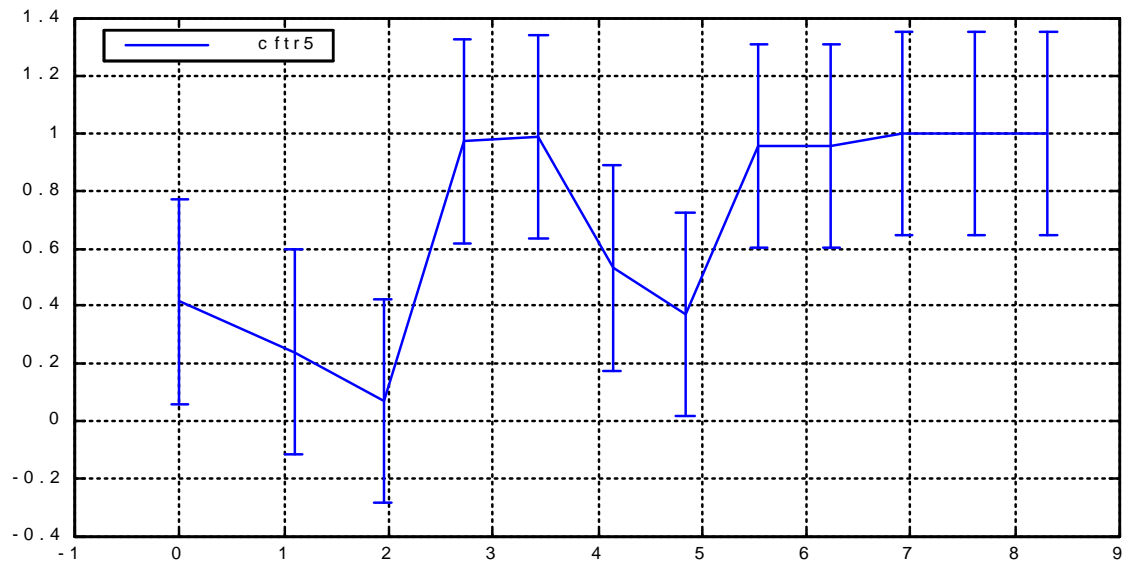

Figure 5-30 loglog plot for the local Hurst exponent

It also changes the local Hurst exponents behavior. 
Sequences cftr6.txt to cftr9.txt are all single base pair changes. Specifically

$\begin{array}{lll}\text { Sequence } & \text { Change } & \text { base pair position } \\ \text { 1. cftr6.txt } & c-t & \text { " } \\ \text { 2. cftr7.txt } & a->t & \text { " } 1681 \\ \text { 3. cftr8.txt } & a->c & \text { " } \\ \text { 4. cftr9.txt } & a->g & \end{array}$

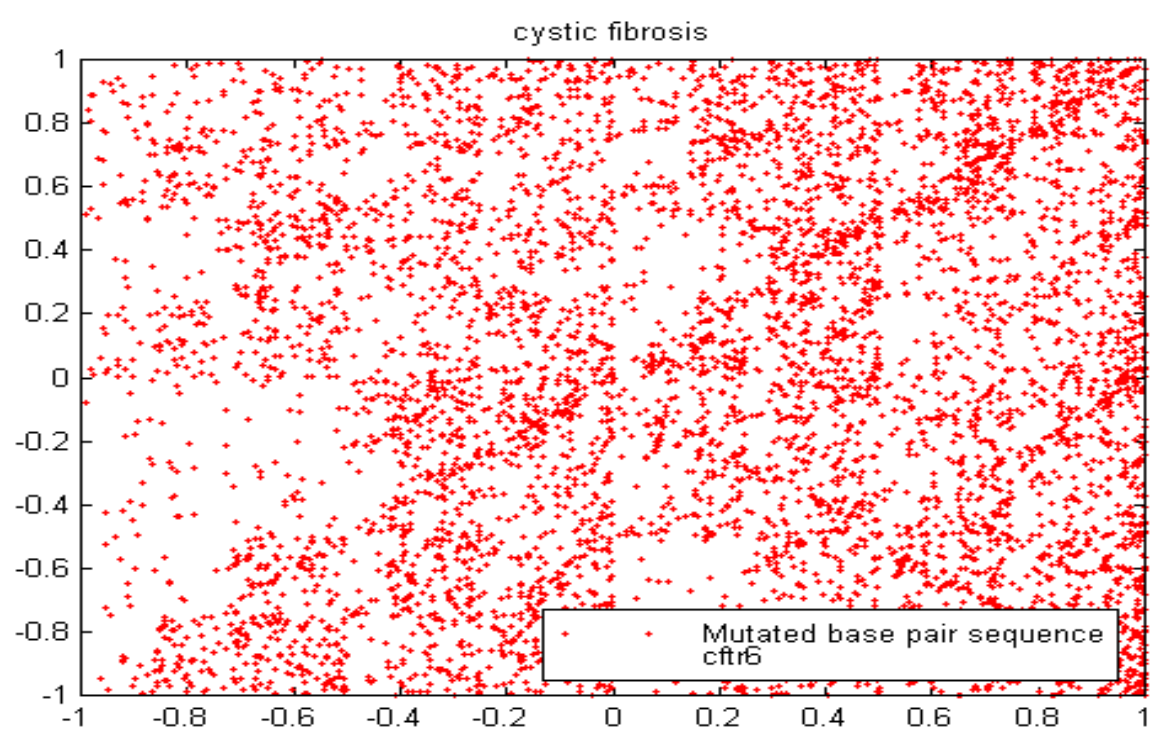

Figure V-31 IFS Mapping of the cftr5.txt sequence 


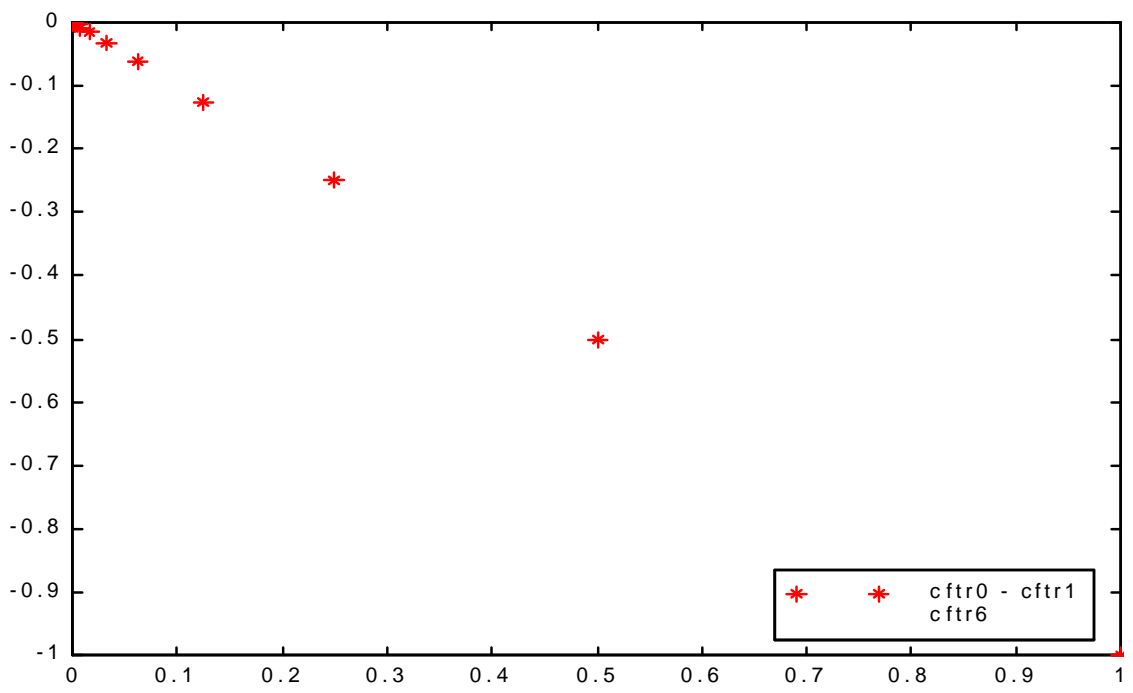

Figure V-32 Difference in IFS Mappings

"Original" - "mutation"

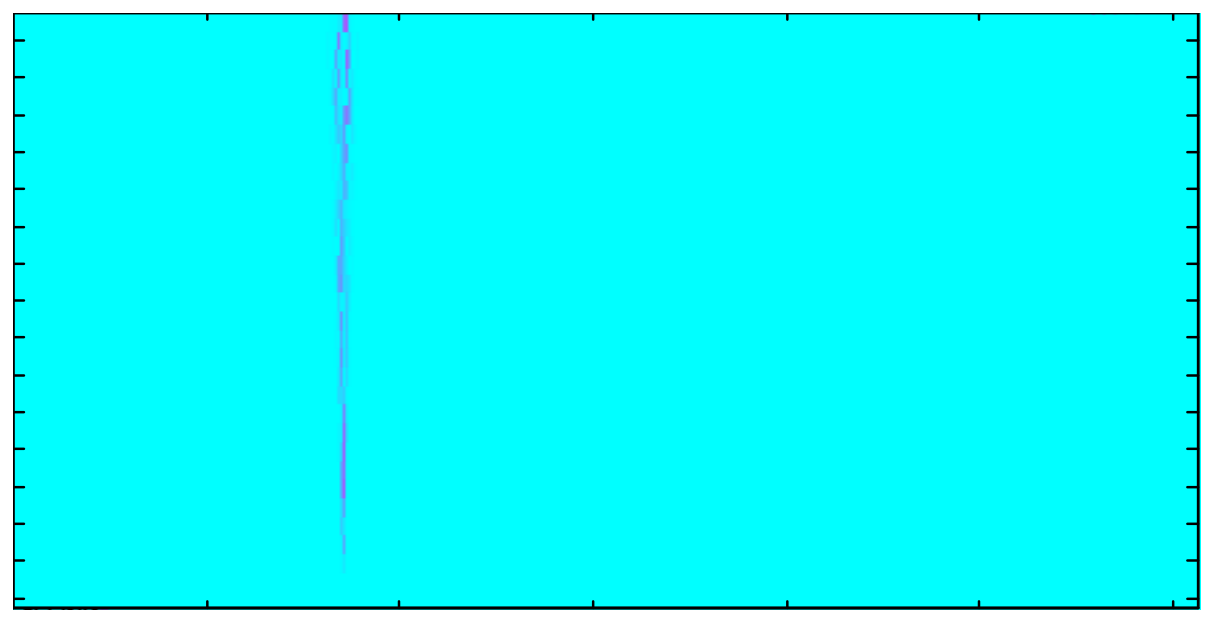

Figure V-33 Difference in the Wavelet coefficients 


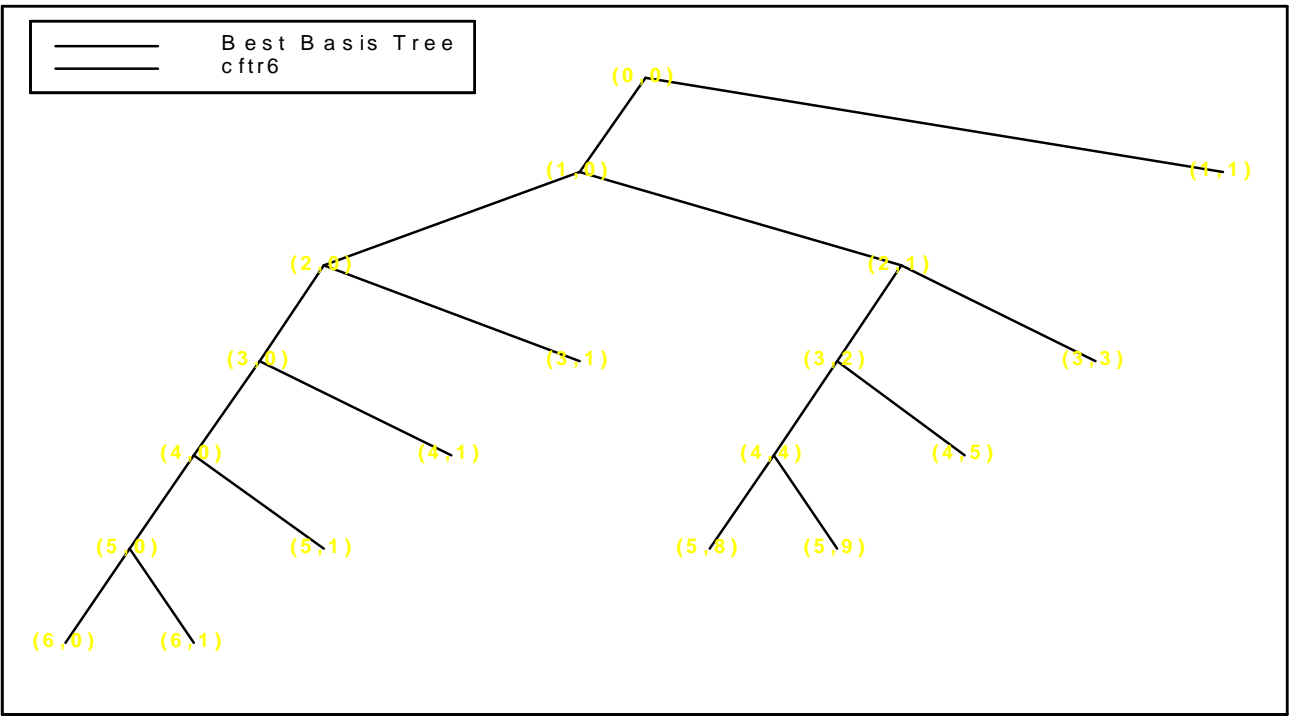

Figure V-34 Best Basis Representation

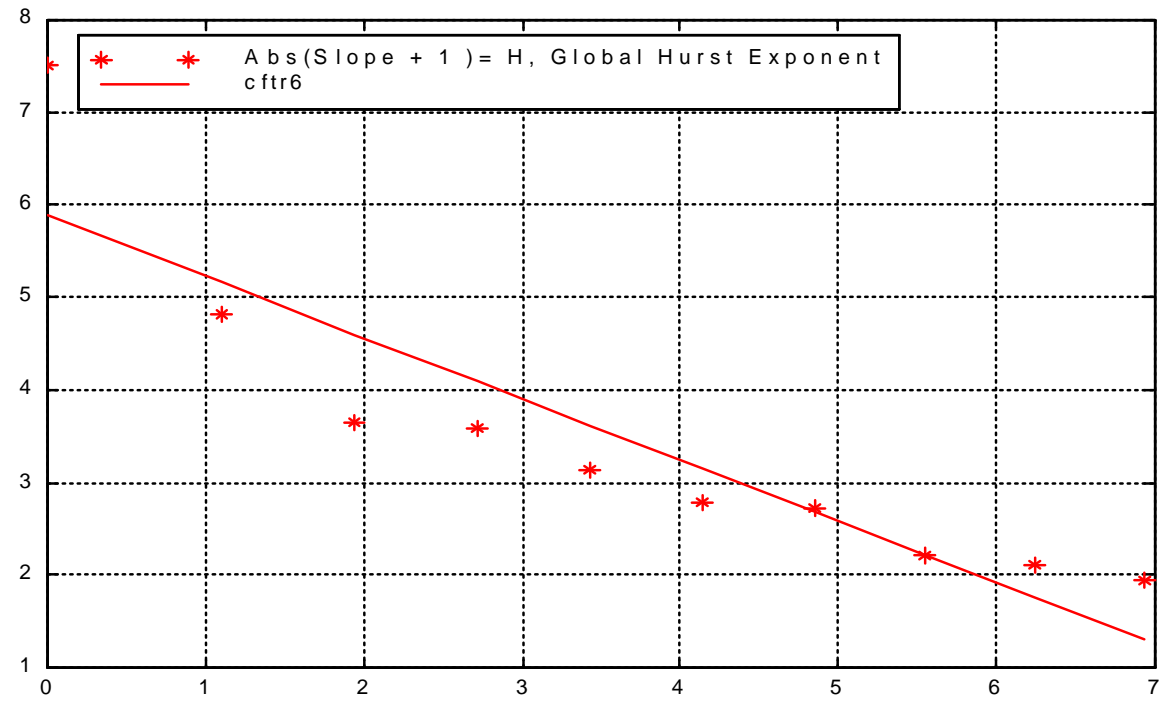

Figure V-35 loglog plot for the global Hurst exponent 


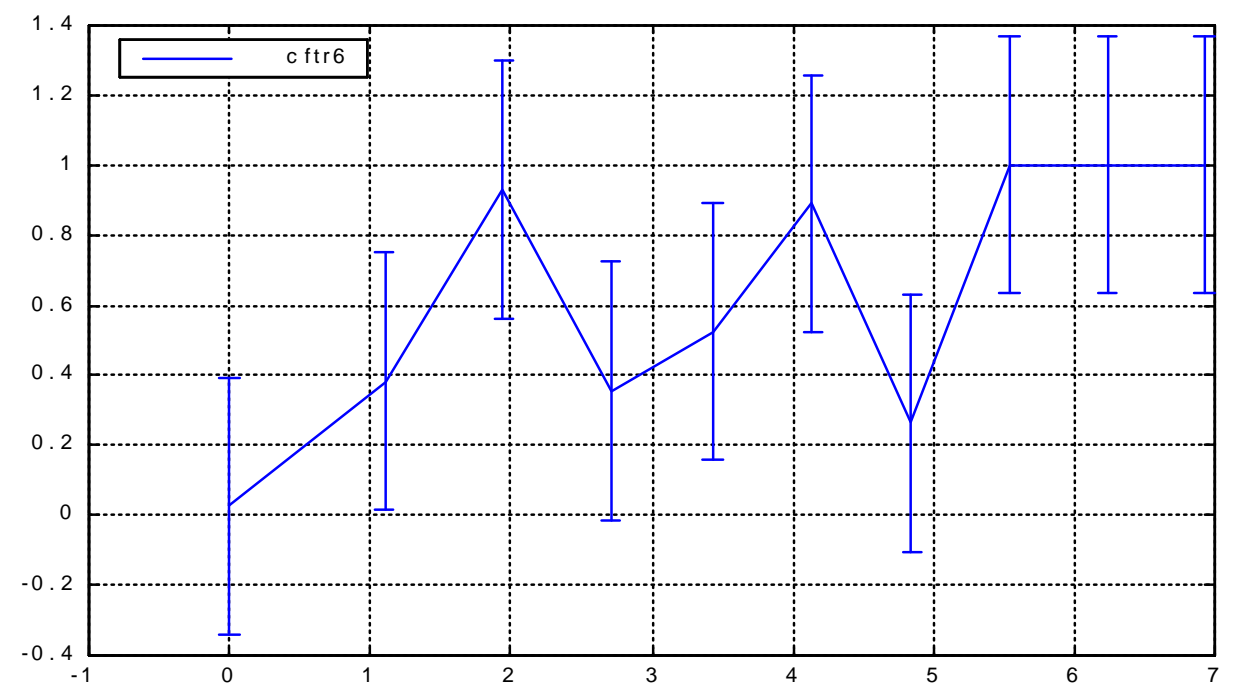

Figure V-36 loglog plot for the local Hurst exponent

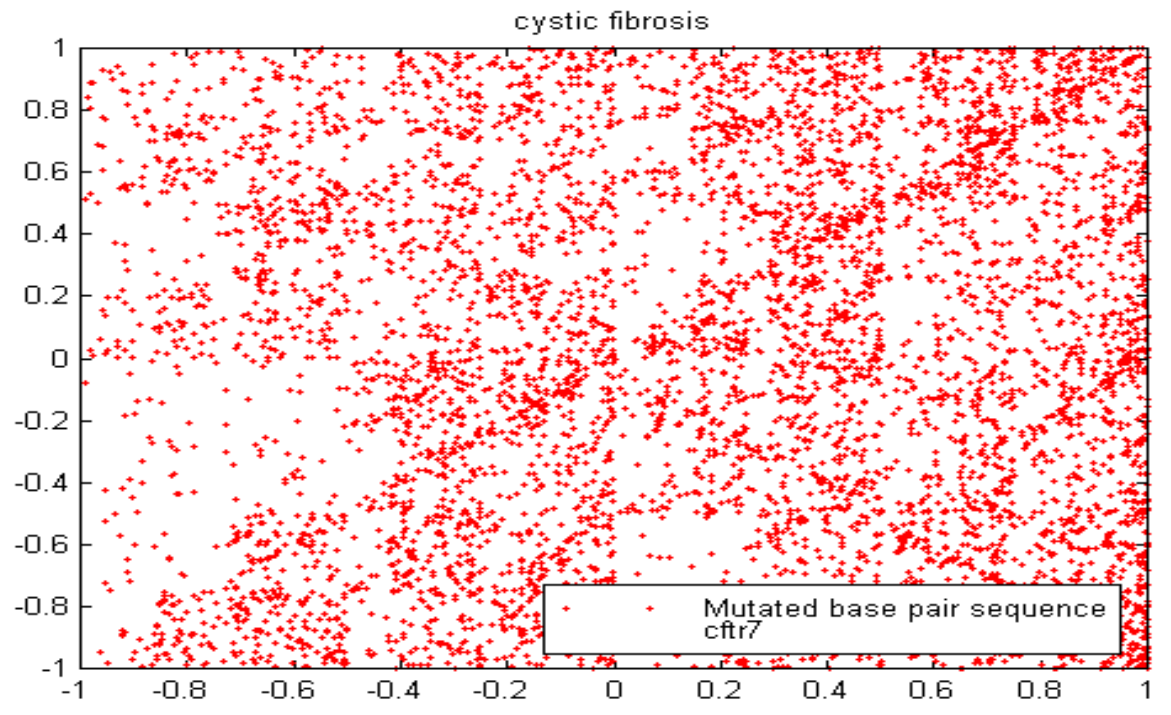

Figure V-37 IFS Mapping of the Cftr7.txt sequence 


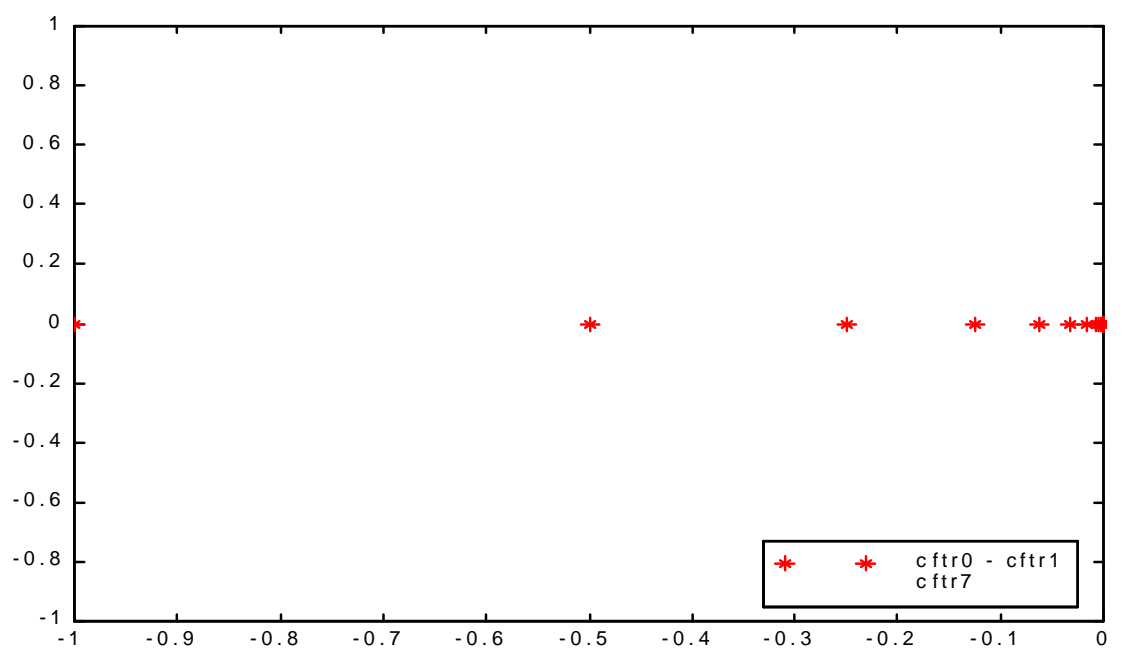

Figure V-38 Difference in IFS Mappings "Original" - "mutation"

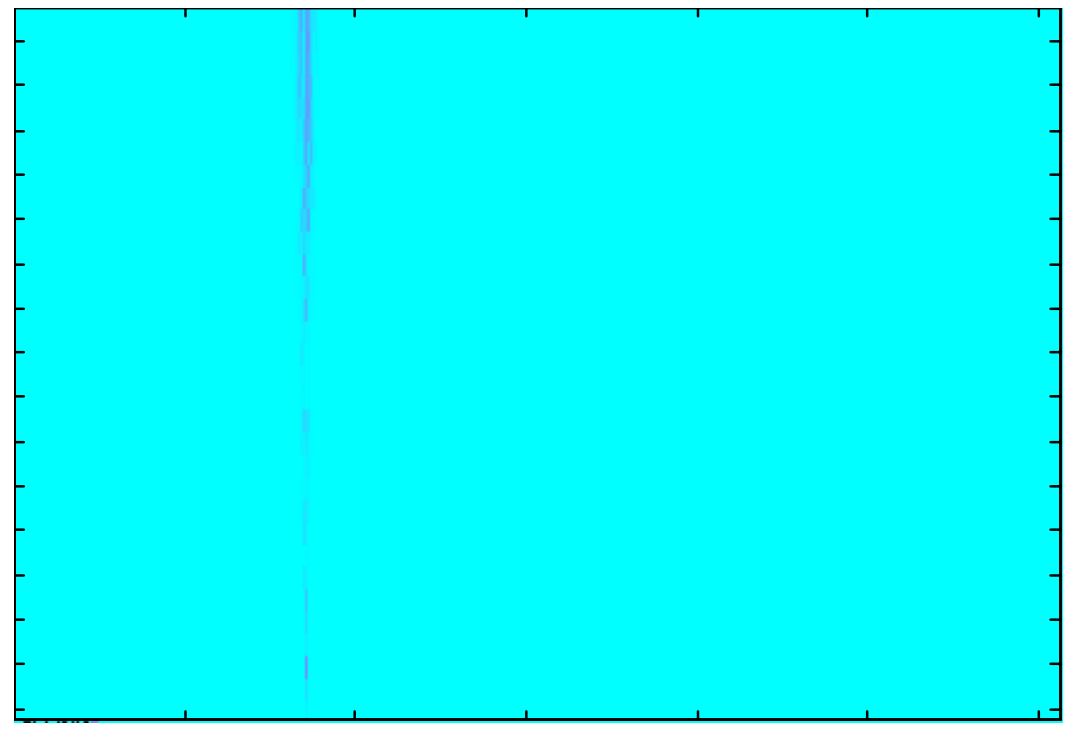

Figure V-39 Difference in the Wavelet coefficients 


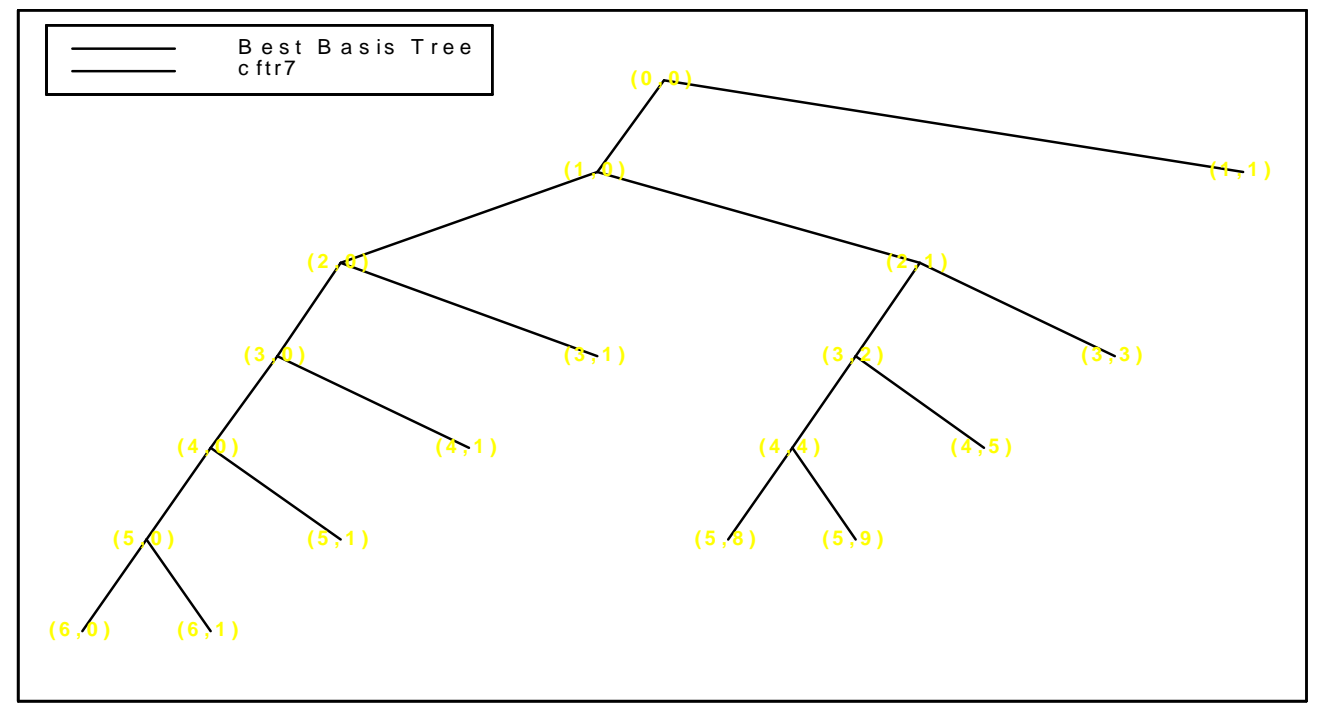

Figure V-40 Best Basis Representation

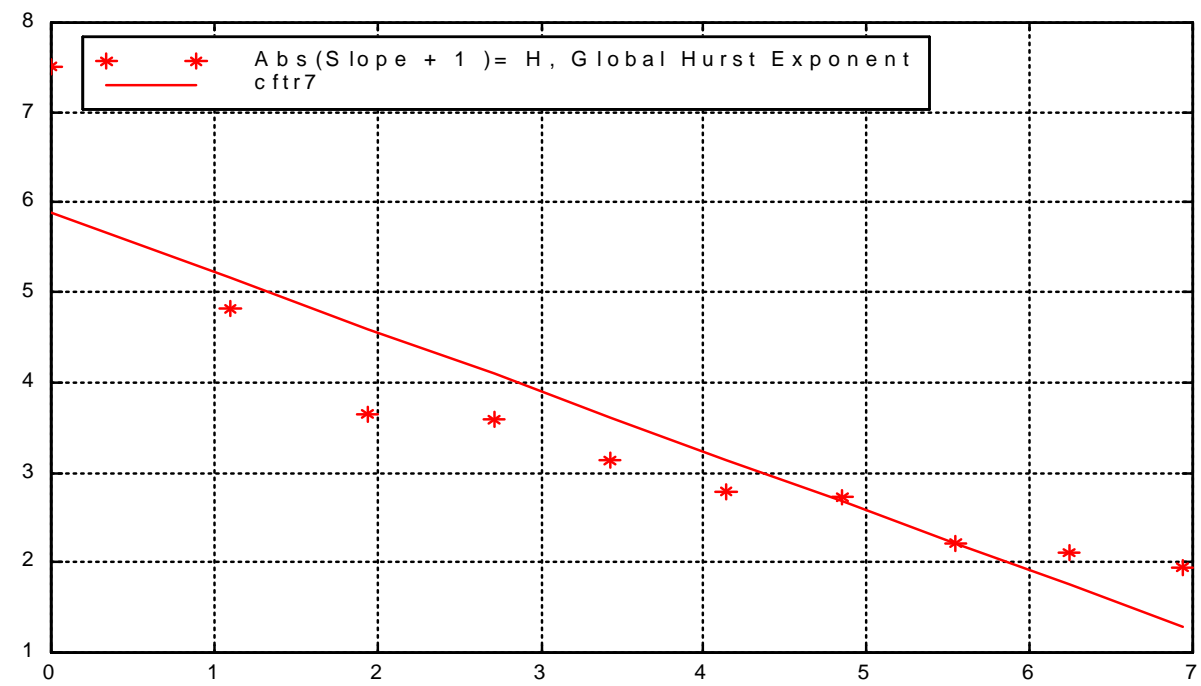

Figure V-41 loglog plot for the global Hurst exponent 


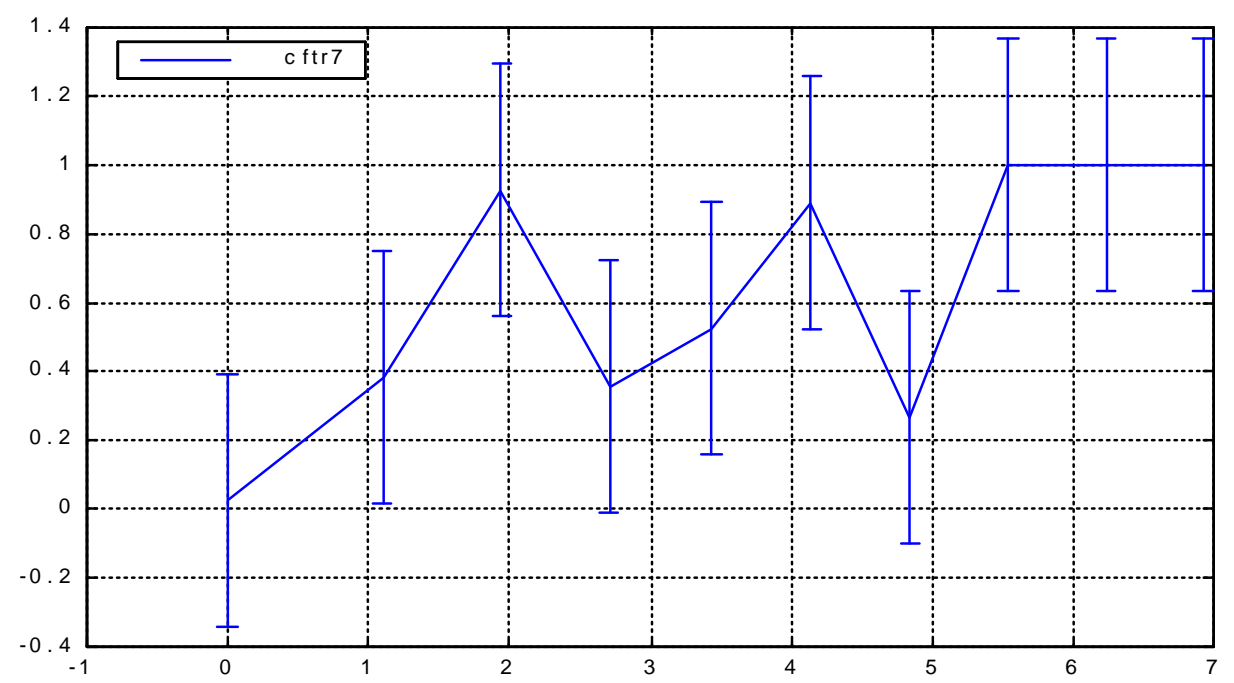

Figure V-42 loglog plot for the local Hurst exponent

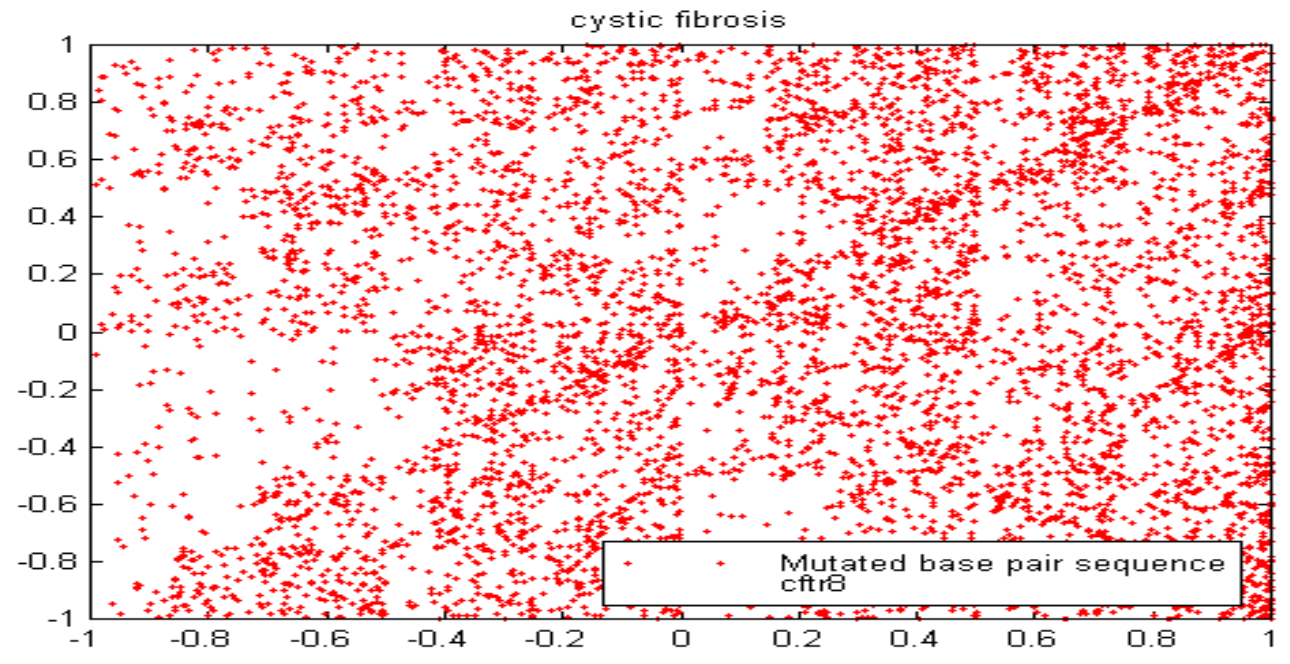

Figure V-43 IFS Mapping of the Cftr8.txt sequence 


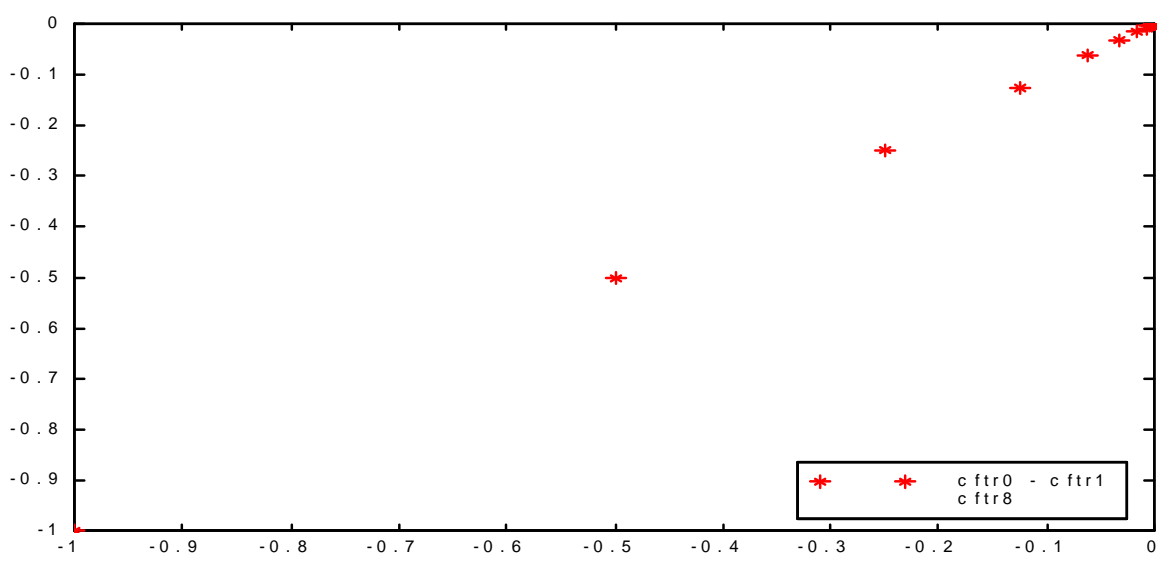

Figure V-44 Difference in IFS Mappings "Original" - "mutation"

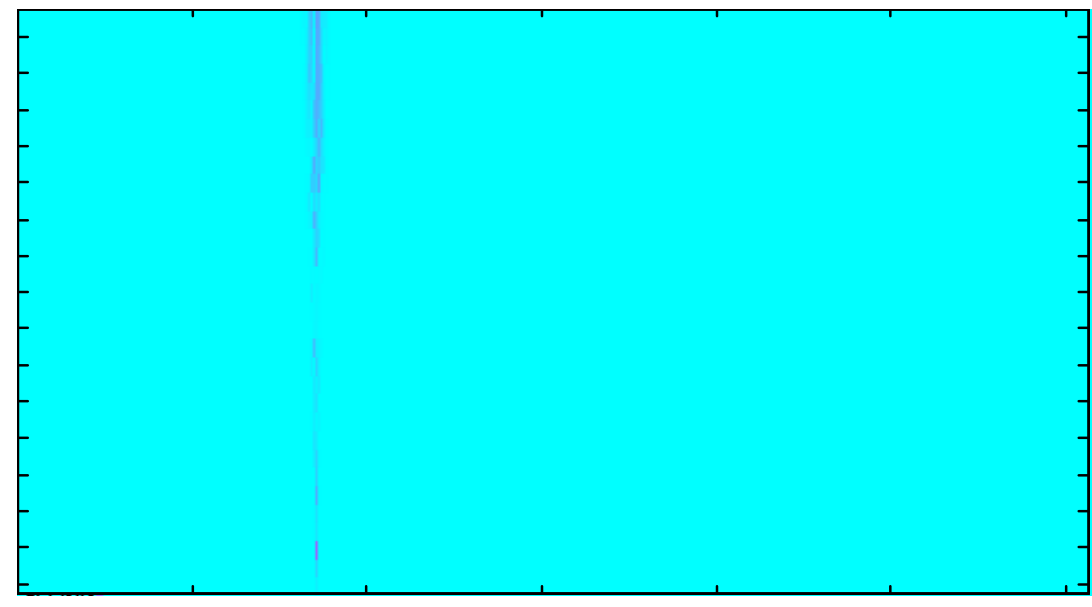

Figure V-45 Difference in the Wavelet coefficients 


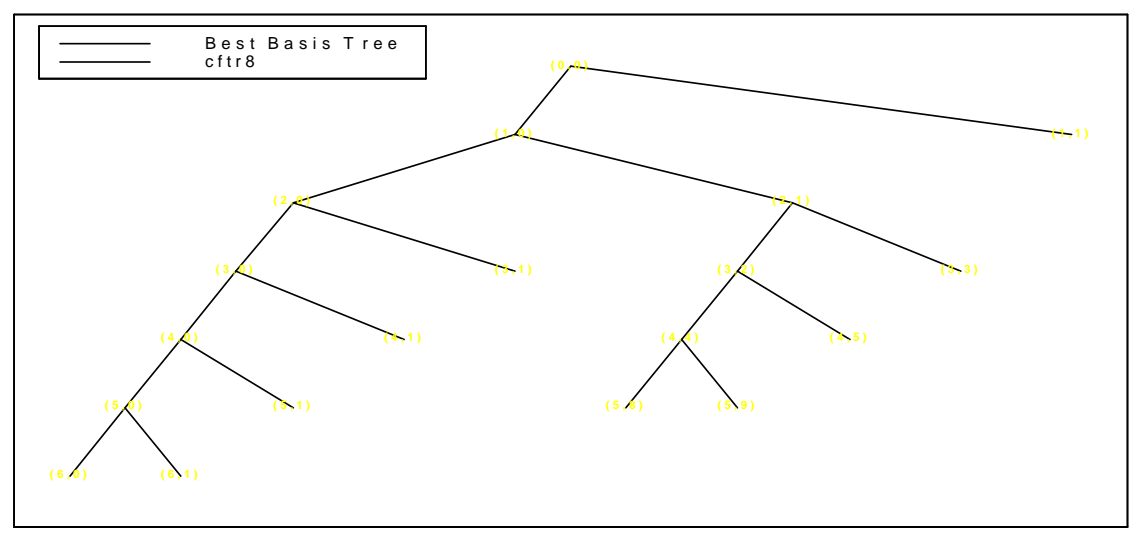

Figure V-46 Best Basis Representation

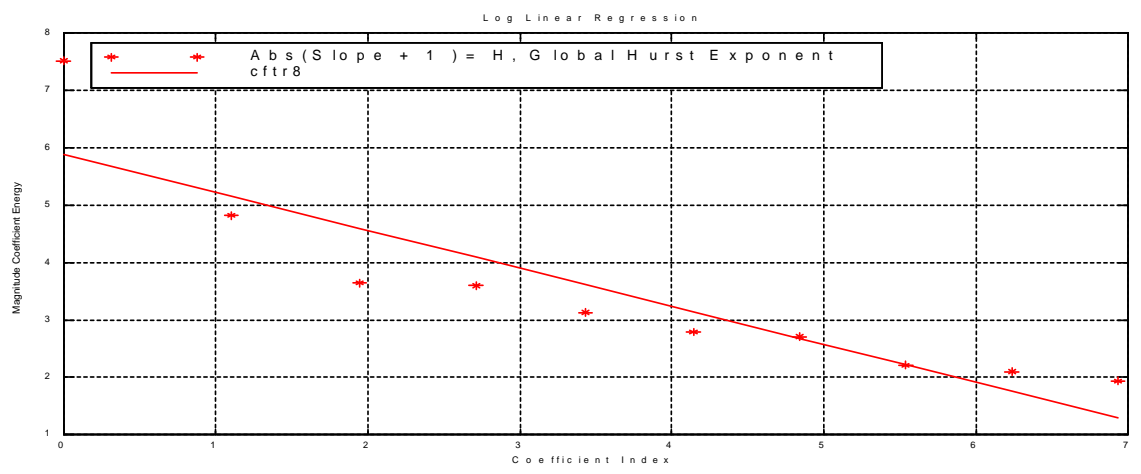

Figure V-47 loglog plot for the global Hurst exponent 


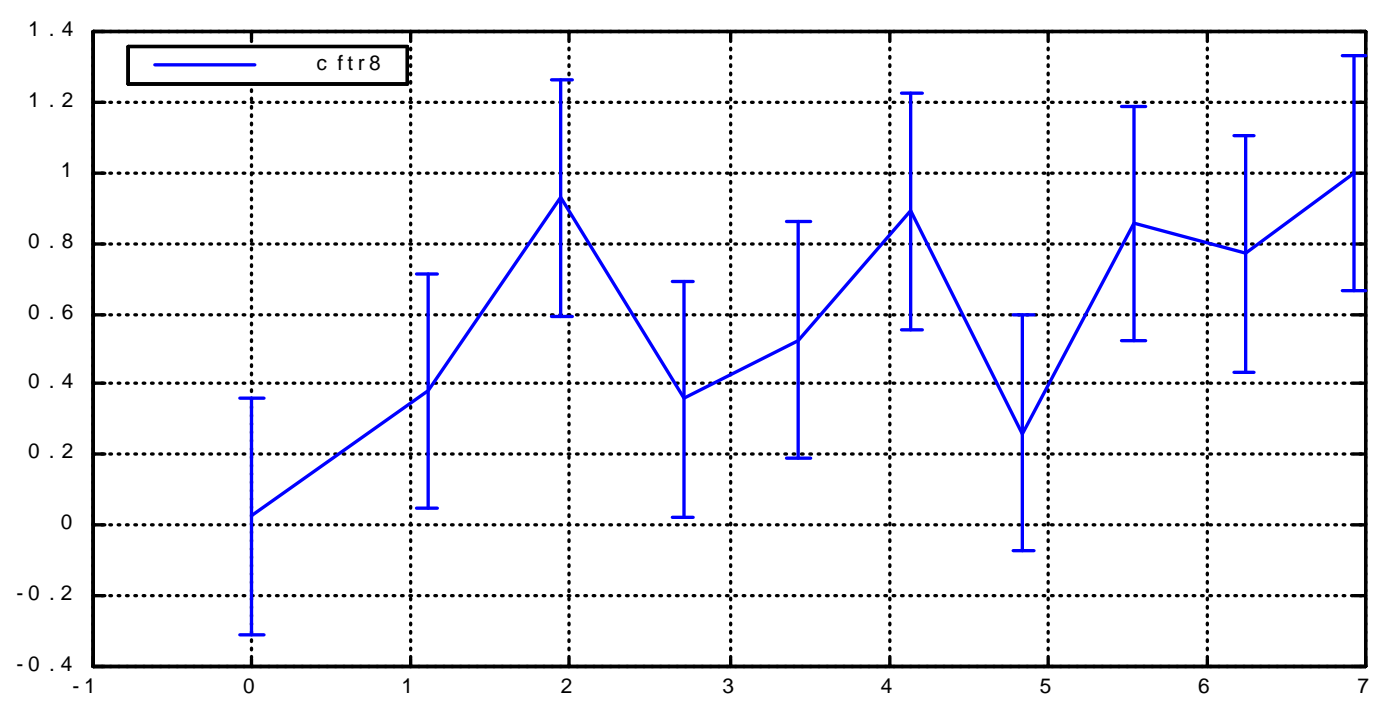

Figure V-48 loglog plot for the local Hurst exponent

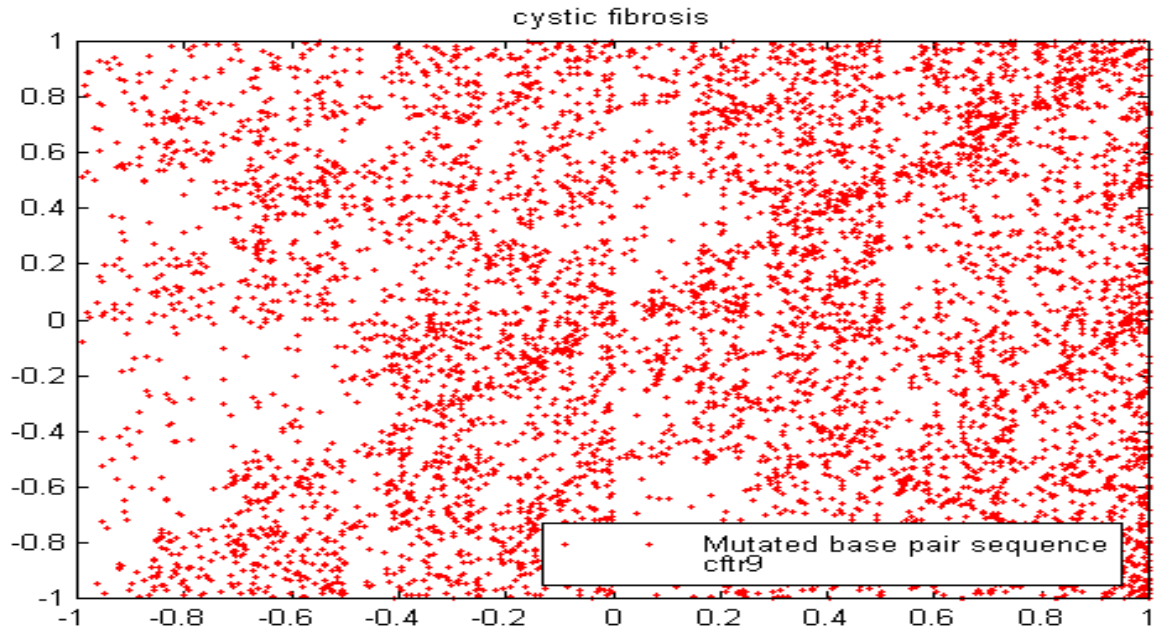

Figure V-49 IFS Mapping of the Cftr9.txt sequence 


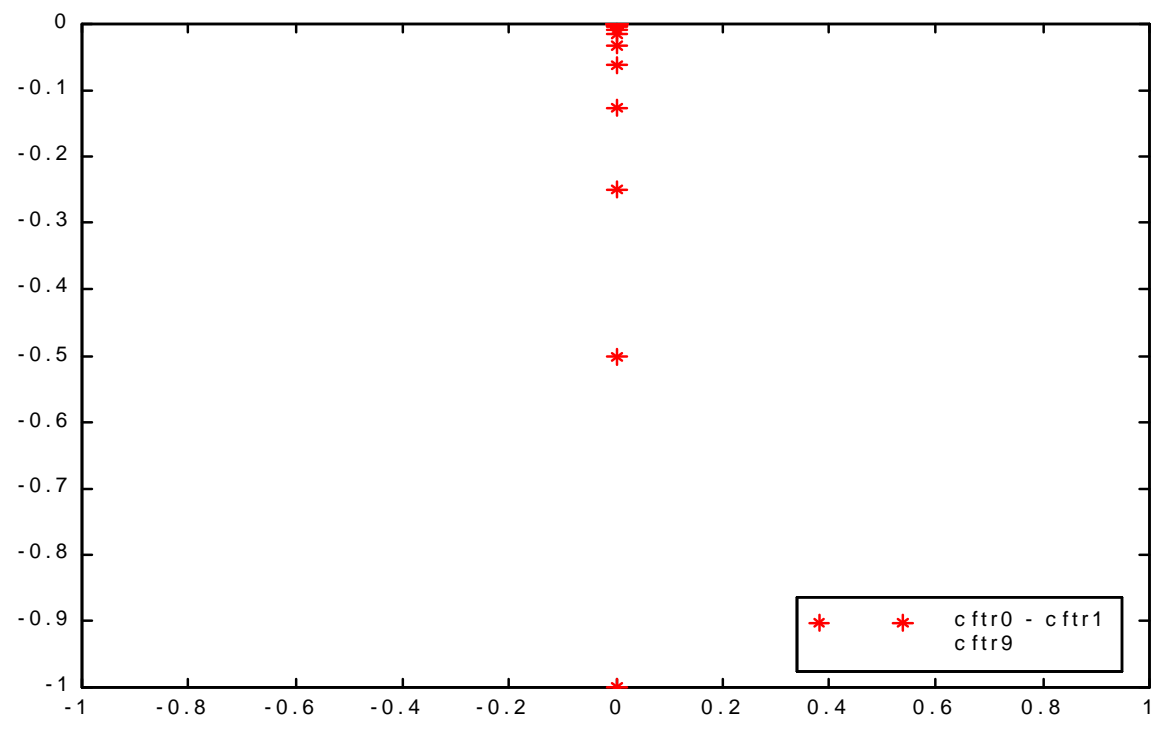

Figure V-50 Difference in IFS Mapings "Original" - "mutation"

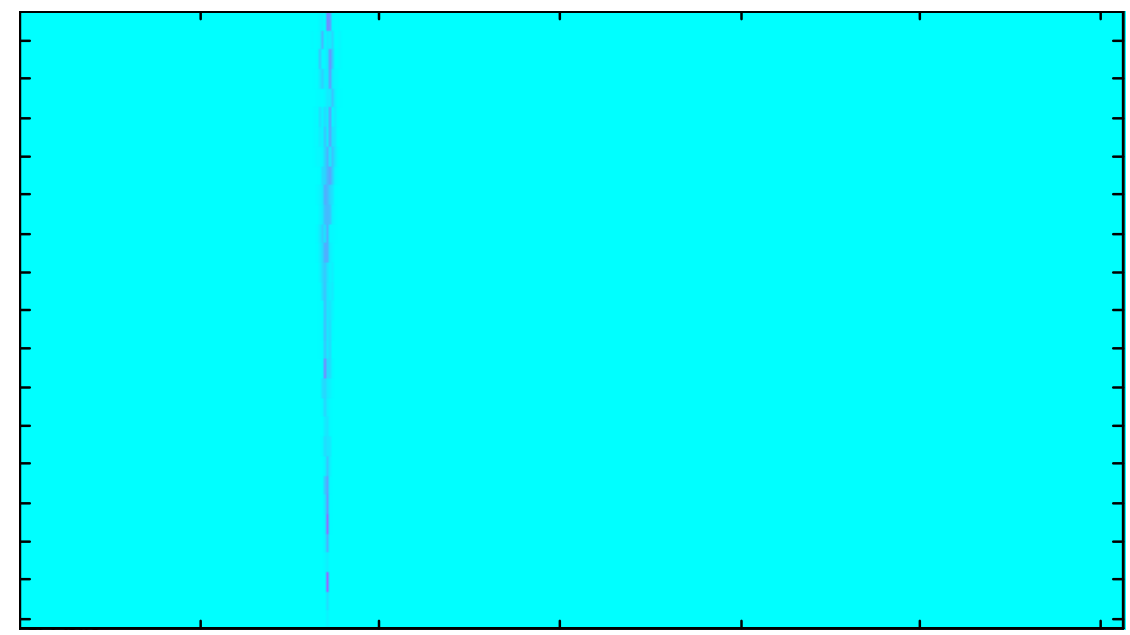

Figure V-51 Difference in the Wavelet coefficients 


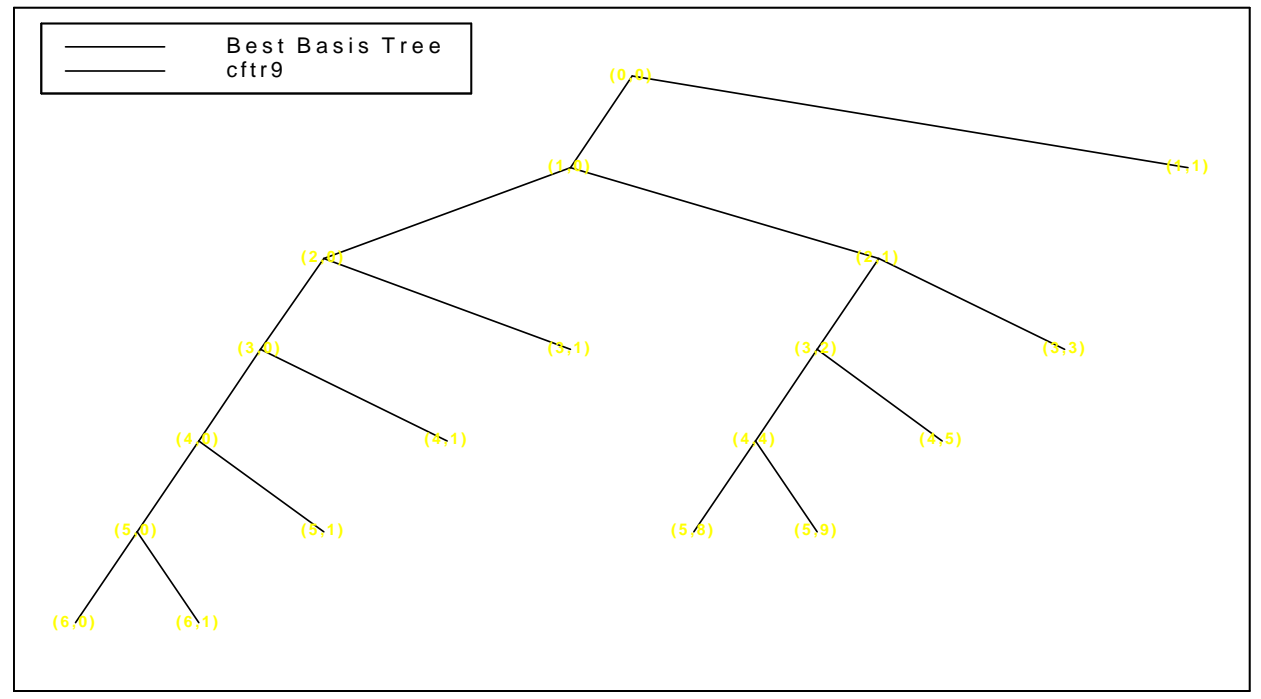

Figure V-52 Best Basis Representation

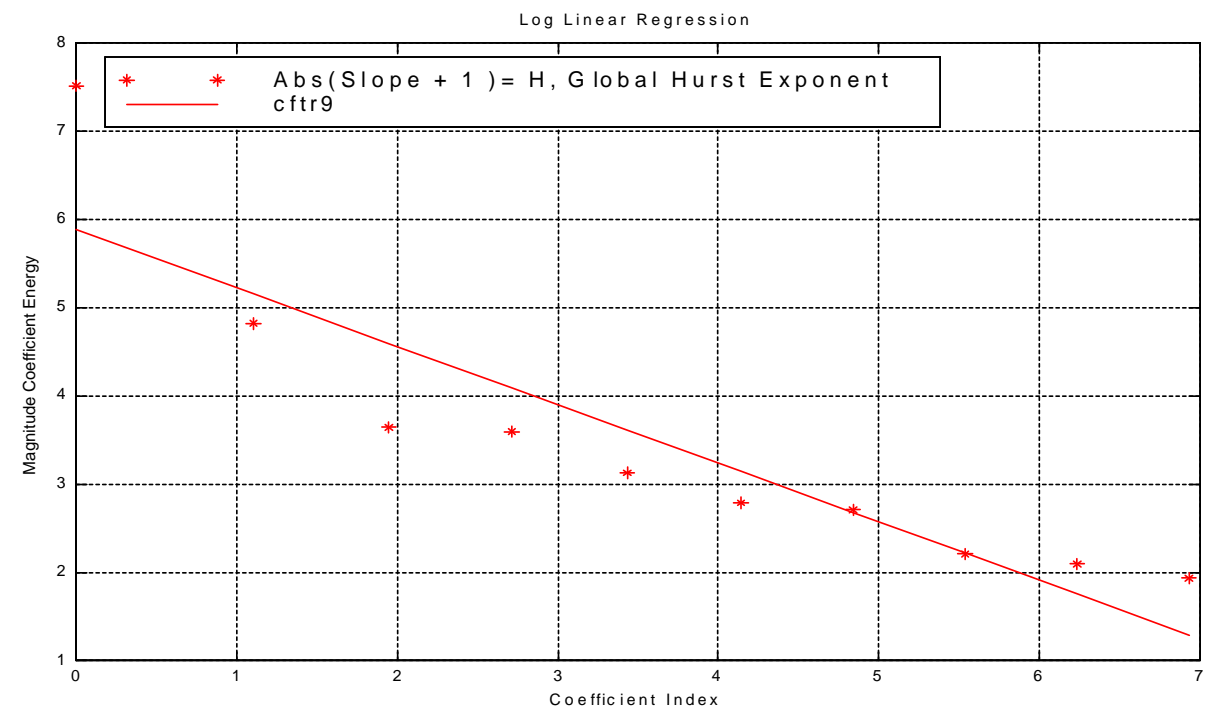

Figure V-53 loglog plot for the global Hurst exponent 


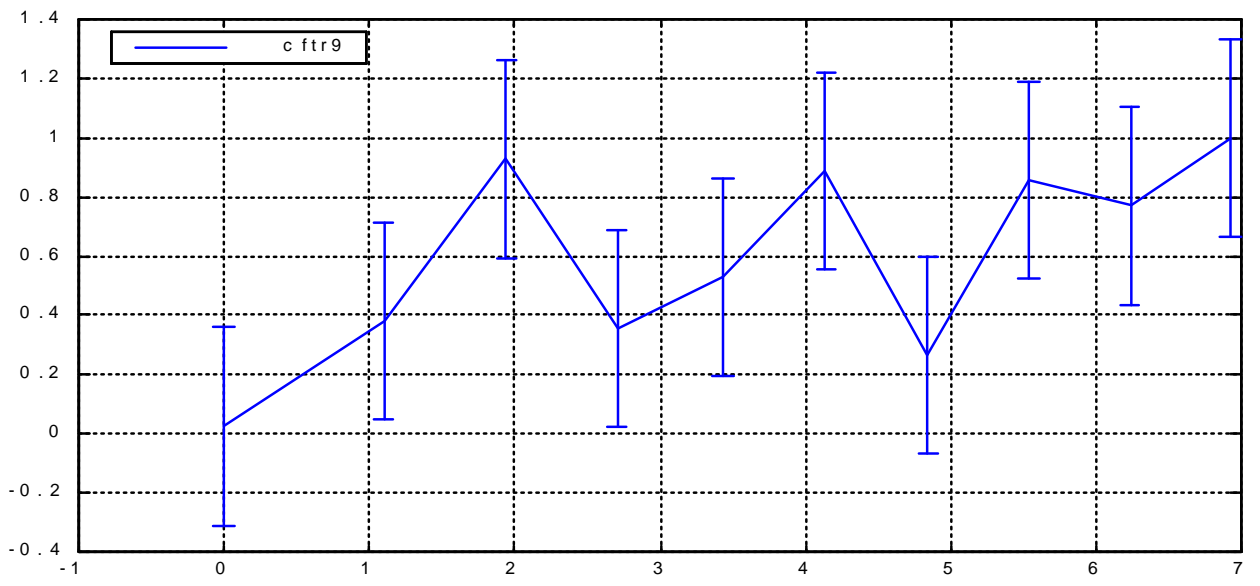

Figure V-54 loglog plot for the local Hurst exponent 
Sequences cftr10.txt to cftr13.txt are all single point deletions. They as did the other deletions before them show dramatically the changes caused by deletions. They are specifically:

$\begin{array}{lll}\text { Sequence } & \text { Change } & \text { base pair position } \\ \text { 1. cftr10.txt } & \text { c deleted } & \text { line } 1021 \text { position } 1032 \\ \text { 2. cftr11.txt } & \text { t deleted } & \text { line } 3361 \text { position } 3382 \\ \text { 3. cftr12.txt } & \text { a deleted } & \text { line } 4861 \text { position } 4893 \\ \text { 4. cftr13.txt } & \text { g deleted } & \text { line } 2581 \text { position } 2597\end{array}$

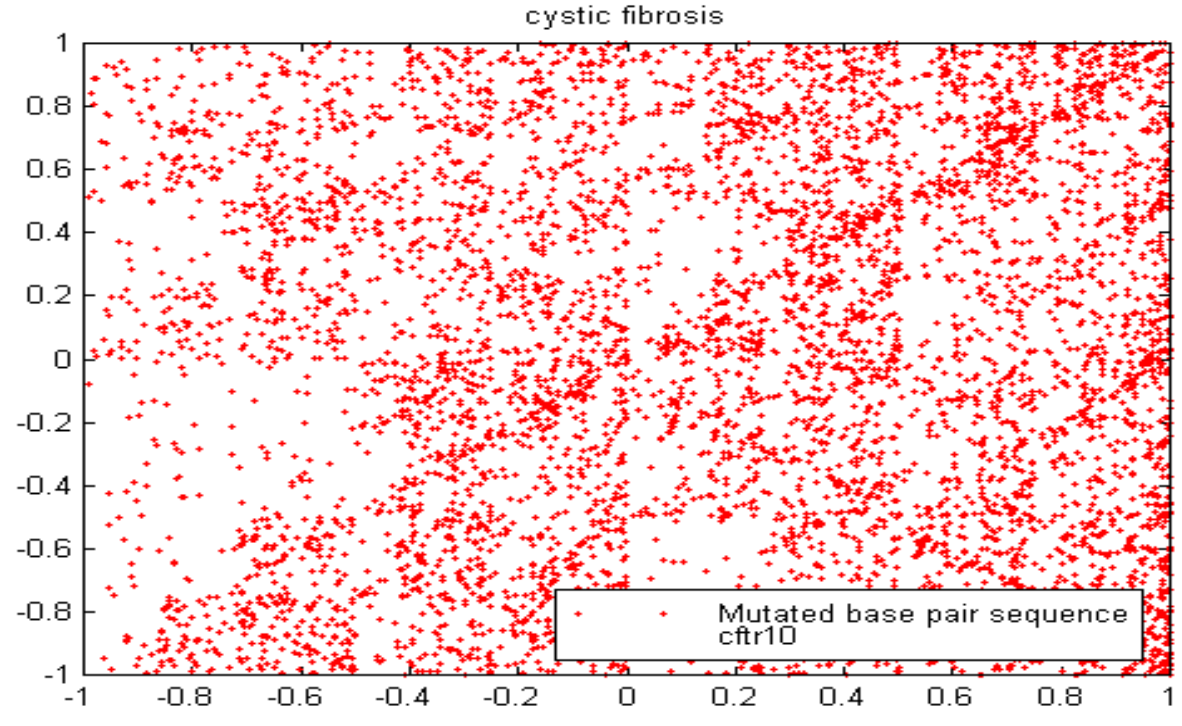

Figure V-55 IFS Mapping of the

Cftr10.txt sequence 
cystic fibrosis

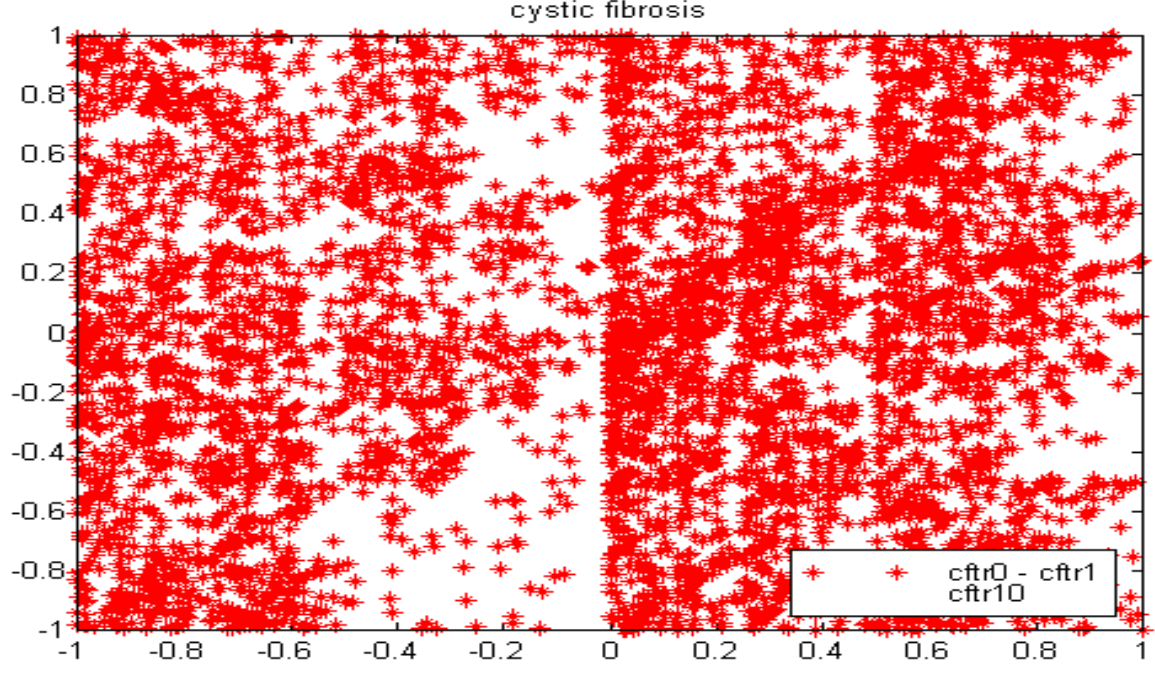

Figure V-56 Difference in IFS Mappings

"Original" - "mutation

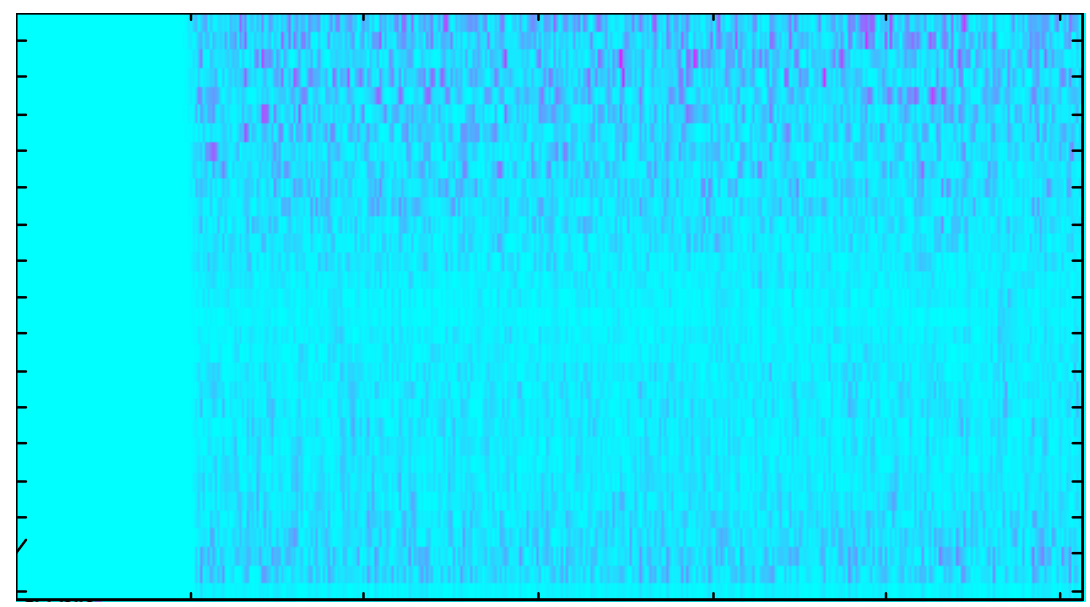

Figure V-57 Difference in the Wavelet coefficients 


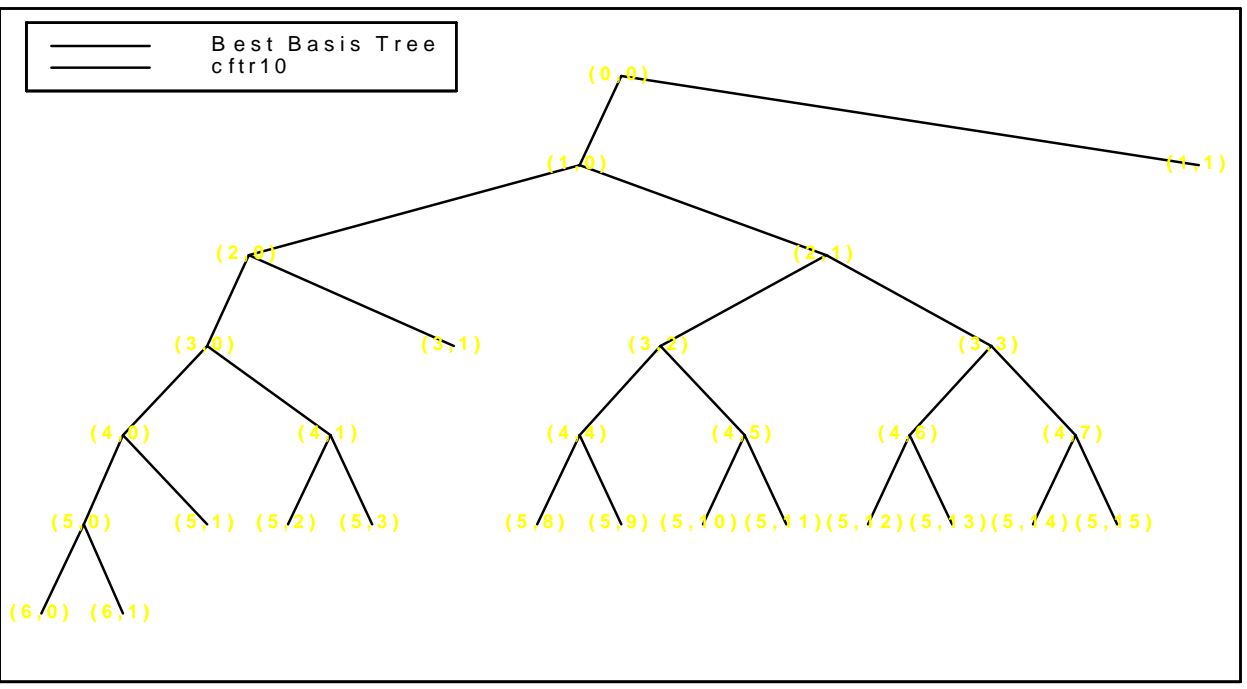

Figure V-58 Best Basis Representation

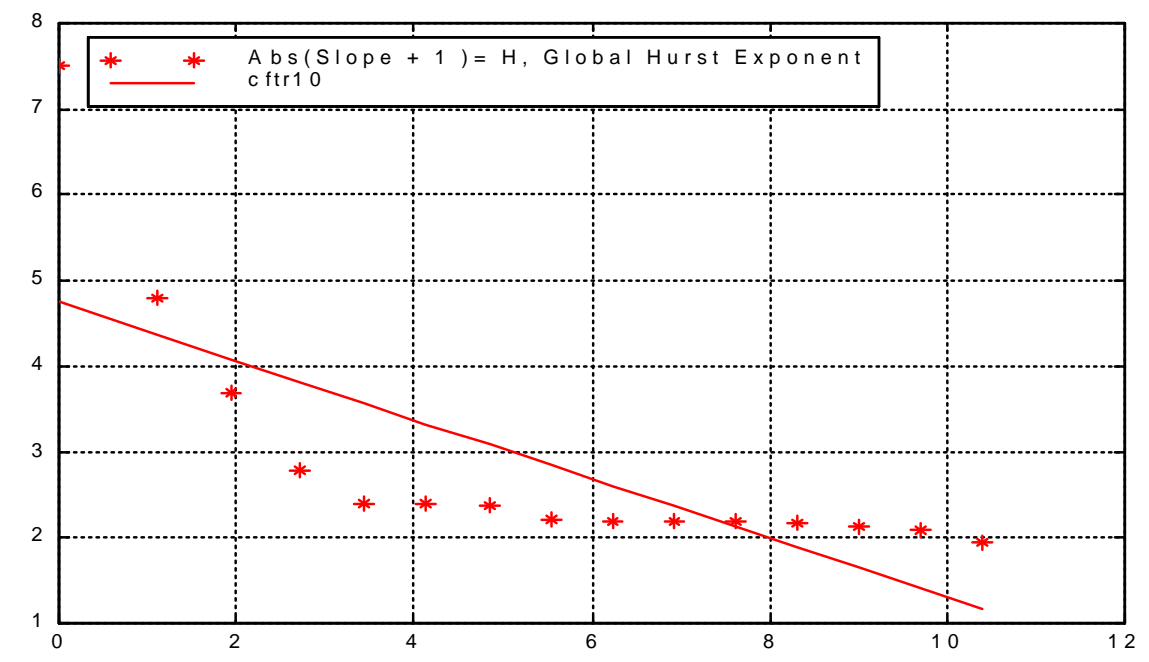

Figure V-59 loglog plot for the global Hurst exponent 


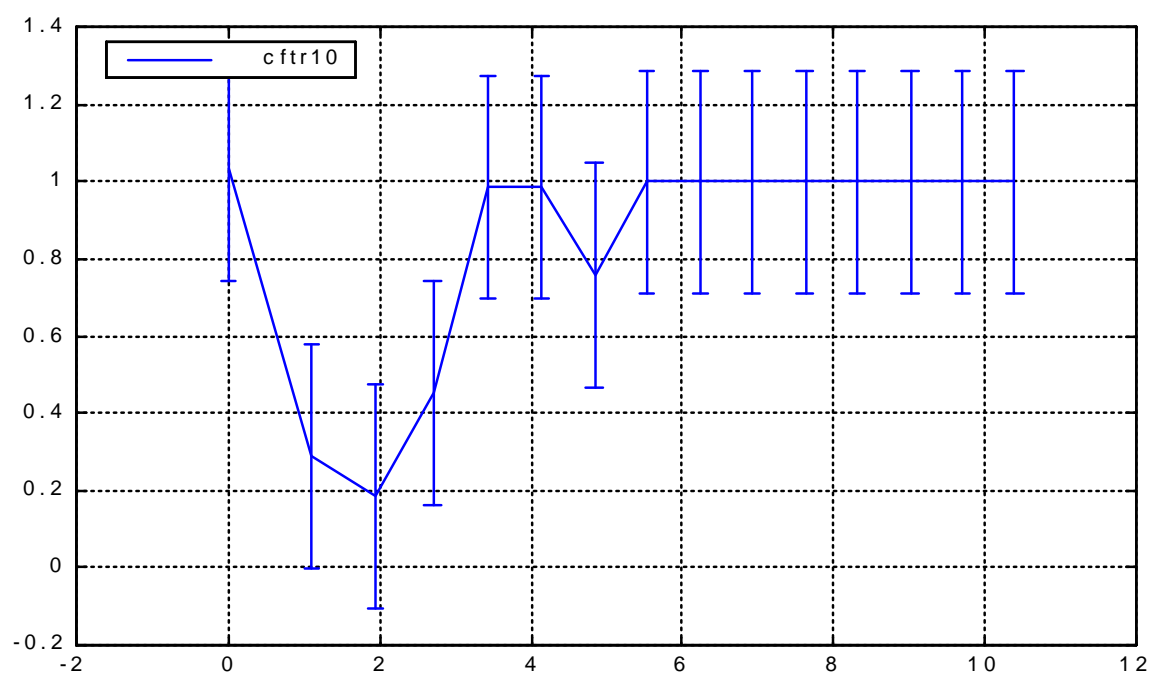

Figure V-60 loglog plot for the local Hurst exponent 


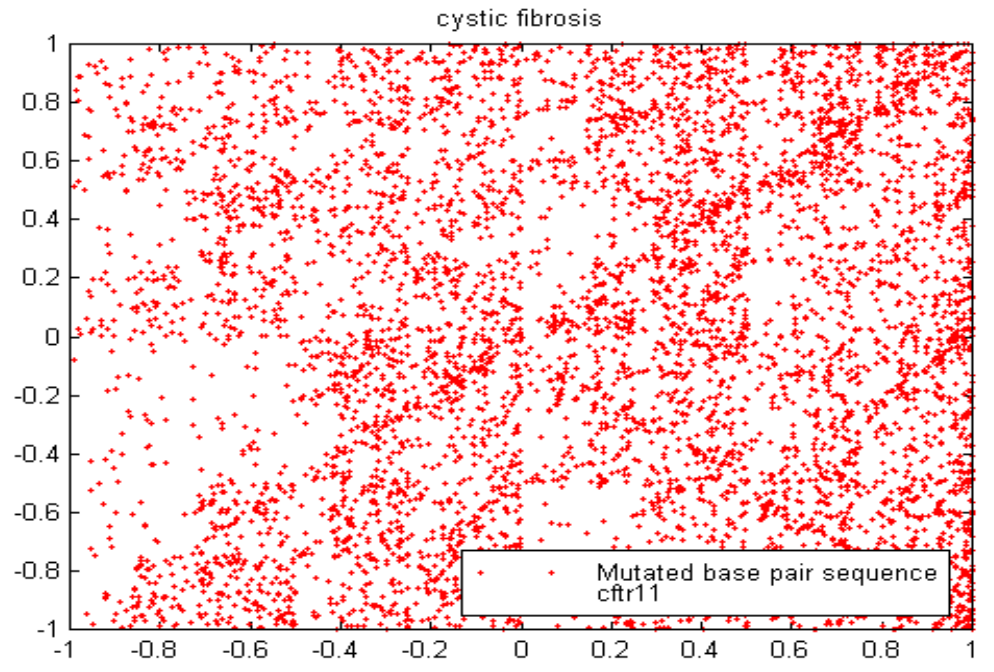

Figure V-61 IFS Maping of the the Cftr10.txt sequence

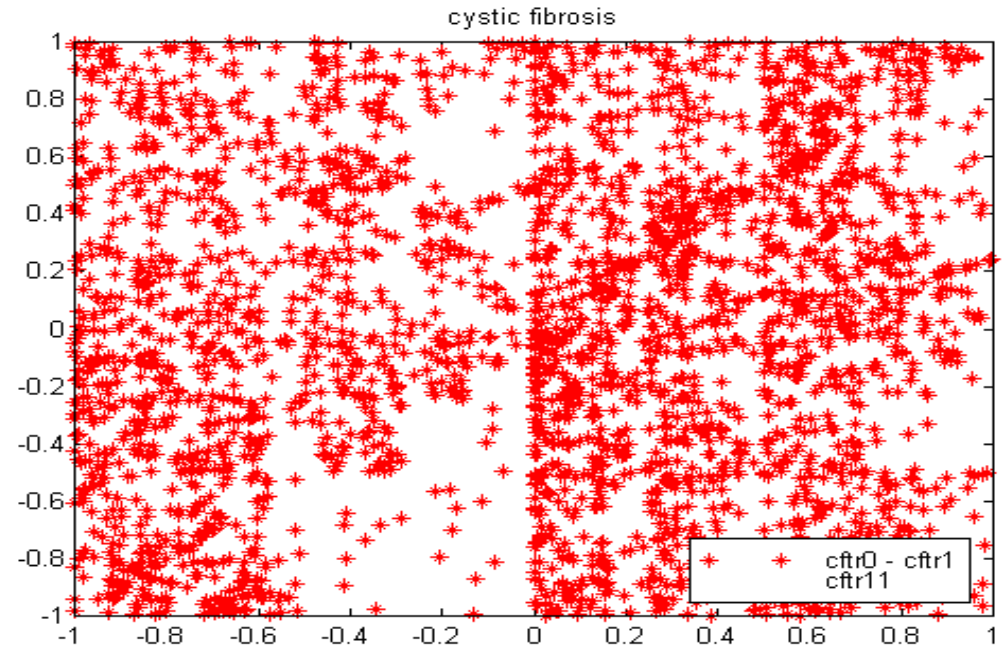

Figure V-62 Difference in IFS Mapings "Original" - "mutation 


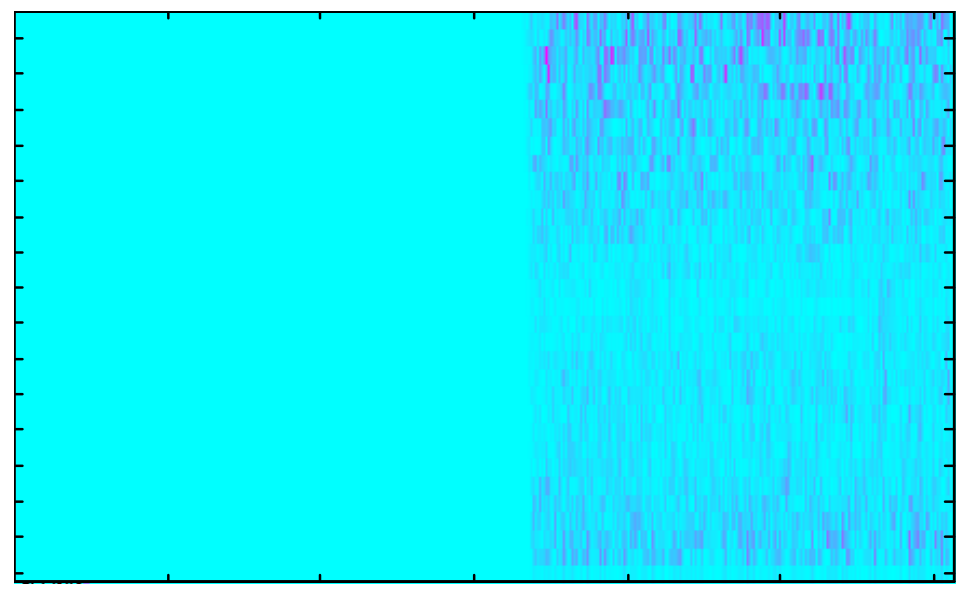

Figure V-64 Difference in the Wavelet coefficients

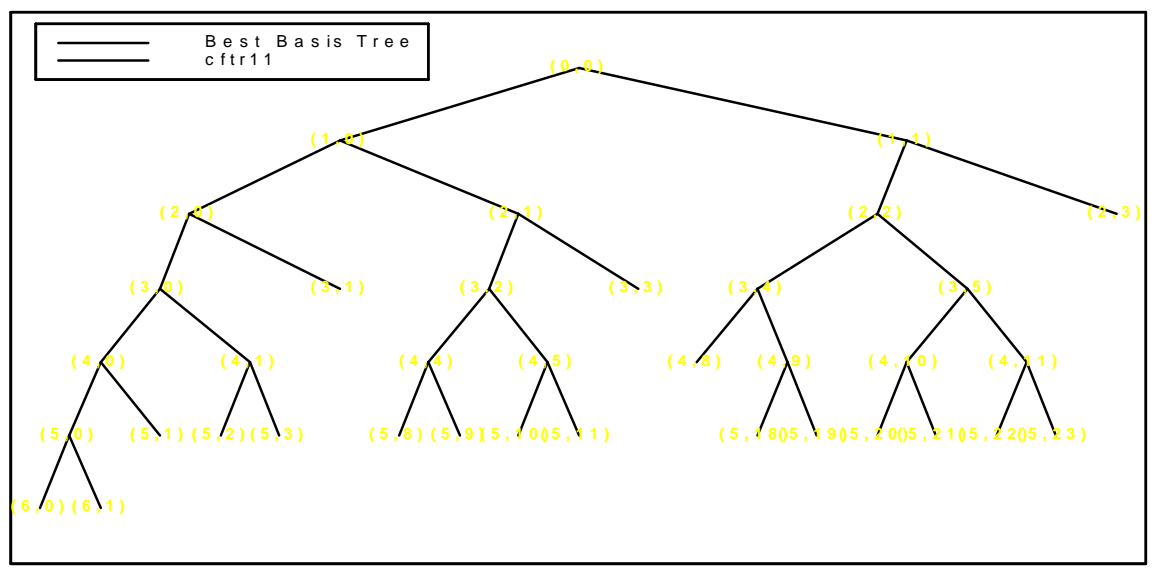

Figure V-65 Best Basis Representation

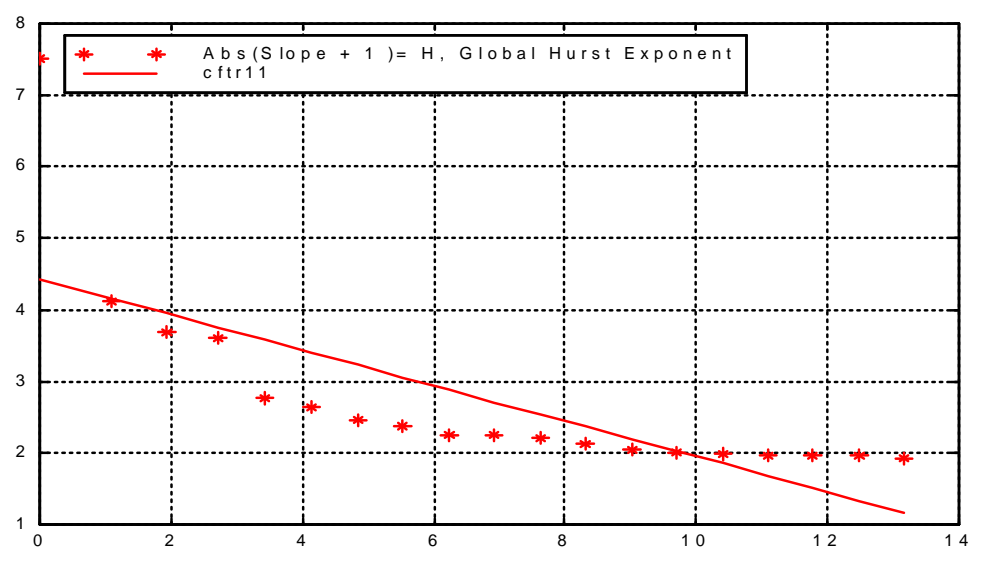

Figure V-66 loglog plot for the global Hurst exponent 


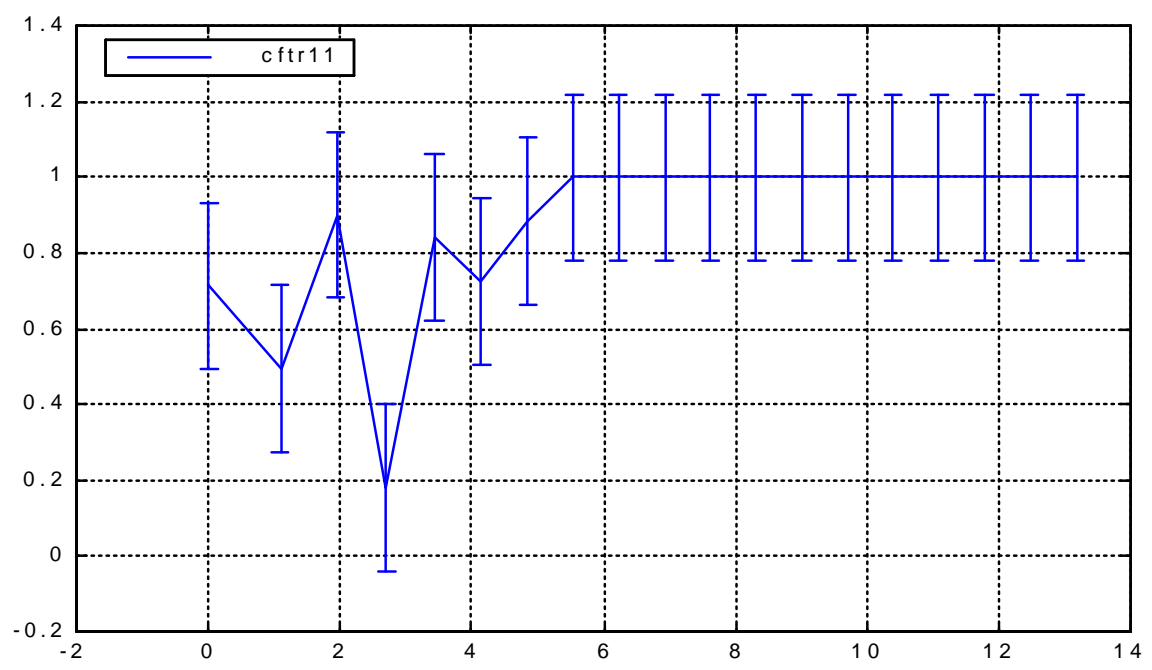

Figure V-67 loglog plot for the local Hurst exponent

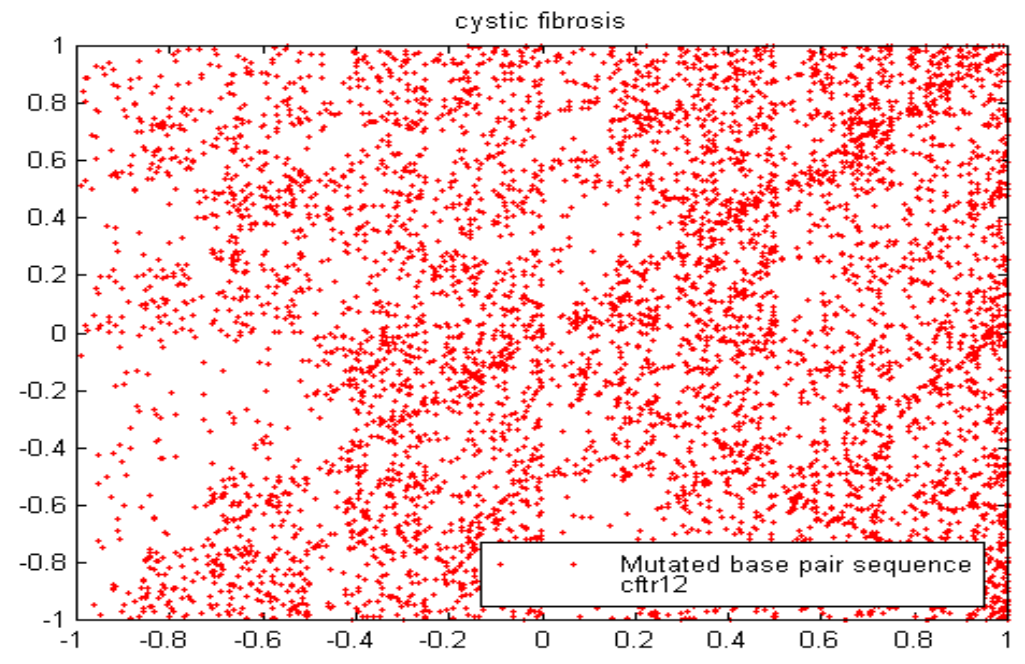

Figure V-68 IFS Maping of the the Cftr12.txt sequence 


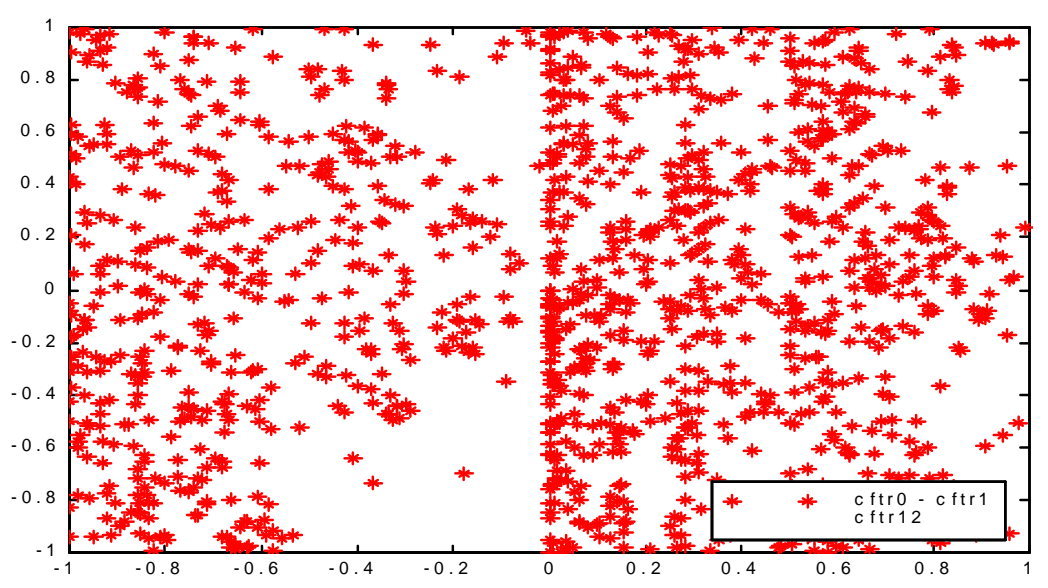

Figure V-69 Difference in IFS Mapings "Original" - "mutation

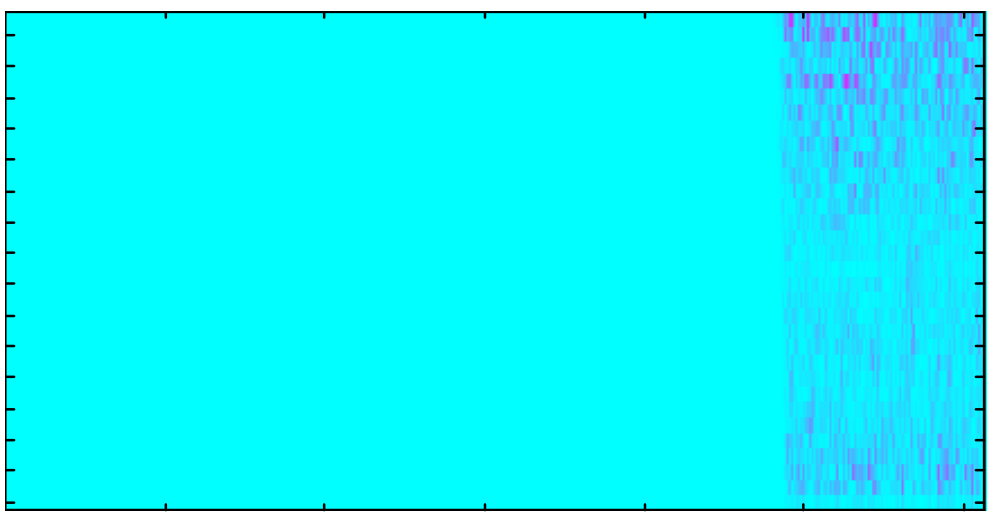

Figure V-70 Difference in the Wavelet coefficients

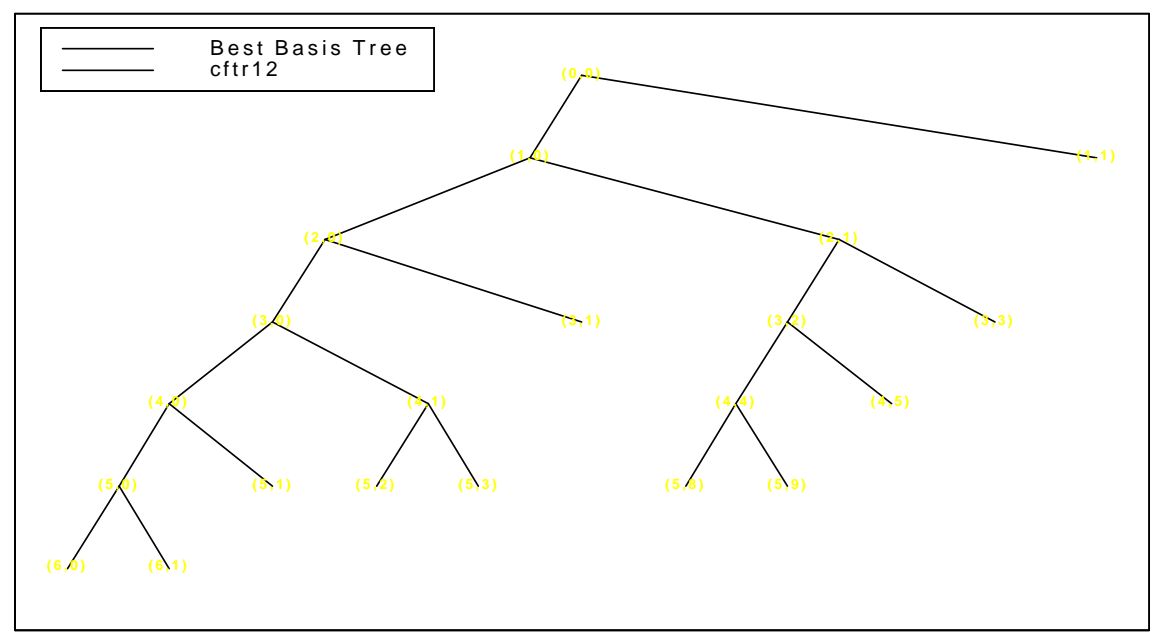

Figure V-71 Best Basis Representation 


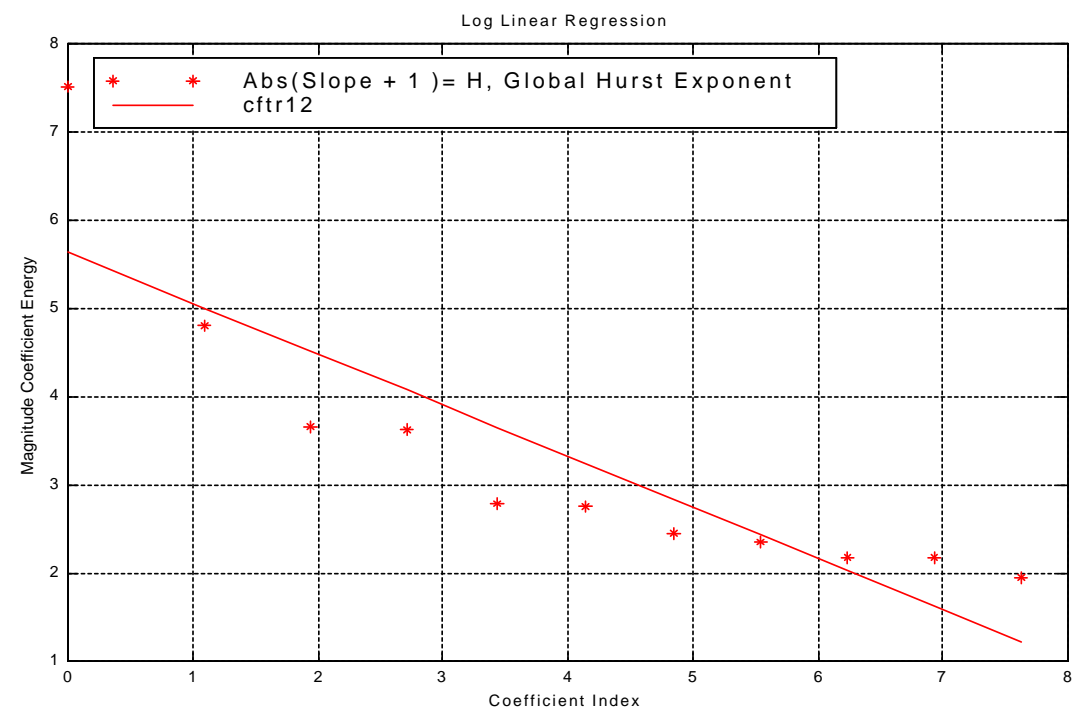

Figure V-72 loglog plot for the global Hurst exponent

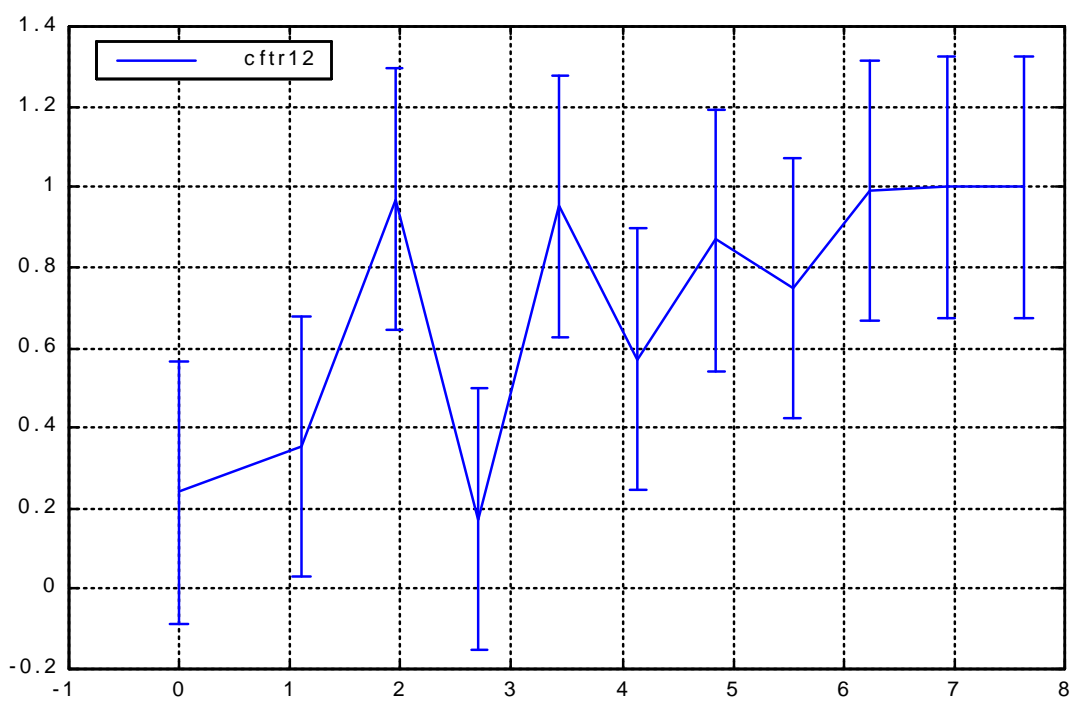

Figure V-73 loglog plot for the local Hurst exponent 


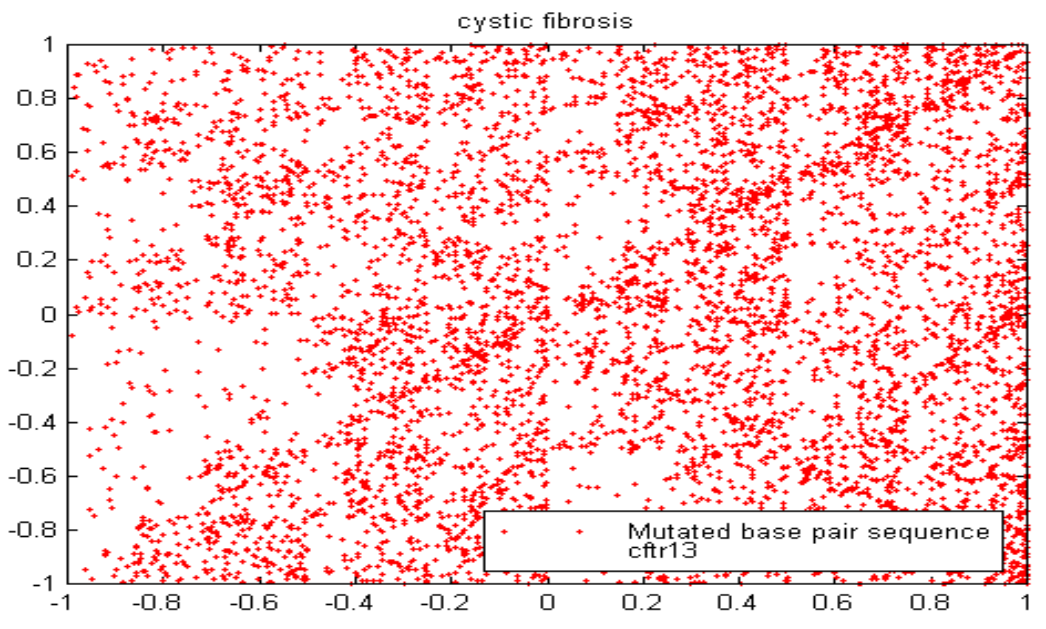

Figure V-74 IFS Maping of the the Cftr13.txt sequence

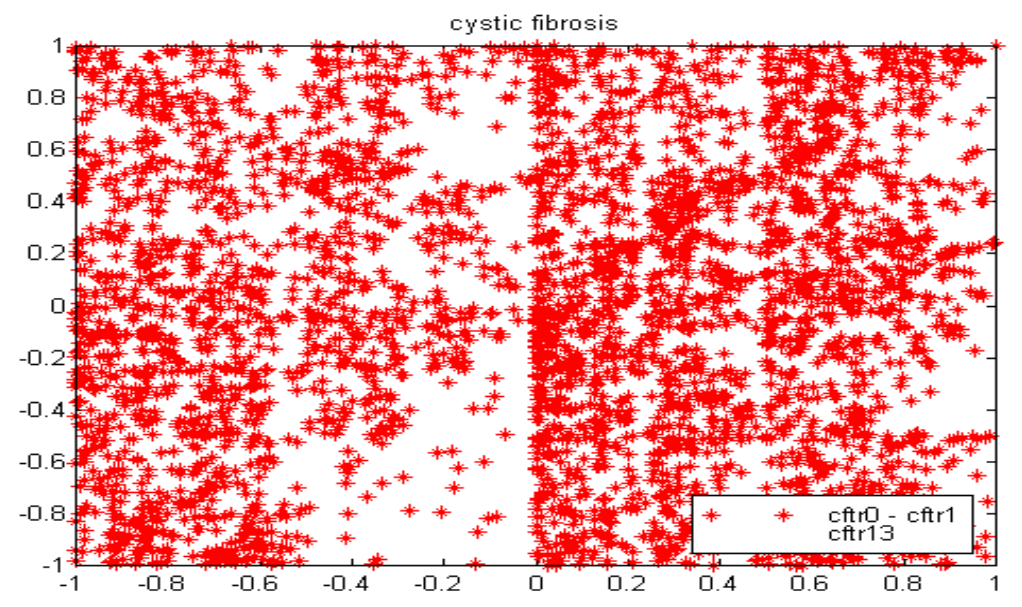

Figure V-75 Difference in IFS Mapings "Original" - "mutation" 


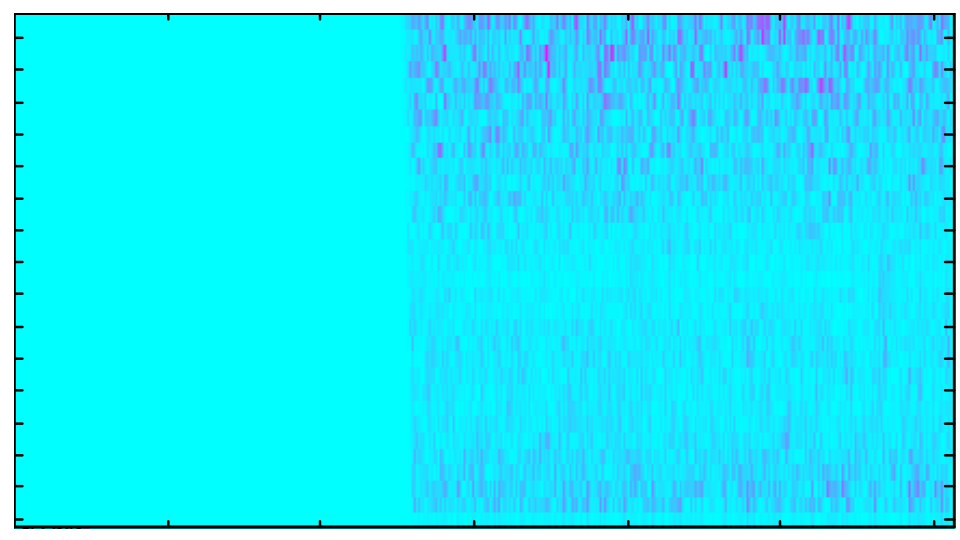

Figure V-76 Difference in the Wavelet coefficients

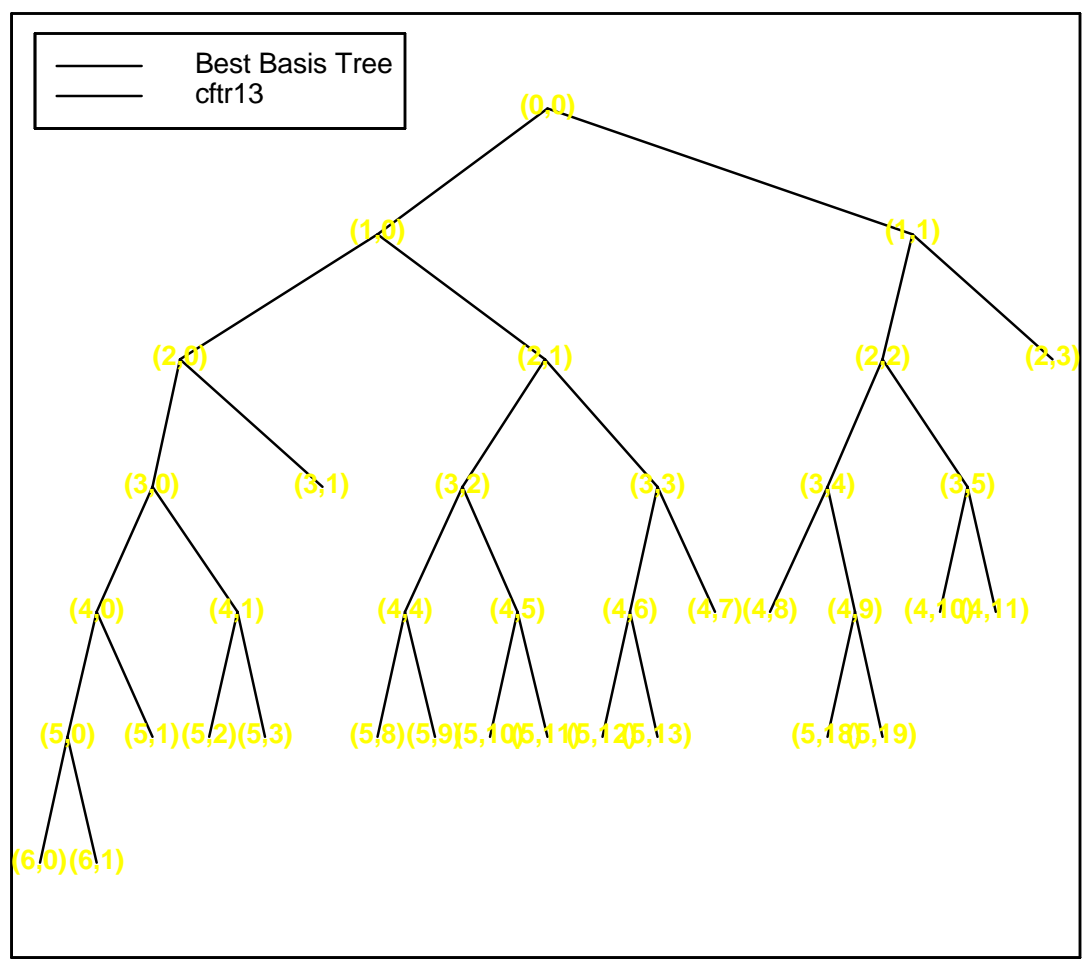

Figure V-77 Best Basis Representation 


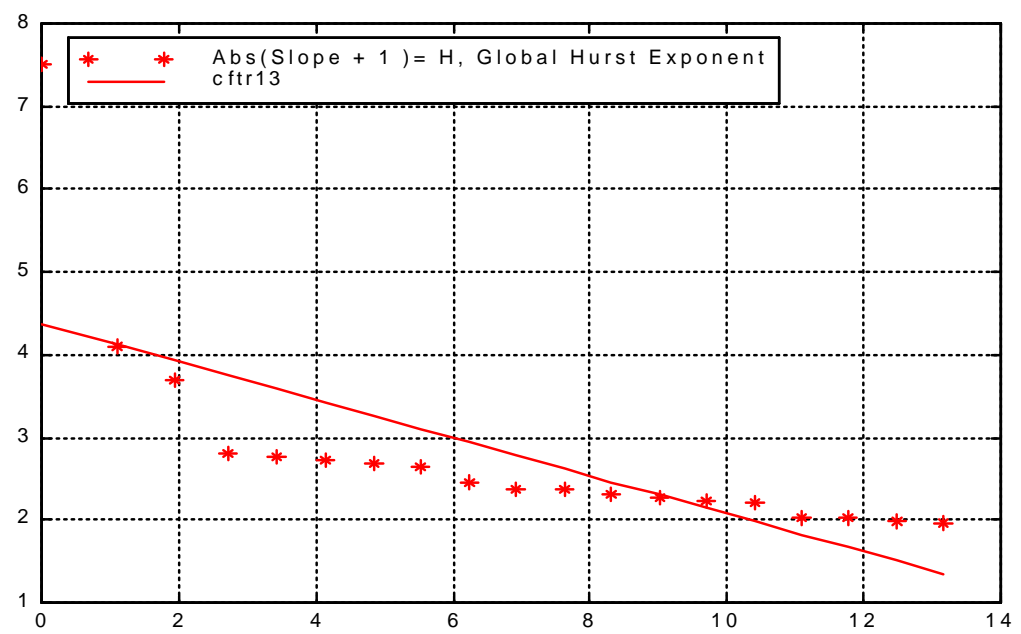

Figure V-78 loglog plot for the global Hurst exponent

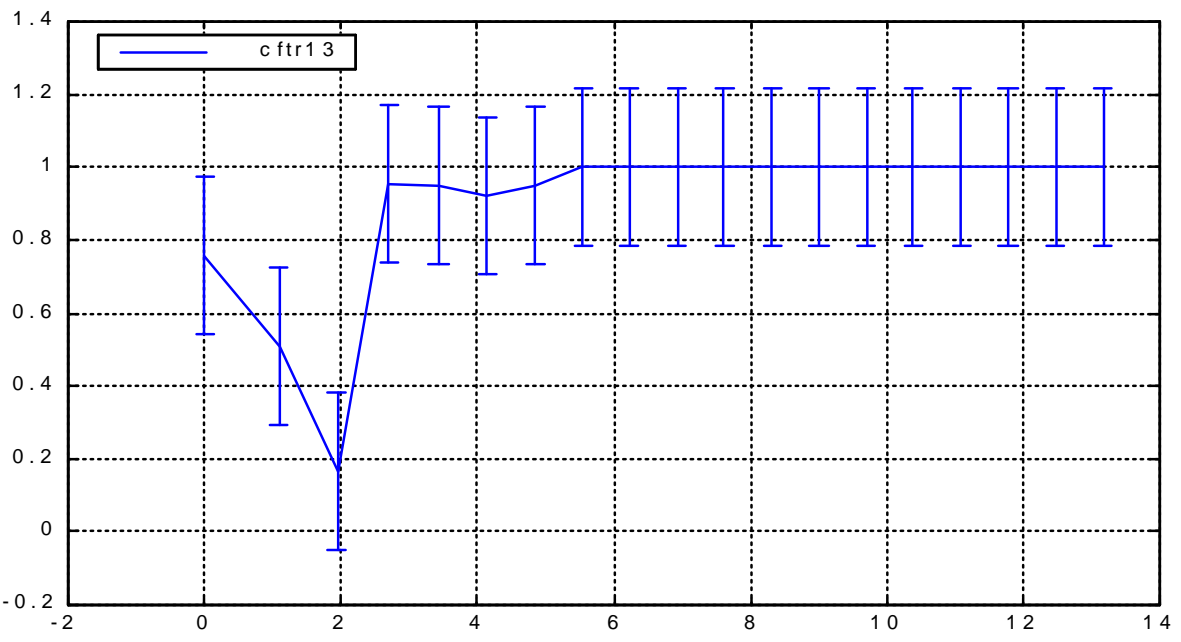

Figure V-79 loglog plot for the local Hurst exponent 
cystic fibrosis

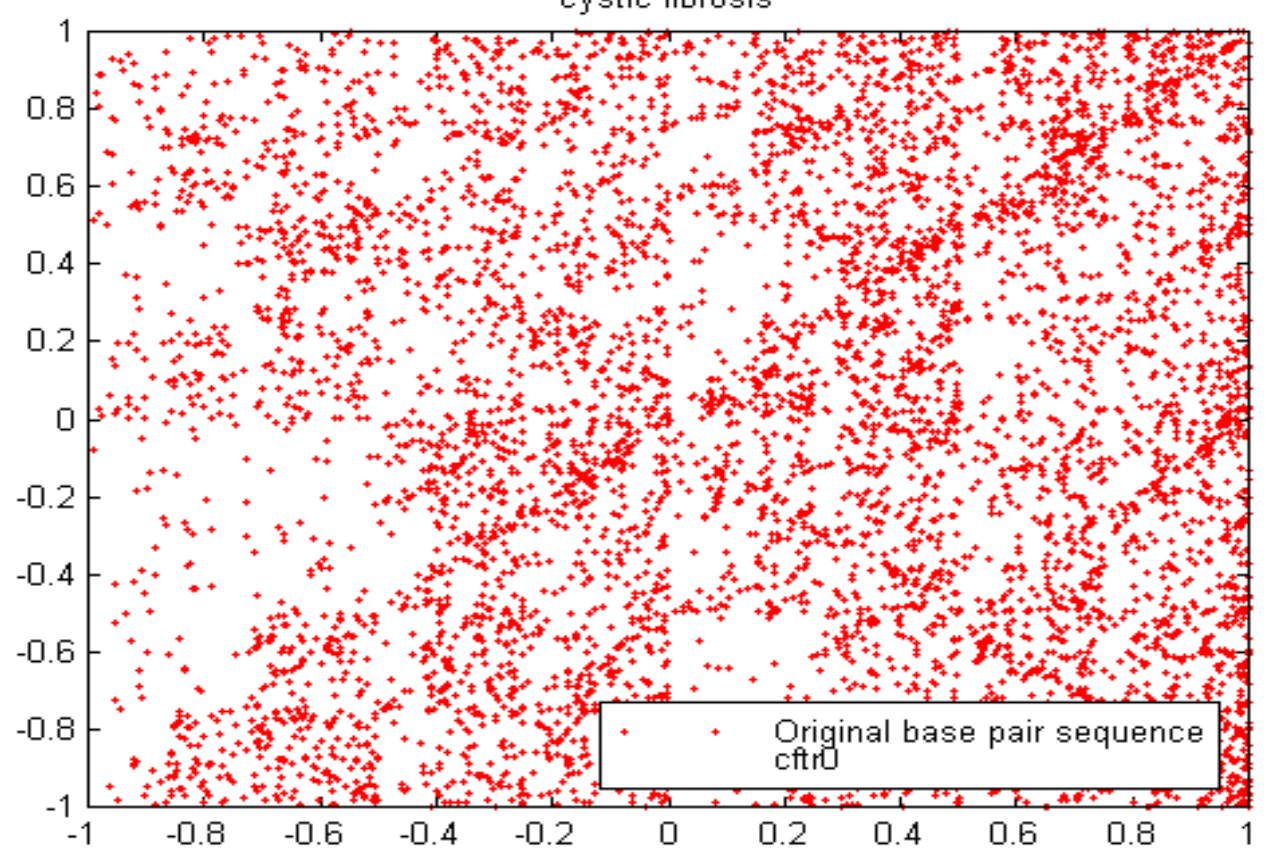

Figure V-80 Original Sequence

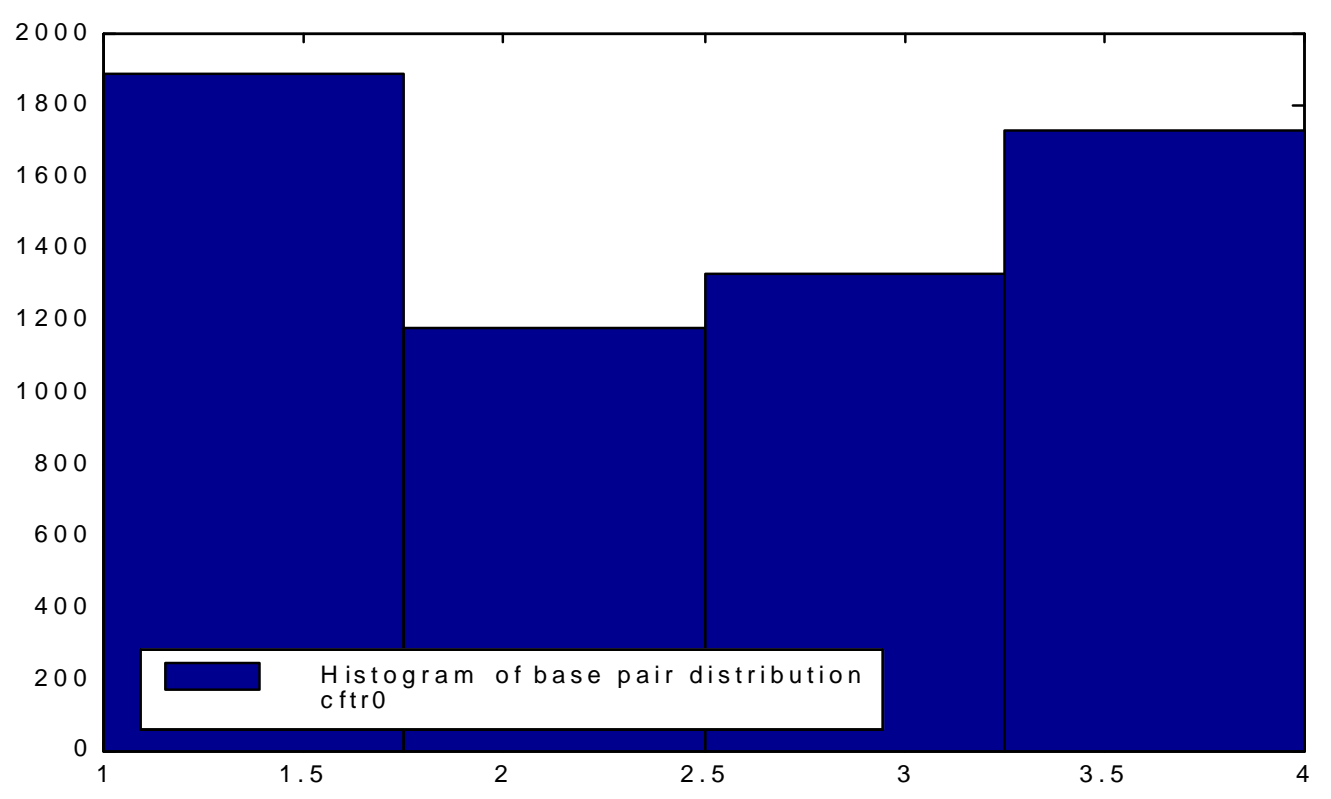

Figure V-81 Base Pair Distribution 
cystic fibrosis

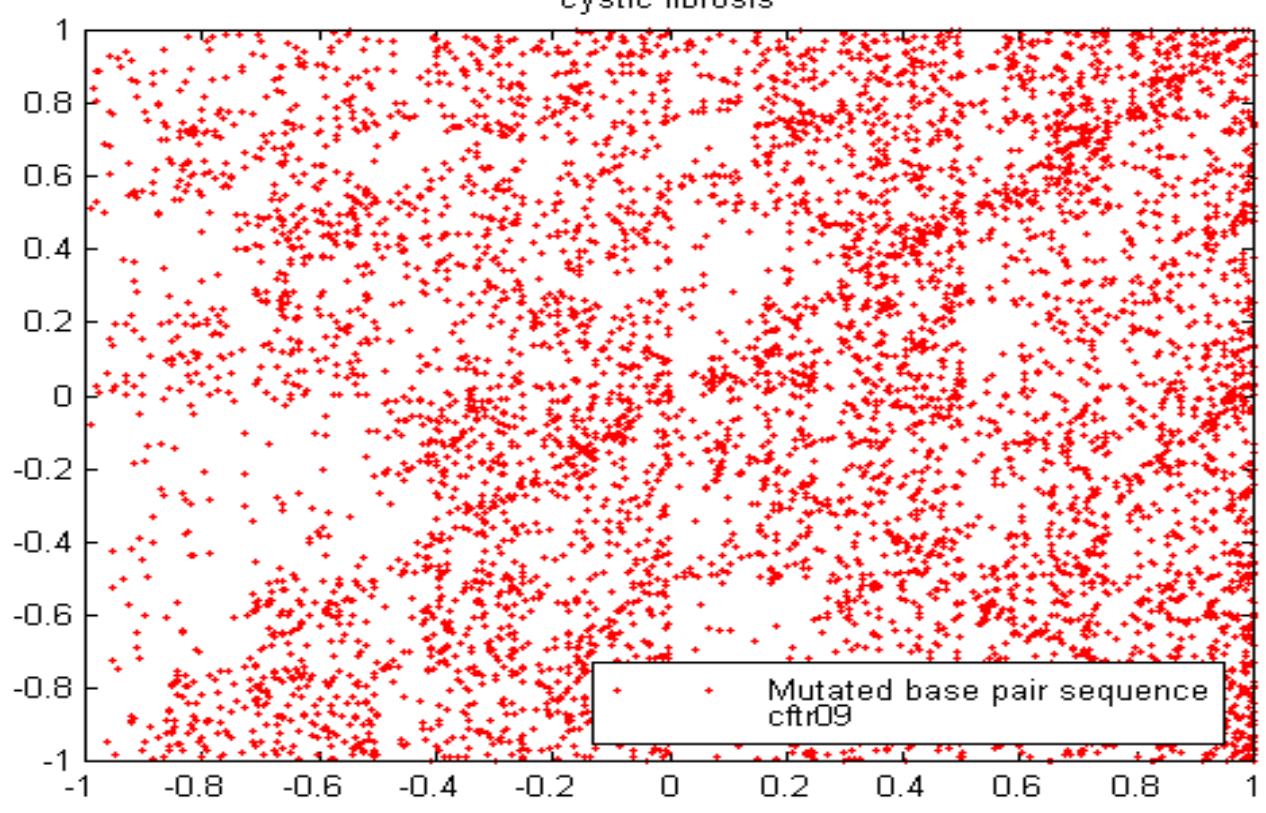

Figure V-82 Artificially Mutated Sequence 1000 -> 1009 Order Reversed

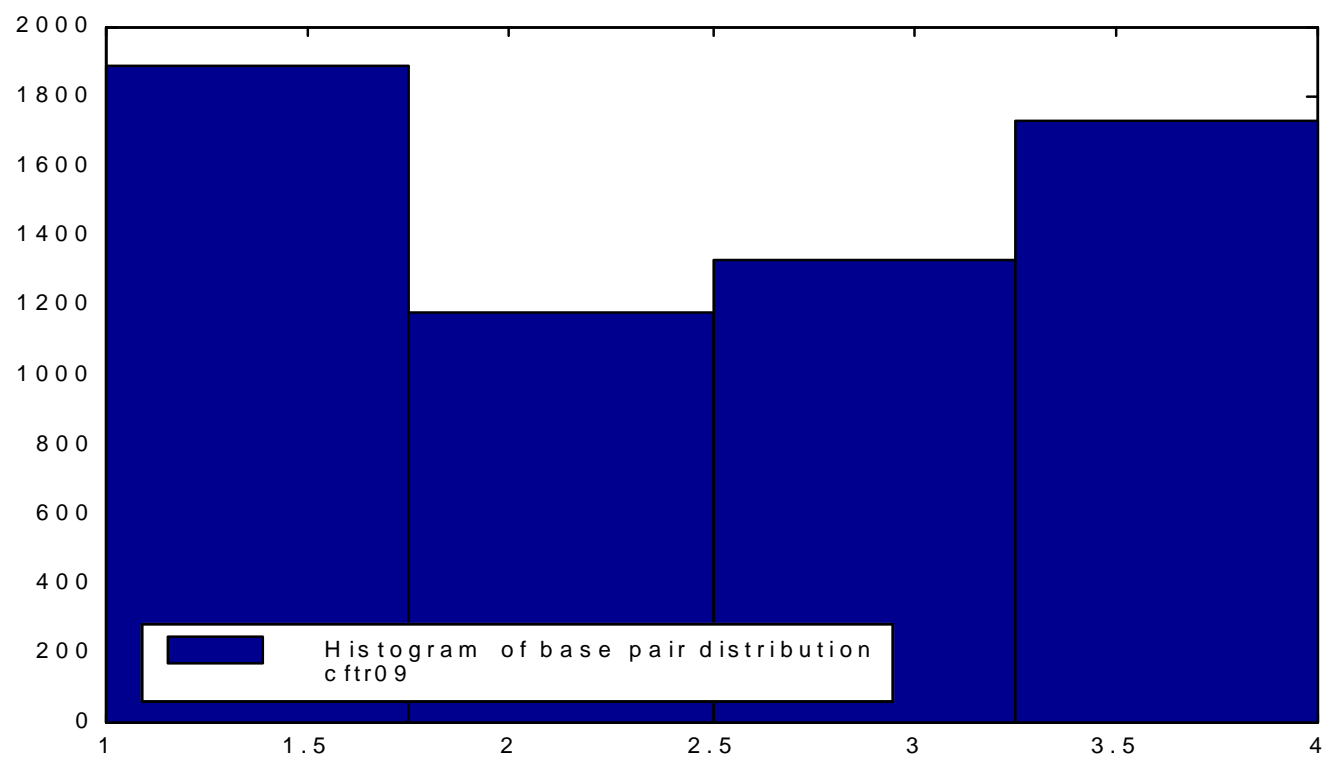

Figure V-83 Base Pair Distribution 


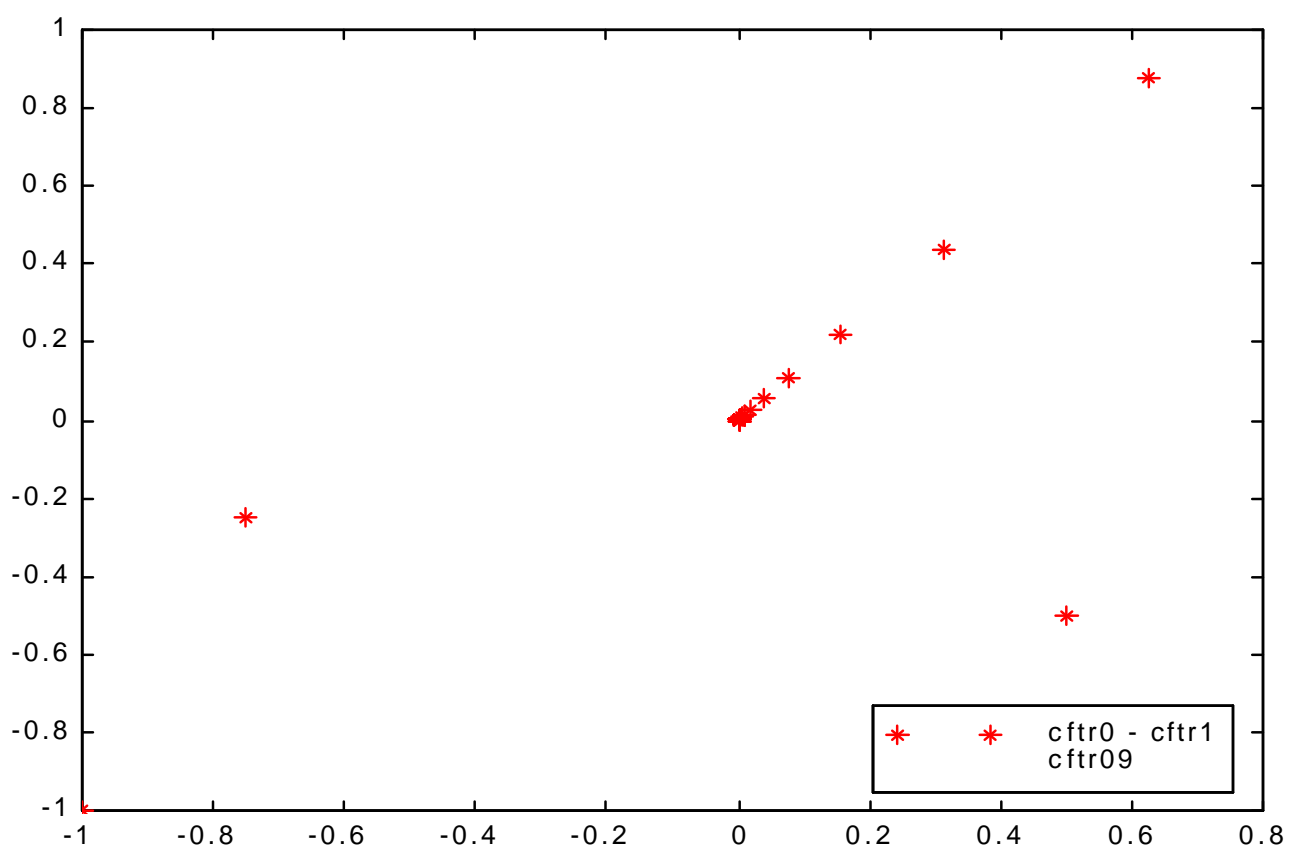

Figure V-84 Difference In IFS Representations I.E. "Cftr0 - Cftr09"

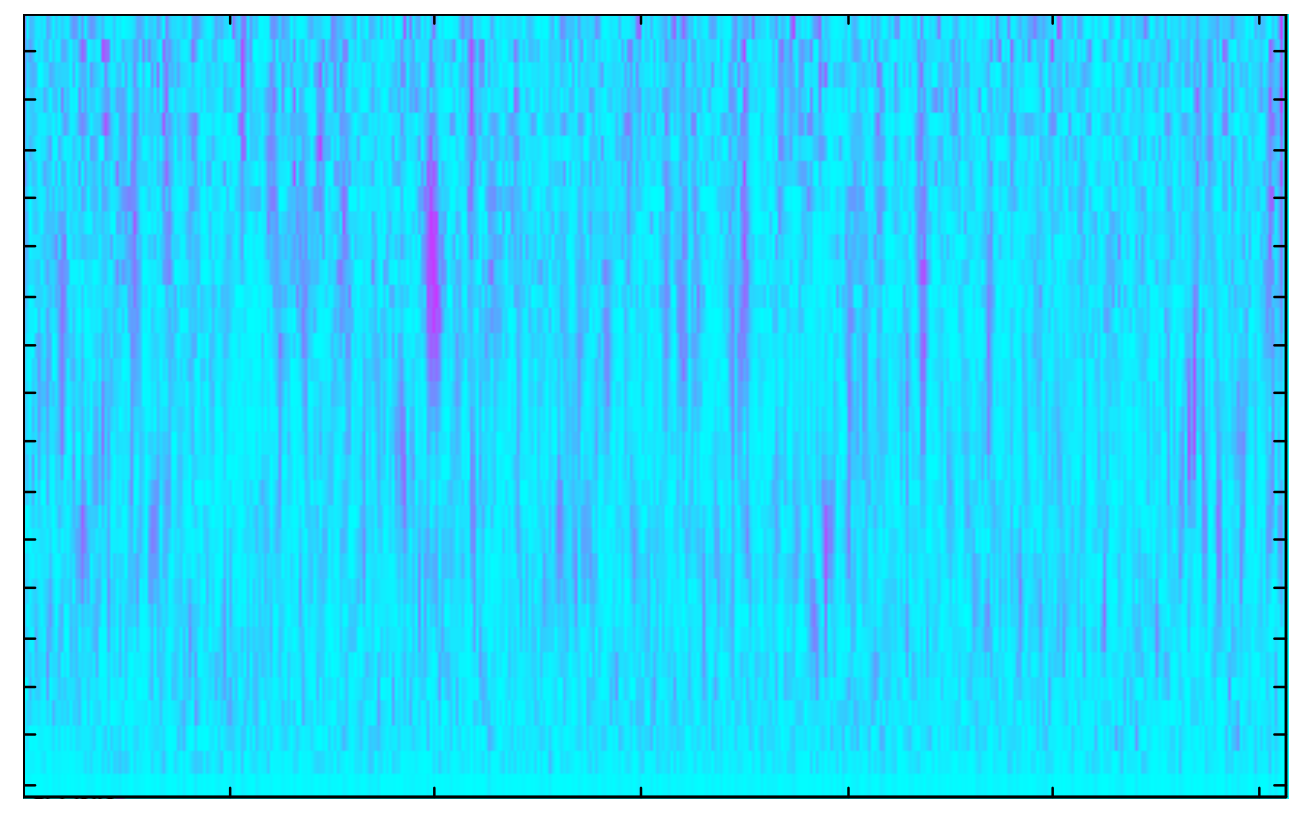

Figure V-85 Continuous Wavelet Transform Of Original Sequence 


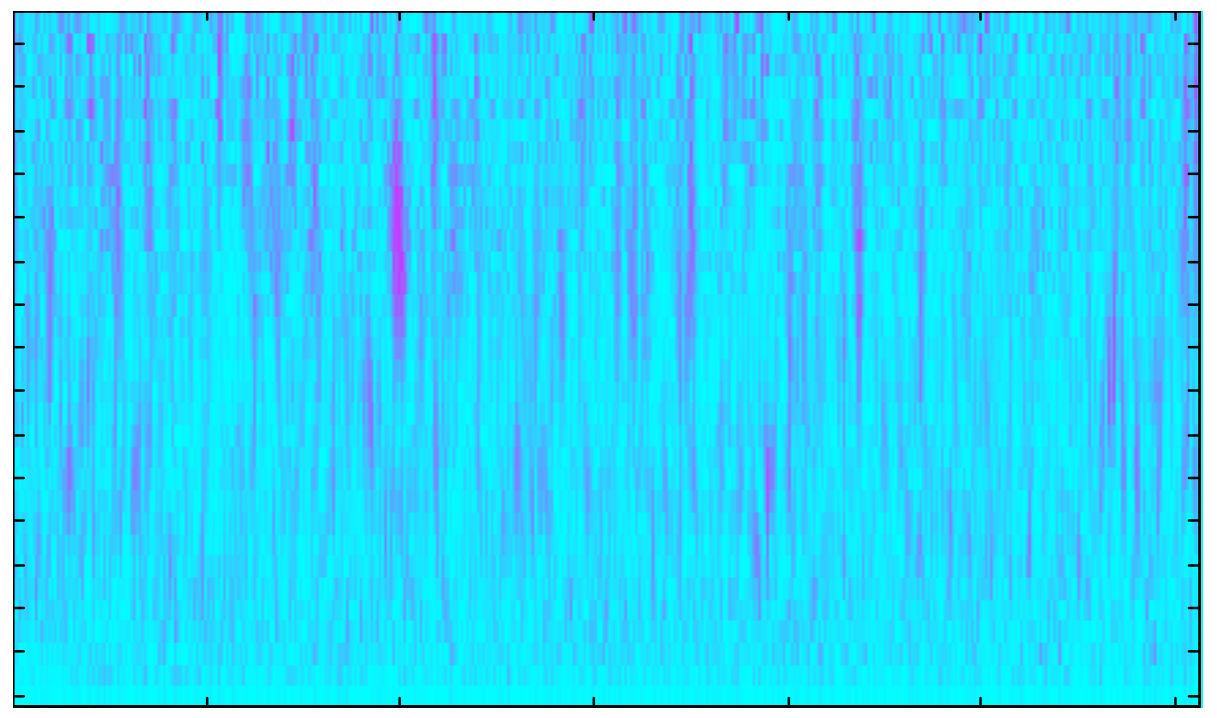

Figure V-86 Continuous Wavelet Transform Of The Mutated Sequence

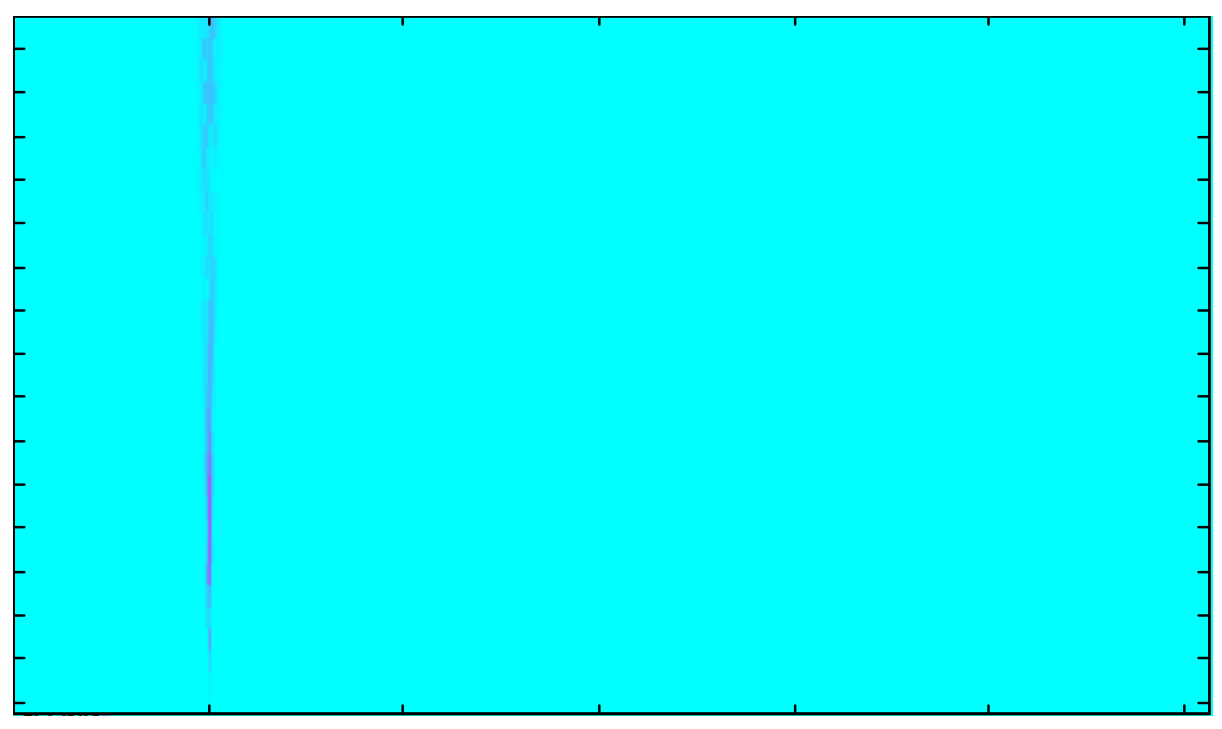

Figure V-87 Difference In The Wavelet Transforms 


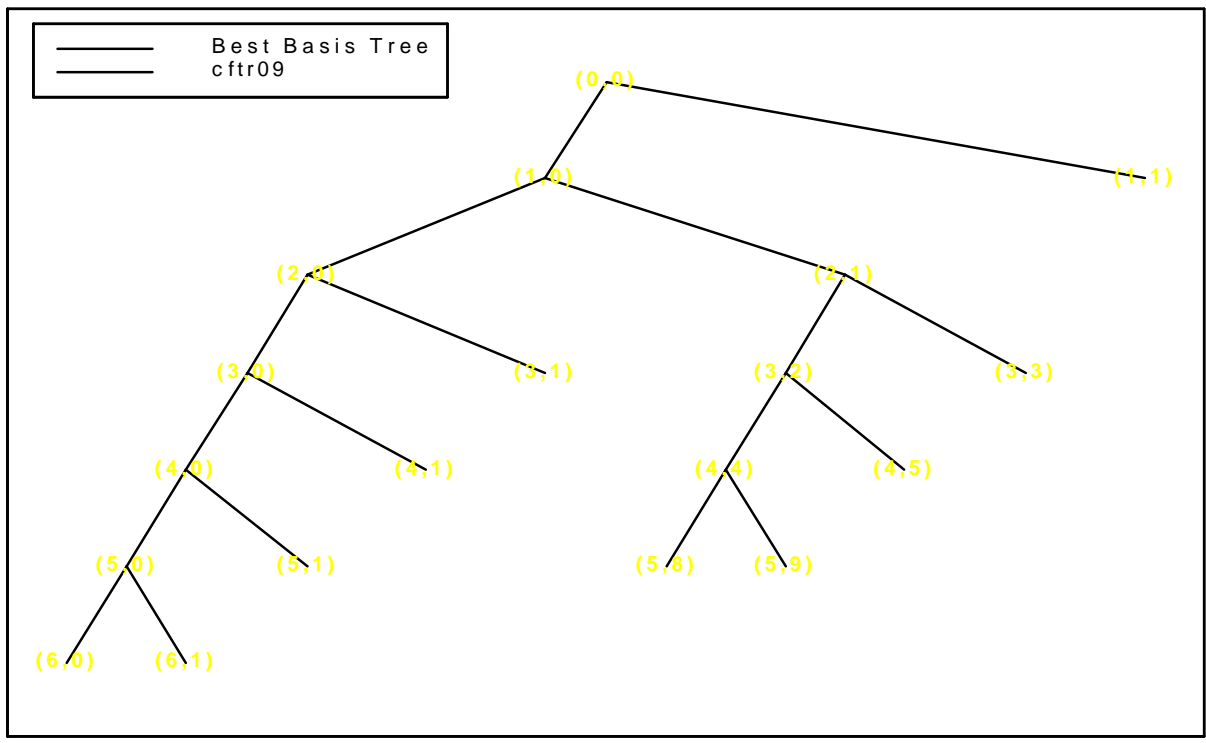

Figure V-88 Best Basis Representation Of The Mutated Sequence

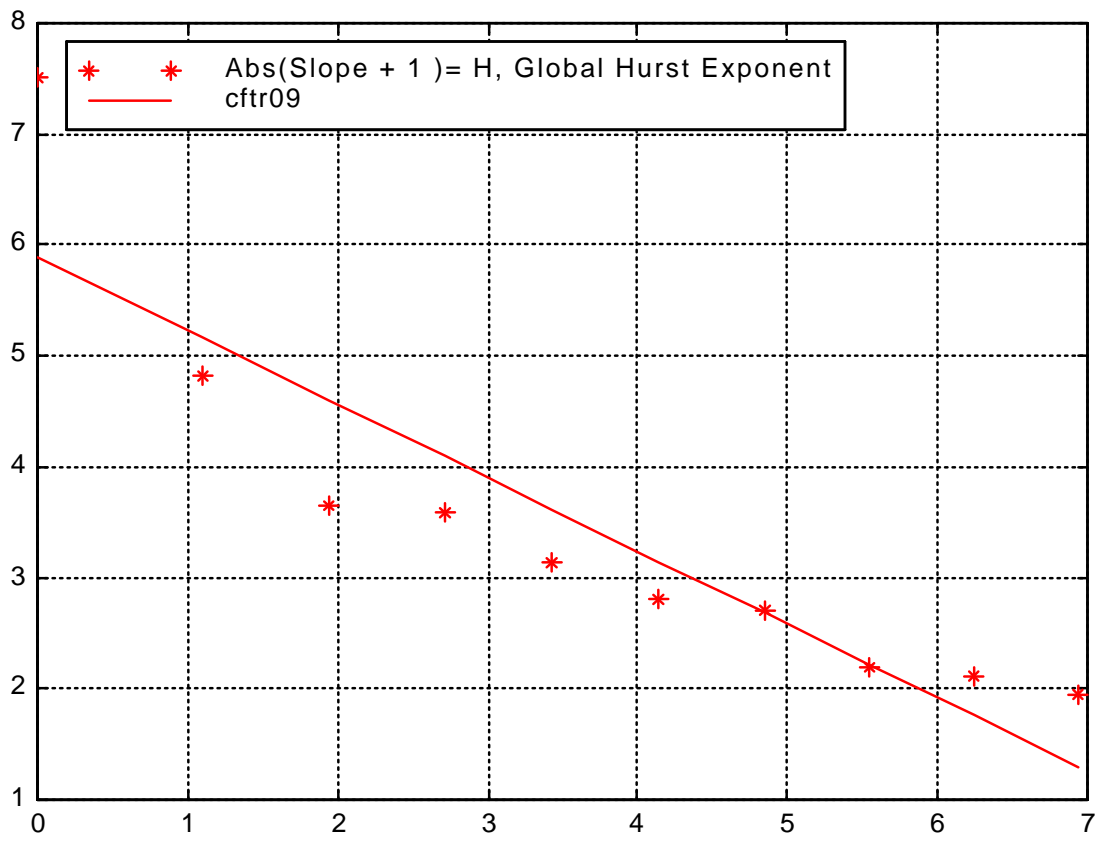

Hglobal $=0.3366$

Figure V-89 Global Hurst Exponent Of The Mutated Sequence 


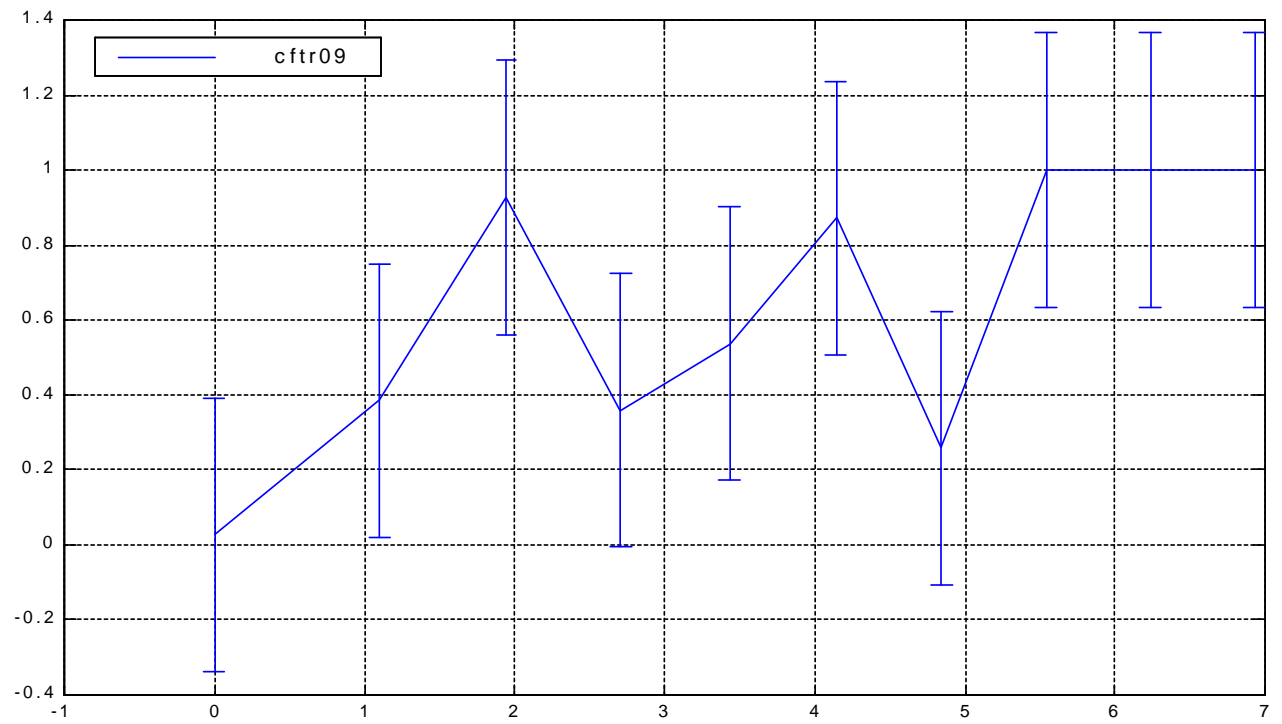

Figure V-90 Local Hurst Exponent Of The Mutated Sequence

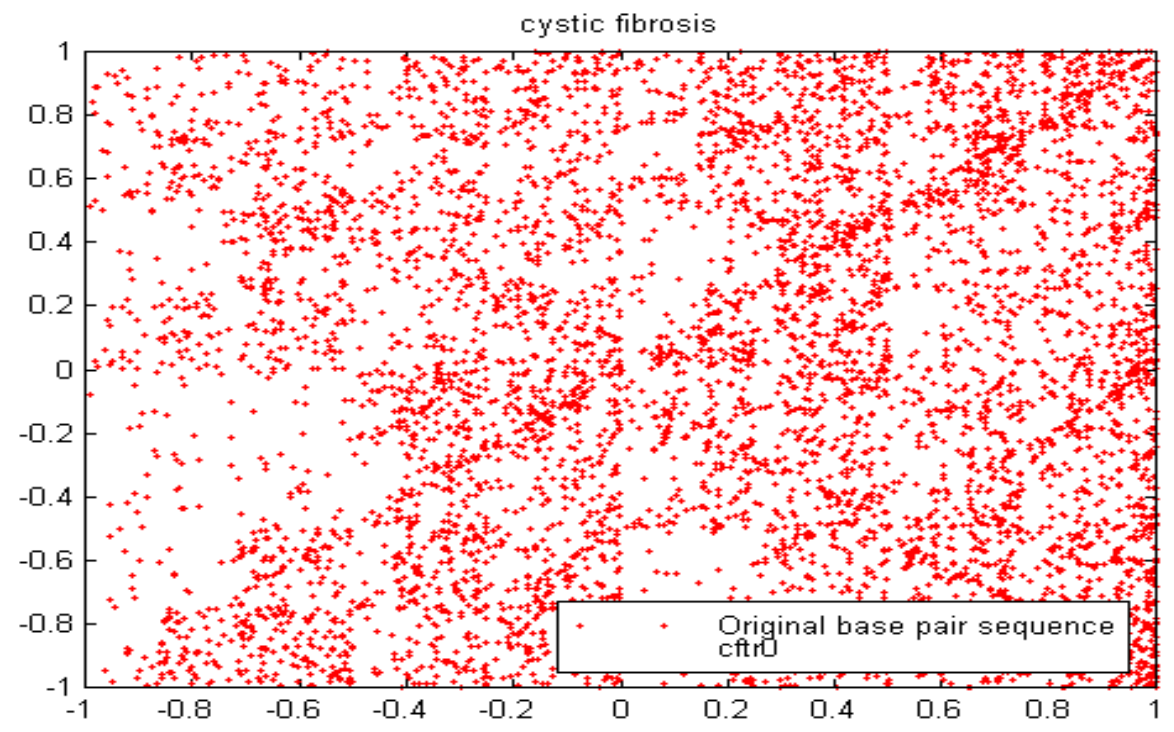

Figure V-91 Original Sequence 


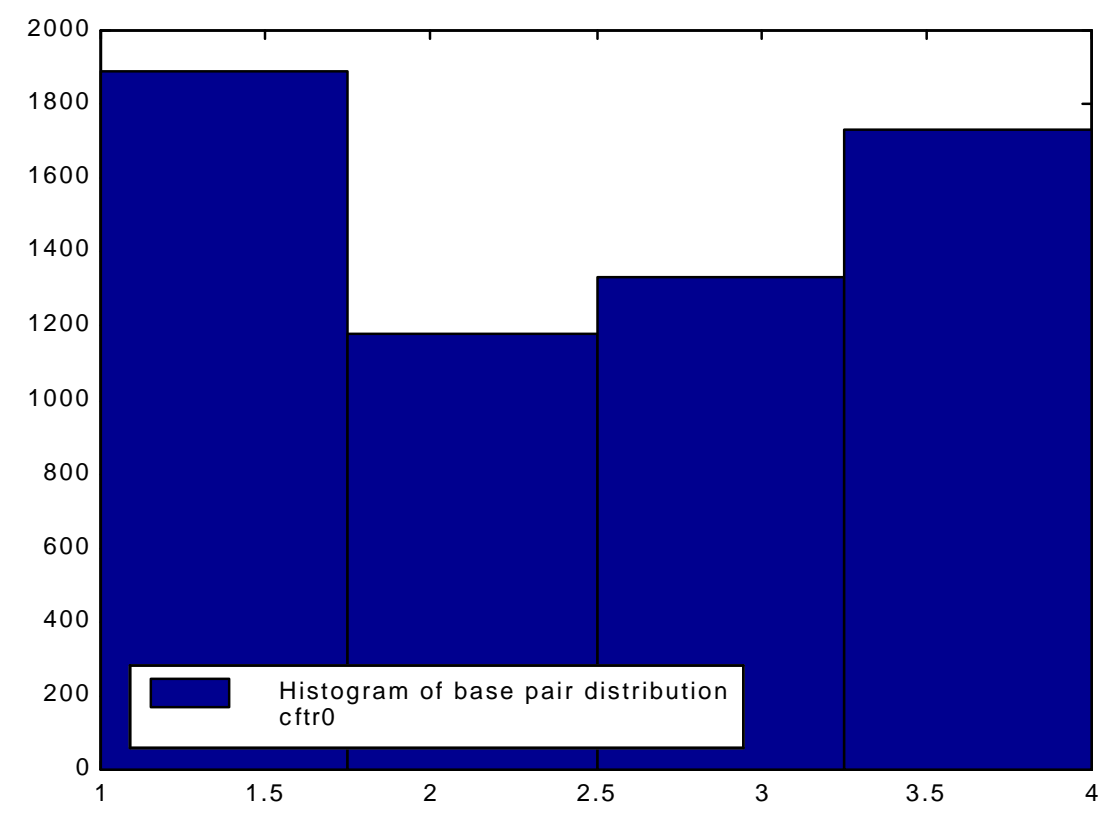

Figure V-92 Base Pair Distribution

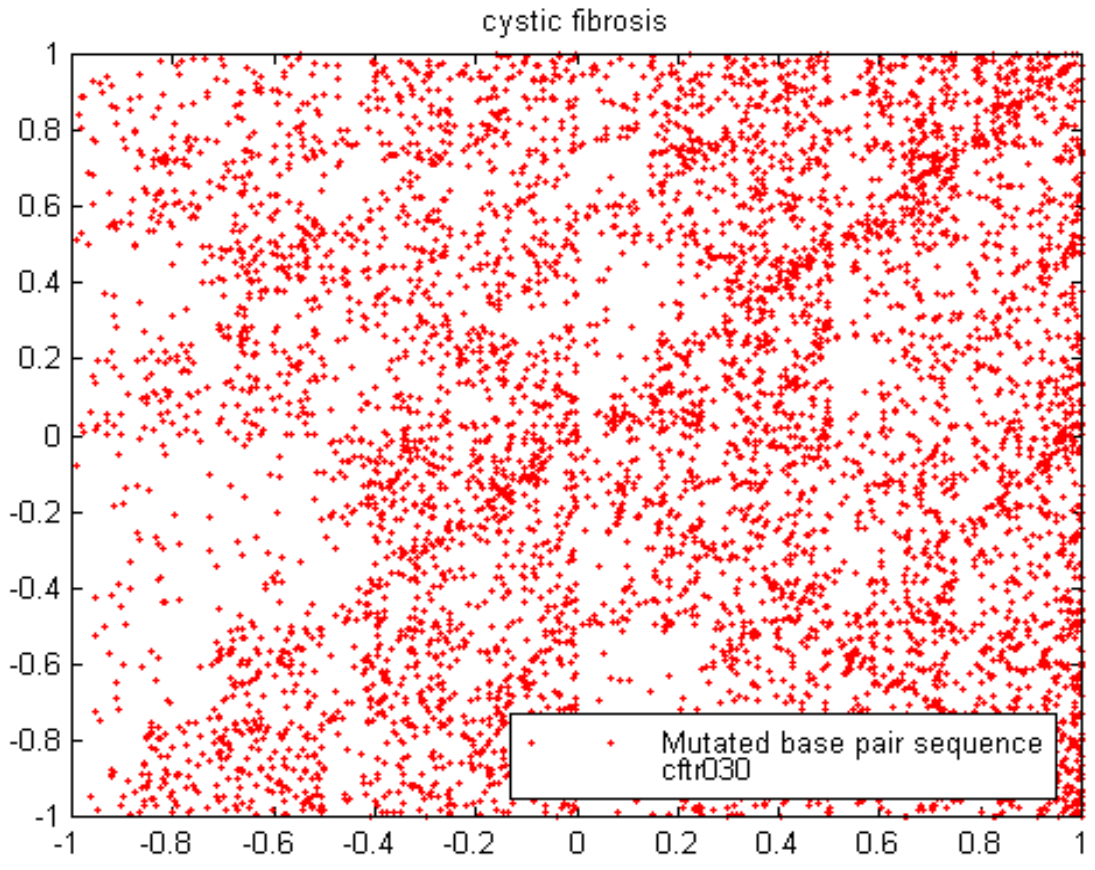

Figure V-93 Artificially Mutated Sequence 1000 -> 1030 Order Reversed 


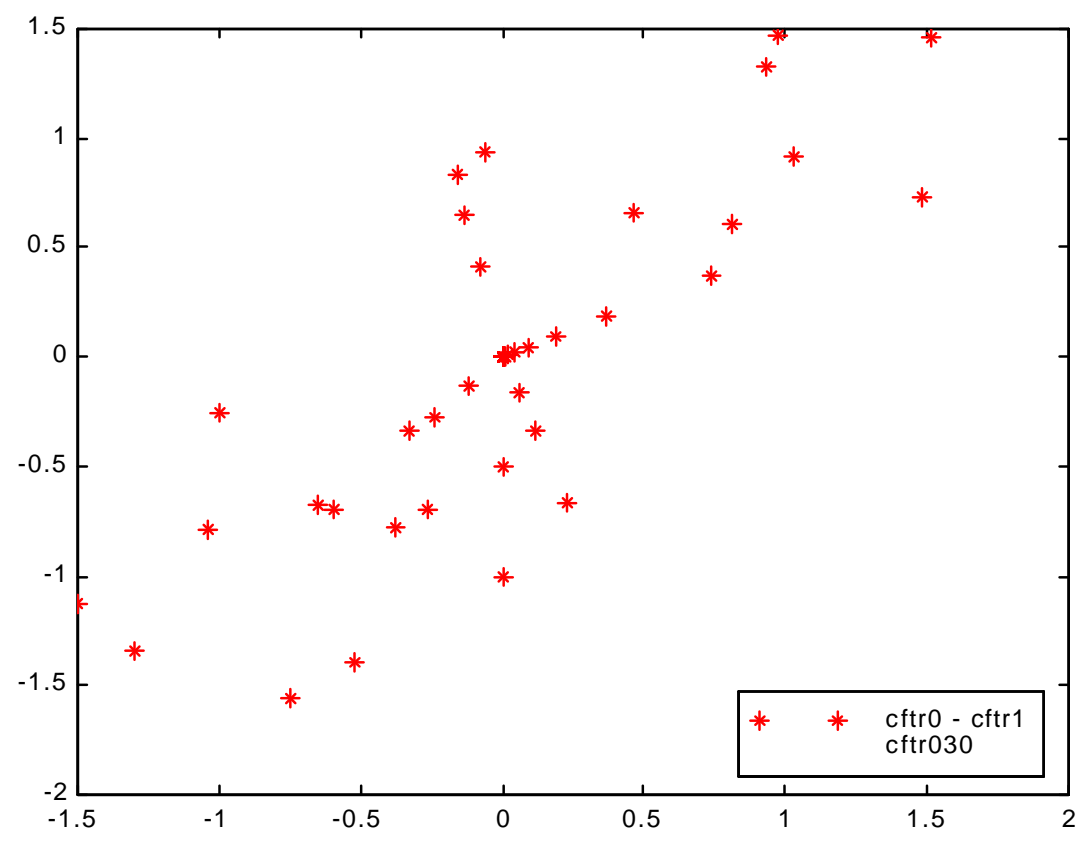

Figure V-94 Difference In IFS

Representation

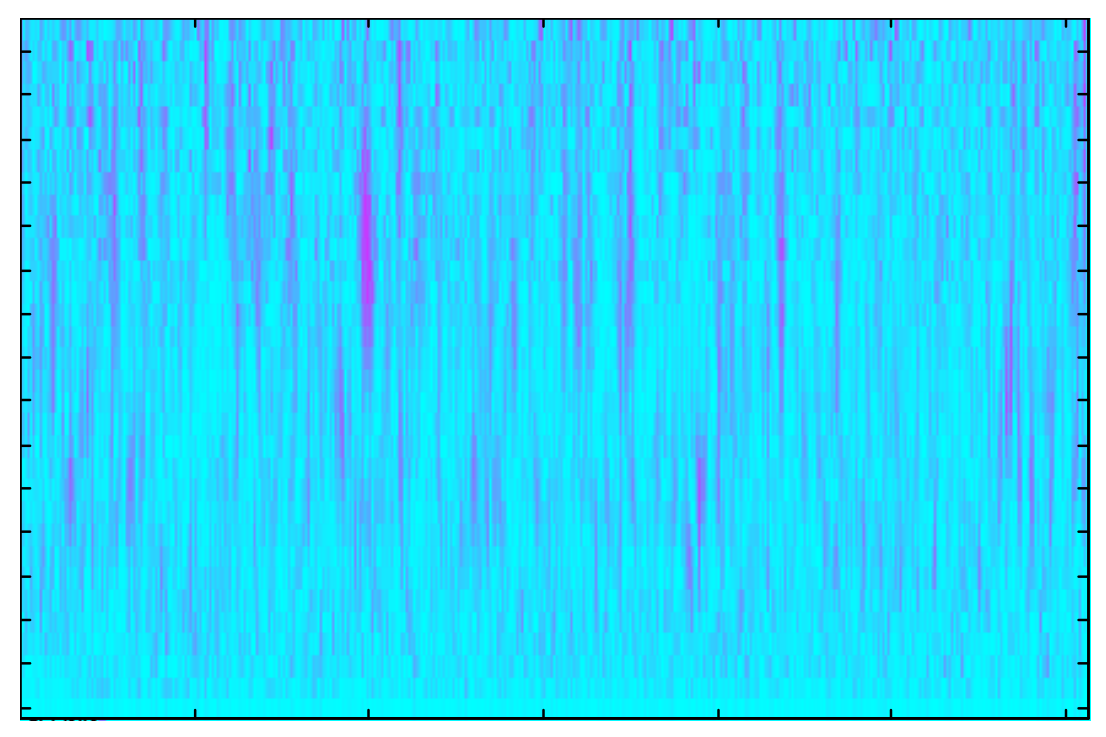

Figure V-95 Continuous Wavelet Transform Of Original Sequence 


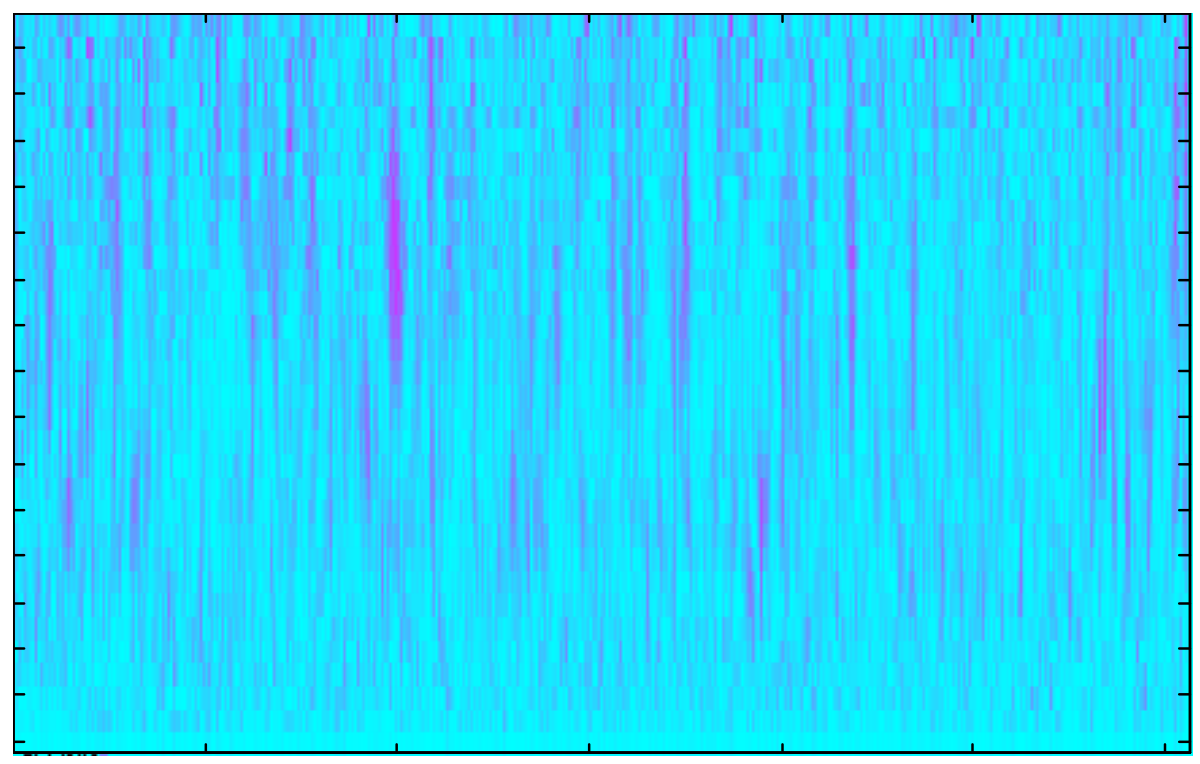

Figure V-96 Continuous Wavelet Transform Of Mutated Sequence $1000->1030$ Order Reversed

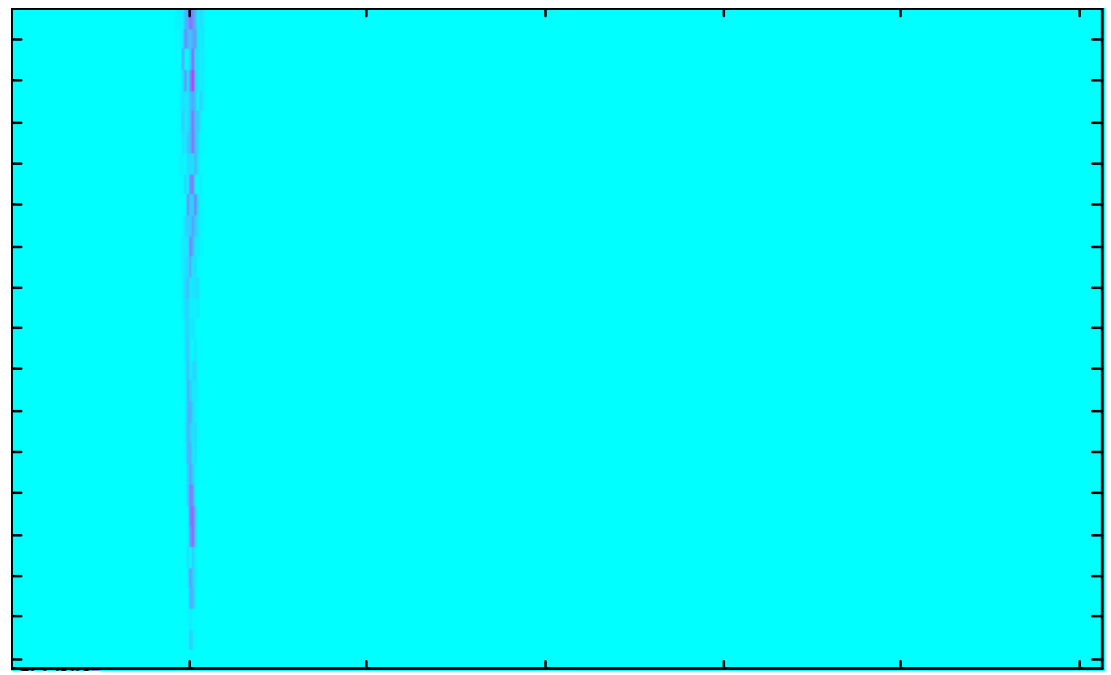

Figure V-97 Difference In The Continuous Wavelet Transforms Of The Two Sequences 


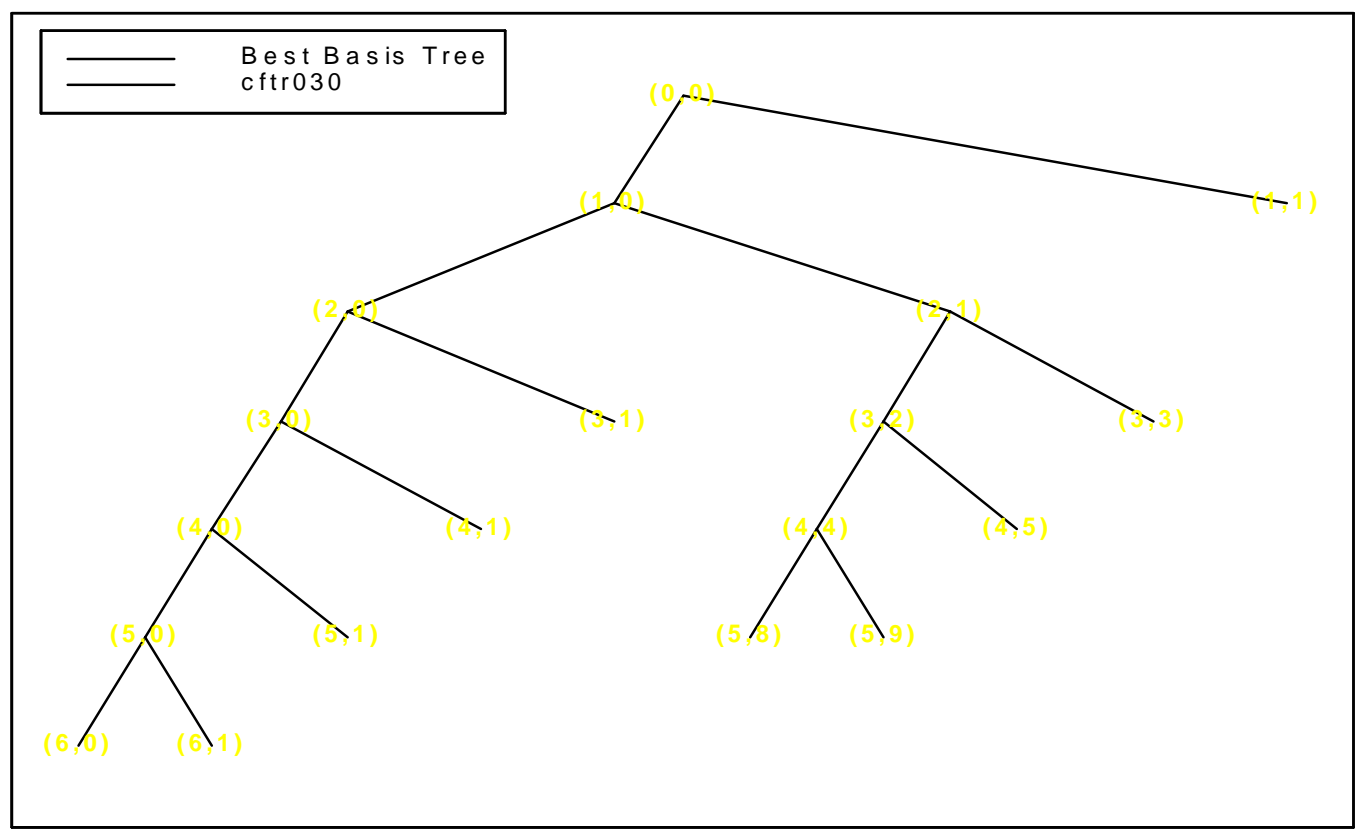

Figure V-98 Best Basis Representation Of The Mutated Sequence

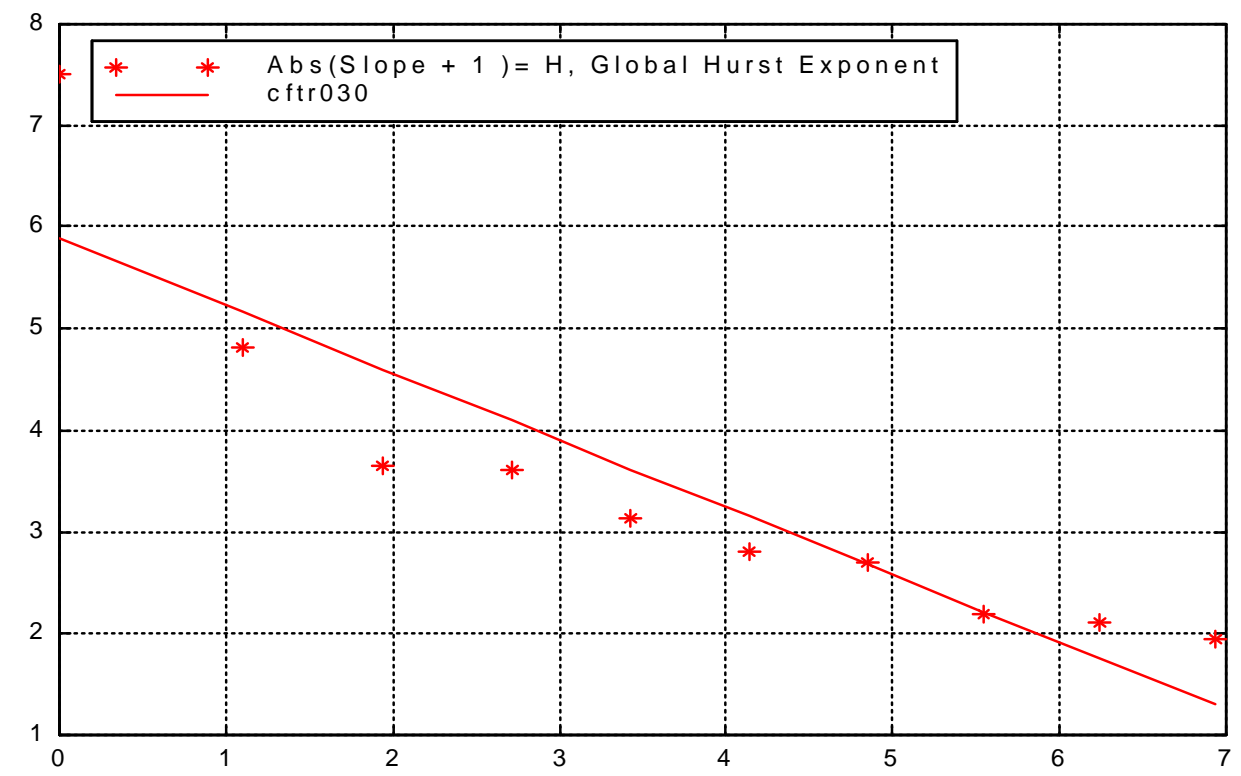

Figure V-99 Global Hurst Exponent Of The Mutated Sequence 


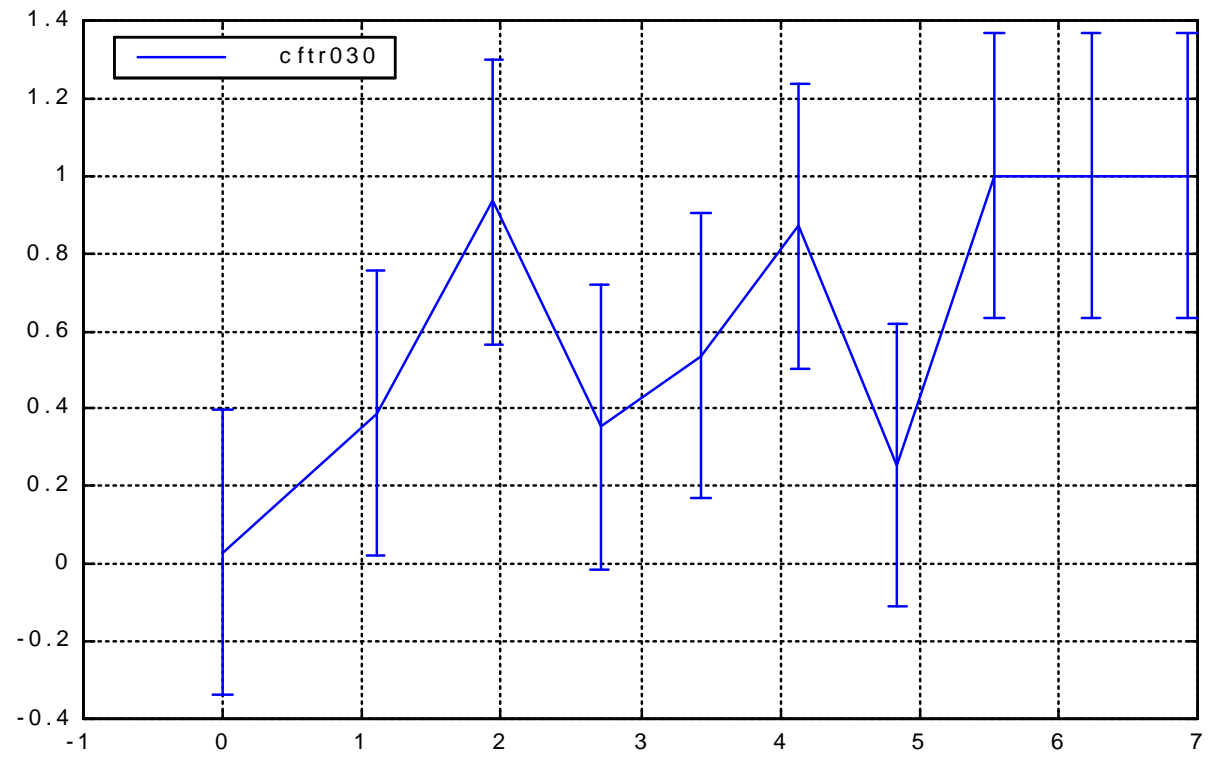

Figure V-100 Local Hurst Exponent Of The Mutated Sequence $1000>1030$

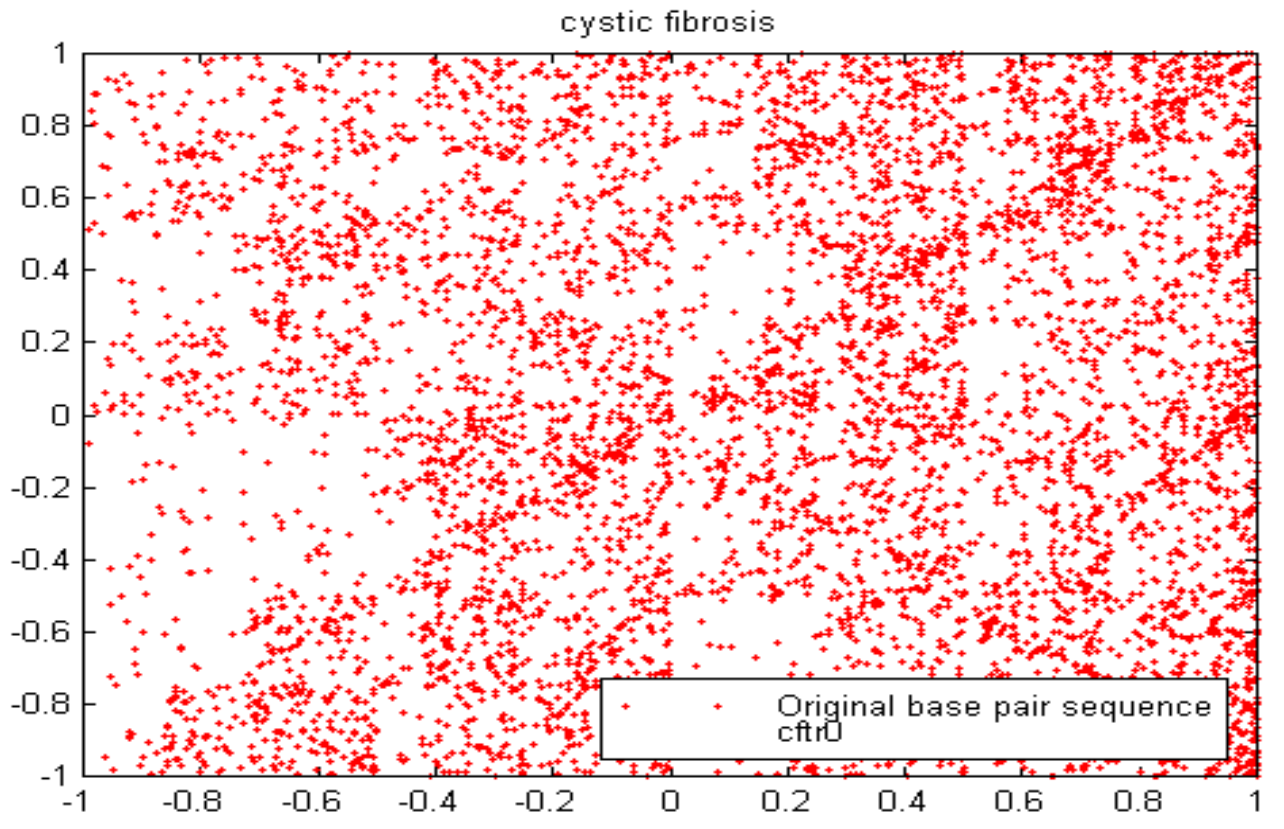

Figure V-101 Original Sequence 


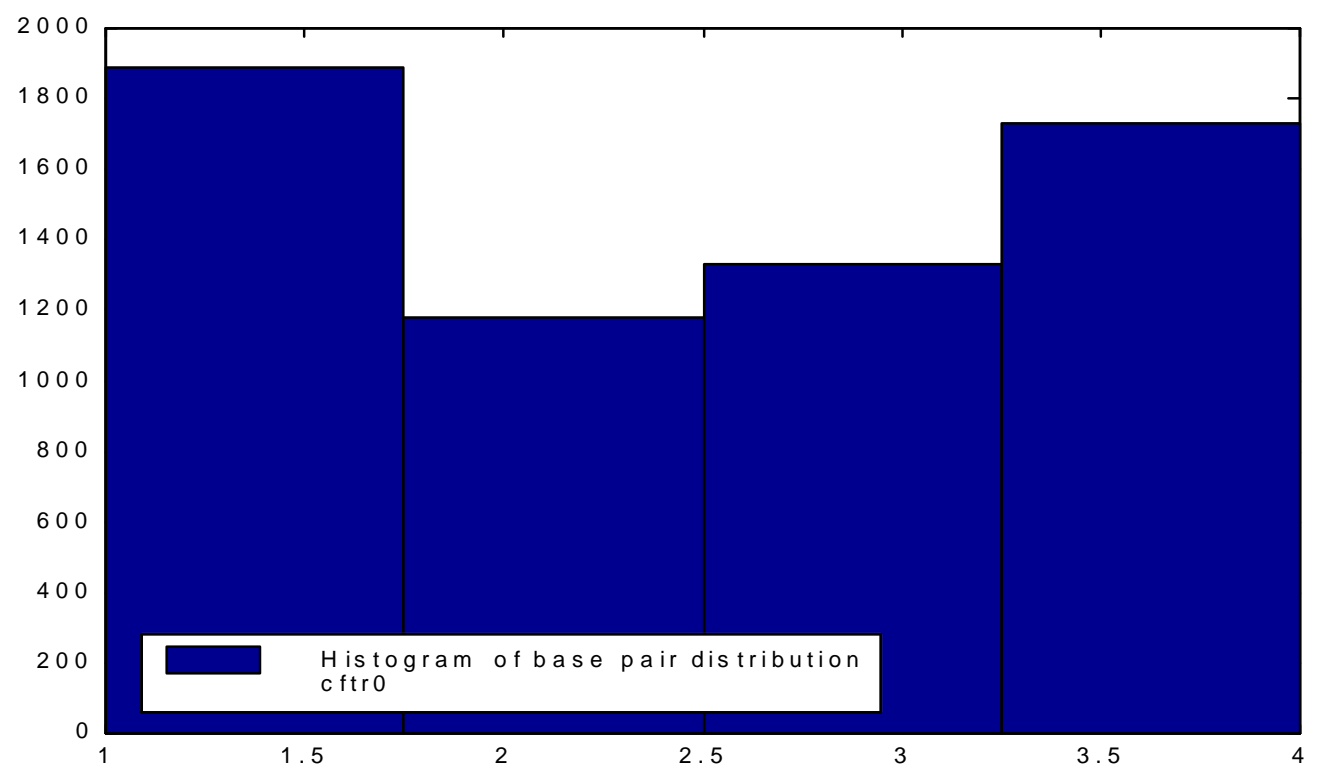

Figure V-102 Base Pair Distribution

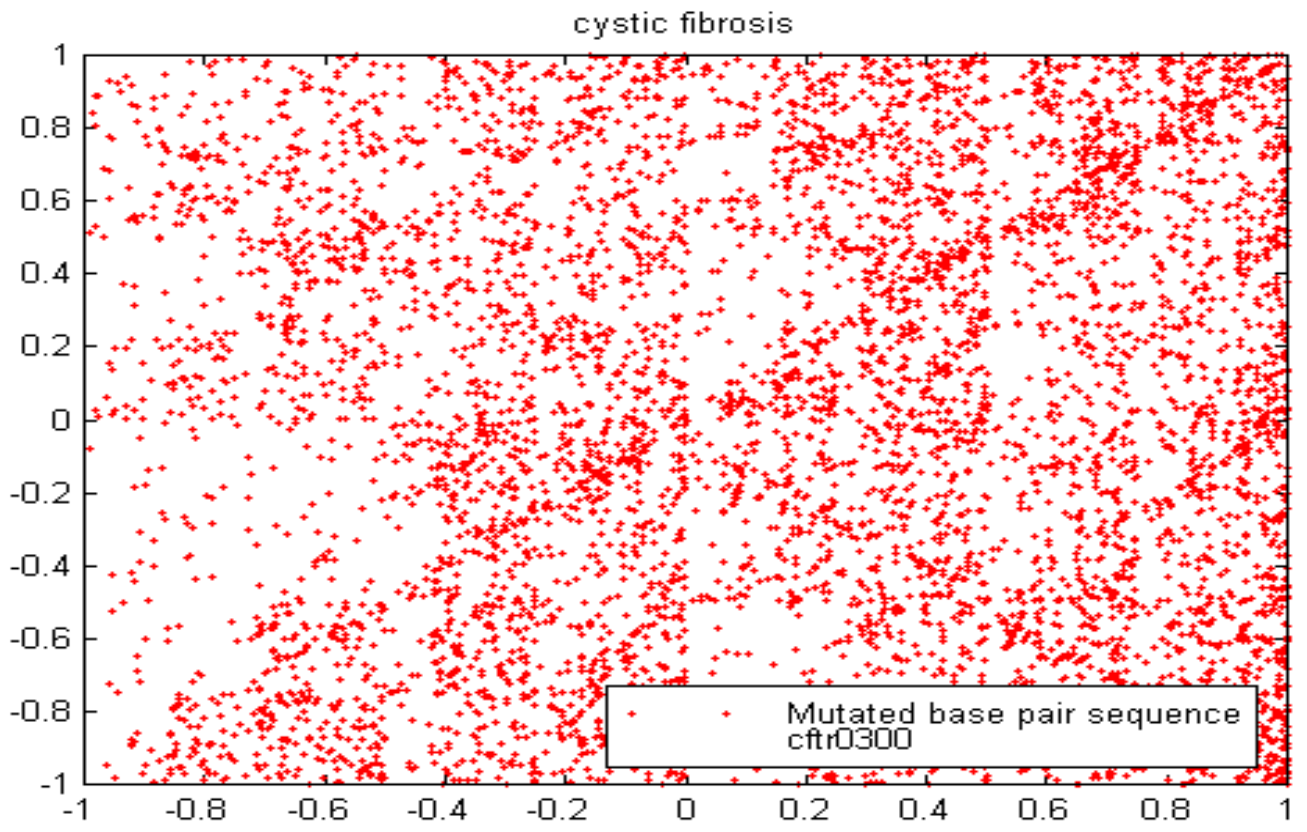

Figure V-103 Mutated Sequence $1000->1300$ 


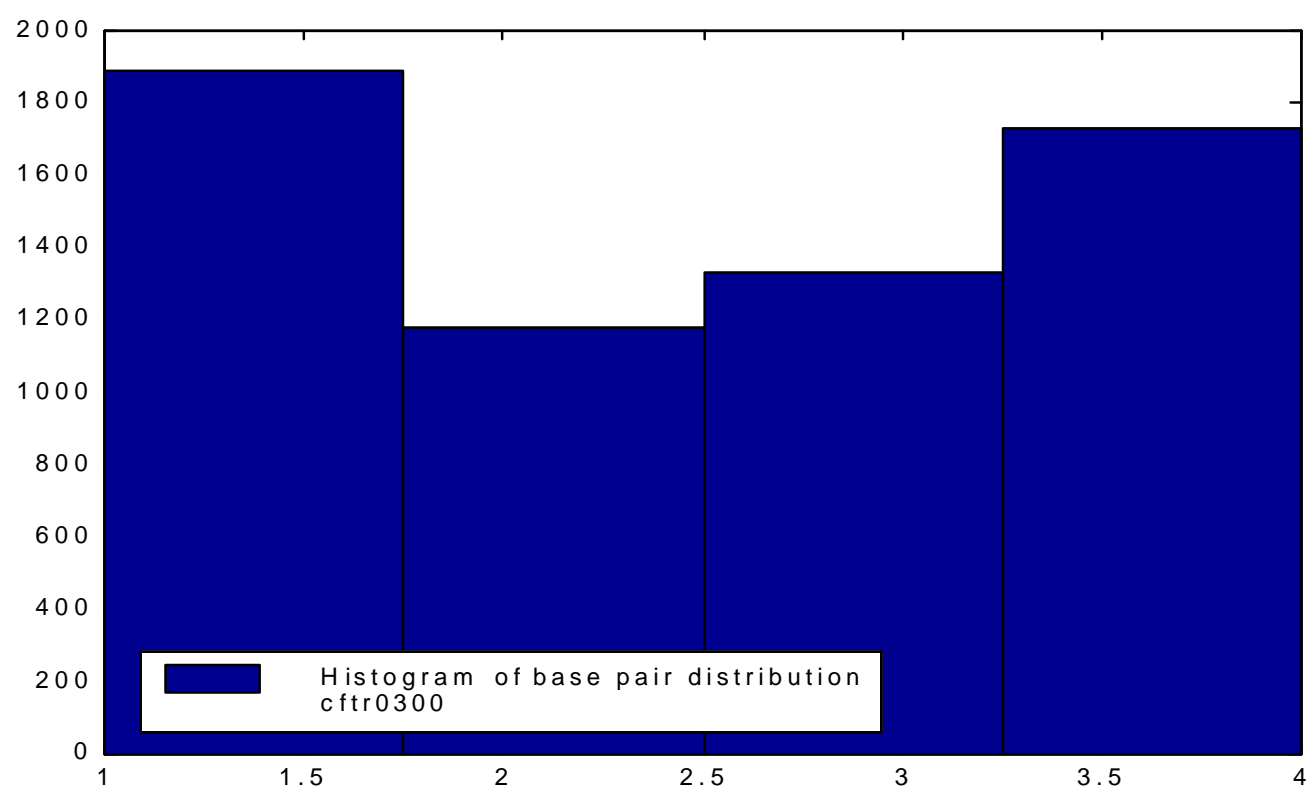

Figure V-104 Base Pair Distribution 1000 ->1300

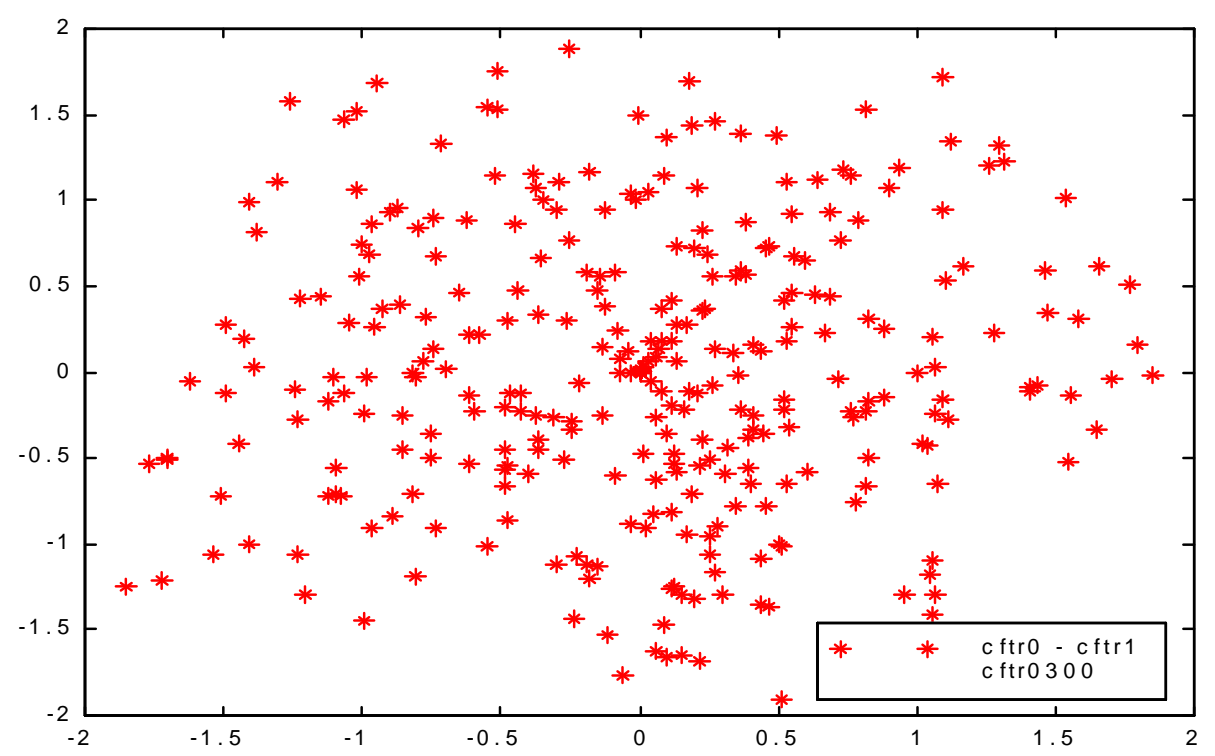

Figure V-105 Difference In The IFS Representations Between Original And Mutated Sequence $1000->1300$ Order Reversed 


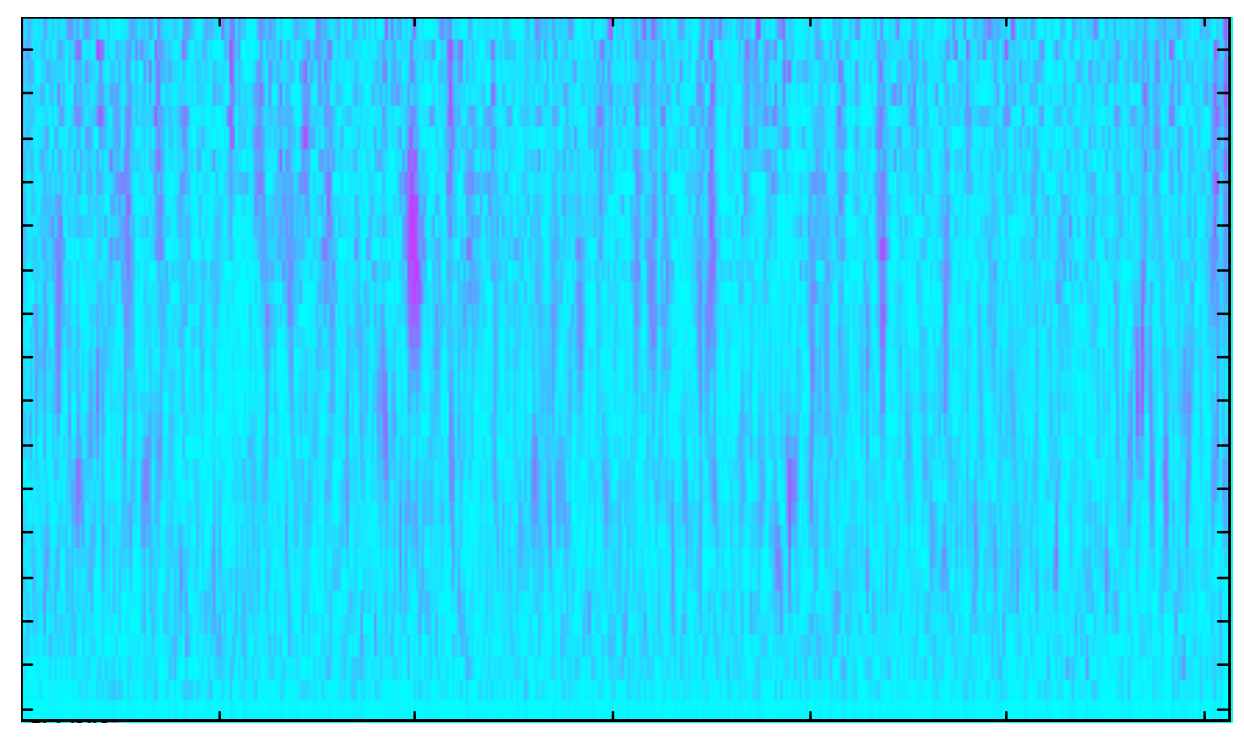

Figure V-106 Continuous Wavelet Transform Of The Original Sequence

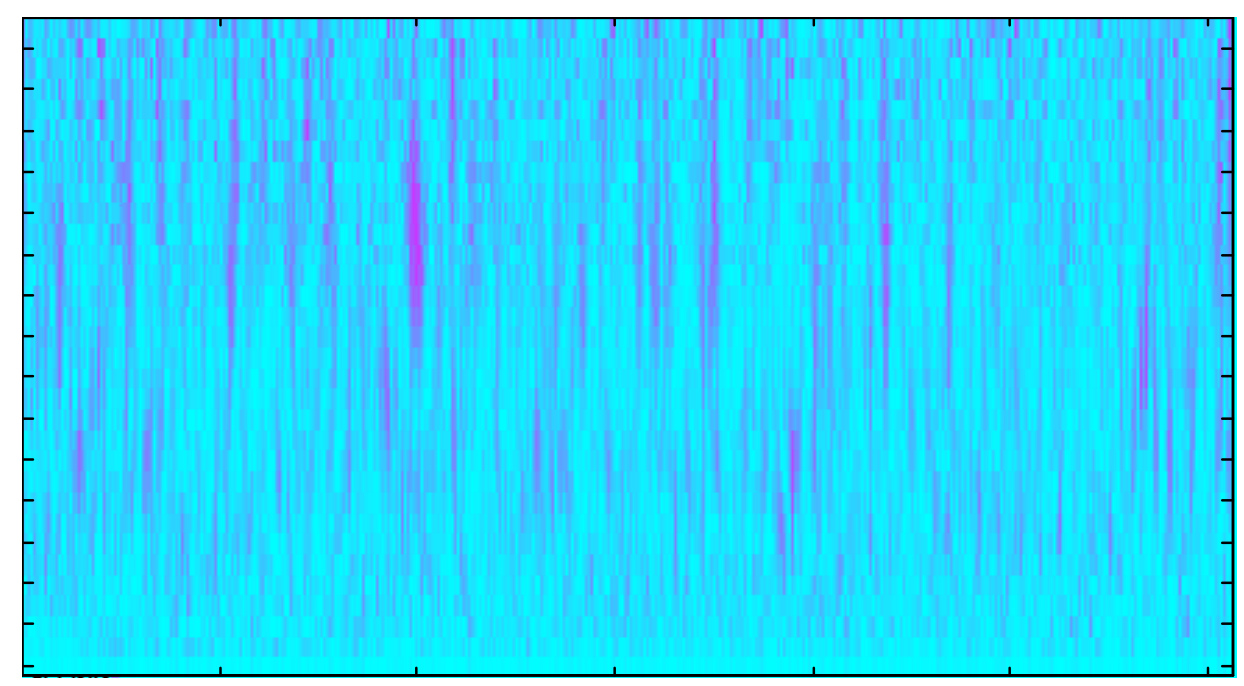

Figure V-107 Continuous Wavelet Transform Of The Mutated Sequence 1000 -> 1300 Order Reversed 


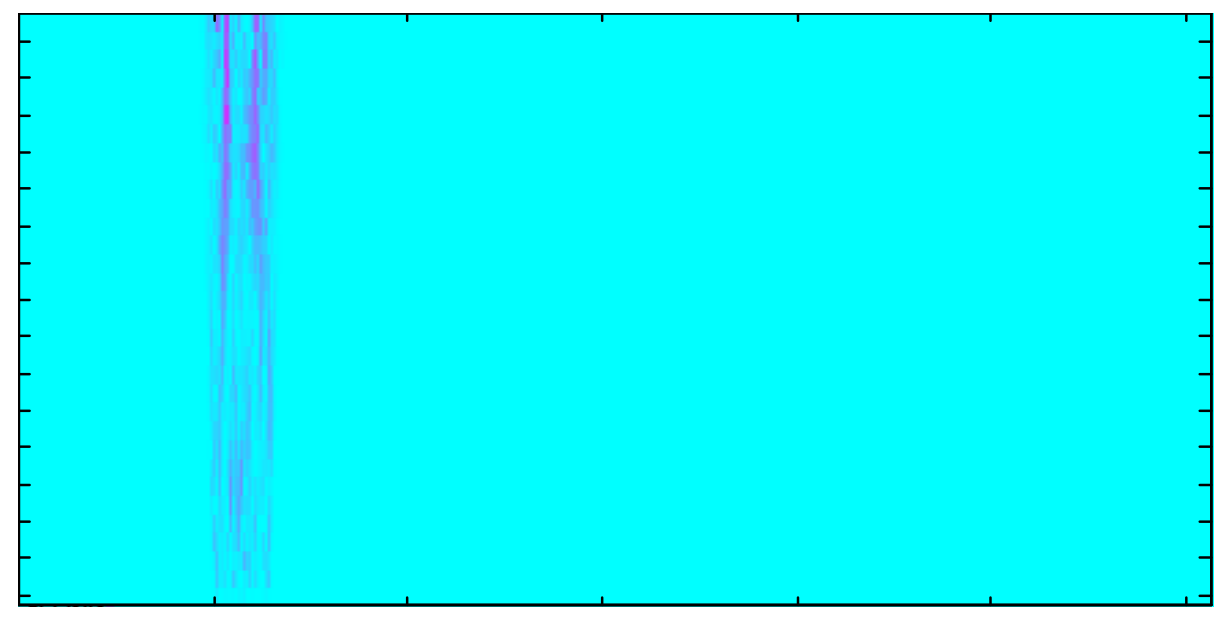

Figure V-108 Difference In The Continuous Wavelet

Transform Of The Mutated Sequence And The Original Sequence

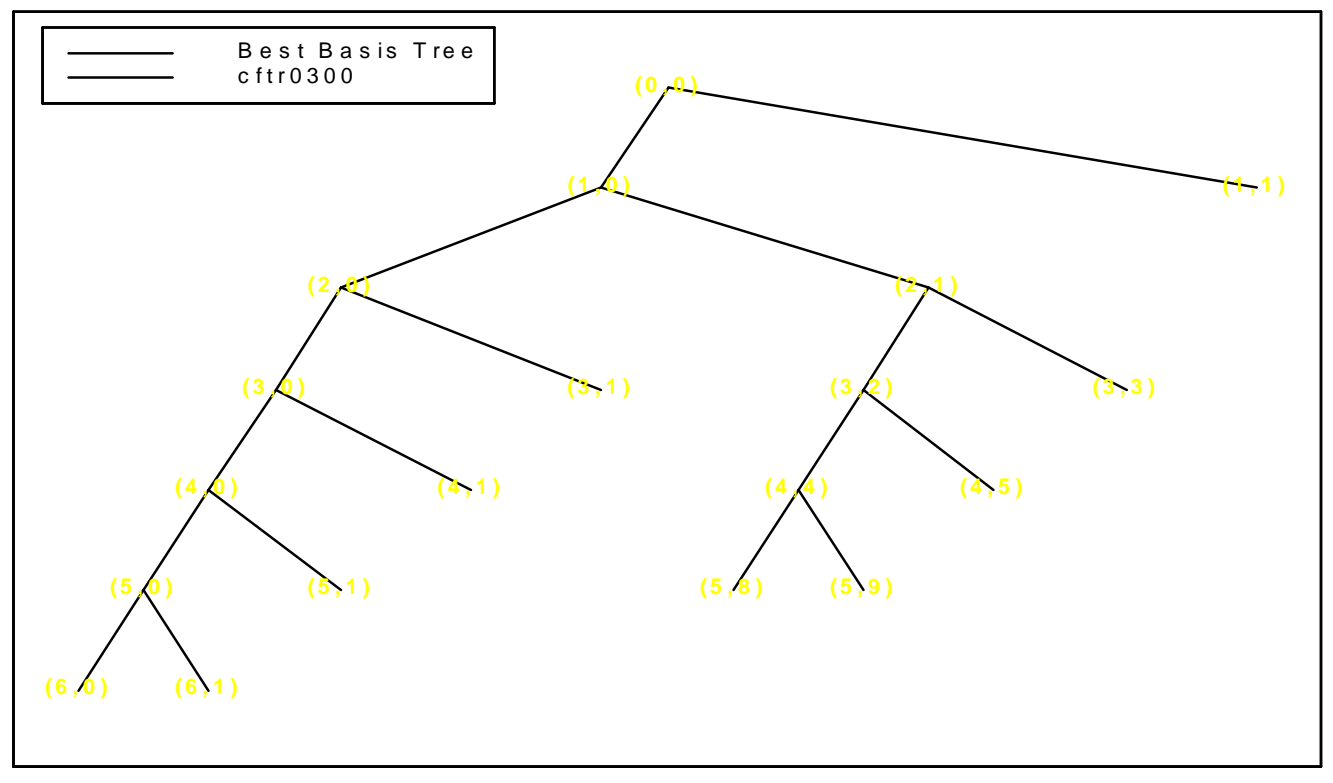

Figure V-109 Best Basis Representation 


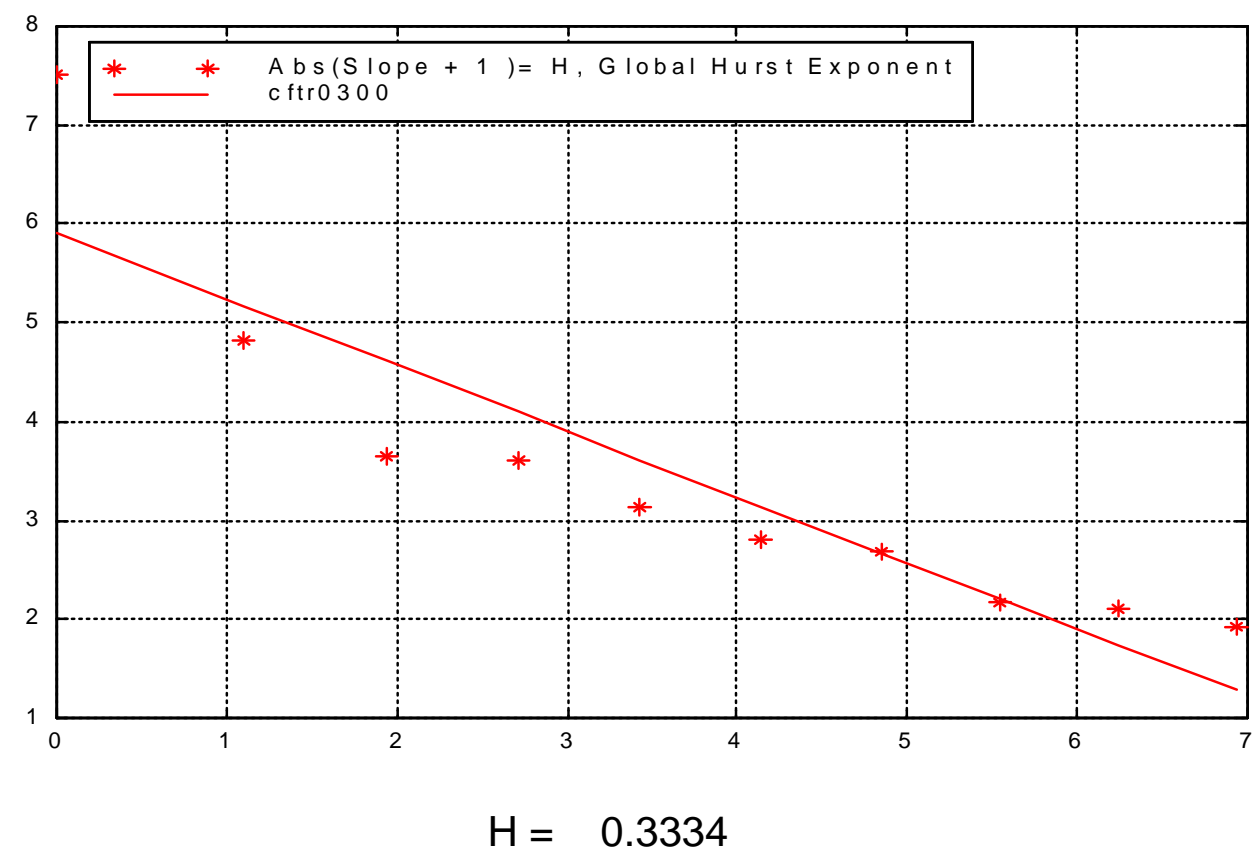

Figure V-110 Global Hurst Of The Mutated Sequence Exponent Of The Mutated Sequence

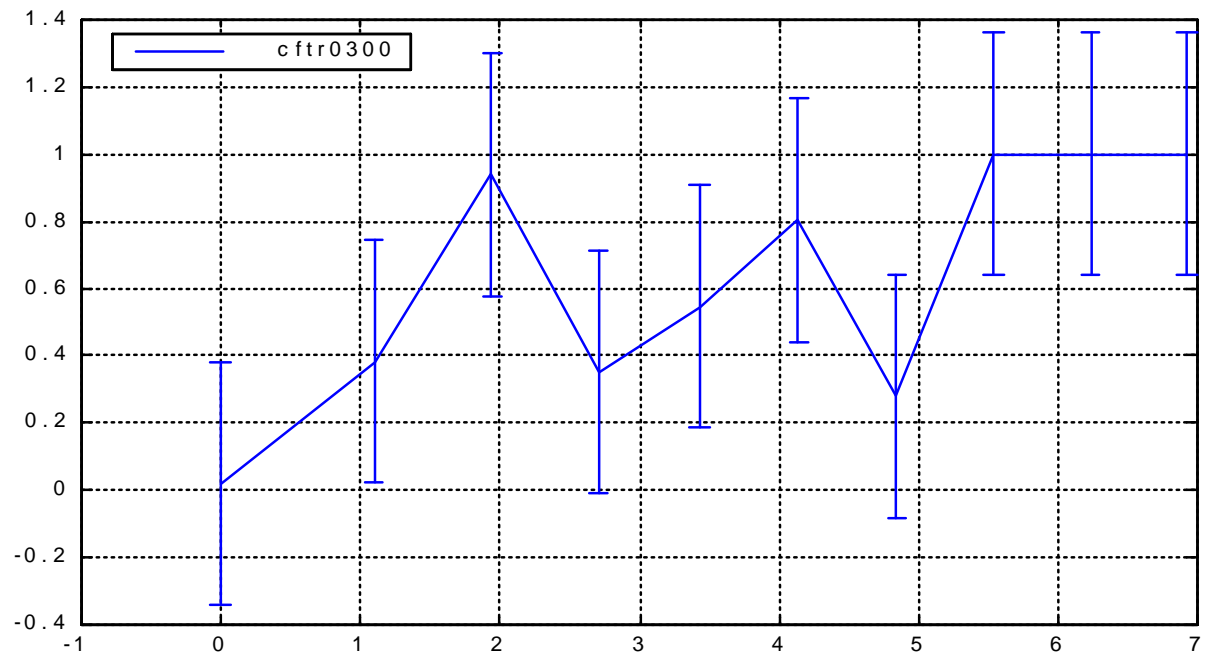

Figure V-111 Local Hurst Exponent Of The Mutated Sequence 
cystic fibrosis

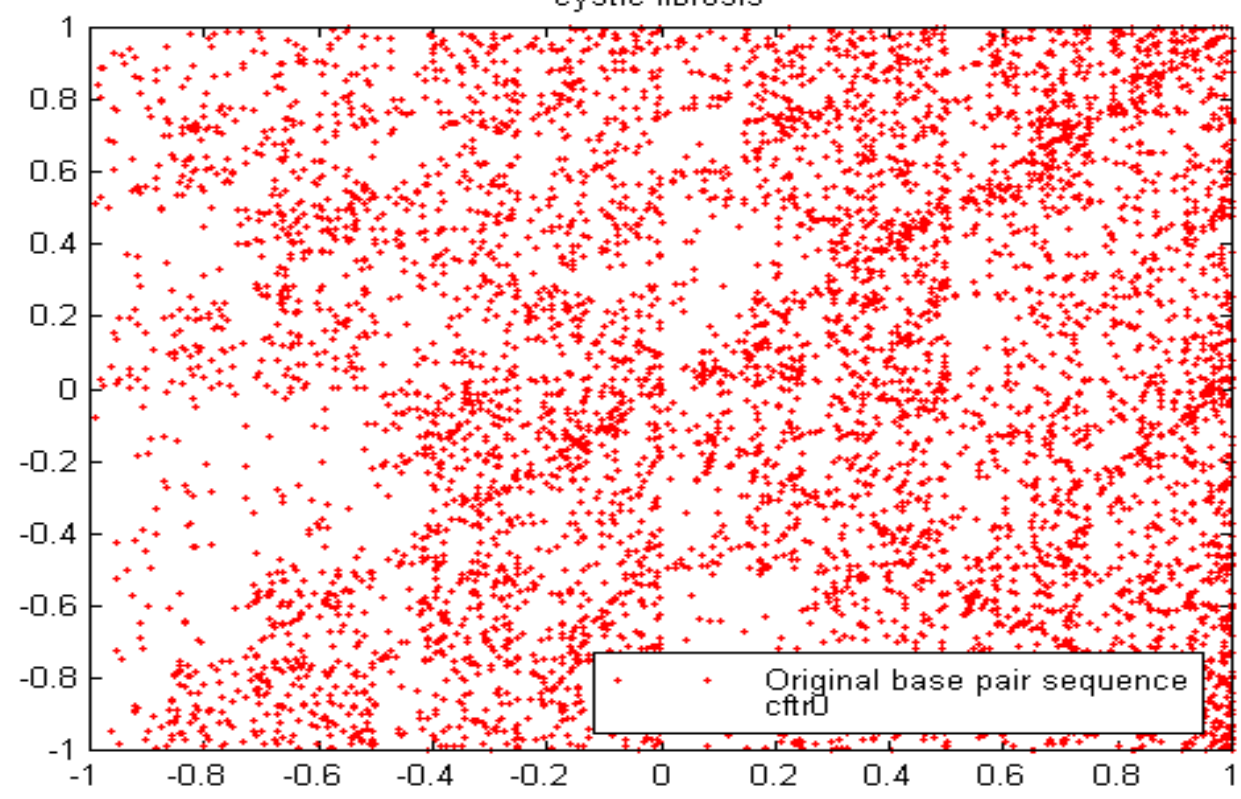

Figure V-112 Original Sequence

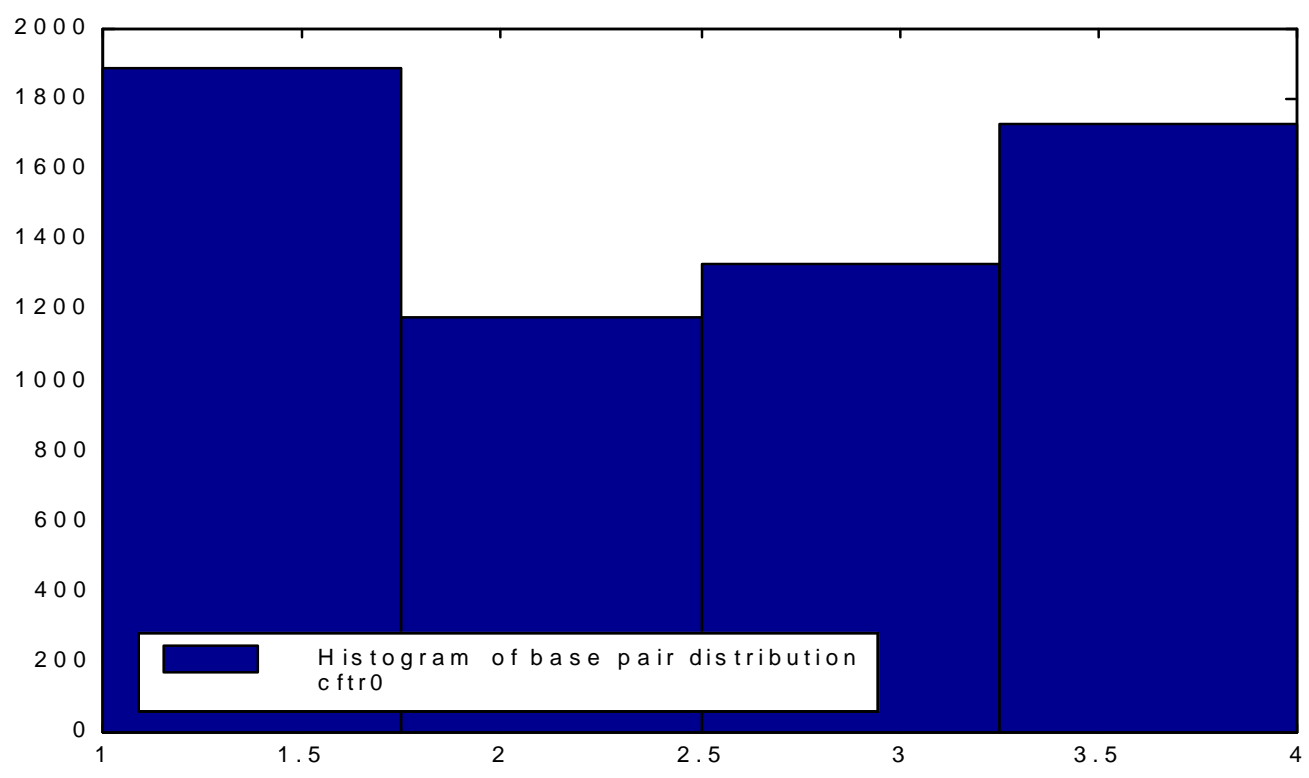

Figure V-113 Base Pair Distribution 
cystic fibrosis

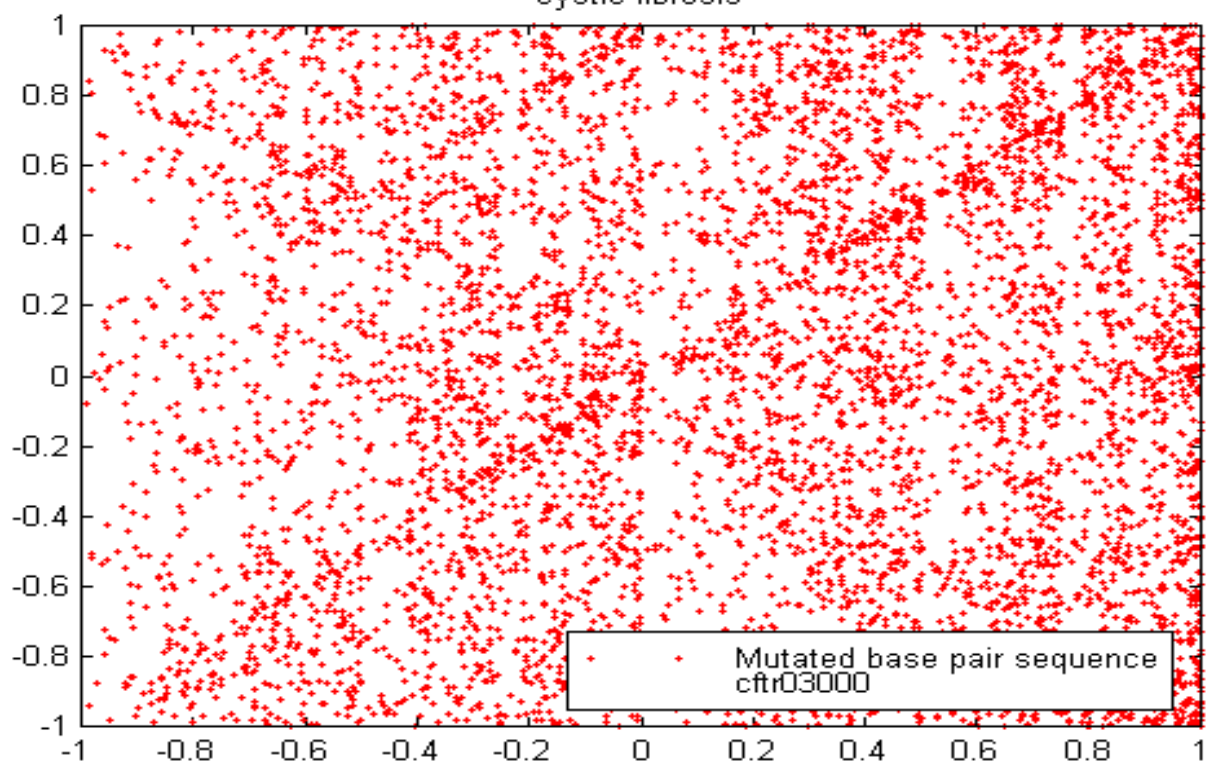

Figure V-114 Mutated Sequence 1000 ->4000

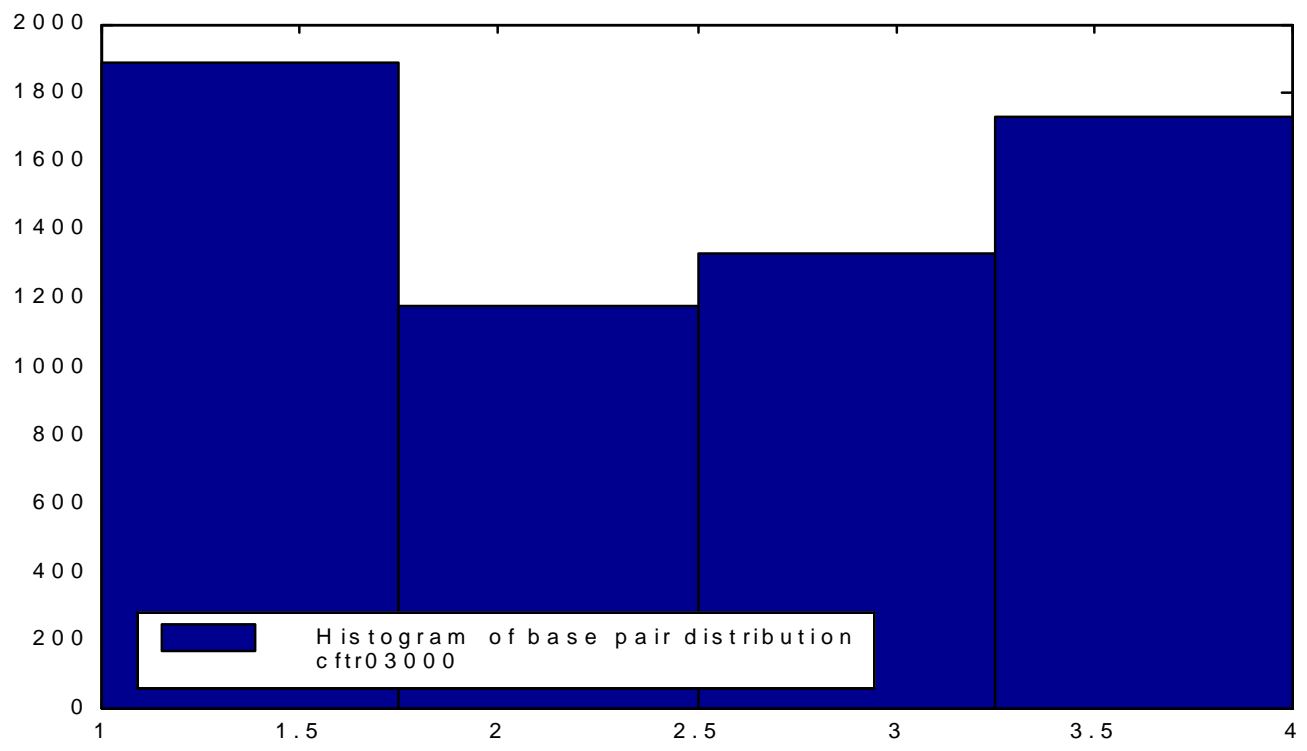

Figure V-115 Base Pair Distribution 1000 ->4000 


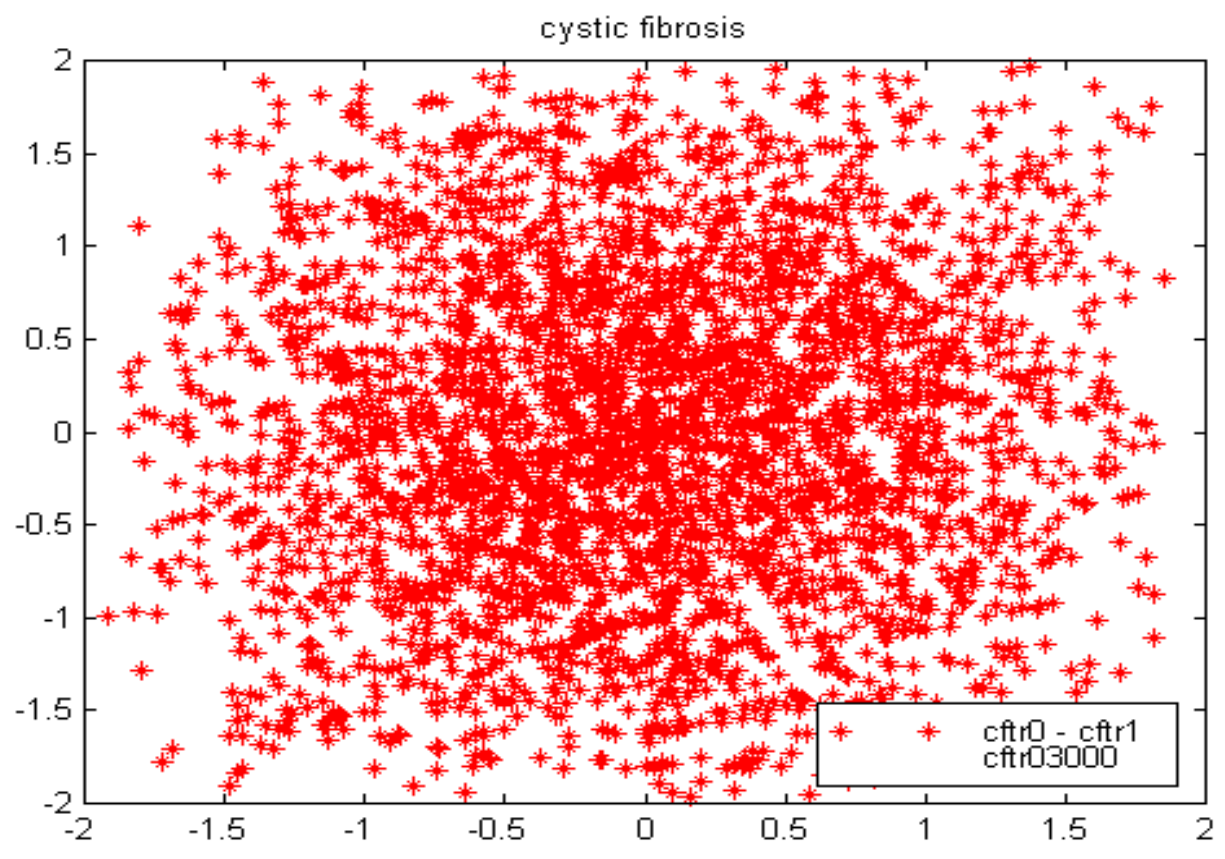

Figure V-116 Difference In The IFS Representations Between Original And Mutated Sequence $1000->4000$ Order Reversed

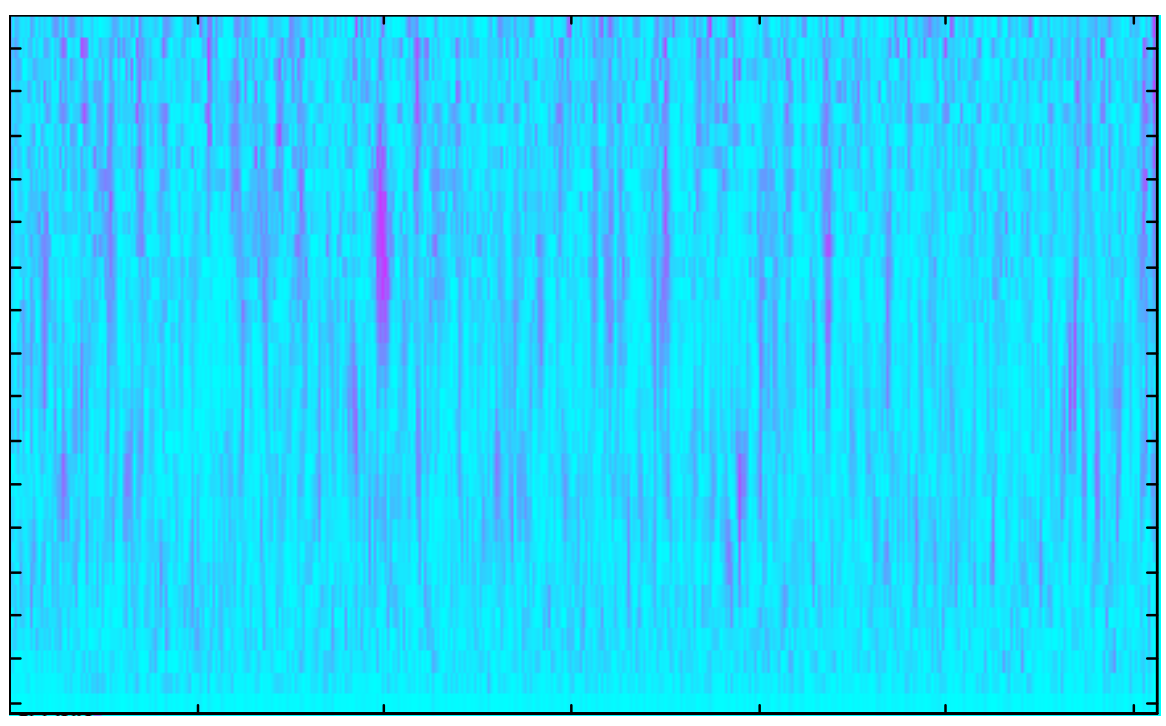

Figure V-117 Continuous Wavelet Transform Of The Original Sequence 


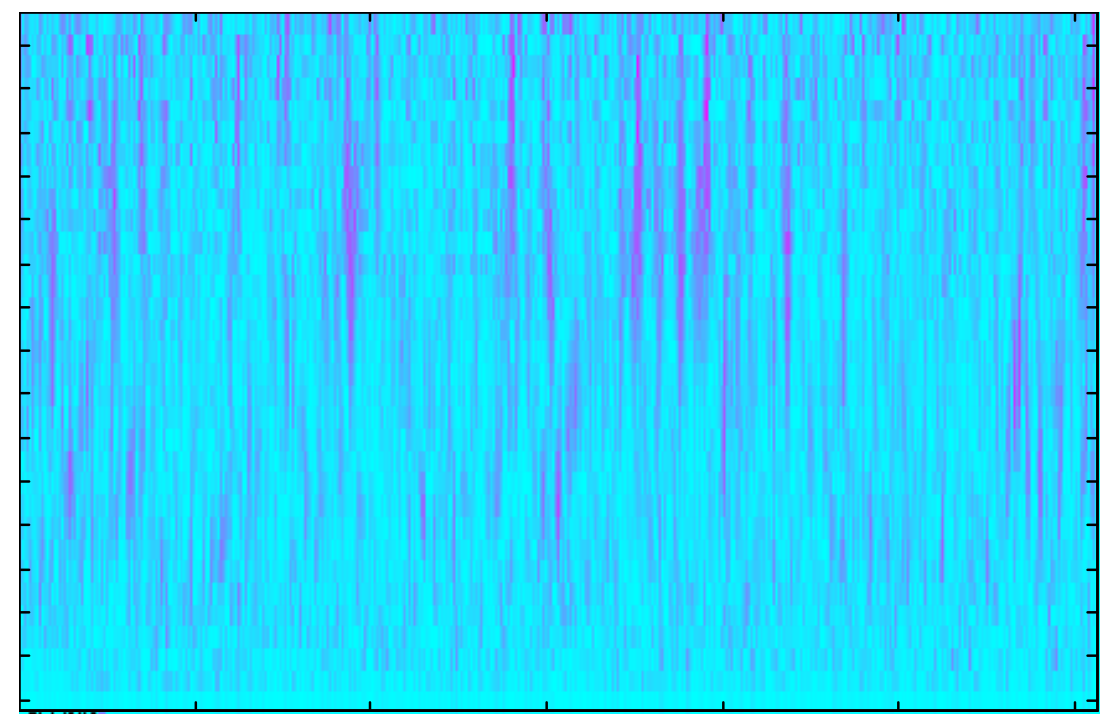

Figure V-118 Continuous Wavelet Transform Of The Mutated Sequence 1000 -> 4000 Order Reversed

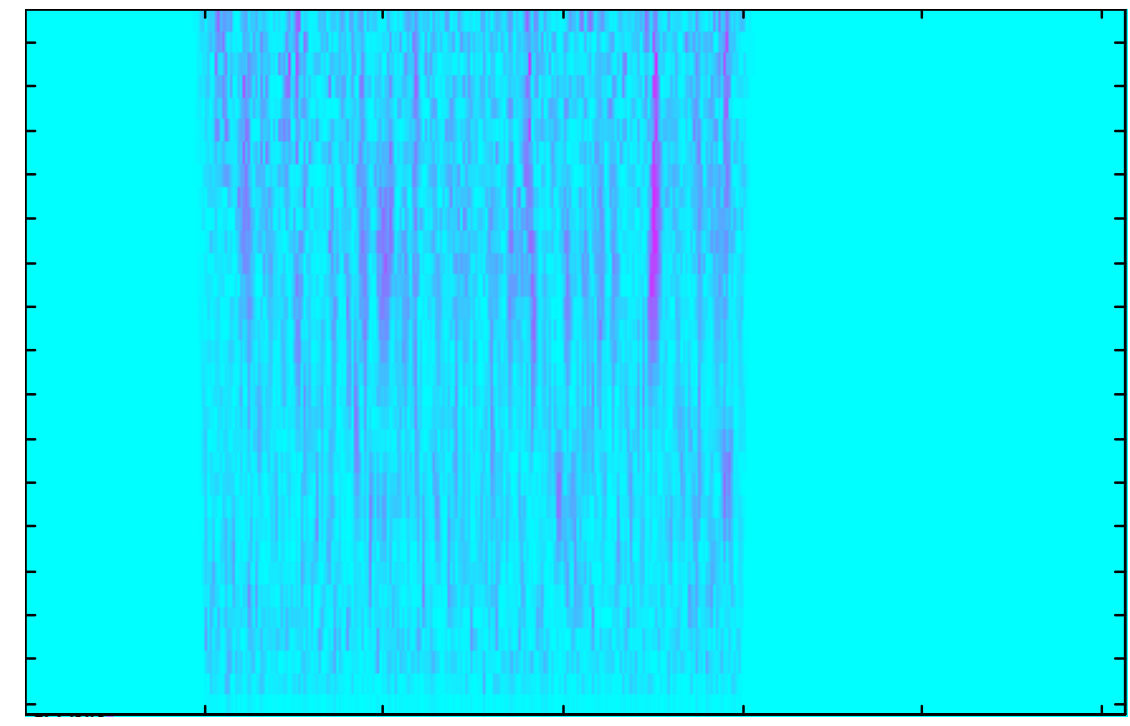

Figure V-119 Difference In The Continuous Wavelet Transform Of The Mutated Sequence And The Original Sequence 


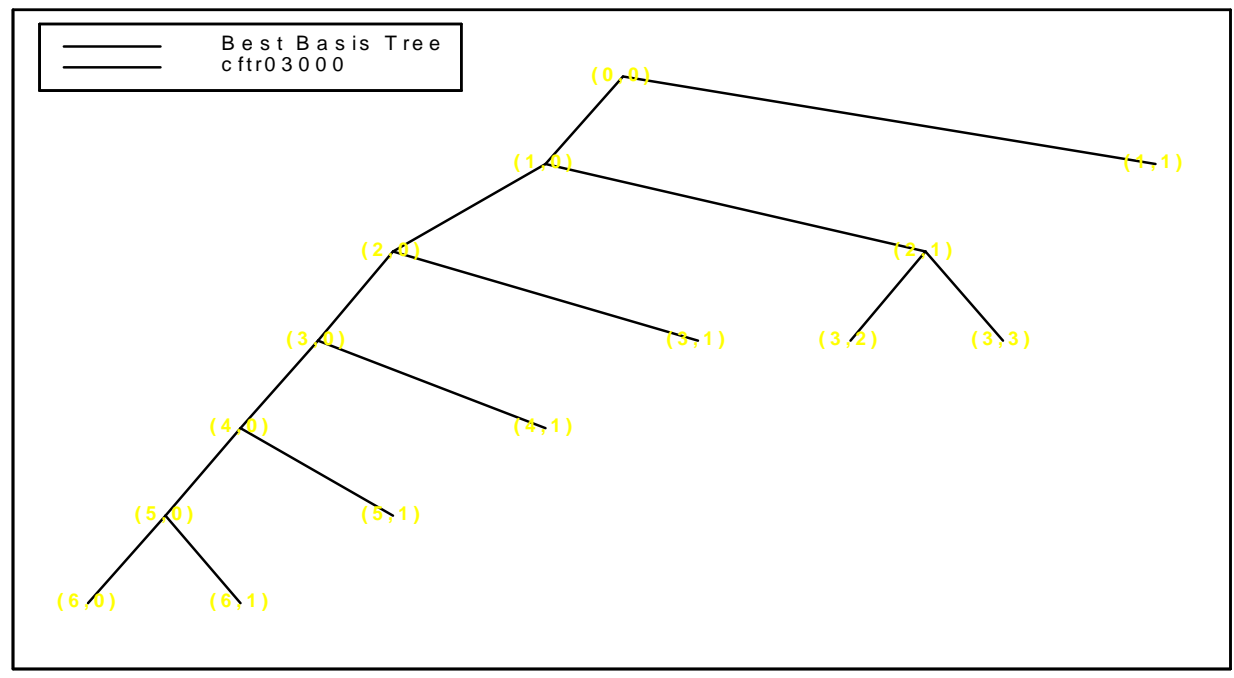

Figure V-120 Best Basis Representation Of The Mutated Sequence $1000->4000$ Order Reversed

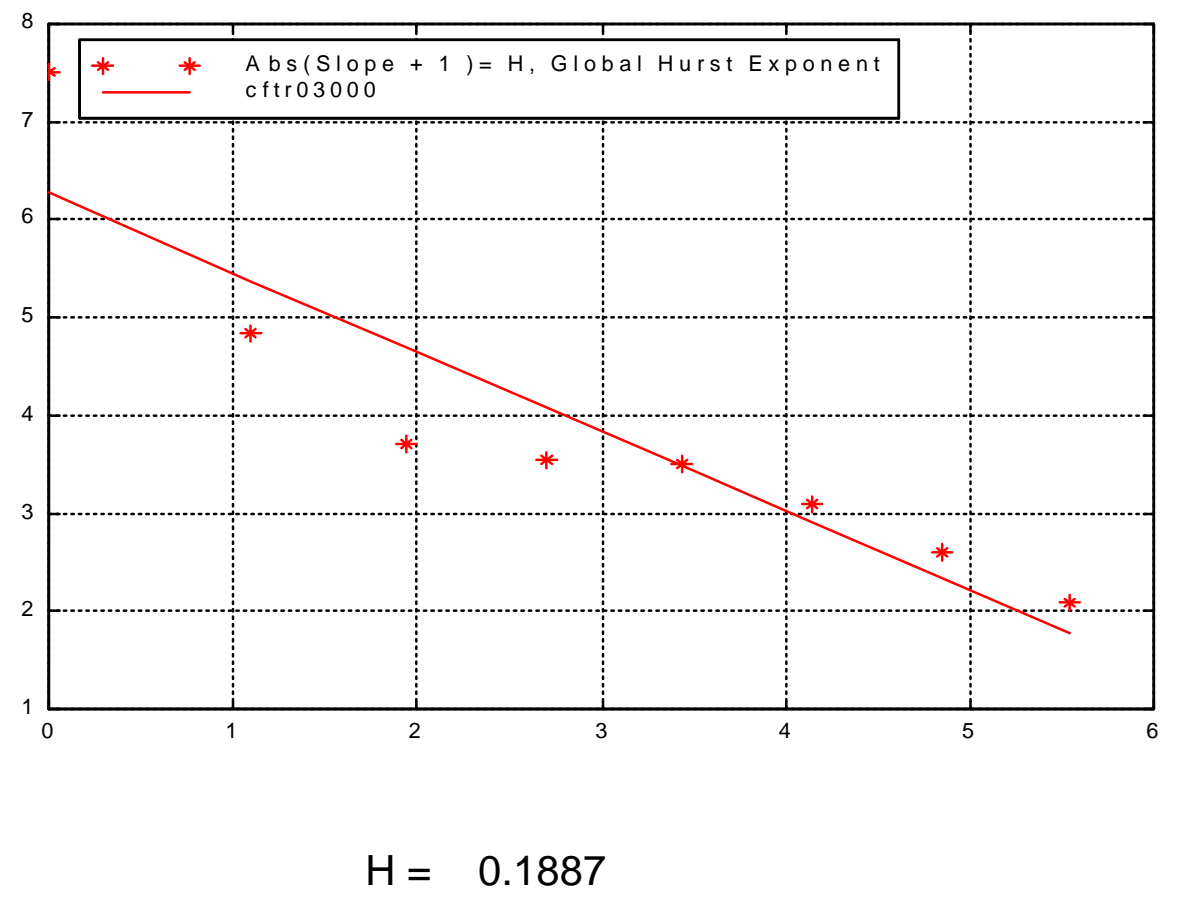


Figure V-121 Global Hurst Exponent Of The Mutated Sequence

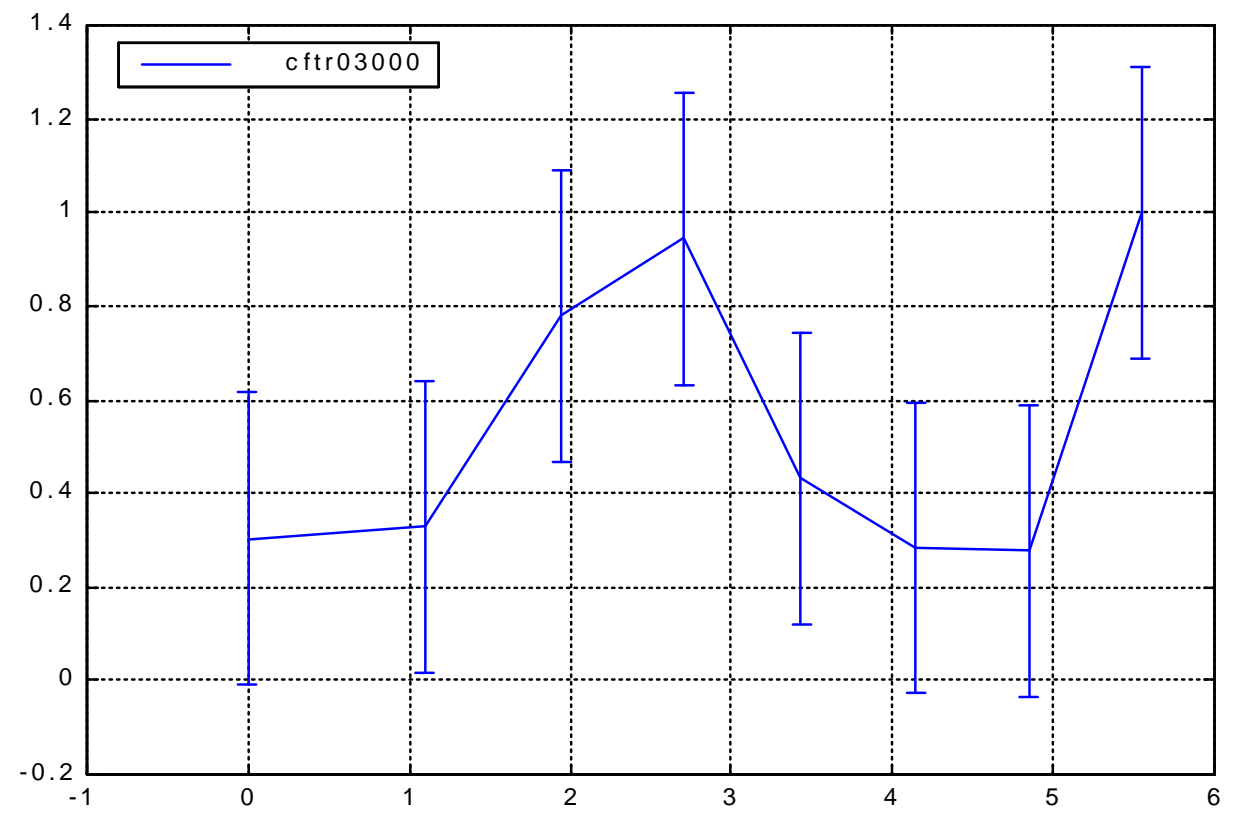

Figure V-122 Local Hurst Exponent Of The Mutated Sequence 1000 -> 4000 Order Reversed 


\section{Conclusions}

In concluding any scientific research endeavor one should always ask have the research goals been met. The goals are listed below for clarity of discussion ${ }^{117,118:}$

1. To determine if non-linear analysis to detect changes of information complexity in DNA sequences. Specifically can different states of "randomness" or "chaos" be categorized?

2. Also can information structures be identified, quantified and the change in these structures be identified and quantified?

3. If 1 ) is true can one distinguish pathogenic from non-pathogenic?

4. Can one distinguish degrees of pathogenicity? Can one distinguish minor illness from catastrophic illness?

5. Can one alter the gene pattern using genetic engineering methods and measure the changes in information content?

6. Can one use the above techniques on diseased sequences and alter the catastrophic nature if the serious defect?

Now examining figures $\mathrm{VI}-1, \mathrm{VI}-2$ and $\mathrm{VI}-3$ regarding the five research goals Remember that the calculations were done from a IFS mapping (or transform) of the base pair sequence. The IFS transform is an algorithm that does a two-dimensional mapping of the sequence. This mapping is a function of how each base pair relates to every other base pair in the sequence. Changes in 
base pairs disturb this relationship and the change is displayed two dimensionally. This information is captured by calculating a Euclidean distance between each displayed point and a reference. All subsequent calculations are done on these distances.

The following three plots summarize the relationship between changes in global and local complexity i.e. Global and Local Hurst Exponent and the IFS spatial pattern represented by the "radius of gyration".

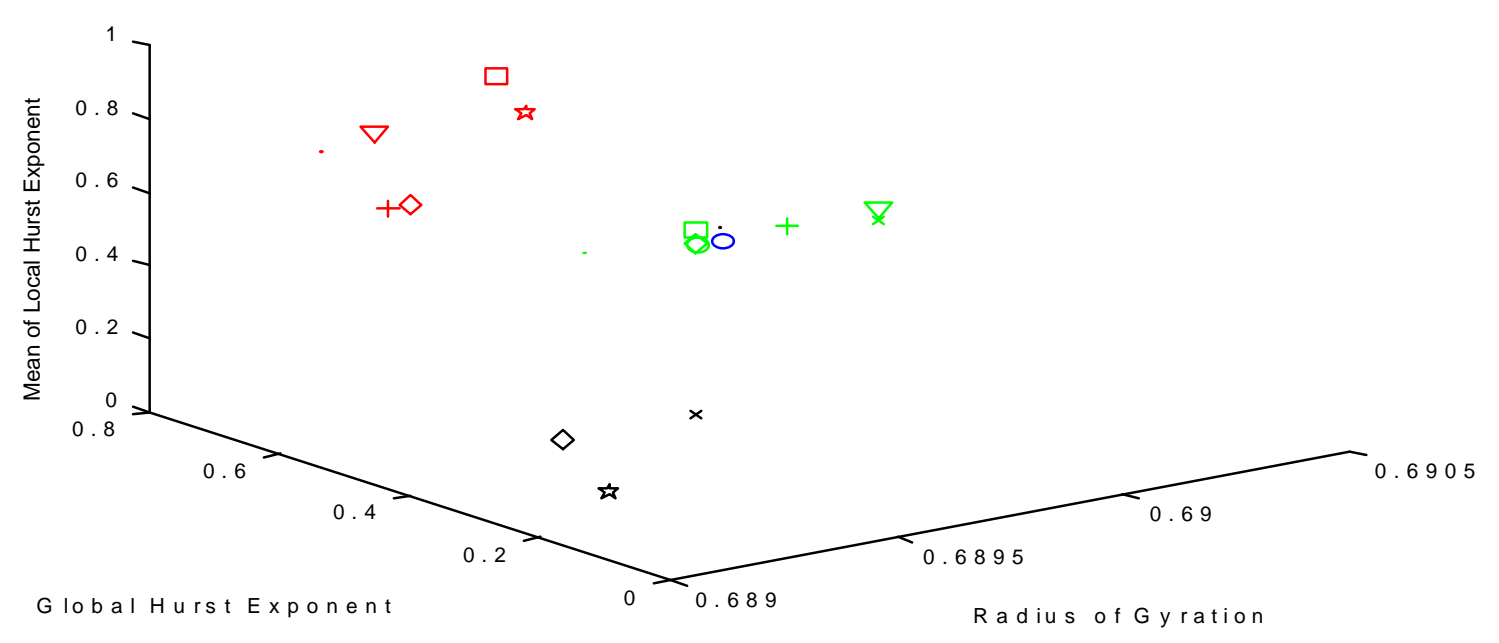

Figure Vl-1

Table VI-1 Legend for Figure VI-1

1. cftro.txt O Original Sequence

2. cftr1.txt $\diamond$ amino acid deletion, CTT deleted 1654-1657

3. $\operatorname{cttr2.txt} \nabla$ transition $1758 \mathrm{~g}->\mathrm{a}$

4. cftr3.txt $x$ transition $1785 \mathrm{~g}->\mathrm{a}$
10. cftr9.txt $O$ transition $1681 \mathrm{a}->\mathrm{g}$

11. $\operatorname{cftr} 10 . \mathrm{txt} \nabla$ point deletion, $\mathrm{C}$ deleted 1032

12. cftr11.txt point deletion, $\mathrm{T}$ deleted 3382

13. cftr12.txt • point deletion, A deleted 4893 
5. cftr4.txt + transition $3638 \mathrm{c}->\mathrm{t}$

14. cftr13.txt is point deletion, $G$ deleted 2597

\section{Table VI-1 Legend for Figure VI-1 continued}

6. cftr5.txt + amino acid deletion,

15. cftr09.txt $\quad$ $1000->1009$ reverse order ATC 1651 -> 1654

7. cftr6.txt - transition $1651 \mathrm{c}->\mathrm{t}$

16. $\operatorname{cftr} 30 . t x t \quad x \quad 1000->1030$ reverse order

8. cftr7.txt transition $1681 \mathrm{a}->\mathrm{t}$

17. cftr300.txt $\quad 1000->1300$ reverse order

9. cftr8.txt $\bullet$ transition $1681 \mathrm{a}->\mathrm{c}$

18. cftr3000.txt is $1000->4000$ reverse order

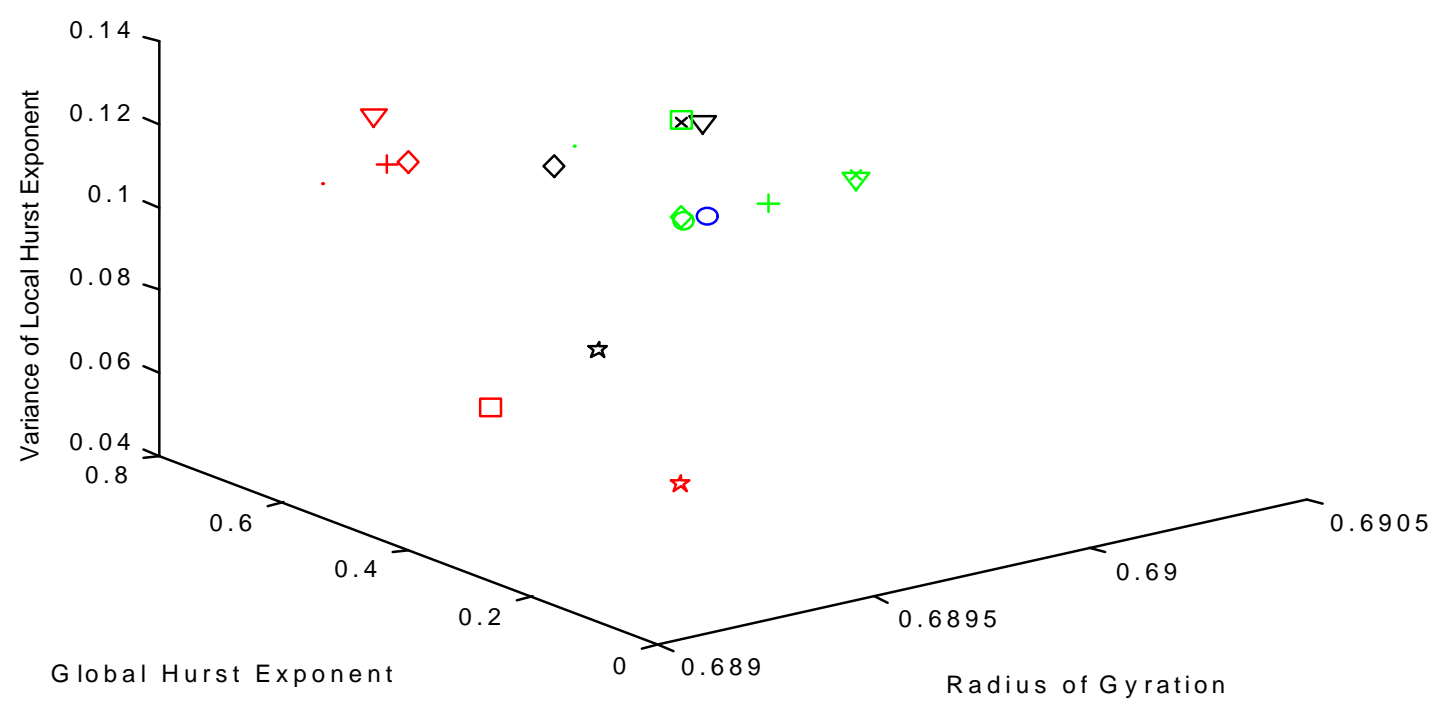

Figure VI-2

Table VI-2 Legend for Figure VI-2

1. cftro.txt O Original Sequence

2. cftr1.txt $\diamond$ amino acid deletion,

CTT deleted 1654-1657

3. $\operatorname{ctt} 2 . \operatorname{txt} \nabla$ transition $1758 \mathrm{~g}->\mathrm{a}$

4. cftr3.txt $x$ transition $1785 \mathrm{~g}->\mathrm{a}$
10. cftr9.txt $O$ transition $1681 \mathrm{a}->\mathrm{g}$

11. cftr10.txt $\nabla$ point deletion, $C$ deleted 1032

12. cftr11.txt point deletion, $\mathrm{T}$ deleted 3382

13. cftr12.txt • point deletion, A deleted 4893 
5. cftr4.txt + transition $3638 \mathrm{c}->\mathrm{t} \quad$ 14. cftr13.txt is point deletion, G deleted 2597

Table VI-2 Legend for Figure VI-2 continued
6. $\operatorname{cftr} 5 . t x t+$ amino acid deletion,
15. cftr09.txt $\quad$ $1000->1009$ reverse order ATC 1651 -> 1654
7. cftr6.txt - transition $1651 \mathrm{c}->\mathrm{t}$
16. $\operatorname{cttr30.txt} \quad x \quad 1000->1030$ reverse order
8. cftr7.txt transition $1681 \mathrm{a}->\mathrm{t}$
17. $\operatorname{cttr300.txt~} \bullet 1000->1300$ reverse order
9. cftr8.txt $\bullet$ transition $1681 \mathrm{a}->\mathrm{c}$
18. cftr3000.txt is1000 -> 4000 reverse order

It is clear in examining Figures $\mathrm{VI}-1$ and $\mathrm{VI}-2$ that the data groups itself into three categories; a) deletions, b) transitions and c) "reverse order". Transitions and "reverse order" categories separate in $\mathrm{VI}-1$, but as strongly as in $\mathrm{VI-2}$. It is important to remember that transitions 7 through 10 are non-destructive mutations. They do not affect the protein and they group together throughout figures VI-1 through VI-3. This suggests a fundamental relationship. Transition 2 and 3 are interesting in the fact that they are mutations that do affect the protein. Transition 2, cftr2.txt at site $1758(\mathrm{~g}->\mathrm{a})$ mutates into a stop codon thus inhibiting proper transcription. At transition site 3 , that is $1785(\mathrm{a}->\mathrm{g})$ in text file in cftr3.txt an amino acid change of Asp mutating to Gly occurs. This mutation is distinguishable in $\mathrm{VI}-1$ because of a slight difference in the mean of the local Hurst exponent. The closeness of these two events suggests a similar information transfer; that should be explored. 


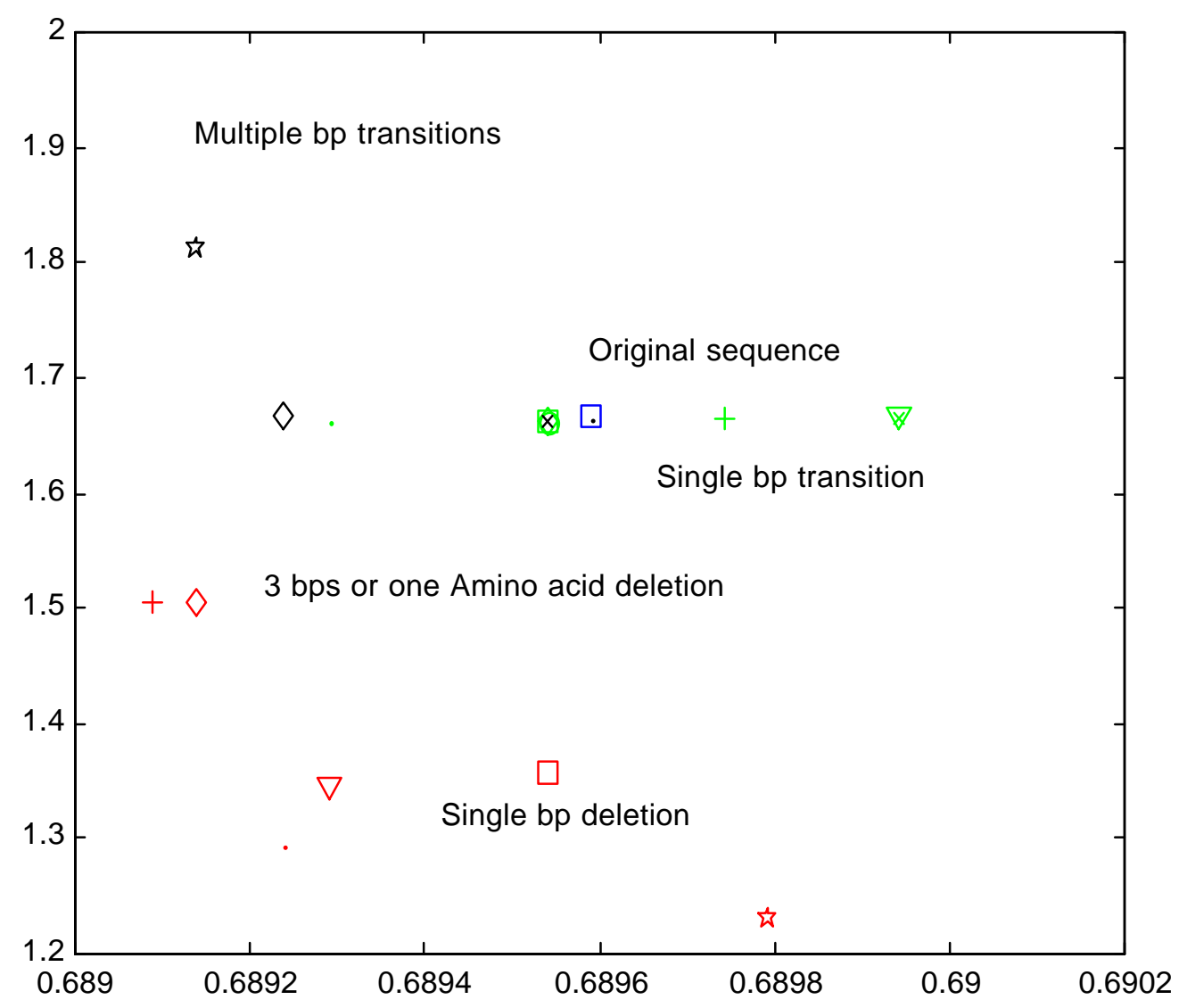

Figure VI-3

\section{Table VI-3 Legend for Figure VI-3}

1. cftro.txt O Original Sequence

2. cftr1.txt $\diamond$ amino acid deletion,

CTT deleted 1654-1657

3. $\operatorname{cftr2.txt} \nabla$ transition $1758 \mathrm{~g} \rightarrow \mathrm{a}$

4. cftr3.txt $x$ transition $1785 \mathrm{~g} \rightarrow \mathrm{a}$

5. cftr4.txt + transition $3638 \mathrm{c}->\mathrm{t}$

6. $\operatorname{ctt} 5 . t x t+$ amino acid deletion,
10. $\operatorname{cftr9.txt~} \mathrm{O}$ transition $1681 \mathrm{a}->\mathrm{g}$

11. cftr10.txt $\nabla$ point deletion, $C$ deleted 1032

12. cftr11.txt point deletion, $T$ deleted 3382

13. cftr12.txt • point deletion, A deleted 4893

14. cftr13.txt is point deletion, $\mathrm{G}$ deleted 2597

15. cftr09.txt $\quad 1000->1009$ reverse order

ATC 1651 -> 1654 
Table VI-3 Legend for Figure VI-3 continued
7. cftr6.txt - transition $1651 \mathrm{c}->\mathrm{t}$
16. $\operatorname{cttr30.txt~} \quad x \quad 1000->1030$ reverse order
8. cftr7.txt transition $1681 \mathrm{a}->\mathrm{t}$
17. $\operatorname{cttr300.txt} \diamond 1000->1300$ reverse order
9. cftr8.txt $\bullet$ transition $1681 \mathrm{a}->\mathrm{c}$
18. cftr3000.txt \&\$1000 -> 4000 reverse order

In V-3 "category c)" becomes strongly distinct at examples cftr300.txt and cftr3000.txt. It is important to remember that the 'reverse order" mutation is defined to be a section of the sequence is excised; then the order of the base pairs is reversed; then the sequence is put back into the same place from where is it was removed. This means "reverse order" mutation is actually a type of group transition and only introduces enough information disturbances with large numbers of transitions such as with sub-sequence lengths 300 and 3000 .

Deletions remain well separated from the others on all the plots. This is because a deletion whether multiple in the case of an amino acid deletion or a point (single base pair) deletion represent a total loss of information and a sequence position disturbance that propagates from the point of the deletion to the end of the sequence. Note "reverse order" transitions have a $25 \%$ chance of matching up correctly at each point in the altered section. There also is a partial loss of information from this type of transition, not the total loss of a deletion. It takes a considerable number of "partial losses" to create a disturbance that does any more then change the spatial relationship IFS transform of the sequence. Therefore global Hurst exponent is very robust to base pair transitions and by implication so is the fractional dimension since they are related linearly, e.g. 


\section{VI-1 $\quad \mathrm{D}=2-\mathrm{H}$}

These sequences remain moderately anti-persistent until the 3000 base pair "reverse order" mutation, (See Figure VI-3). In this case the sequence is driven to a strongly anti-persistent condition, $0<\mathrm{H}<=0.50$. An anti-persistent system covers less distance than a random one. In order for the system to cover less distance, it must reverse itself more frequently than a random process. This behavior mean reverting; however, this is based on the assumption that the system has a stable mean. For these DNA sequences this assumption can not be made. Single point deletions drive the system to a condition toward a persistent condition, $0<=\mathrm{H}<1.00$. A persistent condition is characterized by long term memory effects, i.e. what happens locally in the sequence impacts the entire sequence. In terms of the science of chaotic dynamics, systems such show sensitive dependence on initial conditions. This long-term memory effect occurs regardless of time scale. Using the stock market as an analogy all daily changes correlate with all future daily changes, all weekly changes correlate with all future weekly changes, etc. There is no characteristic time scale. This is a prime characteristic of a fractal time series. 
In the case of the two amino acids deletions (3 BPS) where $\mathrm{D}=1.5$ (or $\mathrm{H}=$ 0.50 ) this would imply an independent process. Its is important to realize that this analysis does not require that the underlying process be Gaussian, just independent. This means that the underlying process is uncorrelated. There is no requirement for this distribution to be any particular shape. The CTT deletion, i.e. cftr1.txt (an amino acid deletion 1654-1657) is an abnormality in the DNA sequence that appears to account for about 70 percent of cystic fibrosis cases. This aberration is often denoted as the AF508. That loss causes the protein product of the gene to lack a single amino acid: phenylalanine at position 508 . The ATC deletion (cftr5.txt) which is an amino acid deletion at sites 1651 -> 1654 this mutation occurs in a much smaller percentage of the cases then the CTT deletion which is a "non-sense" codon this also affects the protein product as do all the other deletions. The grouping of these mutations in solution space shows a similarity in their effect on information transfer and disease production.

Given the above discussion its very clear that goals 1) ,2) and 3) have been met by Figures VI-1 through VI-3 and the IFS plots shown in Section V. Figures VI-1 to VI-3 show clearly that various "states" of chaos can be quantified both visually as well as numerically. IFS plot differences are sensitive down to single base changes this is very clear in Section V. Where not only single base-pair deletions but base-pair transitions show quite distinctive IFS plot differences. As was stated previously Figure VI-3 shows five distinct regions: i) multiple basepair (i.e. an Amino acid) deletions, ii) single base-pair deletions, iii) multiple 
base-pair transitions, iv) single base-pair transition and v) original sequence.

The radius of gyration is most sensitive to base-pair transitions. These divisions into distinct regions clearly satisfy Goal 3 . Goal 4 is satisfied by the analysis done on cystic fibrosis files 'cftr30.txt $->$ cftr3000.txt'. These are artificial mutations they could just as easily be natural helpful mutations. Any number of techniques as discussed in Section IV-F could introduce these 'helpful' mutations. The radius of gyration and Hurst exponents captures the changes in sequence complexity. Sequence complexity changes imply information complexity changes. Goal 5 is can not be satisfied directly because it would require the use of biological and medical resources beyond the scope of this work. However Section VII-B discusses a hypothetical example of how this goal could be met. 


\section{Recommendations}
A. Introduction ${ }^{68}$

Over the past 20 years there has been a major scientific revolution unfolding in the area of genetic engineering. This involves the ability to isolate, study and manipulate the genetic material of all living organisms. Although the origins of genetic engineering come from the study of Microbiology, this new discipline is being enhanced by the introduction of the engineering disciplines as a means of analyzing and solving problems in many diverse areas of the life sciences. For example, at present there is a major international research initiative underway 'The Human Genome Project'. Its aim is to study the genetic script of humans. This could ultimately result in a real understanding of the molecular basis of human disease and genetic disorders.

In the area of Biochemistry, the science of the study of the chemicals that make up living things, there is undergoing another revolution based on genetic engineering. This is known as protein engineering. This is the ability to alter in a rational manner the structure and/or function of proteins or create new proteins that do not occur naturally. This may lead to a revolution bigger than the microelectronics revolution, yet the basis of protein engineering is, as yet, not widely understood by the general public. 
It is obvious enough to the most causal observer that DNA sequence analysis is an area, which is a frontier area for exploration using engineering modeling analysis. The approach used in this work was built on previously developed methods. The uniqueness approach is that it uses information produced by one method e.g. IFS analysis to be "data " for another method Hurst (or R/S) analysis as calculated by wavelet packet transforms procedure. This process could be applied by many of the techniques mentioned in the section III, Literature survey. This work represents just the initial steps in using this approach. Below are some recommended extensions of this effort.

\section{B. A Hypothetical Genetic Engineering Example of How This Research} Might be Applied ${ }^{48}$

Consider the following scenario in which the information generated by this dissertation's approach might be used to prescribe a treatment regime for cystic fibrosis. The operating assumption is that a delivery mechanism exists to deliver the correct CFTR gene to the patients. Two such methods currently being researched offer this possibility. They are:

1. Deliver the CFTR gene to patients via genetically engineered adenovirus vectors,

2. Coat the therapeutic gene with fatty molecules that are not recognized by the immune system but still enable the DNA to enter cells. 
As was discussed previously experiments are currently being done to assess safety of these delivery systems. The operating assumption underlying this hypothetical example is that these two delivery methods are now available. The CFTR mutation to be treated is an amino acid deletion. The codon triplet CTT is deleted at position 1654-1657. In this research document that triplet is in data set cystic.txt. The following table and discussion below shows specifically how the production rules can be used to make a tabular algorithm to specify codons $^{49}$.

An explanation of the above Genetic Code table follows. (1) DNA is a twostranded molecule. (2) Each strand is a polynucleotide composed of $A$ (adenosine), $\mathrm{T}$ (thymidine), $\mathrm{C}($ cytosine), and $\mathrm{G}$ (guanosine) residues polymerized by "dehydration" synthesis in linear chains with specific sequences. (3) Each strand has polarity, such that the 5'-hydroxyl group of the first nucleotide begins the strand and the 3'-hydroxyl group of the final nucleotide ends strand; accordingly, we say that this strand runs $5^{\prime}$ to $3^{\prime}$. It is also essential to know that the two strands of DNA run antiparallel such that one strand runs $5^{\prime}$ $->3^{\prime}$ while the other one runs $3^{\prime}->5^{\prime}$. (4) At each nucleotide residue along the double-stranded DNA molecule, the nucleotides are complementary. That is, A forms two hydrogen-bonds with $\mathrm{T}$; C forms three hydrogen bonds with $\mathrm{G}$. In most 


\begin{tabular}{|c|c|c|c|c|c|c|c|}
\hline \multicolumn{8}{|c|}{ Second Position } \\
\hline \multirow{17}{*}{$\begin{array}{l}\mathrm{Fi} \\
\mathrm{rs} \\
\mathrm{t} \\
\mathrm{P} \\
\text { os } \\
\text { ist } \\
\text { io } \\
\mathrm{n} \\
\end{array}$} & & $\mathrm{T}$ & $\mathrm{C}$ & $\mathrm{A}$ & 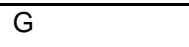 & & \\
\hline & $\mathrm{T}$ & $\begin{array}{l}\text { TTT Phe[F] } \\
\text { TTC Phe[F] } \\
\text { TTA Leu[L] } \\
\text { TTG Leu[L] }\end{array}$ & $\begin{array}{l}\text { TCT Ser[S] } \\
\text { TCC Ser[S] } \\
\text { TCA Ser[S] } \\
\text { TCG Ser[S] }\end{array}$ & $\begin{array}{l}\text { TAT Tyr [Y] } \\
\text { TAC Tyr [Y] } \\
\text { TAA Ter[end] } \\
\text { TAG Ter[end] }\end{array}$ & $\begin{array}{l}\text { TGT Cys [C] } \\
\text { TGC Cys [C] } \\
\text { TGA Ter[end] } \\
\text { TGG Trp [W] }\end{array}$ & $\mathrm{T}$ & $\begin{array}{l}\text { Thi } \\
\text { rd } \\
\text { Po } \\
\text { siti } \\
\text { on } \\
\end{array}$ \\
\hline & & & & & & $\mathrm{C}$ & \\
\hline & & & & & & $A$ & \\
\hline & & & & & & $G$ & \\
\hline & $C$ & $\begin{array}{l}\text { CTT Leu[L] } \\
\text { CTC Leu[L] } \\
\text { CTA Leu[L] } \\
\text { CTG Leu[L] }\end{array}$ & $\begin{array}{l}\text { CCT Pro[P] } \\
\text { CCC Pro[P] } \\
\text { CCA Pro[P] } \\
\text { CCG Pro[P] }\end{array}$ & $\begin{array}{l}\text { CAT His[H] } \\
\text { CAC His[H] } \\
\text { CAA Gln[Q] } \\
\text { CAG GIn[Q] }\end{array}$ & $\begin{array}{l}\text { CGT Arg[R] } \\
\text { CGC Arg[R] } \\
\text { CGA Arg[R] } \\
\text { CGG Arg[R] }\end{array}$ & $\bar{T}$ & \\
\hline & & & & & & $C$ & \\
\hline & & & & & & $A$ & \\
\hline & & & & & & $G$ & \\
\hline & $\mathrm{A}$ & $\begin{array}{l}\text { ATT Ile[l] } \\
\text { ATC Ile[l] } \\
\text { ATA Ile[l] } \\
\text { ATG Met[M] }\end{array}$ & $\begin{array}{l}\text { ACT Thr[T] } \\
\text { ACC Thr[T] } \\
\text { ACA Thr[T] } \\
\text { ACG Thr[T] }\end{array}$ & $\begin{array}{l}\text { AAT Asn[N] } \\
\text { AAC Asn[N] } \\
\text { AAA Lys[K] } \\
\text { AAG Lys[K] }\end{array}$ & $\begin{array}{l}\text { AGT Ser[S] } \\
\text { AGC Ser[S] } \\
\text { AGA Arg[R] } \\
\text { AGG Arg[R] }\end{array}$ & $T$ & \\
\hline & & & & & & C & \\
\hline & & & & & & $A$ & \\
\hline & & & & & & G & \\
\hline & $\bar{G}$ & $\begin{array}{l}\text { GTT Val[V] } \\
\text { GTC Val[V] } \\
\text { GTA Val[V] } \\
\text { GTG Val[V] }\end{array}$ & $\begin{array}{l}\text { GCT Ala[A] } \\
\text { GCC Ala[A] } \\
\text { GCA Ala[A] } \\
\text { GCG Ala[A] }\end{array}$ & $\begin{array}{l}\text { GAT Asp[D] } \\
\text { GAC Asp[D] } \\
\text { GAA Glu[E] } \\
\text { GAG Glu[E] }\end{array}$ & $\begin{array}{l}\text { GGT Gly[G] } \\
\text { GGC Gly[G] } \\
\text { GGA Gly[G] } \\
\text { GGG Gly[G] }\end{array}$ & $\bar{T}$ & \\
\hline & & & & & & $C$ & \\
\hline & & & & & & $\mathrm{A}$ & \\
\hline & & & & & & $\bar{G}$ & \\
\hline
\end{tabular}

Table VII.1 Genetic Code as a Tabular Algorithm to Specify Amino Acid Residues as Triplet Codons 
cases the two-stranded, antiparallel, complementary DNA molecule folds to form a helical structure which resembles a spiral staircase. This is the reason why DNA has been referred to as the "Double Helix".

One strand of DNA holds the information that codes for various genes; this strand is often called the template strand or antisense strand (containing anticodons). The other, and complementary, strand is called the coding strand or sense strand (containing codons). Since mRNA is made from the template strand, it has the same information as the coding strand. The table above refers to the sequence $5^{\prime}->3^{\prime}$ of the coding or sense strand of DNA; the code for the mRNA would be identical but for the fact that RNA contains $U$ (uridine) rather than $\mathrm{T}$.

An example of two complementary strands of DNA would be:

$$
\begin{array}{ll}
\left(5^{\prime}->3^{\prime}\right) \text { ATGGAATTCTCGCTC } & \text { (Coding, sense strand) } \\
\left(3^{\prime}<-5^{\prime}\right) \text { TACCTTAAGAGCGAG } & \text { (Template, antisense strand) } \\
\left(5^{\prime}->3^{\prime}\right) \text { AUGGAAUUCUCGCUC } & \text { (mRNA made from Template strand) }
\end{array}
$$

Since amino acid residues of proteins are specified as triplet codons, the protein sequence made from the above example would be: 
Met-Glu-Phe-Ser-Leu... (MEFSL...). ${ }^{73,76}$

Using the above table and the accompanying explanation one can construct the 20 amino acid groupings ( 21 if you include the stop codons) for humans with a total of 64 possible base pair triplets. This work has shown that the reason that there are 64 total base pair triplets (codons) is a direct result of the L-system grammar rules that govern the construction of the codon. To formulate the codon one must recall that chemically the four nucleotides ( $A, T, G$, and $C)$ belong to 2 classes the pyrimidine ( $T$ and $C$ ) and the purine ( $A$ and $G)$. Applying the "rule of degeneracy" which is:' if two codons have similar two first nucleotides and their third nucleotide belong to the same class (purine or pyrimidine) they encode to one and the same amino acid. This generates the multiple entries in the amino acid families. On the next page, is a table of the 64 possible amino acids grouped into their 21 families. Note the $21^{\text {st }}$ family is the terminating codon. In the table below the first three columns are: the first column is the amino acid base pair triplet; the second column is the human amino acid family and the third column is the associated data file. The last three columns are the non-linear parameters calculated from analytical methodology developed in this research. They are $\mathrm{Rg}$ - radius of gyration, variance Hlocal - variance of the local Hurst exponent, and Global H, - global Hurst exponent. 
Table VII.2 64 “Trial” Amino Acids

\begin{tabular}{|c|c|c|c|c|c|c|c|}
\hline & Amino & & Family & Data File & $\mathbf{R g}$ & variance & Global \\
\hline & Acid & & No. & & & Hlocal & H \\
\hline 1. & TTT & Phe & 1 & cystic1.txt & 1.9923 & .1357 & .3393 \\
\hline 2. & TTC & Phe & & cystic16.txt & 1.9929 & .1351 & .3389 \\
\hline 3. & TTA & Leu & 2 & cystic17.txt & 1.9930 & .1347 & .3375 \\
\hline 4. & TTG & Leu & & cystic18.txt & 1.9923 & .1347 & .3386 \\
\hline 5. & CTT & Leu & & cystic.txt & $1.9929^{\star}$ & .1341 & .3371 \\
\hline 6. & СTC & Leu & & cystic9.txt & 1.9934 & .1340 & .3358 \\
\hline 7. & CTA & Leu & & cystic7.txt & 1.9933 & .1343 & .3363 \\
\hline 8. & CTG & Leu & & cystic8.txt & 1.9928 & .1340 & .3367 \\
\hline 9. & ATT & Ile & 3 & cystic2.txt & 1.9930 & .1350 & .3380 \\
\hline 10. & ATC & Ile & & cystic19.txt & 1.9938 & .1335 & .3367 \\
\hline 11. & ATA & Ile & & cystic20.txt & 1.9936 & .1345 & .3376 \\
\hline 12. & ATG & Met & 4 & cystic21.txt & 1.9929 & .1339 & .3378 \\
\hline 13. & GTT & Val & 5 & cystic22.txt & 1.9924 & .1350 & .3381 \\
\hline 14. & GTC & Val & & cystic $67 . t x t$ & 1.9928 & .1338 & .3363 \\
\hline 15. & GTA & Val & & cystic35.txt & 1.9925 & .1344 & .3369 \\
\hline 16. & GTG & Val & & cystic36.txt & 1.9923 & .1346 & .3377 \\
\hline 17. & TCT & Ser & 6 & cystic23.txt & 1.9929 & .1329 & .3373 \\
\hline 18. & TCC & Ser & & cystic38.txt & 1.9935 & .1331 & .3363 \\
\hline 19. & TCA & Ser & & cystic39.txt & 1.9937 & .1332 & .3366 \\
\hline 20. & TCG & Ser & & cystic $40 . t x t$ & 1.9929 & .1328 & .3371 \\
\hline 21. & AGT & Ser & & cystic61.txt & 1.9929 & .1332 & .3375 \\
\hline 22. & AGC & Ser & & cystic62.txt & 1.9934 & .1332 & .3362 \\
\hline 23. & ССТ & Pro & 7 & cystic41.txt & 1.9935 & .1339 & .3364 \\
\hline 24. & $\operatorname{ccc}$ & Pro & & cystic10.txt & 1.9940 & .1345 & .3365 \\
\hline 25. & CCA & Pro & & cystic42.txt & 1.9941 & .1347 & .3363 \\
\hline 26. & CCG & Pro & & cystic43.txt & 1.9934 & .1344 & .3368 \\
\hline 27. & ACT & Thr & 8 & cystic24.txt & 1.9935 & .1335 & .3372 \\
\hline 28. & ACC & Thr & & cystic44.txt & 1.9941 & .1338 & .3369 \\
\hline 29. & ACA & Thr & & cystic $45 . t x t$ & 1.9942 & .1340 & .3368 \\
\hline 30. & ACG & Thr & & cystic46.txt & 1.9935 & .1334 & .3370 \\
\hline
\end{tabular}


Table VII.2 64 "Trial” Amino Acids continued

\begin{tabular}{|c|c|c|c|c|c|c|c|}
\hline & Amino & & Family & Data File & $\mathbf{R g}$ & variance & Global \\
\hline & Acid & & No. & & & Hlocal & H \\
\hline 31. & GCT & Ala & 9 & cystic25.txt & 1.9928 & .1337 & .3367 \\
\hline 32. & GCC & Ala & & cystic47.txt & 1.9934 & .1341 & .3362 \\
\hline 33. & GCA & Ala & & cystic49.txt & 1.9935 & 1340 & .3361 \\
\hline 34. & GCG & Ala & & cystic68.txt & 1.9928 & .1340 & .3368 \\
\hline 35. & TAT & Tyr & 10 & cystic26.txt & 1.9930 & .1346 & .3383 \\
\hline 36. & TAC & Tyr & & cystic51.txt & 1.9932 & 1337 & .3378 \\
\hline 37. & TAA & Ter & 11 & cystic27.txt & 1.9936 & .1339 & .3363 \\
\hline 38. & TAG & Ter & & cystic52.txt & 1.9929 & .1334 & .3374 \\
\hline 39. & TGA & Ter & & cystic31.txt & 1.9923 & .1335 & .3371 \\
\hline 40. & CAT & His & 12 & cystic4.txt & 1.9935 & .1345 & .3368 \\
\hline 41. & CAC & His & & cystic11.txt & 1.9941 & 1346 & .3366 \\
\hline 42. & CAA & Gln & 13 & cystic29.txt & 1.9943 & 1340 & .3370 \\
\hline 43. & CAG & Gln & & cystic53.txt & 1.9939 & 1939 & .3368 \\
\hline 44. & AAT & Asn & 14 & cystic15.txt & 1.9936 & 1348 & .3380 \\
\hline 45. & AAC & Asn & & cystic54.txt & 1.9938 & 1342 & .3379 \\
\hline 46. & AAA & Lys & 15 & cystic12.txt & 1.9942 & .1342 & .3362 \\
\hline 47. & AAG & Lys & & cystic55.txt & 1.9939 & .1338 & .3365 \\
\hline 48. & GAT & Asp & 16 & cystic70.txt & 1.9929 & 1340 & .3359 \\
\hline 49. & GAC & Asp & & cystic56.txt & 1.9930 & 1340 & .3360 \\
\hline 50. & GAA & Glu & 17 & cystic30.txt & 1.9933 & 1342 & .3364 \\
\hline 51. & GAG & Glu & & cystic57.txt & 1.9934 & 1336 & .3366 \\
\hline 52. & TGT & Cys & 18 & cystic32.txt & 1.9929 & 1343 & .3384 \\
\hline 53. & TGC & Cys & & cystic58.txt & 1.9928 & .1334 & .3382 \\
\hline 54. & TGG & Trp & 19 & cystic33.txt & 1.9920 & 1342 & .3383 \\
\hline 55. & CGT & $\operatorname{Arg}$ & 20 & cystic5.txt & 1.9936 & 1341 & .3369 \\
\hline 56. & CGC & $\operatorname{Arg}$ & & cystic.12.txt & 1.9934 & .1342 & .3362 \\
\hline 57. & CGA & $\operatorname{Arg}$ & & cystic69.txt & 1.9935 & 1341 & .3360 \\
\hline 58. & CGG & $\operatorname{Arg}$ & & cystic60.txt & 1.9934 & .1343 & .3371 \\
\hline 59. & AGA & $\operatorname{Arg}$ & & cystic63.txt & 1.9935 & .1332 & .3366 \\
\hline 60. & AGG & $\operatorname{Arg}$ & & cystic64.txt & 1.9937 & 1332 & .3374 \\
\hline 61. & GGT & Gly & 21 & cystic37.txt & 1.9922 & .1347 & .3378 \\
\hline
\end{tabular}


Table VII.2 64 “Trial” Amino Acids continued

\begin{tabular}{|c|c|c|c|c|c|c|c|}
\hline & Amino & & Family & Data File & $\mathbf{R g}$ & variance & Global \\
\hline & Acid & & No. & & & Hlocal & H \\
\hline 62. & GGC & Gly & & cystic65.txt & 1.9926 & .1342 & .3368 \\
\hline 63. & GGA & Gly & & cystic66.txt & 1.9925 & .1338 & .3364 \\
\hline 64. & GGG & Gly & & cystic14.txt & 1.9921 & .1350 & .3380 \\
\hline
\end{tabular}

The procedure for "cure" is to find the proper base pair triplet to correct the deletion mutation. In this hypothetical case the following is known, there is a 3 base pair deletion starting at site 1654 . This can easily be found by taking the "difference" between a healthy CFTR gene and the mutated CFTR gene in question. This operation tells the analyst that there are three missing base pairs starting at site 1654 , but yields no information about the correct base pair.

The remaining question is "which of the above 64 possible choices is the correct one?" The procedure for finding the correct codon will require the application of the engineering analysis methodology presented in this research. The first step is to create 64 "trial" CFTR sequences. Then calculate the following non-linear parameters: $\mathrm{Rg}$ - radius of gyration; variance Hlocal variance of the local Hurst exponent; and Global $\mathrm{H}$, - global Hurst exponent. The results of these calculations are examined to see how they distribute in threedimensional space as well as in two-dimensional space. If there is a match between the correct healthy CFTR sequence and a "trial" sequence then this will declared to be the proper codon. This codon will then be the "payload" for 
whatever delivery mechanism is selected either through a adenovirus vector or delivered by the "fatty molecule" method.

Shown in the figure below is a three-dimensional distribution of the 64 "trial" CFTR sequences. Notice how all the possible choices distribute in threedimensional cloud around the target sequence. The amino acid family grouping tends not to be very strong. As was said earlier in this document, the variance of the local Hurst exponent parameter captures local variability in the pattern structure. Changes in a single base pair change the relationships in the pattern structure. The change of one base pair can introduce a "disturbance" though the length of the sequence. To get a clearer picture of the base pair distribution the next plot involves the fractal dimension, $\mathrm{D}$ versus the $\mathrm{Rg}$ - radius of gyration. Again this is the topological fractal dimension and is defined to be $\mathrm{D}=2-\mathrm{H}$. 


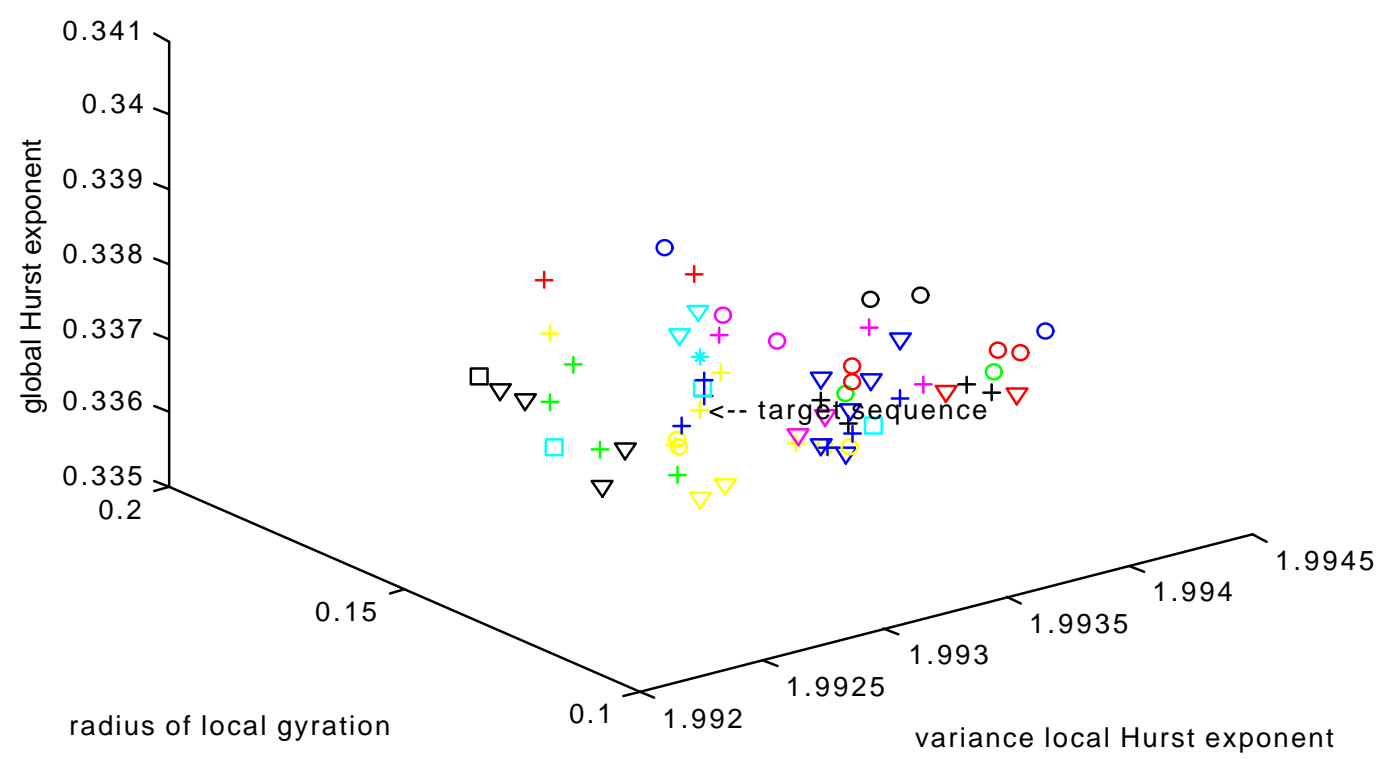

Figure VII-1

Table VII-3 Plot Legend For Figure VII-1 And All Subsequent Figures

\begin{tabular}{|c|c|c|c|c|}
\hline & $\begin{array}{l}\text { Amino } \\
\text { Acid. }\end{array}$ & No. & Data File & Plot Symbol \\
\hline$\pi T$ & Phe & 1 & cystic1.txt & + \\
\hline TTC & Phe & & cystic16.txt & + \\
\hline TTA & Leu & 2 & cystic17.txt & \\
\hline TTG & Leu & & cystic18.txt & \\
\hline CTT & Leu & & cystic.txt & \\
\hline СТC & Leu & & cystic9.txt & \\
\hline CTA & Leu & & cystic7.txt & \\
\hline CTG & Leu & & cystic8.txt & \\
\hline ATT & Ile & 3 & cystic2.txt & + \\
\hline 10. ATC & Ile & & cystic19.txt & + \\
\hline 11. ATA & Ile & & cystic20.txt & + \\
\hline 12. ATG & Met & 4 & cystic21.txt & + \\
\hline 13. GTT & Val & 5 & cystic22.txt & + \\
\hline 14. GTC & Val & & cystic67.txt & + \\
\hline 15. GTA & Val & & cystic35.txt & + \\
\hline 16. GTG & Val & & cystic36.txt & + \\
\hline
\end{tabular}


Table VII-3 Continued Plot Legend For Figure VII-1 And All Subsequent Figures

\begin{tabular}{|c|c|c|c|c|c|}
\hline \multicolumn{2}{|l|}{$\begin{array}{l}\text { Amino } \\
\text { Acid }\end{array}$} & \multirow{2}{*}{$\begin{array}{l}\text { No. } \\
6\end{array}$} & \multicolumn{3}{|c|}{ Data FilePlot Symbol } \\
\hline 17. ТCT & Ser & & cystic23.txt & & + \\
\hline 18. TCC & Ser & & cystic38.txt & & + \\
\hline 19. TCA & Ser & & cystic39.txt & & + \\
\hline 20. TCG & Ser & & cystic40.txt & & + \\
\hline 21. AGT & Ser & & cystic61.txt & & + \\
\hline 22. AGC & Ser & & cystic62.txt & & + \\
\hline 23. СCT & Pro & 7 & cystic41.txt & & + \\
\hline 24. CCC & Pro & & cystic10.txt & & + \\
\hline 25. CCA & Pro & & cystic42.txt & & + \\
\hline 26. CCG & Pro & & cystic43.txt & & + \\
\hline 27. ACT & Thr & 8 & cystic24.txt & & 0 \\
\hline 28. ACC & Thr & & cystic44.txt & & 0 \\
\hline 29. ACA & Thr & & cystic45.txt & & 0 \\
\hline 30. ACG & Thr & & cystic46.txt & & 0 \\
\hline 31. GCT & Ala & 9 & cystic25.txt & & \\
\hline 32. GCC & Ala & & cystic47.txt & & \\
\hline 33. GCA & Ala & & cystic49.txt & & \\
\hline 34. GCG & Ala & & cystic68.txt & & \\
\hline 35. TAT & Tyr & 10 & cystic26.txt & & 0 \\
\hline 36. TAC & Tyr & & cystic51.txt & & 0 \\
\hline 37. TAA & Ter & 11 & cystic27.txt & & 0 \\
\hline 38. TAG & Ter & & cystic52.txt & & 0 \\
\hline 39. TGA & Ter & & cystic31.txt & & 0 \\
\hline 40. CAT & His & 12 & cystic4.txt & 0 & \\
\hline 41. CAC & His & & cystic11.txt & & 0 \\
\hline 42. CAA & Gln & 13 & cystic29.txt & & o \\
\hline 43. CAG & Gln & & cystic53.txt & & o \\
\hline 44. AAT & Asn & 14 & cystic15.txt & & o \\
\hline 45. AAC & Asn & & cystic54.txt & & o \\
\hline 46. $\mathrm{AAA}$ & Lys & 15 & cystic12.txt & & $\nabla$ \\
\hline 47. AAG & Lys & & cystic55.txt & & $\nabla$ \\
\hline 48. GAT & Asp & 16 & cystic70.txt & & \\
\hline 49. GAC & Asp & & cystic56.txt & & \\
\hline 50. GAA & Glu & 17 & cystic30.txt & & $\nabla$ \\
\hline 51. GAG & Glu & & cystic57.txt & & $\nabla$ \\
\hline 52. TGT & Cys & 18 & cystic32.txt & & $\nabla$ \\
\hline 54. TGC & Cys & & cystic58.txt & & $\nabla$ \\
\hline 55. TGG & Trp & 19 & cystic33.txt & & $\nabla$ \\
\hline 56. CGT & Arg & 20 & cystic5.txt & & $\nabla$ \\
\hline 57. CGC & Arg & & cystic.12.txt & & $\nabla$ \\
\hline 58. CGA & Arg & & cystic69.txt & & $\nabla$ \\
\hline 59. CGG & Arg & & cystic60.txt & & $\nabla$ \\
\hline 60. AGA & Arg & & cystic63.txt & & $\nabla$ \\
\hline 61. AGG & Arg & & cystic64.txt & & $\nabla$ \\
\hline 62. GGT & Gly & 21 & cystic37.txt & & $\nabla$ \\
\hline 63. GGC & Gly & & cystic65.txt & & $\nabla$ \\
\hline 64. GGA & Gly & & cystic66.txt & & $\nabla$ \\
\hline 65. GGG & Gly & & cystic14.txt & & $\nabla$ \\
\hline
\end{tabular}




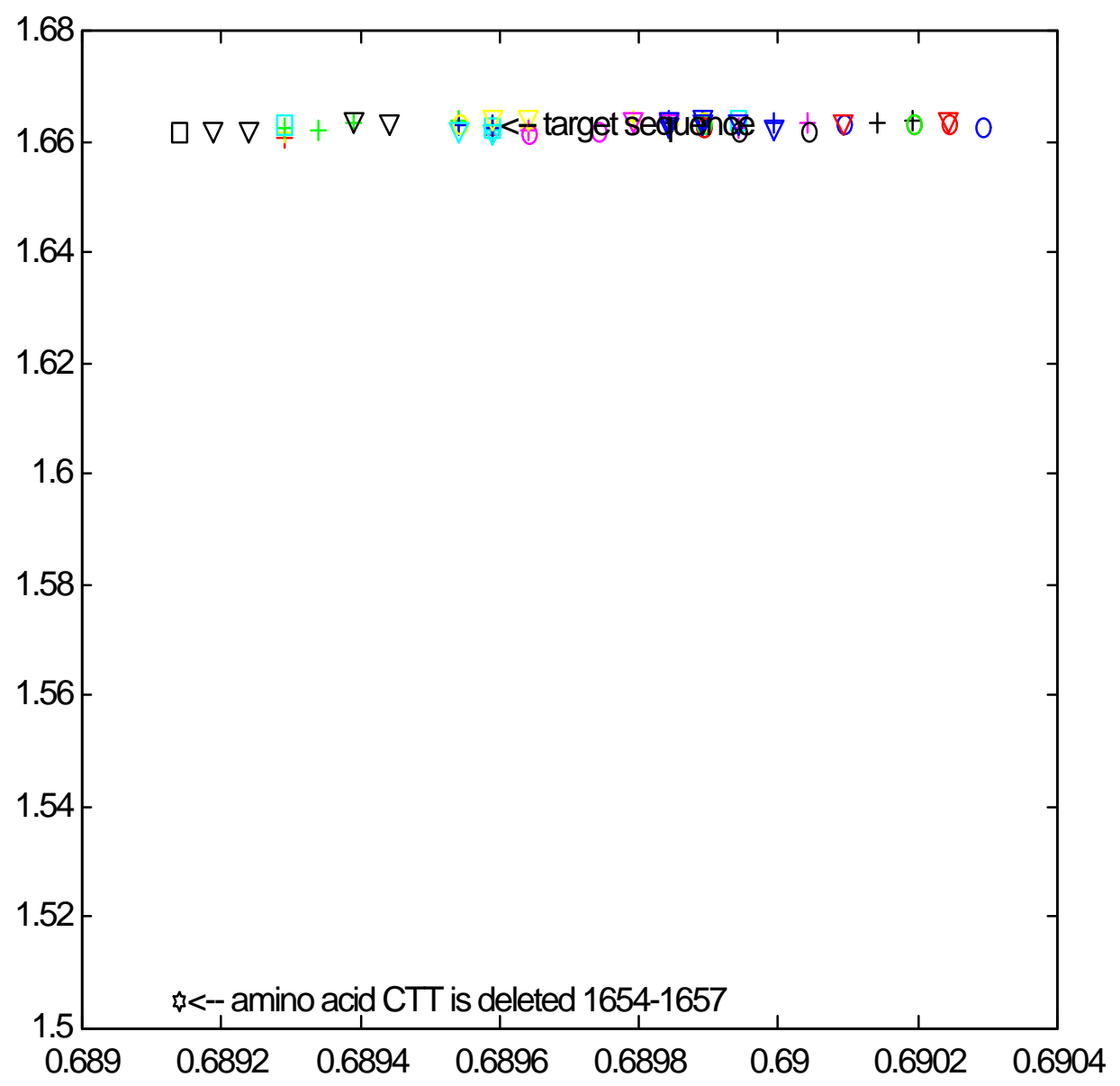

Figure VII-2

This plot shows graphically how devastating a deletion mutation is. It forces a scale on the above plot, that makes the variations in fractal dimensions and radius of gyration calculations of the 64 "trial" sequences look insignificant. The loss of information due to the deletion of three base pairs results in a fractal dimension measurement for the sequence to be 1.5 that represents a Hurst exponent of .5. A Hurst exponent, $\mathrm{H}=.5$ represent mathematical randomness. $\mathrm{H}=.5$ means a sequence that displays neither trend-enforcing bias nor one with trend-reversing bias. This type of result as was pointed out earlier in this work 
and could be a mathematical model of why this type of deletion has such severe biological consequences. As was shown earlier in this document, the amino acid deletion, ATC at sites 1651 -> 1654 gives a similar result. Single base pair deletions show losses of information though not as severe.

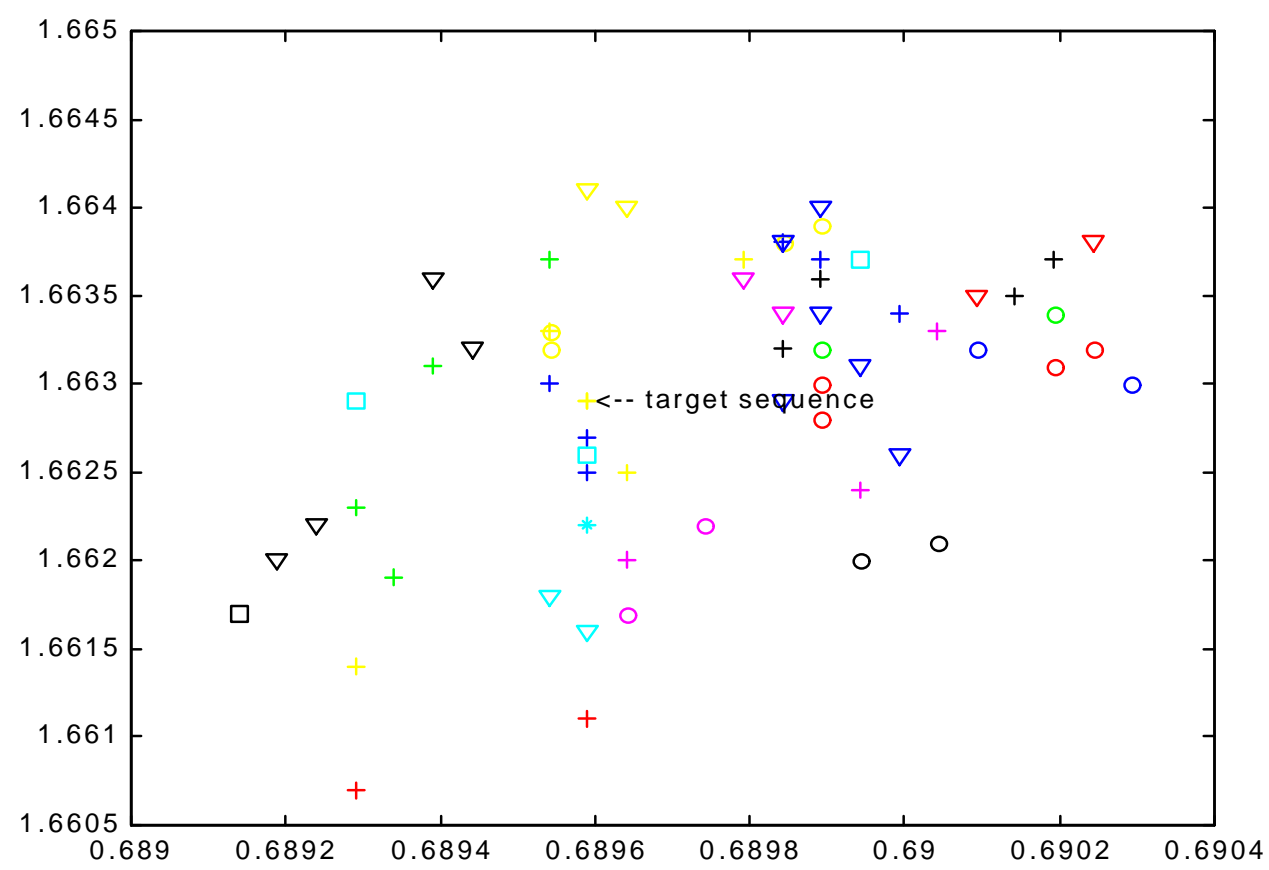

Figure VII-3

B. Further Non-linear Time-frequency Analysis 25,92,108 $^{2}$

All these approaches while building on the traditional linear paradigms that make up the engineering disciplines need to take heed to the advances in non-linear analysis that are forcing a new approach to system analysis and model building. 
Pattern analysis of the DNA base pair sequences is necessary to buttress these new approaches in molecular biology. Patterns describe the interrelation of the physical, chemical and biological properties of DNA. As was done in this work, patterns are analyzed by converting the DNA sequences into a numerical series and then applying non-linear engineering procedures to that numerical series. Consider the example of the human bladder cancer gene. This methodology could also be pursued further by using time-frequency methods such as Villie-Wigner transforms, multi-spectral analysis, etc. These methods could give insight into any non-linear relationships on underlying periodicity that Fourier analysis ignores. This could give further insight into situations like the AT rich region that is thought to be involved in the programming for transcription.

The Ville- Wigner analysis has similarities to both the Fourier analysis and wavelet methods. It is a projection of a data signal onto a family of sinusoids as in the case of the Fourier transform; however it also depends on time or position translations of the series. The Ville-Wigner method is particularly good at uncovering periodic patterns in the data sets where the series is not stationary (i.e. the data may have a strong trend). By using the Wigner-Ville analysis, the data series can be decomposed into its frequency components that vary in temporal or spatial duration.

This work used the IFS transformation as a preprocessor or "data reduction" method. Other methods that could be substituted for the IFS 
transformation step are symmetrized dot patterns and vectorgrams. Both these methods might reveal hidden structure to operator based methods such as Fourier, Ville-Wigner and Wavelet. The sudden changes that reveal mutations and their frequency relationships could then be analyzed.

\section{C) Sequence Complexity ${ }^{30,57}$}

Instead of using the above operator based methods to do R/S analysis an approach can be fashioned using Lyapunov exponents ${ }^{40}$. Lyapunov exponents are the rates at which nearby trajectories diverge or are contracted together. There are as many Lyapunov exponents as there are dimensions in the system. If these exponents are considered they are called the systems Lyapunov's spectrum. In a three dimensional system with a globally attracting fixed point, there will be three negative exponents indicating convergence in three directions. The Lyapunov spectrum can then be represented as three-dimensional coordinate system. In such a three dimensional system with a globally attracting cycle this system will have a Lyapunov's spectrum of $(-,-, 0)$ while an attracting torus will have a spectrum of $(-, 0,0)$. The zero exponents correspond to motions on the attractors. However for chaotic attractors in three dimensions, the sign spectrum is $(-, 0,+)$. Here the positive exponent indicates that there is a direction where the trajectories diverge.

Its is important to understand that Lyapunov exponents are average quantities ${ }^{9,41}$. These can actually be viewed as generalized eigenvalues evaluated over the entire attractor. It 
therefore is possible for local rates of expansion and attraction to vary dramatically. This inherent non-uniformity is why those calculations of Lyapunov's exponents are slow to converge. This is why it is impossible to predict the future behavior of points on the attractor.

Lyapunov exponents can be used as a tool for diagnosing whether or not a system is chaotic. As was said in an earlier paragraph, chaos in deterministic systems implies sensitivity to initial conditions. For ease of discussion time and position in the data sequence will be synonymous.

The characteristic feature of chaotic systems is sensitivity to initial conditions. This implies divergence of initially dynamic adjacent states. The initial state could be known to a high but not infinite degree of precision. The ability to predict future states later decreases because of the diverging trajectories. This means that information is lost. Looking at it conversely, more information is needed to specify the system with the original initial precision. Therefore entropy has increased. ${ }^{13}$

D) Sequence Kolmogorov Entropy ${ }^{9,13,38}$

Using Lyapunov exponent s changes in Kolmogorov entropy ${ }^{21}, \mathrm{~K}$ can be studied for the original sequence and its mutations. This approach can also characterize a dynamic system in three ways:

a) Deterministic, non-chaotic motion, 
b) Deterministic, chaotic motion

c) Random motion.

$\mathrm{K}$ 's value is dependent on the type of motion. Phase space is divided into $\mathrm{N}$ small regions. The system's initial phase is known. The underlying assumptions are the initial phase region exists and the initial probabilities are zero except for the corresponding probability for that phase region and let it equal one. Therefore all the initial information entropy is zero. The above three kinds of motion cause different types of evolution and therefore different changes in the information function.

During a non-chaotic time evolution the system's phase trajectories remain close together. As time $\mathrm{T}$ increases nearby phase points are closely grouped in a small region of phase space and there is no change in information. The Kolmogorov entropy is zero. The categorization is as follows:

a) $K=0$ the motion is regular i.e. periodic, quasi-periodic, or stationary

b) $K>0$ the motion is chaotic

c) $K=\infty$ then the motion is random.

E) L-Systems and DNA Grammars $\mathbf{s}^{49,96,119}$

Formal language theory views languages as sets of strings over some alphabet, and specifies potentially infinite languages with concise sets of rules called grammars. Grammars are an exceptionally well studied methodology, 
familiar to all computer scientists, for the description of complex, higher-order structures embodied in strings of symbols. Moreover, they can be given as input to general-purpose programs called parsers capable of determining whether a given string satisfies the rules of the grammar.

Parser technology is also extensively developed, and has been applied as well to the problem of searching for complex patterns specified by grammars in large amounts of data, in a technique known as syntactic pattern recognition. DNA sequences have been studied from the perspectives of both formal language theory and practical pattern recognition tasks using linguistic tools. A number of results have in the past been presented concerning the mathematical linguistic "complexity" of the language of DNA, e.g. it's position on the Chomsky hierarchy of languages ${ }^{49}$, and the relationship between syntactic structure and secondary structure. This area needs to be related to the dynamical systems analysis methodology of this research effort as well as others.

A novel grammar formalism, called String Variable Grammar, has been defined for DNA It is particularly well suited to the representational needs of $\mathrm{DNA}^{96}$. The practical side entails the development and use of a syntactic pattern recognition system for DNA sequences, called GenLang ${ }^{96}$ that takes advantage of structural and/or hierarchical aspects of a domain by using rule-based methods to describe and discriminate for such structures. The GenLang system has been used successfully to specify and search for tRNA genes, group I 
introns, and most recently, protein-encoding genes, achieving results comparable to other, procedural systems. GenLang studies need to be coordinated with dynamical based studied. Greater insight in the information structure could be gained by this interaction.

F) The Dynamical System Basis of Information Transference in the Action of Antibiotics and Anti-cancer Drugs s7,73,76

Substances that react with DNA to distort its structure and function occupy a central place in present-day cancer chemotherapy. Methods of quantitative measurement of how much information is changed during drug therapy could be of importance in assessing treatment procedures. Future advances in treatment depend crucially upon improving the specificity and selectivity of action of such drugs. Detailed investigation of their molecular basis of action, using purified systems in vitro, and sophisticated biophysical and biochemical techniques, has revealed how DNA can be specifically recognized by drugs and, by extension, reveals possible means for their improvement. Combining multiple centers of reaction within a single molecule provides one such verifiable route. Kinetic and equilibrium DNA-binding data are used to establish the general character of drug interaction with natural and synthetic nucleic acids. One could cooperate with other groups to apply X-ray crystallography and nuclear magnetic resonance spectroscopy to build molecular models, and to identify critical intermolecular contacts involved in the 
recognition of DNA. Then use this information to construct a space-threedimensional model of the drug interaction of quinoxaline and the antibiotic echinomycin. All this involves in one fashion or another information exchange that could be quantified by a methodology used in this research project. Footprinting techniques with sequencing gels can be used detect the preferred sites at which drugs bind to natural DNA fragments.

Sometimes it is necessary to synthesize and clone sequences specifically designed to identify and study strong binding sites or to investigate cooperatively of ligand binding. PCR technology is used to make DNA molecules substituted with unnatural nucleotides as a means of identifying the groups that play a critical role in DNA sequence recognition. The results enable the synthesis of potential drugs that can target critical genes. Both 'digital' and 'analogue' sequence readout mechanisms are involved. Some base-pair substituents are available for recognition by direct ligand binding, such as the 2amino group of guanine. Other sequence-specific features can be recognized indirectly, e.g., by their effect on groove width or helix deformability. By using our growing understanding of the rules that govern the molecular recognition of DNA sequences researchers are beginning to design pharmacologically credible agents to attack targeted genetic elements. This relationship to DNA sequence dynamics should be studied. The results of this study could give molecular biologists more insight into how effective these approaches are. 
G) DNA Computing ${ }^{49,73,74,96}$

Deoxyribonucleic acid (DNA) can be viewed as a digital encoding of information in a biological macromolecule in a manner similar to a computer program. Here are some common molecular biological techniques that are analogous to simple computational operations. DNA may be idealized as a sequence of digits (purine and pyrimidine bases) that are tethered to a chemical backbone that preserves the directionality as well as order of the sequence. All "operations" carried out on information encoded in DNA are performed using enzymes, biological nanomachines that catalyze very specific and reproducible chemical reactions. These reactions are usually carried out under experimental conditions that are far from thermodynamic equilibrium, favoring formation of the products. The advantage of using DNA for computation is one of massive parallelism: in a single reaction $10^{14}$ DNA molecules, each one potentially encoding a different sequence, can be acted upon simultaneously. Any practical strategy employing DNA for computation must take into account the limitations of currently available experimental techniques.

The idea of DNA computing has its roots in the late 1950s, when the late Nobel laureate Richard Feynman first introduced the concept of computing at a molecular level. Feynman's visionary idea was only realized in 1994, when Leonard Adleman performed the first ever truly molecular-level computation using DNA combined with the tools and techniques of molecular biology. Since 
Adleman reported the results of his seminal experiment, there has been a flurry of interest in the idea of using DNA to perform computations. The potential benefits of using this particular molecule are enormous: by harnessing the massive inherent parallelism of performing concurrent operations on trillions of strands, scientists may one day be able to compress the power of today's supercomputer into a single test tube. However, if one compares the development of DNA-based computers to that of their silicon counterparts, it is clear that molecular computers are still in their infancy. Current work in this area is concerned mainly with abstract models of computation and simple proof-ofprinciple experiments.

One goal of this dissertation was to propose a methodology to calculate gross information exchange in DNA sequence due to mutations. Any new results concerning a general model of DNA computation should included a method for quantifying information flow. This is particularly important if an errorresistant implementation of the model is to be defined. Any experimental investigation of the implementation and an assessment of the complexity and viability of DNA computations will demand methods for quantifying structural complexity. If one remembers the history of the search for the structure of DNA molecule it was a struggle to unravel its three-dimensional picture. In providing a detailed description of this molecule and the operations that can be perform by it. One could then describe the basic models of DNA computation. Several such models have been proposed to date, e.g. a parallel filtering model. This 
model was the first to provide a general framework for the elegant expression of algorithms for NP-complete problems. The implementation of such abstract models is crucial to the success of this field. Previous experiments that have been carried out suffer from their reliance on various error-prone laboratory techniques.

There are several open problems regarding DNA computing one is stated as an example for completeness:

1) Take a population of strings, and measure their "fitness" against some criterion. The "most fit" strings have the most descendents in the next generation. Descendants can be mutants, or share material from two (or more) parents. (In practice, such "cross-over" is usually more important than mutation.) Repeat as needed. This is basically a hardware implementation of a "genetic algorithms," but it's the most basic one, and the variations come quickly to mind.

2) Directed molecular evolution, can there be another approach other than artificial selection? In fact, what would natural selection of computer programs look like? 
H) Protein Engineering $27,73,76$

As we have already seen, the biochemical structure of a protein is encoded by the genetic message in DNA that specifies the protein. A unique gene in DNA encodes each protein so that the many thousands of different proteins in living things are encoded by thousands of individual genes. The techniques of genetic engineering allow scientists to decipher individual genes from the thousands found in a chromosome. Once pure, this sequence can be modified or altered using a process called 'in vitro' mutagenesis, which allows one to introduce rational changes into the genetic message. Any change introduced will change the sequence of amino acids in the protein encoded, which may result in the protein folding differently, altering its activity or bestowing a new activity on it. This process of introducing changes into protein molecules is termed protein engineering. In most cases, the changes introduced are done by starting at the genetic level. However, changes can also be introduced via chemical modification in the purified protein using a variety of organic reactions. Such changes are not as stable as those introduced at a genetic level. There is a need to quantify these changes an extension of the work done by this dissertation could be a major step in that direction.

Work on protein engineering that has already been successful has involved altering the chemical properties of protein enzymes. In general, most enzymes work best at an optimum $\mathrm{pH}$ and temperature, usually $\mathrm{pH} 7$ and $37^{\circ} \mathrm{C}$. 
However, many applications of enzymes as hydrolytic agents or in biochemical synthesis require them to work at much higher temperatures, e.g. $95^{\circ} \mathrm{C}$. The higher temperatures may overcome viscosity problems or make the process more economic. Recently biochemists have been able to introduce changes into many proteins, which confers thermostability to the protein. These custom designed proteins are of more use and in some cases allow the development of processes that would not otherwise be viable. Currently the ground rules for engineering proteins are only in the preliminary stages of examination. Future developments will lead to huge advances in the understanding of protein structure and function. Such advances may hinge on being able to quantify the information structure through patterns as well as the physical structure. This information will enable the protein engineer to design new catalytic entities.

I) Forensic DNA ${ }^{58,76,96}$

When DNA is subjected to restriction-enzyme activity, fragments of various sizes are formed and each of these fragments has a dynamical complexity. Each human family line has its own unique pattern of restrictionenzyme DNA fragments. This variation in patterns of DNA fragments found in human genetic lineages is called "restriction-fragment length polymorphism" (RFLP). Because each person, except for identical twins, is formed from different combinations of the genetic material from two family lines, the pattern of sizes of the fragments from an individual is unique and can serve as a "DNA 
fingerprint" of that person. This fact has been crucial in identifying assailants in a number of violent crimes where the victims were unable to do so. In these cases, bloodstains or semen stains on clothing, sperm cells found in a vaginal swab taken after a rape, or root hairs of the assailant were available for analysis. Extraction of DNA from dried blood or from one of the other sources, followed by enzymatic digestion, results in an unambiguous match of DNA fragments with DNA obtained from cells of the accused. Where bloodstains are available, clear-cut DNA fingerprints are obtainable from samples that are as much as four years old. The methodology used in this thesis, since it has the ability to assign a complexity value to whatever DNA sequence that it analyzes could be used in conjunction with standard DNA fingerprinting techniques.

J) Evolution Studies ${ }^{31,58,96}$

The principal contribution of genetics to the understanding of evolution has been the explanation of the inheritance of variability in individuals of the same species. Gregor Mendel discovered the basic principles of inheritance in 1865, but his work was unknown to Darwin. Mendel's work was "rediscovered" by other scientists circa 1900 . From that time to 1925 the science of genetics developed rapidly, and many of Darwin's ideas about the inheritance of variations were found to be incorrect. Only in the years since 1925 has natural selection again been recognized as essential in evolution. The modern theory of evolution combines the findings of modern genetics with the basic framework 
supplied by Darwin and Wallace, creating the basic principle of population genetics. Modern population genetics was developed largely during the 1930s and '40s by the mathematicians J. B. S. Haldane and R. A. Fisher and by the biologists Theodosius Dobzhansky, Julian Huxley, Ernst Mayr, George Gaylord Simpson, Sewall Wright, Berhard Rensch, and G. Ledyard Stebbins. Mathematical complexity theory that is the basis of the dynamical systems model has the potential of be fundamental to evolution. This theory has explained in mathematical systems how complex structures can develop from perturbations in the system dynamics of simpler ones.

According to the modern theory, mutation and genetic recombination produce variability among individuals in a population of sexually reproducing organisms. The resulting genetic variability is subject to natural selection in the environment. Assuming fossil DNA was available its complexity could be quantified and the evolutionary progress as an information flow of, for example the horse could then be tracked. Given the power that non-linear system modeling has over linear methods for prediction, gaps in sequences could possibly be closed with a higher degree of accuracy and thus enable evolutionary microbiologists to better track the evolutionary history of a species. 


\section{Bibliography}

1. Abraham, B., Albano, A.M., Das, B., DeGuzman, G., Young, S., Gioggia, R.S., Puccioni, G.P. and Tredicce, J.R. Phys. Lett. A 114, 217, 1986

2. $\quad$ Alleegrini, P., Barbi,M., Grigolini,P., West, B.J. 'Dynamical Model for DNA Sequences', Phys.Rev.E. Nov. 1995 Vol.52, No.5

3. Altaski, M., Morev, O. and Polzov, R., 'Wavelet Analysis of DNA Sequences', Genetic Analysis:Biomedical Engineering 12 (1196) 165-168

4. Anderson, R.M and R.M. May. 1982 , 'Directly Transmitted Infectious Disease: Control by Vaccination'. Science. 215:1053-1060

5. Aron, J.L. and I.B. Schwartz. 1984. 'Seasonality and Period Doubling Bifurcation in an Epidemiological Model'. j. Theor. Biol. 110: 665-679

6. Arfken, G., Mathematical Methods for Physicists, Academic Press, 1970

7. Argoul, F., Arneodo, A, Grasseau, G., Gagne, Y., Hepfinger, E.J. and Frisch, U. Experimental Evidence for Homoclinic Chaos in the Belousov-Zhabotinski', Nature $338,51,1989$

8. Aubry, N., Holmes, P., Lumley, J.L., and Stone, E., J. 'The Dynamics of Coherent Structures in the Wall Region of a Turbulent Boundary Layer', Fluid Mechanics, 192, 115, 1988

9. Baker, G.L. and Golub, J.P., Chaotic Dynamics, Cambridge University Press, 1990

10. Barnsley, M.F., Devaney, R.L., Mandelbrot, B.B., Peitgen, H.O., Saupe, D. and Voss, R.F., The Science of Fractal Images, Springer-Verlag, New York, 1988

11. Bartlett, M.S., Stochastic Processes, Cambridge University Press, 1955

12. Belair,J., Glass, L. An Der Heiden, U and Milton, J. (eds.), Dynamical Disease: Mathematical Analysis of Human IIIness, AIP, 1995

13. Benettin, G., Galgani,L., and Strelcyn, J.M., 'Kolomogorov Entropy and Numerical Experiments', Phys. Rev. A 14, 2338, 1976

14. Bowen, J.E.., Data Visualization in Molecular Science ,Addison Wesley, 1995

15. Box,G.E.P., Jenkins, G.M. and Reinsel, G.C. Time Series Analysis: Forcasting and Control ,Prentice Hall Englewood Cliffs New Jersey, 1994 
16. Brock, W.A. and Sayers, C.L. 'Is the Business Cycle Characterized by Deterministic Chaos?', Journal of Monetary Economics, 22, 71-90 1988

17. Broomhead, D.S. and King, G.P., 'Extracting Qualitative Dynamics from Experimental Data', Physica D 20, 2171986

18. Broomhead, D.S. and Jones, R., 'Linear Filters and Non-linear Systems', J. Royal Stat. Soc. 54,1992

19. Berge,P., Pomeau,Y. and Vida,C. ,Order Within Chaos: Toward a New Approach to Turbulence, J.Wiley. New York, New York, 1986

20. Cambel, A.B. Applied Chaos Theory, Academic Press, 1993

21. Casdagli, M., 'Non-linear Prediction of Chaotic Time Series', Physica D 35, 33, 1989

22. Casdagli, M., in Non-linear Prediction and Modeling, Casdagli,M., and Eubank, E., (eds.) Addison Wesley, Reading, MA 1991

23. Casey, E.J.., Biophysics, Rheinhold 1962

24. Chui, C.K., An Introduction to Wavelets Analysis, Academic press, Boston,1992

25. Cohen, L. Time-Frequency Analysis, PTR Prentice-Hall, 1996

26. Crandall, R.E., Projects in Scientific Computing, Springer-Verlag, 1994

27. Elliot, W.H. and Elliott, D.C., Biochemistry and Molecular Biology, Oxford University Press, 1997

28. Eckman, J.P., 'Roads to Turbulence in Dissipative Dynamical Systems' Rev. Mod. Phys 54(4) part 1, 643-654

29. Farmer, J.D., Ott, E. and Yorke, 'The Dimension of Chaotic Attractors', Physica D 7, 153,1983

30. Farmer, J.D., Sidorivich, J.J., 'Predicting Chaotic Time Series', Phys. Rev. Lett., $59,845,1987$ D 7, 153, 1983

31. Farmer, J.D. and Sidorovich, J., in Evolution, Learning and Cognition, Lee,Y.C. (ed.) World Scientific, Singapore, 1988

32. Feder, J., Fractals ,Plenum Press New York 1988 
33. Feigenbaum, M.J., 'Qualitative Universality for a Class of Non-linear Transformations', J. Stat. Phys. 19, 25-35 1978

34. Feeny, B.F. and Moon, F.C. , 'Autocorrelation on Symbolic Dynamics for a Chaotic Dry-Friction Oscillator', Phys. Lett. A 141, 397-401 1989

35. Frank-Kamenetskii, M.D., Unraveling DNA, VCH Publishers, 1993

36. Crutchfield, J.P., and McNamara, B.S., 'Equations of Motion from a Data Series', Complx Systems, 1:47-59

37. Gershenfeld, N.A. and Weigend, A.S., The Future of Time Series: Learning and Understanding, Predicting the Future and Understanding the past: A Comparison of Approaches, Addison-Wesley, Reading, MA, 1993

38. Gouyet, J.F., Physics and Fractal Surfaces, Springer-Verlag, 1996

39. Grassbeger, P. and Procaccia, I., 'Measuring the Strangeness of Strange Attractors', Physica, 9D, 189-208

40. Grassbeger, P. and Procaccia, I., Characterization of Strange Attractors', Phys. Rev. Lett. 50, 346-350 1983

41. Grassbeger, P. and Procaccia, I., 'Dimensions and Entropies of Strange Attractors from a Fluctuating Dyanmics Approach', Physica D 13, 34-45, 1984

42. Greenside, H.S., Wolf, A., Swift, J. and Pignataro, T. Phys. Rev. A 25, 3453, 1982

43. Haddad, R.A and Akansu, A.N., Multi-Resolution Signal Decomposition, Academic Press, 1992

44. Halsey, T., Jensen, M.H., Kadanroff, L.P., Procaccia, I., and Shraiman, B.I., 'Fractal Measures and Their Singularities: The Characterization of Strange Sets', Phys, Rev. A., 33, 1141-1151

45. Hamori, E., 'Novel DNA Sequence Representations', nature, Vol. 314, 18-21, April 1985

46. Havstad, J.W. Ehlers, C.L. 'Attractor Dimension of Non-stationary Dynamical Systems from Small Data Sets', Phys. Rev. A 39, 845-850, 1989

47. Holden, A.V. Chaos, Princeton University Press, 1986

48. Hoppensteadt, F.C. and Peskin, C.S., Mathematics in Medicine and the Life Sciences, Springer-Verlag, 1992 
49. Hoftstadter, D.R., Godel, Escher, Bach: An Eternal Golden Braid, Basic Books, 1979

50. Jeffrey, H.J. 'Chaos Game Visualization of Sequences', Com. \& Graph. 16, 25-33 1992

51. Jones, C. L., Lonegan, G.T., Mainwaring, D.E., 'Wavelet Packet Computation of the Hurst Exponent', Journal of Physics, A:292509-2527, 1996

52. Kasper, F. and Schuster, H.G., 'Easily Calculable Measure for the Complexity of Spatio-Temporal Patterns', Phys. Rev. A 36, 842-848 1987

53. Kennel, M.B. Brown, R. and Abarbanel, H.D.L. 'Determining Embedding Dimension for Phase-Space Reconstruction Using a Geometrical Construction', Phys. Rev. A 45, 3403-3411, 1992

54. Kennel, M.B. and Isabell, S., 'Method to dsitinguis Possible Chaos from Colored Noise and to Determine the Embedding Parameters. Phys. Rev. A 46, 3111-3123 1992

55. Laeri, F. Comp. Phys. 4, 6271990

56. Lauwerier, H. A., 'Two-Dimensional Iterations', pp 58-95. In. Holden. A. V. (ed.) Chaos. Manchester University Press, 1986

57. Lempel, A. and Ziv, J., 'On Complexity of Finite Sequences', IEEE Transactions in Information Theory IT-22, 751976

58. Levin,B., Genes, Oxford University Press, 1994

59. Linder, E.S., 'Mapping Heredity: Using Probabilistic Models and Algorithms to Genes and Genomes', (Part I). Notices Am. Math. Soc. ,42,7 July 1995

60. Linder, E.S., 'Mapping Heredity: Using Probabilistic Models and Algorithms to Genes and Genomes', (Part II). Notices Am. Math. Soc. ,42,8 August 1995

61. Lorenz, E. N., 'Deterministic Non-periodic Flow', J. Atm. Sci. 357: 130-141, 1963

62. Lorenz, E. N., 'On the Prevalence of Aperiodicity in Simple Systems', pp53 In. Global Analysis. Grmela, M., Marsden, J. E. (eds) Lect. Notes in Math. 755. Springer-Verlag, Berlin 1979

63. Lumley, J.L. Stochastic Tools in Turbulence, Academic Press, New York, 1970

64. Mackey, M.C., and Glass. Leon 1977,' Oscillation and Chaos in Physiological Control Systems', Science. 197: 287-289. 
65. Mandelbrot, B.B., The Fractal Geometry of Nature, Freeman Press, San Francisco 1982

66. Marotto, F., 'Chaotic Behavior in the Henon Mapping', Commun. Math. Physics 63: 187,1979

67. May, R.M., 'Simple Mathematical Models with Very Complicated Dynamics', Nature 261, 4591976

68. May, W.D., Edges of Reality, Insight Books, 1996

69. Misti, M., Misti, Y., Oppenheim, G. and Poggi, J.M., 'Wavelet Toolbox Users Guide', Mathworks, pp 8-30, 1996

70. Mullin,T. (ed.) The Nature of Chaos., Clarendon Press Oxford, 1993

71. Moon. F. C., 'Fractal Basin Boundary for Chaos in a Two-State Mechanical Oscillator', Phys. Rev. Lett. 53: 962-964, 1984

72. Moon, F.C., Chaotic and Fractal Dynamics, John Wiley \& Sons, 1992

73. Nicholl, D.S.T., An Introduction to Genetic Engineering, Cambridge University Press, 1996

74. Nicholis, G., and Prigogine, I., Exploring Complexity, W.H.Freeman \& Co. 1989

75. Nussbaum, J. and Ruina, A. 'A Two Degree-of-Freedom Earthquake Model with Static/Dynamic Friction', Pure \& Applied Geophysics, 125(4), 629-656, 1996

76. Old, R.W., Principles of Gene Manipulation, Blackwell Scientific Press, 1994

77. Ott, E., Sauer, T. and Yorke, J. (eds.), Coping With Chaos, John Wiley \& Sons,NewYork, 1994

78. Pancham, S., 'Evidence of the Multi-Fractal Market Hypothesis Using Wavelet Transforms', Ph.D. Dissertation in Business Administration, Florida International University, 1994

79. Parker, T.S., and Chua, L.O., Practical Numerical Algorithms for Chaotic Systems, Springer-Verlag, 1989

80. Peitgen. H.O. and Richter P.H., The Beauty of Fractals: Images of Complex Dynamical Systems. Springer-Verlag. Berlin, 1986

81. Peters, E.E., Chaos and Order in the Capital Markets, John Wiley \& Sons, 1991 
82. Peters, E.E., Fractal Market Analysis, John Wiley \& Sons, 1994

83. Piccardo, J.C., 'A Transforming Function for the Generation of Fractal Functions from Nucleotide Sequences', from Visualizing Biological Information, pp 96-118 (ed) Pickover, C.A., Wolrd Scientific, 1995

84. Pickover, C.A., Computers, Pattern, Chaos and Beauty, St. Martin's Press, New York, 1990

85. Pickover, C.A., Fractal Horizons:The Future Use of Fractal, St. Martin's Press, New York, 1996

86. Pickover, C.A., 'Use of Symmetrized Dot Patterns in Characterizations and Detection of Cardiac Abnormalities',IBM Technical Disclosure Bulletin, Vol. 29 No. 6, Nov. 1986

87. Pickover, C.A., 'DNA Vectorgrams Representations of Cancer Genes as Movements on a Two-Dimensional Cellular Lattice', IBM Technical Disclosure Bulletin , Vol. 29 No. 6, Nov. 1986

88. Pickover, C.A., 'Frequency Spectra of Gene Sequences: An Application to a Human Bladder Cancer Gene', Journal of Molecular Graphics, 2:50-52 1976

89. Pickover, C.A., 'Computer Drawn Faces Characterizing Nucleic Acid Sequences' Journal of Molecular Graphics, 2:107-110, 1980

90. Pickover, C.A., 'On Genes and Graphics', Speculations in Science and Technology, Vol.12, No.1 Page 5, 1983

91. Press, W.H., Flannery, B.P., Teukolsky, and Vetterling, W.T., Numerical Recipes ,Cambridge University Press 1992

92. Qian, S, and Chen, D., Joint-Time Frequency Analysis: Methods and Applications, PTR Prentice-Hall, 1996

93. Rasband, S.N., Chaotic Dynamics of Non-linear Systems, Wiley-Interscience, 1990

94. Rowlands, G., Non-Linear Phenomena in Science and Engineering, Ellis Horwood, London, 1990

95. Saltzman, B., 'Finite Amplitude Convection as an Initial Value Problem', I.J. Atm. Sci. 19: 29-341 1993

96. Saunders, P. and Goodwin, B. (ed) Theoretical Biology: Epigentic and Evolutionary Order from Complex Systems, Johns Hopkins University Press, 1989 
97. Sayers, C.L. ,'Chaos and the Business Cycle', pp115-125. In, Krasner, S. (ed.) The Ubiquity of Chaos, AAAS. Washington D.C., 1990

98. Schaffer, W.M.,' Can Non-liner Dynamics Help Us Infer Mechanisms in Ecology and Epidemiology? 'IMA J. Math Apll. Med. Biol. 2: 221-252, 1985

99. Schaffer, W.M. and Truty, G.L. ,' Chaos vs. Noise-Driven Dynamics', pps. 77-96. In. Hastings, A.M. (ed.) Some Questions In Biology Amer. Math Soc., 1989

100. Schaffer W.M and Tidd C.W. , NLF: Non-linear Forcasting for a Dynamic System. Dynamical Science Corp., 1991

101. Silverman, B.D. and Linsker, R., 'A Measure of DNA Periodicity', Theor. Biology, 1986, 118,295-300

102. Simo,C. ,' On the Heon-Pomeau Attractor',. J. Stat. Phys. 21:465-494, 1979

103. Singh, A. and Joseph, D.D., 'Auto-Regressive Methods for Chaos on Binary Sequences for the Lorentz Attractor', Phys. Lett. A 135, 247, 1989

104. Sirovich, L and Rodriguez, J.D., Phys. Lett. A 120, 211, 1987

105. Smith, L.A. 'Intrinsic Limits on Dimenskion Calcuations', Phys. Lett. A 133, 283, 1988

106. Sparrow, C. The Lorentz Equaiotns: Bifurcations, Chaos and Stranger Attractors. Springer-Verlag, New York, 1982

107. Sprott, J.C., Strange Attractors: Creating Patterns in Chaos M \& T Books, New York, 1993

108. J. C. Sprott and Rowlands, George, Chaos Data Analyzer Manual Physics Academy Software, 1995

109. Stanley, E., Buldyrev, S.V., Goldberger, A.L., Havlin, S. Mantegena, R.N., Peng,C.K., and Simpons, M., 'Scale Invariant Features of Coding and Non-coding DNA Sequences', from Fractal Geometry in Biological Systems, pp 15-27, (eds.) lannaccone, P.M. and Khokha, M., CRC Press, 1995

110. Stone, L., 'Colored Noise or Low Dimensional Chaos', Proc., Royal Soc. London, B $330,235-240,1990$

111. Stoop, R. and Parisi, J., 'Calculation of Lyanpunov Exponents Avoiding Spurious Elements', Physica D 50 89-93, 1991 
112. Strogatz, S.H., Non-linear Dynamics and Chaos, Addison-Wesley, 1994

113. Sugihara, G. , Grenfell, B., and May, R.M., 'Non-linear Forecasting as a Way of Distinguishing Chaos from Measurement Error in Time Series', Nature, 344, 734, 1990

114. Takens, F. ,Lecture Notes in Mathematics, 898, Rand, D.A. and Young, L. S., (eds.)Springer-Verlag, 1981, Berlin

115. Tsonis, A.A., Chaos: From Theory to Applications, Plenum Press, New York, 1992

116. Vautard, R. and Ghil, M., 'Singular Spectrum Analysis: A Toolkit for Short Noisy Signals', Physica D. 35, 3951989

117. Vehel, J.L., Lutton, E. and Tricot, C., (eds.), Fractals in Engineering: Theory to Application, Springer-verlag, 1997

118. Wornell, G.W., Signal Processing with Fractals: A Wavelet Based Approach, PTR Prentice Hall, 1996

119. Yockey, H.P., Information Theory and Molecular Biology, Cambridge University Press, 1992

120. Yorke, J.A. and Alligood, K.T., 'Period Doubling Cascades of Attractors: A Prequisite for Horseshoes', Commun. Math Phys. 101: 305-321, 1985

121. Welsh, M.J. and Smith, A.E., 'Cystic Fibrosis', Scientific American, December, 1995

122. West, B.J., Fractal Physiology and Chaos in Medicine, World Scientific, 1990

123. Williams, A, Chenault, K.D. and Melcher, U., 'Graphical Representation of Amino Acid Sequences' from Visualizing Biological Information, pp 6-14 (ed) Pickover, C.A., World Scientific, 1995

124. Wolf, A., Swift, J.B. ,Swinney, H.L., and Vastano, J.A. , ' Determining Lyanpunov Exponents from a Time Series', Physica D 16, 285-290, 1985

125. Zhang, C.T., 'Diagrammatic Representation of Base Composition in DNA Sequences', from Visualizing Biological Information, pp 84-95 (ed.) Pickover, C.A., World Scientific, 1995 


\section{Appendix A. Gene Data Set}


Reference: Science 245, 1066-1080 and Human Biology 67, 561-576

Data and reference kindly provided by Dr. Mike R. Miller.

Start codon is on line 121 and is in upper case, i.e. ATG.

laaftggaagc aaatgacatc acagcaggtc agagaaaaag ggttgagcgg caggcaccca 61 gagtagtagg tctttggcat taggagcttg agcccagacg gccctagcag ggaccccagc 121 gcccgagaga cc ATGcagag gtcgcctctg gaaaaggcca gcgftgtctc caaactttt

181 ttcagctgga ccagaccaat tttgaggaaa ggatacagac agcgcctgga attgtcagac

241 atataccaaa tcccttctgt tgaftctgct gacaatctat ctgaaaaft ggaaagagaa

301 tgggatagag agctggcttc aaagaaaat cctaaactca ttaatgccct tcggcgatgt

361 tttftctgga gatttatgft ctatggaatc tttttataft taggggaagt caccaaagca

421 gtacagcctc tcttactggg aagaatcata gcttcctatg acccggataa caaggaggaa

481 cgctctatcg cgatttatct aggcataggc ttatgccftc tctttaftgt gaggacactg

541 ctcctacacc cagccattt tggccftcat cacattggaa tgcagatgag aatagctatg

601 tttagtttga tttataagaa gactttaaag ctgtcaagcc gtgttctaga taaataagt

661 attggacaac ttgttagtct ccttccaac aacctgaaca aatttgatga aggacttgca

721 ttggcacatt tcgtgtggat cgctcctttg caagtggcac tcctcatggg gctaatctgg

781 gagftgftac aggcgtctgc cttctgtgga cttggtttcc tgatagtcct tgccctttt

841 caggctgggc tagggagaat gatgatgaag tacagagatc agagagctgg gaagateagt

901 gaaagacttg tgattacctc agaaatgaft gaaaatatcc aatctgftaa ggcatactgc

961 tgggaagaag caatggaaa aatgattgaa aacttaagac aaacagaact gaactgact

1021 cggaaggcag cctatgtgag atacttcaat agctcagcct tcttcttctc agggftctt

1081 gtggtgttt tatctgtgct tccctatgca ctaatcaaag gaatcatcct ccggaaata

1141 ttcaccacca tctcaftctg caftgftctg cgcatggcgg tcactcggca atttccctgg

1201 gctgtacaaa catggtatga ctctcttgga gcaataaca aaatacagga tttcttacaa

1261 aagcaagaat ataagacatt ggaatataac ttaacgacta cagaagtagt gatggagaat

1321 gtaacagcct tctgggagga gggatttggg gaattatttg agaaagcaaa acaaacaat

1381 aacaatagaa aacttctaa tggtgatgac agcctcttct tcagtaattt ctcacttct

1441 ggtactcctg tcctgaaaga taftaatttc aagatagaaa gaggacagtt gttggcggtt

1501 gctggatcca ctggagcagg caagacttca cttctaatga tgaftatggg agaactggag

1561 ccttcagagg gtaaaaftaa gcacagtgga agaatttcat tctgttctca gttttcctgg

1621 aftatgcctg gcaccattaa agaaatatc atctttggtg tttcctatga tgaatataga

1681 tacagaagcg teatcaaagc atgccaacta gaagaggaca tctccaagft tgcagagaaa

1741 gacaatatag ftcttggaga aggtggaatc acactgagtg gaggtcaacg agcaagaatt 1801 tctttagcaa gagcagtata caaggatgct gatttgtaft taftagactc tccftftgga

1861 tacctagatg ttttaacaga aaagaaata tttgaaagct gtgtctgtaa actgatggct 1921 aacaaacta ggattttggt cacttctaaa atggaacatt taagaago tgacaaata

1981 ttaatttga atgaaggtag cagctattt tatgggacat tttcagaact ccaaatcta 2041 cagccagact ftagctcaaa actcatggga tgtgaftctt tcgaccaaft tagtgcagaa 2101 agaagaaaft caatcctaac tgagacctta caccgtttct caftagaagg agatgctcct 2161 gtctcctgga cagaaacaaa aaacaatct tttaaacaga ctggagagft tggggaaaaa 2221 aggaagaaft ctaftctcaa tccaatcaac tctatacgaa aattttccat tgtgcaaaag 2281 actcccttac aaatgaatgg catcgaagag gaftctgatg agcctttaga gagaaggctg 2341 tccttagtac cagaftctga gcagggagag gcgatactgc ctcgcatcag cgtgatcagc

2401 actggoccca cgcttcaggc acgaaggagg cagtctgtcc tgaacctgat gacacactca 2461 gftaaccaag gtcagaacat tcaccgaaag acaacagcat ccacacgaaa agtgtcactg 2521 gcccctcagg caaacttgac tgaactggat atataftcaa gaaggttatc tcaagaaact 2581 ggcftggaaa taagtgaaga aaftaacgaa gaagacttaa aggagtgcct ttttgatgat 2641 atggagagca taccagcagt gactacatgg aacacatacc ttcgatatat tactgtccac 2701 aagagcttaa tttttgtgct aatttggtgc ftagtaattt ftctggcaga ggtggctgct 2761 tctttggftg tgctgtggct ccttggaaac actcctettc aagacaaagg gaatagtact 2821 catagtagaa ataacagcta tgcagtgaft atcaccagca ccagftcgta ftatgtgttt 2881 tacatttacg tgggagtagc cgacactttg cttgctatgg gaftcttcag aggtctacca 2941 ctggtgcata ctctaatcac agtgtcgaaa attttacacc acaaatgft acattctgft 
3001 cttcaagcac ctatgtcaac cctcaacacg ftgaaagcag gtgggaftct taatagaftc 3061 tccaagata tagcaatttt ggatgaccft ctgcctctta ccatatttga cttcatccag 3121 ftgftaftaa ftgtgaftgg agctatagca gftgtcgcag ttttacaacc ctacatcttt

3181 gftgcaacag tgccagtgat agtggcttt aftatgftga gagcatattt cctccaaacc 3241 tcacagcaac tcaaacaact ggaatctgaa ggcaggagtc caattttcac tcatcttgft 3301 acaagcttaa aaggactatg gacacttcgt gccttcggac ggcagccfta ctttgaaact 3361 ctgftccaca aagctctgaa tttacatact gccaactggt tcttgtacct gtcaacactg 3421 cgctggftcc aaatgagaat agaaatgaft tttgtcatct tcttcaftgc tgftaccftc 3481 atttccattt taacaacagg agaaggagaa ggaagagftg gtaftatcct gactttagcc 3541 atgaatatca tgagtacaft gcagtgggct gtaaactcca gcatagatgt ggatagcttg 3601 atgcgatctg tgagccgagt ctttaagftc aftgacatgc caacagaagg taaacctacc 3661 aagtcaacca aaccatacaa gaatggccaa ctctcgaaag ttatgattat tgagaaftca 3721 cacgtgaaga aagatgacat ctggccctca gggggccaaa tgactgtcaa agatctcaca 3781 gcaaataca cagaaggtgg aaatgccata ftagagaaca tttccttctc aataagtcct 3841 ggccagaggg tgggcctctt gggaagaact ggatcaggga agagtacttt gftatcagct 3901 tttttgagac tactgaacac tgaaggagaa atccagatcg atggtgtgtc ftgggaftca

3961 ataactttgc aacagtggag gaaagcctt ggagtgatac cacagaaagt atttatttt 4021 tctggaacat.ftagaaaaa cttggatccc tatgaacagt ggagtgatca agaaatatgg 4081 aaagftgcag atgaggftgg gctcagatct gtgatagaac agtttcctgg gaagcttgac 4141 tttgtccttg tggatggggg ctgtgtccta agccatggcc acaagcagft gatgtgcttg 4201 gctagatctg ftctcagtaa ggcgaagate ftgctgcttg atgaacccag tgctcatttg 4261 gatccagtaa cataccaaat aaftagaaga actctaaac aagcatttgc tgaftgcaca

4321 gtaaftotct gtgaacacag gatagaagca atgctggaat gccaacaaft tttggtcata 4381 gaagagaaca aagtgcggca gtacgaftcc atccagaaac tgctgaacga gaggagcctc 4441 ftccggcaag ccatcagccc ctccgacagg gtgaagctct ftccccaccg gaactcaagc 4501 aagtgcaagt ctaagcccca gaftgctgct ctgaaagagg agacagaaga agaggtgcaa 4561 gatacaagg $\mathrm{c}$ ft tagagagc agcataaatg ftgacatggg acatttgctc atggaaftgg 4621 agctcgtggg acagtcacet catggaaftg gagctcgtgg aacagftacc tctgcctcag 4681 aaacaagga tgaaftaagt tft=aa aaaagaaca tttggtaagg ggaaftgagg 4741 acactgatat gggtcftgat aaatggcttc ctggcaatag tcaajtgtg tgaaaggtac 4801 ftcaaatcct tgaagattta ccacttgtgt tttgcaagcc agattttcct gaaaaccctt 4861 gccatgtgct agtaaftgga aaggcagctc taaatgtcaa tcagcctagt tgatcagctt 4921 aftgtctagt gaactcgft aattgtagt gftggagaag aactgaaatc atacftctta 4981 gggftatgat taagtaatga taactggaaa cttcagcggt ftatataagc ftgtaftcct 5041 ttttctctcc tctccccatg atgtttagaa acacaactat aftgtttgct aagcaftcca 5101 actatctcat ttccaagcaa gtaftagaat accacaggaa ccacaagact gcacatcaaa 5161 atatgeccca ttcaacatct agtgagcagt caggaaagag aacttccaga tcctggaaat 5221 cagggttagt attgtccagg tctaccaaaa atctcaatat ftcagataat cacaatacat 5281 ccct tacctg ggaaagggct gftataatct ftcacagggg acaggatggt tcccttgatg 5341 aagaagftga tatgccttt cccaactcca gaagtgaca agctcacaga cctttgaact 5401 agagtttagc tggaaagta tglagtgca aaftgtcaca ggacagccct tctttccaca 5461 gaagctccag gtagagggtg tgtaagtaga taggccatgg gcactgtggg tagacacaca 5521 tgaagtccaa gcatttagat gtataggftg atggtggtat gttttcaggc tagatgtatg 5581 tacftcatgc tgtctacact aagagagaat gagagacaca ctgaagaagc accaatcatg 5641 aaftagftet atatgcttct gftftataat tttgtgaagc aaaal ctctaggaaa 5701 tatttaftft aataatgttt caacatata ftacaatgct gtaftftaaa agaatgafta 5761 tgaaftacat ftgtataaa taattttat atttgaaata ftgactttt atggcactag 5821 tattttatg aaataftatg ftaaactgg gacaggggag aacctagggt gataftaacc 5881 aggggccatg aatcaccttt tggtctggag ggaagccftg gggctgatcg agftgftgcc 5941 cacagctgta tgaftcccag ccagacacag cctcttagat gcagftctga agaagatggt 6001 accaccagtc tgactgtttc catcaagggt acactgcctt ctcaactcca aactgactct 6061 taagaagact gcaftataft taftactgta agaaatatc acftgtcaat aaatccata 6121 catttgtgt 


\section{Appendix \\ B. Mutation Data Set}




\section{DNA Sequence Mutations Both Natural and Artificial}

Used in this Analysis

\begin{tabular}{|c|c|c|c|c|}
\hline case & data file & $\begin{array}{l}\text { Line } \\
\text { No. }\end{array}$ & $\begin{array}{l}\text { BP Position in } \\
\text { sequence }\end{array}$ & comment \\
\hline 1 & cftro.txt & & & $\begin{array}{l}\text { original } \\
\text { sequence }\end{array}$ \\
\hline 2 & Cftr.txt & 1621 & $\begin{array}{l}\text { 1654-> } 1657 \\
\text { deletion }\end{array}$ & $\begin{array}{l}\text { i.e. "tc } \\
\text { atcTTtggtg } \\
\text { ttt'CTT is } \\
\text { deleted }\end{array}$ \\
\hline 3 & cftr2.txt & 1741 & $1758 \quad g->a$ & $\begin{array}{l}\text { a AGA mutation } \\
\text { i.e. a "stop" } \\
\text { codon }\end{array}$ \\
\hline 4 & $\operatorname{cftr} 3 . t x t$ & 1741 & 1785 a $\rightarrow$ g & $\begin{array}{l}\text { ie "tg } \\
\text { gaggtcaacg' 'tg } \\
\text { gagatcaacg" } \\
\text { i.e Asp-> Gly }\end{array}$ \\
\hline 5 & cftr4.txt & 3601 & $3638 c->t$ & $\begin{array}{l}\text { in'aftgacatgc" } \\
\text {->"aftgatatgc' } \\
\text { i.e. c->t }\end{array}$ \\
\hline 6 & cftr5.txt & 1621 & $\begin{array}{l}\text { 1651-> } 1654 \\
\text { deletion }\end{array}$ & $\begin{array}{l}\text { in "agaaatatc } \\
\text { ATCtttggtg" } \\
\text { ATC is delete } \\
\text { d }\end{array}$ \\
\hline 7 & cftr6.txt & 1681 & $1709 \mathrm{c}->\mathrm{t}$ & $\begin{array}{l}\text { nondestructive } \\
\text { mutation in } \\
\text { "atgccaaCTA } \\
\text { gaagaggaca" } \\
\text { CTA ->TTA i.e. } \\
\text { c -> t no } \\
\text { effect to } \\
\text { protein }\end{array}$ \\
\hline 8 & cftr7.txt & 1681 & 1711 a->t & $\begin{array}{l}\text { nondestructive } \\
\text { mutation in } \\
\text { "atgccaaCTA } \\
\text { gaagaggaca' } \\
\text { CTA -> CTT } \\
\text { i.e. a -> t no } \\
\text { effect to } \\
\text { protein }\end{array}$ \\
\hline 9 & cftr8.txt & 1681 & 1711 a $\rightarrow c$ & $\begin{array}{l}\text { nondestructive } \\
\text { mutation in } \\
\text { "atgcCaaCTA } \\
\text { gaagaggaca" } \\
\text { CTA i.e. a -> } \\
\text { c, CTC }\end{array}$ \\
\hline 10 & cftr9.txt & 1681 & 1711 a-> g & $\begin{array}{l}\text { non- } \\
\text { destructive } \\
\text { mutation in } \\
\text { in 'atgccaactA } \\
\text { gaagaggaca' } \\
\text { CTA-> CTG i.e. } \\
\text { a }->\text { g }\end{array}$ \\
\hline 12 & cftr11.txt & 3361 & $\begin{array}{l}3382 \text { point } \\
\text { deletion }\end{array}$ & $\begin{array}{l}\text { in "aagctctgaa } \\
\text { Tttacatact" a }\end{array}$ \\
\hline
\end{tabular}




\begin{tabular}{|c|c|c|c|c|}
\hline & & & & $\begin{array}{l}\text { "T' is deleted } \\
\text {-> 'aagctctgaa } \\
\text { ftacatact" }\end{array}$ \\
\hline 13 & cftr12.txt & 4861 & $\begin{array}{l}4893 \text { point } \\
\text { deletion }\end{array}$ & $\begin{array}{l}\text { in "aaggcagctc } \\
\text { taaatgtcaa" a } \\
\text { "N'is deleted } \\
\text {-> "aaggcagctc } \\
t \text { aatgtcaa" }\end{array}$ \\
\hline 14 & cftr13.txt & 2581 & $\begin{array}{l}2597 \text { point } \\
\text { deletion }\end{array}$ & $\begin{array}{l}\text { in "taagtgaaga } \\
\text { aaftaaqaa" a } \\
\text { 'G' is deleted } \\
\text {-> taagt aaga } \\
\text { aaftaacgaa' }\end{array}$ \\
\hline 15 & Cftr09.txt & 1000 & $\begin{array}{l}1000->1009 \\
\text { order reversed }\end{array}$ & $\begin{array}{l}\text { e.g. tag cac } \\
\text { ttg -> gtt cac } \\
\text { gat that is } 3 \\
\text { amino acids } \\
\text { are reversed } \\
\text { from their } \\
\text { natural } \\
\text { order** }\end{array}$ \\
\hline 16 & cftr030txt & 1000 & $\begin{array}{l}1000->1030 \\
\text { order reversed }\end{array}$ & $\begin{array}{l}10 \text { ammo acids } \\
\text { are reversed } \\
\text { from their } \\
\text { natural } \\
\text { order** }\end{array}$ \\
\hline 17 & cftro300.txt & 1000 & $\begin{array}{l}1000->1300 \\
\text { order reversed }\end{array}$ & $\begin{array}{l}100 \text { amino } \\
\text { acids are } \\
\text { reversed from } \\
\text { their natural } \\
\text { order* }\end{array}$ \\
\hline 18 & cftro3000.txt & 1000 & $\begin{array}{l}1000->4000 \\
\text { order reversed }\end{array}$ & $\begin{array}{l}1000 \text { amino } \\
\text { acids are } \\
\text { reversed from } \\
\text { their natural } \\
\text { order** }\end{array}$ \\
\hline
\end{tabular}

**artificial mutation 


\section{Appendix \\ C. Matlab Script Files}




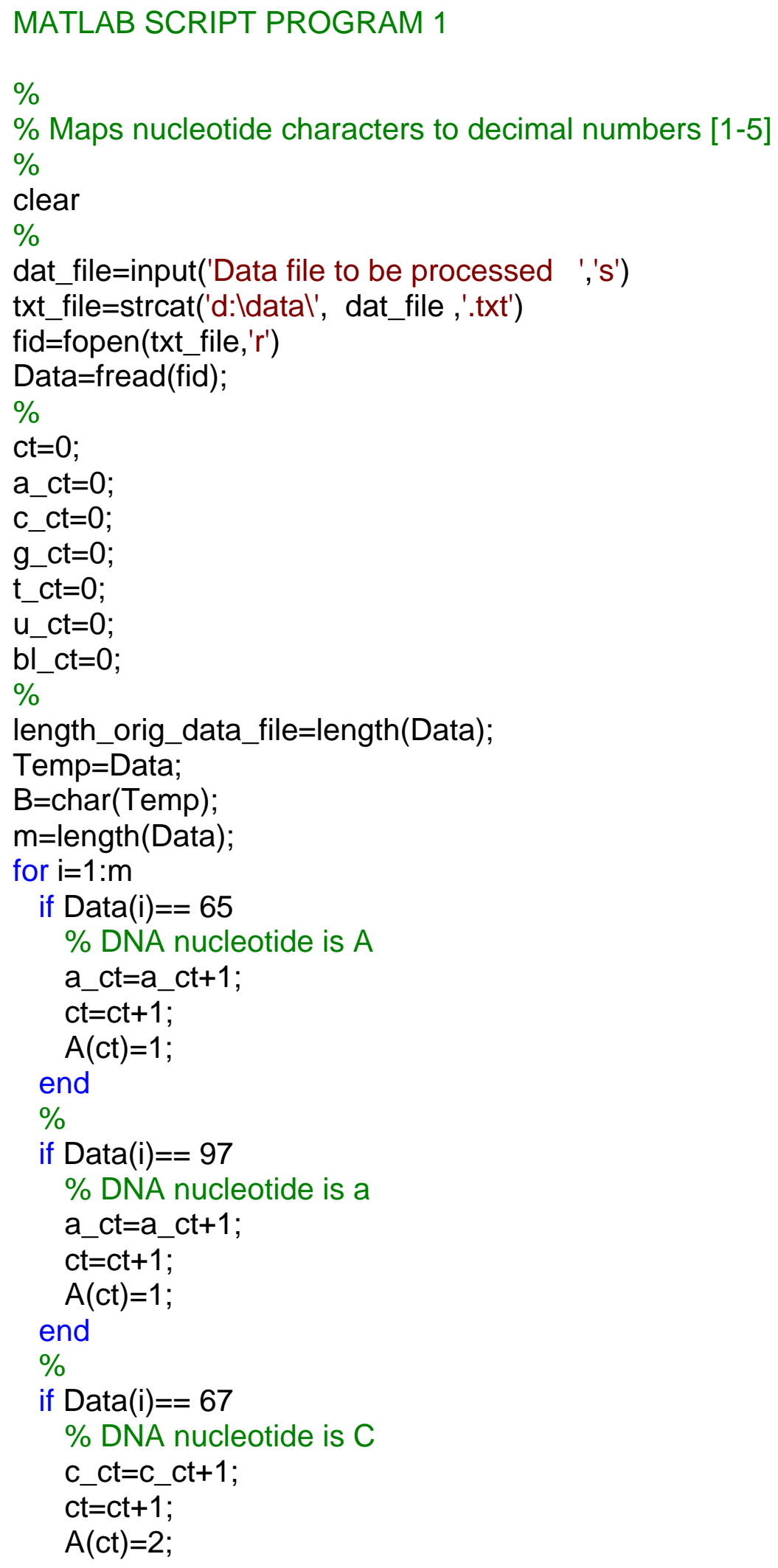




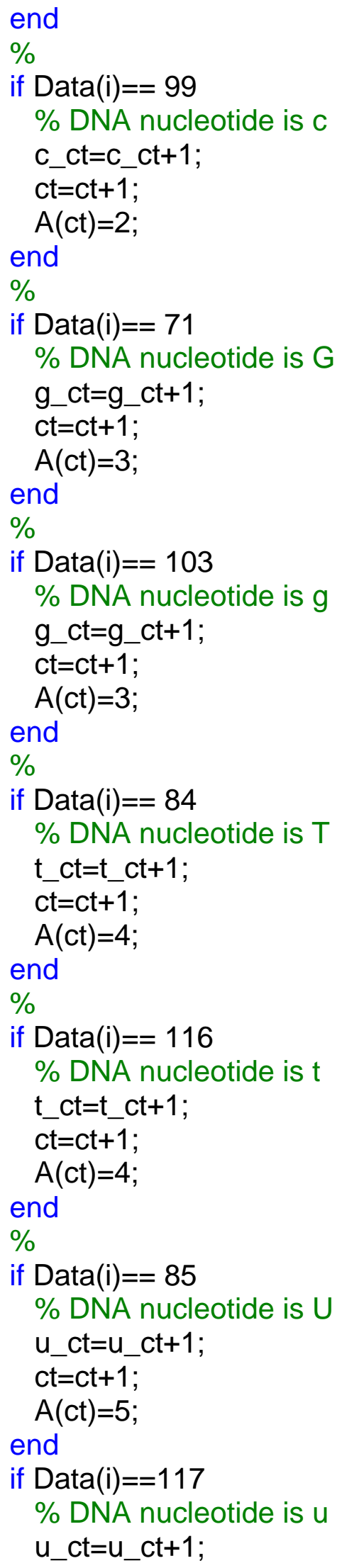




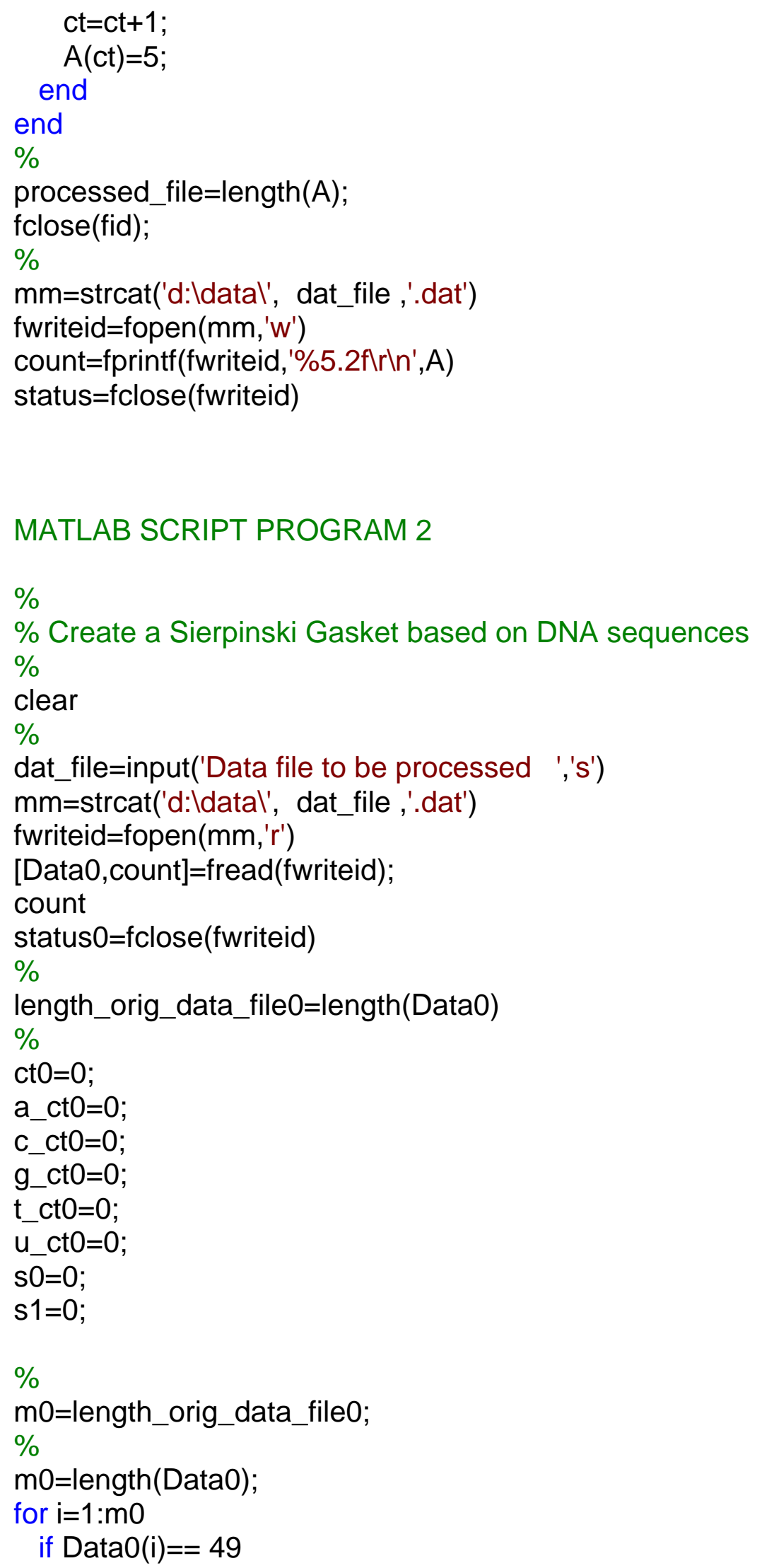

\section{MATLAB SCRIPT PROGRAM 2}

$\%$

$\%$ Create a Sierpinski Gasket based on DNA sequences

$\%$

clear

$\%$

dat_file=input('Data file to be processed ','s')

$\mathrm{mm}=$ strcat('d:Idatal', dat_file, '.dat')

fwriteid=fopen ( $\left.\mathrm{mm}, \mathrm{r}^{\prime}\right)$

[Data0, count]=fread(fwriteid);

count

status $0=$ fclose (fwriteid)

$\%$

length_orig_data_file0=length(Data0)

$\%$

ct $0=0$;

a_ct $0=0$;

c_ct $0=0$;

g_ct $0=0$;

t_ct $0=0$;

u_ct $0=0$;

$\mathrm{s} 0=0$;

$\mathrm{s} 1=0$;

$\%$

$\mathrm{m} 0=$ length_orig_data_file0;

$\%$

$\mathrm{m} 0=$ length(Data0);

for $\mathrm{i}=1: \mathrm{m} 0$

if DataO(i) $==49$ 


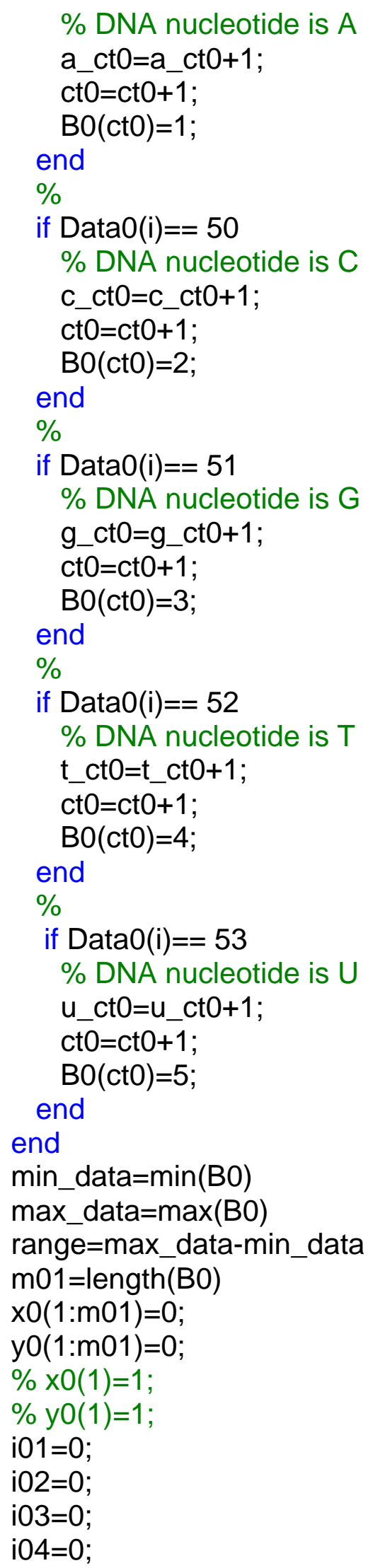




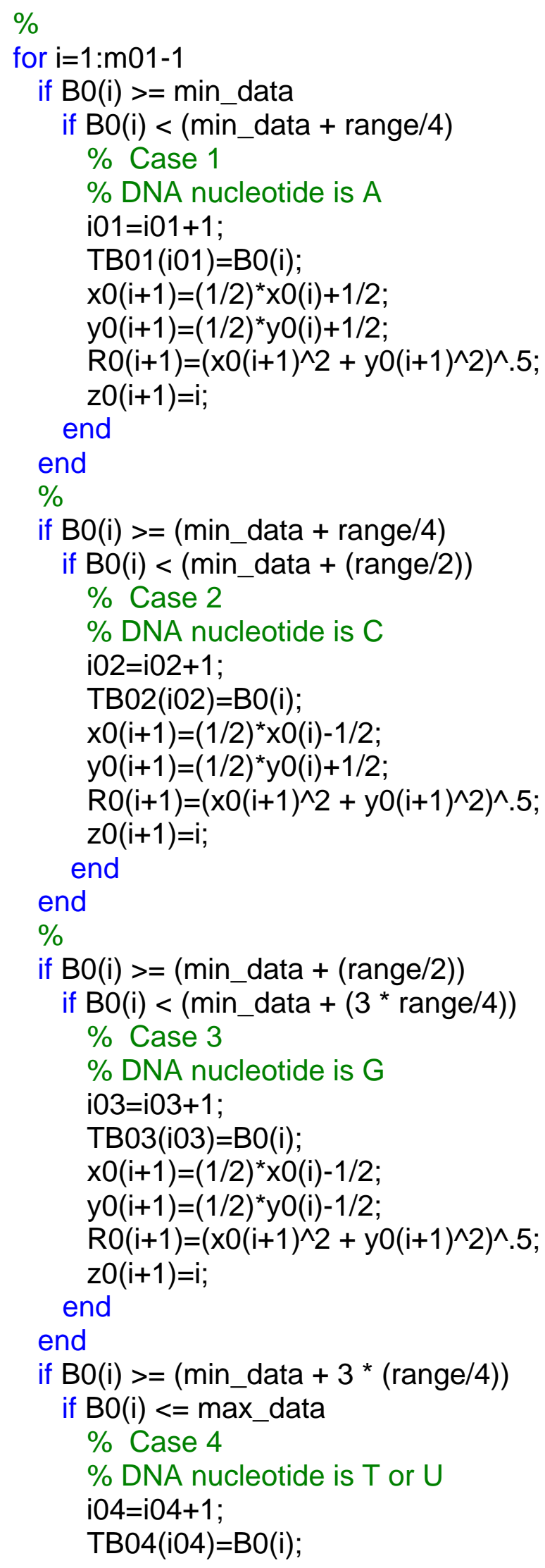




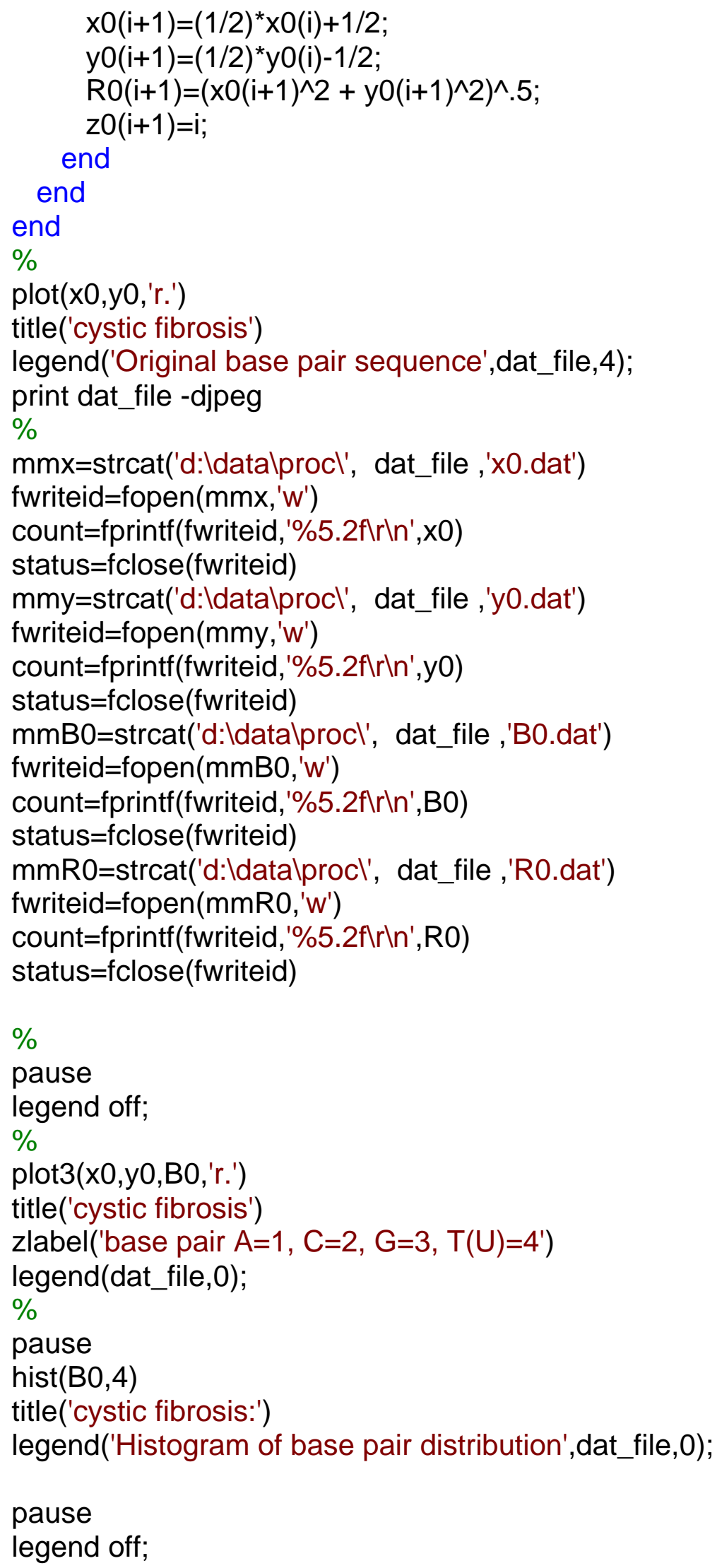




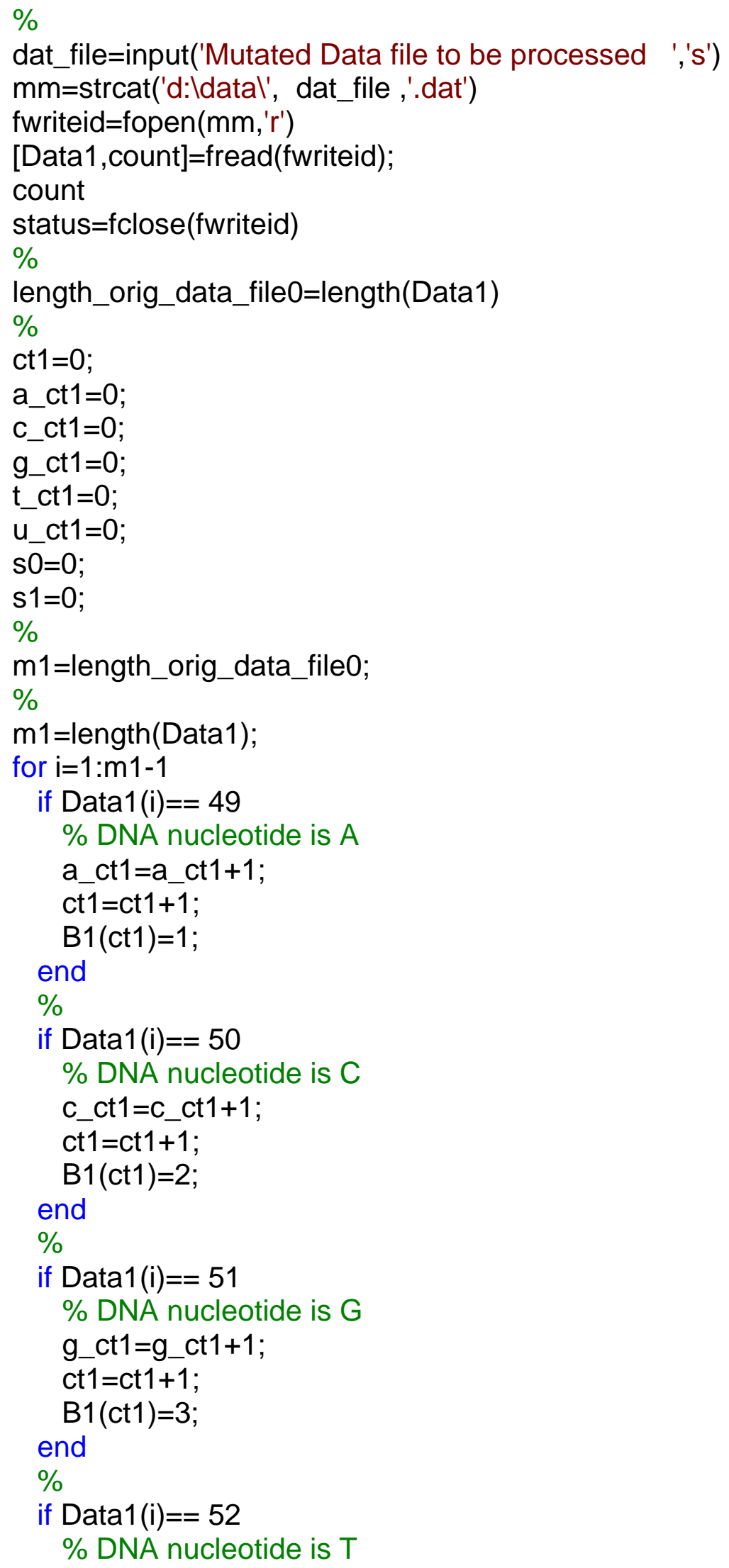




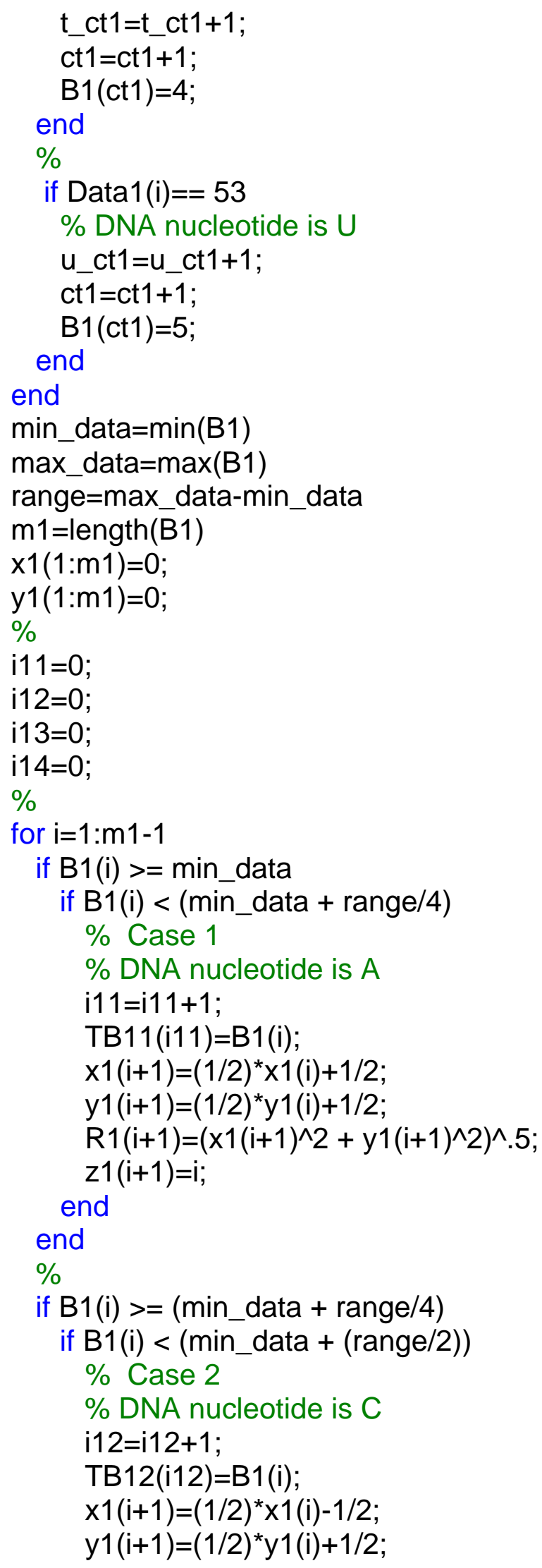




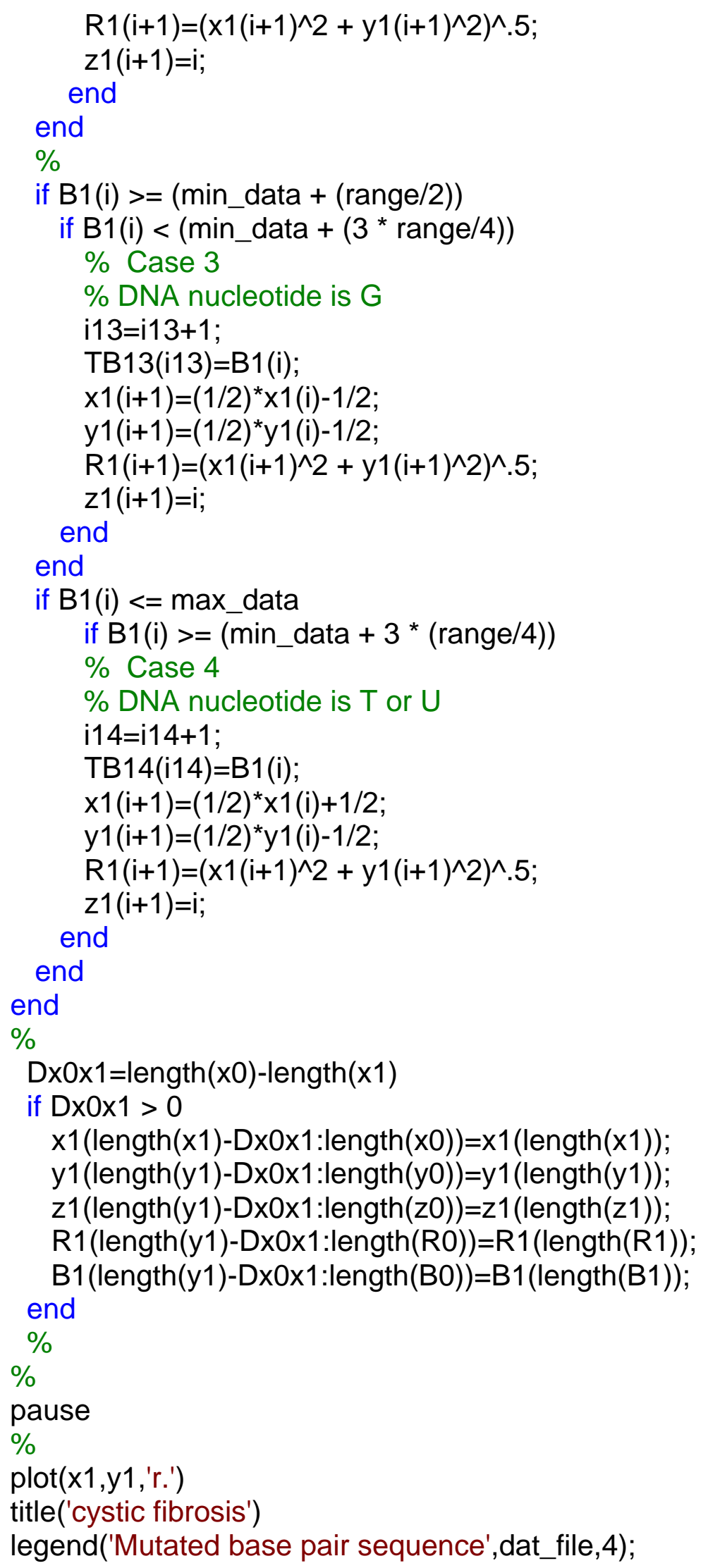




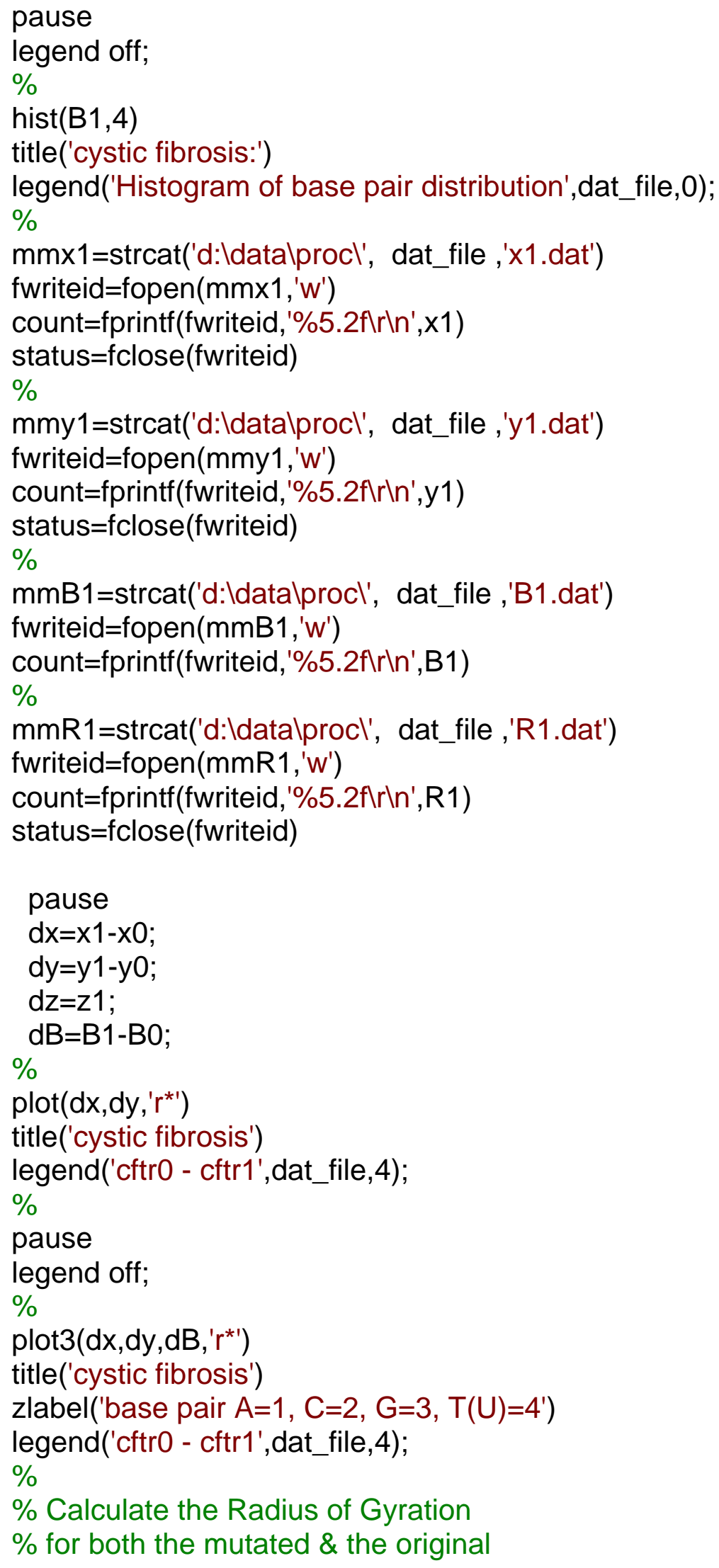




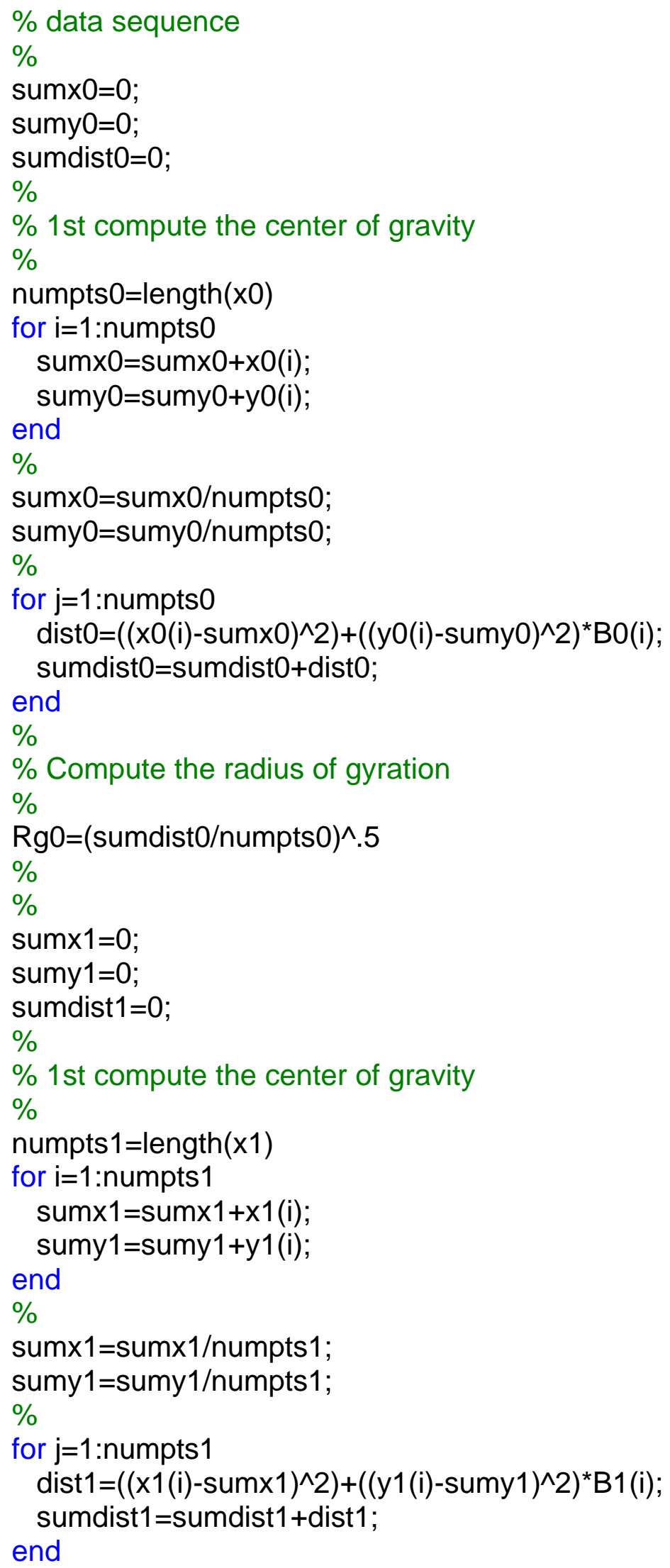




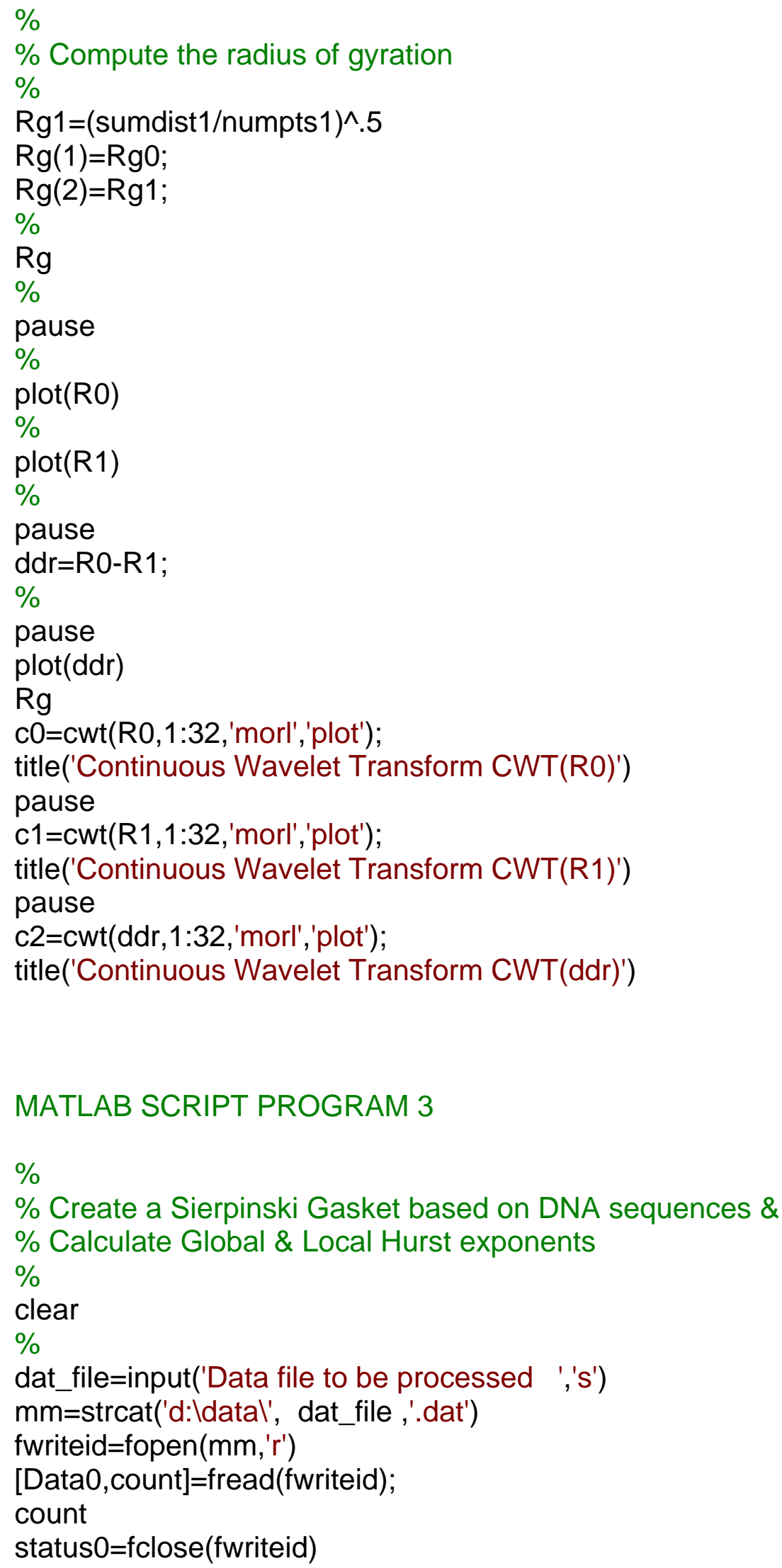

\section{MATLAB SCRIPT PROGRAM 3}

$\%$

$\%$ Create a Sierpinski Gasket based on DNA sequences \& $\%$ Calculate Global \& Local Hurst exponents

$\%$

clear

$\%$

dat_file=input('Data file to be processed ','s')

$\mathrm{mm}=$ strcat('d:Idatal', dat_file, '.dat')

fwriteid=fopen ( $\left.\mathrm{mm}, \mathrm{r}^{\prime}\right)$

[Data0, count]=fread(fwriteid);

count

status $0=$ fclose(fwriteid) 


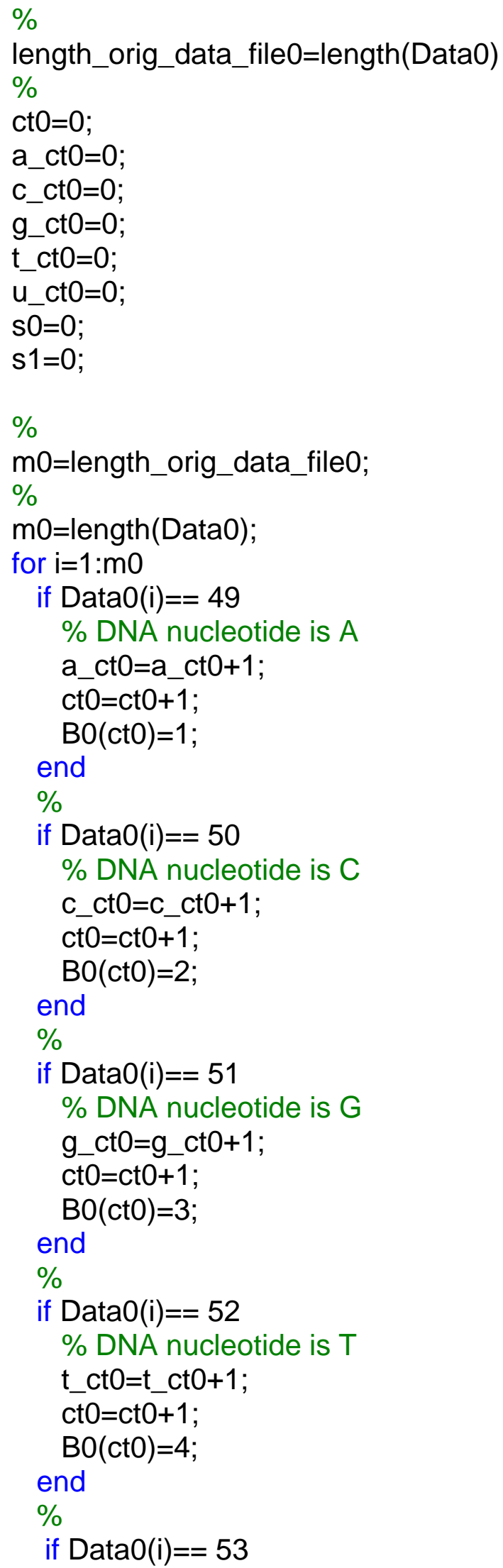




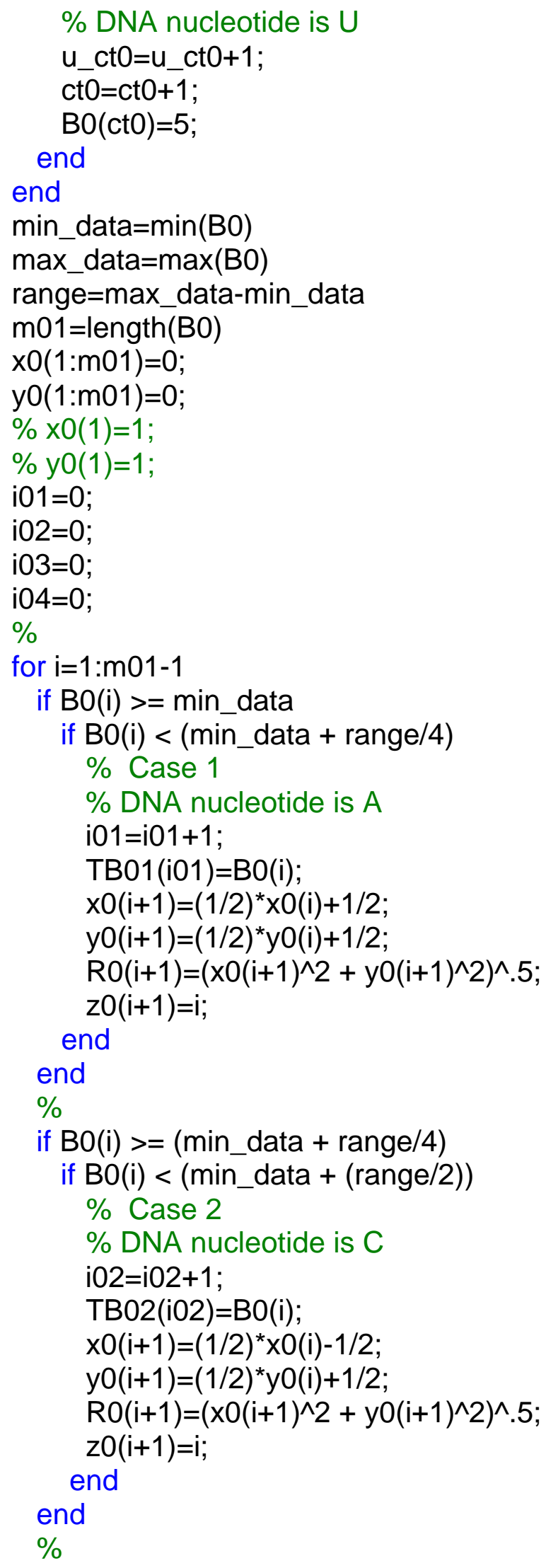




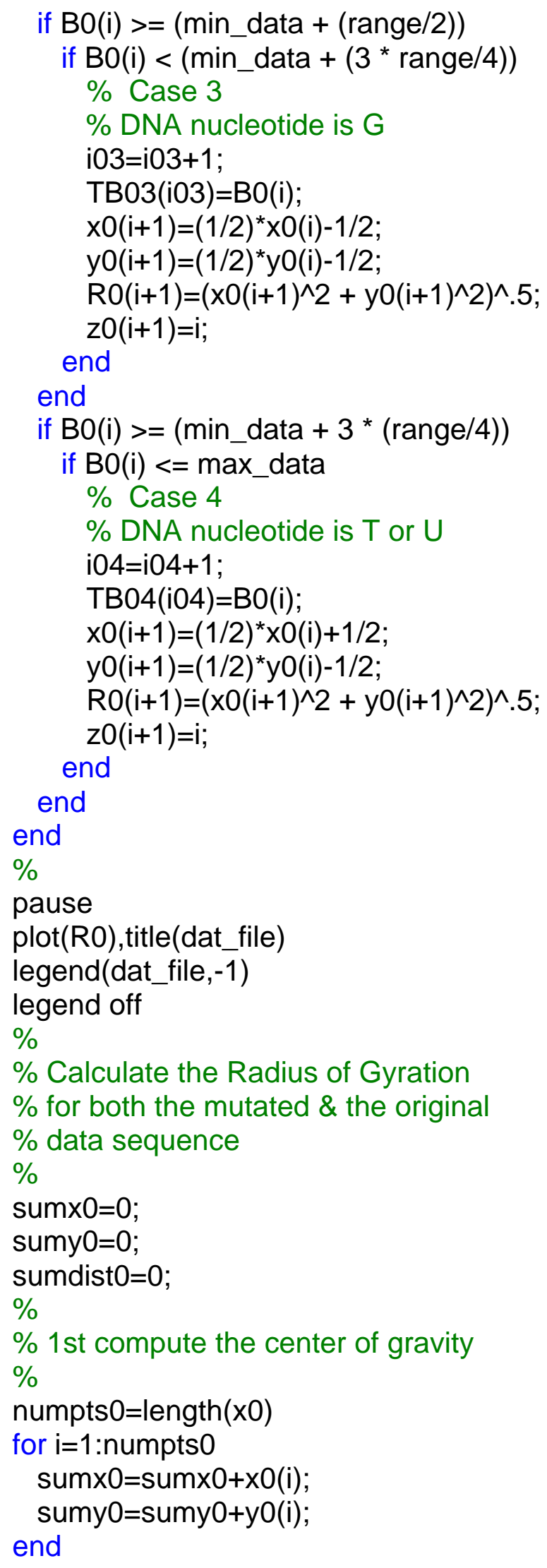




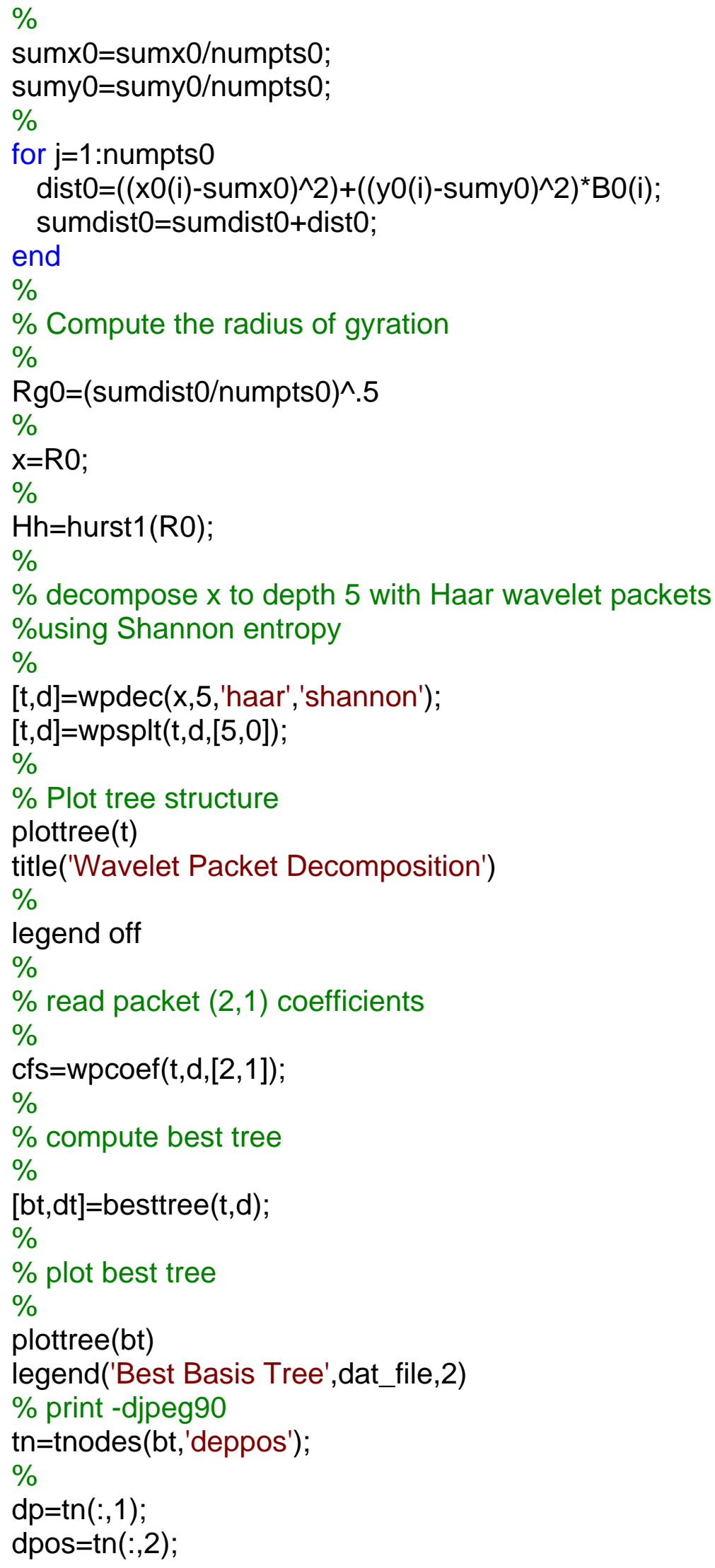




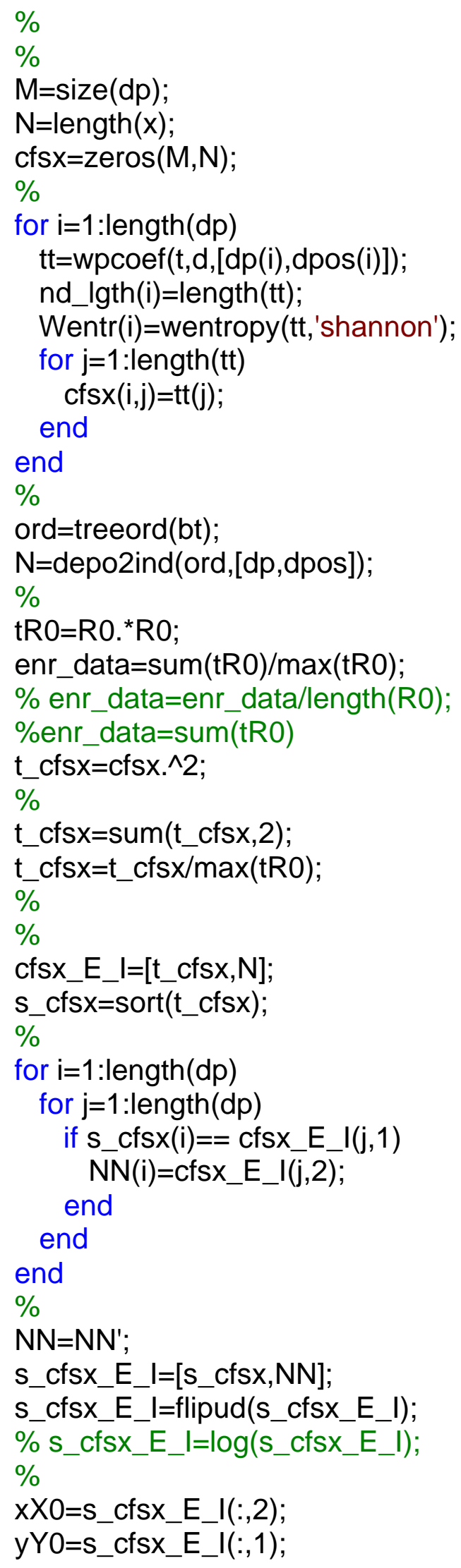




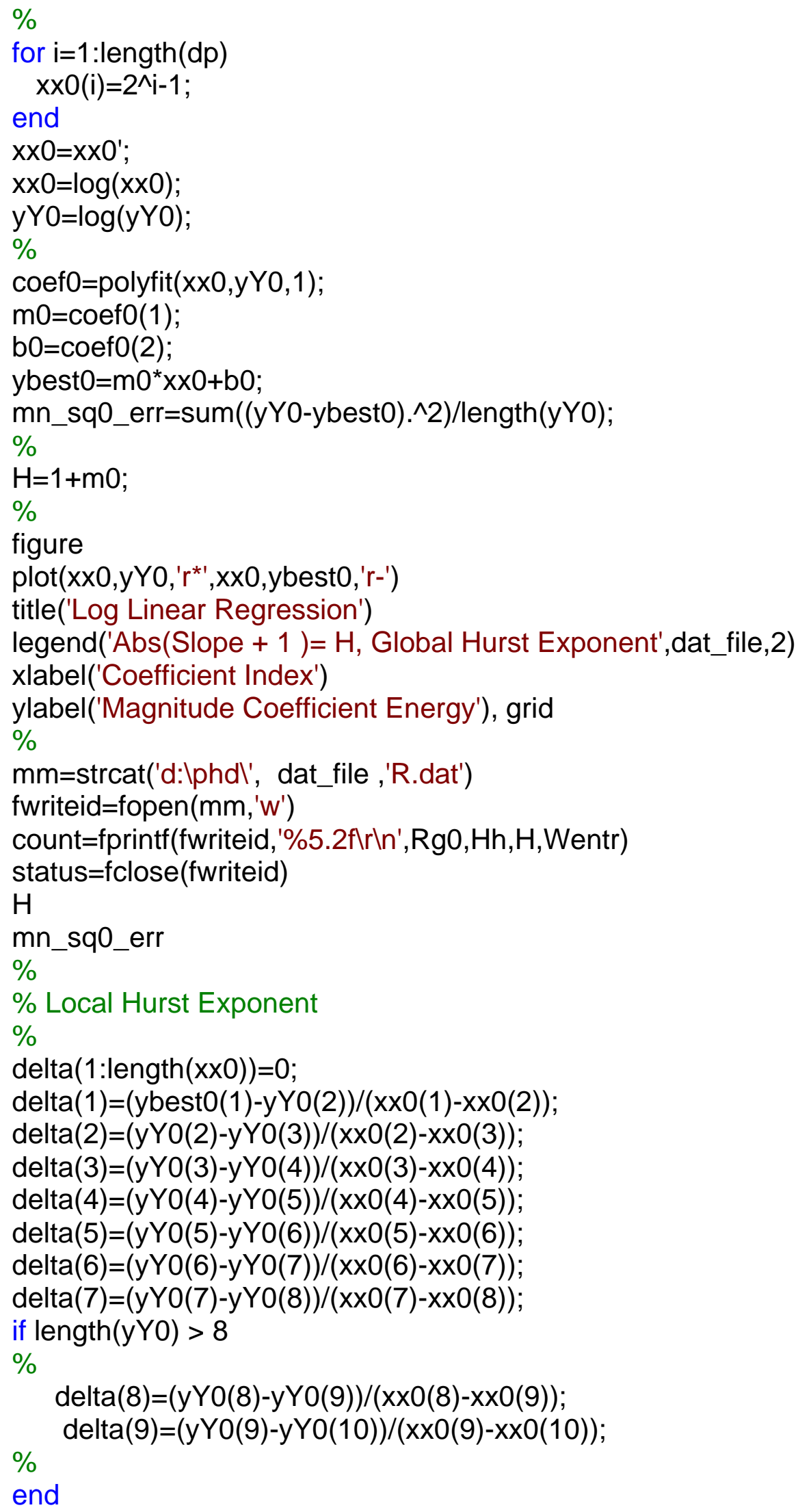




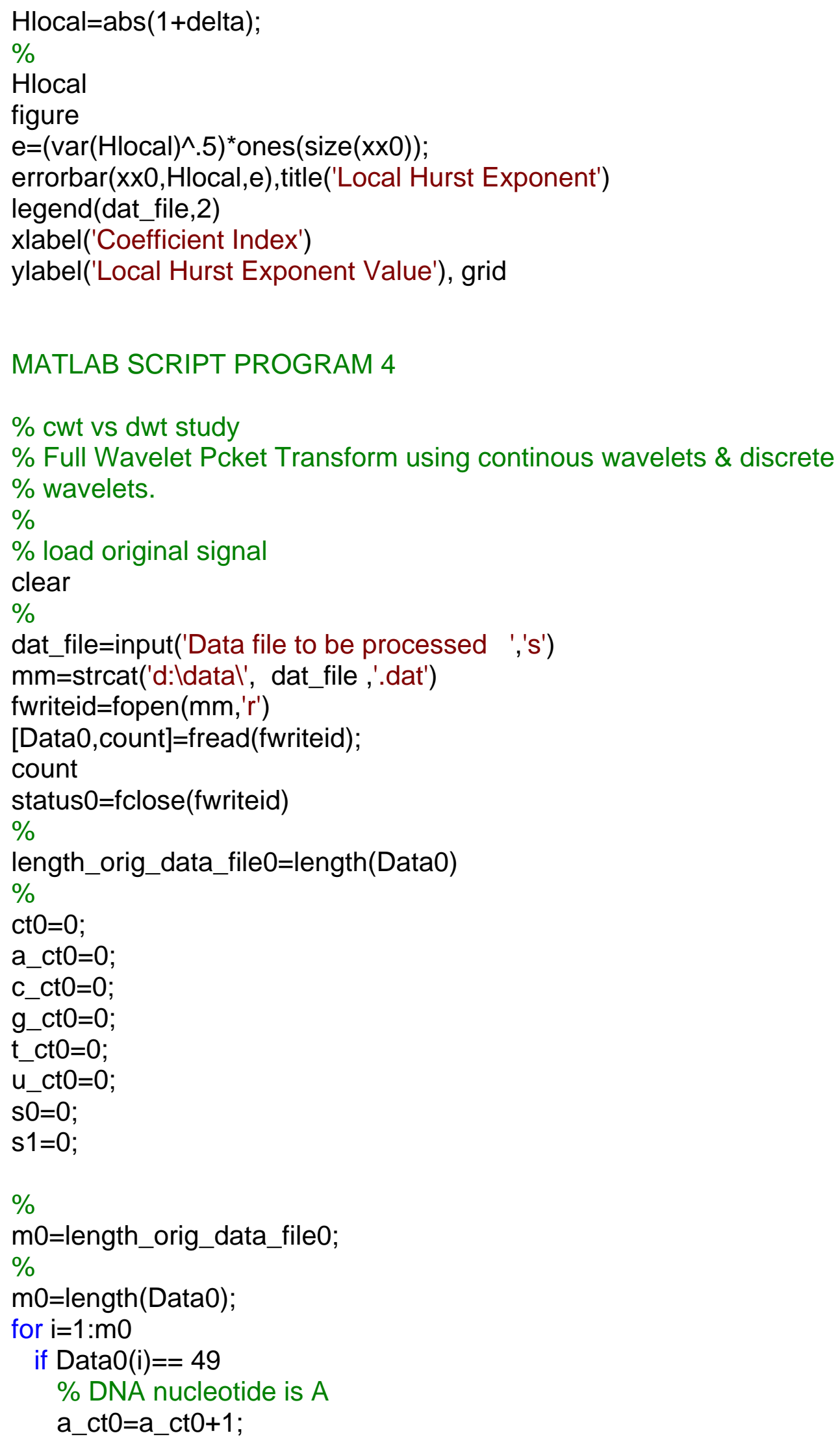




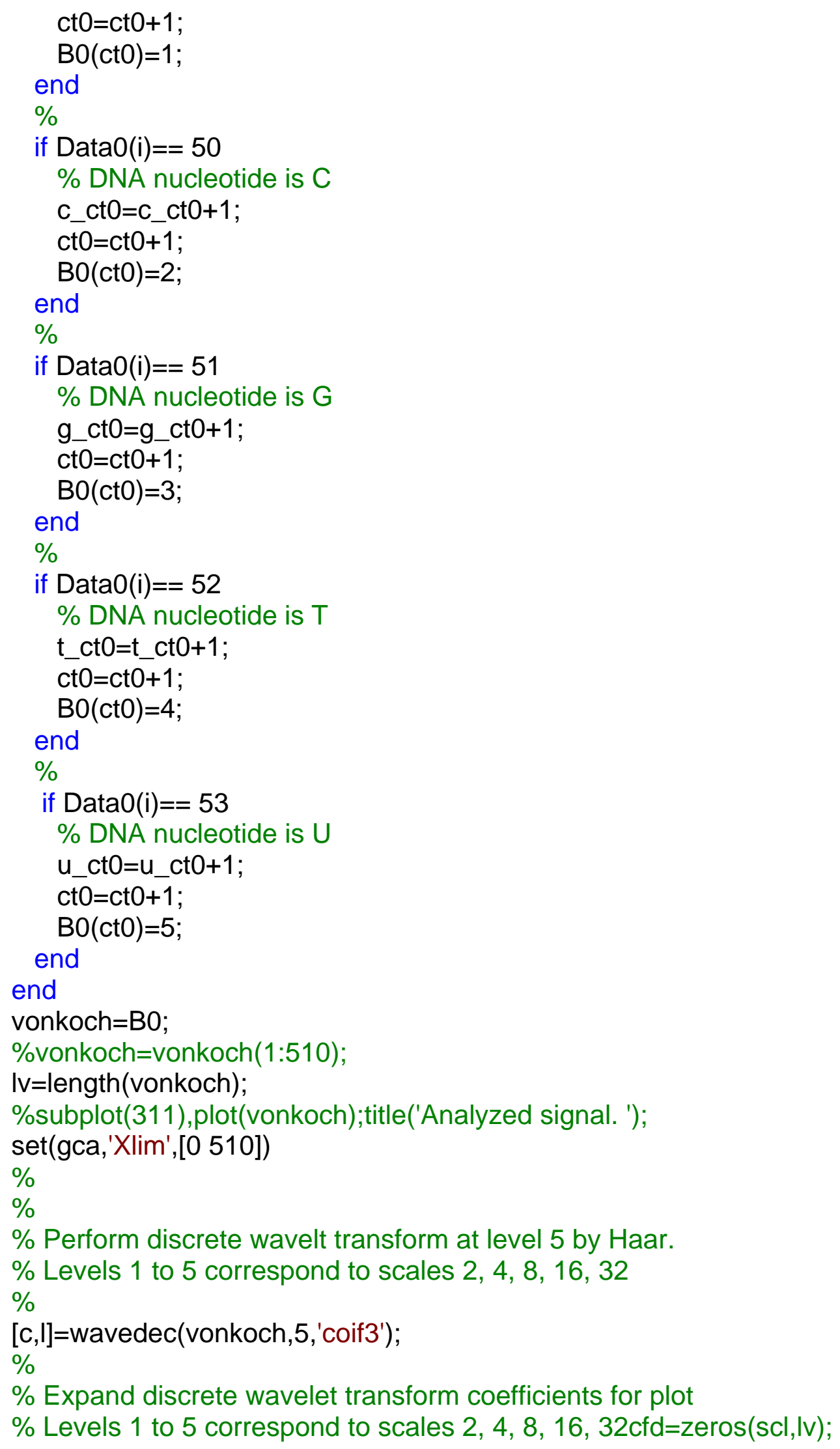




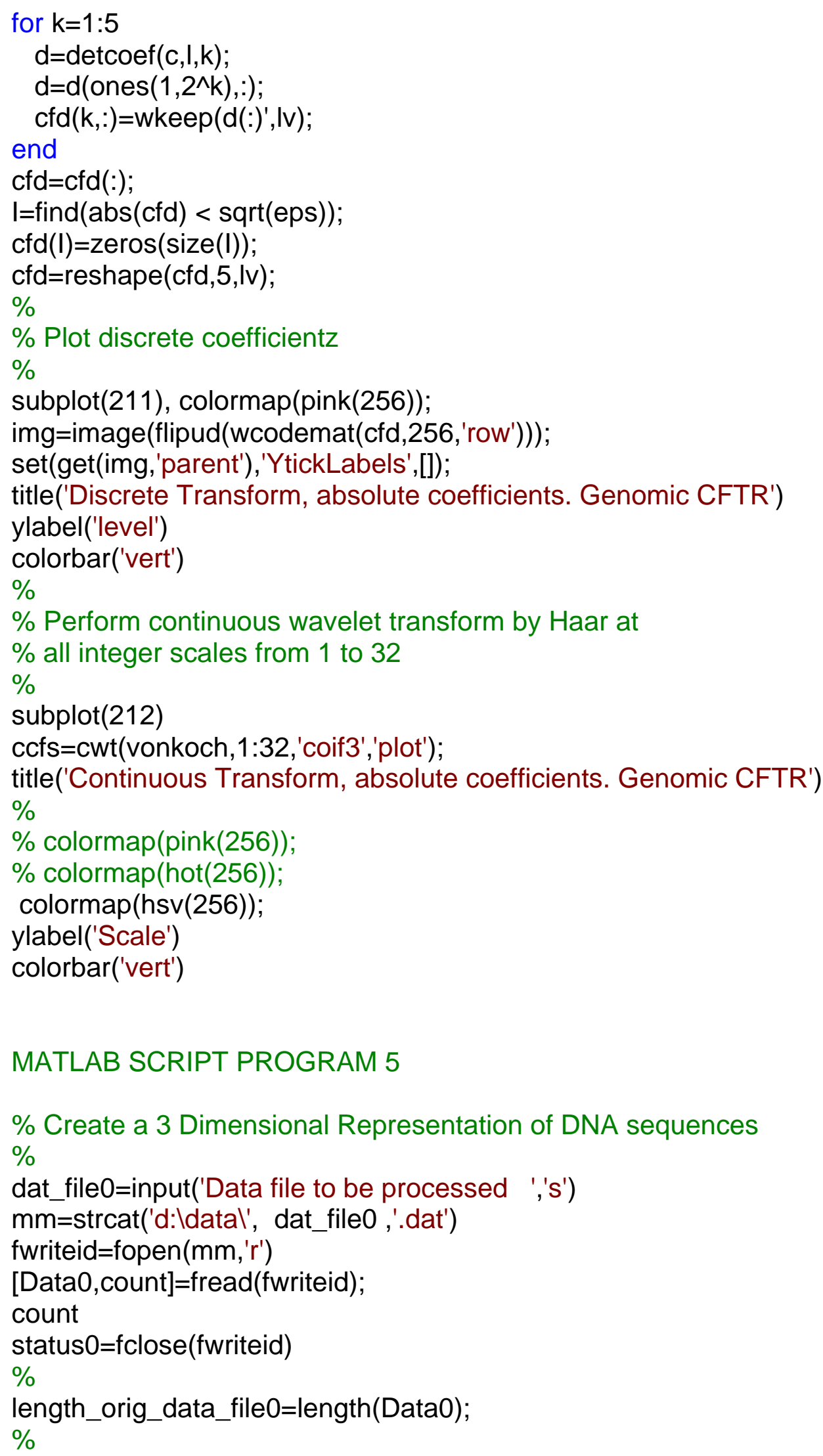




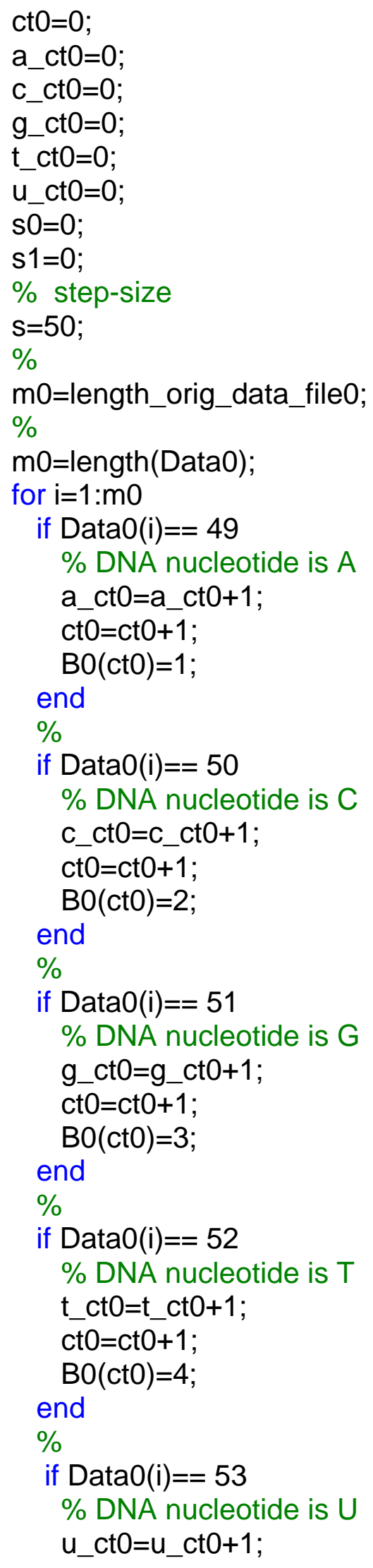




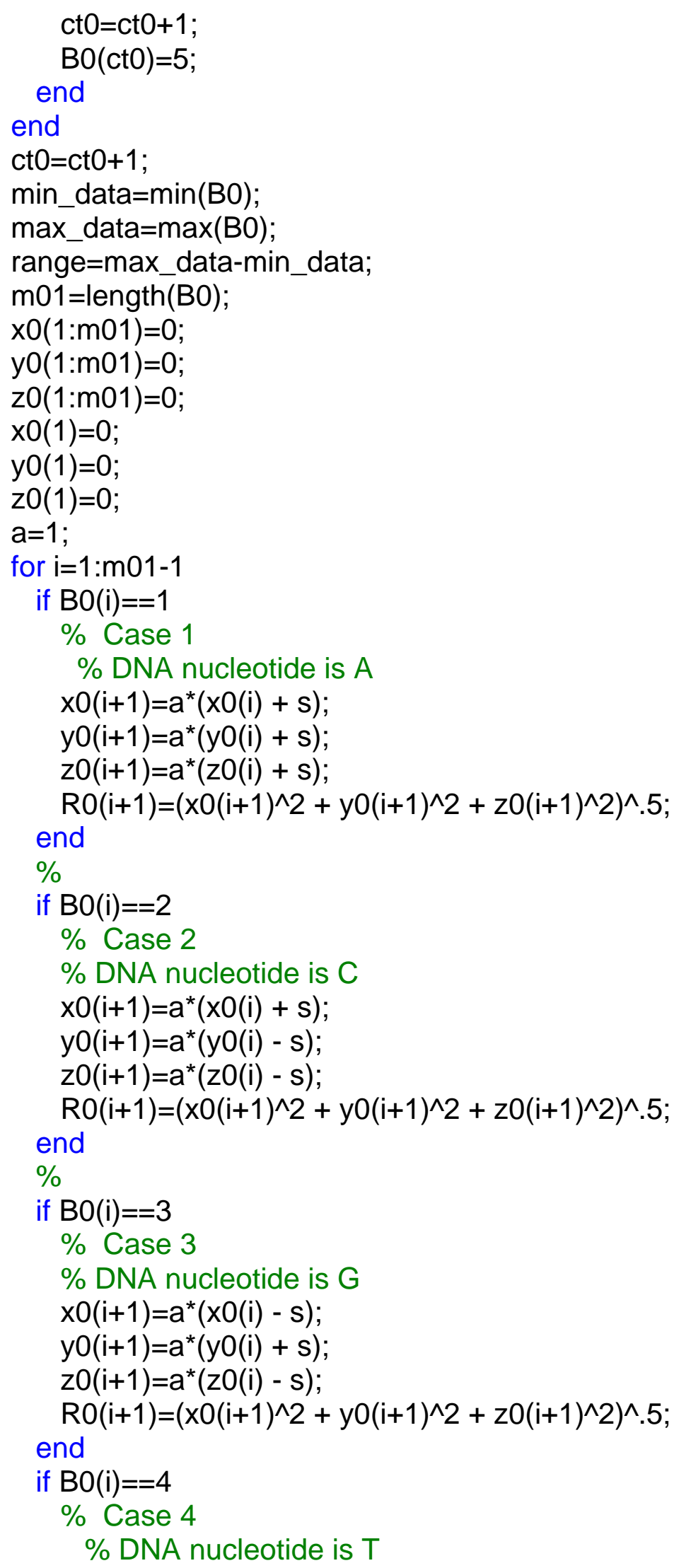




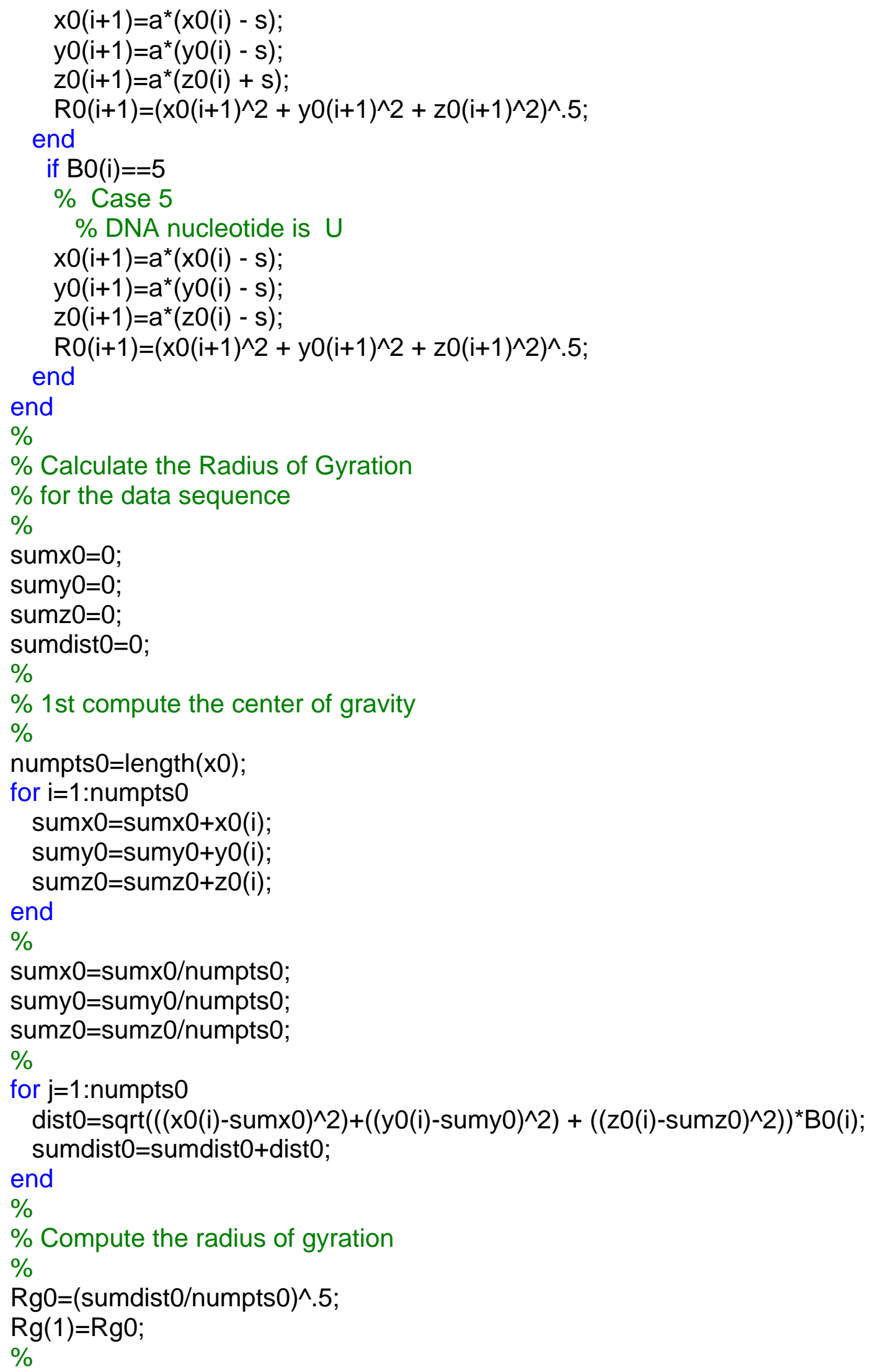




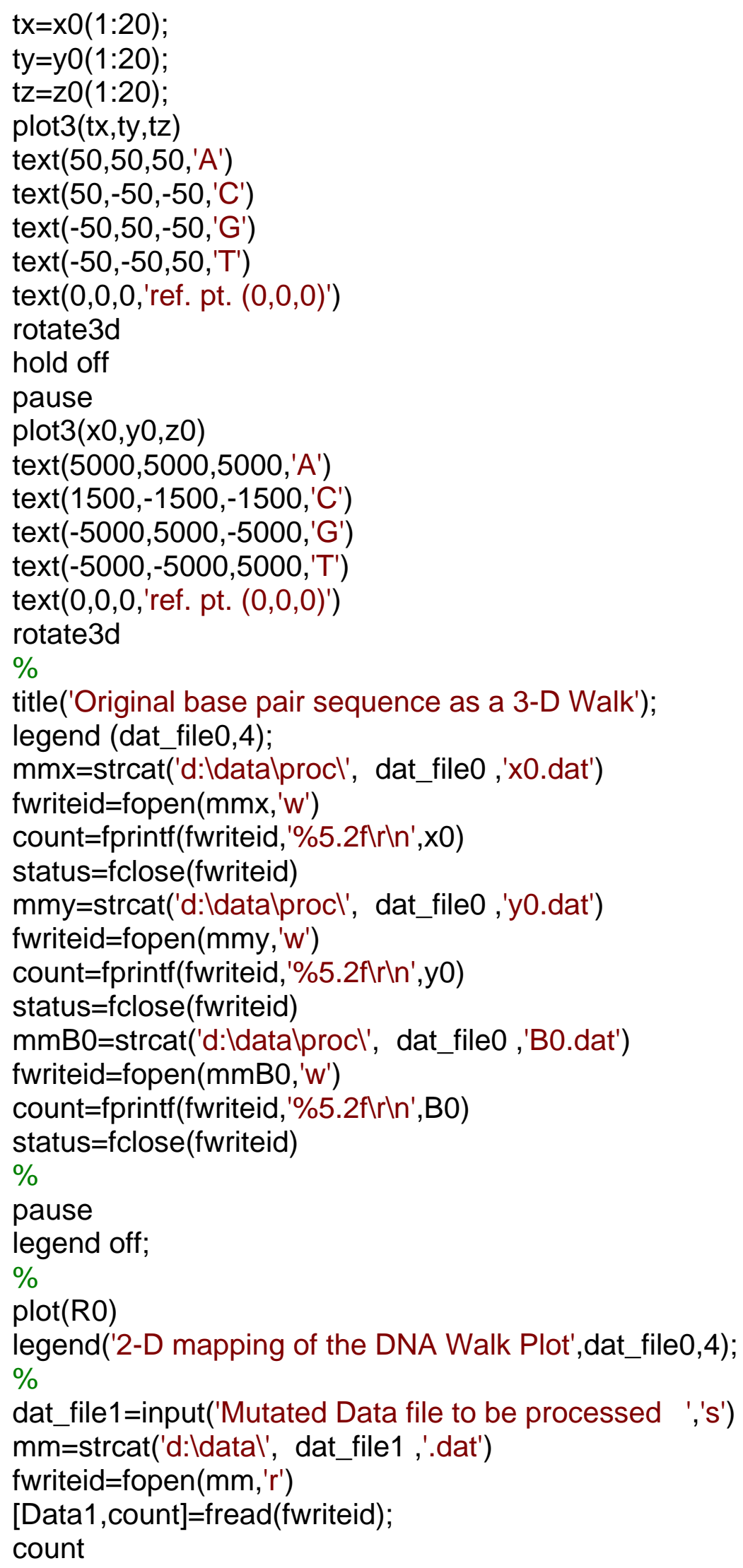




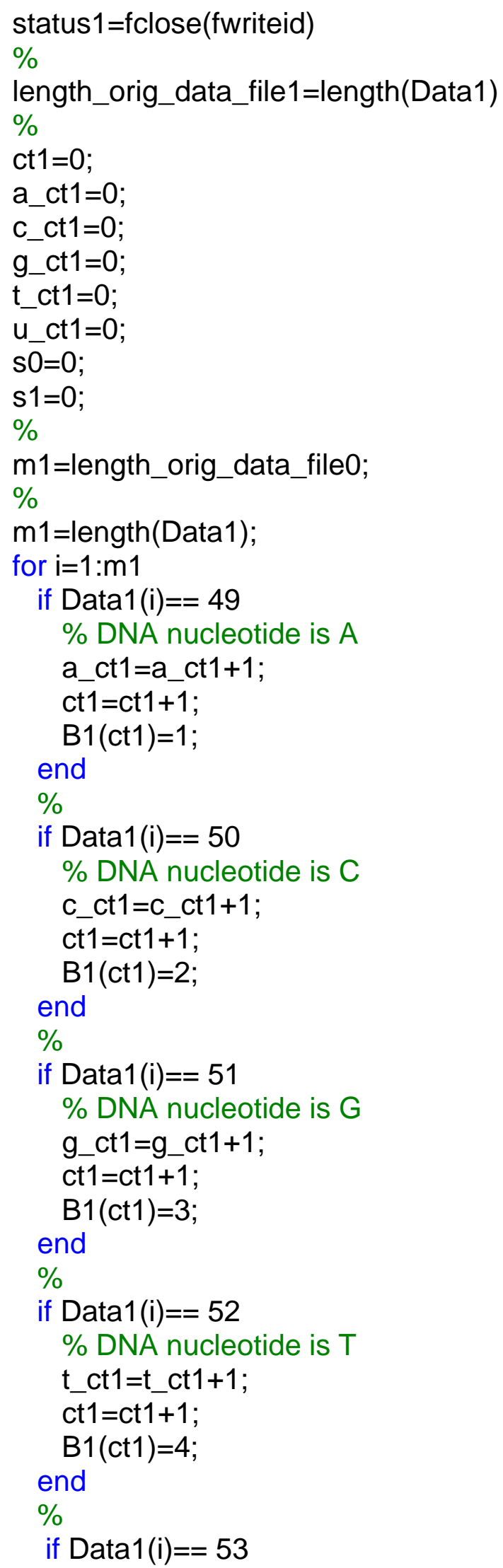




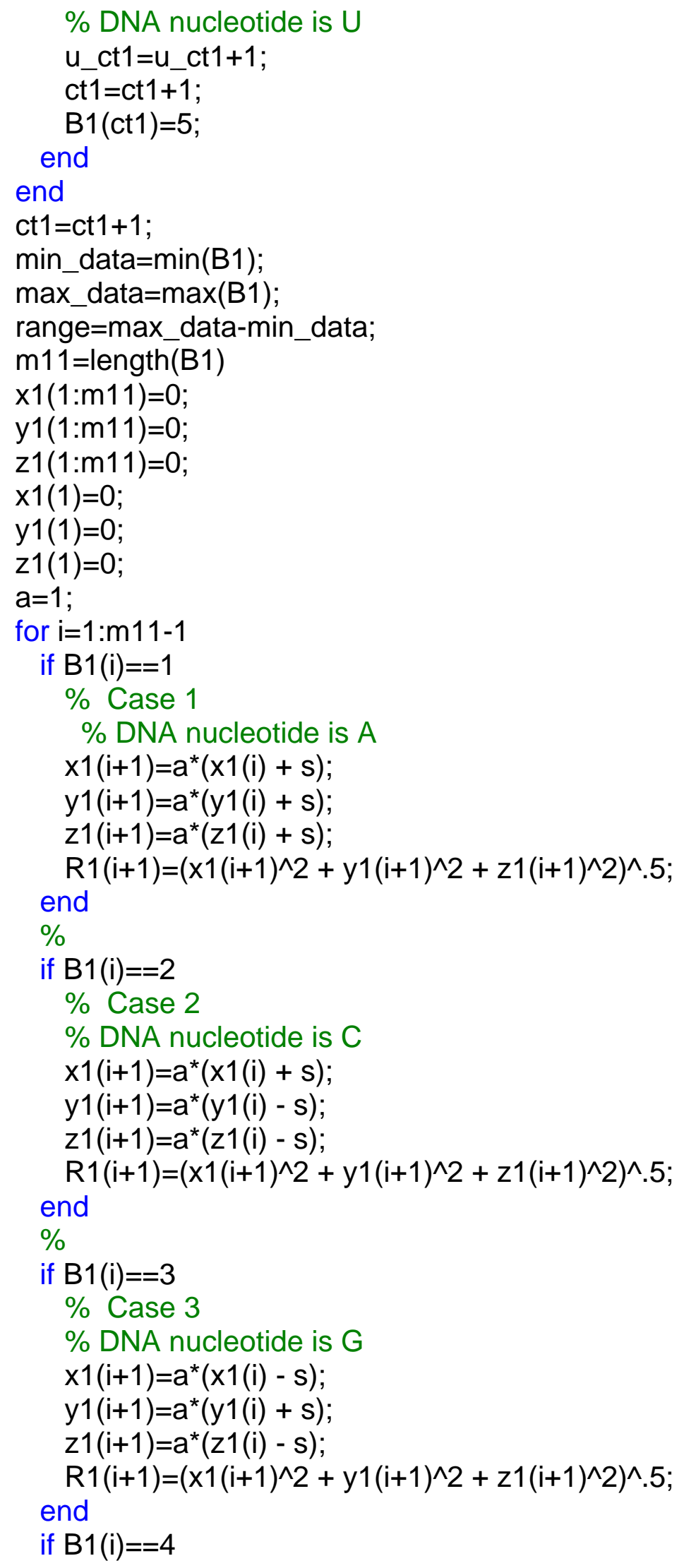




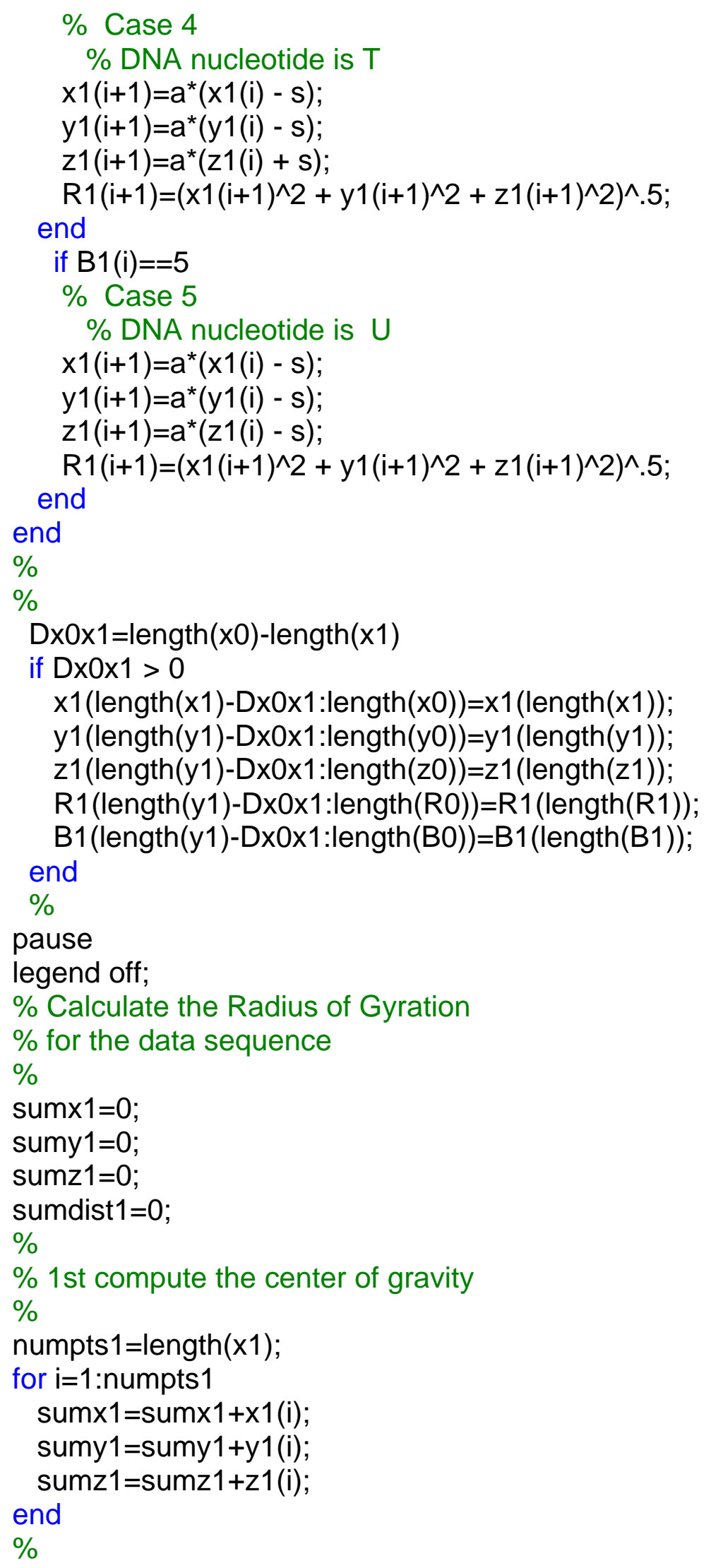




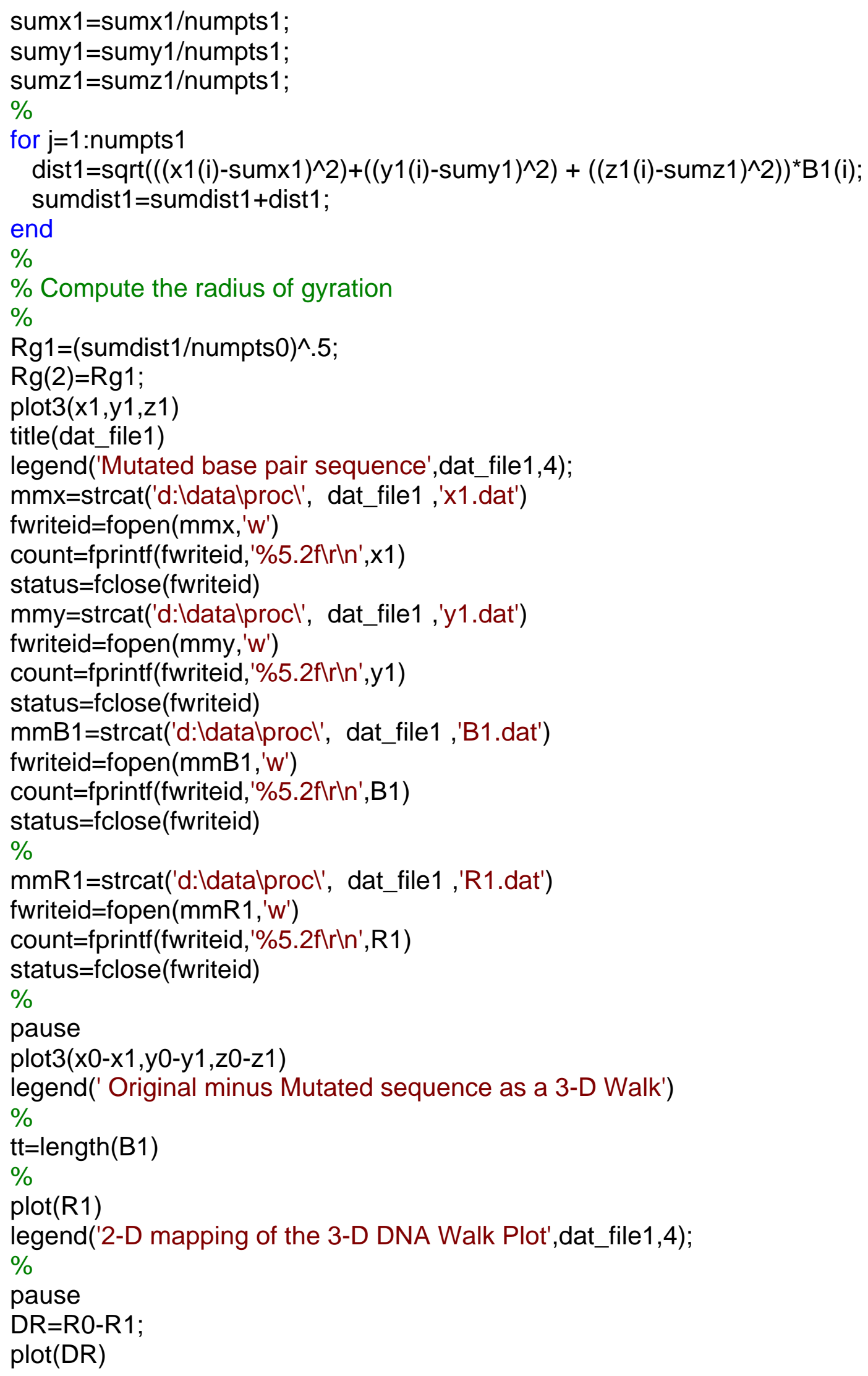




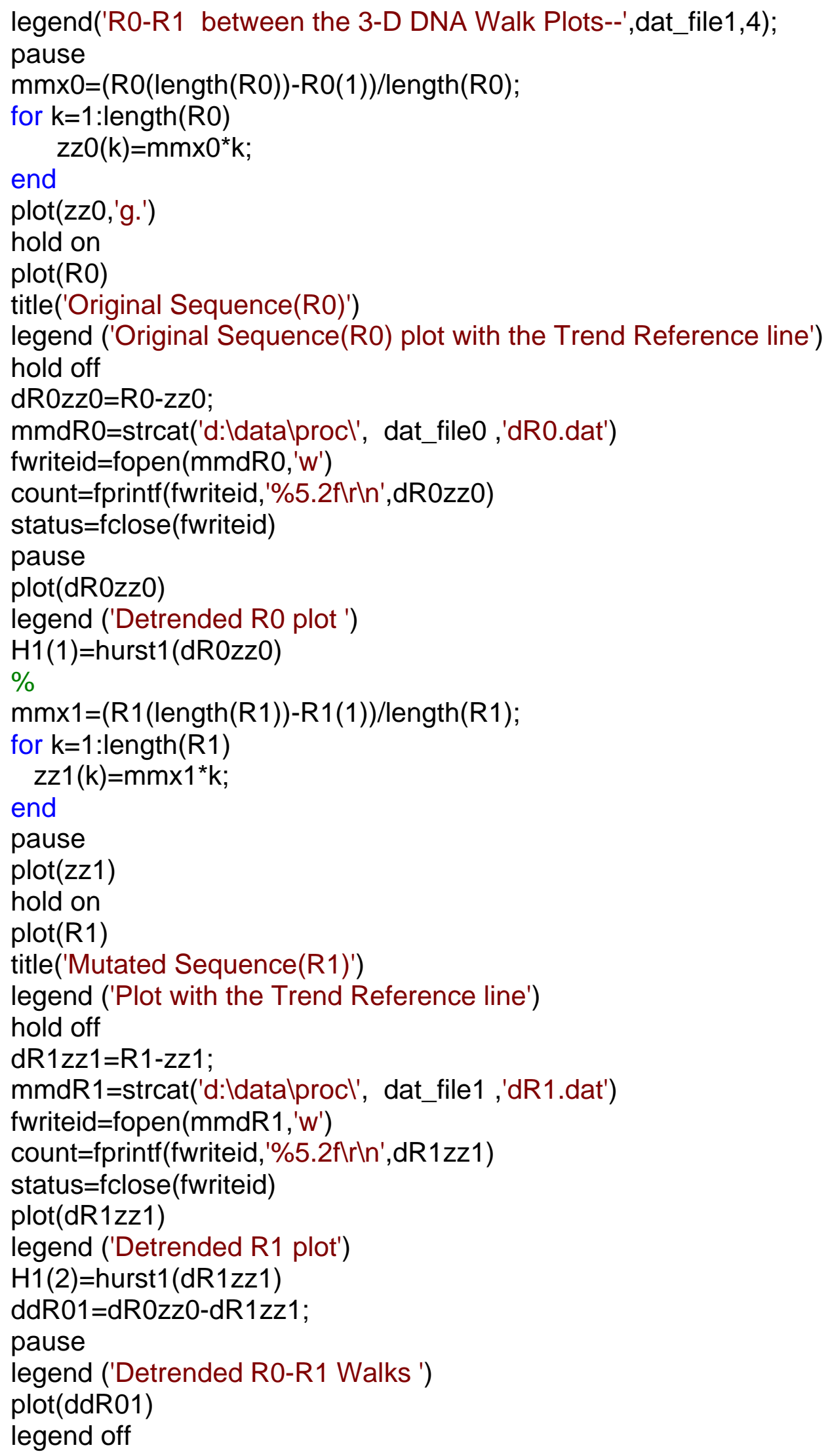




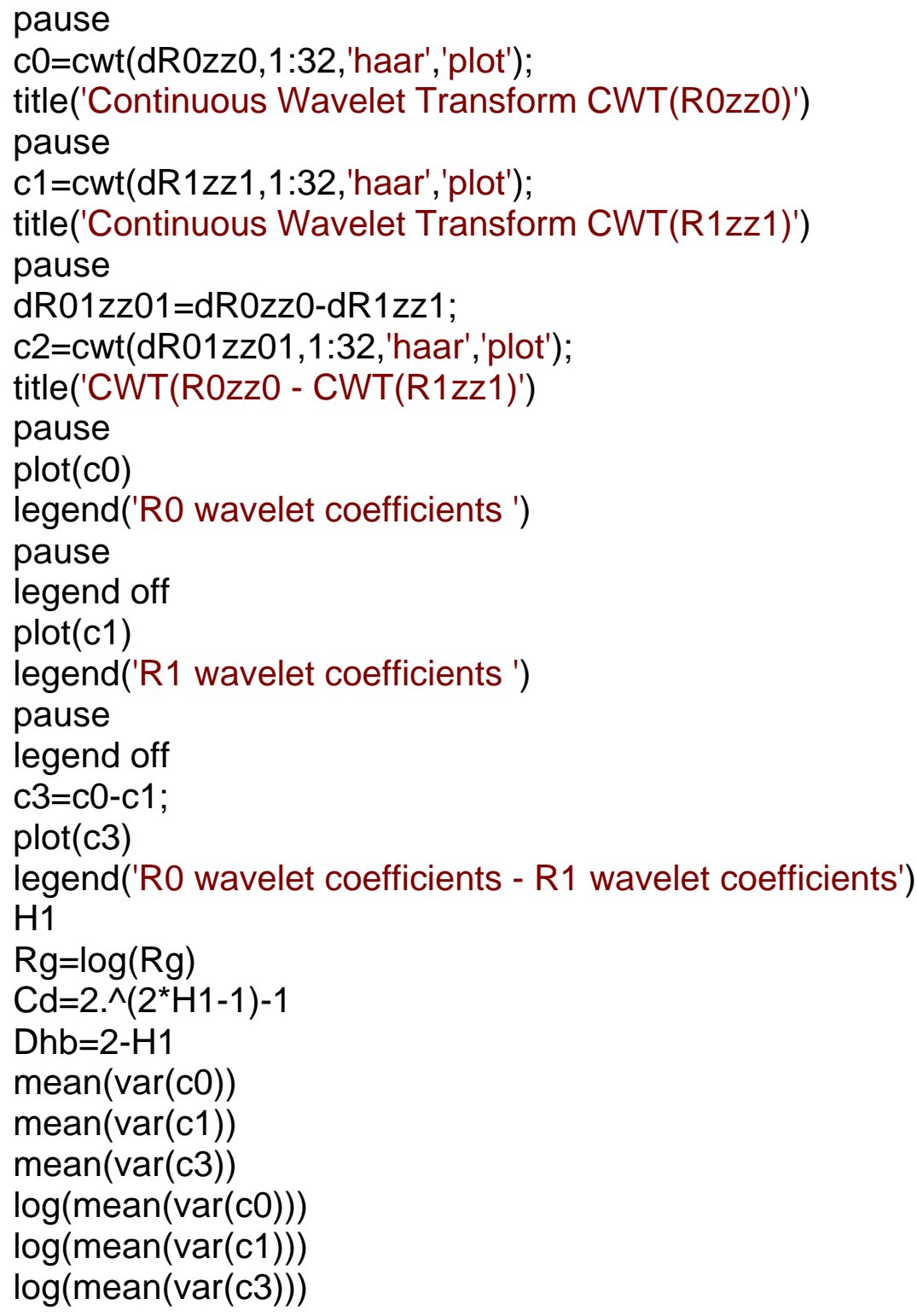




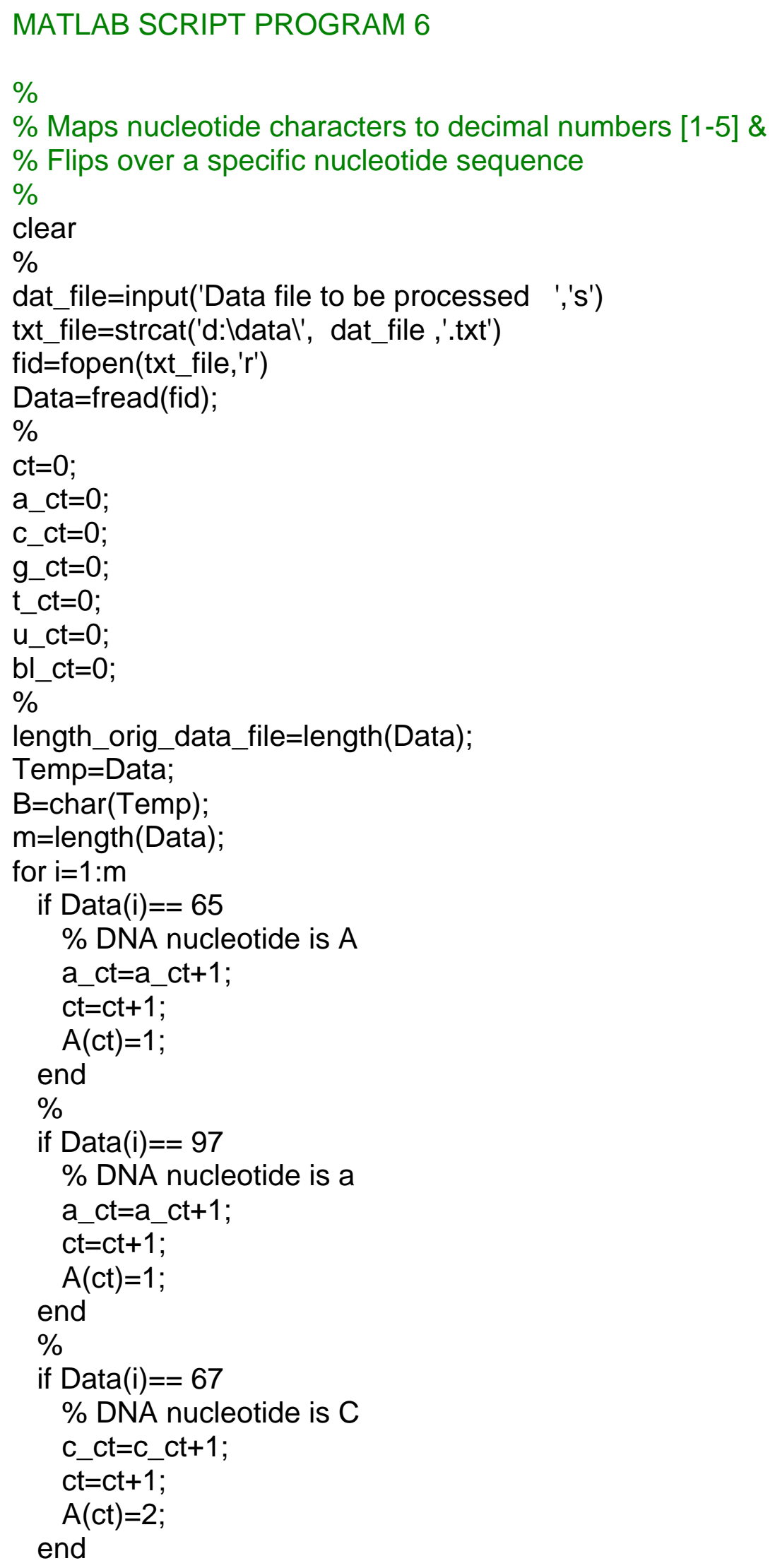




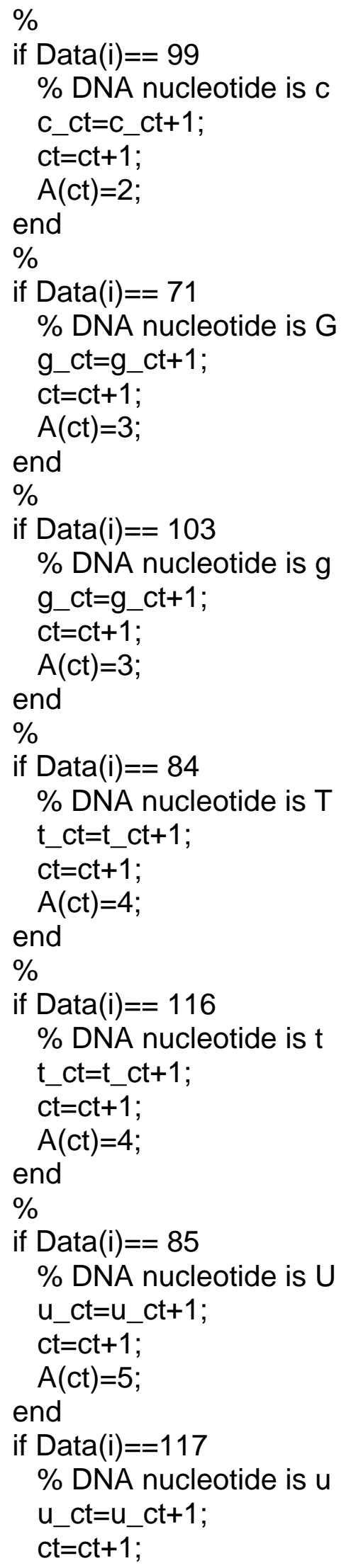




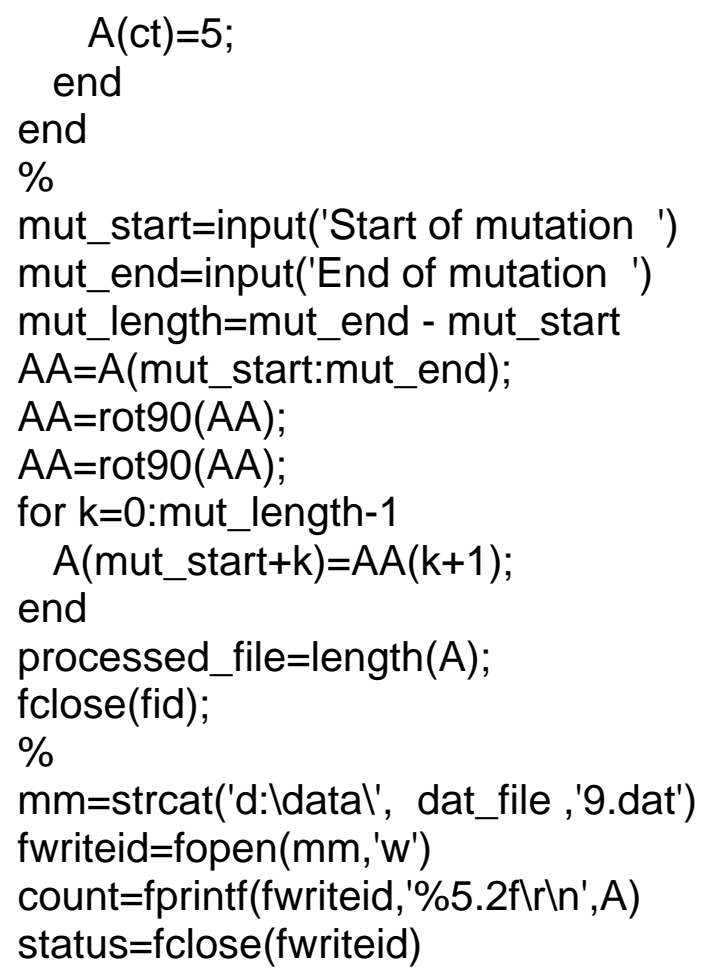

